United States Department of Commerce Technology Administration

National Institute of Standards and Technology

NISTIR 3953

\title{
TABLES OF EXPERIMENTAL DATA USED FOR THE CORRELATION OF THE THERMOPHYSICAL PROPERTIES OF ETHANE
}

Daniel G. Friend James F. Ely Hepburn Ingham

QC

100

.456

\#3953

1993 



\section{TABLES OF EXPERIMENTAL DATA USED FOR THE CORRELATION OF THE THERMOPHYSICAL PROPERTIES OF ETHANE}

Daniel G. Friend

James F. Ely

Hepburn Ingham

Thermophysics Division

Chemical Science and Technology Laboratory National Institute of Standards and Technology

Boulder, Colorado 80303-3328

January 1993

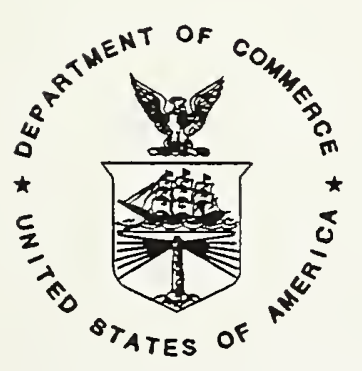

U.S. DEPARTMENT OF COMMERCE, Barbara Hackman Franklin, Secretary TECHNOLOGY ADMINISTRATION, Robert M. White, Under Secretary for Technology NATIONAL INSTITUTE OF STANDARDS AND TECHNOLOGY, John W. Lyons, Director 

1. Introduction . . . . . . . . . . . . . . . . . . . . . 2

2. Two-Phase Boundary . . . . . . . . . . . . . . . . . 3

3. Ideal Gas properties . . . . . . . . . . . . . . . . . . 46 46

4. Thermodynamic Properties in the Single-Phase Region . . . . . . 55

5. Dilute Gas Transport Properties . . . . . . . . . . . . . . . . 183

6. Transport Properties at Elevated Pressures . . . . . . . . . . 198

7. References ........................ . 296 


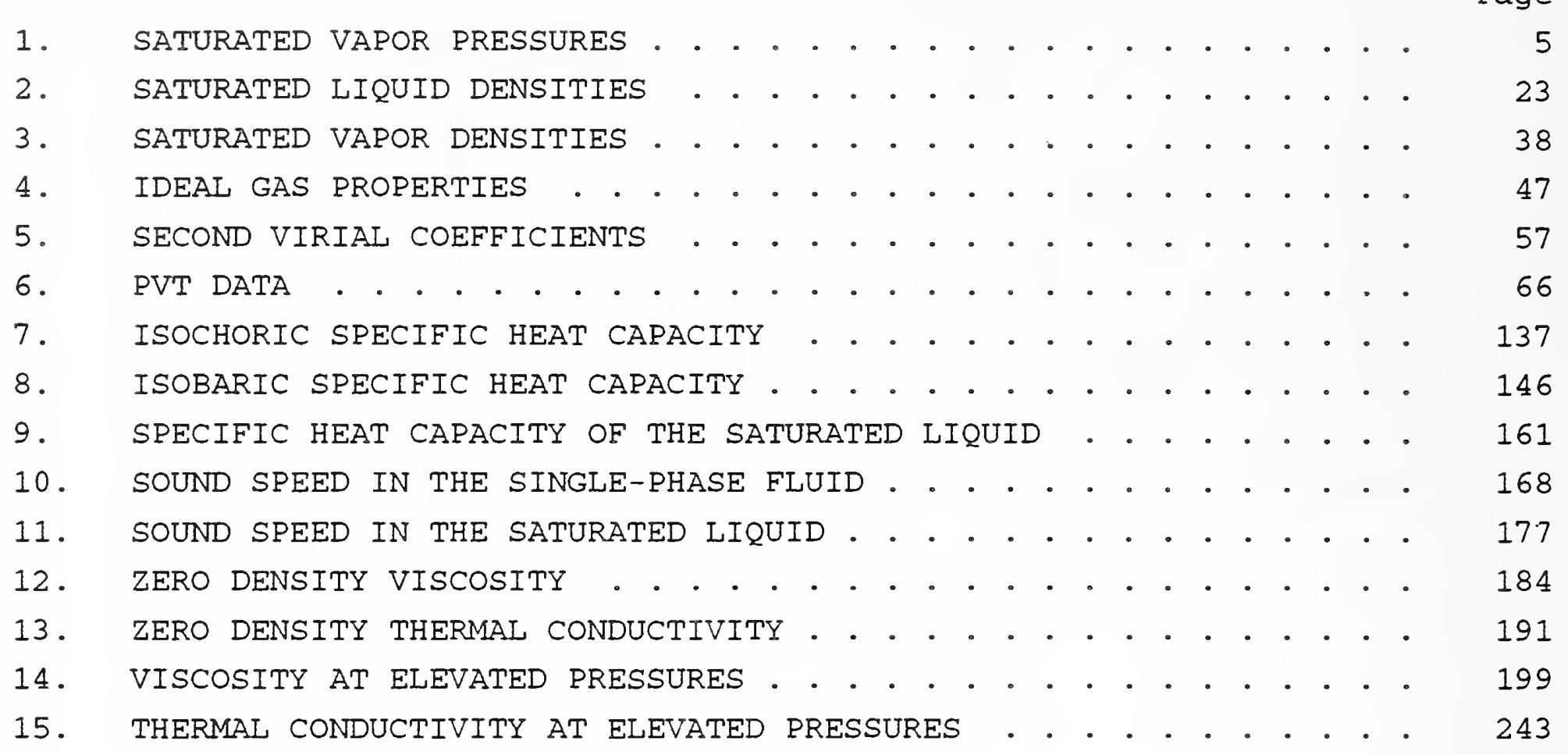




\section{Tables of Experimental Data \\ Used for the Correlation \\ of the Thermophysical Properties of Ethane}

Daniel G. Friend, James F. Ely, and Hepburn Ingham

Thermophysics Division

National Institute of Standards and Technology

Boulder, Colorado 80303

We tabulate experimental data for the thermophysical properties of ethane from an extensive selection of the published literature. This report provides a complete tabulation of the data on ethane properties which were used in the development of correlating equations for the fluid state properties. The tables give comparisons between the correlations and the data as well as the weight which was assigned to each point in the development of the correlations. The properties include pressure and densities of the saturated liquid and vapor, the PVT relationship in the single phase, isochoric and isobaric heat capacities, sound speed, viscosity, and thermal conductivity. The general range of the data is from the triple point, near $90.4 \mathrm{~K}$ and $1.1 \mathrm{MPa}$, to about $625 \mathrm{~K}$ with pressures to about $100 \mathrm{MPa}$.

Key words: density; equation of state; heat capacity; ethane; experimental data; phase boundary; pressure; speed of sound; tables; thermal conductivity; thermophysical properties; transport properties; virial coefficients; viscosity. 


\section{Introduction}

We have recently completed a study of the thermophysical properties of fluid ethane [1,2]. In [1], we described correlations for these properties, discussed their development, and provided a limited comparison between the equations and experimental data as well as short tables of properties. In a companion Technical Note [2], we included extensive graphical comparisons between experimental data and their correlating equations and more complete tables of the thermophysical properties of fluid ethane. This report provides a complete tabulation of the data on ethane properties which were considered in the study. We provide comparisons with the correlation for each point. In addition, the tables give the numerical relative weight for each point as used in the development of the correlations; both primary data, used to determine the final coefficients in the correlating equations, and secondary data are included in these tables.

With only a few exceptions, the experimental values for both independent and dependent variables reported in these tables were taken directly from the cited literature source. Those exceptions include conversion to SI units (and, in a few cases, to IPTS-68), correction of obvious typographical errors, and adjustments as described in the table notes. A few data from the cited sources were omitted because the correction for obvious typographical errors was not clear, they lay outside the range of state variables which we considered, or they lay inside the solid, metastable, or two-phase region according to our correlations. These tables include some, especially older, data which were not included in the statistical summaries or in the figures of [1] and [2].

We make several prefatory notes applicable to the tables in this report. Additional specific notes are in the following sections and at the head of some of the tables. In several instances, only first authors are given; complete citations can be found in the numbered reference. Deviations (Dev) are given on a percentage basis according to $\mathrm{dev}=100$ (calc - expt)/expt where expt is the experimental value and $c a l c$ is a quantity calculated from the appropriate correlation using the experimental value(s) of the independent variable(s). The weights (wt) refer to weighting of individual data points in the least squares 
algorithms for the determination of the coefficients of the correlating equations as discussed in detail in [1]. The summary statistics given for each reference and for the cumulative experimental data of each type are defined as follows:

$$
\begin{aligned}
A A D & =\frac{I}{N} \sum\left|c a l c_{i}-\exp t_{i}\right|, \\
B I A S & =\frac{1}{N} \sum\left(\operatorname{cal} c_{i}-\exp t_{i}\right),
\end{aligned}
$$

and

$$
R M S=\left[\frac{1}{N} \sum\left(\operatorname{cal} c_{i}-\exp t_{i}\right)^{2}-B I A S^{2}\right]^{1 / 2}
$$

The quantity $N$ above represents the number of points in the data set, and the summation over the index $i$ is over all $N$ points. These statistics give dimensioned quantities; the analogously defined dimensionless statistics are based on percentage deviations. Thus, AAD $\%$, BIAS $\%$, and RMS $\%$ are defined as above, but with the quantity (calc $c_{i}-e^{e x p t_{i}}$ ) replaced by 100 (calc $c_{i}-$ expt $\left.t_{i}\right) / e x p t_{i}$. Some of the entries in the tables have digits beyond the range of significance, including trailing zeroes; these serve only to maintain uniform appearance of the columns and the quantities can be truncated according to the error esitimates in the original experimental publications and the discussion in $[1]$.

We have not included the correlating equations and their coefficients in this work. We refer the interested reader to [1] and [2]. A similar study of the methane fluid was described in [3] and [4].

\section{Two-Phase Boundary}

The three tables in this section (1-3) give data for the pressure of the saturated fluid, the density of the saturated liquid, and the density of the saturated vapor. Comparisons are given for both the ancillary equations (anc.), eqs. (4-6) in [1], and for the saturation properties calculated from the full equation of state (Schmidt-Wagner equation of state or SWEOS) using the Maxwell 
construction. Comparisons and statistics based on the ancillary equations have the suffix 1, and those based on the SwEOS are denoted 2. The weights which are given were those used in the development of the ancillary equations. Points generated from the ancillary equations were used in the development of the SWEOS and are included in these tables but excluded from the summary statistics. Tables for the heat capacity of the liquid along the saturation boundary and the speed of sound in the saturated liquid are deferred to section 4. 
Table 1. SATURATED VAPOR PRESSURES

Data from Barkelew et al. [5]

\begin{tabular}{|c|c|c|c|c|c|c|}
\hline $\begin{array}{l}\mathrm{T} \\
\mathrm{K}\end{array}$ & $\begin{array}{l}\mathrm{P}_{\sigma}, \operatorname{expt} \\
\mathrm{MPa}\end{array}$ & $\begin{array}{c}\mathrm{P}_{\sigma} \text {, anc. } \\
\mathrm{MPa}\end{array}$ & $\begin{array}{c}\operatorname{dev} 1 \\
8\end{array}$ & $\begin{array}{c}\mathrm{P}_{\sigma}, \underset{\mathrm{MPa}}{\mathrm{SWEOS}} \\
\mathrm{MPa}\end{array}$ & $\begin{array}{c}\text { dev2 } \\
8\end{array}$ & wt \\
\hline 110.000 & $0.7701 \mathrm{E}-04$ & $0.7469 E-04$ & -3.012 & $0.7468 E-04$ & -3.020 & 0.0 \\
\hline 120.000 & $0.3506 E-03$ & $0.3546 E-03$ & 1.141 & $0.3546 E-03$ & 1.155 & 0.0 \\
\hline 130.000 & $0.1289 \mathrm{E}-02$ & $0.1291 E-02$ & 0.204 & $0.1292 \mathrm{E}-02$ & 0.219 & 0.0 \\
\hline 140.000 & $0.3835 E-02$ & $0.3831 E-02$ & -0.103 & $0.3831 E-02$ & -0.096 & 0.0 \\
\hline 150.000 & $0.9687 \mathrm{E}-02$ & $0.9670 \mathrm{E}-02$ & -0.172 & $0.9670 \mathrm{E}-02$ & -0.174 & 0.0 \\
\hline 160.000 & $0.2148 \mathrm{E}-01$ & $0.2145 E-01$ & -0.126 & $0.2145 E-01$ & -0.133 & 0.0 \\
\hline 170.000 & $0.4292 E-01$ & $0.4288 E-01$ & -0.087 & $0.4288 E-01$ & -0.093 & 0.0 \\
\hline 180.000 & $0.7881 \mathrm{E}-01$ & $0.7872 E-01$ & -0.116 & $0.7872 E-01$ & -0.117 & 0.0 \\
\hline 190.000 & $0.1348 \mathrm{E}+00$ & $0.1347 E+00$ & -0.054 & $0.1347 E+00$ & -0.049 & 0.0 \\
\hline 200.000 & $0.2175 \mathrm{E}+00$ & $0.2174 E+00$ & -0.049 & $0.2174 E+00$ & -0.039 & 0.0 \\
\hline 210.000 & $0.3341 E+00$ & $0.3340 \mathrm{E}+00$ & -0.032 & $0.3340 E+00$ & -0.019 & 0.0 \\
\hline 220.000 & $0.4922 \mathrm{E}+00$ & $0.4923 E+00$ & 0.007 & $0.4923 E+00$ & 0.022 & 0.0 \\
\hline 230.000 & $0.7004 \mathrm{E}+00$ & $0.7005 E+00$ & 0.017 & $0.7006 \mathrm{E}+00$ & 0.034 & 0.0 \\
\hline 240.000 & $0.9678 \mathrm{E}+00$ & $0.9671 E+00$ & -0.064 & $0.9673 E+00$ & -0.045 & 0.0 \\
\hline 250.000 & $0.1302 \mathrm{E}+01$ & $0.1301 \mathrm{E}+01$ & -0.066 & $0.1301 E+01$ & -0.043 & 0.0 \\
\hline 260.000 & $0.1713 E+01$ & $0.1712 E+01$ & -0.084 & $0.1712 \mathrm{E}+01$ & -0.053 & 0.0 \\
\hline 270.000 & $0.2209 E+01$ & $0.2210 \mathrm{E}+01$ & 0.035 & $0.2211 E+01$ & 0.083 & 0.0 \\
\hline 280.000 & $0.2802 E+01$ & $0.2806 E+01$ & 0.147 & $0.2808 \mathrm{E}+01$ & 0.211 & 0.0 \\
\hline 290.000 & $0.3511 E+01$ & $0.3514 \mathrm{E}+01$ & 0.100 & $0.3517 E+01$ & 0.165 & 0.0 \\
\hline 300.000 & $0.4366 \mathrm{E}+01$ & $0.4356 \mathrm{E}+01$ & -0.230 & $0.4357 \mathrm{E}+01$ & -0.219 & 0.0 \\
\hline
\end{tabular}

Number of Points [5] 20

$\begin{array}{llll}\mathrm{AAD} \% & =0.292 & \text { BIAS } 8=-0.127 & \mathrm{RMS} z=0.717 \\ \mathrm{AAD} 2 \%=0.300 & \text { BIAS2r }=-0.111 & \mathrm{RMS} 2 \%=0.724\end{array}$

Absolute Deviations:

$\begin{array}{lrllll}\mathrm{AAD} & =1.101 & \mathrm{BIAS} & =-0.242 & \mathrm{RMS} & =2.578 \mathrm{kPa} \\ \mathrm{AAD} 2 & =1.289 & \mathrm{BIAS} 2 & =0.099 & \mathrm{RMS} 2 & =2.870 \mathrm{kPa}\end{array}$

Data from Carruth and Kobayashi [6]

\begin{tabular}{ccccccc}
\hline $\mathrm{T}$ & $\mathrm{P}_{\sigma}$, expt & $\mathrm{P}_{\sigma}$, anc. & dev1 \\
$\mathrm{KPa}$ & $\mathrm{MPa}$ & $\mathrm{P}_{\sigma}, \begin{array}{c}\text { SWEOS } \\
\mathrm{MPa}\end{array}$ & $\begin{array}{c}\text { dev2 } \\
\text { s }\end{array}$ & wt \\
\hline 91.340 & $0.1543 \mathrm{E}-05$ & $0.1463 \mathrm{E}-05$ & -5.173 & $0.1464 \mathrm{E}-05$ & -5.135 & 0.0 \\
93.700 & $0.2733 \mathrm{E}-05$ & $0.2647 \mathrm{E}-05$ & -3.143 & $0.2647 \mathrm{E}-05$ & -3.149 & 0.0 \\
96.240 & $0.4977 \mathrm{E}-05$ & $0.4835 \mathrm{E}-05$ & -2.836 & $0.4834 \mathrm{E}-05$ & -2.867 & 0.0 \\
100.700 & $0.1313 \mathrm{E}-04$ & $0.1285 \mathrm{E}-04$ & -2.118 & $0.1285 \mathrm{E}-04$ & -2.154 & 0.0 \\
105.600 & $0.3304 \mathrm{E}-04$ & $0.3390 \mathrm{E}-04$ & 2.598 & $0.3389 \mathrm{E}-04$ & 2.574 & 0.0
\end{tabular}


Table 1. SATURATED VAPOR PRESSURES (continued)

Data from Carruth and Kobayashi [6] (continued)

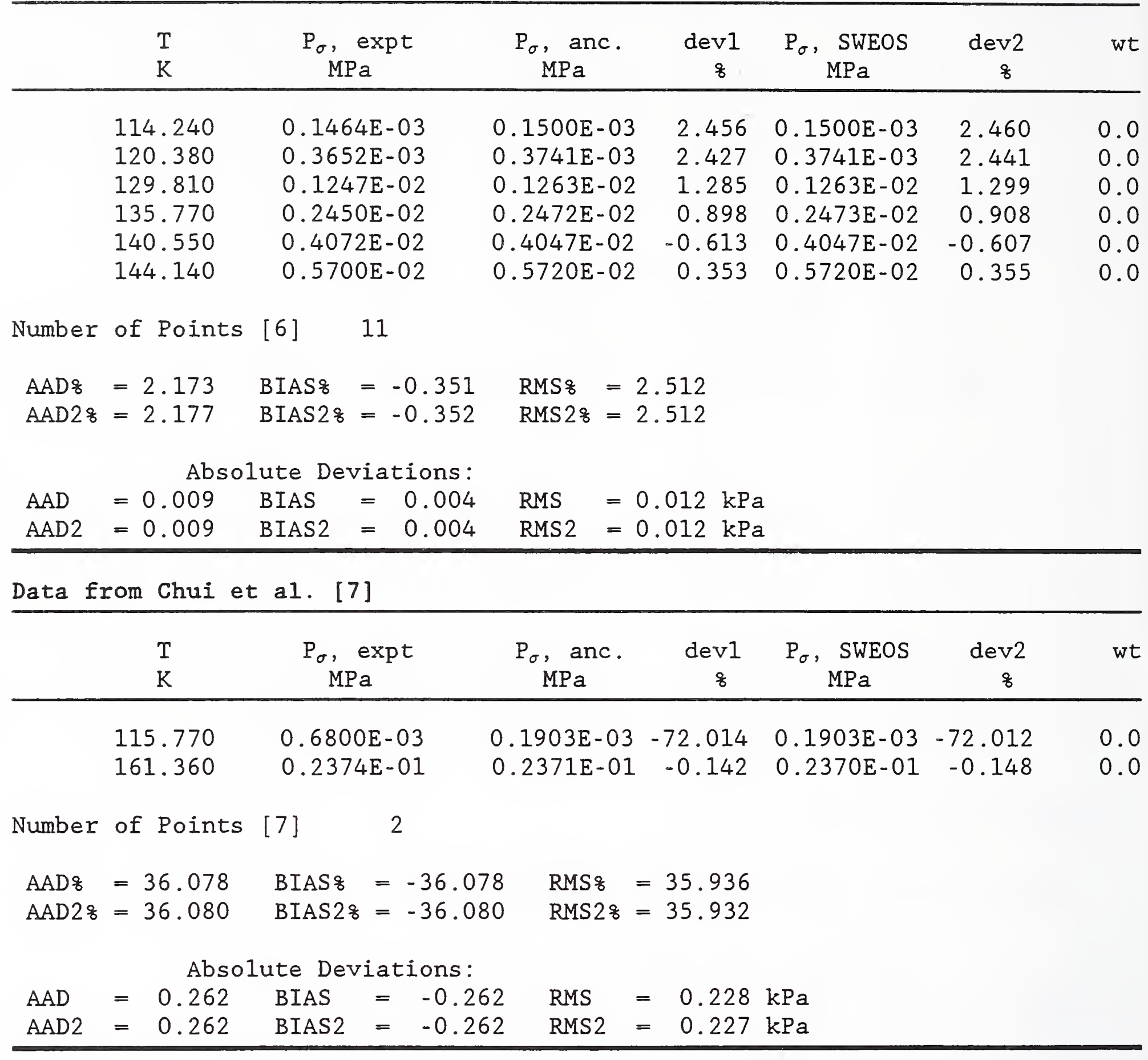


Table 1. SATURATED VAPOR PRESSURES (continued)

Data from Djordjevich and Budenholzer [8]

\begin{tabular}{|c|c|c|c|c|c|c|}
\hline $\begin{array}{l}\mathrm{T} \\
\mathrm{K}\end{array}$ & $\begin{array}{l}\mathrm{P}_{\sigma} \text {, expt } \\
\mathrm{MPa}\end{array}$ & $\begin{array}{c}\mathrm{P}_{\sigma} \text {, anc. } \\
\mathrm{MPa}\end{array}$ & $\begin{array}{c}\text { dev1 } \\
\frac{8}{8}\end{array}$ & $\begin{array}{cl}\mathrm{P}_{\sigma}, & \text { SWEOS } \\
\text { MPA }\end{array}$ & $\begin{array}{r}\operatorname{dev} 2 \\
\frac{9}{8}\end{array}$ & wt \\
\hline 255.370 & $0.1503 E+01$ & $0.1512 \mathrm{E}+01$ & 0.567 & $0.1512 \mathrm{E}+01$ & 0.594 & 0.0 \\
\hline 227.590 & $0.6405 E+00$ & $0.6453 E+00$ & 0.743 & $0.6454 \mathrm{E}+00$ & 0.759 & 0.0 \\
\hline 199.817 & $0.2161 E+00$ & $0.2156 \mathrm{E}+00$ & -0.241 & $0.2156 \mathrm{E}+00$ & -0.231 & 0.0 \\
\hline 172.039 & $0.4837 \mathrm{E}-01$ & $0.4885 \mathrm{E}-01$ & 0.995 & $0.4885 \mathrm{E}-01$ & 0.990 & 0.0 \\
\hline 144.261 & $0.5853 E-02$ & $0.5785 E-02$ & -1.156 & $0.5786 \mathrm{E}-02$ & -1.154 & 0.0 \\
\hline 127.594 & $0.1013 \mathrm{E}-02$ & $0.9661 E-03$ & -4.599 & $0.9662 E-03$ & -4.584 & 0.0 \\
\hline
\end{tabular}

Number of Points [8] 6

$$
\begin{array}{lll}
\mathrm{AAD} \%=1.384 & \text { BIAS } 8=-0.615 & \text { RMS } \%=1.921 \\
\mathrm{AAD} 2 \%=1.385 & \text { BIAS2\% }=-0.604 & \text { RMS2\% } 8=1.920
\end{array}
$$

Absolute Deviations:

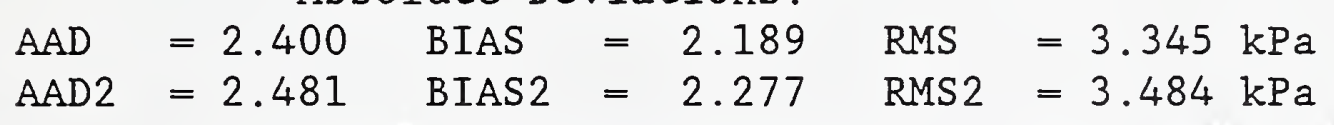

\begin{tabular}{|c|c|c|c|c|c|c|}
\hline $\begin{array}{l}\mathrm{T} \\
\mathrm{K}\end{array}$ & $\begin{array}{l}\mathrm{P}_{\sigma}, \operatorname{expt} \\
\mathrm{MPa}\end{array}$ & $\begin{array}{c}\mathrm{P}_{\sigma} \text {, anc. } \\
\mathrm{MPa}\end{array}$ & $\begin{array}{c}\operatorname{dev} 1 \\
8\end{array}$ & $\mathrm{P}_{\sigma}, \begin{array}{l}\text { SWEOS } \\
\text { MPA }\end{array}$ & $\begin{array}{r}\operatorname{dev} 2 \\
\frac{8}{8}\end{array}$ & wt \\
\hline 238.150 & $0.9129 E+00$ & $0.9130 E+00$ & 0.010 & $0.9132 E+00$ & 0.028 & 1.0 \\
\hline 243.150 & $0.1065 \mathrm{E}+01$ & $0.1065 \mathrm{E}+01$ & 0.010 & $0.1065 \mathrm{E}+01$ & 0.029 & 1.0 \\
\hline 248.150 & $0.1234 \mathrm{E}+01$ & $0.1234 \mathrm{E}+01$ & 0.015 & $0.1234 \mathrm{E}+01$ & 0.037 & 1.0 \\
\hline 253.150 & $0.1422 E+01$ & $0.1422 E+01$ & 0.010 & $0.1422 E+01$ & 0.035 & 1.0 \\
\hline 258.150 & $0.1630 \mathrm{E}+01$ & $0.1630 \mathrm{E}+01$ & 0.004 & $0.1630 \mathrm{E}+01$ & 0.033 & 1.0 \\
\hline 263.150 & $0.1859 \mathrm{E}+01$ & $0.1859 \mathrm{E}+01$ & -0.005 & $0.1860 \mathrm{E}+01$ & 0.031 & 1.0 \\
\hline 268.150 & $0.2111 E+01$ & $0.2111 \mathrm{E}+01$ & -0.011 & $0.2111 E+01$ & 0.033 & 1.0 \\
\hline 273.150 & $0.2387 \mathrm{E}+01$ & $0.2386 \mathrm{E}+01$ & -0.020 & $0.2387 E+01$ & 0.033 & 1.0 \\
\hline 278.150 & $0.2688 \mathrm{E}+01$ & $0.2687 E+01$ & -0.028 & $0.2689 E+01$ & 0.033 & 1.0 \\
\hline 283.150 & $0.3017 \mathrm{E}+01$ & $0.3016 \mathrm{E}+01$ & -0.033 & $0.3018 E+01$ & 0.033 & 1.0 \\
\hline 288.150 & $0.3375 \mathrm{E}+01$ & $0.3374 E+01$ & -0.035 & $0.3376 E+01$ & 0.031 & 1.0 \\
\hline 293.150 & $0.3765 \mathrm{E}+01$ & $0.3764 \mathrm{E}+01$ & -0.030 & $0.3766 \mathrm{E}+01$ & 0.025 & 1.0 \\
\hline 298.150 & $0.4190 \mathrm{E}+01$ & $0.4189 \mathrm{E}+01$ & -0.018 & $0.4190 E+01$ & 0.008 & 1.0 \\
\hline 302.150 & $0.4558 \mathrm{E}+01$ & $0.4558 \mathrm{E}+01$ & -0.004 & $0.4557 \mathrm{E}+01$ & -0.010 & 1.0 \\
\hline 303.150 & $0.4654 \mathrm{E}+01$ & $0.4654 \mathrm{E}+01$ & 0.000 & $0.4654 \mathrm{E}+01$ & -0.013 & 1.0 \\
\hline 304.150 & $0.4753 E+01$ & $0.4753 E+01$ & 0.003 & $0.4752 E+01$ & -0.011 & 1.0 \\
\hline 305.150 & $0.4853 \mathrm{E}+01$ & $0.4853 \mathrm{E}+01$ & 0.000 & $0.4853 E+01$ & -0.004 & 1.0 \\
\hline 305.250 & $0.4864 \mathrm{E}+01$ & $0.4864 E+01$ & 0.002 & $0.4863 E+01$ & -0.001 & 1.0 \\
\hline
\end{tabular}

Data from Douslin and Harrison [9] 
Table 1. SATURATED VAPOR PRESSURES (continued)

Data from Douslin and Harrison [9] (continued)

Number of Points [9] 18

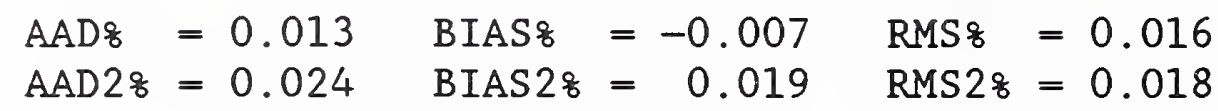

Absolute Deviations:

$\begin{array}{lrllll}\mathrm{AAD} & =0.369 & \mathrm{BIAS} & =-0.279 & \mathrm{RMS} & =0.464 \mathrm{kPa} \\ \mathrm{AAD} 2 & =0.567 & \text { BIAS2 } & =0.361 & \text { RMS2 } & =0.519 \mathrm{kPa}\end{array}$

Weighted Data:

Number of Points [9] 18

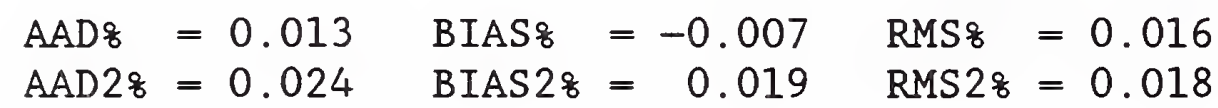

Absolute Deviations:

$\mathrm{AAD}=0.369$ BIAS $=-0.279$ RMS $=0.464 \mathrm{kPa}$

AAD2 $=0.567$ BIAS2 $=0.361$ RMS2 $=0.519 \mathrm{kPa}$

Data from Kahre [10]

\begin{tabular}{cccrcrrr}
\hline $\mathrm{T}$ & $\begin{array}{c}\mathrm{P}_{\sigma} \text {, expt } \\
\mathrm{MPa}\end{array}$ & $\begin{array}{c}\mathrm{P}_{\sigma}, \text { anc. } \\
\mathrm{MPa}\end{array}$ & $\begin{array}{c}\text { dev1 } \\
\text { \& }\end{array}$ & $\begin{array}{c}\mathrm{P}_{\sigma}, \text { SWEOS } \\
\mathrm{MPA}\end{array}$ & $\begin{array}{r}\text { dev2 } \\
\text { \& }\end{array}$ & wt \\
\hline 267.450 & $0.2079 \mathrm{E}+01$ & $0.2074 \mathrm{E}+01$ & -0.255 & $0.2075 \mathrm{E}+01$ & -0.211 & 0.0 \\
277.550 & $0.2641 \mathrm{E}+01$ & $0.2650 \mathrm{E}+01$ & 0.356 & $0.2652 \mathrm{E}+01$ & 0.416 & 0.0 \\
288.750 & $0.3395 \mathrm{E}+01$ & $0.3419 \mathrm{E}+01$ & 0.698 & $0.3421 \mathrm{E}+01$ & 0.764 & 0.0 \\
294.250 & $0.3833 \mathrm{E}+01$ & $0.3854 \mathrm{E}+01$ & 0.552 & $0.3856 \mathrm{E}+01$ & 0.603 & 0.0 \\
299.850 & $0.4302 \mathrm{E}+01$ & $0.4342 \mathrm{E}+01$ & 0.931 & $0.4343 \mathrm{E}+01$ & 0.942 & 0.0
\end{tabular}

Number of Points [10] 5

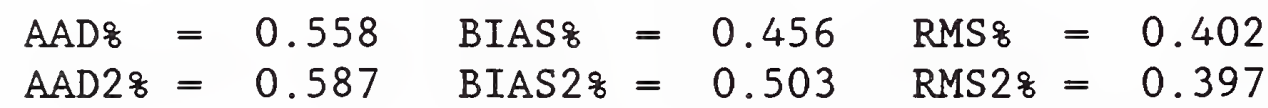

Absolute Deviations:

$\mathrm{AAD}=19.916$ BIAS $=17.798 \quad \mathrm{RMS}=15.129 \mathrm{kPa}$

$\mathrm{AAD} 2=20.998$ BIAS2 $=19.241$ RMS2 $=15.103 \mathrm{kPa}$ 
Table 1. SATURATED VAPOR PRESSURES (continued)

Data from Loomis and Walters [11]

\begin{tabular}{|c|c|c|c|c|c|c|}
\hline $\begin{array}{l}\mathrm{T} \\
\mathrm{K}\end{array}$ & $\begin{array}{l}\mathrm{P}_{\sigma}, \exp t \\
\mathrm{MPa}\end{array}$ & $\begin{array}{c}\mathrm{P}_{\sigma}, \text { anc. } \\
\mathrm{MPa}\end{array}$ & $\begin{array}{c}\operatorname{dev} 1 \\
8\end{array}$ & 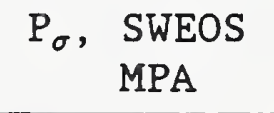 & $\begin{array}{r}\operatorname{dev} 2 \\
\frac{8}{8}\end{array}$ & wt \\
\hline 167.836 & $0.3742 \mathrm{E}-01$ & $0.3720 E-01$ & -0.574 & $0.3720 \mathrm{E}-01$ & -0.581 & 0.0 \\
\hline 165.629 & $0.3202 \mathrm{E}-01$ & $0.3205 \mathrm{E}-01$ & 0.094 & $0.3205 E-01$ & 0.087 & 0.0 \\
\hline 162.629 & $0.2607 \mathrm{E}-01$ & $0.2598 \mathrm{E}-01$ & -0.359 & $0.2598 \mathrm{E}-01$ & -0.366 & 0.0 \\
\hline 158.385 & $0.1911 \mathrm{E}-01$ & $0.1901 E-01$ & -0.536 & $0.1901 \mathrm{E}-01$ & -0.542 & 0.0 \\
\hline 154.546 & $0.1419 \mathrm{E}-01$ & $0.1409 \mathrm{E}-01$ & -0.656 & $0.1409 \mathrm{E}-01$ & -0.661 & 0.0 \\
\hline 147.324 & $0.7691 \mathrm{E}-02$ & $0.7653 E-02$ & -0.488 & $0.7653 \mathrm{E}-02$ & -0.489 & 0.0 \\
\hline 143.267 & $0.5289 \mathrm{E}-02$ & $0.5268 \mathrm{E}-02$ & -0.400 & $0.5268 \mathrm{E}-02$ & -0.397 & 0.0 \\
\hline 135.736 & $0.2482 \mathrm{E}-02$ & $0.2463 E-02$ & -0.771 & $0.2464 E-02$ & -0.761 & 0.0 \\
\hline 169.175 & $0.4086 \mathrm{E}-01$ & $0.4064 \mathrm{E}-01$ & -0.543 & $0.4064 \mathrm{E}-01$ & -0.549 & 0.0 \\
\hline 171.700 & $0.4806 \mathrm{E}-01$ & $0.4781 E-01$ & -0.510 & $0.4781 E-01$ & -0.515 & 0.0 \\
\hline 170.602 & $0.4489 \mathrm{E}-01$ & $0.4458 \mathrm{E}-01$ & -0.682 & $0.4458 \mathrm{E}-01$ & -0.687 & 0.0 \\
\hline 174.062 & $0.5571 \mathrm{E}-01$ & $0.5540 \mathrm{E}-01$ & -0.555 & $0.5540 \mathrm{E}-01$ & -0.559 & 0.0 \\
\hline 175.708 & $0.6153 \mathrm{E}-01$ & $0.6123 \mathrm{E}-01$ & -0.499 & $0.6123 \mathrm{E}-01$ & -0.503 & 0.0 \\
\hline 177.623 & $0.6894 \mathrm{E}-01$ & $0.6861 E-01$ & -0.487 & $0.6860 \mathrm{E}-01$ & -0.489 & 0.0 \\
\hline 178.621 & $0.7306 \mathrm{E}-01$ & $0.7272 \mathrm{E}-01$ & -0.460 & $0.7272 \mathrm{E}-01$ & -0.462 & 0.0 \\
\hline 179.750 & $0.7798 \mathrm{E}-01$ & $0.7760 \mathrm{E}-01$ & -0.482 & $0.7760 \mathrm{E}-01$ & -0.484 & 0.0 \\
\hline 181.506 & $0.8612 \mathrm{E}-01$ & $0.8571 E-01$ & -0.474 & $0.8571 \mathrm{E}-01$ & -0.474 & 0.0 \\
\hline 182.463 & $0.9082 \mathrm{E}-01$ & $0.9040 \mathrm{E}-01$ & -0.465 & $0.9040 \mathrm{E}-01$ & -0.464 & 0.0 \\
\hline 183.778 & $0.9762 \mathrm{E}-01$ & $0.9716 \mathrm{E}-01$ & -0.467 & $0.9716 \mathrm{E}-01$ & -0.466 & 0.0 \\
\hline 184.539 & $0.1017 \mathrm{E}+00$ & $0.1013 E+00$ & -0.470 & $0.1013 \mathrm{E}+00$ & -0.468 & 0.0 \\
\hline 185.137 & $0.1050 \mathrm{E}+00$ & $0.1046 \mathrm{E}+00$ & -0.450 & $0.1046 \mathrm{E}+00$ & -0.448 & 0.0 \\
\hline 185.914 & $0.1094 \mathrm{E}+00$ & $0.1090 \mathrm{E}+00$ & -0.409 & $0.1090 E+00$ & -0.407 & 0.0 \\
\hline 186.609 & $0.1136 \mathrm{E}+00$ & $0.1131 E+00$ & -0.443 & $0.1131 \mathrm{E}+00$ & -0.440 & 0.0 \\
\hline 187.302 & $0.1177 \mathrm{E}+00$ & $0.1172 E+00$ & -0.411 & $0.1172 \mathrm{E}+00$ & -0.408 & 0.0 \\
\hline 187.726 & $0.1204 \mathrm{E}+00$ & $0.1199 \mathrm{E}+00$ & -0.432 & $0.1199 \mathrm{E}+00$ & -0.428 & 0.0 \\
\hline 188.379 & $0.1245 \mathrm{E}+00$ & $0.1240 \mathrm{E}+00$ & -0.428 & $0.1240 \mathrm{E}+00$ & -0.424 & 0.0 \\
\hline 189.114 & $0.1293 E+00$ & $0.1288 E+00$ & -0.392 & $0.1288 E+00$ & -0.387 & 0.0 \\
\hline 189.858 & $0.1342 E+00$ & $0.1337 \mathrm{E}+00$ & -0.381 & $0.1337 E+00$ & -0.376 & 0.0 \\
\hline 190.791 & $0.1407 \mathrm{E}+00$ & $0.1402 E+00$ & -0.373 & $0.1402 E+00$ & -0.368 & 0.0 \\
\hline 191.430 & $0.1452 \mathrm{E}+00$ & $0.1447 \mathrm{E}+00$ & -0.362 & $0.1447 \mathrm{E}+00$ & -0.357 & 0.0 \\
\hline 192.286 & $0.1515 \mathrm{E}+00$ & $0.1510 \mathrm{E}+00$ & -0.349 & $0.1510 \mathrm{E}+00$ & -0.343 & 0.0 \\
\hline 192.777 & $0.1552 \mathrm{E}+00$ & $0.1547 \mathrm{E}+00$ & -0.347 & $0.1547 E+00$ & -0.341 & 0.0 \\
\hline 199.909 & $0.2170 E+00$ & $0.2165 \mathrm{E}+00$ & -0.251 & $0.2165 \mathrm{E}+00$ & -0.241 & 0.0 \\
\hline 196.244 & $0.1833 \mathrm{E}+00$ & $0.1827 \mathrm{E}+00$ & -0.299 & $0.1827 E+00$ & -0.290 & 0.0 \\
\hline
\end{tabular}


Table 1. SATURATED VAPOR PRESSURES (continued)

Data from Loomis and Walters [11] (continued)

Number of Points [11] 34

$$
\begin{aligned}
& \mathrm{AAD} z=0.450 \quad \text { BIAS } 8=-0.444 \quad \text { RMS } 8=0.140 \\
& \mathrm{AAD} 28=0.449 \quad \text { BIAS } 28=-0.444 \quad \text { RMS } 28=0.141
\end{aligned}
$$

Absolute Deviations:

$\begin{array}{llllll}\mathrm{AAD} & =0.353 & \mathrm{BIAS} & =-0.351 & \mathrm{RMS} & =0.182 \mathrm{kPa} \\ \mathrm{AAD} 2 & =0.350 & \text { BIAS2 } & =-0.349 & \text { RMS2 } & =0.179 \mathrm{kPa}\end{array}$

$\mathrm{AAD} 2=0.350 \quad \mathrm{BIAS} 2=-0.349 \quad$ RMS2 $=0.179 \mathrm{kPa}$

\begin{tabular}{|c|c|c|c|c|c|c|}
\hline $\begin{array}{l}\mathrm{T} \\
\mathrm{K}\end{array}$ & $\begin{array}{c}\mathrm{P}_{\sigma}, \operatorname{expt} \\
\mathrm{MPa}\end{array}$ & $\begin{array}{c}\mathrm{P}_{\sigma}, \text { anc. } \\
\mathrm{MPa}\end{array}$ & $\begin{array}{c}\operatorname{dev} 1 \\
8\end{array}$ & $\begin{array}{ll}\mathrm{P}_{\sigma}, & \text { SWEOS } \\
\text { MPA }\end{array}$ & $\begin{array}{r}\operatorname{dev} 2 \\
8\end{array}$ & wt \\
\hline 302.988 & $0.4641 E+01$ & $0.4638 E+01$ & -0.060 & $0.4638 \mathrm{E}+01$ & -0.072 & 0.0 \\
\hline 303.880 & $0.4727 E+01$ & $0.4726 E+01$ & -0.031 & $0.4725 E+01$ & -0.045 & 0.0 \\
\hline 304.568 & $0.4796 E+01$ & $0.4795 E+01$ & -0.032 & $0.4794 E+01$ & -0.044 & 0.0 \\
\hline 305.025 & $0.4842 E+01$ & $0.4841 E+01$ & -0.032 & $0.4840 E+01$ & -0.039 & 0.0 \\
\hline 305.262 & $0.4866 \mathrm{E}+01$ & $0.4865 E+01$ & -0.033 & $0.4865 E+01$ & -0.035 & 0.0 \\
\hline 305.325 & $0.4872 E+01$ & $0.4871 E+01$ & -0.012 & $0.4871 E+01$ & -0.013 & 0.0 \\
\hline 305.260 & $0.4866 E+01$ & $0.4865 E+01$ & -0.019 & $0.4865 E+01$ & -0.021 & 0.0 \\
\hline 305.035 & $0.4842 E+01$ & $0.4842 E+01$ & -0.003 & $0.4841 E+01$ & -0.010 & 0.0 \\
\hline 304.663 & $0.4802 \mathrm{E}+01$ & $0.4804 E+01$ & 0.046 & $0.4804 \mathrm{E}+01$ & 0.035 & 0.0 \\
\hline 303.937 & $0.4732 E+01$ & $0.4732 E+01$ & -0.011 & $0.4731 E+01$ & -0.025 & 0.0 \\
\hline 302.872 & $0.4628 E+01$ & $0.4627 E+01$ & -0.023 & $0.4627 E+01$ & -0.035 & 0.0 \\
\hline
\end{tabular}

Data from Miniovich and Sorina [12]

Number of Points [12] 11

$$
\begin{aligned}
& \mathrm{AAD} \frac{8}{8}=0.028 \quad \text { BIAS\& }=-0.019 \quad \mathrm{RMS} \%=0.025 \\
& \mathrm{AAD} 2 \%=0.034 \quad \text { BIAS2 } 8=-0.028 \quad \text { RMS } 28=0.026
\end{aligned}
$$

Absolute Deviations:

\begin{tabular}{|c|c|c|c|c|c|c|}
\hline $\begin{array}{l}\mathrm{T} \\
\mathrm{K}\end{array}$ & $\begin{array}{l}\mathrm{P}_{\sigma}, \exp t \\
\mathrm{MPa}\end{array}$ & $\begin{array}{c}\mathrm{P}_{\sigma} \text {, anc. } \\
\mathrm{MPa}\end{array}$ & $\begin{array}{c}\operatorname{dev} 1 \\
8\end{array}$ & $\mathrm{P}_{\sigma}, \begin{array}{l}\text { SWEOS } \\
\text { MPA }\end{array}$ & $\begin{array}{r}\text { dev2 } \\
8\end{array}$ & wt \\
\hline 224.102 & $0.5711 E+00$ & $0.5712 E+00$ & 0.004 & $0.5713 E+00$ & 0.020 & 1.0 \\
\hline 229.756 & $0.6948 E+00$ & $0.6947 E+00$ & -0.006 & $0.6949 E+00$ & 0.011 & 1.0 \\
\hline 234.558 & $0.8140 E+00$ & $0.8142 E+00$ & 0.031 & $0.8144 E+00$ & 0.048 & 1.0 \\
\hline 239.844 & $0.9622 E+00$ & $0.9625 E+00$ & 0.032 & $0.9627 E+00$ & 0.050 & 1.0 \\
\hline
\end{tabular}

$\mathrm{AAD}=1.312 \quad \mathrm{BIAS}=-0.908 \quad \mathrm{RMS}=1.198 \mathrm{kPa}$

$A$ AD2 $=1.621$ BIAS2 $=-1.316$ RMS2 $=1.213 \mathrm{kPa}$

Data from $\mathrm{Pal}$ et al. [13] 
Table 1. SATURATED VAPOR PRESSURES (continued)

Data from Pal et al. [13] (continued)

\begin{tabular}{|c|c|c|c|c|c|c|}
\hline $\begin{array}{l}\mathrm{T} \\
\mathrm{K}\end{array}$ & $\mathrm{P}_{\sigma}$, expt & $\begin{array}{c}\mathrm{P}_{\sigma}, \underset{\mathrm{MPa}}{\text { anc. }} . \\
.\end{array}$ & $\begin{array}{c}\text { dev1 } \\
\frac{8}{8}\end{array}$ & 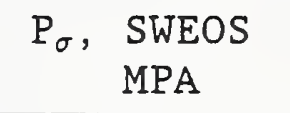 & $\begin{array}{r}\text { dev2 } \\
8\end{array}$ & wt \\
\hline 240.514 & $0.9824 E+00$ & $0.9826 \mathrm{E}+00$ & 0.014 & $0.9828 E+00$ & 0.033 & 1.0 \\
\hline 243.359 & $0.1072 E+01$ & $0.1071 E+01$ & -0.023 & $0.1072 E+01$ & -0.004 & 1.0 \\
\hline 246.814 & $0.1187 \mathrm{E}+01$ & $0.1187 \mathrm{E}+01$ & -0.001 & $0.1187 E+01$ & 0.020 & 1.0 \\
\hline 247.816 & $0.1221 E+01$ & $0.1222 E+01$ & 0.083 & $0.1222 E+01$ & 0.104 & 1.0 \\
\hline 249.741 & $0.1293 E+01$ & $0.1292 \mathrm{E}+01$ & -0.117 & $0.1292 E+01$ & -0.094 & 1.0 \\
\hline 250.146 & $0.1307 \mathrm{E}+01$ & $0.1307 \mathrm{E}+01$ & -0.027 & $0.1307 \mathrm{E}+01$ & -0.004 & 1.0 \\
\hline 251.587 & $0.1362 \mathrm{E}+01$ & $0.1361 E+01$ & -0.080 & $0.1361 E+01$ & -0.056 & 1.0 \\
\hline 252.544 & $0.1399 \mathrm{E}+01$ & $0.1398 \mathrm{E}+01$ & -0.067 & $0.1398 E+01$ & -0.043 & 1.0 \\
\hline 254.290 & $0.1468 \mathrm{E}+01$ & $0.1467 \mathrm{E}+01$ & -0.051 & $0.1468 E+01$ & -0.025 & 1.0 \\
\hline 257.543 & $0.1603 E+01$ & $0.1603 E+01$ & -0.007 & $0.1604 \mathrm{E}+01$ & 0.022 & 1.0 \\
\hline 263.380 & $0.1870 \mathrm{E}+01$ & $0.1870 \mathrm{E}+01$ & 0.002 & $0.1871 E+01$ & 0.039 & 1.0 \\
\hline 267.536 & $0.2079 E+01$ & $0.2078 E+01$ & -0.038 & $0.2079 E+01$ & 0.006 & 1.0 \\
\hline 271.749 & $0.2307 E+01$ & $0.2306 \mathrm{E}+01$ & -0.013 & $0.2308 E+01$ & 0.037 & 1.0 \\
\hline 275.922 & $0.2549 E+01$ & $0.2550 E+01$ & 0.032 & $0.2551 E+01$ & 0.089 & 1.0 \\
\hline 276.363 & $0.2579 E+01$ & $0.2577 E+01$ & -0.099 & $0.2578 E+01$ & -0.040 & 1.0 \\
\hline 276.385 & $0.2579 E+01$ & $0.2578 \mathrm{E}+01$ & -0.020 & $0.2580 E+01$ & 0.038 & 1.0 \\
\hline 276.514 & $0.2589 E+01$ & $0.2586 E+01$ & -0.100 & $0.2588 E+01$ & -0.041 & 1.0 \\
\hline 277.813 & $0.2667 \mathrm{E}+01$ & $0.2666 E+01$ & -0.014 & $0.2668 \mathrm{E}+01$ & 0.046 & 1.0 \\
\hline 280.041 & $0.2807 E+01$ & $0.2808 E+01$ & 0.048 & $0.2810 \mathrm{E}+01$ & 0.111 & 1.0 \\
\hline 282.247 & $0.2954 E+01$ & $0.2955 E+01$ & 0.021 & $0.2957 \mathrm{E}+01$ & 0.087 & 1.0 \\
\hline 284.635 & $0.3117 \mathrm{E}+01$ & $0.3119 \mathrm{E}+01$ & 0.058 & $0.3121 E+01$ & 0.126 & 1.0 \\
\hline 287.653 & $0.3337 E+01$ & $0.3337 \mathrm{E}+01$ & 0.017 & $0.3339 E+01$ & 0.084 & 1.0 \\
\hline 288.263 & $0.3383 E+01$ & $0.3382 E+01$ & -0.022 & $0.3385 E+01$ & 0.045 & 1.0 \\
\hline 290.040 & $0.3515 \mathrm{E}+01$ & $0.3518 \mathrm{E}+01$ & 0.080 & $0.3520 E+01$ & 0.145 & 1.0 \\
\hline 292.236 & $0.3693 E+01$ & $0.3690 \mathrm{E}+01$ & -0.069 & $0.3692 \mathrm{E}+01$ & -0.011 & 1.0 \\
\hline 293.098 & $0.3757 \mathrm{E}+01$ & $0.3760 \mathrm{E}+01$ & 0.064 & $0.3762 \mathrm{E}+01$ & 0.120 & 1.0 \\
\hline 296.347 & $0.4029 E+01$ & $0.4031 E+01$ & 0.068 & $0.4033 E+01$ & 0.107 & 1.0 \\
\hline 299.665 & $0.4322 E+01$ & $0.4325 E+01$ & 0.080 & $0.4326 \mathrm{E}+01$ & 0.093 & 1.0 \\
\hline 300.205 & $0.4374 E+01$ & $0.4375 E+01$ & 0.028 & $0.4375 \mathrm{E}+01$ & 0.037 & 1.0 \\
\hline 301.251 & $0.4469 E+01$ & $0.4472 E+01$ & 0.067 & $0.4472 E+01$ & 0.067 & 1.0 \\
\hline 303.471 & $0.4682 E+01$ & $0.4686 \mathrm{E}+01$ & 0.085 & $0.4685 \mathrm{E}+01$ & 0.071 & 1.0 \\
\hline 303.477 & $0.4689 E+01$ & $0.4686 \mathrm{E}+01$ & -0.068 & $0.4685 E+01$ & -0.082 & 1.0 \\
\hline 304.049 & $0.4739 E+01$ & $0.4743 E+01$ & 0.078 & $0.4742 E+01$ & 0.064 & 1.0 \\
\hline 304.360 & $0.4772 \mathrm{E}+01$ & $0.4774 E+01$ & 0.040 & $0.4773 E+01$ & 0.027 & 1.0 \\
\hline 304.446 & $0.4785 \mathrm{E}+01$ & $0.4782 E+01$ & -0.048 & $0.4782 \mathrm{E}+01$ & -0.061 & 1.0 \\
\hline 304.519 & $0.4783 \mathrm{E}+01$ & $0.4790 \mathrm{E}+01$ & 0.142 & $0.4789 \mathrm{E}+01$ & 0.129 & 1.0 \\
\hline
\end{tabular}


Table 1. SATURATED VAPOR PRESSURES (continued)

Data from Pal et al. [13] (continued)

\begin{tabular}{|c|c|c|c|c|c|c|}
\hline $\begin{array}{l}\mathrm{T} \\
\mathrm{K}\end{array}$ & $\begin{array}{c}\mathrm{P}_{\sigma}, \operatorname{expt} \\
\mathrm{MPa}\end{array}$ & $\begin{array}{c}\mathrm{P}_{\sigma}, \text { anc. } \\
\mathrm{MPa}\end{array}$ & $\begin{array}{c}\operatorname{dev} 1 \\
\frac{8}{8}\end{array}$ & 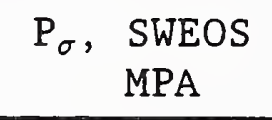 & $\begin{array}{r}\operatorname{dev} 2 \\
8\end{array}$ & wt \\
\hline 304.734 & $0.4806 E+01$ & $0.4811 E+01$ & 0.110 & $0.4811 E+01$ & 0.099 & 1.0 \\
\hline 304.796 & $0.4815 E+01$ & $0.4817 \mathrm{E}+01$ & 0.055 & $0.4817 \mathrm{E}+01$ & 0.045 & 1.0 \\
\hline 304.924 & $0.4832 E+01$ & $0.4830 E+01$ & -0.025 & $0.4830 E+01$ & -0.033 & 1.0 \\
\hline 304.980 & $0.4835 E+01$ & $0.4836 \mathrm{E}+01$ & 0.033 & $0.4836 E+01$ & 0.025 & 1.0 \\
\hline 305.121 & $0.4848 E+01$ & $0.4850 \mathrm{E}+01$ & 0.043 & $0.4850 E+01$ & 0.038 & 1.0 \\
\hline 305.135 & $0.4846 E+01$ & $0.4852 E+01$ & 0.124 & $0.4852 E+01$ & 0.119 & 1.0 \\
\hline 305.153 & $0.4852 E+01$ & $0.4854 \mathrm{E}+01$ & 0.045 & $0.4853 E+01$ & 0.041 & 1.0 \\
\hline 214.302 & $0.3973 E+00$ & $0.3965 E+00$ & -0.205 & $0.3965 E+00$ & -0.191 & 0.0 \\
\hline 221.101 & $0.5153 E+00$ & $0.5126 \mathrm{E}+00$ & -0.524 & $0.5127 E+00$ & -0.509 & 0.0 \\
\hline 300.443 & $0.4388 E+01$ & $0.4397 \mathrm{E}+01$ & 0.201 & $0.4397 E+01$ & 0.207 & 0.0 \\
\hline
\end{tabular}

Number of Points [13] 50

$$
\begin{aligned}
& \mathrm{AAD} \%=0.066 \quad \text { BIAS\& }=0.001 \quad \mathrm{RMS} \%=0.103 \\
& \mathrm{AAD} 2 \%=0.073 \quad \text { BIAS } 2 \%=0.025 \quad \text { RMS } 2 \%=0.103
\end{aligned}
$$

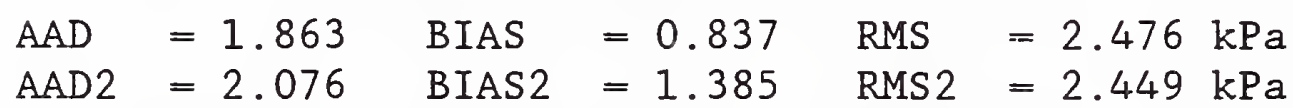

Weighted Data:

Number of Points [13] 47
$\begin{array}{lll}\text { AAD } 8=0.051 & \text { BIAS } 8=0.013 & \text { RMS } \%=0.060 \\ \text { AAD2\% }=0.058 & \text { BIAS2\% }=0.037 & \text { RMS } 2 \%=0.058\end{array}$

Absolute Deviations:

$\mathrm{AAD}=1.720 \quad \mathrm{BIAS}=0.778 \quad \mathrm{RMS}=2.201 \mathrm{kPa}$

\begin{tabular}{|c|c|c|c|c|c|c|}
\hline $\begin{array}{l}\mathrm{T} \\
\mathrm{K}\end{array}$ & $\begin{array}{l}\mathrm{P}_{\sigma}, \operatorname{expt} \\
\mathrm{MPa}\end{array}$ & $\begin{array}{c}\mathrm{P}_{\sigma}, \text { anc. } \\
\mathrm{MPa}\end{array}$ & $\begin{array}{c}\operatorname{dev} 1 \\
8\end{array}$ & $\begin{array}{ll}\mathrm{P}_{\sigma}, & \text { SWEOS } \\
\text { MPA }\end{array}$ & $\begin{array}{r}\text { dev2 } \\
8\end{array}$ & wt \\
\hline 198.181 & $0.2000 \mathrm{E}+00$ & $0.2000 \mathrm{E}+00$ & 0.015 & $0.2000 \mathrm{E}+00$ & 0.024 & 1.0 \\
\hline 234.692 & $0.8181 E+00$ & $0.8178 E+00$ & -0.040 & $0.8179 E+00$ & -0.023 & 1.0 \\
\hline 238.771 & $0.9306 E+00$ & $0.9309 E+00$ & 0.034 & $0.9311 E+00$ & 0.052 & 1.0 \\
\hline 272.949 & $0.2376 E+01$ & $0.2375 E+01$ & -0.066 & $0.2376 \mathrm{E}+01$ & -0.013 & 1.0 \\
\hline 284.845 & $0.3137 \mathrm{E}+01$ & $0.3134 \mathrm{E}+01$ & -0.081 & $0.3136 \mathrm{E}+01$ & -0.014 & 1.0 \\
\hline
\end{tabular}

$\mathrm{AAD} 2=1.943$ BIAS2 $=1.352$ RMS2 $=2.162 \mathrm{kPa}$

Data from Pope [14] 
Table 1. SATURATED VAPOR PRESSURES (continued)

Data from Pope [14] (continued)

\begin{tabular}{|c|c|c|c|c|c|c|}
\hline $\begin{array}{l}\mathrm{T} \\
\mathrm{K}\end{array}$ & $\begin{array}{l}\mathrm{P}_{\sigma} \text {, expt } \\
\mathrm{MPa}\end{array}$ & $\begin{array}{c}\mathrm{P}_{\sigma} \text {, anc. } \\
\mathrm{MPa}\end{array}$ & $\begin{array}{c}\operatorname{dev} 1 \\
8\end{array}$ & $\begin{array}{ll}\mathrm{P}_{\sigma}, & \text { SWEOS } \\
\text { MPA }\end{array}$ & $\begin{array}{r}\text { dev2 } \\
\frac{9}{\delta}\end{array}$ & wt \\
\hline 290.214 & $0.3534 \mathrm{E}+01$ & $0.3531 \mathrm{E}+01$ & -0.077 & $0.3533 E+01$ & -0.014 & 1.0 \\
\hline 293.266 & $0.3776 \mathrm{E}+01$ & $0.3773 E+01$ & -0.072 & $0.3775 \mathrm{E}+01$ & -0.017 & 1.0 \\
\hline 299.863 & $0.4345 \mathrm{E}+01$ & $0.4343 \mathrm{E}+01$ & -0.045 & $0.4344 \mathrm{E}+01$ & -0.034 & 1.0 \\
\hline 304.012 & $0.4739 E+01$ & $0.4739 \mathrm{E}+01$ & -0.008 & $0.4738 \mathrm{E}+01$ & -0.022 & 1.0 \\
\hline 254.807 & $0.1493 E+01$ & $0.1488 E+01$ & -0.284 & $0.1489 E+01$ & -0.258 & 0.0 \\
\hline 209.534 & $0.3259 E+00$ & $0.3277 E+00$ & 0.543 & $0.3277 \mathrm{E}+00$ & 0.557 & 0.0 \\
\hline
\end{tabular}

Number of Points [14] 11

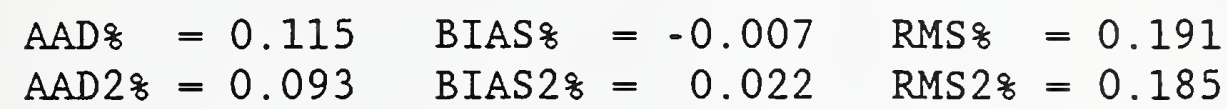

Absolute Deviations:

$\mathrm{AAD}=1.692 \quad \mathrm{BIAS}=-1.306$

RMS $=1.663 \mathrm{kPa}$

$\mathrm{AAD} 2=0.981$ BIAS2 $=-0.554$ RMS2 $=1.323 \mathrm{kPa}$

Weighted Data:

Number of Points [14] 9

$$
\begin{array}{lll}
\mathrm{AAD} \gamma=0.049 & \text { BIAS } 8=-0.038 & \text { RMS } 8=0.040 \\
\mathrm{AAD} 2 \%=0.024 & \text { BIAS2 } 8=-0.007 & \text { RMS2 } 8=0.026
\end{array}
$$

Absolute Deviations:

$\begin{array}{llllll}\mathrm{AAD} & =1.399 & \text { BIAS } & =-1.322 & \text { RMS } & =1.170 \mathrm{kPa} \\ \mathrm{AAD} 2 & =0.569 & \text { BIAS2 } & =-0.450 & \text { RMS2 } & =0.544 \mathrm{kPa}\end{array}$

\begin{tabular}{|c|c|c|c|c|c|c|}
\hline $\begin{array}{l}\mathrm{T} \\
\mathrm{K}\end{array}$ & $\begin{array}{l}\mathrm{P}_{\sigma}, \text { expt } \\
\mathrm{MPa}\end{array}$ & $\begin{array}{c}\mathrm{P}_{\sigma} \text {, anc. } \\
\mathrm{MPa}\end{array}$ & $\begin{array}{c}\operatorname{dev} 1 \\
\frac{8}{8}\end{array}$ & 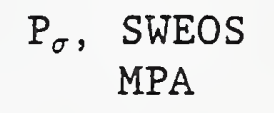 & $\begin{array}{r}\text { dev2 } \\
8\end{array}$ & wt \\
\hline 184.470 & $0.1013 \mathrm{E}+00$ & $0.1009 \mathrm{E}+00$ & -0.384 & $0.1009 E+00$ & -0.382 & 0.0 \\
\hline 203.490 & $0.2529 E+00$ & $0.2538 \mathrm{E}+00$ & 0.357 & $0.2538 E+00$ & 0.368 & 0.0 \\
\hline 205.620 & $0.2769 \mathrm{E}+00$ & $0.2782 \mathrm{E}+00$ & 0.471 & $0.2783 E+00$ & 0.483 & 0.0 \\
\hline 210.960 & $0.3459 \mathrm{E}+00$ & $0.3472 E+00$ & 0.375 & $0.3473 E+00$ & 0.389 & 0.0 \\
\hline 216.310 & $0.4281 E+00$ & $0.4285 \mathrm{E}+00$ & 0.091 & $0.4286 \mathrm{E}+00$ & 0.106 & 0.0 \\
\hline 221.880 & $0.5276 \mathrm{E}+00$ & $0.5274 E+00$ & -0.047 & $0.5274 E+00$ & -0.031 & 0.0 \\
\hline 225.100 & $0.5915 E+00$ & $0.5917 E+00$ & 0.027 & $0.5918 E+00$ & 0.043 & 0.0 \\
\hline 226.180 & $0.6153 E+00$ & $0.6145 \mathrm{E}+00$ & -0.135 & $0.6146 \mathrm{E}+00$ & -0.119 & 0.0 \\
\hline 234.580 & $0.8151 \mathrm{E}+00$ & $0.8148 E+00$ & -0.029 & $0.8150 \mathrm{E}+00$ & -0.011 & 0.0 \\
\hline 238.900 & $0.9351 E+00$ & $0.9347 E+00$ & -0.049 & $0.9348 E+00$ & -0.031 & 0.0 \\
\hline
\end{tabular}

Data from Porter [15] 
Table 1. SATURATED VAPOR PRESSURES (continued)

Data from Porter [15] (continued)

\begin{tabular}{cccrcrr}
\hline $\mathrm{T}$ & $\begin{array}{c}\mathrm{P}_{\sigma}, \text { expt } \\
\mathrm{MPa}\end{array}$ & $\begin{array}{c}\mathrm{P}_{\sigma}, \text { anc. } \\
\mathrm{MPa}\end{array}$ & $\begin{array}{c}\text { dev1 } \\
8\end{array}$ & $\begin{array}{c}\mathrm{P}_{\sigma}, \text { SWEOS } \\
\mathrm{MPA}\end{array}$ & $\begin{array}{r}\text { dev2 } \\
\text { \& }\end{array}$ & wt \\
\hline 243.220 & $0.1068 \mathrm{E}+01$ & $0.1067 \mathrm{E}+01$ & -0.062 & $0.1067 \mathrm{E}+01$ & -0.042 & 0.0 \\
248.650 & $0.1252 \mathrm{E}+01$ & $0.1252 \mathrm{E}+01$ & 0.003 & $0.1252 \mathrm{E}+01$ & 0.025 & 0.0 \\
253.030 & $0.1423 \mathrm{E}+01$ & $0.1417 \mathrm{E}+01$ & -0.409 & $0.1417 \mathrm{E}+01$ & -0.384 & 0.0 \\
258.800 & $0.1664 \mathrm{E}+01$ & $0.1658 \mathrm{E}+01$ & -0.335 & $0.1659 \mathrm{E}+01$ & -0.305 & 0.0 \\
263.280 & $0.1869 \mathrm{E}+01$ & $0.1865 \mathrm{E}+01$ & -0.221 & $0.1866 \mathrm{E}+01$ & -0.185 & 0.0 \\
& & & & & & \\
268.730 & $0.2147 \mathrm{E}+01$ & $0.2141 \mathrm{E}+01$ & -0.247 & $0.2142 \mathrm{E}+01$ & -0.202 & 0.0 \\
273.090 & $0.2386 \mathrm{E}+01$ & $0.2383 \mathrm{E}+01$ & -0.119 & $0.2384 \mathrm{E}+01$ & -0.066 & 0.0 \\
278.840 & $0.2719 \mathrm{E}+01$ & $0.2731 \mathrm{E}+01$ & 0.437 & $0.2733 \mathrm{E}+01$ & 0.500 & 0.0 \\
283.580 & $0.3050 \mathrm{E}+01$ & $0.3046 \mathrm{E}+01$ & -0.158 & $0.3048 \mathrm{E}+01$ & -0.091 & 0.0 \\
288.260 & $0.3391 \mathrm{E}+01$ & $0.3382 \mathrm{E}+01$ & -0.262 & $0.3385 \mathrm{E}+01$ & -0.195 & 0.0
\end{tabular}

Number of Points [15] 20

$\begin{array}{llll}\mathrm{AAD} \% & =0.211 & \text { BIAS } 8=-0.035 & \mathrm{RMS} \%=0.259 \\ \mathrm{AAD} 28=0.198 & \text { BIAS28 }=-0.007 & \mathrm{RMS} 2 \%=0.256\end{array}$

Absolute Deviations:

$\mathrm{AAD}=2.808 \quad \mathrm{BIAS}=-1.209 \quad \mathrm{RMS}=4.114 \mathrm{kPa}$

$A A D 2=2.482$ BIAS2 $=-0.659$ RMS2 $=4.015 \mathrm{kPa}$

Data from Regnier [16]

\begin{tabular}{|c|c|c|c|c|c|c|}
\hline $\begin{array}{l}\mathrm{T} \\
\mathrm{K}\end{array}$ & $\begin{array}{l}\mathrm{P}_{\sigma}, \operatorname{expt} \\
\mathrm{MPa}\end{array}$ & $\begin{array}{c}\mathrm{P}_{\sigma} \text {, anc. } \\
\mathrm{MPa}\end{array}$ & $\begin{array}{c}\text { dev1 } \\
8\end{array}$ & $\mathrm{P}_{\sigma}, \begin{array}{l}\text { SWEOS } \\
\text { MPA }\end{array}$ & $\begin{array}{r}\text { dev2 } \\
8\end{array}$ & wt \\
\hline 95.000 & $0.3990 E-05$ & $0.3619 E-05$ & -9.297 & $0.3618 E-05$ & -9.317 & 0.0 \\
\hline 100.000 & $0.1161 E-04$ & $0.1110 \mathrm{E}-04$ & -4.429 & $0.1109 E-04$ & -4.466 & 0.0 \\
\hline 105.000 & $0.3051 E-04$ & $0.3027 \mathrm{E}-04$ & -0.792 & $0.3026 \mathrm{E}-04$ & -0.818 & 0.0 \\
\hline 110.000 & $0.7342 E-04$ & $0.7469 E-04$ & 1.727 & $0.7468 E-04$ & 1.718 & 0.0 \\
\hline 115.000 & $0.1637 \mathrm{E}-03$ & $0.1690 \mathrm{E}-03$ & 3.230 & $0.1690 \mathrm{E}-03$ & 3.236 & 0.0 \\
\hline 120.000 & $0.3414 \mathrm{E}-03$ & $0.3546 E-03$ & 3.862 & $0.3546 E-03$ & 3.876 & 0.0 \\
\hline 125.000 & $0.6713 E-03$ & $0.6966 E-03$ & 3.766 & $0.6967 \mathrm{E}-03$ & 3.782 & 0.0 \\
\hline 130.000 & $0.1253 E-02$ & $0.1291 E-02$ & 3.071 & $0.1292 \mathrm{E}-02$ & 3.086 & 0.0 \\
\hline 135.000 & $0.2233 \mathrm{E}-02$ & $0.2275 E-02$ & 1.893 & $0.2276 \mathrm{E}-02$ & 1.904 & 0.0 \\
\hline
\end{tabular}


Table 1. SATURATED VAPOR PRESSURES (continued)

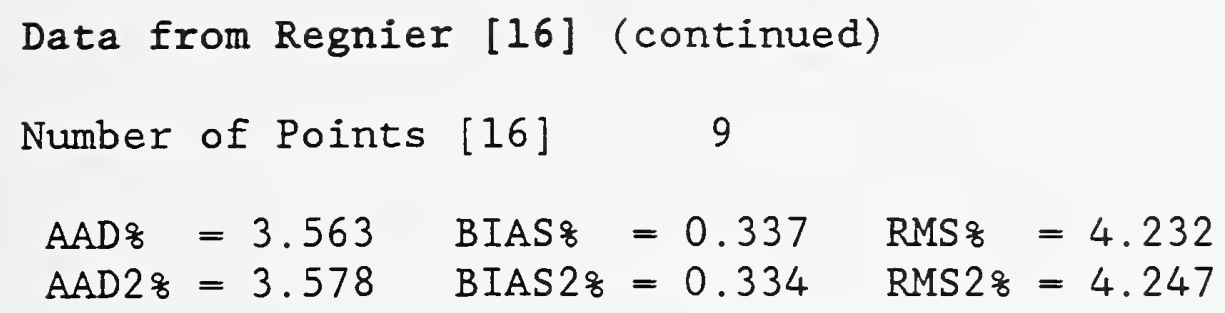

Absolute Deviations:

$\begin{array}{llllll}A A D & =0.014 & \text { BIAS } & =0.014 & \text { RMS } & =0.016 \\ A P D 2 & =0.014 & \text { BIAS2 } & =0.014 & \text { RMS2 } & =0.016 \mathrm{kPa}\end{array}$

Data from Rossini (via Ziegler et al. [17])

\begin{tabular}{|c|c|c|c|c|c|c|}
\hline $\begin{array}{l}\mathrm{T} \\
\mathrm{K}\end{array}$ & $\begin{array}{l}\mathrm{P}_{\sigma} \text {, expt } \\
\mathrm{MPa}\end{array}$ & $\begin{array}{c}\mathrm{P}_{\sigma}, \text { anc. } \\
\mathrm{MPa}\end{array}$ & $\begin{array}{c}\text { dev1 } \\
\xi\end{array}$ & $\mathrm{P}_{\sigma}, \begin{array}{l}\text { SWEOS } \\
\text { MPA }\end{array}$ & $\begin{array}{r}\text { dev2 } \\
8\end{array}$ & wt \\
\hline 130.270 & $0.1332 E-02$ & $0.1333 E-02$ & 0.062 & $0.1333 E-02$ & 0.077 & 0.0 \\
\hline 136.460 & $0.2665 E-02$ & $0.2661 E-02$ & -0.145 & $0.2661 \mathrm{E}-02$ & -0.136 & 0.0 \\
\hline 140.410 & $0.3997 E-02$ & $0.3991 E-02$ & -0.155 & $0.3991 E-02$ & -0.149 & 0.0 \\
\hline 143.370 & $0.5330 E-02$ & $0.5320 E-02$ & -0.187 & $0.5320 E-02$ & -0.184 & 0.0 \\
\hline 145.760 & $0.6662 E-02$ & $0.6645 E-02$ & -0.253 & $0.6645 E-02$ & -0.252 & 0.0 \\
\hline 147.790 & $0.7995 E-02$ & $0.7977 E-02$ & -0.223 & $0.7977 E-02$ & -0.224 & 0.0 \\
\hline 151.120 & $0.1066 \mathrm{E}-01$ & $0.1064 E-01$ & -0.226 & $0.1064 \mathrm{E}-01$ & -0.229 & 0.0 \\
\hline 153.820 & $0.1332 E-01$ & $0.1329 E-01$ & -0.241 & $0.1329 E-01$ & -0.245 & 0.0 \\
\hline 159.030 & $0.1999 \mathrm{E}-01$ & $0.1996 \mathrm{E}-01$ & -0.153 & $0.1995 \mathrm{E}-01$ & -0.160 & 0.0 \\
\hline 162.960 & $0.2665 E-01$ & $0.2660 E-01$ & -0.191 & $0.2660 \mathrm{E}-01$ & -0.198 & 0.0 \\
\hline 166.170 & $0.3331 E-01$ & $0.3326 E-01$ & -0.164 & $0.3325 E-01$ & -0.171 & 0.0 \\
\hline 168.900 & $0.3997 \mathrm{E}-01$ & $0.3992 E-01$ & -0.141 & $0.3991 E-01$ & -0.147 & 0.0 \\
\hline 173.410 & $0.5330 \mathrm{E}-01$ & $0.5322 E-01$ & -0.151 & $0.5321 E-01$ & -0.156 & 0.0 \\
\hline 177.100 & $0.6662 E-01$ & $0.6652 \mathrm{E}-01$ & -0.145 & $0.6652 E-01$ & -0.147 & 0.0 \\
\hline 180.250 & $0.7995 \mathrm{E}-01$ & $0.7985 E-01$ & -0.123 & $0.7985 E-01$ & -0.124 & 0.0 \\
\hline 183.010 & $0.9327 E-01$ & $0.9316 E-01$ & -0.114 & $0.9316 E-01$ & -0.113 & 0.0 \\
\hline 184.520 & $0.1013 E+00$ & $0.1011 E+00$ & -0.114 & $0.1011 E+00$ & -0.113 & 0.0 \\
\hline 185.480 & $0.1066 \mathrm{E}+00$ & $0.1065 \mathrm{E}+00$ & -0.093 & $0.1065 \mathrm{E}+00$ & -0.091 & 0.0 \\
\hline 187.710 & $0.1199 \mathrm{E}+00$ & $0.1198 \mathrm{E}+00$ & -0.128 & $0.1198 \mathrm{E}+00$ & -0.125 & 0.0 \\
\hline 189.770 & $0.1332 \mathrm{E}+00$ & $0.1331 E+00$ & -0.085 & $0.1331 \mathrm{E}+00$ & -0.080 & 0.0 \\
\hline 193.440 & $0.1599 \mathrm{E}+00$ & $0.1598 \mathrm{E}+00$ & -0.081 & $0.1598 E+00$ & -0.074 & 0.0 \\
\hline 198.150 & $0.1999 E+00$ & $0.1997 E+00$ & -0.068 & $0.1997 \mathrm{E}+00$ & -0.059 & 0.0 \\
\hline
\end{tabular}


Table 1. SATURATED VAPOR PRESSURES (continued)

Data from Rossini (via Ziegler et al. [17]) (continued)

Number of Points [17] 22

$$
\begin{aligned}
& \mathrm{AAD} \%=0.147 \quad \text { BIAS\% }=-0.142 \quad \mathrm{RMS} \%=0.067 \\
& \operatorname{AAD} 2 \%=0.148 \quad \text { BIAS2\% }=-0.141 \quad \text { RMS } 2 \%=0.071
\end{aligned}
$$

\begin{tabular}{|c|c|c|c|c|c|c|}
\hline $\begin{array}{l}\mathrm{T} \\
\mathrm{K}\end{array}$ & $\begin{array}{l}\mathrm{P}_{\sigma} \text {, expt } \\
\mathrm{MPa}\end{array}$ & $\mathrm{P}_{\sigma}$, anc. & $\begin{array}{c}\operatorname{dev} 1 \\
8\end{array}$ & $\begin{array}{ll}\mathrm{P}_{\sigma}, & \text { SWEOS } \\
\text { MPA }\end{array}$ & $\begin{array}{r}\text { dev2 } \\
8\end{array}$ & wt \\
\hline 130.650 & $0.1332 \mathrm{E}-02$ & $0.1394 \mathrm{E}-02$ & 4.620 & $0.1394 E-02$ & 4.635 & 0.0 \\
\hline 125.550 & $0.6662 E-03$ & $0.7475 E-03$ & 12.208 & $0.7477 \mathrm{E}-03$ & 12.226 & 0.0 \\
\hline 119.050 & $0.2665 E-03$ & $0.3097 \mathrm{E}-03$ & 16.202 & $0.3097 E-03$ & 16.216 & 0.0 \\
\hline 114.650 & $0.1332 E-03$ & $0.1600 \mathrm{E}-03$ & 20.091 & $0.1600 E-03$ & 20.096 & 0.0 \\
\hline 110.550 & $0.6662 \mathrm{E}-04$ & $0.8204 \mathrm{E}-04$ & 23.138 & $0.8203 E-04$ & 23.130 & 0.0 \\
\hline 105.350 & $0.2665 E-04$ & $0.3234 E-04$ & 21.371 & $0.3234 E-04$ & 21.342 & 0.0 \\
\hline 101.850 & $0.1332 \mathrm{E}-04$ & $0.1629 E-04$ & 22.257 & $0.1628 E-04$ & 22.214 & 0.0 \\
\hline 98.550 & $0.6662 E-05$ & $0.8121 E-05$ & 21.904 & $0.8118 \mathrm{E}-05$ & 21.850 & 0.0 \\
\hline 94.450 & $0.2665 E-05$ & $0.3174 E-05$ & 19.109 & $0.3174 E-05$ & 19.089 & 0.0 \\
\hline 91.350 & $0.1332 E-05$ & $0.1467 \mathrm{E}-05$ & 10.094 & $0.1468 \mathrm{E}-05$ & 10.138 & 0.0 \\
\hline
\end{tabular}

Data from Tickner and Lossing [18]

Number of Points [18] 10

\begin{tabular}{|c|c|c|c|c|c|c|}
\hline $\begin{array}{l}\mathrm{T} \\
\mathrm{K}\end{array}$ & $\mathrm{P}_{\sigma}$, expt & $\begin{array}{c}\mathrm{P}_{\sigma} \text {, anc. } \\
\mathrm{MPa}\end{array}$ & $\begin{array}{c}\operatorname{dev} 1 \\
8\end{array}$ & $\mathrm{P}_{\sigma}, \begin{array}{l}\text { SWEOS } \\
\text { MPA }\end{array}$ & $\begin{array}{r}\operatorname{dev} 2 \\
8\end{array}$ & wt \\
\hline 165.000 & $0.3067 \mathrm{E}-01$ & $0.3069 \mathrm{E}-01$ & 0.064 & $0.3069 E-01$ & 0.058 & 1.0 \\
\hline 170.000 & $0.4287 \mathrm{E}-01$ & $0.4288 \mathrm{E}-01$ & 0.032 & $0.4288 \mathrm{E}-01$ & 0.027 & 1.0 \\
\hline 175.000 & $0.5864 \mathrm{E}-01$ & $0.5866 \mathrm{E}-01$ & 0.048 & $0.5866 \mathrm{E}-01$ & 0.044 & 1.0 \\
\hline 180.000 & $0.7873 \mathrm{E}-01$ & $0.7872 \mathrm{E}-01$ & -0.019 & $0.7872 \mathrm{E}-01$ & -0.020 & 1.0 \\
\hline 180.000 & $0.7871 \mathrm{E}-01$ & $0.7872 E-01$ & 0.017 & $0.7872 \mathrm{E}-01$ & 0.016 & 1.0 \\
\hline
\end{tabular}

$$
\begin{aligned}
& \mathrm{AADq}=17.099 \quad \text { BIAS\% }=17.099 \quad \mathrm{RMS} \%=5.890 \\
& A A D 2 \%=17.094 \quad \text { BIAS2 } 8=17.094 \quad \text { RMS } 2 \%=5.868
\end{aligned}
$$

Absolute Deviations:

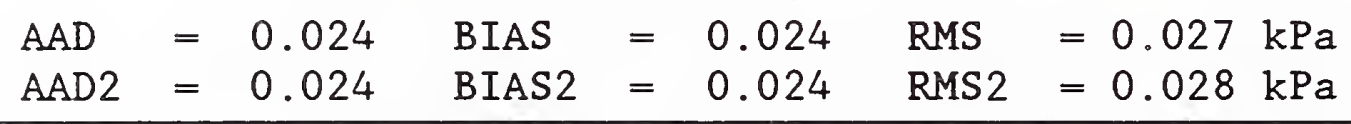

Data from Straty and Tsumura [19] 
Table 1. SATURATED VAPOR PRESSURES (continued)

Data from Straty and Tsumura [19] (continued)

\begin{tabular}{|c|c|c|c|c|c|c|}
\hline $\begin{array}{l}\mathrm{T} \\
\mathrm{K}\end{array}$ & $\mathrm{P}_{\sigma}$, expt & $\begin{array}{c}\mathrm{P}_{\sigma}, \text { anc. } \\
\mathrm{MPa}\end{array}$ & $\begin{array}{c}\operatorname{dev} 1 \\
8\end{array}$ & $\begin{array}{ll}P_{\sigma}, & \text { SWEOS } \\
\text { MPA }\end{array}$ & $\begin{array}{r}\operatorname{dev} 2 \\
8\end{array}$ & wt \\
\hline 185.000 & $0.1038 \mathrm{E}+00$ & $0.1038 \mathrm{E}+00$ & -0.043 & $0.1038 \mathrm{E}+00$ & -0.042 & 1.0 \\
\hline 190.000 & $0.1346 \mathrm{E}+00$ & $0.1347 E+00$ & 0.044 & $0.1347 \mathrm{E}+00$ & 0.049 & 1.0 \\
\hline 190.000 & $0.1347 \mathrm{E}+00$ & $0.1347 E+00$ & -0.023 & $0.1347 \mathrm{E}+00$ & -0.018 & 1.0 \\
\hline 195.000 & $0.1722 E+00$ & $0.1722 E+00$ & 0.022 & $0.1723 E+00$ & 0.029 & 1.0 \\
\hline 195.000 & $0.1723 E+00$ & $0.1722 \mathrm{E}+00$ & -0.007 & $0.1723 \mathrm{E}+00$ & 0.000 & 1.0 \\
\hline 200.000 & $0.2173 E+00$ & $0.2174 \mathrm{E}+00$ & 0.045 & $0.2174 \mathrm{E}+00$ & 0.055 & 1.0 \\
\hline 200.000 & $0.2173 E+00$ & $0.2174 E+00$ & 0.017 & $0.2174 E+00$ & 0.027 & 1.0 \\
\hline 205.000 & $0.2709 E+00$ & $0.2709 E+00$ & 0.004 & $0.2710 E+00$ & 0.017 & 1.0 \\
\hline 205.000 & $0.2710 E+00$ & $0.2709 E+00$ & -0.021 & $0.2710 E+00$ & -0.009 & 1.0 \\
\hline 210.000 & $0.3341 E+00$ & $0.3340 E+00$ & -0.051 & $0.3340 \mathrm{E}+00$ & -0.037 & 1.0 \\
\hline 210.000 & $0.3342 E+00$ & $0.3340 E+00$ & -0.063 & $0.3340 \mathrm{E}+00$ & -0.049 & 1.0 \\
\hline 210.000 & $0.3340 \mathrm{E}+00$ & $0.3340 \mathrm{E}+00$ & -0.006 & $0.3340 \mathrm{E}+00$ & 0.008 & 1.0 \\
\hline 210.000 & $0.3340 \mathrm{E}+00$ & $0.3340 E+00$ & -0.009 & $0.3340 \mathrm{E}+00$ & 0.005 & 1.0 \\
\hline 215.000 & $0.4073 E+00$ & $0.4074 E+00$ & 0.014 & $0.4075 E+00$ & 0.029 & 1.0 \\
\hline 220.000 & $0.4922 \mathrm{E}+00$ & $0.4923 E+00$ & 0.022 & $0.4923 E+00$ & 0.038 & 1.0 \\
\hline 225.000 & $0.5897 \mathrm{E}+00$ & $0.5896 \mathrm{E}+00$ & -0.020 & $0.5897 \mathrm{E}+00$ & -0.004 & 1.0 \\
\hline 230.000 & $0.7005 E+00$ & $0.7005 E+00$ & 0.000 & $0.7006 \mathrm{E}+00$ & 0.016 & 1.0 \\
\hline 235.000 & $0.8260 E+00$ & $0.8260 \mathrm{E}+00$ & -0.001 & $0.8261 \mathrm{E}+00$ & 0.017 & 1.0 \\
\hline 240.000 & $0.9666 \mathrm{E}+00$ & $0.9671 \mathrm{E}+00$ & 0.056 & $0.9673 E+00$ & 0.074 & 1.0 \\
\hline 245.000 & $0.1124 \mathrm{E}+01$ & $0.1125 E+01$ & 0.067 & $0.1125 \mathrm{E}+01$ & 0.088 & 1.0 \\
\hline 245.000 & $0.1125 E+01$ & $0.1125 \mathrm{E}+01$ & 0.032 & $0.1125 \mathrm{E}+01$ & 0.052 & 1.0 \\
\hline 250.000 & $0.1300 E+01$ & $0.1301 E+01$ & 0.090 & $0.1301 \mathrm{E}+01$ & 0.113 & 1.0 \\
\hline 250.000 & $0.1302 E+01$ & $0.1301 E+01$ & -0.056 & $0.1301 E+01$ & -0.033 & 1.0 \\
\hline 250.000 & $0.1302 \mathrm{E}+01$ & $0.1301 \mathrm{E}+01$ & -0.071 & $0.1301 E+01$ & -0.049 & 1.0 \\
\hline 250.000 & $0.1302 \mathrm{E}+01$ & $0.1301 E+01$ & -0.048 & $0.1301 E+01$ & -0.026 & 1.0 \\
\hline 255.000 & $0.1495 \mathrm{E}+01$ & $0.1496 \mathrm{E}+01$ & 0.091 & $0.1497 \mathrm{E}+01$ & 0.117 & 1.0 \\
\hline 260.000 & $0.1710 E+01$ & $0.1712 \mathrm{E}+01$ & 0.097 & $0.1712 \mathrm{E}+01$ & 0.128 & 1.0 \\
\hline 265.000 & $0.1948 E+01$ & $0.1949 \mathrm{E}+01$ & 0.071 & $0.1950 \mathrm{E}+01$ & 0.110 & 1.0 \\
\hline 270.000 & $0.2208 E+01$ & $0.2210 \mathrm{E}+01$ & 0.076 & $0.2211 E+01$ & 0.123 & 1.0 \\
\hline 275.000 & $0.2493 E+01$ & $0.2495 E+01$ & 0.061 & $0.2496 E+01$ & 0.117 & 1.0 \\
\hline 275.000 & $0.2493 E+01$ & $0.2495 \mathrm{E}+01$ & 0.057 & $0.2496 \mathrm{E}+01$ & 0.113 & 1.0 \\
\hline 280.000 & $0.2805 E+01$ & $0.2806 \mathrm{E}+01$ & 0.042 & $0.2808 \mathrm{E}+01$ & 0.105 & 1.0 \\
\hline 280.000 & $0.2806 \mathrm{E}+01$ & $0.2806 \mathrm{E}+01$ & -0.015 & $0.2808 E+01$ & 0.048 & 1.0 \\
\hline 285.000 & $0.3144 \mathrm{E}+01$ & $0.3145 \mathrm{E}+01$ & 0.021 & $0.3147 E+01$ & 0.089 & 1.0 \\
\hline 290.000 & $0.3513 E+01$ & $0.3514 E+01$ & 0.027 & $0.3517 E+01$ & 0.091 & 1.0 \\
\hline
\end{tabular}


Table 1. SATURATED VAPOR PRESSURES (continued)

Data from Straty and Tsumura [19] (continued)

\begin{tabular}{cccrcrrr}
\hline $\mathrm{T}$ & $\mathrm{P}_{\sigma}$, expt & $\mathrm{P}_{\sigma}$, anc. \\
$\mathrm{MPa}$ & $\mathrm{MPa}$ & $\begin{array}{r}\operatorname{dev1} \\
\text { o }\end{array}$ & $\begin{array}{c}\mathrm{P}_{\sigma}, \text { SWEOS } \\
\mathrm{MPA}\end{array}$ & $\begin{array}{r}\operatorname{dev} 2 \\
\text { o }\end{array}$ & wt \\
\hline 298.150 & $0.4191 \mathrm{E}+01$ & $0.4189 \mathrm{E}+01$ & -0.046 & $0.4190 \mathrm{E}+01$ & -0.020 & 1.0 \\
298.150 & $0.4189 \mathrm{E}+01$ & $0.4189 \mathrm{E}+01$ & 0.002 & $0.4190 \mathrm{E}+01$ & 0.028 & 1.0 \\
300.000 & $0.4353 \mathrm{E}+01$ & $0.4356 \mathrm{E}+01$ & 0.059 & $0.4357 \mathrm{E}+01$ & 0.069 & 1.0 \\
160.000 & $0.2150 \mathrm{E}-01$ & $0.2145 \mathrm{E}-01$ & -0.218 & $0.2145 \mathrm{E}-01$ & -0.224 & 0.0
\end{tabular}

Number of Points [19] 44

$$
\begin{aligned}
& \mathrm{AAD} \%=0.043 \quad \text { BIAS\% }=0.010 \quad \mathrm{RMS} \%=0.056 \\
& \text { AAD2 } 8=0.055 \quad \text { BIAS2\% }=0.031 \quad \text { RMS } 2 \%=0.064
\end{aligned}
$$

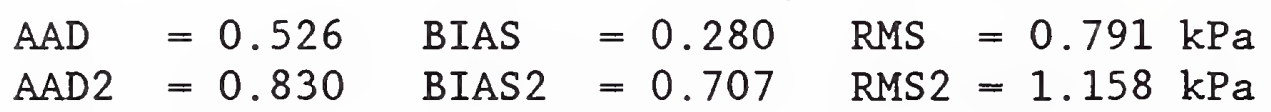

Weighted Data:

Number of Points [19] 43

$$
\begin{aligned}
& \mathrm{AAD} \%=0.039 \quad \text { BIAS\% }=0.016 \quad \text { RMS } \%=0.044 \\
& \text { AAD2 } 8=0.051 \quad \text { BIAS } 2 \%=0.037 \quad \text { RMS } 2 \%=0.052
\end{aligned}
$$

Absolute Deviations:

$\begin{array}{llllll}\mathrm{AAD} & =0.537 & \text { BIAS } & =0.288 & \text { RMS } & =0.799 \mathrm{kPa} \\ \mathrm{AAD2} & =0.848 & \text { BIAS2 } & =0.724 & \text { RMS2 } & =1.166 \mathrm{kPa}\end{array}$

Data from Ziegler et al. [17]

These data were adjusted by Goodwin et al. [20].

\begin{tabular}{cccrcrr}
\hline $\mathrm{T}$ & $\begin{array}{c}\mathrm{P}_{\sigma}, \text { expt } \\
\mathrm{MPa}\end{array}$ & $\begin{array}{c}\mathrm{P}_{\sigma}, \text { anc } \\
\mathrm{MPa}\end{array}$ & $\begin{array}{c}\text { dev1 } \\
\text { o }\end{array}$ & $\begin{array}{c}\mathrm{P}_{\sigma}, \text { SWEOS } \\
\mathrm{MPA}\end{array}$ & $\begin{array}{r}\text { dev2 } \\
\text { o }\end{array}$ & wt \\
\hline 100.010 & $0.1112 \mathrm{E}-04$ & $0.1112 \mathrm{E}-04$ & -0.034 & $0.1112 \mathrm{E}-04$ & -0.072 & 1.0 \\
109.998 & $0.7462 \mathrm{E}-04$ & $0.7466 \mathrm{E}-04$ & 0.056 & $0.7466 \mathrm{E}-04$ & 0.048 & 1.0 \\
119.989 & $0.3540 \mathrm{E}-03$ & $0.3540 \mathrm{E}-03$ & 0.018 & $0.3541 \mathrm{E}-03$ & 0.031 & 1.0 \\
129.987 & $0.1290 \mathrm{E}-02$ & $0.1289 \mathrm{E}-02$ & -0.010 & $0.1290 \mathrm{E}-02$ & 0.005 & 1.0 \\
139.992 & $0.3829 \mathrm{E}-02$ & $0.3828 \mathrm{E}-02$ & -0.030 & $0.3828 \mathrm{E}-02$ & -0.024 & 1.0 \\
& & & & & & \\
150.000 & $0.9674 \mathrm{E}-02$ & $0.9670 \mathrm{E}-02$ & -0.039 & $0.9670 \mathrm{E}-02$ & -0.042 & 1.0 \\
160.010 & $0.2148 \mathrm{E}-01$ & $0.2147 \mathrm{E}-01$ & -0.034 & $0.2147 \mathrm{E}-01$ & -0.041 & 1.0 \\
170.019 & $0.4295 \mathrm{E}-01$ & $0.4294 \mathrm{E}-01$ & -0.029 & $0.4293 \mathrm{E}-01$ & -0.034 & 1.0 \\
180.027 & $0.7885 \mathrm{E}-01$ & $0.7884 \mathrm{E}-01$ & -0.018 & $0.7884 \mathrm{E}-01$ & -0.019 & 1.0 \\
184.550 & $0.1013 \mathrm{E}+00$ & $0.1013 \mathrm{E}+00$ & -0.013 & $0.1013 \mathrm{E}+00$ & -0.011 & 1.0
\end{tabular}


Table 1. SATURATED VAPOR PRESSURES (continued)

Data from Ziegler et al. [17] (continued)

Number of Points [17] 10

$$
\begin{array}{lll}
\mathrm{AAD} \%=0.028 & \text { BIAS } 8=-0.013 & \text { RMS } 8=0.028 \\
\mathrm{AAD} 2 \%=0.033 & \text { BIAS2\% }=-0.016 & \text { RMS2\% }=0.034
\end{array}
$$

Absolute Deviations:

$\begin{array}{lllll}\mathrm{AAD} & =0.005 & \mathrm{BIAS}=-0.005 & \mathrm{RMS} & =0.006 \mathrm{kPa} \\ \mathrm{AAD2} & =0.006 & \mathrm{BIAS} 2=-0.005 & \mathrm{RMS} 2=0.006 \mathrm{kPa}\end{array}$

Weighted Data:

Number of Points [17] 10

$$
\begin{array}{lll}
\mathrm{AAD} \gamma=0.028 & \text { BIAS } \%=-0.013 & \text { RMS } 8=0.028 \\
\mathrm{AAD} 2 \%=0.033 & \text { BIAS2\% }=-0.016 & \text { RMS2\% }=0.034
\end{array}
$$

\begin{tabular}{|c|c|c|c|c|c|c|}
\hline $\begin{array}{l}\mathrm{T} \\
\mathrm{K}\end{array}$ & $\begin{array}{l}\mathrm{P}_{\sigma}, \operatorname{expt} \\
\mathrm{MPa}\end{array}$ & $\begin{array}{c}\mathrm{P}_{\sigma} \text {, anc. } \\
\mathrm{MPa}\end{array}$ & $\begin{array}{c}\operatorname{dev} 1 \\
\frac{8}{8}\end{array}$ & $\begin{array}{ll}P_{\sigma}, & \text { SWEOS } \\
\text { MPA }\end{array}$ & $\begin{array}{r}\operatorname{dev} 2 \\
\frac{9}{8}\end{array}$ & wt \\
\hline 89.890 & $0.9767 \mathrm{E}-06$ & $0.9995 E-06$ & 2.336 & $0.1000 E-05$ & 2.423 & 0.0 \\
\hline 90.000 & $0.1006 \mathrm{E}-05$ & $0.1029 E-05$ & 2.316 & $0.1030 E-05$ & 2.399 & 0.0 \\
\hline 92.000 & $0.1692 \mathrm{E}-05$ & $0.1733 E-05$ & 2.398 & $0.1733 E-05$ & 2.422 & 0.0 \\
\hline 94.000 & $0.2785 \mathrm{E}-05$ & $0.2847 \mathrm{E}-05$ & 2.251 & $0.2847 E-05$ & 2.240 & 0.0 \\
\hline 96.000 & $0.4464 \mathrm{E}-05$ & $0.4575 E-05$ & 2.488 & $0.4573 E-05$ & 2.456 & 0.0 \\
\hline 98.000 & $0.7022 \mathrm{E}-05$ & $0.7196 \mathrm{E}-05$ & 2.475 & $0.7193 \mathrm{E}-05$ & 2.432 & 0.0 \\
\hline 100.000 & $0.1082 \mathrm{E}-04$ & $0.1110 \mathrm{E}-04$ & 2.555 & $0.1109 \mathrm{E}-04$ & 2.516 & 0.0 \\
\hline 102.000 & $0.1639 \mathrm{E}-04$ & $0.1679 \mathrm{E}-04$ & 2.470 & $0.1679 \mathrm{E}-04$ & 2.434 & 0.0 \\
\hline 104.000 & $0.2438 \mathrm{E}-04$ & $0.2498 \mathrm{E}-04$ & 2.429 & $0.2497 E-04$ & 2.399 & 0.0 \\
\hline 106.000 & $0.3571 E-04$ & $0.3654 \mathrm{E}-04$ & 2.316 & $0.3653 E-04$ & 2.293 & 0.0 \\
\hline 108.000 & $0.5143 \mathrm{E}-04$ & $0.5262 \mathrm{E}-04$ & 2.315 & $0.5261 E-04$ & 2.300 & 0.0 \\
\hline 110.000 & $0.7288 \mathrm{E}-04$ & $0.7469 \mathrm{E}-04$ & 2.476 & $0.7468 \mathrm{E}-04$ & 2.467 & 0.0 \\
\hline 112.000 & $0.1022 \mathrm{E}-03$ & $0.1045 E-03$ & 2.300 & $0.1045 E-03$ & 2.298 & 0.0 \\
\hline 114.000 & $0.1412 \mathrm{E}-03$ & $0.1444 \mathrm{E}-03$ & 2.270 & $0.1444 \mathrm{E}-03$ & 2.273 & 0.0 \\
\hline 116.000 & $0.1928 \mathrm{E}-03$ & $0.1971 E-03$ & 2.234 & $0.1971 \mathrm{E}-03$ & 2.241 & 0.0 \\
\hline
\end{tabular}

Absolute Deviations:

$\begin{array}{llllll}\mathrm{AAD} & =0.005 & \mathrm{BIAS} & =-0.005 & \mathrm{RMS} & =0.006 \mathrm{kPa} \\ \mathrm{AAD2} & =0.006 & \mathrm{BIAS} 2 & =-0.005 & \text { RMS2 } & =0.006 \mathrm{kPa}\end{array}$

Data from Ziegler et al. (unadj) [17] 
Table 1. SATURATED VAPOR PRESSURES (continued)

Data from Ziegler et a1. (unadj) [17] (continued)

\begin{tabular}{|c|c|c|c|c|c|c|}
\hline $\begin{array}{l}\mathrm{T} \\
\mathrm{K}\end{array}$ & $\begin{array}{c}\mathrm{P}_{\sigma}, \text { expt } \\
\mathrm{MPa}\end{array}$ & $\begin{array}{c}\mathrm{P}_{\sigma}, \text { anc. } \\
\mathrm{MPa}\end{array}$ & $\begin{array}{c}\text { dev1 } \\
8 \%\end{array}$ & 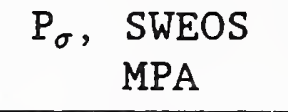 & $\begin{array}{r}\operatorname{dev} 2 \\
8\end{array}$ & wt \\
\hline 118.000 & $0.2602 \mathrm{E}-03$ & $0.2658 \mathrm{E}-03$ & 2.160 & $0.2659 E-03$ & 2.171 & 0.0 \\
\hline 120.000 & $0.3472 \mathrm{E}-03$ & $0.3546 \mathrm{E}-03$ & 2.118 & $0.3546 \mathrm{E}-03$ & 2.132 & 0.0 \\
\hline 122.000 & $0.4588 \mathrm{E}-03$ & $0.4680 E-03$ & 2.016 & $0.4681 E-03$ & 2.031 & 0.0 \\
\hline 124.000 & $0.5999 E-03$ & $0.6116 \mathrm{E}-03$ & 1.950 & $0.6117 \mathrm{E}-03$ & 1.966 & 0.0 \\
\hline 126.000 & $0.7771 \mathrm{E}-03$ & $0.7916 \mathrm{E}-03$ & 1.869 & $0.7917 E-03$ & 1.886 & 0.0 \\
\hline 128.000 & $0.9975 E-03$ & $0.1015 E-02$ & 1.804 & $0.1016 \mathrm{E}-02$ & 1.820 & 0.0 \\
\hline 130.000 & $0.1270 \mathrm{E}-02$ & $0.1291 E-02$ & 1.718 & $0.1292 \mathrm{E}-02$ & 1.733 & 0.0 \\
\hline 132.000 & $0.1603 \mathrm{E}-02$ & $0.1629 \mathrm{E}-02$ & 1.641 & $0.1629 \mathrm{E}-02$ & 1.654 & 0.0 \\
\hline 134.000 & $0.2008 E-02$ & $0.2039 E-02$ & 1.556 & $0.2040 \mathrm{E}-02$ & 1.568 & 0.0 \\
\hline 136.000 & $0.2497 \mathrm{E}-02$ & $0.2534 \mathrm{E}-02$ & 1.480 & $0.2534 \mathrm{E}-02$ & 1.490 & 0.0 \\
\hline 138.000 & $0.3083 E-02$ & $0.3126 \mathrm{E}-02$ & 1.396 & $0.3127 \mathrm{E}-02$ & 1.404 & 0.0 \\
\hline 140.000 & $0.3781 \mathrm{E}-02$ & $0.3831 E-02$ & 1.317 & $0.3831 \mathrm{E}-02$ & 1.323 & 0.0 \\
\hline 142.000 & $0.4608 \mathrm{E}-02$ & $0.4665 \mathrm{E}-02$ & 1.240 & $0.4665 E-02$ & 1.244 & 0.0 \\
\hline 144.000 & $0.5581 \mathrm{E}-02$ & $0.5646 \mathrm{E}-02$ & 1.160 & $0.5646 \mathrm{E}-02$ & 1.163 & 0.0 \\
\hline 146.000 & $0.6719 \mathrm{E}-02$ & $0.6792 \mathrm{E}-02$ & 1.085 & $0.6792 \mathrm{E}-02$ & 1.085 & 0.0 \\
\hline 148.000 & $0.8045 E-02$ & $0.8126 \mathrm{E}-02$ & 1.011 & $0.8126 \mathrm{E}-02$ & 1.010 & 0.0 \\
\hline 150.000 & $0.9580 \mathrm{E}-02$ & $0.9670 E-02$ & 0.936 & $0.9670 E-02$ & 0.933 & 0.0 \\
\hline 152.000 & $0.1135 \mathrm{E}-01$ & $0.1145 \mathrm{E}-01$ & 0.865 & $0.1145 \mathrm{E}-01$ & 0.861 & 0.0 \\
\hline 154.000 & $0.1338 \mathrm{E}-01$ & $0.1349 E-01$ & 0.798 & $0.1349 \mathrm{E}-01$ & 0.793 & 0.0 \\
\hline 156.000 & $0.1570 \mathrm{E}-01$ & $0.1581 E-01$ & 0.728 & $0.1581 E-01$ & 0.722 & 0.0 \\
\hline 158.000 & $0.1834 \mathrm{E}-01$ & $0.1846 \mathrm{E}-01$ & 0.661 & $0.1846 \mathrm{E}-01$ & 0.654 & 0.0 \\
\hline 160.000 & $0.2133 E-01$ & $0.2145 E-01$ & 0.589 & $0.2145 \mathrm{E}-01$ & 0.582 & 0.0 \\
\hline 162.000 & $0.2470 E-01$ & $0.2483 E-01$ & 0.527 & $0.2483 E-01$ & 0.520 & 0.0 \\
\hline 164.000 & $0.2849 \mathrm{E}-01$ & $0.2862 E-01$ & 0.465 & $0.2862 \mathrm{E}-01$ & 0.458 & 0.0 \\
\hline 166.000 & $0.3274 \mathrm{E}-01$ & $0.3287 \mathrm{E}-01$ & 0.401 & $0.3287 \mathrm{E}-01$ & 0.394 & 0.0 \\
\hline 168.000 & $0.3749 E-01$ & $0.3761 E-01$ & 0.340 & $0.3761 \mathrm{E}-01$ & 0.334 & 0.0 \\
\hline 170.000 & $0.4276 \mathrm{E}-01$ & $0.4288 E-01$ & 0.280 & $0.4288 \mathrm{E}-01$ & 0.274 & 0.0 \\
\hline 172.000 & $0.4862 E-01$ & $0.4873 E-01$ & 0.223 & $0.4873 E-01$ & 0.218 & 0.0 \\
\hline 174.000 & $0.5510 \mathrm{E}-01$ & $0.5519 E-01$ & 0.167 & $0.5519 \mathrm{E}-01$ & 0.163 & 0.0 \\
\hline 176.000 & $0.6224 \mathrm{E}-01$ & $0.6231 E-01$ & 0.110 & $0.6231 \mathrm{E}-01$ & 0.107 & 0.0 \\
\hline 178.000 & $0.7010 \mathrm{E}-01$ & $0.7014 \mathrm{E}-01$ & 0.055 & $0.7014 \mathrm{E}-01$ & 0.053 & 0.0 \\
\hline 180.000 & $0.7872 E-01$ & $0.7872 \mathrm{E}-01$ & 0.003 & $0.7872 \mathrm{E}-01$ & 0.002 & 0.0 \\
\hline 182.000 & $0.8815 \mathrm{E}-01$ & $0.8810 \mathrm{E}-01$ & -0.050 & $0.8810 \mathrm{E}-01$ & -0.050 & 0.0 \\
\hline 184.000 & $0.9844 \mathrm{E}-01$ & $0.9834 \mathrm{E}-01$ & -0.101 & $0.9834 \mathrm{E}-01$ & -0.099 & 0.0 \\
\hline 184.520 & $0.1013 E+00$ & $0.1011 E+00$ & -0.114 & $0.1011 E+00$ & -0.113 & 0.0 \\
\hline
\end{tabular}


Table 1. SATURATED VAPOR PRESSURES (continued)

Data from Ziegler et al. (unadj) [17] (continued)

Number of Points [17] 50

$\begin{array}{lll}\mathrm{AAD} \%=1.371 & \text { BIAS } 8=1.361 & \text { RMS } 8=0.889 \\ \mathrm{AAD} 2 \%=1.372 & \text { BIAS2\% }=1.362 & \text { RMS2\% }=0.891\end{array}$

Absolute Deviations:

$\begin{array}{llllll}A A D & =0.049 & \text { BIAS } & =0.038 & \text { RMS } & =0.057 \mathrm{kPa} \\ \mathrm{AAD} 2 & =0.048 & \text { BIAS2 } & =0.038 & \text { RMS2 } & =0.056 \mathrm{kPa}\end{array}$

Daid from ancillary equation [1]

\begin{tabular}{|c|c|c|c|c|c|c|}
\hline $\begin{array}{l}\mathrm{T} \\
\mathrm{K}\end{array}$ & $\begin{array}{c}\mathrm{P}_{\sigma}, \operatorname{expt} \\
\mathrm{MPa}\end{array}$ & $\begin{array}{c}\mathrm{P}_{\sigma}, \underset{\mathrm{MPa}}{\text { anc }} \\
.\end{array}$ & $\begin{array}{r}\operatorname{dev} 1 \\
\frac{8}{8}\end{array}$ & 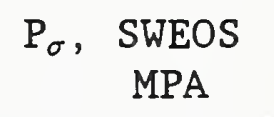 & ${ }_{8}^{\operatorname{dev} 2}$ & wt* \\
\hline 91.000 & & $0.1340 \mathrm{E}-05$ & & $0.1340 \mathrm{E}-05$ & 0.043 & 531.0 \\
\hline 96.000 & & $0.4575 \mathrm{E}-05$ & & $0.4573 E-05$ & -0.031 & 531.0 \\
\hline 101.000 & & $0.1368 \mathrm{E}-04$ & & $0.1368 \mathrm{E}-04$ & -0.037 & 531.0 \\
\hline 106.000 & & $0.3654 \mathrm{E}-04$ & & $0.3653 E-04$ & -0.022 & 531.0 \\
\hline 111.000 & & $0.8851 \mathrm{E}-04$ & & $0.8851 E-04$ & -0.005 & 531.0 \\
\hline 116.000 & & $0.1971 \mathrm{E}-03$ & & $0.1971 E-03$ & 0.008 & 531.0 \\
\hline 121.000 & & $0.4079 E-03$ & & $0.4079 E-03$ & 0.014 & 531.0 \\
\hline 126.000 & & $0.7916 \mathrm{E}-03$ & & $0.7917 \mathrm{E}-03$ & 0.016 & 531.0 \\
\hline 131.000 & & $0.1452 \mathrm{E}-02$ & & $0.1452 \mathrm{E}-02$ & 0.014 & 531.0 \\
\hline 136.000 & & $0.2534 \mathrm{E}-02$ & & $0.2534 \mathrm{E}-02$ & 0.010 & 531.0 \\
\hline 141.000 & & $0.4231 \mathrm{E}-02$ & & $0.4231 E-02$ & 0.005 & 531.0 \\
\hline 146.000 & & $0.6792 \mathrm{E}-02$ & & $0.6792 E-02$ & 0.001 & 531.0 \\
\hline 151.000 & & $0.1053 \mathrm{E}-01$ & & $0.1053 E-01$ & -0.003 & 531.0 \\
\hline 156.000 & & $0.1581 \mathrm{E}-01$ & & $0.1581 \mathrm{E}-01$ & -0.005 & 531.0 \\
\hline 161.000 & & $0.2309 E-01$ & & $0.2309 E-01$ & -0.007 & 531.0 \\
\hline 166.000 & & $0.3287 \mathrm{E}-01$ & & $0.3287 \mathrm{E}-01$ & -0.007 & 531.0 \\
\hline 171.000 & & $0.4573 E-01$ & & $0.4573 E-01$ & -0.005 & 531.0 \\
\hline 176.000 & & $0.6231 \mathrm{E}-01$ & & $0.6231 E-01$ & -0.003 & 531.0 \\
\hline 181.000 & & $0.8331 \mathrm{E}-01$ & & $0.8331 E-01$ & -0.001 & 531.0 \\
\hline 186.000 & & $0.1095 \mathrm{E}+00$ & & $0.1095 \mathrm{E}+00$ & 0.002 & 493.0 \\
\hline 191.000 & & $0.1416 \mathrm{E}+00$ & & $0.1416 \mathrm{E}+00$ & 0.005 & 371.0 \\
\hline 196.000 & & $0.1806 \mathrm{E}+00$ & & $0.1807 E+00$ & 0.008 & 284.0 \\
\hline 201.000 & & $0.2274 \mathrm{E}+00$ & & $0.2274 \mathrm{E}+00$ & 0.010 & 221.0 \\
\hline 206.000 & & $0.2828 E+00$ & & $0.2828 E+00$ & 0.012 & 174.0 \\
\hline 211.000 & & $0.3478 \mathrm{E}+00$ & & $0.3478 E+00$ & 0.014 & 138.0 \\
\hline
\end{tabular}


Table 1. SATURATED VAPOR PRESSURES (continued)

Data from ancillary equation [1] (continued)

\begin{tabular}{|c|c|c|c|c|c|c|}
\hline $\begin{array}{l}\mathrm{T} \\
\mathrm{K}\end{array}$ & $\begin{array}{c}\mathrm{P}_{\sigma}, \operatorname{expt} \\
\mathrm{MPa}\end{array}$ & $\mathrm{P}_{\sigma}, \underset{\mathrm{MPa}}{\text { anc }}$. & $\begin{array}{r}\operatorname{dev1} \\
8\end{array}$ & 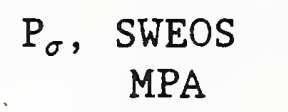 & $\operatorname{dev}_{8}$ & wt* \\
\hline 216.000 & & $0.4234 \mathrm{E}+00$ & & $0.4235 E+00$ & 0.015 & 112.0 \\
\hline 221.000 & & $0.5107 E+00$ & & $0.5108 E+00$ & 0.016 & 90.8 \\
\hline 226.000 & & $0.6107 \mathrm{E}+00$ & & $0.6108 \mathrm{E}+00$ & 0.016 & 74.6 \\
\hline 231.000 & & $0.7244 E+00$ & & $0.7245 E+00$ & 0.017 & 61.9 \\
\hline 236.000 & & $0.8529 E+00$ & & $0.8530 \mathrm{E}+00$ & 0.017 & 51.8 \\
\hline 241.000 & & $0.9974 \mathrm{E}+00$ & & $0.9975 E+00$ & 0.019 & 43.7 \\
\hline 246.000 & & $0.1159 \mathrm{E}+01$ & & $0.1159 E+01$ & 0.021 & 37.2 \\
\hline 251.000 & & $0.1339 E+01$ & & $0.1339 \mathrm{E}+01$ & 0.024 & 69.7 \\
\hline 256.000 & & $0.1538 E+01$ & & $0.1538 \mathrm{E}+01$ & 0.027 & 60.2 \\
\hline 261.000 & & $0.1758 \mathrm{E}+01$ & & $0.1758 \mathrm{E}+01$ & 0.033 & 52.2 \\
\hline 266.000 & & $0.1999 \mathrm{E}+01$ & & $0.2000 \mathrm{E}+01$ & 0.041 & 45.7 \\
\hline 271.000 & & $0.2265 E+01$ & & $0.2266 \mathrm{E}+01$ & 0.050 & 40.2 \\
\hline 276.000 & & $0.2555 \mathrm{E}+01$ & & $0.2556 \mathrm{E}+01$ & 0.058 & 35.7 \\
\hline 281.000 & & $0.2871 \mathrm{E}+01$ & & $0.2873 E+01$ & 0.065 & 31.9 \\
\hline 286.000 & & $0.3216 \mathrm{E}+01$ & & $0.3219 \mathrm{E}+01$ & 0.068 & 28.8 \\
\hline 291.000 & & $0.3592 \mathrm{E}+01$ & & $0.3594 \mathrm{E}+01$ & 0.062 & 26.2 \\
\hline 296.000 & & $0.4002 \mathrm{E}+01$ & & $0.4003 E+01$ & 0.041 & 24.1 \\
\hline 301.000 & & $0.4449 \mathrm{E}+01$ & & $0.4449 E+01$ & 0.002 & 22.6 \\
\hline
\end{tabular}

Number of Points from ancillary equation. 43

*These weights refer to development of the SWEOS.

These data are not included in the overall statistics below.

$$
\mathrm{AAD} 28=0.020 \quad \text { BIAS2 } 8=0.015 \quad \text { RMS } 28=0.023
$$

Absolute Deviations:

$$
\mathrm{AAD} 2=0.321 \quad \text { BIAS2 }=0.321 \quad \mathrm{RMS} 2=0.617 \mathrm{kPa}
$$

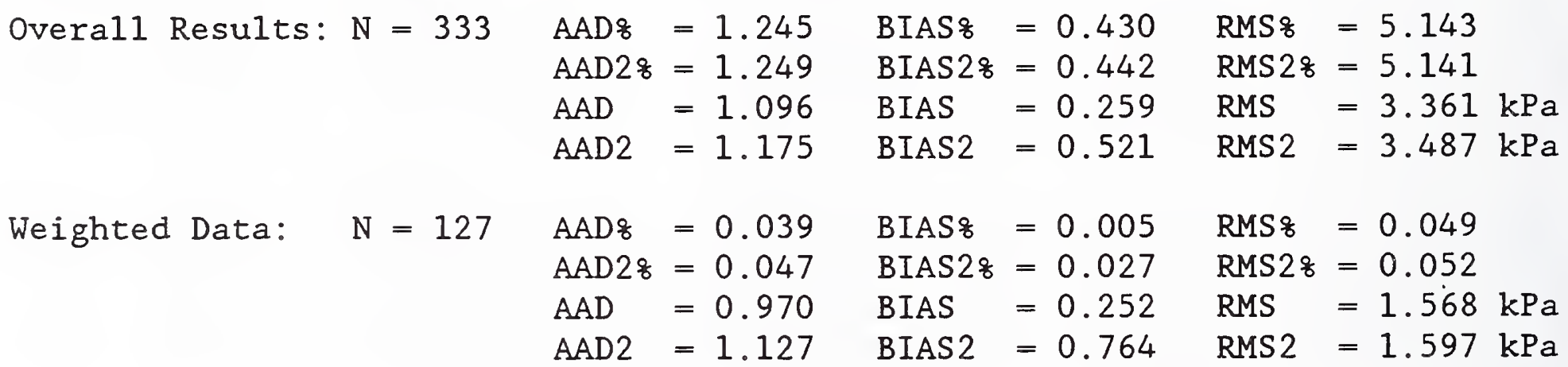


Table 2. SATURATED LIQUID DENSITIES

Data from Chui et al. [7]

\begin{tabular}{ccccccc}
\hline $\mathrm{T}$ & $\begin{array}{c}\rho_{\sigma \mathrm{L}}, \operatorname{expt} \\
\mathrm{mol} \cdot \mathrm{dm}^{-3}\end{array}$ & $\begin{array}{c}\rho_{\sigma \mathrm{L}}, \mathrm{anc} . \\
\mathrm{mol} \cdot \mathrm{dm}^{-3}\end{array}$ & $\begin{array}{c}\text { dev1 } \\
8\end{array}$ & $\begin{array}{c}\rho_{\sigma \mathrm{L}}, \begin{array}{c}\text { SWEOS } \\
\mathrm{mol} \cdot \mathrm{dm}^{-3}\end{array} \\
\begin{array}{c}\text { dev2 } \\
8\end{array}\end{array}$ & wt \\
\hline 161.360 & 19.03 & 19.03 & 0.004 & 19.03 & 0.013 & 1.0 \\
115.770 & 20.75 & 20.75 & 0.025 & 20.75 & 0.020 & 1.0 \\
108.150 & 21.03 & 21.03 & 0.011 & 21.03 & 0.014 & 1.0
\end{tabular}

Number of Points [7] 3

$$
\begin{array}{lll}
\mathrm{AAD} z=0.013 & \text { BIAS } 8=0.013 & \text { RMS } 8=0.009 \\
\mathrm{AAD} 2 \%=0.015 & \text { BIAS2\% }=0.015 & \text { RMS2\% } 2 \% 0.003
\end{array}
$$

Absolute Deviations:

$$
\begin{array}{llllll}
A A D & =0.00 & \text { BIAS } & =0.00 & \text { RMS } & =0.00 \mathrm{~mol} \cdot \mathrm{dm}^{-3} \\
A A D 2 & =0.00 & \text { BIAS2 } & =0.00 & \text { RMS2 } & =0.00 \mathrm{~mol} \cdot \mathrm{dm}^{-3}
\end{array}
$$

Weighted Data:

Number of Points [7] 3

$$
\begin{array}{llll}
\mathrm{AAD} z=0.013 & \text { BIAS } 8=0.013 & \text { RMS } 8=0.009 \\
\mathrm{AAD} 2 \%=0.015 & \text { BIAS2\% }=0.015 & \text { RMS } 2 \%=0.003
\end{array}
$$

\begin{tabular}{|c|c|c|c|c|c|c|}
\hline $\begin{array}{l}\mathrm{T} \\
\mathrm{K}\end{array}$ & $\begin{array}{l}\rho_{\sigma \mathrm{L}}, \operatorname{expt} \\
\mathrm{mol} \cdot \mathrm{dm}^{-3}\end{array}$ & $\begin{array}{l}\rho_{\sigma \mathrm{L}}, \text { anc. } \\
\mathrm{mol} \cdot \mathrm{dm}^{-3}\end{array}$ & $\begin{array}{c}\operatorname{dev} 1 \\
\delta\end{array}$ & $\begin{array}{c}\rho_{\sigma \mathrm{L}}, \quad \text { SWEOS } \\
\mathrm{mOI} \cdot \mathrm{dm}^{-3}\end{array}$ & $\begin{array}{c}\operatorname{dev} 2 \\
8\end{array}$ & $w t$ \\
\hline 248.150 & 15.06 & 15.00 & -0.366 & 15.00 & -0.356 & 0.0 \\
\hline 305.250 & 7.60 & 7.58 & -0.250 & 7.63 & 0.362 & 0.0 \\
\hline 305.150 & 7.83 & 7.82 & -0.136 & 7.89 & 0.717 & 0.0 \\
\hline 304.150 & 8.74 & 8.73 & -0.046 & 8.76 & 0.290 & 1.0 \\
\hline 303.150 & 9.20 & 9.20 & -0.042 & 9.20 & -0.008 & 1.0 \\
\hline 302.150 & 9.54 & 9.54 & -0.029 & 9.53 & -0.137 & 1.0 \\
\hline 298.150 & 10.50 & 10.48 & -0.178 & 10.47 & -0.300 & 0.0 \\
\hline 293.150 & 11.30 & 11.28 & -0.175 & 11.28 & -0.118 & 0.0 \\
\hline 283.150 & 12.46 & 12.42 & -0.265 & 12.44 & -0.104 & 0.0 \\
\hline 273.150 & 13.34 & 13.31 & -0.271 & 13.32 & -0.156 & 0.0 \\
\hline
\end{tabular}

Absolute Deviations:
$A A D=0.00 \quad$ BIAS $=0.00$
RMS $=0.00 \mathrm{~mol} \cdot \mathrm{dm}^{-3}$
AAD2 $=0.00 \quad$ BIAS2 $=0.00 \quad$ RMS2 $=0.00 \mathrm{~mol} \cdot \mathrm{dm}^{-3}$

Data from Douslin and Harrison [9] 
Table 2. SATURATED LIQUID DENSITIES (continued)

Data from Douslin and Harrison [9] (continued)

\begin{tabular}{|c|c|c|c|c|c|c|}
\hline $\begin{array}{l}\mathrm{T} \\
\mathrm{K}\end{array}$ & $\begin{array}{l}\rho_{\sigma \mathrm{L}}, \operatorname{expt} \\
\mathrm{mol} \cdot \mathrm{dm}^{-3}\end{array}$ & $\begin{array}{l}\rho_{\sigma \mathrm{L}}, \text { anc. } \\
\mathrm{mol} \cdot \mathrm{dm}^{-3}\end{array}$ & $\begin{array}{c}\operatorname{dev} 1 \\
\frac{8}{\delta}\end{array}$ & $\begin{array}{c}\rho_{\sigma \mathrm{L}}, \quad \text { SWEOS } \\
\mathrm{mol} \cdot \mathrm{dm}^{-3}\end{array}$ & $\begin{array}{c}\text { dev2 } \\
8\end{array}$ & wt \\
\hline 263.150 & 14.09 & 14.05 & -0.296 & 14.06 & -0.238 & 0.0 \\
\hline 253.150 & 14.75 & 14.70 & -0.359 & 14.70 & -0.338 & 0.0 \\
\hline
\end{tabular}

Number of Points [9] 12

$\begin{array}{lll}\text { AAD } \%=0.201 & \text { BIAS } \%=-0.201 & \text { RMS } z=0.114 \\ \text { AAD2 } z=0.260 & \text { BIAS2\% }=-0.032 & \text { RMS2\% } 2 \% 0.313\end{array}$

Absolute Deviations:
$\mathrm{AAD}=0.02$
BIAS $=-0.02$
RMS $=0.02 \mathrm{~mol} \cdot \mathrm{dm}^{-3}$
$A A D 2=0.03$
BIAS2 $=-0.01$
RMS2 $=0.03 \mathrm{~mol} \cdot \mathrm{dm}^{-3}$

Weighted Data:

Number of Points [9] 3

$\begin{array}{lll}\mathrm{AAD} \%=0.039 & \text { BIAS } 8=-0.039 & \mathrm{RMS} \%=0.007 \\ \mathrm{AAD} 2 \%=0.145 & \text { BIAS2 } 8=0.049 & \mathrm{RMS} 2 \%=0.178\end{array}$

Absolute Deviations:

\begin{tabular}{|c|c|}
\hline$A D$ & BTAS \\
\hline$A D 2$ & BIAS 2 \\
\hline
\end{tabular}

Data from Goodwin et a1. [20]

Using isochores from [13]

\begin{tabular}{|c|c|c|c|c|c|c|}
\hline $\begin{array}{l}\mathrm{T} \\
\mathrm{K}\end{array}$ & $\begin{array}{l}\rho_{\sigma \mathrm{L}}, \operatorname{expt} \\
\mathrm{mol} \cdot \mathrm{dm}^{-3}\end{array}$ & $\begin{array}{l}\rho_{\sigma \mathrm{L}}, \text { anc. } \\
\mathrm{mol} \cdot \mathrm{dm}^{-3}\end{array}$ & $\begin{array}{c}\text { devl } \\
8\end{array}$ & $\begin{array}{c}\rho_{\sigma \mathrm{L}}, \quad \text { SWEOS } \\
\mathrm{mol} \cdot \mathrm{dm}^{-3}\end{array}$ & $\begin{array}{c}\text { dev2 } \\
8\end{array}$ & wt \\
\hline 255.963 & 14.55 & 14.52 & -0.209 & 14.53 & -0.180 & 0.0 \\
\hline 247.962 & 15.05 & 15.01 & -0.246 & 15.01 & -0.236 & 0.0 \\
\hline 240.700 & 15.46 & 15.43 & -0.175 & 15.43 & -0.176 & 0.0 \\
\hline 229.917 & 16.04 & 16.00 & -0.210 & 16.00 & -0.215 & 0.0 \\
\hline 222.618 & 16.42 & 16.37 & -0.320 & 16.37 & -0.323 & 0.0 \\
\hline 214.942 & 16.75 & 16.74 & -0.080 & 16.74 & -0.079 & 0.0 \\
\hline 207.941 & 17.13 & 17.07 & -0.345 & 17.07 & -0.339 & 0.0 \\
\hline 197.888 & 17.53 & 17.52 & -0.075 & 17.52 & -0.063 & 0.0 \\
\hline 188.451 & 17.94 & 17.92 & -0.102 & 17.93 & -0.086 & 0.0 \\
\hline 176.512 & 18.45 & 18.42 & -0.142 & 18.42 & -0.126 & 0.0 \\
\hline
\end{tabular}


Table 2. SATURATED LIQUID DENSITIES (continued)

Data from Goodwin et al. [20] (continued)

\begin{tabular}{ccccccc}
$\mathrm{T}$ & $\begin{array}{c}\rho_{\sigma \mathrm{L}}, \operatorname{expt} \\
\mathrm{mol} \cdot \mathrm{dm}^{-3}\end{array}$ & $\begin{array}{c}\rho_{\sigma \mathrm{L}}, \mathrm{anc} . \\
\mathrm{mol} \cdot \mathrm{dm}^{-3}\end{array}$ & $\begin{array}{c}\mathrm{dev} 1 \\
8\end{array}$ & $\begin{array}{c}\rho_{\sigma \mathrm{L}}, \begin{array}{c}\mathrm{SWEOS} \\
\mathrm{mol} \cdot \mathrm{dm}^{-3}\end{array} \\
\begin{array}{c}\text { dev2 } \\
\text { \% }\end{array}\end{array}$ & wt \\
\hline 167.366 & 18.82 & 18.79 & -0.179 & 18.79 & -0.166 & 0.0 \\
156.875 & 19.23 & 19.20 & -0.117 & 19.20 & -0.111 & 0.0
\end{tabular}

Number of Points [20] 12

$$
\begin{aligned}
& \text { AiDi }=0.183 \text { BIAS } 8=-0.183 \quad \text { RMS } 8=0.084 \\
& A A D 28=0.175 \quad \text { BIAS2 } 8=-0.175 \quad \text { RMS } 28=0.087
\end{aligned}
$$

Absolute Deviations:

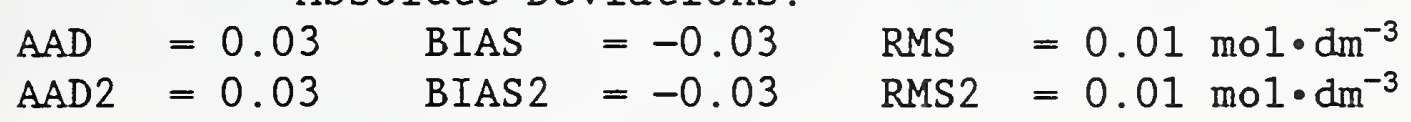

\begin{tabular}{|c|c|c|c|c|c|c|}
\hline $\begin{array}{l}\mathrm{T} \\
\mathrm{K}\end{array}$ & $\begin{array}{l}\rho_{\sigma \mathrm{L}}, \operatorname{expt} \\
\mathrm{mol} \cdot \mathrm{dm}^{-3}\end{array}$ & $\begin{array}{l}\rho_{\sigma \mathrm{L}}, \text { anc. } \\
\mathrm{mol} \cdot \mathrm{dm}^{-3}\end{array}$ & $\begin{array}{c}\operatorname{dev} 1 \\
8\end{array}$ & $\begin{array}{c}\rho_{\sigma \mathrm{L}}, \quad \text { SWEOS } \\
\mathrm{mol} \cdot \mathrm{dm}^{-3}\end{array}$ & $\begin{array}{c}\text { dev2 } \\
\frac{8}{8}\end{array}$ & $w t$ \\
\hline 269.250 & 13.80 & 13.61 & -1.377 & 13.62 & -1.287 & 0.0 \\
\hline 283.150 & 12.41 & 12.42 & 0.137 & 12.44 & 0.298 & 0.0 \\
\hline 255.372 & 14.47 & 14.56 & 0.608 & 14.57 & 0.636 & 0.0 \\
\hline 241.483 & 15.34 & 15.38 & 0.263 & 15.38 & 0.263 & 0.0 \\
\hline
\end{tabular}

Data from Gugnoni et a1. [21]

Number of Points [21] 4

$$
\begin{array}{lll}
\mathrm{AAD} \%=0.596 & \text { BIAS } 8=-0.092 & \mathrm{RMS} \%=0.762 \\
\mathrm{AAD} 2 \%=0.621 & \text { BIAS2\% }=-0.022 & \text { RMS2\% }=0.745
\end{array}
$$

Absolute Deviations:
$\mathrm{AAD}=0.08 \quad \mathrm{BIAS}=-0.01$

\begin{tabular}{|c|c|c|c|c|c|c|}
\hline $\begin{array}{l}\mathrm{T} \\
\mathrm{K}\end{array}$ & $\begin{array}{l}\rho_{\sigma \mathrm{L}}, \operatorname{expt} \\
\mathrm{mol} \cdot \mathrm{dm}^{-3}\end{array}$ & $\begin{array}{l}\rho_{\sigma \mathrm{L}}, \text { anc. } \\
\mathrm{mol} \cdot \mathrm{dm}^{-3}\end{array}$ & $\begin{array}{c}\text { devl } \\
\&\end{array}$ & $\begin{array}{c}\rho_{\sigma \mathrm{L}}, \quad \text { SWEOS } \\
\mathrm{mol} \cdot \mathrm{dm}^{-3}\end{array}$ & $\begin{array}{c}\text { dev2 } \\
\frac{8}{8}\end{array}$ & wt \\
\hline 270.000 & 13.55 & 13.55 & 0.017 & 13.56 & 0.113 & 1.0 \\
\hline 260.000 & 14.26 & 14.26 & -0.001 & 14.27 & 0.044 & 1.0 \\
\hline 250.000 & 14.89 & 14.89 & 0.013 & 14.89 & 0.026 & 1.0 \\
\hline 240.000 & 15.46 & 15.47 & 0.017 & 15.47 & 0.016 & 1.0 \\
\hline 230.000 & 16.00 & 16.00 & 0.013 & 16.00 & 0.008 & 1.0 \\
\hline
\end{tabular}
RMS $=0.11 \mathrm{~mol} \cdot \mathrm{dm}^{-3}$
AAD2 $=0.09 \quad$ BIAS2 $=0.00 \quad$ RMS2 $=0.10 \mathrm{~mol} \cdot \mathrm{dm}^{-3}$

Data from Haynes and Hiza [22] 
Table 2. SATURATED IIQUID DENSITIES (continued)

Data from Haynes and Hiza [22] (continued)

\begin{tabular}{|c|c|c|c|c|c|c|}
\hline $\begin{array}{l}\mathrm{T} \\
\mathrm{K}\end{array}$ & $\begin{array}{l}\rho_{\sigma L}, \operatorname{expt} \\
\mathrm{mol} \cdot \mathrm{dm}^{-3}\end{array}$ & $\begin{array}{l}\rho_{\sigma \mathrm{L}}, \text { anc. } \\
\mathrm{mol} \cdot \mathrm{dm}^{-3}\end{array}$ & $\begin{array}{c}\operatorname{dev} 1 \\
8\end{array}$ & $\begin{array}{c}\rho_{\sigma \mathrm{L}}, \quad \text { SWEOS } \\
\mathrm{mol} \cdot \mathrm{dm}^{-3}\end{array}$ & $\begin{array}{c}\text { dev2 } \\
\frac{8}{8}\end{array}$ & wt \\
\hline 220.000 & 16.50 & 16.50 & -0.004 & 16.50 & -0.005 & 1.0 \\
\hline 210.000 & 16.97 & 16.97 & 0.002 & 16.97 & 0.007 & 1.0 \\
\hline 200.000 & 17.43 & 17.42 & -0.035 & 17.42 & -0.024 & 1.0 \\
\hline 190.000 & 17.86 & 17.86 & -0.023 & 17.86 & -0.008 & 1.0 \\
\hline 180.000 & 18.28 & 18.28 & -0.014 & 18.28 & 0.002 & 1.0 \\
\hline 170.000 & 18.69 & 18.68 & -0.017 & 18.69 & -0.003 & 1.0 \\
\hline 160.000 & 19.09 & 19.08 & -0.025 & 19.08 & -0.017 & 1.0 \\
\hline 150.000 & 19.48 & 19.47 & -0.026 & 19.47 & -0.025 & 1.0 \\
\hline 140.000 & 19.86 & 19.85 & -0.027 & 19.85 & -0.033 & 1.0 \\
\hline 135.000 & 20.05 & 20.04 & -0.029 & 20.04 & -0.038 & 1.0 \\
\hline 130.000 & 20.23 & 20.23 & -0.024 & 20.23 & -0.034 & 1.0 \\
\hline 125.000 & 20.42 & 20.41 & -0.031 & 20.41 & -0.040 & 1.0 \\
\hline 120.000 & 20.60 & 20.60 & -0.024 & 20.60 & -0.032 & 1.0 \\
\hline 115.000 & 20.79 & 20.78 & -0.061 & 20.78 & -0.066 & 1.0 \\
\hline 110.000 & 20.98 & 20.96 & -0.061 & 20.96 & -0.060 & 1.0 \\
\hline 105.000 & 21.16 & 21.14 & -0.075 & 21.14 & -0.069 & 1.0 \\
\hline 100.000 & 21.34 & 21.32 & -0.085 & 21.32 & -0.076 & 1.0 \\
\hline
\end{tabular}

Number of Points [22] 22

$$
\begin{aligned}
& \mathrm{AAD} z=0.028 \quad \text { BIAS\& }=-0.023 \quad \text { RMS } 8=0.028 \\
& \text { AAD2 } 8=0.034 \quad \text { BIAS2 } 8=-0.014 \quad \text { RMS } 28=0.041 \\
& \begin{array}{llllll}
A A D & =0.01 & \text { BIAS } & =0.00 & \text { RMS } & =0.01 \mathrm{~mol} \cdot \mathrm{dm}^{-3} \\
A A D 2 & =0.01 & \text { BIAS2 } & =0.00 & \text { RMS2 } & =0.01 \mathrm{~mol} \cdot \mathrm{dm}^{-3}
\end{array}
\end{aligned}
$$

Weighted Data:

Number of Points [22] 22

$$
\begin{aligned}
& \mathrm{AAD} z=0.028 \quad \text { BIAS } 8=-0.023 \quad \text { RMS } \%=0.028 \\
& \text { AAD2 } z=0.034 \quad \text { BIAS2 } z=-0.014 \quad \text { RMS } 28=0.041
\end{aligned}
$$


Table 2. SATURATED LIQUID DENSITIES (continued)

Data from Kahre [10]

\begin{tabular}{cccrrrr}
\hline $\mathrm{T}$ & $\begin{array}{c}\rho_{\sigma \mathrm{L}}, \operatorname{expt} \\
\mathrm{mol} \cdot \mathrm{dm}^{-3}\end{array}$ & $\begin{array}{c}\rho_{\sigma \mathrm{I}}, \mathrm{anc} \\
\mathrm{mol} \cdot \mathrm{dm}^{-3}\end{array}$ & $\begin{array}{c}\mathrm{dev1} \\
\text { \% }\end{array}$ & $\begin{array}{c}\rho_{\sigma \mathrm{L}}, \mathrm{SWEOS} \\
\mathrm{mol} \cdot \mathrm{dm}^{-3}\end{array}$ & $\begin{array}{c}\text { dev2 } \\
\text { \% }\end{array}$ & wt \\
\hline 299.850 & 10.19 & 10.13 & -0.504 & 10.12 & -0.672 & 0.0 \\
294.250 & 11.15 & 11.12 & -0.292 & 11.12 & -0.269 & 0.0 \\
288.750 & 11.85 & 11.83 & -0.129 & 11.85 & 0.010 & 0.0 \\
277.550 & 12.96 & 12.94 & -0.177 & 12.96 & -0.037 & 0.0 \\
267.450 & 13.74 & 13.74 & -0.018 & 13.75 & 0.063 & 0.0
\end{tabular}

Number of Points [10] 5

$\begin{array}{llll}\mathrm{AAD} \% & =0.224 & \text { BIAS } 8=-0.224 & \text { RMS } 8=0.165 \\ \mathrm{AAD} 2 \%=0.210 & \text { BIAS2\% }=-0.181 & \text { RMS2\% } 280.270\end{array}$

Absolute Deviations:

$A A D=0.02 \quad$ BIAS $=-0.02$ RMS $=0.02 \mathrm{~mol} \cdot \mathrm{dm}^{-3}$

AAD2 $=0.02 \quad$ BIAS2 $=-0.02 \quad \mathrm{RMS} 2=0.03 \mathrm{~mol} \cdot \mathrm{dm}^{-3}$

Data from Klosek and McKinley [23]

\begin{tabular}{cccrrrr}
\hline $\mathrm{T}$ & $\begin{array}{c}\rho_{\sigma \mathrm{I}}, \operatorname{expt} \\
\mathrm{mol} \cdot \mathrm{dm}^{-3}\end{array}$ & $\begin{array}{c}\rho_{\sigma \mathrm{I}}, \mathrm{anc} \\
\mathrm{mol} \cdot \mathrm{dm}^{-3}\end{array}$ & $\begin{array}{c}\text { dev1 } \\
8\end{array}$ & $\begin{array}{c}\rho_{\sigma \mathrm{L}}, \mathrm{SWEOS} \\
\mathrm{mol} \cdot \mathrm{dm}^{-3}\end{array}$ & $\begin{array}{c}\text { dev2 } \\
8\end{array}$ & wt \\
\hline 133.150 & 20.13 & 20.11 & -0.082 & 20.11 & -0.091 & 0.0 \\
127.594 & 20.32 & 20.32 & -0.031 & 20.31 & -0.041 & 0.0 \\
122.039 & 20.52 & 20.52 & 0.005 & 20.52 & -0.004 & 0.0 \\
116.483 & 20.72 & 20.73 & 0.044 & 20.72 & 0.038 & 0.0 \\
110.928 & 20.91 & 20.93 & 0.065 & 20.93 & 0.065 & 0.0 \\
105.372 & 21.11 & 21.13 & 0.117 & 21.13 & 0.123 & 0.0 \\
99.817 & 21.30 & 21.33 & 0.148 & 21.33 & 0.157 & 0.0 \\
94.261 & 21.49 & 21.53 & 0.176 & 21.53 & 0.180 & 0.0
\end{tabular}

Number of Points [23] 8

$\begin{array}{llll}A A D & =0.084 & \text { BIAS } 8=0.055 & \text { RMS } 8=0.084 \\ A A D 2 \%=0.087 & \text { BIAS28 }=0.053 & \text { RMS28 }=0.090\end{array}$

Absolute Deviations:

$\mathrm{AAD}=0.02 \quad \mathrm{BIAS}=0.01 \quad \mathrm{RMS}=0.02 \mathrm{~mol} \cdot \mathrm{dm}^{-3}$

$A A D 2=0.02 \quad$ BIAS2 $=0.01 \quad$ RMS2 $=0.02 \mathrm{~mol} \cdot \mathrm{dm}^{-3}$ 
Table 2. SATURATED LIQUID DENSITIES (continued)

Data from McClune [24]

\begin{tabular}{|c|c|c|c|c|c|c|}
\hline $\begin{array}{l}\mathrm{T} \\
\mathrm{K}\end{array}$ & $\begin{array}{l}\rho_{\sigma L}, \quad \exp t \\
\mathrm{~mol} \cdot \mathrm{dm}^{-3}\end{array}$ & $\begin{array}{l}\rho_{\sigma \mathrm{L}}, \text { anc. } \\
\mathrm{mol} \cdot \mathrm{dm}^{-3}\end{array}$ & $\underset{8}{\operatorname{dev} 1}$ & $\begin{array}{c}\rho_{\sigma \mathrm{L}}, \quad \text { SWEOS } \\
\mathrm{mol} \cdot \mathrm{dm}^{-3}\end{array}$ & $\begin{array}{c}\operatorname{dev} 2 \\
8\end{array}$ & wt \\
\hline 173.150 & 18.57 & 18.56 & -0.045 & 18.56 & -0.030 & 1.0 \\
\hline 168.150 & 18.77 & 18.76 & -0.037 & 18.76 & -0.024 & 1.0 \\
\hline 163.150 & 18.96 & 18.96 & -0.037 & 18.96 & -0.027 & 1.0 \\
\hline 158.150 & 19.16 & 19.15 & -0.022 & 19.16 & -0.015 & 1.0 \\
\hline 153.150 & 19.35 & 19.35 & -0.029 & 19.35 & -0.026 & 1.0 \\
\hline 148.150 & 19.55 & 19.54 & -0.030 & 19.54 & -0.030 & 1.0 \\
\hline 143.150 & 19.74 & 19.73 & -0.030 & 19.73 & -0.034 & 1.0 \\
\hline 138.150 & 19.92 & 19.92 & 0.003 & 19.92 & -0.004 & 1.0 \\
\hline 133.150 & 20.11 & 20.11 & 0.022 & 20.11 & 0.013 & 1.0 \\
\hline 128.150 & 20.29 & 20.30 & 0.024 & 20.29 & 0.014 & 1.0 \\
\hline 123.150 & 20.48 & 20.48 & 0.025 & 20.48 & 0.016 & 1.0 \\
\hline 118.150 & 20.66 & 20.67 & 0.015 & 20.66 & 0.008 & 1.0 \\
\hline 113.150 & 20.85 & 20.85 & 0.008 & 20.85 & 0.006 & 1.0 \\
\hline 108.150 & 21.03 & 21.03 & 0.006 & 21.03 & 0.009 & 1.0 \\
\hline 103.150 & 21.21 & 21.21 & 0.004 & 21.21 & 0.011 & 1.0 \\
\hline 98.150 & 21.39 & 21.39 & 0.005 & 21.39 & 0.014 & 1.0 \\
\hline 93.150 & 21.57 & 21.57 & -0.003 & 21.57 & -0.001 & 1.0 \\
\hline
\end{tabular}

Number of Points [24] 17

$$
\begin{aligned}
& \mathrm{AAD} \%=0.020 \quad \mathrm{BIAS} \%=-0.007 \quad \mathrm{RMS} \%=0.023 \\
& \text { AAD2 } \%=0.017 \quad \text { BIAS2 } z=-0.006 \quad \text { RMS } 2 \%=0.018
\end{aligned}
$$

\begin{tabular}{|c|c|c|c|c|c|}
\hline $\mathrm{AAD}$ & 0.00 & BIAS & 0.00 & RMS & $=0.00 \mathrm{~mol} \cdot \mathrm{dm}^{-3}$ \\
\hline AAD2 & $=0.00$ & BIAS2 & 0.00 & RMS2 & $=0.00 \mathrm{~mol} \cdot \mathrm{dm}^{-3}$ \\
\hline
\end{tabular}

Weighted Data:

Number of Points [24] 17

$$
\begin{array}{llll}
\text { AAD } 8=0.020 & \text { BIAS } z=-0.007 & \text { RMS } q=0.023 \\
\text { AAD2 } 8=0.017 & \text { BIAS2 } 8=-0.006 & \text { RMS2 } 8=0.018
\end{array}
$$

Absolute Deviations:

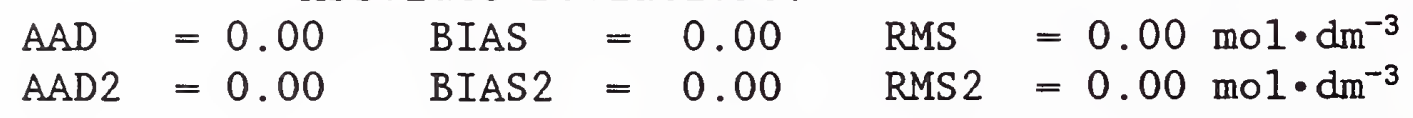


Table 2. SATURATED LIQUID DENSITIES (continued)

Data from Miniovich and Sorina [12]

\begin{tabular}{|c|c|c|c|c|c|c|c|c|c|c|}
\hline & $\begin{array}{l}\mathrm{T} \\
\mathrm{K}\end{array}$ & & $\begin{array}{l}\sigma L, \quad \operatorname{expt} \\
0 I \cdot \mathrm{dm}^{-3}\end{array}$ & $\begin{array}{l}\rho_{\sigma \mathrm{L}}, \quad \text { an } \\
\mathrm{mol} \cdot \mathrm{dm}\end{array}$ & nc. & $\begin{array}{c}\text { devl } \\
\frac{8}{8}\end{array}$ & $\begin{array}{l}\rho_{\sigma L}, \\
\text { mol }\end{array}$ & $\begin{array}{l}\text { SWEOS } \\
\cdot \mathrm{dm}^{-3}\end{array}$ & $\begin{array}{c}\text { dev2 } \\
8\end{array}$ & wt \\
\hline & 305.262 & & 7.62 & 7.54 & & -1.074 & & 7.59 & -0.430 & 0.0 \\
\hline & 305.025 & & 8.05 & 8.02 & & -0.446 & & 8.08 & 0.356 & 0.0 \\
\hline & 304.568 & & 8.49 & 8.46 & & -0.314 & & 8.51 & 0.227 & 0.0 \\
\hline & 303.880 & & 8.89 & 8.88 & & -0.184 & & 8.90 & 0.046 & 0.0 \\
\hline & 302.983 & & 9.27 & 9.26 & & -0.112 & & 9.26 & -0.110 & 0.0 \\
\hline Number & of Points & [12] & 5 & & & & & & & \\
\hline$A A D \&$ & $=0.426$ & BIAS 8 & $=-0.426$ & RMS 8 & $=$ & 0.343 & & & & \\
\hline $\mathrm{AAD} 2 \%$ & $=0.234$ & BIAS $2 \%$ & $=0.018$ & RMS 28 & $=$ & 0.274 & & & & \\
\hline & Abs & Lute $\mathrm{De}$ & viations: & & & & & & & \\
\hline$A A D$ & $=0.03$ & BIAS & $=-0.03$ & RMS & $=$ & $0.03 \mathrm{mo}$ & $d^{-3}$ & & & \\
\hline $\mathrm{AAD} 2$ & $=0.02$ & BIAS 2 & $=0.00$ & RMS 2 & $=$ & $0.02 \mathrm{mo}$ & $\mathrm{dm}^{-3}$ & & & \\
\hline
\end{tabular}

Data from Orrit et al. [25]

\begin{tabular}{|c|c|c|c|c|c|c|}
\hline $\begin{array}{l}\mathrm{T} \\
\mathrm{K}\end{array}$ & $\begin{array}{l}\rho_{\sigma L}, \operatorname{expt} \\
\mathrm{mol} \cdot \mathrm{dm}^{-3}\end{array}$ & $\begin{array}{l}\rho_{\sigma L}, \text { anc. } \\
\text { mol } \cdot \mathrm{dm}^{-3}\end{array}$ & $\underset{\frac{8}{8}}{\operatorname{dev} 1}$ & $\begin{array}{c}\rho_{\sigma \mathrm{L}}, \quad \text { SWEOS } \\
\mathrm{mol} \cdot \mathrm{dm}^{-3}\end{array}$ & $\begin{array}{c}\text { dev2 } \\
\frac{8}{8}\end{array}$ & wt \\
\hline 228.361 & 16.07 & 16.08 & 0.074 & 16.08 & 0.070 & 0.0 \\
\hline 222.937 & 16.35 & 16.35 & 0.035 & 16.35 & 0.032 & 0.0 \\
\hline 203.192 & 17.27 & 17.28 & 0.034 & 17.28 & 0.043 & 0.0 \\
\hline 194.567 & 17.65 & 17.66 & 0.049 & 17.66 & 0.063 & 0.0 \\
\hline 187.951 & 17.94 & 17.94 & 0.044 & 17.95 & 0.060 & 0.0 \\
\hline 182.416 & 18.16 & 18.18 & 0.067 & 18.18 & 0.084 & 0.0 \\
\hline 176.870 & 18.40 & 18.41 & 0.039 & 18.41 & 0.055 & 0.0 \\
\hline 165.650 & 18.85 & 18.86 & 0.063 & 18.86 & 0.075 & 0.0 \\
\hline 154.664 & 19.28 & 19.29 & 0.050 & 19.29 & 0.054 & 0.0 \\
\hline 149.049 & 19.50 & 19.51 & 0.060 & 19.51 & 0.060 & 0.0 \\
\hline 143.718 & 19.70 & 19.71 & 0.069 & 19.71 & 0.065 & 0.0 \\
\hline 138.355 & 19.90 & 19.91 & 0.080 & 19.91 & 0.073 & 0.0 \\
\hline 130.318 & 20.20 & 20.22 & 0.051 & 20.21 & 0.041 & 0.0 \\
\hline 124.997 & 20.40 & 20.41 & 0.063 & 20.41 & 0.053 & 0.0 \\
\hline 119.623 & 20.60 & 20.61 & 0.058 & 20.61 & 0.050 & 0.0 \\
\hline 114.221 & 20.80 & 20.81 & 0.066 & 20.81 & 0.062 & 0.0 \\
\hline 108.932 & 20.99 & 21.00 & 0.048 & 21.00 & 0.049 & 0.0 \\
\hline 103.710 & 21.18 & 21.19 & 0.022 & 21.19 & 0.029 & 0.0 \\
\hline 218.526 & 16.56 & 16.57 & 0.058 & 16.57 & 0.057 & 0.0 \\
\hline 207.229 & 17.08 & 17.10 & 0.131 & 17.10 & 0.138 & 0.0 \\
\hline
\end{tabular}


Table 2. SATURATED LIQUID DENSITIES (continued)

Data from Orrit et al. [25] (continued)

\begin{tabular}{|c|c|c|c|c|c|c|}
\hline $\begin{array}{l}\mathrm{T} \\
\mathrm{K}\end{array}$ & $\begin{array}{l}\rho_{\sigma \mathrm{L}}, \operatorname{expt} \\
\mathrm{mol} \cdot \mathrm{dm}^{-3}\end{array}$ & $\begin{array}{l}\rho_{\sigma \mathrm{L}}, \text { anc. } \\
\mathrm{mol} \cdot \mathrm{dm}^{-3}\end{array}$ & $\begin{array}{c}\text { devl } \\
\frac{8}{8}\end{array}$ & $\begin{array}{c}\rho_{\sigma \mathrm{L}}, \quad \text { SWEOS } \\
\mathrm{mol} \cdot \mathrm{dm}^{-3}\end{array}$ & $\begin{array}{c}\operatorname{dev} 2 \\
8\end{array}$ & wt \\
\hline 199.012 & 17.46 & 17.47 & 0.060 & 17.47 & 0.072 & 0.0 \\
\hline 190.724 & 17.82 & 17.83 & 0.039 & 17.83 & 0.054 & 0.0 \\
\hline 185.157 & 18.06 & 18.06 & 0.015 & 18.06 & 0.031 & 0.0 \\
\hline 179.604 & 18.28 & 18.29 & 0.053 & 18.30 & 0.070 & 0.0 \\
\hline 174.114 & 18.51 & 18.52 & 0.046 & 18.52 & 0.061 & 0.0 \\
\hline 168.639 & 18.73 & 18.74 & 0.045 & 18.74 & 0.059 & 0.0 \\
\hline 162.861 & 18.96 & 18.97 & 0.060 & 18.97 & 0.070 & 0.0 \\
\hline 157.490 & 19.18 & 19.18 & 0.013 & 19.18 & 0.020 & 0.0 \\
\hline 151.915 & 19.39 & 19.40 & 0.052 & 19.40 & 0.055 & 0.0 \\
\hline 140.648 & 19.81 & 19.83 & 0.071 & 19.83 & 0.066 & 0.0 \\
\hline 127.679 & 20.30 & 20.31 & 0.056 & 20.31 & 0.046 & 0.0 \\
\hline 117.003 & 20.70 & 20.71 & 0.034 & 20.71 & 0.028 & 0.0 \\
\hline 106.165 & 21.09 & 21.10 & 0.043 & 21.10 & 0.048 & 0.0 \\
\hline 232.107 & 15.90 & 15.89 & -0.089 & 15.89 & -0.094 & 1.0 \\
\hline 225.698 & 16.22 & 16.22 & -0.016 & 16.22 & -0.020 & 1.0 \\
\hline 212.621 & 16.84 & 16.85 & 0.081 & 16.85 & 0.084 & 1.0 \\
\hline 203.191 & 17.27 & 17.28 & 0.034 & 17.28 & 0.043 & 1.0 \\
\hline 190.723 & 17.82 & 17.83 & 0.039 & 17.83 & 0.055 & 1.0 \\
\hline 182.415 & 18.16 & 18.18 & 0.062 & 18.18 & 0.079 & 1.0 \\
\hline 171.338 & 18.62 & 18.63 & 0.048 & 18.63 & 0.062 & 1.0 \\
\hline 160.100 & 19.07 & 19.08 & 0.043 & 19.08 & 0.052 & 1.0 \\
\hline 146.454 & 19.59 & 19.61 & 0.077 & 19.61 & 0.076 & 1.0 \\
\hline 132.767 & 20.11 & 20.12 & 0.049 & 20.12 & 0.039 & 1.0 \\
\hline 122.296 & 20.50 & 20.51 & 0.071 & 20.51 & 0.062 & 1.0 \\
\hline 111.491 & 20.90 & 20.91 & 0.053 & 20.91 & 0.052 & 1.0 \\
\hline 102.578 & 21.23 & 21.23 & 0.002 & 21.23 & 0.009 & 1.0 \\
\hline
\end{tabular}

Number of Points [25] 46

$$
\begin{aligned}
& \mathrm{AAD} ;=0.052 \quad \mathrm{BIAS} \%=0.048 \quad \mathrm{RMS} \%=0.031 \\
& \text { AAD2\% } 280.057 \quad \text { BIAS2\% }=0.052 \quad \text { RMS } 2 \%=0.032
\end{aligned}
$$

Absolute Deviations:

$$
\begin{array}{llllll}
A A D & =0.01 & \text { BIAS } & =0.01 & \text { RMS } & =0.01 \mathrm{~mol} \cdot \mathrm{dm}^{-3} \\
A A D 2 & =0.01 & \text { BIAS2 } & =0.01 & \text { RMS2 } & =0.01 \mathrm{~mol} \cdot \mathrm{dm}^{-3}
\end{array}
$$


Table 2. SATURATED LIQUID DENSITIES (continued)

Data from Orrit et al. [25] (continued)

Weighted Data:

Number of Points [25] 13

$$
\begin{array}{llll}
\mathrm{AAD} \% & =0.051 & \text { BIAS } 8=0.035 & \mathrm{RMS} \%=0.044 \\
\mathrm{AAD} 2 \%=0.056 & \text { BIAS2\% } & =0.038 & \mathrm{RMS} 2 \%=0.047
\end{array}
$$

Absolute Deviations:

$\begin{array}{llllll}A A D & =0.01 & \text { BIAS } & =0.01 & \text { RMS } & =0.01 \mathrm{~mol} \cdot \mathrm{dm}^{-3} \\ A A D 2 & =0.01 & \text { BIAS2 } & =0.01 & \text { RMS2 } & =0.01 \mathrm{~mol} \cdot \mathrm{dm}^{-3}\end{array}$

\begin{tabular}{|c|c|c|c|c|c|c|c|c|c|}
\hline & $\begin{array}{l}\mathrm{T} \\
\mathrm{K}\end{array}$ & & $\begin{array}{l}\rho_{\sigma L}, \operatorname{expt} \\
\text { mol } \cdot \mathrm{dm}^{-3}\end{array}$ & $\begin{array}{l}\rho_{\sigma \mathrm{L}}, \text { anc } \\
\mathrm{mol} \cdot \mathrm{dm}^{-3}\end{array}$ & & $\begin{array}{c}\operatorname{dev} 1 \\
8\end{array}$ & $\begin{array}{c}\rho_{\sigma \mathrm{I}}, \quad \text { SWEOS } \\
\mathrm{mol} \cdot \mathrm{dm}^{-3}\end{array}$ & $\begin{array}{c}\text { dev2 } \\
8\end{array}$ & wt \\
\hline & 304.038 & & 8.81 & 8.80 & & -0.172 & 8.82 & 0.116 & 0.0 \\
\hline & 301.727 & & 9.69 & 9.67 & & -0.228 & 9.65 & -0.366 & 0.0 \\
\hline & 292.736 & & 11.38 & 11.33 & & -0.371 & 11.34 & -0.304 & 0.0 \\
\hline & 277.858 & & 12.99 & 12.91 & & -0.575 & 12.93 & -0.434 & 0.0 \\
\hline & 268.412 & & 13.71 & 13.67 & & -0.324 & 13.68 & -0.238 & 0.0 \\
\hline & 256.493 & & 14.55 & 14.49 & & -0.435 & 14.49 & -0.403 & 0.0 \\
\hline & 248.479 & & 15.05 & 14.98 & & -0.435 & 14.98 & -0.425 & 0.0 \\
\hline & 241.202 & & 15.45 & 15.40 & & -0.343 & 15.40 & -0.343 & 0.0 \\
\hline & 230.486 & & 16.03 & 15.97 & & -0.368 & 15.97 & -0.373 & 0.0 \\
\hline & 223.500 & & 16.42 & 16.33 & & -0.579 & 16.33 & -0.582 & 0.0 \\
\hline & 215.775 & & 16.75 & 16.70 & & -0.304 & 16.70 & -0.303 & 0.0 \\
\hline Number & of Points & [13] & 11 & & & & & & \\
\hline $\mathrm{AAD} \&$ & $=0.376$ & BIAS 8 & $=-0.376$ & RMS 8 & & 0.121 & & & \\
\hline$A A D 28$ & $=0.353$ & BIAS 28 & $8=-0.332$ & RMS28 $=$ & $=$ & 0.166 & & & \\
\hline & Abs & lute $\mathrm{De}$ & eviations: & & & & & & \\
\hline$A A D$ & $=0.05$ & BIAS & $=-0.05$ & RMS & & $0.02 \mathrm{~mol}$ & $\mathrm{~m}^{-3}$ & & \\
\hline AAD2 & $=0.05$ & BIAS2 & $=-0.05$ & RMS2 & $=$ & $0.02 \mathrm{~mol}$ & $\mathrm{~lm}^{-3}$ & & \\
\hline
\end{tabular}

Data from Pal et al. [13] 
Table 2. SATURATED LIQUID DENSITIES (continued)

Data from Pestak et al. [26]

\begin{tabular}{|c|c|c|c|c|c|c|}
\hline $\begin{array}{l}\mathrm{T} \\
\mathrm{K}\end{array}$ & $\begin{array}{l}\rho_{\sigma \mathrm{L}}, \operatorname{expt} \\
\mathrm{mol} \cdot \mathrm{dm}^{-3}\end{array}$ & $\begin{array}{l}\rho_{\sigma \mathrm{L}}, \text { anc. } \\
\mathrm{mol} \cdot \mathrm{dm}^{-3}\end{array}$ & $\begin{array}{c}\text { devl } \\
8\end{array}$ & $\begin{array}{c}\rho_{\sigma \mathrm{L}}, \quad \text { SWEOS } \\
\mathrm{mol} \cdot \mathrm{dm}^{-3}\end{array}$ & $\begin{array}{c}\text { dev2 } \\
\frac{8}{8}\end{array}$ & wt \\
\hline 305.173 & 7.65 & 7.77 & 1.639 & 7.84 & 2.469 & 0.0 \\
\hline 305.166 & 7.67 & 7.79 & 1.598 & 7.85 & 2.443 & 0.0 \\
\hline 305.149 & 7.70 & 7.82 & 1.557 & 7.89 & 2.426 & 0.0 \\
\hline 305.134 & 7.74 & 7.85 & 1.365 & 7.91 & 2.194 & 0.0 \\
\hline 305.117 & 7.77 & 7.88 & 1.389 & 7.94 & 2.249 & 0.0 \\
\hline 305.102 & 7.81 & 7.90 & 1.210 & 7.97 & 2.067 & 0.0 \\
\hline 305.084 & 7.84 & 7.93 & 1.131 & 8.00 & 1.983 & 0.0 \\
\hline 305.053 & 7.90 & 7.98 & 0.990 & 8.04 & 1.836 & 0.0 \\
\hline 305.021 & 7.95 & 8.02 & $0.9 n 8$ & 8.09 & 1.739 & 0.0 \\
\hline 304.987 & 8.00 & 8.07 & 0.838 & 8.13 & 1.630 & 0.0 \\
\hline 304.964 & 8.02 & 8.09 & 0.879 & 8.16 & 1.670 & 0.0 \\
\hline 304.932 & 8.08 & 8.13 & 0.660 & 8.19 & 1.424 & 0.0 \\
\hline 304.900 & 8.11 & 8.17 & 0.708 & 8.23 & 1.453 & 0.0 \\
\hline 304.868 & 8.15 & 8.20 & 0.646 & 8.26 & 1.369 & 0.0 \\
\hline 304.836 & 8.17 & 8.23 & 0.714 & 8.29 & 1.421 & 0.0 \\
\hline 304.805 & 8.21 & 8.26 & 0.603 & 8.32 & 1.290 & 0.0 \\
\hline 304.757 & 8.25 & 8.31 & 0.666 & 8.36 & 1.329 & 0.0 \\
\hline 304.709 & 8.30 & 8.35 & 0.540 & 8.40 & 1.172 & 0.0 \\
\hline 304.662 & 8.34 & 8.39 & 0.575 & 8.44 & 1.174 & 0.0 \\
\hline 304.457 & 8.50 & 8.54 & 0.487 & 8.58 & 0.970 & 0.0 \\
\hline 304.379 & 8.56 & 8.59 & 0.435 & 8.63 & 0.878 & 0.0 \\
\hline 304.301 & 8.61 & 8.64 & 0.328 & 8.68 & 0.732 & 0.0 \\
\hline 304.146 & 8.70 & 8.74 & 0.413 & 8.76 & 0.747 & 0.0 \\
\hline 303.988 & 8.79 & 8.82 & 0.303 & 8.85 & 0.573 & 0.0 \\
\hline 303.838 & 8.87 & 8.90 & 0.365 & 8.92 & 0.583 & 0.0 \\
\hline 303.686 & 8.94 & 8.97 & 0.352 & 8.99 & 0.520 & 0.0 \\
\hline 303.533 & 9.01 & 9.04 & 0.295 & 9.05 & 0.420 & 0.0 \\
\hline 303.383 & 9.08 & 9.10 & 0.215 & 9.11 & 0.301 & 0.0 \\
\hline 303.234 & 9.14 & 9.16 & 0.296 & 9.17 & 0.348 & 0.0 \\
\hline 302.865 & 9.29 & 9.30 & 0.144 & 9.30 & 0.126 & 0.0 \\
\hline 302.503 & 9.41 & 9.43 & 0.215 & 9.42 & 0.145 & 0.0 \\
\hline 302.143 & 9.53 & 9.54 & 0.175 & 9.53 & 0.067 & 0.0 \\
\hline 301.722 & 9.65 & 9.67 & 0.192 & 9.65 & 0.052 & 0.0 \\
\hline 301.310 & 9.77 & 9.78 & 0.082 & 9.77 & -0.077 & 0.0 \\
\hline 300.770 & 9.91 & 9.92 & 0.144 & 9.90 & -0.028 & 0.0 \\
\hline
\end{tabular}


Table 2. SATURATED LIQUID DENSITIES (continued)

Data from Pestak et al. [26] (continued)

\begin{tabular}{|c|c|c|c|c|c|c|c|c|}
\hline & $\begin{array}{l}\mathrm{T} \\
\mathrm{K}\end{array}$ & & $\begin{array}{l}\sigma L, \operatorname{expt} \\
01 \cdot \mathrm{dm}^{-3}\end{array}$ & $\begin{array}{l}\rho_{\sigma \mathrm{L}}, \text { anc. } \\
\mathrm{mol} \cdot \mathrm{dm}^{-3}\end{array}$ & $\begin{array}{c}\operatorname{dev} 1 \\
8\end{array}$ & $\begin{array}{c}\rho_{\sigma \mathrm{L}}, \quad \text { SWEOS } \\
\mathrm{mol} \cdot \mathrm{dm}^{-3}\end{array}$ & $\begin{array}{c}\text { dev2 } \\
\frac{8}{8}\end{array}$ & wt \\
\hline & 300.238 & & 10.05 & 10.05 & 0.010 & 10.03 & -0.163 & 0.0 \\
\hline & 299.722 & & 10.15 & 10.16 & 0.155 & 10.15 & -0.012 & 0.0 \\
\hline & 299.219 & & 10.27 & 10.27 & 0.035 & 10.25 & -0.121 & 0.0 \\
\hline & 298.730 & & 10.36 & 10.37 & 0.073 & 10.35 & -0.070 & 0.0 \\
\hline Number & of Points & [26] & 39 & & & & & \\
\hline $\mathrm{AAD} \&$ & $=0.598$ & BIAS 8 & $=0.598$ & RMS क \& & & & & \\
\hline $\mathrm{AAD} 28$ & $=1.033$ & BIAS $2 \%$ & $=1.008$ & $\operatorname{RMS} 28=0$ & & & & \\
\hline & Abs & lute $\mathrm{De}$ & viations: & & & & & \\
\hline$A A D$ & $=0.05$ & BIAS & $=0.05$ & RMS & $3 \mathrm{~mol} \cdot$ & & & \\
\hline AAD2 & $=0.08$ & BIAS2 & $=0.08$ & RMS2 & $6 \mathrm{~mol}$. & & & \\
\hline
\end{tabular}

Data from Rodosevich and Miller [27]

\begin{tabular}{|c|c|c|c|c|c|c|}
\hline $\begin{array}{l}\mathrm{T} \\
\mathrm{K}\end{array}$ & $\begin{array}{l}\rho_{\sigma \mathrm{L}}, \exp t \\
\mathrm{~mol} \cdot \mathrm{dm}^{-3}\end{array}$ & $\begin{array}{l}\rho_{\sigma \mathrm{L}}, \text { anc. } \\
\mathrm{mol} \cdot \mathrm{dm}^{-3}\end{array}$ & $\begin{array}{c}\operatorname{dev} 1 \\
8\end{array}$ & $\begin{array}{c}\rho_{\sigma \mathrm{L}}, \quad \text { SWEOS } \\
\mathrm{mol} \cdot \mathrm{dm}^{-3}\end{array}$ & $\begin{array}{c}\text { dev2 } \\
8\end{array}$ & wt \\
\hline 115.050 & 20.77 & 20.78 & 0.031 & 20.78 & 0.027 & 1.0 \\
\hline 108.110 & 21.02 & 21.03 & 0.027 & 21.03 & 0.030 & 1.0 \\
\hline 100.020 & 21.31 & 21.32 & 0.043 & 21.32 & 0.052 & 1.0 \\
\hline 91.010 & 21.64 & 21.64 & 0.020 & 21.64 & 0.013 & 1.0 \\
\hline
\end{tabular}

Number of Points [27] 4
$\mathrm{AAD} 8=0.030$
BIAS $8=0.030$
BIAS28 $=0.030$
RMS $8=0.008$
$\mathrm{AAD} 28=0.030$
RMS $28=0.014$
Absolute Deviations:
$\mathrm{AAD}=0.01$
BIAS $=0.01$
RMS $=0.00 \mathrm{~mol} \cdot \mathrm{dm}^{-3}$
$\mathrm{AAD} 2=0.01$
BIAS2 $=0.01$
RMS2 $=0.00 \mathrm{~mol} \cdot \mathrm{dm}^{-3}$ 
Table 2. SATURATED LIQUID DENSITIES (continued)

Data from Rodosevich and Miller [27] (continued)

Weighted Data:

Number of Points [27] 4

$$
\begin{aligned}
& \text { AAD } 8=0.030 \quad \text { BIAS } 8=0.030 \quad \text { RMS } 8=0.008 \\
& \text { AAD2 } z=0.030 \quad \text { BIAS2 } z=0.030 \quad \text { RMS } 2 z=0.014
\end{aligned}
$$

Absolute Deviations:

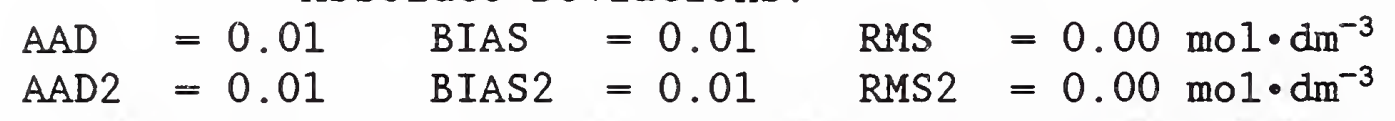

\begin{tabular}{|c|c|c|c|c|c|c|}
\hline $\begin{array}{l}\mathrm{T} \\
\mathrm{K}\end{array}$ & $\begin{array}{l}\rho_{\sigma \mathrm{L}}, \operatorname{expt} \\
\mathrm{mol} \cdot \mathrm{dm}^{-3}\end{array}$ & $\begin{array}{l}\rho_{\sigma \mathrm{L}} \text {, anc. } \\
\mathrm{mol} \cdot \mathrm{dm}^{-3}\end{array}$ & $\begin{array}{c}\text { devl } \\
\text { \& }\end{array}$ & $\begin{array}{c}\rho_{\sigma \mathrm{L}}, \quad \text { SWEOS } \\
\mathrm{mol} \cdot \mathrm{dm}^{-3}\end{array}$ & $\begin{array}{c}\text { dev2 } \\
8\end{array}$ & wt \\
\hline 305.150 & 7.83 & 7.82 & -0.103 & 7.89 & 0.750 & 0.0 \\
\hline 304.650 & 8.40 & 8.40 & 0.006 & 8.45 & 0.599 & 0.0 \\
\hline 304.150 & 8.73 & 8.73 & 0.016 & 8.76 & 0.352 & 0.0 \\
\hline 303.160 & 9.19 & 9.19 & 0.037 & 9.20 & 0.072 & 0.0 \\
\hline 302.160 & 9.53 & 9.54 & 0.045 & 9.53 & -0.061 & 0.0 \\
\hline 300.660 & 9.94 & 9.95 & 0.102 & 9.93 & -0.071 & 0.0 \\
\hline 298.170 & 10.47 & 10.48 & 0.044 & 10.46 & -0.079 & 1.0 \\
\hline 295.670 & 10.90 & 10.91 & 0.066 & 10.90 & 0.039 & 0.0 \\
\hline 293.180 & 11.27 & 11.27 & 0.028 & 11.28 & 0.083 & 1.0 \\
\hline 288.190 & 11.89 & 11.89 & -0.002 & 11.91 & 0.143 & 1.0 \\
\hline 283.200 & 12.42 & 12.42 & -0.024 & 12.44 & 0.138 & 1.0 \\
\hline
\end{tabular}

Data from Sliwinski [28]

Number of Points [28] 11

$$
\begin{aligned}
& \mathrm{AAD} 8=0.043 \quad \mathrm{BIAS} \&=0.019 \quad \mathrm{RMS} 8=0.051 \\
& A A D 28=0.217 \quad \text { BIAS } 28=0.179 \quad \text { RMS } 28=0.263 \\
& \text { Absolute Deviations: } \\
& \mathrm{AAD}=0.00 \quad \mathrm{BIAS}=0.00 \mathrm{RMS}=0.00 \mathrm{~mol} \cdot \mathrm{dm}^{-3} \\
& \text { AAD2 }=0.02 \quad \text { BIAS2 }=0.02 \quad \text { RMS2 }=0.02 \mathrm{~mol} \cdot \mathrm{dm}^{-3}
\end{aligned}
$$


Table 2. SATURATED LIQUID DENSITIES (continued)

Data from Sliwinski [28] (continued)

Weighted Data:

Number of Points [28] 4

$$
\begin{aligned}
& \mathrm{AAD} \&=0.024 \quad \text { BIAS\& }=0.011 \quad \mathrm{RMS} \%=0.026 \\
& \mathrm{AAD} 2 \%=0.111 \quad \text { BIAS2 } z=0.071 \quad \mathrm{RMS} 2 \%=0.090
\end{aligned}
$$

\begin{tabular}{|c|c|c|c|c|c|}
\hline & & ute & viation & & \\
\hline AI & $=0.00$ & BIAS & $=0.00$ & RMS & $=0.00 \mathrm{~mol} \cdot \mathrm{dm}^{-3}$ \\
\hline $\mathrm{AAD} 2$ & $=0.01$ & BIAS2 & $=0.01$ & RMS2 & $=0.01 \mathrm{~mol} \cdot \mathrm{dm}^{-3}$ \\
\hline
\end{tabular}

\begin{tabular}{|c|c|c|c|c|c|c|}
\hline $\begin{array}{l}\mathrm{T} \\
\mathrm{K}\end{array}$ & $\begin{array}{l}\rho_{\sigma L}, \operatorname{expt} \\
\mathrm{mol} \cdot \mathrm{dm}^{-3}\end{array}$ & $\begin{array}{l}\rho_{\sigma L}, \text { anc. } \\
\mathrm{mol} \cdot \mathrm{dm}^{-3}\end{array}$ & $\begin{array}{c}\operatorname{dev} 1 \\
\frac{\gamma}{\delta}\end{array}$ & $\begin{array}{c}\rho_{\sigma \mathrm{L}}, \quad \text { SWEOS } \\
\mathrm{mol} \cdot \mathrm{dm}^{-3}\end{array}$ & $\begin{array}{c}\operatorname{dev} 2 \\
\frac{8}{8}\end{array}$ & wt \\
\hline 302.160 & 9.58 & 9.54 & -0.416 & 9.53 & -0.522 & 0.0 \\
\hline 300.660 & 9.98 & 9.95 & -0.332 & 9.93 & -0.503 & 0.0 \\
\hline 298.170 & 10.50 & 10.48 & -0.242 & 10.46 & -0.365 & 0.0 \\
\hline 295.670 & 10.93 & 10.91 & -0.172 & 10.90 & -0.199 & 0.0 \\
\hline 293.180 & 11.29 & 11.27 & -0.150 & 11.28 & -0.094 & 0.0 \\
\hline 288.190 & 11.91 & 11.89 & -0.119 & 11.91 & 0.026 & 0.0 \\
\hline 283.200 & 12.43 & 12.42 & -0.112 & 12.44 & 0.049 & 0.0 \\
\hline
\end{tabular}

Data from Tomlinson [29]

Number of Points [29] 7

$$
\begin{array}{llll}
\mathrm{AAD} \% & =0.220 & \text { BIAS } 8=-0.220 & \text { RMS } 8=0.107 \\
\mathrm{AAD} 2 \%=0.251 & \text { BIAS2\% }=-0.230 & \text { RMS2\% }=0.221
\end{array}
$$

\begin{tabular}{|c|c|c|c|c|c|c|}
\hline $\begin{array}{l}\mathrm{T} \\
\mathrm{K}\end{array}$ & $\begin{array}{l}\rho_{\sigma \mathrm{L}}, \operatorname{expt} \\
\mathrm{mol} \cdot \mathrm{dm}^{-3}\end{array}$ & $\begin{array}{l}\rho_{\sigma \mathrm{L}}, \text { anc. } \\
\mathrm{mol} \cdot \mathrm{dm}^{-3}\end{array}$ & $\begin{array}{c}\operatorname{dev} 1 \\
8\end{array}$ & $\begin{array}{c}\rho_{\sigma \mathrm{L}}, \quad \text { SWEOS } \\
\mathrm{mol} \cdot \mathrm{dm}^{-3}\end{array}$ & $\begin{array}{c}\text { dev2 } \\
\frac{8}{8}\end{array}$ & wt* \\
\hline 91.000 & & 21.644 & & 21.642 & -0.007 & 2.55 \\
\hline 96.000 & & 21.466 & & 21.467 & 0.007 & 2.55 \\
\hline 101.000 & & 21.287 & & 21.289 & 0.009 & 2.55 \\
\hline 106.000 & & 21.107 & & 21.108 & 0.005 & 2.55 \\
\hline 111.000 & & 20.926 & & 20.926 & 0.000 & 2.55 \\
\hline
\end{tabular}

Absolute Deviations:

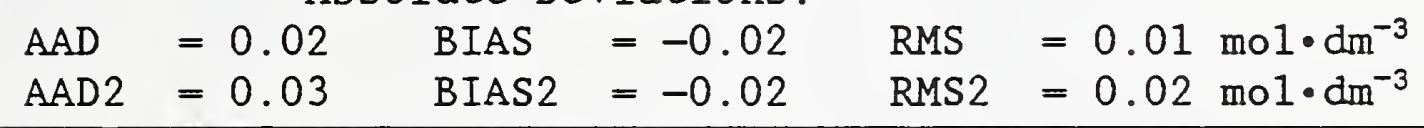

Data from ancillary equation [1] 
Table 2. SATURATED LIQUID DENSITIES (continued)

Data from ancillary equation [1] (continued)

\begin{tabular}{|c|c|c|c|c|c|c|}
\hline $\begin{array}{l}\mathrm{T} \\
\mathrm{K}\end{array}$ & $\begin{array}{l}\rho_{\sigma \mathrm{L}}, \operatorname{expt} \\
\mathrm{mol} \cdot \mathrm{dm}^{-3}\end{array}$ & $\begin{array}{l}\rho_{\sigma \mathrm{L}}, \text { anc. } \\
\mathrm{mol} \cdot \mathrm{dm}^{-3}\end{array}$ & $\begin{array}{c}\text { dev1 } \\
\frac{8}{8}\end{array}$ & $\begin{array}{c}\rho_{\sigma \mathrm{L}}, \quad \text { SWEOS } \\
\mathrm{mol} \cdot \mathrm{dm}^{-3}\end{array}$ & $\begin{array}{c}\text { dev2 } \\
8\end{array}$ & wt* \\
\hline 116.000 & & 20.744 & & 20.743 & -0.005 & 2.55 \\
\hline 121.000 & & 20.560 & & 20.559 & -0.008 & 2.55 \\
\hline 126.000 & & 20.376 & & 20.374 & -0.010 & 2.55 \\
\hline 131.000 & & 20.190 & & 20.188 & -0.010 & 2.55 \\
\hline 136.000 & & 20.003 & & 20.001 & -0.008 & 2.55 \\
\hline 141.000 & & 19.814 & & 19.813 & -0.005 & 2.55 \\
\hline 146.000 & & 19.623 & & 19.623 & -0.002 & 2.55 \\
\hline 151.000 & & 19.431 & & 19.432 & 0.002 & 2.55 \\
\hline 156.000 & & 19.238 & & 19.239 & 0.005 & 2.55 \\
\hline 161.000 & & 19.042 & & 19.044 & 0.009 & 2.55 \\
\hline 166.000 & & 18.844 & & 18.846 & 0.012 & 2.55 \\
\hline 171.000 & & 18.644 & & 18.646 & 0.014 & 2.55 \\
\hline 176.000 & & 18.441 & & 18.444 & 0.016 & 2.55 \\
\hline 181.000 & & 18.235 & & 18.238 & 0.017 & 2.55 \\
\hline 186.000 & & 18.026 & & 18.029 & 0.016 & 2.55 \\
\hline 191.000 & & 17.814 & & 17.817 & 0.015 & 2.55 \\
\hline 196.000 & & 17.598 & & 17.601 & 0.013 & 2.55 \\
\hline 201.000 & & 17.379 & & 17.380 & 0.011 & 2.55 \\
\hline 206.000 & & 17.154 & & 17.156 & 0.007 & 2.55 \\
\hline 211.000 & & 16.925 & & 16.926 & 0.004 & 2.55 \\
\hline 216.000 & & 16.690 & & 16.691 & 0.001 & 2.55 \\
\hline 221.000 & & 16.450 & & 16.449 & -0.002 & 2.55 \\
\hline 226.000 & & 16.202 & & 16.202 & -0.004 & 2.55 \\
\hline 231.000 & & 15.947 & & 15.947 & -0.005 & 2.55 \\
\hline 236.000 & & 15.684 & & 15.683 & -0.004 & 2.55 \\
\hline 241.000 & & 15.411 & & 15.411 & -0.001 & 2.55 \\
\hline 246.000 & & 15.128 & & 15.129 & 0.006 & 2.55 \\
\hline 251.000 & & 14.832 & & 14.834 & 0.016 & 2.55 \\
\hline 256.000 & & 14.521 & & 14.526 & 0.030 & 2.55 \\
\hline 261.000 & & 14.194 & & 14.201 & 0.049 & 2.55 \\
\hline 266.000 & & 13.847 & & 13.857 & 0.073 & 2.55 \\
\hline 271.000 & & 13.475 & & 13.488 & 0.101 & 2.55 \\
\hline 276.000 & & 13.072 & & 13.090 & 0.132 & 2.55 \\
\hline 281.000 & & 12.630 & & 12.650 & 0.156 & 2.55 \\
\hline 286.000 & & 12.135 & & 12.154 & 0.159 & 2.55 \\
\hline
\end{tabular}


Table 2. SATURATED LIQUID DENSITIES (continued)

Data from ancillary equation [1] (continued)

\begin{tabular}{|c|c|c|c|c|c|c|}
\hline $\begin{array}{l}\mathrm{T} \\
\mathrm{K}\end{array}$ & $\begin{array}{l}\rho_{\sigma \mathrm{L}}, \operatorname{expt} \\
\mathrm{mol} \cdot \mathrm{dm}^{-3}\end{array}$ & $\begin{array}{l}\rho_{\sigma \mathrm{L}}, \text { anc. } \\
\mathrm{mol} \cdot \mathrm{dm}^{-3}\end{array}$ & $\begin{array}{c}\text { dev1 } \\
\frac{8}{8}\end{array}$ & $\begin{array}{c}\rho_{\sigma \mathrm{L}}, \quad \text { SWEOS } \\
\mathrm{mol} \cdot \mathrm{dm}^{-3}\end{array}$ & $\begin{array}{c}\text { dev2 } \\
\frac{8}{8}\end{array}$ & wt* \\
\hline 291.000 & & 11.560 & & 11.572 & 0.107 & 2.55 \\
\hline 296.000 & & 10.854 & & 10.849 & -0.040 & 12.70 \\
\hline 301.000 & & 9.862 & & 9.846 & -0.167 & 12.70 \\
\hline
\end{tabular}

Number of Points from ancillary equation. 43

$*$ These weights refer to development of the SWEOS.

These data are not included in the overall statistics below.

$$
\mathrm{AAD} 2 \%=0.030 \quad \mathrm{BIAS} 2 \%=0.017 \quad \mathrm{RMS} 2 \%=0.052
$$

Absolute Deviations:

$\mathrm{AAD2}=0.004$ BIAS2 $=0.002$ RMS2 $=0.006 \mathrm{~mol} \cdot \mathrm{dm}^{-3}$

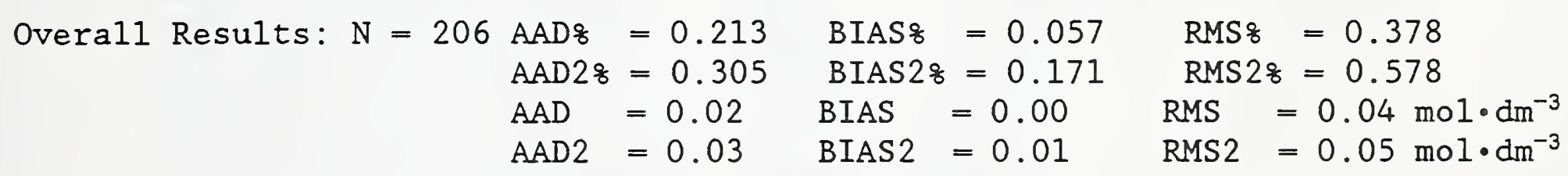

Weighted Data: $\quad \mathrm{N}=66 \mathrm{AAD} 8=0.030$ BIAS $8=-0.001 \quad$ RMS $8=0.037$ $\mathrm{AAD} 2 \%=0.042 \quad$ BIAS $2 \%=0.010 \quad$ RMS $2 \%=0.061$ $A A D=0.01 \quad B I A S=0.00 \quad$ RMS $=0.01 \mathrm{~mol} \cdot \mathrm{dm}^{-3}$ $A A D 2=0.01 \quad$ BIAS2 $=0.00 \quad$ RMS2 $=0.01 \mathrm{~mol} \cdot \mathrm{dm}^{-3}$ 
Table 3. SATURATED VAPOR DENSITIES

Data from Douslin and Harrison [9]

\begin{tabular}{|c|c|c|c|c|c|c|}
\hline $\begin{array}{l}\mathrm{T} \\
\mathrm{K}\end{array}$ & $\begin{array}{r}\rho_{\sigma \mathrm{V}}, \quad \operatorname{expt} \\
\mathrm{mol} \cdot \mathrm{dm}^{-3}\end{array}$ & $\begin{array}{c}\rho_{\text {ov }}, \text { anc. } \\
\mathrm{mol} \cdot \mathrm{dm}^{-3}\end{array}$ & $\begin{array}{c}\text { dev1 } \\
8\end{array}$ & $\begin{array}{c}\rho_{\sigma \mathrm{V}}, \quad \text { SWEOS } \\
\mathrm{mol} \cdot \mathrm{dm}^{-3}\end{array}$ & $\begin{array}{c}\text { dev2 } \\
8\end{array}$ & wt \\
\hline 248.150 & 0.745 & 0.745 & -0.053 & 0.745 & 0.050 & 1.0 \\
\hline 253.150 & 0.863 & 0.863 & 0.015 & 0.864 & 0.108 & 1.0 \\
\hline 263.150 & 1.153 & 1.153 & 0.026 & 1.154 & 0.115 & 1.0 \\
\hline 273.150 & 1.537 & 1.537 & 0.009 & 1.539 & 0.129 & 1.0 \\
\hline 283.150 & 2.067 & 2.066 & -0.047 & 2.070 & 0.130 & 1.0 \\
\hline 293.150 & 2.880 & 2.868 & -0.418 & 2.873 & -0.248 & 0.0 \\
\hline 298.150 & 3.502 & 3.495 & -0.188 & 3.496 & -0.181 & 0.0 \\
\hline 302.150 & 4.307 & 4.302 & -0.112 & 4.284 & -0.523 & 0.0 \\
\hline 303.150 & 4.604 & 4.614 & 0.214 & 4.586 & -0.393 & 1.0 \\
\hline 304.150 & 5.035 & 5.046 & 0.219 & 5.003 & -0.636 & 1.0 \\
\hline 305.150 & 5.913 & 5.928 & 0.248 & 5.878 & -0.584 & 0.0 \\
\hline 305.250 & 6.150 & 6.162 & 0.203 & 6.122 & -0.455 & 0.0 \\
\hline
\end{tabular}

Number of Points [9] 12

$$
\begin{aligned}
& \mathrm{AAD} \&=0.146 \quad \text { BIAS } 8=0.010 \quad \mathrm{RMS} \%=0.188 \\
& \text { AAD2 } 8=0.296 \quad \text { BIAS2 } 8=-0.207 \quad \text { RMS2 } 8=0.291
\end{aligned}
$$

Weighted Data:

Number of Points [9] 7

$$
\begin{array}{llll}
\mathrm{AAD} z=0.084 & \text { BIAS } z=0.055 & \text { RMS } z=0.106 \\
\mathrm{AAD} 28=0.223 & \text { BIAS2 } z=-0.071 & \text { RMS28 }=0.289
\end{array}
$$

Absolute Deviations:

$$
\begin{array}{lllll}
A A D & =0.003 & \text { BIAS }=0.003 & \text { RMS }=0.005 \mathrm{~mol} \cdot \mathrm{dm}^{-3} \\
\text { AAD2 } & =0.008 & \text { BIAS2 }=-0.006 & \text { RMS2 }=0.013 \mathrm{~mol} \cdot \mathrm{dm}^{-3}
\end{array}
$$


Table 3. SATURATED VAPOR DENSITIES (continued)

Data from Goodwin et al. [20]

Using virial equation of state and fitted saturation pressures.

\begin{tabular}{|c|c|c|c|c|c|c|}
\hline $\begin{array}{l}\mathrm{T} \\
\mathrm{K}\end{array}$ & $\begin{array}{r}\rho_{\sigma \mathrm{V}}, \quad \operatorname{expt} \\
\mathrm{mol} \cdot \mathrm{dm}^{-3}\end{array}$ & $\begin{array}{c}\rho_{\text {ov }}, \text { anc. } \\
\mathrm{mol} \cdot \mathrm{dm}^{-3}\end{array}$ & $\begin{array}{c}\text { devl } \\
8\end{array}$ & $\begin{array}{c}\rho_{\sigma \mathrm{V}}, \quad \text { SWEOS } \\
\mathrm{mol} \cdot \mathrm{dm}^{-3}\end{array}$ & $\begin{array}{c}\text { dev2 } \\
\frac{8}{8}\end{array}$ & wt \\
\hline $90.348 x$ & $0.150 E-05$ & $0.150 \mathrm{E}-05$ & -0.117 & $0.150 \mathrm{E}-05$ & -0.046 & 0.0 \\
\hline 100.000 & $0.133 E-04$ & $0.133 E-04$ & -0.034 & $0.133 \mathrm{E}-04$ & -0.072 & 0.0 \\
\hline 110.000 & $0.817 E-04$ & $0.817 E-04$ & 0.000 & $0.816 \mathrm{E}-04$ & -0.023 & 1.0 \\
\hline 120.000 & $0.356 \mathrm{E}-03$ & $0.356 E-03$ & 0.004 & $0.355 \mathrm{E}-03$ & -0.038 & 1.0 \\
\hline 130.000 & $0.120 \mathrm{E}-02$ & $0.120 E-02$ & 0.001 & $0.120 \mathrm{E}-02$ & -0.103 & 1.0 \\
\hline 140.000 & $0.330 E-02$ & $0.330 E-02$ & -0.011 & $0.330 E-02$ & -0.191 & 1.0 \\
\hline 150.000 & $0.780 E-02$ & $0.780 E-02$ & -0.020 & $0.779 E-02$ & -0.250 & 1.0 \\
\hline 160.000 & $0.163 \mathrm{E}-01$ & $0.163 E-01$ & -0.007 & $0.163 \mathrm{E}-01$ & -0.239 & 1.0 \\
\hline 170.000 & $0.309 E-01$ & $0.309 E-01$ & -0.005 & $0.308 E-01$ & -0.192 & 1.0 \\
\hline 180.000 & $0.541 E-01$ & $0.541 E-01$ & -0.012 & $0.541 E-01$ & -0.120 & 1.0 \\
\hline 190.000 & $0.889 \mathrm{E}-01$ & $0.889 E-01$ & -0.018 & $0.889 E-01$ & -0.035 & 1.0 \\
\hline 200.000 & 0.139 & 0.139 & -0.016 & 0.139 & 0.050 & 1.0 \\
\hline 210.000 & 0.208 & 0.208 & 0.007 & 0.208 & 0.131 & 1.0 \\
\hline 220.000 & 0.300 & 0.300 & 0.053 & 0.301 & 0.204 & 1.0 \\
\hline 230.000 & 0.422 & 0.422 & 0.119 & 0.423 & 0.267 & 1.0 \\
\hline 240.000 & 0.580 & 0.581 & 0.162 & 0.582 & 0.288 & 1.0 \\
\hline 250.000 & 0.787 & 0.787 & -0.008 & 0.787 & 0.091 & 1.0 \\
\hline 260.000 & 1.055 & 1.053 & -0.116 & 1.054 & -0.029 & 1.0 \\
\hline 270.000 & 1.406 & 1.404 & -0.166 & 1.405 & -0.060 & 1.0 \\
\hline 280.000 & 1.881 & 1.879 & -0.116 & 1.882 & 0.042 & 1.0 \\
\hline
\end{tabular}

*This point is below the currently accepted triple point temperature. Number of Points [20] 20

$$
\begin{array}{lll}
\mathrm{AAD} \%=0.050 & \text { BIAS } 8=-0.015 & \text { RMS } \%=0.074 \\
\mathrm{AAD} 2 \%=0.124 & \text { BIAS2\% }=-0.016 & \text { RMS } 2 \%=0.151
\end{array}
$$

Absolute Deviations:
$\mathrm{AAD}=0.000$
BIAS $=0.000$
RMS $=0.001 \mathrm{~mol} \cdot \mathrm{dm}^{-3}$
$\mathrm{AAD2}=0.000$
BIAS2 $=0.000$
RMS2 $=0.001 \mathrm{~mol} \cdot \mathrm{dm}^{-3}$ 
Table 3. SATURATED VAPOR DENSITIES (continued)

Data from Goodwin et al. [20] (continued)

Weighted Data:

Number of Points [20] 18

$$
\begin{array}{llll}
\text { AAD } 8=0.047 & \text { BIAS } 8=-0.008 & \text { RMS } q=0.074 \\
\text { AAD2 }=0.131 & \text { BIAS2\% }=-0.011 & \text { RMS2\% }=0.158
\end{array}
$$

Absolute Deviations:

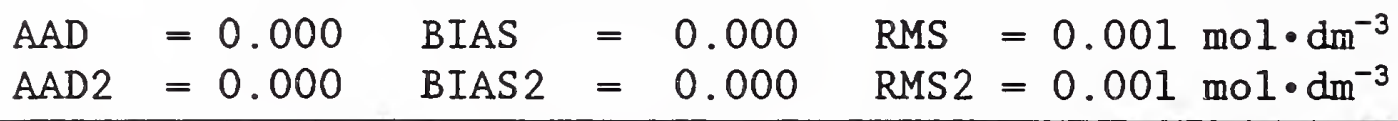

\begin{tabular}{|c|c|c|c|c|c|c|}
\hline $\begin{array}{l}\mathrm{T} \\
\mathrm{K}\end{array}$ & $\begin{array}{r}\rho_{\sigma \mathrm{v}}, \quad \operatorname{expt} \\
\mathrm{mol} \cdot \mathrm{dm}^{-3}\end{array}$ & $\begin{array}{l}\rho_{\sigma \mathrm{V}}, \text { anc. } \\
\mathrm{mol} \cdot \mathrm{dm}^{-3}\end{array}$ & $\begin{array}{c}\text { devl } \\
8\end{array}$ & $\begin{array}{c}\rho_{\text {ov }}, \quad \text { SWEOS } \\
\mathrm{mol} \cdot \mathrm{dm}^{-3}\end{array}$ & $\begin{array}{c}\operatorname{dev} 2 \\
8\end{array}$ & wt \\
\hline 305.325 & 6.317 & 6.605 & 4.565 & 6.456 & 2.208 & 0.0 \\
\hline 305.260 & 6.059 & 6.195 & 2.255 & 6.161 & 1.688 & 0.0 \\
\hline 305.035 & 5.653 & 5.748 & 1.691 & 5.692 & 0.703 & 0.0 \\
\hline 304.633 & 5.277 & 5.352 & 1.423 & 5.300 & 0.435 & 0.0 \\
\hline 303.937 & 4.873 & 4.938 & 1.319 & 4.898 & 0.512 & 0.0 \\
\hline 302.872 & 4.455 & 4.519 & 1.436 & 4.494 & 0.883 & 0.0 \\
\hline
\end{tabular}

Data from Miniovich and Sorina [12]

Number of Points [12] 6

$\begin{array}{lll}\mathrm{AAD} \%=2.115 & \text { BIAS } \%=2.115 & \mathrm{RMS} \%=1.138 \\ \mathrm{AAD} 2 \%=1.071 & \text { BIAS2\% } 8=1.071 & \mathrm{RMS} 2 \%=0.653\end{array}$

Absolute Deviations:

$A A D=0.121$ BIAS $=0.121$ RMS $=0.079 \mathrm{~mol} \cdot \mathrm{dm}^{-3}$

AAD2 $=0.061$ BIAS2 $=0.061 \quad$ RMS2 $=0.044 \mathrm{mo} \cdot \mathrm{dm}^{-3}$

Data from Pestak et al. [26]

\begin{tabular}{ccccccc}
$\mathrm{T}$ & $\begin{array}{c}\rho_{\sigma \mathrm{V}}, \operatorname{expt} \\
\mathrm{mol} \cdot \mathrm{dm}^{-3}\end{array}$ & $\begin{array}{c}\rho_{\sigma \mathrm{V}}, \mathrm{anc} \\
\mathrm{mol} \cdot \mathrm{dm}^{-3}\end{array}$ & $\begin{array}{c}\text { dev1 } \\
\text { \% }\end{array}$ & $\begin{array}{c}\rho_{\sigma \mathrm{V}}, \mathrm{SWEOS} \\
\mathrm{mol} \cdot \mathrm{dm}^{-3}\end{array}$ & $\begin{array}{c}\text { dev2 } \\
\text { \% }\end{array}$ & wt \\
\hline 298.730 & 3.593 & 3.588 & -0.141 & 3.587 & -0.172 & 0.0 \\
299.219 & 3.677 & 3.670 & -0.187 & 3.668 & -0.253 & 0.0 \\
299.722 & 3.765 & 3.761 & -0.121 & 3.756 & -0.229 & 0.0 \\
300.238 & 3.867 & 3.859 & -0.196 & 3.853 & -0.354 & 0.0 \\
300.770 & 3.966 & 3.969 & 0.080 & 3.960 & -0.135 & 0.0
\end{tabular}


Table 3. SATURATED VAPOR DENSITIES (continued)

Data from Pestak et al. [26] (continued)

\begin{tabular}{|c|c|c|c|c|c|c|}
\hline $\begin{array}{l}\mathrm{T} \\
\mathrm{K}\end{array}$ & $\begin{array}{r}\rho_{\sigma \mathrm{v}}, \quad \text { expt } \\
\mathrm{mol} \cdot \mathrm{dm}^{-3}\end{array}$ & $\begin{array}{l}\rho_{\sigma \mathrm{v}}, \text { anc. } \\
\mathrm{mol} \cdot \mathrm{dm}^{-3}\end{array}$ & $\begin{array}{c}\text { dev1 } \\
\frac{8}{8}\end{array}$ & $\begin{array}{c}\rho_{\sigma \mathrm{V}}, \quad \text { SWEOS } \\
\mathrm{mol} \cdot \mathrm{dm}^{-3}\end{array}$ & $\begin{array}{c}\text { dev2 } \\
\frac{8}{8}\end{array}$ & wt \\
\hline 301.310 & 4.095 & 4.089 & -0.131 & 4.078 & -0.415 & 0.0 \\
\hline 301.722 & 4.198 & 4.189 & -0.201 & 4.175 & -0.543 & 0.0 \\
\hline 302.143 & 4.302 & 4.300 & -0.031 & 4.283 & -0.441 & 0.0 \\
\hline 302.503 & 4.411 & 4.403 & -0.165 & 4.382 & -0.639 & 0.0 \\
\hline 302.865 & 4.523 & 4.516 & -0.138 & 4.492 & -0.681 & 0.0 \\
\hline 303.234 & 4.651 & 4.644 & -0.153 & 4.615 & -0.776 & 0.0 \\
\hline 303.383 & 4.710 & 4.700 & -0.200 & 4.669 & -0.858 & 0.0 \\
\hline 303.533 & 4.767 & 4.759 & -0.160 & 4.726 & -0.852 & 0.0 \\
\hline 303.686 & 4.832 & 4.823 & -0.174 & 4.788 & -0.906 & 0.0 \\
\hline 303.838 & 4.899 & 4.891 & -0.156 & 4.853 & -0.927 & 0.0 \\
\hline 303.988 & 4.971 & 4.963 & -0.178 & 4.922 & -0.989 & 0.0 \\
\hline 304.146 & 5.052 & 5.044 & -0.152 & 5.001 & -1.008 & 0.0 \\
\hline 304.301 & 5.145 & 5.131 & -0.271 & 5.085 & -1.166 & 0.0 \\
\hline 304.379 & 5.190 & 5.178 & -0.230 & 5.131 & -1.143 & 0.0 \\
\hline 304.457 & 5.238 & 5.228 & -0.191 & 5.179 & -1.127 & 0.0 \\
\hline 304.662 & 5.389 & 5.375 & -0.254 & 5.322 & -1.236 & 0.0 \\
\hline 304.709 & 5.433 & 5.413 & -0.378 & 5.359 & -1.357 & 0.0 \\
\hline 304.757 & 5.470 & 5.453 & -0.303 & 5.399 & -1.290 & 0.0 \\
\hline 304.805 & 5.517 & 5.496 & -0.379 & 5.441 & -1.382 & 0.0 \\
\hline 304.836 & 5.544 & 5.525 & -0.333 & 5.469 & -1.338 & 0.0 \\
\hline 304.868 & 5.579 & 5.556 & -0.401 & 5.500 & -1.414 & 0.0 \\
\hline 304.900 & 5.617 & 5.589 & -0.490 & 5.533 & -1.497 & 0.0 \\
\hline 304.932 & 5.655 & 5.624 & -0.555 & 5.567 & -1.559 & 0.0 \\
\hline 304.964 & 5.690 & 5.660 & -0.528 & 5.604 & -1.513 & 0.0 \\
\hline 304.987 & 5.722 & 5.687 & -0.605 & 5.630 & -1.607 & 0.0 \\
\hline 305.021 & 5.767 & 5.730 & -0.645 & 5.675 & -1.592 & 0.0 \\
\hline 305.053 & 5.819 & 5.773 & -0.784 & 5.719 & -1.713 & 0.0 \\
\hline 305.084 & 5.871 & 5.818 & -0.910 & 5.764 & -1.828 & 0.0 \\
\hline 305.102 & 5.901 & 5.846 & -0.936 & 5.793 & -1.835 & 0.0 \\
\hline 305.117 & 5.933 & 5.870 & -1.059 & 5.818 & -1.941 & 0.0 \\
\hline 305.134 & 5.969 & 5.899 & -1.181 & 5.845 & -2.092 & 0.0 \\
\hline 305.149 & 6.001 & 5.926 & -1.246 & 5.877 & -2.066 & 0.0 \\
\hline 305.166 & 6.036 & 5.958 & -1.293 & 5.909 & -2.112 & 0.0 \\
\hline 305.173 & 6.064 & 5.972 & -1.524 & 5.922 & -2.345 & 0.0 \\
\hline
\end{tabular}


Table 3. SATURATED VAPOR DENSITIES (continued)

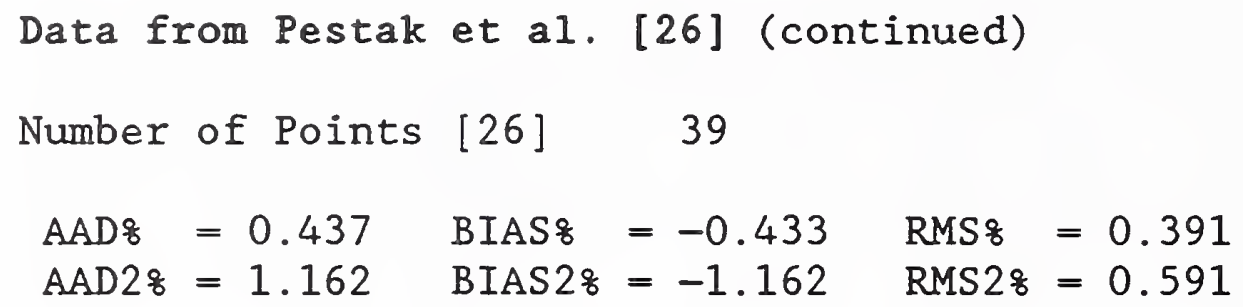

Absolute Deviations:

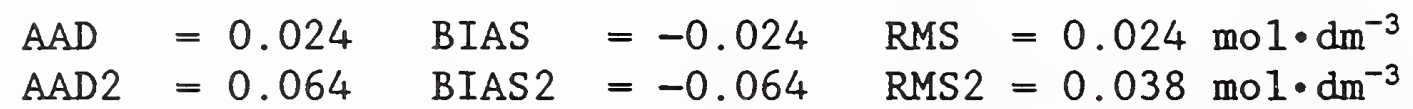

Data from Porter [30]

\begin{tabular}{|c|c|c|c|c|c|c|c|c|}
\hline & $\begin{array}{l}\mathrm{T} \\
\mathrm{K}\end{array}$ & & $\begin{array}{l}\sigma \mathrm{v}, \operatorname{expt} \\
\mathrm{mol} \cdot \mathrm{dm}^{-3}\end{array}$ & $\begin{array}{l}\rho_{\sigma \mathrm{V}}, \text { anc. } \\
\mathrm{mol} \cdot \mathrm{dm}^{-3}\end{array}$ & $\begin{array}{c}\operatorname{dev} 1 \\
\frac{8}{8}\end{array}$ & $\begin{array}{c}\rho_{\sigma \mathrm{V}}, \quad \mathrm{SWEOS} \\
\mathrm{mol} \cdot \mathrm{dm}^{-3}\end{array}$ & $\begin{array}{c}\text { dev2 } \\
8\end{array}$ & wt \\
\hline & 288.260 & & 2.412 & 2.427 & 0.620 & 2.431 & 0.818 & 0.0 \\
\hline & 283.580 & & 2.086 & 2.093 & 0.341 & 2.097 & 0.522 & 0.0 \\
\hline & 278.840 & & 1.811 & 1.815 & 0.231 & 1.818 & 0.383 & 0.0 \\
\hline & 273.090 & & 1.540 & 1.534 & -0.378 & 1.536 & -0.259 & 0.0 \\
\hline & 268.730 & & 1.357 & 1.353 & -0.273 & 1.355 & -0.172 & 0.0 \\
\hline & 263.280 & & 1.161 & 1.158 & -0.309 & 1.159 & -0.220 & 0.0 \\
\hline & 258.800 & & 1.032 & 1.018 & -1.442 & 1.018 & -1.356 & 0.0 \\
\hline & 253.030 & & 0.864 & 0.860 & -0.382 & 0.861 & -0.290 & 0.0 \\
\hline & 248.650 & & 0.757 & 0.756 & -0.197 & 0.756 & -0.096 & 0.0 \\
\hline & 243.220 & & 0.643 & 0.642 & -0.200 & 0.642 & -0.084 & 0.0 \\
\hline & 238.900 & & 0.564 & 0.561 & -0.524 & 0.562 & -0.397 & 0.0 \\
\hline & 234.580 & & 0.494 & 0.490 & -0.767 & 0.490 & -0.629 & 0.0 \\
\hline & 226.180 & & 0.377 & 0.372 & -1.453 & 0.372 & -1.303 & 0.0 \\
\hline & 184.470 & 0.68 & $39 E-01$ & $0.681 \mathrm{E}-01$ & -1.247 & $0.680 \mathrm{E}-01$ & -1.314 & 0.0 \\
\hline Number & of Points & {$[30]$} & 14 & & & & & \\
\hline AAD 8 & $=0.598$ & BIAS 8 & $=-0.427$ & \multicolumn{5}{|c|}{ RMS $8=0.606$} \\
\hline $\mathrm{AAD} 28$ & $=0.560$ & BIAS $2 \%$ & $=-0.314$ & \multicolumn{5}{|c|}{$\operatorname{RMS} 28=0.642$} \\
\hline \multicolumn{9}{|c|}{ Absolute Deviations: } \\
\hline $\mathrm{AAD}$ & $=0.005$ & BIAS & $=-0.001$ & \multicolumn{5}{|c|}{ RMS $=0.007 \mathrm{~mol} \cdot \mathrm{dm}^{-3}$} \\
\hline $\mathrm{AAD} 2$ & $=0.005$ & BIAS2 & $=0.000$ & RMS2 $=0$. & $008 \mathrm{~mol}$ & $m^{-3}$ & & \\
\hline
\end{tabular}


Table 3. SATURATED VAPOR DENSITIES (continued)

Data from Sliwinski [28]

\begin{tabular}{|c|c|c|c|c|c|c|}
\hline $\begin{array}{l}\mathrm{T} \\
\mathrm{K}\end{array}$ & $\begin{array}{r}\rho_{\sigma \mathrm{v}}, \operatorname{expt} \\
\mathrm{mol} \cdot \mathrm{dm}^{-3}\end{array}$ & $\begin{array}{c}\rho_{\text {ov }}, \text { anc. } \\
\mathrm{mol} \cdot \mathrm{dm}^{-3}\end{array}$ & $\begin{array}{c}\text { devl } \\
\frac{8}{8}\end{array}$ & $\begin{array}{c}\rho_{\sigma \mathrm{V}}, \quad \text { SWEOS } \\
\mathrm{mol} \cdot \mathrm{dm}^{-3}\end{array}$ & $\begin{array}{c}\text { dev2 } \\
\frac{q}{8}\end{array}$ & wt \\
\hline 283.200 & 2.068 & 2.069 & 0.033 & 2.073 & 0.211 & 1.0 \\
\hline 288.190 & 2.416 & 2.421 & 0.208 & 2.426 & 0.406 & 0.0 \\
\hline 293.180 & 2.872 & 2.871 & -0.018 & 2.876 & 0.152 & 1.0 \\
\hline 295.670 & 3.154 & 3.153 & -0.035 & 3.157 & 0.081 & 1.0 \\
\hline 298.170 & 3.500 & 3.499 & -0.048 & 3.499 & -0.042 & 1.0 \\
\hline 300.660 & 3.941 & 3.945 & 0.115 & 3.937 & -0.088 & 1.0 \\
\hline 302.160 & 4.294 & 4.305 & 0.248 & 4.287 & -0.166 & 1.0 \\
\hline 303.160 & 4.605 & 4.617 & 0.263 & 4.589 & -0.347 & 1.0 \\
\hline 304.150 & 5.020 & 5.046 & 0.513 & 5.003 & -0.345 & 0.0 \\
\hline 304.650 & 5.328 & 5.366 & 0.713 & 5.313 & -0.266 & 0.0 \\
\hline 305.150 & 5.866 & 5.928 & 1.046 & 5.878 & 0.208 & 0.0 \\
\hline
\end{tabular}

Number of Points [28] 11

$$
\begin{aligned}
& \mathrm{AAD} \&=0.295 \quad \text { BIAS }=0.276 \quad \text { RMS } 8=0.333 \\
& A A D 2 \%=0.210 \quad \text { BIAS } 2 \%=-0.018 \quad \text { RMS } 2 \%=0.238
\end{aligned}
$$

Absolute Deviations:

$$
\begin{array}{lllll}
\mathrm{AAD}=0.015 & \text { BIAS }=0.014 & \mathrm{RMS}=0.019 \mathrm{~mol} \cdot \mathrm{dm}^{-3} \\
\mathrm{AAD2}=0.008 & \text { BIAS2 } & =-0.002 & \mathrm{RMS} 2=0.010 \mathrm{~mol} \cdot \mathrm{dm}^{-3}
\end{array}
$$

Weighted Data:

Number of Points [28] 7

$$
\begin{array}{lll}
\mathrm{AAD} z=0.109 & \text { BIAS } q=0.080 & \text { RMS } q=0.122 \\
\mathrm{AAD} 2 \%=0.155 & \text { BIAS2\% }=-0.029 & \text { RMS } 2 \%=0.180
\end{array}
$$

Absolute Deviations:

\begin{tabular}{|c|c|c|c|c|c|c|}
\hline $\begin{array}{l}\mathrm{T} \\
\mathrm{K}\end{array}$ & $\begin{array}{r}\rho_{\sigma \mathrm{V}}, \quad \operatorname{expt} \\
\mathrm{mol} \cdot \mathrm{dm}^{-3}\end{array}$ & $\begin{array}{c}\rho_{\sigma \mathrm{v}}, \text { anc } \\
\mathrm{mol} \cdot \mathrm{dm}^{-3}\end{array}$ & $\begin{array}{c}\operatorname{dev} 1 \\
\frac{8}{8}\end{array}$ & $\begin{array}{c}\rho_{\sigma \mathrm{V}}, \quad \text { SWEOS } \\
\mathrm{mol} \cdot \mathrm{dm}^{-3}\end{array}$ & $\begin{array}{c}\operatorname{dev} 2 \\
\frac{8}{\delta}\end{array}$ & wtx \\
\hline 91.000 & & $0.177 E-05$ & & $0.177 E-05$ & 0.051 & 531.0 \\
\hline 96.000 & & $0.573 E-05$ & & $0.573 E-05$ & -0.028 & 531.0 \\
\hline 101.000 & & $0.163 E-04$ & & $0.163 E-04$ & -0.037 & 531.0 \\
\hline 106.000 & & $0.415 E-04$ & & $0.414 E-04$ & -0.028 & 531.0 \\
\hline 111.000 & & $0.959 E-04$ & & $0.959 E-04$ & -0.022 & 531.0 \\
\hline
\end{tabular}
$\mathrm{AAD}=0.004 \quad \mathrm{BIAS}=0.004$
RMS $=0.005 \mathrm{~mol} \cdot \mathrm{dm}^{-3}$
$\mathrm{AAD2}=0.006 \quad \mathrm{BIAS} 2=-0.002$
RMS2 $=0.007 \mathrm{~mol} \cdot \mathrm{dm}^{-3}$

Data from ancillary equation [1] 
Table 3. SATURATED VAPOR DENSITIES (continued)

Data from ancillary equation [1] (continued)

\begin{tabular}{|c|c|c|c|c|c|c|}
\hline $\begin{array}{l}\mathrm{T} \\
\mathrm{K}\end{array}$ & $\begin{array}{r}\rho_{\sigma v}, \quad \operatorname{expt} \\
\mathrm{mol} \cdot \mathrm{dm}^{-3}\end{array}$ & $\begin{array}{l}\rho_{\text {ov }}, \text { anc } \\
\mathrm{mol} \cdot \mathrm{dm}^{-3}\end{array}$ & $\begin{array}{c}\text { dev1 } \\
\frac{8}{8}\end{array}$ & $\begin{array}{c}\rho_{\sigma \mathrm{V}}, \quad \text { SWEOS } \\
\mathrm{mol} \cdot \mathrm{dm}^{-3}\end{array}$ & $\begin{array}{c}\operatorname{dev} 2 \\
8\end{array}$ & wt* \\
\hline 116.000 & & $0.204 E-03$ & & $0.204 E-03$ & -0.028 & 531.0 \\
\hline 121.000 & & $0.406 \mathrm{E}-03$ & & $0.405 \mathrm{E}-03$ & -0.046 & 531.0 \\
\hline 126.000 & & $0.756 \mathrm{E}-03$ & & $0.756 \mathrm{E}-03$ & -0.076 & 531.0 \\
\hline 131.000 & & $0.134 \mathrm{E}-02$ & & $0.133 \mathrm{E}-02$ & -0.112 & 531.0 \\
\hline 136.000 & & $0.225 \mathrm{E}-02$ & & $0.224 \mathrm{E}-02$ & -0.151 & 531.0 \\
\hline 141.000 & & $0.362 E-02$ & & $0.361 E-02$ & -0.186 & 531.0 \\
\hline 146.000 & & $0.562 \mathrm{E}-02$ & & $0.561 \mathrm{E}-02$ & -0.215 & 531.0 \\
\hline 151.000 & & $0.844 \mathrm{E}-02$ & & $0.842 \mathrm{E}-02$ & -0.232 & 531.0 \\
\hline 156.000 & & $0.123 E-01$ & & $0.123 E-01$ & -0.237 & 531.0 \\
\hline 161.000 & & $0.175 \mathrm{E}-01$ & & $0.174 \mathrm{E}-01$ & -0.230 & 531.0 \\
\hline 166.000 & & $0.242 \mathrm{E}-01$ & & $0.241 E-01$ & -0.210 & 531.0 \\
\hline 171.000 & & $0.328 \mathrm{E}-01$ & & $0.327 \mathrm{E}-01$ & -0.181 & 531.0 \\
\hline 176.000 & & $0.436 \mathrm{E}-01$ & & $0.436 \mathrm{E}-01$ & -0.142 & 531.0 \\
\hline 181.000 & & $0.570 \mathrm{E}-01$ & & $0.570 \mathrm{E}-01$ & -0.099 & 531.0 \\
\hline 186.000 & & $0.734 \mathrm{E}-01$ & & $0.734 \mathrm{E}-01$ & -0.053 & 493.0 \\
\hline 191.000 & & $0.932 \mathrm{E}-01$ & & $0.932 \mathrm{E}-01$ & -0.008 & 371.0 \\
\hline 196.000 & & 0.117 & & 0.117 & 0.035 & 284.0 \\
\hline 201.000 & & 0.145 & & 0.145 & 0.073 & 221.0 \\
\hline 206.000 & & 0.177 & & 0.178 & 0.104 & 174.0 \\
\hline 211.000 & & 0.216 & & 0.216 & 0.128 & 138.0 \\
\hline 216.000 & & 0.260 & & 0.260 & 0.143 & 112.0 \\
\hline 221.000 & & 0.311 & & 0.311 & 0.151 & 90.8 \\
\hline 226.000 & & 0.369 & & 0.370 & 0.152 & 74.6 \\
\hline 231.000 & & 0.436 & & 0.437 & 0.146 & 61.9 \\
\hline 236.000 & & 0.512 & & 0.513 & 0.136 & 51.8 \\
\hline 241.000 & & 0.599 & & 0.600 & 0.122 & 43.7 \\
\hline 246.000 & & 0.698 & & 0.699 & 0.109 & 37.2 \\
\hline 251.000 & & 0.810 & & 0.811 & 0.096 & 69.7 \\
\hline 256.000 & & 0.938 & & 0.939 & 0.089 & 60.2 \\
\hline 261.000 & & 1.084 & & 1.085 & 0.087 & 52.2 \\
\hline 266.000 & & 1.252 & & 1.253 & 0.094 & 45.7 \\
\hline 271.000 & & 1.445 & & 1.446 & 0.109 & 40.2 \\
\hline 276.000 & & 1.670 & & 1.672 & 0.134 & 35.7 \\
\hline 281.000 & & 1.936 & & 1.939 & 0.164 & 31.9 \\
\hline 286.000 & & 2.257 & & 2.261 & 0.191 & 28.8 \\
\hline
\end{tabular}


Table 3. SATURATED VAPOR DENSITIES (continued)

Data from ancillary equation [1] (continued)

\begin{tabular}{|c|c|c|c|c|c|c|}
\hline $\begin{array}{l}\mathrm{T} \\
\mathrm{K}\end{array}$ & $\begin{array}{c}\rho_{\sigma \mathrm{v}}, \operatorname{expt} \\
\mathrm{mol} \cdot \mathrm{dm}^{-3}\end{array}$ & $\begin{array}{c}\rho_{\sigma \mathrm{v}}, \text { anc. } \\
\mathrm{mol} \cdot \mathrm{dm}^{-3}\end{array}$ & $\begin{array}{c}\text { devl } \\
\frac{8}{8}\end{array}$ & $\begin{array}{c}\rho_{\sigma \mathrm{V}}, \mathrm{SWEOS} \\
\mathrm{mol} \cdot \mathrm{dm}^{-3}\end{array}$ & $\begin{array}{c}\operatorname{dev} 2 \\
\frac{8}{8}\end{array}$ & wt* \\
\hline 291.000 & & 2.659 & & 2.664 & 0.191 & 26.2 \\
\hline 296.000 & & 3.195 & & 3.198 & 0.105 & 24.1 \\
\hline 301.000 & & 4.019 & & 4.009 & -0.244 & 22.6 \\
\hline
\end{tabular}

Number of Points from ancillary equation.

$*$ These weights refer to development of the SWEOS.

These data are not included in the overall statistics below.

$$
\mathrm{AAD} 2 \%=0.120 \quad \text { BIAS } 2 \%=0.001 \quad \text { RMS } 2 \%=0.137
$$

Absolute Deviations:

$$
\text { AAD2 }=0.001 \text { BIAS2 }=0.000 \quad \text { RMS2 }=0.002 \mathrm{~mol} \cdot \mathrm{dm}^{-3}
$$

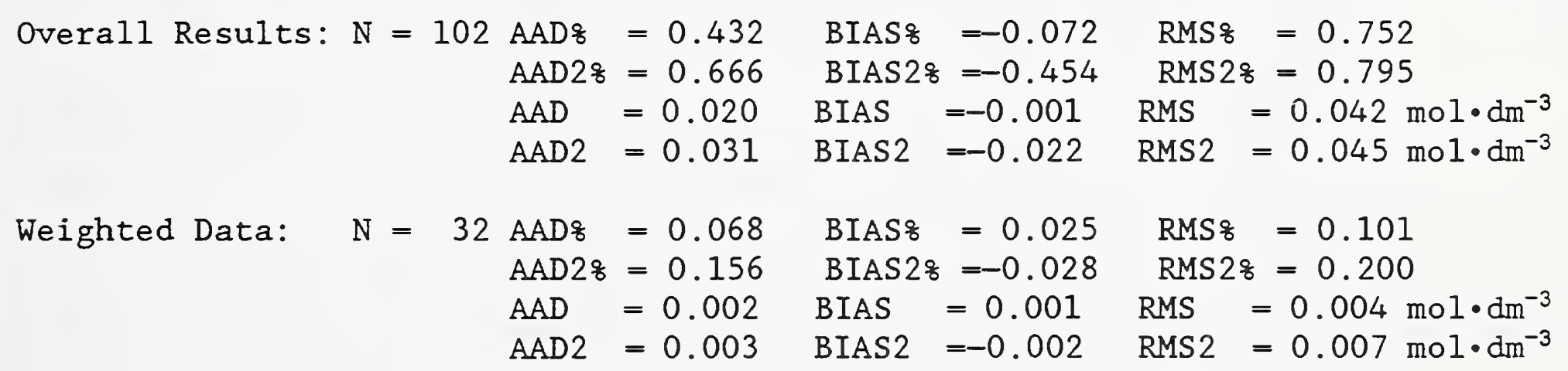


Table 4 gives a comparison between our correlation for the ideal gas properties, derived from eq. (3) of [1], and other tabulations. We have included ideal gas entropies (at $0.101325 \mathrm{MPa}$ for [31] and [32], but at $0.1 \mathrm{MPa}$ for [33]), ideal gas isobaric heat capacities, and ideal gas enthalpies. Most of the data were actually calculated from spectroscopic models; some of the heat capacities [34, 35] were determined from extrapolation of thermodynamic measurements. The two points indicated with weights of 100 were fixed point integration constants to which the correlating equations were rigorously constrained. 
Table 4. IDEAL GAS PROPERTIES

Data from Chao et al. [31]

Entropy at $0.101325 \mathrm{MPa}$

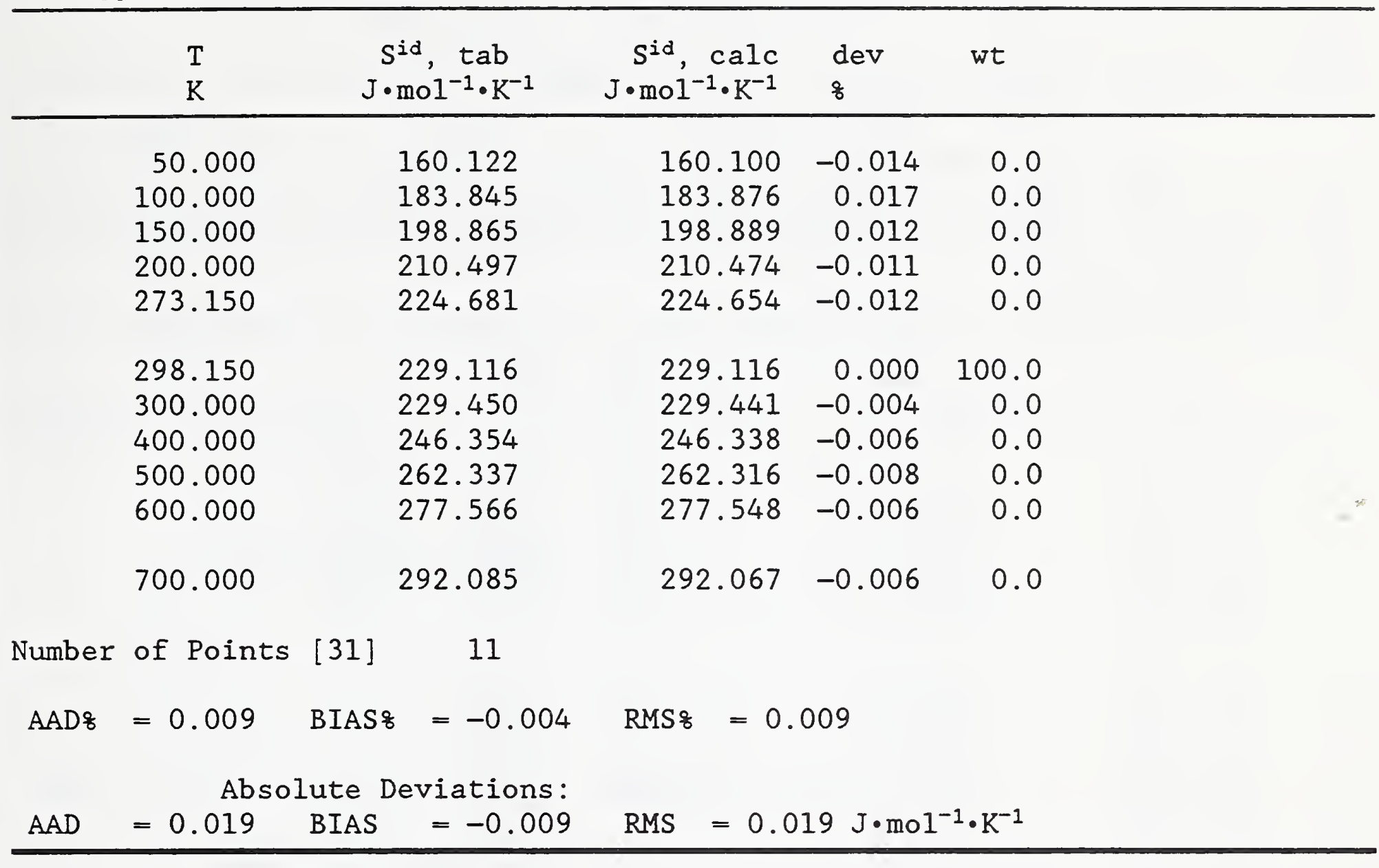

Data from Pamidimukkala et al. [32]

Entropy at $0.101325 \mathrm{MPa}$

\begin{tabular}{|c|c|c|c|c|}
\hline $\begin{array}{l}\mathrm{T} \\
\mathrm{K}\end{array}$ & $\begin{array}{c}\mathrm{S}^{\mathrm{id}}, \quad \mathrm{tab} \\
\mathrm{J} \cdot \mathrm{mol}^{-1} \cdot \mathrm{K}^{-1}\end{array}$ & $\begin{array}{r}\mathrm{S}^{\mathrm{id}}, \mathrm{calc} \\
\mathrm{J} \cdot \mathrm{mol}^{-1} \cdot \mathrm{K}^{-1}\end{array}$ & $\begin{array}{l}\text { dev } \\
8\end{array}$ & wt \\
\hline 100.000 & 183.761 & 183.876 & 0.063 & 0.0 \\
\hline 200.000 & 210.497 & 210.474 & -0.011 & 0.0 \\
\hline 298.000 & 229.074 & 229.090 & 0.007 & 0.0 \\
\hline 300.000 & 229.450 & 229.441 & -0.004 & 0.0 \\
\hline 400.000 & 246.395 & 246.338 & -0.023 & 0.0 \\
\hline 500.000 & 262.295 & 262.316 & 0.008 & 0.0 \\
\hline 600.000 & 277.483 & 277.548 & 0.023 & 0.0 \\
\hline 700.000 & 292.001 & 292.067 & 0.022 & 0.0 \\
\hline
\end{tabular}


Table 4. IDEAL GAS PROPERTIES (continued)

Data from Pamidimukkala et al. [32] (continued)

Number of Points [32] 8

$\mathrm{AAD} \%=0.020 \quad$ BIAS $8=0.011 \quad$ RMS $8=0.025$

Absolute Deviations:

$\mathrm{AAD}=0.046 \mathrm{BIAS}=0.024 \mathrm{RMS}=0.052 \mathrm{~J} \cdot \mathrm{mol}^{-1} \cdot \mathrm{K}^{-1}$

Data from TRC [33]

Entropy at $0.1 \mathrm{MPa}$

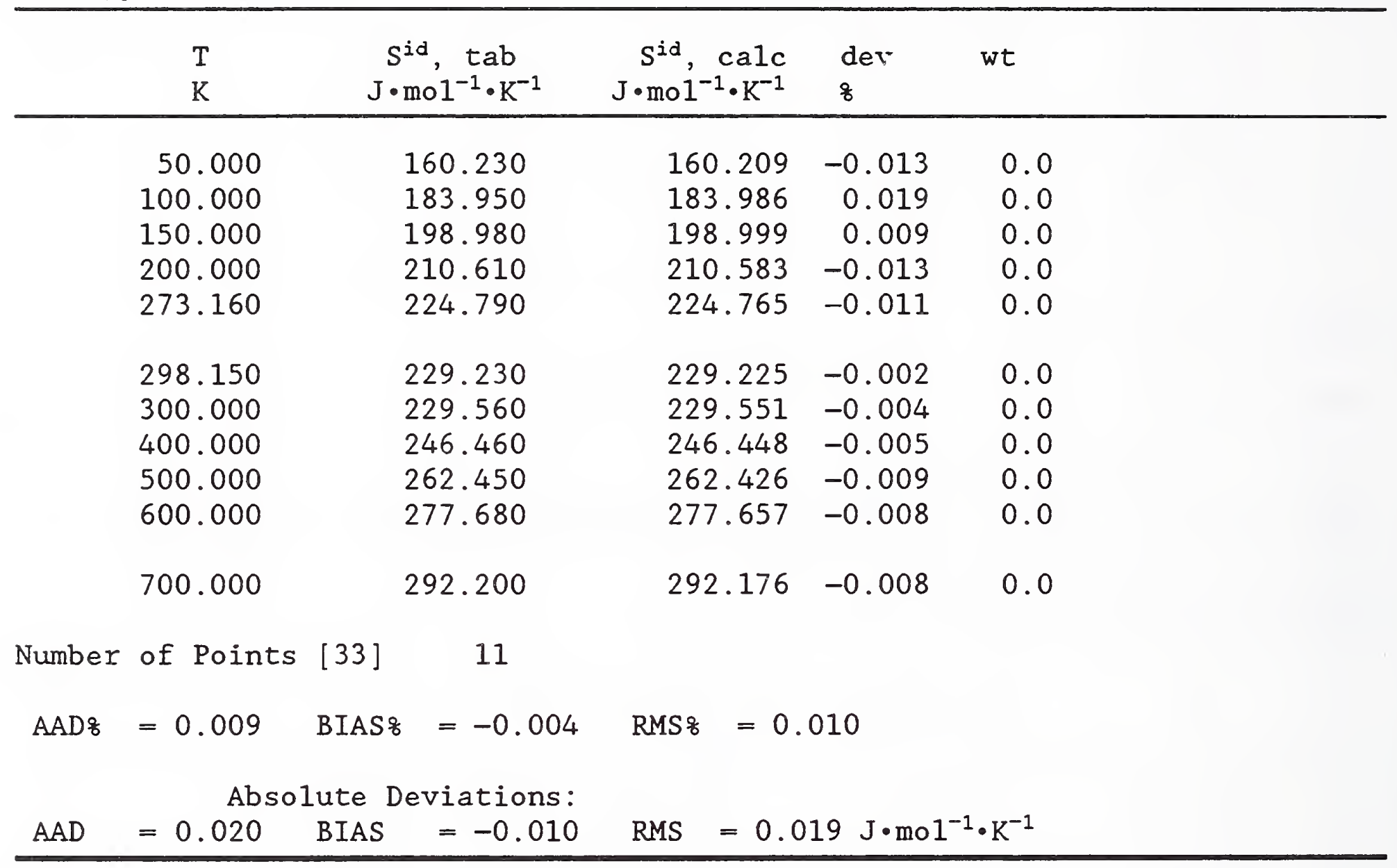

Data from Chao et al. [31]

\begin{tabular}{|c|c|c|c|c|}
\hline $\begin{array}{l}\mathrm{T} \\
\mathrm{K}\end{array}$ & $\begin{array}{l}\mathrm{C}_{\mathrm{P}}^{\mathrm{id}}, \quad \text { tab } \\
\mathrm{J} \cdot \mathrm{mol}^{-1} \cdot \mathrm{K}^{-1}\end{array}$ & $\begin{array}{l}\mathrm{C}_{\mathrm{P}}^{\mathrm{id}}, \mathrm{calc} \\
\mathrm{J} \cdot \mathrm{mol}^{-1} \cdot \mathrm{K}^{-1}\end{array}$ & $\begin{array}{l}\mathrm{dev} \\
8\end{array}$ & wt \\
\hline 50.000 & 33.388 & 33.321 & -0.201 & 1.0 \\
\hline 100.000 & 35.648 & 35.698 & 0.140 & 1.0 \\
\hline 150.000 & 38.660 & 38.628 & -0.084 & 1.0 \\
\hline 200.000 & 42.258 & 42.243 & -0.037 & 1.0 \\
\hline 273.150 & 49.538 & 49.510 & -0.057 & 1.0 \\
\hline
\end{tabular}


Table 4. IDEAL GAS PROPERTIES (continued)

nata from Chao et al. [31]

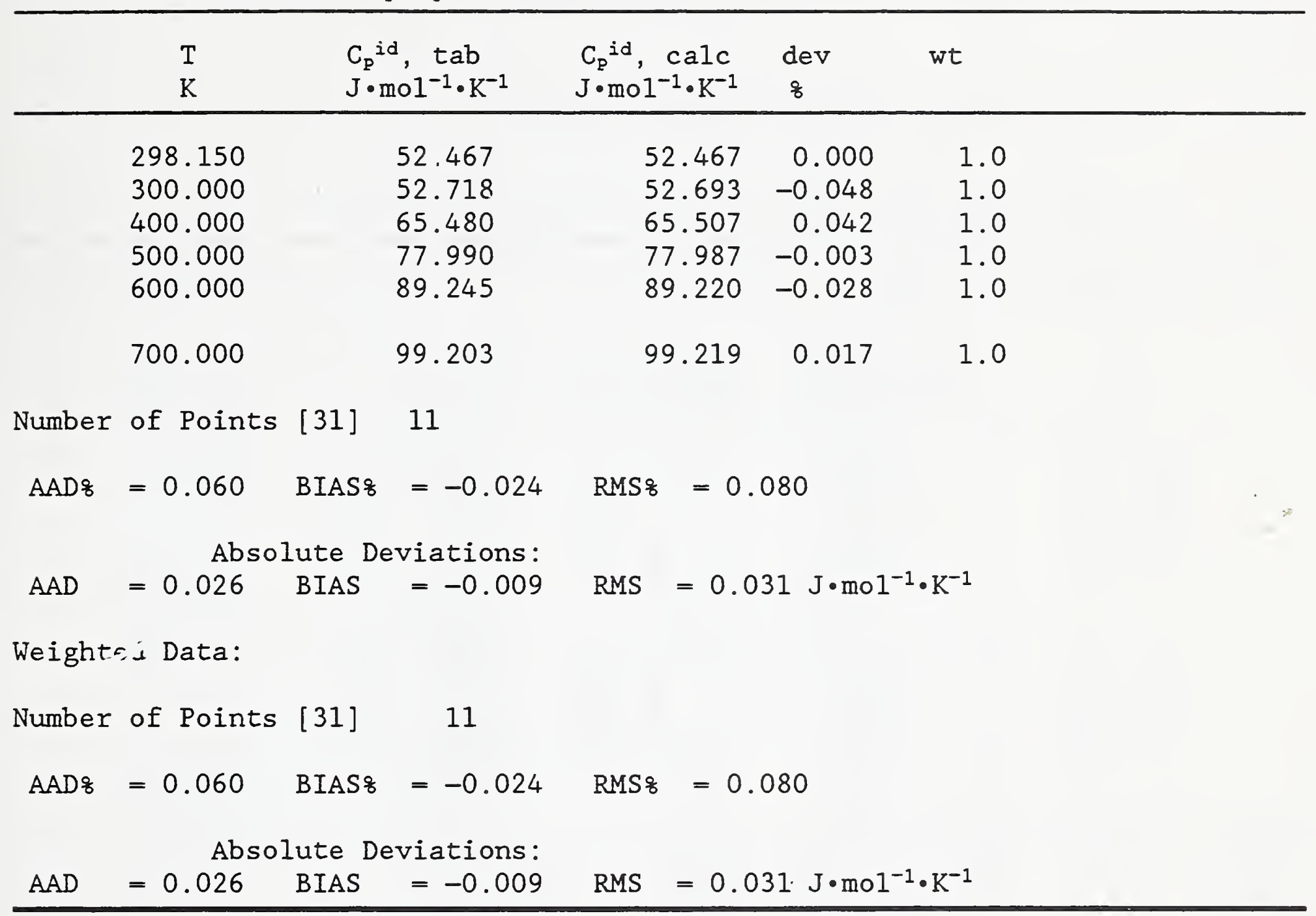

Data from Pamidimukkala et al. [32]

\begin{tabular}{|c|c|c|c|c|}
\hline $\begin{array}{l}\mathrm{T} \\
\mathrm{K}\end{array}$ & $\begin{array}{c}\mathrm{C}_{\mathrm{P}}^{\mathrm{id}}, \mathrm{tab} \\
\mathrm{J} \cdot \mathrm{mol}^{-1} \cdot \mathrm{K}^{-1}\end{array}$ & $\begin{array}{l}\mathrm{C}_{\mathrm{p}}^{\mathrm{id}}, \mathrm{calc} \\
\mathrm{J} \cdot \mathrm{mol}^{-1} \cdot \mathrm{K}^{-1}\end{array}$ & $\begin{array}{l}\text { dev } \\
8\end{array}$ & wt \\
\hline 100.000 & 35.731 & 35.698 & -0.094 & 0.0 \\
\hline 200.000 & 42.384 & 42.243 & -0.333 & 0.0 \\
\hline 298.000 & 52.467 & 52.449 & -0.035 & 0.0 \\
\hline 300.000 & 52.718 & 52.693 & -0.048 & 0.0 \\
\hline 400.000 & 65.438 & 65.507 & 0.106 & 0.0 \\
\hline 500.000 & 77.906 & 77.987 & 0.104 & 0.0 \\
\hline 600.000 & 89.161 & 89.220 & 0.066 & 0.0 \\
\hline 700.000 & 99.119 & 99.219 & 0.101 & 0.0 \\
\hline
\end{tabular}


Table 4. IDEAL GAS PROPERTIES (continued)

Data from Pamidimukkala et al. [32] (continued)

Number of Points [32] 8

$\mathrm{AAD} \%=0.111 \quad \mathrm{BIAS} \%=-0.017 \quad \mathrm{RMS} \%=0.140$

Absolute Deviations:

$\mathrm{AAD}=0.066$ BIAS $=0.011 \mathrm{RMS}=0.076 \mathrm{~J} \cdot \mathrm{mol}^{-1} \cdot \mathrm{K}^{-1}$

Data from Bier et al. [34]

\begin{tabular}{|c|c|c|c|c|}
\hline $\begin{array}{l}\mathrm{T} \\
\mathrm{K}\end{array}$ & $\begin{array}{l}\mathrm{CP}_{\mathrm{P}}^{\mathrm{id}}, \operatorname{expt} \\
\mathrm{J} \cdot \mathrm{mo} \mathrm{I}^{-1} \cdot \mathrm{K}^{-1}\end{array}$ & $\begin{array}{l}\mathrm{C}_{\mathrm{P}}^{\mathrm{id}}, \quad \mathrm{calc} \\
\mathrm{J} \cdot \mathrm{mol}^{-1} \cdot \mathrm{K}^{-1}\end{array}$ & $\begin{array}{l}\mathrm{dev} \\
8\end{array}$ & wt \\
\hline 283.150 & 50.486 & 50.671 & 0.365 & 0.0 \\
\hline 298.150 & 52.471 & 52.467 & -0.007 & 0.0 \\
\hline 305.350 & 53.283 & 53.350 & 0.125 & 0.0 \\
\hline 323.150 & 55.478 & 55.576 & 0.176 & 0.0 \\
\hline 348.150 & 58.154 & 58.778 & 1.072 & 0.0 \\
\hline 373.150 & 61.161 & 62.022 & 1.407 & 0.0 \\
\hline 398.150 & 64.589 & 65.268 & 1.051 & 0.0 \\
\hline 423.150 & 67.776 & 68.484 & 1.044 & 0.0 \\
\hline 473.150 & 74.091 & 74.746 & 0.884 & 0.0 \\
\hline
\end{tabular}

Number of Points [34] 9

$\mathrm{AAD} \%=0.681 \quad \mathrm{BIAS} \%=0.680 \quad \mathrm{RMS} \%=0.486$

Absolute Deviations:

$\mathrm{AAD}=0.431 \quad \mathrm{BIAS}=0.430 \mathrm{RMS}=0.317 \mathrm{~J} \cdot \mathrm{mol}^{-1} \cdot \mathrm{K}^{-1}$

Data from Furtado [35]

\begin{tabular}{ccrrr}
\hline $\mathrm{T}$ & $\begin{array}{c}\mathrm{C}_{\mathrm{P}}{ }^{i d}, \operatorname{expt} \\
\mathrm{J} \cdot \mathrm{mol}^{-1} \cdot \mathrm{K}^{-1}\end{array}$ & $\begin{array}{c}\mathrm{C}_{\mathrm{P}}{ }^{\mathrm{id}}, \mathrm{calc} \\
\mathrm{J} \cdot \mathrm{mol}^{-1} \cdot \mathrm{K}^{-1}\end{array}$ & $\begin{array}{c}\text { dev } \\
\text { o }\end{array}$ & wt \\
\hline 99.817 & 35.856 & 35.688 & -0.470 & 0.0 \\
110.928 & 36.493 & 36.301 & -0.527 & 0.0 \\
118.372 & 36.981 & 36.723 & -0.696 & 0.0 \\
122.039 & 37.243 & 36.934 & -0.828 & 0.0 \\
133.150 & 37.993 & 37.588 & -1.064 & 0.0 \\
& & & & \\
144.261 & 38.742 & 38.266 & -1.229 & 0.0 \\
155.372 & 39.249 & 38.974 & -0.700 & 0.0 \\
166.483 & 40.017 & 39.717 & -0.749 & 0.0 \\
177.594 & 40.767 & 40.504 & -0.646 & 0.0 \\
186.872 & 41.385 & 41.197 & -0.454 & 0.0
\end{tabular}


Table 4. IDEAL GAS PROPERTIES (continued)

Lata from Furtado [35] (continued)

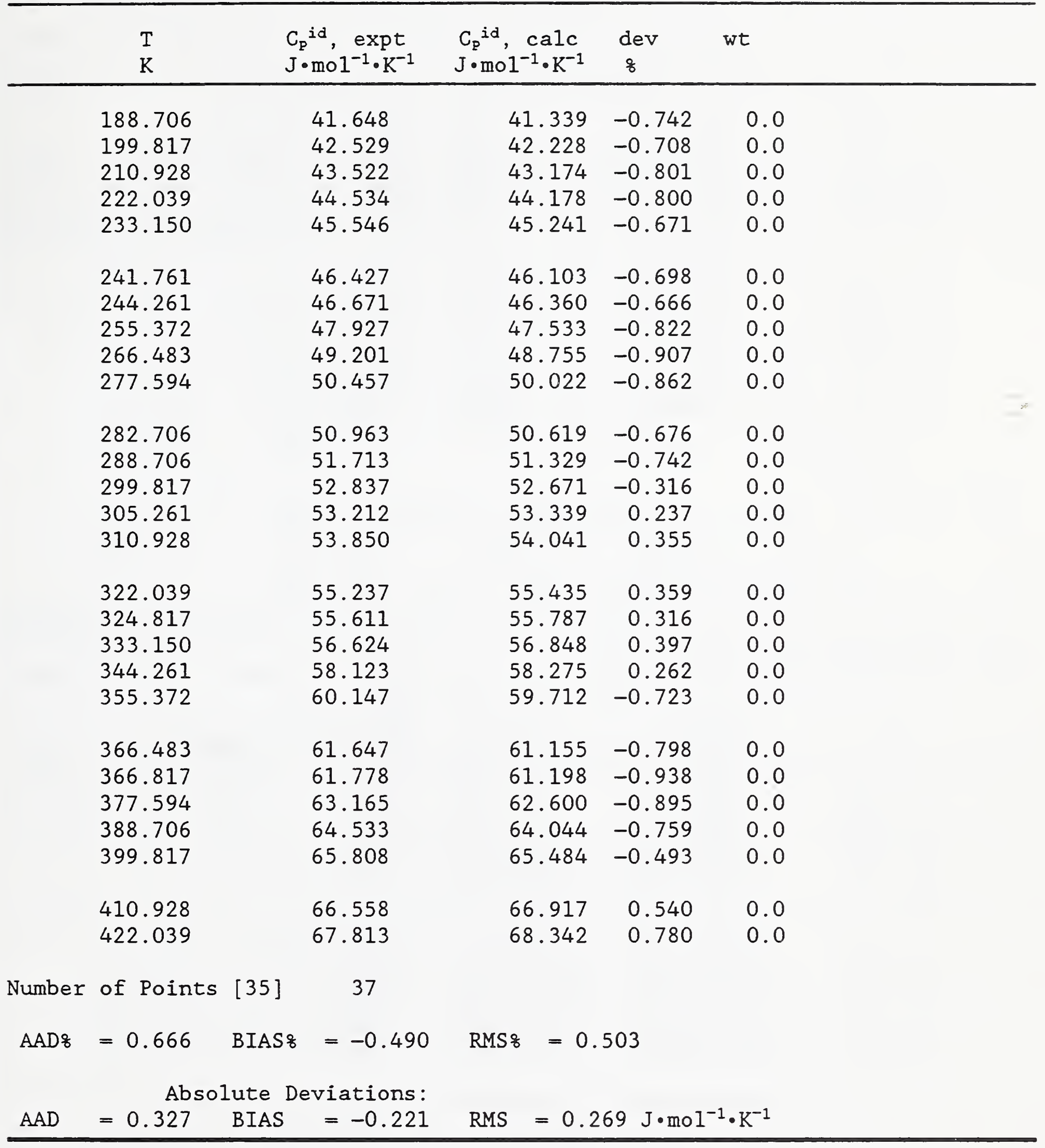


Table 4. IDEAL GAS PROPERTIES (continued)

Data from TRC [33]

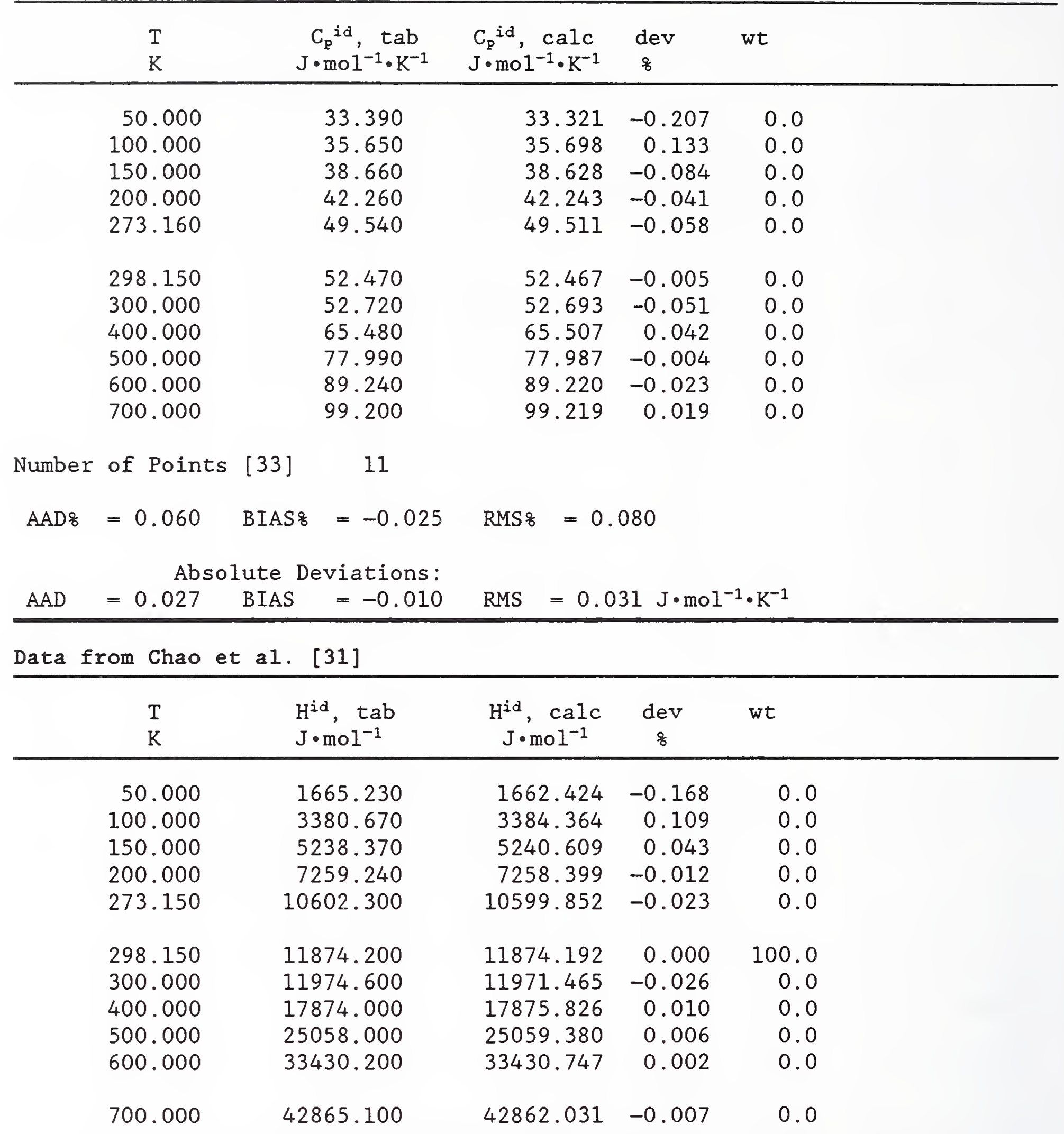


Table 4. IDEAL GAS PROPERTIES (continued)

Data from Chao et al. [31] (continued)

Number of Points [31] 11

$\mathrm{AAD} z=0.037 \quad \mathrm{BIAS} \%=-0.006 \quad$ RMS $8=0.063$

Absoluta Deviations:

$A A D=1.999 \quad B I A S=-0.238 \quad \mathrm{RMS}=2.284 \mathrm{~J} \cdot \mathrm{mol}^{-1}$

Data from Pamidimukkala et al. [32]

\begin{tabular}{crrrr}
$\mathrm{T}$ & $\begin{array}{c}\mathrm{H}^{\mathrm{id}}, \mathrm{tab} \\
\mathrm{J} \cdot \mathrm{mo} 1^{-1}\end{array}$ & $\begin{array}{c}\mathrm{H}^{\mathrm{id}}, \mathrm{calc} \\
\mathrm{J} \cdot \mathrm{mol}^{-1}\end{array}$ & $\begin{array}{c}\mathrm{dev} \\
\mathrm{s}\end{array}$ & wt \\
\hline $\mathrm{K}$ & 3389.040 & 3384.364 & -0.138 & 0.0 \\
200.000 & 7280.160 & 7258.399 & -0.299 & 0.0 \\
298.000 & 11882.600 & 11866.323 & -0.137 & 0.0 \\
300.000 & 12008.100 & 11971.465 & -0.305 & 0.0 \\
400.000 & 17907.500 & 17875.826 & -0.177 & 0.0 \\
500.000 & 25062.200 & 25059.380 & -0.011 & 0.0 \\
600.000 & 33430.200 & 33430.747 & 0.002 & 0.0 \\
700.000 & 42844.200 & 42862.031 & 0.042 & 0.0
\end{tabular}

Number of Points [32] 8

$\mathrm{AAD} \&=0.139 \quad \mathrm{BIAS} \%=-0.128 \quad \mathrm{RMS} \%=0.124$

Absolute Deviations:

$A A D=16.528 \quad$ BIAS $=-11.933 \quad$ RMS $=16.929 \mathrm{~J} \cdot \mathrm{mol}^{-1}$

Data from TRC [33]

\begin{tabular}{|c|c|c|c|c|}
\hline $\begin{array}{l}\mathrm{T} \\
\mathrm{K}\end{array}$ & $\begin{array}{l}\mathrm{H}^{\mathrm{id}}, \quad \mathrm{tab} \\
\mathrm{J} \cdot \mathrm{mol}^{-1}\end{array}$ & $\begin{array}{c}\mathrm{H}^{\mathrm{id}}, \mathrm{calc} \\
\mathrm{J} \cdot \mathrm{mol}^{-1}\end{array}$ & $\begin{array}{c}\mathrm{dev} \\
8\end{array}$ & wt \\
\hline 50.000 & 1665.000 & 1662.424 & -0.155 & 0.0 \\
\hline 100.000 & 3381.000 & 3384.364 & 0.099 & 0.0 \\
\hline 150.000 & 5238.000 & 5240.609 & 0.050 & 0.0 \\
\hline 200.000 & 7259.000 & 7258.399 & -0.008 & 0.0 \\
\hline 273.160 & 10602.000 & 10600.347 & -0.016 & 0.0 \\
\hline 298.150 & 11874.000 & 11874.192 & 0.002 & 0.0 \\
\hline 300.000 & 11975.000 & 11971.465 & -0.030 & 0.0 \\
\hline 400.000 & 17874.000 & 17875.826 & 0.010 & 0.0 \\
\hline 500.000 & 25058.000 & 25059.380 & 0.006 & 0.0 \\
\hline 600.000 & 33430.000 & 33430.747 & 0.002 & 0.0 \\
\hline 700.000 & 42840.000 & 42862.031 & 0.051 & 0.0 \\
\hline
\end{tabular}


Table 4. IDEAL GAS PROPERTIES (continued)

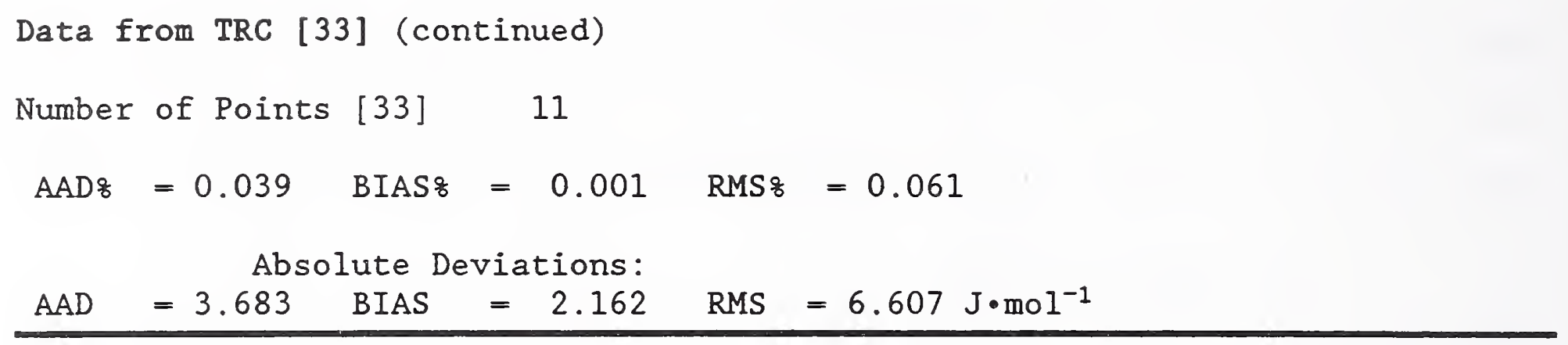


4. Thermodynamic Properties in the Single-Phase Region

Tables 5-11 give comparisons for the thermodynamic properties of the single-phase fluid as well as the heat capacity and sound speed in the saturated liquid.

Some of the data for the second virial coefficient, as tabulated in table 5, have been obtained from model potentials or were originally presented graphically or algebraically; consult the references for details. Some of these data have been adjusted or tabulated by other correlators as indicated in the table notes.

For the PVT data, table 6, we have presented comparisons based on both calculation of density using experimental temperature and pressure, and calculation of pressure using experimental temperature and density. The stati,tics with the suffix 2 refer to density calculations, and the statistics without the suffix refer to pressure calculations.

In table 7, for the isochoric specific heat capacity, we have presented several comparisons: $\mathrm{dev}$ is the percent difference between the heat capacity calculated from the tabulated temperature and density and the experimental value, dev2 is based on calculation from the tabulated temperature and pressure, dev3 compares densities calculated from the SWEOS with the tabulated values, and dev 4 compares pressures calculated from the SWEOS with the tabulated values. The two sets of statistics compare the experimental isochoric heat capacities with calculations based on both density input and pressure input.

Table 9, for the heat capacity along the saturated liquid boundary, includes a column for the density of the liquid; this quantity was calculated from the ancillary equation and was used as input into the SWEOS to generate the calculated heat capacity.

We have presented two sets of statistics in table 10 for the speed of sound in the single phase fluid. The density, in column 3, was calculated from the SWEOS and the experimental temperature and pressure (given in the table) and was 
used to calculate $\mathrm{W}$ and $\partial \mathrm{P} /\left.\partial \rho\right|_{\mathrm{T}}$. The isothermal density derivatives are included in the table because these quantities, rather than the sound speed itself, were used to fit the SWEOS [1]. The experimental value of $\partial P /\left.\partial \rho\right|_{\mathrm{T}}$ is defined by $\partial \mathrm{P} /\left.\partial \rho\right|_{\mathrm{T}}=\mathrm{N}_{\mathrm{A}} \mathrm{u} \mathrm{M} \mathrm{M}_{\mathrm{T}} \mathrm{W}^{2} \mathrm{C}_{\mathrm{V}} / \mathrm{C}_{\mathrm{P}}$ where $\mathrm{N}_{\mathrm{A}}$ is the Avogadro number, $\mathrm{u}$ is the unified atomic mass unit, $M_{r}$ is the relative molecular mass of ethane, $W$ is the experimental value of the speed of sound, and the heat capacities are calculated from the SWEOS at the experimental state point. The statistics with the suffix 2 refer to comparisons between this "experimental" value of $\partial \mathrm{P} /\left.\partial \rho\right|_{\mathrm{T}}$ and the value calculated directly from the SWEOS.

Table 11 is similar in form to table 10. The essential difference is that the pressure and density at saturation were calculated from the experimental temperature using the SWEOS and the Maxwell construction. These densities were used for the calculations of sound speed and the isothermal pressure derivative. The suffix 2 on the statistics has the same meaning as in table 10: the comparisons are for the experimental value of $\partial \mathrm{P} /\left.\partial \rho\right|_{\mathrm{T}}$ and that calculated by the SWEOS (for the saturated liquid). 
Table 5. SECOND VIRIAL COEFFICIENTS

Data from Douslin and Harrison [9]

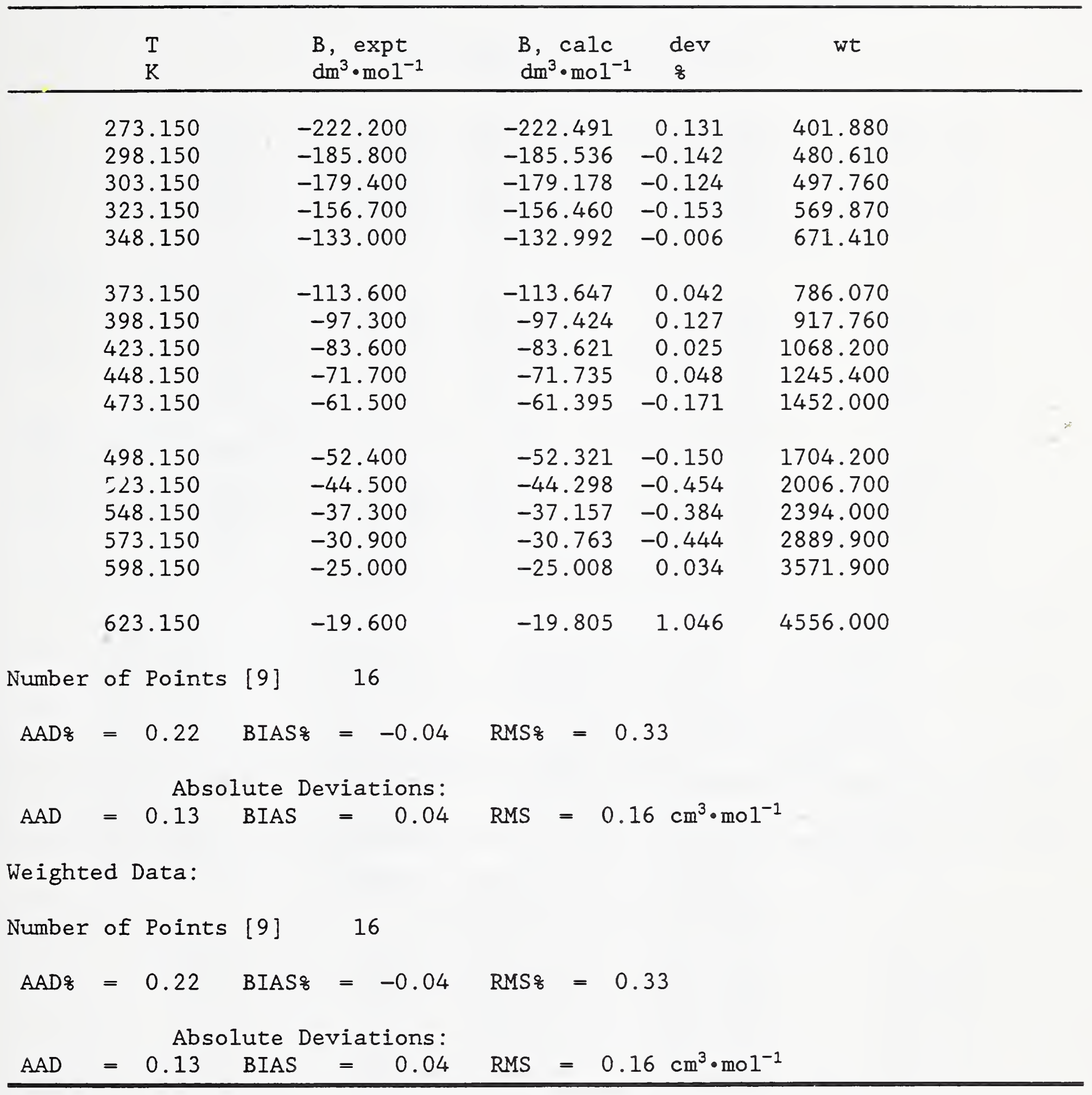


Table 5. SECOND VIRIAL COEFFICIENTS (continued)

Data from Dymond and Smith [36]

\begin{tabular}{ccccc}
$T$ & $\begin{array}{c}\text { B, expt } \\
\mathrm{dm} \cdot \mathrm{mol}^{-1}\end{array}$ & $\begin{array}{c}\mathrm{B}, \mathrm{calc} \\
\mathrm{dm} \cdot \mathrm{mol}^{-1}\end{array}$ & $\begin{array}{c}\mathrm{d} \text { dev } \\
\text { o }\end{array}$ & wt \\
\hline 200.000 & -410.000 & -420.798 & 2.634 & 0.000 \\
210.000 & -370.000 & -381.032 & 2.982 & 0.000 \\
220.000 & -336.000 & -346.514 & 3.129 & 0.000 \\
240.000 & -282.000 & -290.060 & 2.858 & 0.000 \\
260.000 & -243.000 & -246.228 & 1.328 & 0.000 \\
& & & & \\
280.000 & -211.000 & -211.407 & 0.193 & 0.000 \\
300.000 & -182.000 & -183.149 & 0.631 & 0.000 \\
325.000 & -154.000 & -154.553 & 0.359 & 0.000 \\
350.000 & -130.500 & -131.433 & 0.715 & 0.000 \\
375.000 & -111.000 & -112.350 & 1.216 & 0.000 \\
400.000 & -96.000 & -96.327 & 0.340 & 0.000 \\
450.000 & -71.000 & -70.921 & -0.112 & 0.000 \\
500.000 & -52.000 & -51.694 & -0.589 & 0.000 \\
550.000 & -36.500 & -36.659 & 0.437 & 0.000 \\
600.000 & -24.500 & -24.606 & 0.431 & 0.000
\end{tabular}

Number of Points [36] 15

$\mathrm{AAD} \%=1.20 \quad$ BIAS $8=1.10 \quad$ RMS $8=1.17$

Absolute Deviations:

$\mathrm{AAD}=3.27$ BIAS $=-3.22$ RMS $=4.27 \mathrm{~cm}^{3} \cdot \mathrm{mol}^{-1}$

Data from Eucken and Parts [37]

\begin{tabular}{ccccc}
\hline & $\begin{array}{c}\mathrm{B}, \text { expt } \\
\mathrm{dm} \cdot \mathrm{mol}^{-1}\end{array}$ & $\begin{array}{c}\mathrm{B}, \mathrm{calc} \\
\mathrm{dm}^{3} \cdot \mathrm{mol}^{-1}\end{array}$ & $\begin{array}{c}\mathrm{dev} \\
8\end{array}$ & wt \\
\hline 191.860 & -498.000 & -457.664 & -8.100 & 0.000 \\
193.650 & -487.000 & -449.183 & -7.765 & 0.000 \\
201.610 & -446.000 & -414.004 & -7.174 & 0.000 \\
202.170 & -443.000 & -411.678 & -7.070 & 0.000 \\
211.330 & -404.000 & -376.157 & -6.892 & 0.000 \\
213.340 & -395.000 & -368.962 & -6.592 & 0.000 \\
222.580 & -360.000 & -338.358 & -6.012 & 0.000 \\
224.500 & -354.000 & -332.471 & -6.082 & 0.000 \\
233.410 & -325.000 & -307.049 & -5.523 & 0.000 \\
236.670 & -316.000 & -298.465 & -5.549 & 0.000
\end{tabular}


Table 5. SECOND VIRIAL COEFFICIENTS (continued)

Data from Eucken and Parts [37] (continued)

\begin{tabular}{ccccc}
\hline $\begin{array}{c}\mathrm{T} \\
\mathrm{K}\end{array}$ & $\begin{array}{l}\mathrm{B}, \operatorname{expt} \\
\mathrm{dm}^{3} \cdot \mathrm{mo1}^{-1}\end{array}$ & $\begin{array}{l}\mathrm{B}, \mathrm{calc} \\
\mathrm{dm}^{3} \cdot \mathrm{mol}^{-1}\end{array}$ & $\begin{array}{l}\mathrm{dev} \\
8\end{array}$ & wt \\
\hline 244.700 & -293.000 & -278.781 & -4.853 & 0.000 \\
247.000 & -287.000 & -273.497 & -4.705 & 0.000 \\
257.090 & -262.000 & -251.974 & -3.827 & 0.000 \\
259.030 & -258.000 & -248.122 & -3.829 & 0.000 \\
273.200 & -227.500 & -222.407 & -2.239 & 0.000
\end{tabular}

Number of Points [37] 15

$\mathrm{AAD} \&=5.75 \quad$ BIAS\& $=-5.75$ RMS $8=1.58$

Absolute Deviations:

$\mathrm{AAD}=21.78$ BIAS $=21.78$ RMS $=10.23 \mathrm{~cm}^{3} \cdot \mathrm{mol}^{-1}$

Data from Eucken via Tester [38]

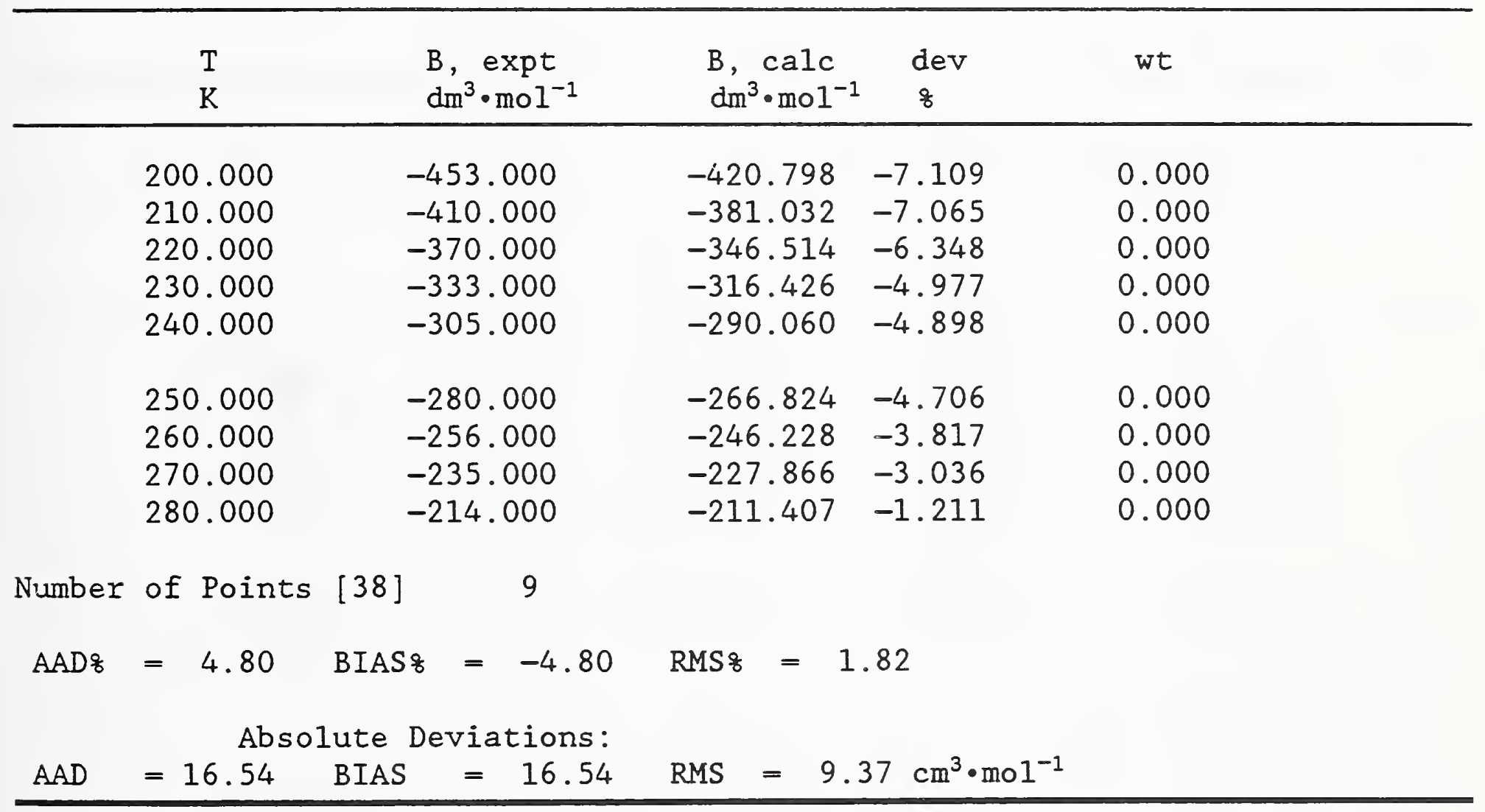


Table 5. SECOND VIRIAL COEFFICIENTS (continued)

Data from Gunn [39] reported by Huff and Reed [40]

\begin{tabular}{|c|c|c|c|c|c|c|c|}
\hline & & $\begin{array}{l}\mathrm{T} \\
\mathrm{K}\end{array}$ & & $\begin{array}{l}B, \operatorname{expt} \\
\mathrm{dm}^{3} \cdot \mathrm{mol}^{-1}\end{array}$ & $\begin{array}{l}\mathrm{B}, \mathrm{calc} \\
\mathrm{dm}^{3} \cdot \mathrm{mol}^{-1}\end{array}$ & $\begin{array}{l}\text { dev } \\
8\end{array}$ & wt \\
\hline & 273 & 3.200 & & -222.200 & -222.407 & 0.093 & 0.000 \\
\hline & 298 & 8.200 & & -186.900 & -185.471 & -0.765 & 0.000 \\
\hline & 323 & 3.200 & & -157.500 & -156.408 & -0.693 & 0.000 \\
\hline & 377 & 7.600 & & -109.400 & -110.555 & 1.055 & 0.000 \\
\hline & 410 & 0.900 & & -89.600 & -90.117 & 0.577 & 0.000 \\
\hline & 444 & 4.300 & & -74.000 & -73.456 & -0.735 & 0.000 \\
\hline & 477 & 7.600 & & -61.600 & -59.694 & -3.094 & 0.000 \\
\hline & 510 & 0.900 & & -51.000 & -48.110 & -5.667 & 0.000 \\
\hline Number & of & Points & [39] & 8 & & & \\
\hline$A A D$ 용 & $=$ & 1.58 & BIAS क्ष & $=-1.15$ & RMS 8 & .06 & \\
\hline & & $\mathrm{Abs}$ & Lute D & Deviations: & & & \\
\hline$A A D$ & $=$ & 1.22 & BIAS & $=0.75$ & $=1$. & $26 \mathrm{~cm}^{3} \cdot \mathrm{mol}^{-1}$ & \\
\hline
\end{tabular}

Data from Hamann and McManamey [41]

Using data from Reamer et al. [42], as reported by Dymond and Smith [36]

\begin{tabular}{|c|c|c|c|c|c|c|}
\hline & $\begin{array}{l}\mathrm{T} \\
\mathrm{K}\end{array}$ & & $\begin{array}{l}B, \operatorname{expt} \\
\mathrm{dm}^{3} \cdot \mathrm{mol}^{-1}\end{array}$ & $\begin{array}{l}\mathrm{B}, \text { calc } \\
\mathrm{dm}^{3} \cdot \mathrm{mol}^{-1}\end{array}$ & $\begin{array}{l}\text { dev } \\
q 8\end{array}$ & wt \\
\hline & 310.940 & & -164.900 & -169.843 & 2.997 & 0.000 \\
\hline & 344.270 & & -135.500 & -136.333 & 0.615 & 0.000 \\
\hline & 377.600 & & -110.000 & -110.555 & 0.504 & 0.000 \\
\hline & 410.940 & & -90.400 & -90.095 & -0.337 & 0.000 \\
\hline & 444.270 & & -74.200 & -73.470 & -0.984 & 0.000 \\
\hline & 477.600 & & -59.900 & -59.094 & -0.344 & 0.000 \\
\hline & 510.940 & & -47.400 & -48.097 & 1.470 & 0.000 \\
\hline Number & of Points & [41] & 7 & & & \\
\hline $\mathrm{AAD} \%$ & $=1.04$ & BIAS 웅 & 0.56 & RMS 8 & .24 & \\
\hline & Abs & -ute D & Deviations: & & & \\
\hline$A A D$ & $=1.18$ & BIAS & $=-0.83$ & $=1$. & $76 \mathrm{~cm}^{3} \cdot \mathrm{mol}^{-1}$ & \\
\hline
\end{tabular}


Table 5. SECOND VIRIAL COEFFICIENTS (continued)

Data from Hoover et al. [43]

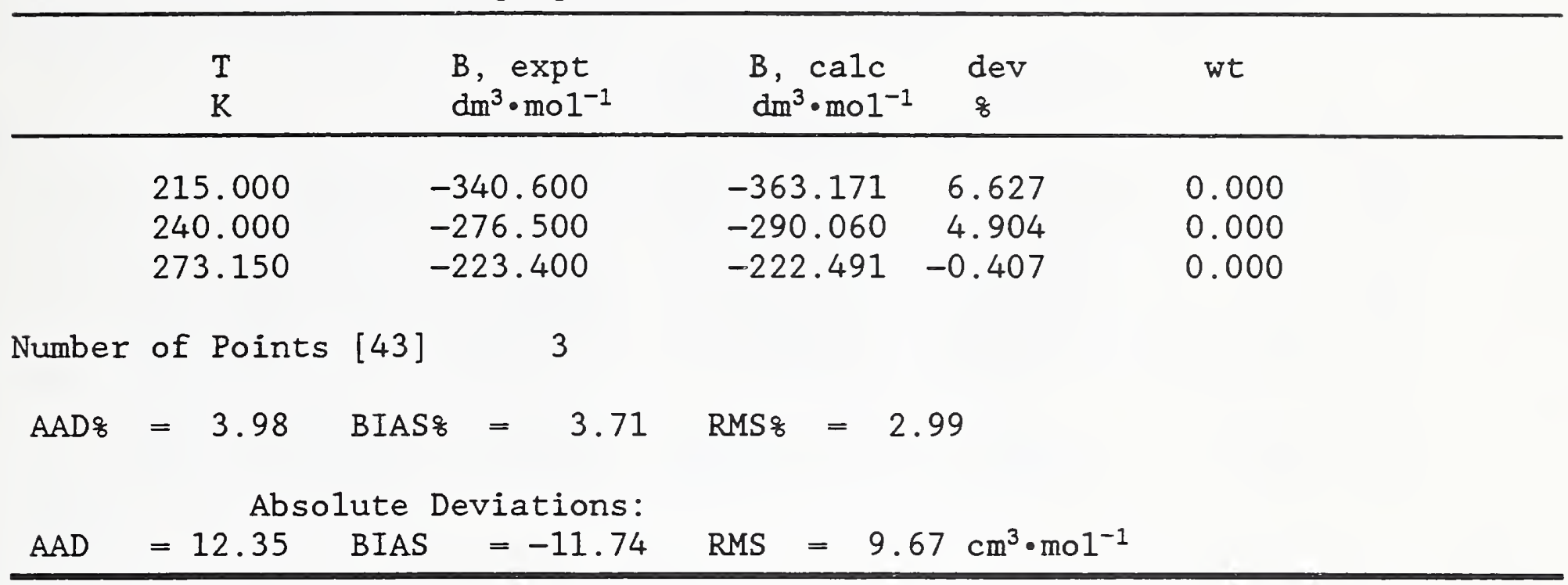

Data from Lambert et al. [44]

Reported by Tester [38]

\begin{tabular}{|c|c|c|c|c|c|c|}
\hline & $\begin{array}{l}\mathrm{T} \\
\mathrm{K}\end{array}$ & & $\begin{array}{l}B, \operatorname{expt} \\
\mathrm{dm}^{3} \cdot \mathrm{mol}^{-1}\end{array}$ & $\begin{array}{l}\text { B, calc } \\
\mathrm{dm}^{3} \cdot \mathrm{mol}^{-1}\end{array}$ & $\begin{array}{l}\text { dev } \\
q\end{array}$ & wt \\
\hline & 200.000 & & -454.000 & -420.798 & -7.313 & 0.000 \\
\hline & 210.000 & & -410.000 & -381.032 & -7.065 & 0.000 \\
\hline & 220.000 & & -375.000 & -346.514 & -7.596 & 0.000 \\
\hline & 230.000 & & -341.000 & -316.426 & -7.207 & 0.000 \\
\hline & 240.000 & & -308.000 & -290.060 & -5.825 & 0.000 \\
\hline & 250.000 & & -283.000 & -266.824 & -5.716 & 0.000 \\
\hline & 260.000 & & -260.000 & -246.228 & -5.297 & 0.000 \\
\hline & 270.000 & & -240.000 & -227.866 & -5.056 & 0.000 \\
\hline & 280.000 & & -220.000 & -211.407 & -3.906 & 0.000 \\
\hline & 290.000 & & -205.000 & -196.577 & -4.109 & 0.000 \\
\hline & 300.000 & & -190.000 & -183.149 & -3.606 & 0.000 \\
\hline Number & of Points & [44] & 11 & & & \\
\hline $\mathrm{AAD} \&$ & $=5.70$ & BIAS 8 & $g=-5.70$ & RMS 8 & .38 & \\
\hline & Abso & Lute D & Deviations: & & & \\
\hline$A A D$ & $=18.10$ & BIAS & $=18.10$ & RMS & $87 \mathrm{~cm}^{3} \cdot \mathrm{mol}^{-1}$ & \\
\hline
\end{tabular}


Table 5. SECOND VIRIAL COEFFICIENTS (continued)

Data from Mansoorian et al. [45]

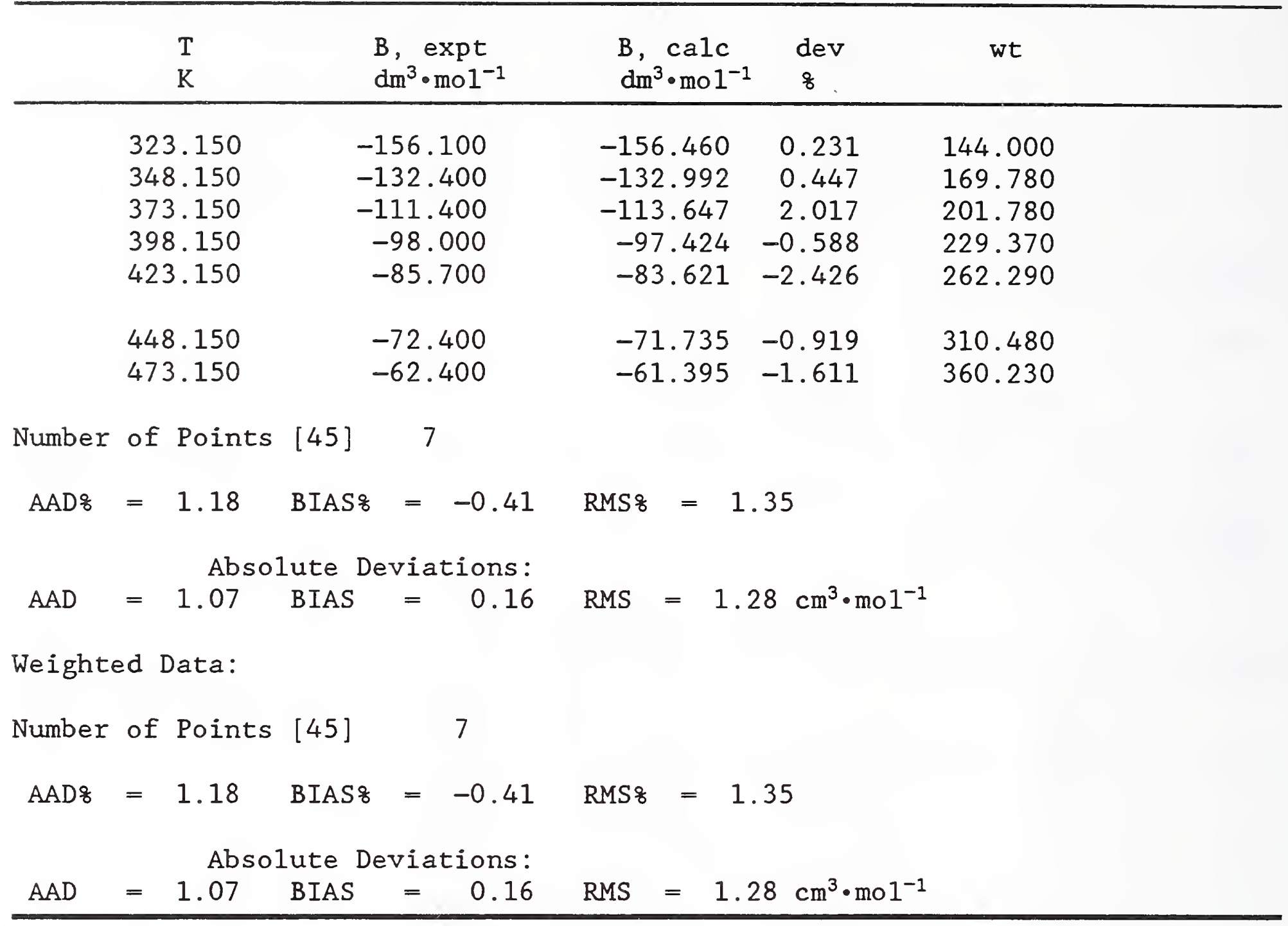

Data from McGlashan and Potter [46]

Reported by Goodwin et al. [20]

\begin{tabular}{ccccc}
\hline & $\begin{array}{c}\text { B, expt } \\
\mathrm{dm} \cdot \mathrm{mol}^{-1}\end{array}$ & $\begin{array}{c}\text { B, calc } \\
\mathrm{dm}^{3} \cdot \mathrm{mol}^{-1}\end{array}$ & $\begin{array}{c}\mathrm{dev} \\
\text { 8 }\end{array}$ & wt \\
\hline 150.000 & -769.000 & -719.208 & -6.475 & 0.000 \\
160.000 & -666.030 & -647.669 & -2.757 & 0.000 \\
170.000 & -583.830 & -580.113 & -0.637 & 0.000 \\
180.000 & -517.070 & -519.635 & 0.496 & 0.000 \\
190.000 & -461.730 & -466.711 & 1.079 & 0.000 \\
200.000 & -415.440 & -420.798 & 1.290 & 0.000 \\
210.000 & -376.270 & -381.032 & 1.266 & 0.000 \\
220.000 & -342.290 & -346.514 & 1.234 & 0.000 \\
230.000 & -313.060 & -316.426 & 1.075 & 0.000 \\
240.000 & -287.390 & -290.060 & 0.929 & 0.000
\end{tabular}


Table 5. SECOND VIRIAL COEFFICIENTS (continued)

Data from McGlashan and Potter [46]

Reported by Goodwin et al. [20]

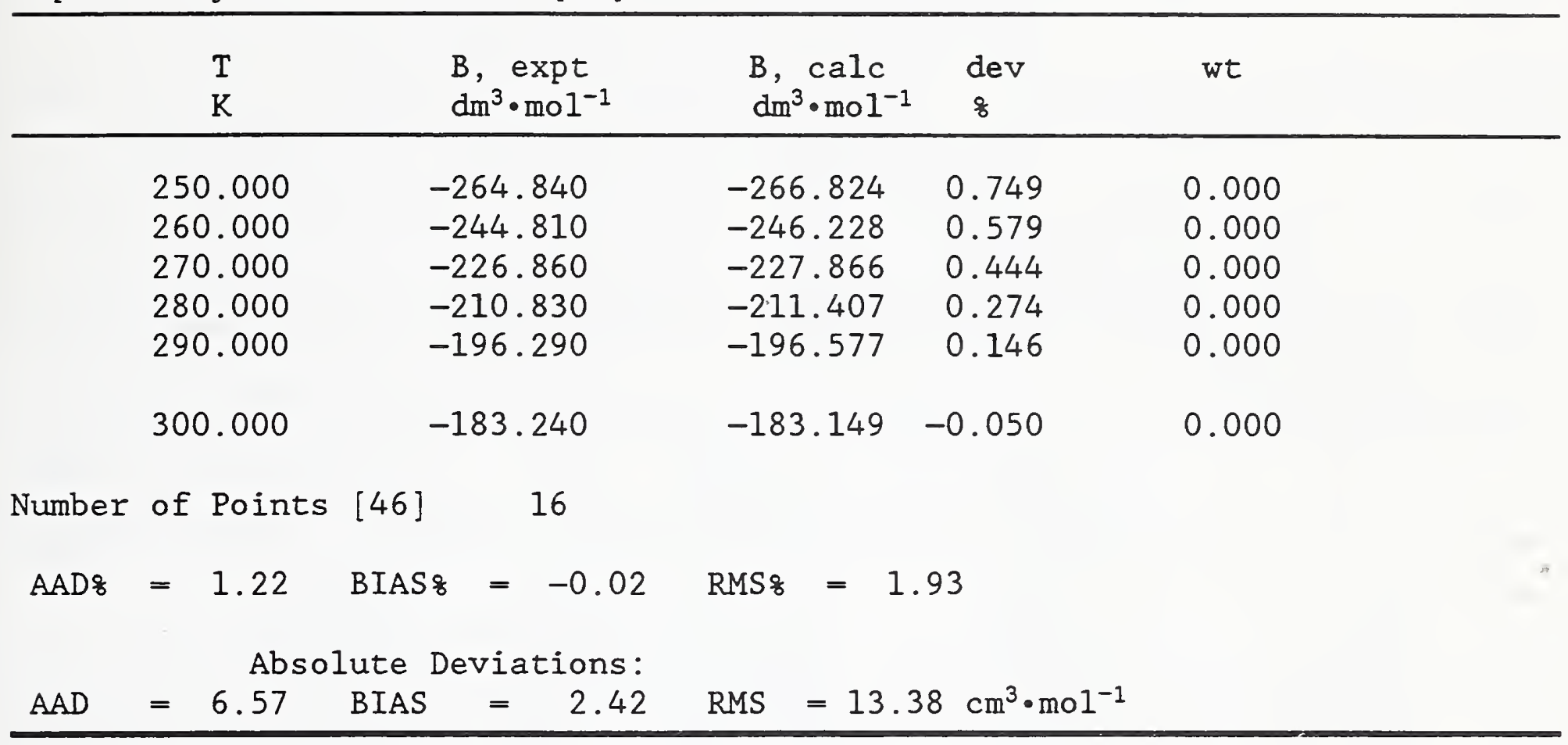

Data from Michels et a1. [47]

\begin{tabular}{ccrrr}
$T$ & $\begin{array}{c}\text { B, expt } \\
\mathrm{dm}^{3} \cdot \mathrm{mol}^{-1}\end{array}$ & \multicolumn{1}{c}{$\begin{array}{l}\text { B, calc } \\
\mathrm{dm}^{3} \cdot \mathrm{mol}^{-1}\end{array}$} & \multicolumn{1}{c}{$\begin{array}{c}\mathrm{dev} \\
\text { 8 }\end{array}$} & wt \\
\hline 273.150 & -221.450 & -22.491 & 0.470 & 125.140 \\
298.130 & -185.600 & -185.562 & -0.020 & 149.320 \\
322.738 & -156.920 & -156.889 & -0.020 & 176.610 \\
347.562 & -133.280 & -133.492 & 0.159 & 207.930 \\
372.522 & -114.060 & -114.092 & 0.028 & 242.970 \\
397.854 & -97.720 & -97.601 & -0.122 & 283.600 \\
422.720 & -83.910 & -83.841 & -0.083 & 330.270
\end{tabular}

Number of Points [47] 7

$\mathrm{AAD} \%=0.13 \quad \mathrm{BIAS} \%=0.06 \quad \mathrm{RMS} \%=0.19$

Absolute Deviations:

$\mathrm{AAD}=0.22$ BIAS $=-0.15$ RMS $=0.38 \mathrm{~cm}^{3} \cdot \mathrm{mol}^{-1}$ 
Table 5. SECOND VIRIAL COEFFICIENTS (continued)

Data from Michels et al. [47] (continued)

Weighted Data:

Number of Points [47] 7

$\mathrm{AAD} \&=0.13 \quad$ BIAS\& $=0.06 \quad$ RMS $8=0.19$

Absolute Deviations:

$\mathrm{AAD}=0.22$ BIAS $=-0.15$ RMS $=0.38 \mathrm{~cm}^{3} \cdot \mathrm{mol}^{-1}$

Data from Pope [14]

\begin{tabular}{cllll}
\hline & $\begin{array}{c}\mathrm{B}, \text { expt } \\
\mathrm{dm} \cdot \mathrm{mol}^{-1}\end{array}$ & $\begin{array}{c}\mathrm{B}, \mathrm{calc} \\
\mathrm{dm}^{3} \cdot \mathrm{mol}^{-1}\end{array}$ & $\begin{array}{l}\mathrm{dev} \\
8\end{array}$ & wt \\
\hline 209.534 & -368.660 & -382.762 & 3.825 & 25.058 \\
238.769 & -287.050 & -293.126 & 2.117 & 32.182 \\
254.807 & -252.270 & -256.621 & 1.725 & 36.618 \\
273.150 & -219.380 & -222.491 & 1.418 & 42.108 \\
306.062 & -175.270 & -175.610 & 0.194 & 52.706
\end{tabular}

Number of Points [14] 5

$\mathrm{AAD} \%=1.86 \quad \mathrm{BIAS} \%=1.86 \quad \mathrm{RMS} \%=1.18$

Absolute Deviations:

$\mathrm{AAD}=5.60$ BIAS $=-5.60$ RMS $=4.65 \mathrm{~cm}^{3} \cdot \mathrm{mol}^{-1}$

Weighted Data:

Number of Points [14] 5

$\mathrm{AAD}$ \% $=1.86 \quad \mathrm{BIAS} \%=1.86 \quad \mathrm{RMS} \%=1.18$

Absolute Deviations:

$A A D=5.60$ BIAS $=-5.60$ RMS $=4.65 \mathrm{~cm}^{3} \cdot \mathrm{mol}^{-1}$

Data from Strein et al. [48]

\begin{tabular}{cllll}
\hline & $\begin{array}{c}\mathrm{B}, \text { expt } \\
\mathrm{dm}\end{array}$ & $\begin{array}{c}\mathrm{B}, \mathrm{calc} \mathrm{mol}^{-1} \\
\mathrm{dm}^{3} \cdot \mathrm{mol}^{-1}\end{array}$ & $\begin{array}{c}\mathrm{dev} \\
\text { 8 }\end{array}$ & wt \\
\hline 296.100 & -188.000 & -188.232 & 0.123 & 0.000 \\
307.600 & -172.000 & -173.764 & 1.025 & 0.000 \\
333.600 & -144.300 & -146.067 & 1.225 & 0.000 \\
353.400 & -126.200 & -128.627 & 1.923 & 0.000 \\
373.700 & -111.900 & -113.260 & 1.215 & 0.000
\end{tabular}


Table 5. SECOND VIRIAL COEFFICIENTS (continued)

Data from Strein et al. [48] (continued)

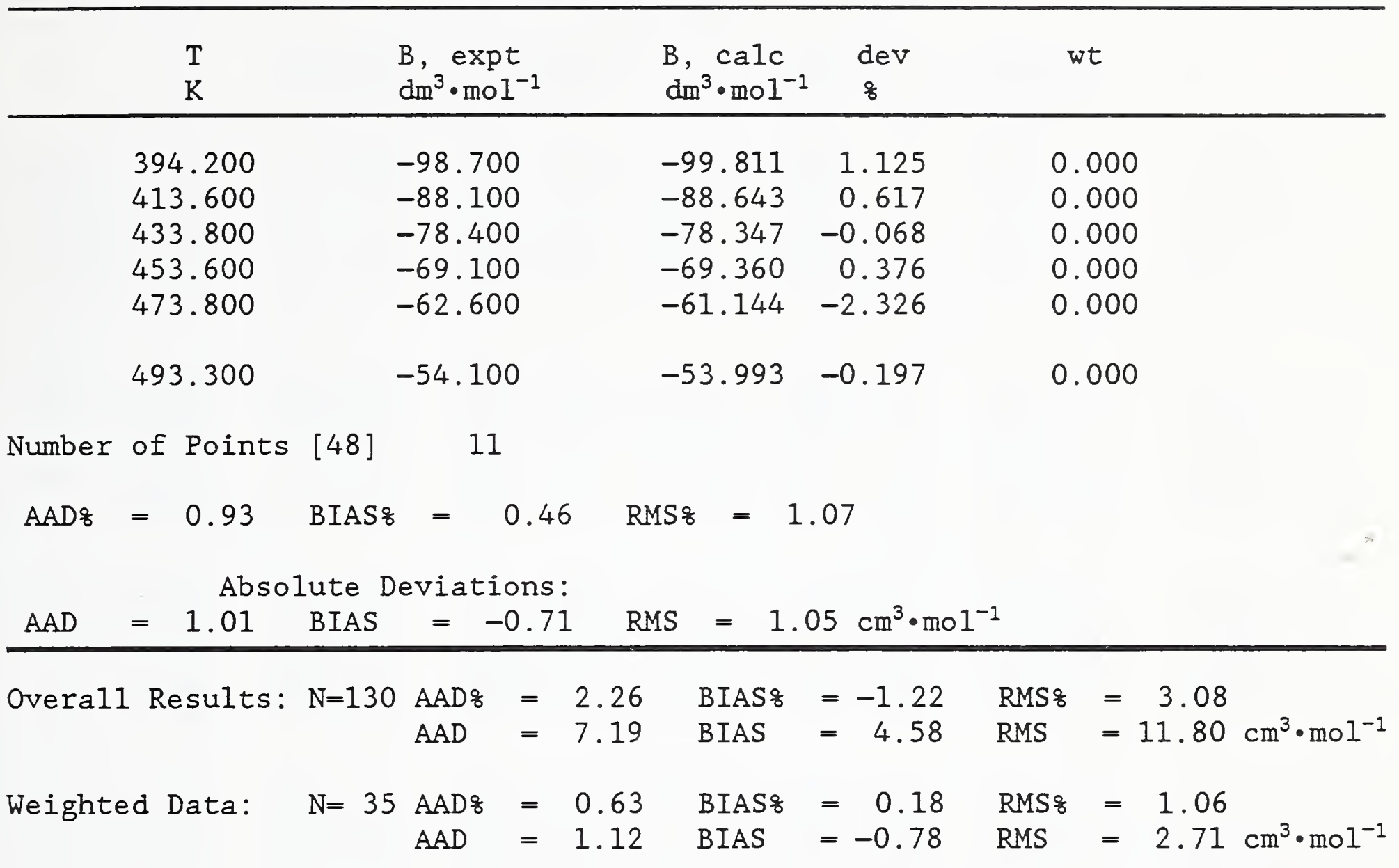


Table 6. PVT DATA

Data from Beattie et al. [49]

\begin{tabular}{|c|c|c|c|c|c|c|c|}
\hline $\begin{array}{l}\mathrm{T} \\
\mathrm{K}\end{array}$ & $\mathrm{P}$, expt & 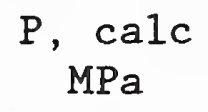 & $\begin{array}{c}\text { dev } \\
8\end{array}$ & $\begin{array}{l}\rho, \quad \operatorname{expt} \\
\mathrm{mol} 1 \cdot \mathrm{dm}^{-3}\end{array}$ & $\begin{array}{c}\rho, \quad \text { calc } \\
\mathrm{mol} \cdot \mathrm{dm}^{-3}\end{array}$ & $\begin{array}{c}\text { dev } \\
8\end{array}$ & wt \\
\hline 298.150 & 1.126 & 1.128 & 0.17 & 0.500 & 0.499 & -0.19 & 0.0 \\
\hline 298.150 & 2.041 & 2.045 & 0.20 & 1.000 & 0.998 & -0.25 & 0.0 \\
\hline 298.150 & 2.770 & 2.772 & 0.05 & 1.500 & 1.499 & -0.07 & 0.0 \\
\hline 298.150 & 3.327 & 3.329 & 0.04 & 2.000 & 1.999 & -0.07 & 0.0 \\
\hline 298.150 & 3.737 & 3.737 & 0.01 & 2.500 & 2.500 & -0.01 & 0.0 \\
\hline 298.150 & 4.019 & 4.018 & -0.02 & 3.000 & 3.002 & 0.07 & 0.0 \\
\hline 323.150 & 1.240 & 1.241 & 0.10 & 0.500 & 0.499 & -0.11 & 0.0 \\
\hline 323.150 & 2.288 & 2.292 & 0.17 & 1.000 & 0.998 & -0.20 & 0.0 \\
\hline 323.150 & 3.165 & 3.171 & 0.17 & 1.500 & 1.497 & -0.22 & 0.0 \\
\hline 323.150 & 3.892 & 3.898 & 0.16 & 2.000 & 1.995 & -0.23 & 0.0 \\
\hline 323.150 & 4.486 & 4.493 & 0.17 & 2.500 & 2.493 & -0.29 & 0.0 \\
\hline 323.150 & 4.967 & 4.976 & 0.18 & 3.000 & 2.989 & -0.35 & 0.0 \\
\hline 323.150 & 5.357 & 5.365 & 0.15 & 3.500 & 3.489 & -0.32 & 0.0 \\
\hline 323.150 & 5.671 & 5.678 & 0.12 & 4.000 & 3.988 & -0.29 & 0.0 \\
\hline 323.150 & 5.926 & 5.931 & 0.08 & 4.500 & 4.489 & -0.24 & 0.0 \\
\hline 323.150 & 6.136 & 6.141 & 0.08 & 5.000 & 4.988 & -0.25 & 0.0 \\
\hline 348.150 & 1.352 & 1.354 & 0.18 & 0.500 & 0.499 & -0.19 & 0.0 \\
\hline 348.150 & 2.528 & 2.534 & 0.25 & 1.000 & 0.997 & -0.28 & 0.0 \\
\hline 348.150 & 3.554 & 3.559 & 0.14 & 1.500 & 1.497 & -0.17 & 0.0 \\
\hline 348.150 & 4.438 & 4.448 & 0.22 & 2.000 & 1.994 & -0.30 & 0.0 \\
\hline 348.150 & 5.205 & 5.218 & 0.25 & 2.500 & 2.491 & -0.37 & 0.0 \\
\hline 348.150 & 5.875 & 5.888 & 0.23 & 3.000 & 2.989 & -0.36 & 0.0 \\
\hline 348.150 & 6.463 & 6.475 & 0.19 & 3.500 & 3.489 & -0.32 & 0.0 \\
\hline 348.150 & 6.980 & 6.995 & 0.20 & 4.000 & 3.986 & -0.36 & 0.0 \\
\hline 348.150 & 7.449 & 7.463 & 0.18 & 4.500 & 4.485 & -0.33 & 0.0 \\
\hline 348.150 & 7.882 & 7.895 & 0.16 & 5.000 & 4.985 & -0.30 & 0.0 \\
\hline 373.150 & 1.462 & 1.466 & 0.27 & 0.500 & 0.499 & -0.29 & 0.0 \\
\hline 373.150 & 2.764 & 2.774 & 0.35 & 1.000 & 0.996 & -0.39 & 0.0 \\
\hline 373.150 & 3.930 & 3.941 & 0.27 & 1.500 & 1.495 & -0.32 & 0.0 \\
\hline 373.150 & 4.968 & 4.986 & 0.36 & 2.000 & 1.991 & -0.45 & 0.0 \\
\hline 373.150 & 5.906 & 5.925 & 0.33 & 2.500 & 2.489 & -0.43 & 0.0 \\
\hline 373.150 & 6.756 & 6.777 & 0.30 & 3.000 & 2.987 & -0.42 & 0.0 \\
\hline 373.150 & 7.534 & 7.556 & 0.29 & 3.500 & 3.486 & -0.41 & 0.0 \\
\hline 373.150 & 8.258 & 8.279 & 0.26 & 4.000 & 3.985 & -0.38 & 0.0 \\
\hline 373.150 & 8.942 & 8.962 & 0.22 & 4.500 & 4.485 & -0.33 & 0.0 \\
\hline
\end{tabular}


Table 6. PVT DATA (continued)

Data from Beattie et al. [49] (continued)

\begin{tabular}{|c|c|c|c|c|c|c|c|}
\hline $\begin{array}{l}\mathrm{T} \\
\mathrm{K}\end{array}$ & $\begin{array}{l}\mathrm{P}, \operatorname{expt} \\
\mathrm{MPa}\end{array}$ & $\begin{array}{c}\mathrm{P}, \mathrm{calc} \\
\mathrm{MPa}\end{array}$ & $\begin{array}{c}\mathrm{dev} \\
8\end{array}$ & $\begin{array}{l}\rho, \text { expt } \\
\mathrm{mol} 1 \cdot \mathrm{dm}^{-3}\end{array}$ & $\begin{array}{c}\rho, \mathrm{calc} \\
\mathrm{mol} \cdot \mathrm{dm}^{-3}\end{array}$ & $\begin{array}{c}\mathrm{dev} \\
\frac{8}{8}\end{array}$ & wt \\
\hline 373.150 & 9.598 & 9.619 & 0.22 & 5.000 & 4.984 & -0.32 & 0.0 \\
\hline 398.150 & 1.573 & 1.577 & 0.31 & 0.500 & 0.498 & -0.32 & 0.0 \\
\hline 398.150 & 3.003 & 3.011 & 0.25 & 1.000 & 0.997 & -0.28 & 0.0 \\
\hline 398.150 & 4.305 & 4.318 & 0.29 & 1.500 & 1.495 & -0.34 & 0.0 \\
\hline 398.150 & 5.497 & 5.516 & 0.34 & 2.000 & 1.992 & -0.41 & 0.0 \\
\hline 398.150 & 6.599 & 6.621 & 0.33 & 2.500 & 2.490 & -0.40 & 0.0 \\
\hline 398.150 & 7.626 & 7.650 & 0.32 & 3.000 & 2.988 & -0.41 & 0.0 \\
\hline 398.150 & 8.593 & 8.619 & 0.30 & 3.500 & 3.486 & -0.39 & 0.0 \\
\hline 398.150 & 9.515 & 9.544 & 0.30 & 4.000 & 3.984 & -0.39 & 0.0 \\
\hline 398.150 & 10.409 & 10.441 & 0.31 & 4.500 & 4.482 & -0.40 & 0.0 \\
\hline 398.150 & 11.294 & 11.327 & 0.29 & 5.000 & 4.981 & -0.37 & 0.0 \\
\hline 423.150 & 1.682 & 1.688 & 0.38 & 0.500 & 0.498 & -0.39 & 0.0 \\
\hline 423.150 & 3.231 & 3.246 & 0.47 & 1.000 & 0.995 & -0.50 & 0.0 \\
\hline 423.150 & 4.669 & 4.691 & 0.47 & 1.500 & 1.492 & -0.53 & 0.0 \\
\hline 423.150 & 6.010 & 6.039 & 0.50 & 2.000 & 1.989 & -0.57 & 0.0 \\
\hline 423.150 & 7.273 & 7.308 & 0.47 & 2.500 & 2.486 & -0.56 & 0.0 \\
\hline 423.150 & 8.472 & 8.512 & 0.47 & 3.000 & 2.983 & -0.57 & 0.0 \\
\hline 423.150 & 9.625 & 9.669 & 0.46 & 3.500 & 3.481 & -0.55 & 0.0 \\
\hline 423.150 & 10.747 & 10.794 & 0.44 & 4.000 & 3.979 & -0.53 & 0.0 \\
\hline 423.150 & 11.855 & 11.905 & 0.42 & 4.500 & 4.477 & -0.50 & 0.0 \\
\hline 423.150 & 12.968 & 13.020 & 0.40 & 5.000 & 4.977 & -0.46 & 0.0 \\
\hline 448.150 & 1.790 & 1.799 & 0.48 & 0.500 & 0.498 & -0.49 & 0.0 \\
\hline 448.150 & 3.462 & 3.481 & 0.53 & 1.000 & 0.994 & -0.56 & 0.0 \\
\hline 448.150 & 5.032 & 5.062 & 0.59 & 1.500 & 1.490 & -0.65 & 0.0 \\
\hline 448.150 & 6.521 & 6.558 & 0.57 & 2.000 & 1.987 & -0.64 & 0.0 \\
\hline 448.150 & 7.944 & 7.988 & 0.55 & 2.500 & 2.484 & -0.62 & 0.0 \\
\hline 448.150 & 9.315 & 9.365 & 0.54 & 3.000 & 2.981 & -0.62 & 0.0 \\
\hline 448.150 & 10.652 & 10.708 & 0.52 & 3.500 & 3.479 & -0.60 & 0.0 \\
\hline 448.150 & 11.973 & 12.033 & 0.50 & 4.000 & 3.977 & -0.57 & 0.0 \\
\hline 448.150 & 13.294 & 13.357 & 0.48 & 4.500 & 4.476 & -0.53 & 0.0 \\
\hline 448.150 & 14.632 & 14.700 & 0.46 & 5.000 & 4.975 & -0.50 & 0.0 \\
\hline 473.150 & 1.896 & 1.909 & 0.71 & 0.500 & 0.496 & -0.73 & 0.0 \\
\hline 473.150 & 3.692 & 3.714 & 0.58 & 1.000 & 0.994 & -0.61 & 0.0 \\
\hline 473.150 & 5.396 & 5.430 & 0.63 & 1.500 & 1.490 & -0.68 & 0.0 \\
\hline 473.150 & 7.030 & 7.074 & 0.62 & 2.000 & 1.986 & -0.68 & 0.0 \\
\hline
\end{tabular}


Table 6. PVT DATA (continued)

Data from Beattie et al. [49] (continued)

\begin{tabular}{|c|c|c|c|c|c|c|c|}
\hline $\begin{array}{l}\mathrm{T} \\
\mathrm{K}\end{array}$ & $\mathrm{P}, \underset{\mathrm{MPa}}{\exp t}$ & $\begin{array}{c}\mathrm{P}, \underset{\mathrm{MPa}}{\mathrm{calc}} \\
\text { cal }\end{array}$ & $\begin{array}{c}\mathrm{dev} \\
\frac{8}{8}\end{array}$ & $\begin{array}{l}\rho, \operatorname{expt} \\
\mathrm{mol} \cdot \mathrm{dm}^{-3}\end{array}$ & $\begin{array}{c}\rho, \text { calc } \\
\mathrm{mol} \cdot \mathrm{dm}^{-3}\end{array}$ & $\begin{array}{c}\text { dev } \\
8\end{array}$ & $w t$ \\
\hline 473.150 & 8.610 & 8.662 & 0.61 & 2.500 & 2.483 & -0.67 & 0.0 \\
\hline 473.150 & 10.152 & 10.212 & 0.59 & 3.000 & 2.981 & -0.65 & 0.0 \\
\hline 473.150 & 11.673 & 11.739 & 0.57 & 3.500 & 3.478 & -0.62 & 0.0 \\
\hline 473.150 & 13.191 & 13.262 & 0.54 & 4.000 & 3.977 & -0.58 & 0.0 \\
\hline 473.150 & 14.723 & 14.799 & 0.52 & 4.500 & 4.475 & -0.55 & 0.0 \\
\hline 473.150 & 16.290 & 16.370 & 0.49 & 5.000 & 4.975 & -0.50 & 0.0 \\
\hline 498.150 & 2.006 & 2.019 & 0.65 & 0.500 & 0.497 & -0.67 & 0.0 \\
\hline 498.150 & 3.915 & 3.946 & 0.79 & 1.000 & 0.992 & -0.82 & 0.0 \\
\hline 498.150 & 5.748 & 5.796 & 0.83 & 1.500 & 1.487 & -0.88 & 0.0 \\
\hline 498.150 & 7.525 & 7.586 & 0.81 & 2.000 & 1.983 & -0.86 & 0.0 \\
\hline 498.150 & 9.259 & 9.333 & 0.79 & 2.500 & 2.479 & -0.85 & 0.0 \\
\hline 498.150 & 10.967 & 11.052 & 0.78 & 3.000 & 2.975 & -0.83 & 0.0 \\
\hline 498.150 & 12.668 & 12.763 & 0.75 & 3.500 & 3.472 & -0.80 & 0.0 \\
\hline 498.150 & 14.378 & 14.483 & 0.73 & 4.000 & 3.970 & -0.76 & 0.0 \\
\hline 498.150 & 16.119 & 16.232 & 0.70 & 4.500 & 4.468 & -0.71 & 0.0 \\
\hline 498.150 & 17.907 & 18.031 & 0.69 & 5.000 & 4.966 & -0.68 & 0.0 \\
\hline 523.150 & 2.117 & 2.129 & 0.59 & 0.500 & 0.497 & -0.60 & 0.0 \\
\hline 523.150 & 4.141 & 4.178 & 0.88 & 1.000 & 0.991 & -0.90 & 0.0 \\
\hline 523.150 & 6.103 & 6.161 & 0.95 & 1.500 & 1.485 & -0.99 & 0.0 \\
\hline 523.150 & 8.021 & 8.096 & 0.94 & 2.000 & 1.980 & -0.98 & 0.0 \\
\hline 523.150 & 9.911 & 9.999 & 0.90 & 2.500 & 2.477 & -0.94 & 0.0 \\
\hline 523.150 & 11.784 & 11.888 & 0.88 & 3.000 & 2.972 & -0.92 & 0.0 \\
\hline 523.150 & 13.663 & 13.781 & 0.87 & 3.500 & 3.469 & -0.89 & 0.0 \\
\hline 523.150 & 15.563 & 15.697 & 0.86 & 4.000 & 3.965 & -0.87 & 0.0 \\
\hline 523.150 & 17.516 & 17.656 & 0.80 & 4.500 & 4.465 & -0.79 & 0.0 \\
\hline 523.150 & 19.532 & 19.683 & 0.77 & 5.000 & 4.964 & -0.73 & 0.0 \\
\hline
\end{tabular}

Number of Points [49] 96

Pressure Calculation:

$\mathrm{AAD} ;=0.43 \quad \mathrm{BIAS} \%=0.43 \quad \mathrm{RMS} \%=0.25$

Density Calculation:

$A A D 2 z=0.49 \quad$ BIAS $2 \%=-0.49 \quad \operatorname{RMS} 2 \%=0.23$ 
Table 6. PVT DATA (continued)

Data from Beattie et al. [49] (continued)

\begin{tabular}{cccccccc}
\hline $\mathrm{T}$ & $\mathrm{P}, \operatorname{expt}$ & $\mathrm{P}, \mathrm{calc}$ & $\mathrm{dev}$ & $\rho, \operatorname{expt}$ & $\rho, \mathrm{calc}$ & $\mathrm{dev}$ & wt \\
$\mathrm{K}$ & $\mathrm{MPa}$ & $\mathrm{MPa}$ & 8 & $\mathrm{~mol} \cdot \mathrm{dm}^{-3}$ & $\mathrm{~mol} \cdot \mathrm{dm}^{-3}$ & 8 & \\
\hline
\end{tabular}

Absolute Deviations:

Pressure Calculation:

$\mathrm{AAD}=0.04 \mathrm{BIAS}=0.04 \mathrm{RMS}=0.04 \mathrm{MPa}$

Density Calculation:

$\mathrm{AAD2}=0.013$ BIAS2 $=-0.013$ RMS2 $=0.009 \mathrm{~mol} \cdot \mathrm{dm}^{-3}$

Data from Besserer and Robinson [50]

\begin{tabular}{|c|c|c|c|c|c|c|c|}
\hline $\begin{array}{l}\mathrm{T} \\
\mathrm{K}\end{array}$ & $\begin{array}{l}\mathrm{P}, \operatorname{MPa}_{\mathrm{MPa}} \\
\text { expt }\end{array}$ & $\begin{array}{c}\mathrm{P}, \mathrm{calc} \\
\mathrm{MPa}\end{array}$ & $\begin{array}{c}\mathrm{dev} \\
\frac{8}{8}\end{array}$ & $\begin{array}{l}\rho, \operatorname{expt} \\
\mathrm{mol} \cdot \mathrm{dm}^{-3}\end{array}$ & $\begin{array}{l}\rho, \quad \mathrm{calc} \\
\mathrm{mol} \cdot \mathrm{dm}^{-3}\end{array}$ & $\begin{array}{r}\text { dev } \\
\frac{8}{8}\end{array}$ & $w t$ \\
\hline 311.094 & 0.690 & 0.690 & 0.03 & 0.280 & 0.280 & -0.03 & 0.0 \\
\hline 311.094 & 1.379 & 1.381 & 0.12 & 0.591 & 0.590 & -0.14 & 0.0 \\
\hline 311.094 & 2.068 & 2.074 & 0.29 & 0.945 & 0.942 & -0.35 & 0.0 \\
\hline 311.094 & 2.758 & 2.760 & 0.09 & 1.353 & 1.351 & -0.12 & 0.0 \\
\hline 311.094 & 3.447 & 3.446 & -0.03 & 1.849 & 1.849 & 0.04 & 0.0 \\
\hline 311.094 & 4.137 & 4.137 & 0.00 & 2.503 & 2.503 & 0.00 & 0.0 \\
\hline 311.094 & 4.826 & 4.851 & 0.50 & 3.581 & 3.530 & -1.43 & 0.0 \\
\hline 311.094 & 5.171 & 5.196 & 0.47 & 4.617 & 4.512 & -2.27 & 0.0 \\
\hline 311.094 & 5.516 & 5.555 & 0.72 & 7.698 & 7.327 & -4.82 & 0.0 \\
\hline 311.094 & 5.861 & 5.926 & 1.11 & 9.210 & 9.059 & -1.64 & 0.0 \\
\hline 311.094 & 6.205 & 6.282 & 1.24 & 9.792 & 9.689 & -1.05 & 0.0 \\
\hline 311.094 & 6.895 & 6.921 & 0.38 & 10.412 & 10.392 & -0.19 & 0.0 \\
\hline 311.094 & 7.584 & 7.475 & -1.44 & 10.780 & 10.842 & 0.57 & 0.0 \\
\hline 311.094 & 8.274 & 8.111 & -1.96 & 11.107 & 11.179 & 0.65 & 0.0 \\
\hline 311.094 & 8.963 & 8.722 & -2.69 & 11.361 & 11.451 & 0.79 & 0.0 \\
\hline 311.094 & 9.653 & 9.366 & -2.97 & 11.589 & 11.681 & 0.79 & 0.0 \\
\hline 311.094 & 10.342 & 10.035 & -2.97 & 11.794 & 11.880 & 0.73 & 0.0 \\
\hline 344.483 & 0.690 & 0.690 & 0.06 & 0.249 & 0.249 & -0.06 & 0.0 \\
\hline 344.483 & 1.379 & 1.379 & 0.00 & 0.517 & 0.517 & 0.00 & 0.0 \\
\hline 344.483 & 2.068 & 2.068 & -0.01 & 0.806 & 0.806 & 0.02 & 0.0 \\
\hline 344.483 & 2.758 & 2.755 & -0.09 & 1.121 & 1.122 & 0.11 & 0.0 \\
\hline 344.483 & 3.447 & 3.446 & -0.04 & 1.470 & 1.470 & 0.05 & 0.0 \\
\hline 344.483 & 4.137 & 4.141 & 0.09 & 1.861 & 1.859 & -0.13 & 0.0 \\
\hline 344.483 & 4.826 & 4.845 & 0.39 & 2.311 & 2.299 & -0.56 & 0.0 \\
\hline 344.483 & 5.171 & 5.197 & 0.49 & 2.561 & 2.542 & -0.73 & 0.0 \\
\hline
\end{tabular}


Table 6. PVT DATA (continued)

Data from Besserer and Robinson [50] (continued)

\begin{tabular}{|c|c|c|c|c|c|c|c|}
\hline $\begin{array}{l}\mathrm{T} \\
\mathrm{K}\end{array}$ & $\begin{array}{l}\mathrm{P}, \operatorname{expt} \\
\mathrm{MPa}\end{array}$ & $\begin{array}{c}\mathrm{P}, \mathrm{MPa} \\
\mathrm{MPa}\end{array}$ & $\begin{array}{c}\text { dev } \\
8\end{array}$ & $\begin{array}{l}\rho, \operatorname{expt} \\
\mathrm{mol} \cdot \mathrm{dm}^{-3}\end{array}$ & $\begin{array}{l}\rho, \quad \mathrm{calc} c \\
\mathrm{~mol} \cdot \mathrm{dm}^{-3}\end{array}$ & $\begin{array}{r}\mathrm{dev} \\
\%\end{array}$ & wt \\
\hline 344.483 & 5.516 & 5.540 & 0.44 & 2.824 & 2.804 & -0.68 & 0.0 \\
\hline 344.483 & 5.861 & 5.885 & 0.42 & 3.110 & 3.088 & -0.68 & 0.0 \\
\hline 344.483 & 6.205 & 6.233 & 0.44 & 3.423 & 3.397 & -0.75 & 0.0 \\
\hline 344.483 & 6.895 & 6.915 & 0.29 & 4.122 & 4.100 & -0.54 & 0.0 \\
\hline 344.483 & 7.584 & 7.608 & 0.31 & 4.959 & 4.928 & -0.62 & 0.0 \\
\hline 344.483 & 8.274 & 8.295 & 0.26 & 5.883 & 5.854 & -0.50 & 0.0 \\
\hline 344.483 & 8.963 & 8.962 & -0.01 & 6.774 & 6.775 & 0.02 & 0.0 \\
\hline 344.483 & 9.653 & 9.631 & -0.22 & 7.556 & 7.579 & 0.30 & 0.0 \\
\hline 344.483 & 10.342 & 10.287 & -0.53 & 8.188 & 8.235 & 0.58 & 0.0 \\
\hline 372.372 & 0.690 & 0.688 & -0.16 & 0.228 & 0.229 & 0.16 & 0.0 \\
\hline 372.372 & 1.379 & 1.375 & -0.30 & 0.468 & 0.470 & 0.32 & 0.0 \\
\hline 372.372 & 2.068 & 2.059 & -0.47 & 0.722 & 0.725 & 0.51 & 0.0 \\
\hline 372.372 & 2.758 & 2.738 & -0.71 & 0.989 & 0.997 & 0.81 & 0.0 \\
\hline 372.372 & 3.447 & 3.420 & -0.80 & 1.274 & 1.286 & 0.94 & 0.0 \\
\hline 372.372 & 4.137 & 4.094 & -1.04 & 1.576 & 1.595 & 1.26 & 0.0 \\
\hline 372.372 & 4.826 & 4.772 & -1.12 & 1.901 & 1.928 & 1.42 & 0.0 \\
\hline 372.372 & 5.171 & 5.105 & -1.27 & 2.070 & 2.104 & 1.65 & 0.0 \\
\hline 372.372 & 5.516 & 5.442 & -1.33 & 2.247 & 2.286 & 1.76 & 0.0 \\
\hline 372.372 & 5.861 & 5.784 & -1.31 & $2.43 ?$ & 2.476 & 1.76 & 0.0 \\
\hline 372.372 & 6.205 & 6.114 & -1.47 & 2.620 & 2.673 & 2.02 & 0.0 \\
\hline 372.372 & 6.895 & 6.784 & -1.60 & 3.022 & 3.091 & 2.29 & 0.0 \\
\hline 372.372 & 7.584 & 7.441 & -1.89 & 3.445 & 3.542 & 2.79 & 0.0 \\
\hline 372.372 & 8.274 & 8.102 & -2.08 & 3.901 & 4.025 & 3.16 & 0.0 \\
\hline 372.372 & 8.963 & 8.712 & -2.80 & 4.347 & 4.536 & 4.36 & 0.0 \\
\hline 372.372 & 9.653 & 9.410 & -2.51 & 4.879 & 5.067 & 3.86 & 0.0 \\
\hline 372.372 & 10.342 & 10.052 & -2.81 & 5.379 & 5.605 & 4.20 & 0.0 \\
\hline 394.039 & 0.690 & 0.689 & 0.00 & 0.215 & 0.215 & 0.00 & 0.0 \\
\hline 394.039 & 1.379 & 1.380 & 0.05 & 0.440 & 0.440 & -0.05 & 0.0 \\
\hline 394.039 & 2.068 & 2.068 & -0.02 & 0.674 & 0.675 & 0.02 & 0.0 \\
\hline 394.039 & 2.758 & 2.758 & 0.00 & 0.921 & 0.921 & 0.00 & 0.0 \\
\hline 394.039 & 3.447 & 3.447 & 0.00 & 1.180 & 1.180 & 0.01 & 0.0 \\
\hline 394.039 & 4.137 & 4.132 & -0.11 & 1.450 & 1.451 & 0.12 & 0.0 \\
\hline 394.039 & 4.826 & 4.820 & -0.12 & 1.735 & 1.738 & 0.14 & 0.0 \\
\hline 394.039 & 5.171 & 5.165 & -0.12 & 1.883 & 1.886 & 0.15 & 0.0 \\
\hline 394.039 & 5.516 & 5.509 & -0.12 & 2.036 & 2.039 & 0.15 & 0.0 \\
\hline
\end{tabular}


Table 6. PVT DATA (continued)

Data from Besserer and Robinson [50] (continued)

\begin{tabular}{|c|c|c|c|c|c|c|c|}
\hline $\begin{array}{l}\mathrm{T} \\
\mathrm{K}\end{array}$ & $\begin{array}{c}\mathrm{P}, \operatorname{MPa}_{\mathrm{MPa}} \\
\text { expt }\end{array}$ & $\begin{array}{c}\mathrm{P}, \mathrm{calc} \\
\mathrm{MPa}\end{array}$ & $\begin{array}{c}\mathrm{dev} \\
\frac{8}{8}\end{array}$ & $\begin{array}{l}\rho, \operatorname{expt} \\
\mathrm{mol} \cdot \mathrm{dm}^{-3}\end{array}$ & $\begin{array}{l}\rho, \quad \mathrm{calc} \\
\mathrm{mol} \cdot \mathrm{dm}^{-3}\end{array}$ & $\begin{array}{r}\text { dev } \\
\%\end{array}$ & wt \\
\hline 394.039 & 5.861 & 5.854 & -0.12 & 2.192 & 2.195 & 0.14 & 0.0 \\
\hline 394.039 & 6.205 & 6.199 & -0.11 & 2.353 & 2.356 & 0.13 & 0.0 \\
\hline 394.039 & 6.895 & 6.891 & -0.06 & 2.688 & 2.690 & 0.07 & 0.0 \\
\hline 394.039 & 7.584 & 7.580 & -0.05 & 3.038 & 3.040 & 0.07 & 0.0 \\
\hline 394.039 & 8.274 & 8.277 & 0.04 & 3.408 & 3.406 & -0.05 & 0.0 \\
\hline 394.039 & 8.963 & 8.966 & 0.03 & 3.789 & 3.788 & -0.04 & 0.0 \\
\hline 394.039 & 9.653 & 9.659 & 0.06 & 4.185 & 4.181 & -0.08 & 0.0 \\
\hline 394.039 & 10.342 & 10.338 & -0.04 & 4.582 & 4.584 & 0.06 & 0.0 \\
\hline
\end{tabular}

Number of Points [50] 68

Pressure Calculation:

$\mathrm{AAD}$ \% $=0.67$ BIAS웅 $=-0.43$ RMS

Density Calculation:

$A A D 2 \%=0.83 \quad$ BIAS28 $=0.32 \quad$ RMS28 $=1.37$

Absolute Deviations:

Pressure Calculation:

$\mathrm{AAD}=0.05$ BIAS $=-0.04 \mathrm{RMS}=0.09 \mathrm{MPa}$

Density Calculation:

$\mathrm{AAD2}=0.039$ BIAS2 $=0.009 \quad \mathrm{RMS} 2=0.075 \mathrm{~mol} \cdot \mathrm{dm}^{-3}$

Data from Douslin and Harrison [9]

\begin{tabular}{cccccccc}
\hline $\begin{array}{c}\mathrm{T} \\
\mathrm{K}\end{array}$ & $\begin{array}{c}\mathrm{P}, \text { expt } \\
\mathrm{MPa}\end{array}$ & $\begin{array}{c}\mathrm{P}, \mathrm{calc} \\
\mathrm{MPa}\end{array}$ & $\begin{array}{c}\mathrm{dev} \\
8\end{array}$ & $\begin{array}{c}\rho, \operatorname{expt} \\
\mathrm{mol} \cdot \mathrm{dm}^{-3}\end{array}$ & $\begin{array}{c}\rho, \mathrm{calc} \\
\mathrm{mol} \cdot \mathrm{dm}^{-3}\end{array}$ & $\begin{array}{c}\mathrm{dev} \\
8\end{array}$ & wt \\
\hline 273.150 & 1.430 & 1.429 & -0.03 & 0.750 & 0.750 & 0.04 & 71.2 \\
298.150 & 1.612 & 1.611 & -0.02 & 0.750 & 0.750 & 0.02 & 62.0 \\
303.150 & 1.648 & 1.647 & -0.02 & 0.750 & 0.750 & 0.03 & 60.4 \\
323.150 & 1.790 & 1.789 & -0.02 & 0.750 & 0.750 & 0.03 & 55.1 \\
348.150 & 1.965 & 1.965 & -0.02 & 0.750 & 0.750 & 0.02 & 49.6 \\
373.150 & 2.139 & 2.139 & -0.02 & 0.750 & 0.750 & 0.03 & 45.2 \\
398.150 & 2.312 & 2.311 & -0.02 & 0.750 & 0.750 & 0.02 & 41.6 \\
423.150 & 2.483 & 2.483 & -0.02 & 0.750 & 0.750 & 0.02 & 38.5 \\
448.150 & 2.654 & 2.653 & -0.02 & 0.750 & 0.750 & 0.02 & 35.9 \\
473.150 & 2.824 & 2.824 & -0.01 & 0.750 & 0.750 & 0.01 & 33.6
\end{tabular}


Table 6. PVT DATA (continued)

Data from Douslin and Harrison [9] (continued)

\begin{tabular}{|c|c|c|c|c|c|c|c|}
\hline $\begin{array}{l}\mathrm{T} \\
\mathrm{K}\end{array}$ & $\begin{array}{l}\mathrm{P}, \exp t \\
\mathrm{MPa}\end{array}$ & $\begin{array}{c}\mathrm{P}, \underset{\mathrm{MPa}}{\mathrm{calc}} \\
\text { cal }\end{array}$ & $\begin{array}{c}\mathrm{dev} \\
8\end{array}$ & $\begin{array}{l}\rho, \quad \operatorname{expt} \\
\mathrm{mol} \cdot \mathrm{dm}^{-3}\end{array}$ & $\begin{array}{l}\rho, \quad \text { calc } \\
\mathrm{mol} \cdot \mathrm{dm}^{-3}\end{array}$ & $\begin{array}{r}\mathrm{dev} \\
8\end{array}$ & wt \\
\hline 498.150 & 2.993 & 2.993 & 0.00 & 0.750 & 0.750 & 0.01 & 31.6 \\
\hline 523.150 & 3.163 & 3.163 & -0.01 & 0.750 & 0.750 & 0.01 & 29.8 \\
\hline 548.150 & 3.331 & 3.331 & 0.00 & 0.750 & 0.750 & 0.00 & 28.2 \\
\hline 573.150 & 3.500 & 3.500 & -0.01 & 0.750 & 0.750 & 0.01 & 26.8 \\
\hline 598.150 & 3.669 & 3.668 & -0.02 & 0.750 & 0.750 & 0.02 & 25.5 \\
\hline 623.150 & 3.837 & 3.836 & -0.03 & 0.750 & 0.750 & 0.03 & 24.3 \\
\hline 273.150 & 1.791 & 1.790 & -0.03 & 1.000 & 1.000 & 0.04 & 58.8 \\
\hline 298.150 & 2.045 & 2.045 & -0.01 & 1.000 & 1.000 & 0.01 & 50.1 \\
\hline 303.150 & 2.095 & 2.095 & -0.01 & 1.000 & 1.000 & 0.01 & 48.7 \\
\hline 323.150 & 2.292 & 2.292 & 0.00 & 1.000 & 1.000 & 0.01 & 43.9 \\
\hline 348.150 & 2.534 & 2.534 & 0.00 & 1.000 & 1.000 & 0.00 & 39.1 \\
\hline 373.150 & 2.774 & 2.774 & 0.00 & 1.000 & 1.000 & 0.00 & 35.4 \\
\hline 398.150 & 3.011 & 3.011 & 0.00 & 1.000 & 1.000 & 0.00 & 32.3 \\
\hline 423.150 & 3.246 & 3.246 & 0.01 & 1.000 & 1.000 & -0.01 & 29.7 \\
\hline 448.150 & 3.480 & 3.481 & 0.01 & 1.000 & 1.000 & -0.01 & 27.5 \\
\hline 473.150 & 3.713 & 3.714 & 0.02 & 1.000 & 1.000 & -0.02 & 25.7 \\
\hline 498.150 & 3.945 & 3.946 & 0.03 & 1.000 & 1.000 & -0.03 & 24.1 \\
\hline 523.150 & 4.176 & 4.178 & 0.03 & 1.000 & 1.000 & -0.03 & 22.6 \\
\hline 548.150 & 4.407 & 4.408 & 0.03 & 1.000 & 1.000 & -0.03 & 21.4 \\
\hline 573.150 & 4.638 & 4.639 & 0.03 & 1.000 & 1.000 & -0.03 & 20.2 \\
\hline 598.150 & 4.867 & 4.869 & 0.03 & 1.000 & 1.000 & -0.03 & 19.2 \\
\hline 623.150 & 5.098 & 5.098 & 0.00 & 1.000 & 1.000 & 0.00 & 18.3 \\
\hline 273.150 & 2.353 & 2.352 & -0.03 & 1.500 & 1.501 & 0.05 & 48.1 \\
\hline 298.150 & 2.772 & 2.772 & -0.01 & 1.500 & 1.500 & 0.02 & 39.0 \\
\hline 303.150 & 2.853 & 2.853 & -0.01 & 1.500 & 1.500 & 0.01 & 37.6 \\
\hline 323.150 & 3.171 & 3.171 & -0.01 & 1.500 & 1.500 & 0.01 & 33.0 \\
\hline 348.150 & 3.559 & 3.559 & 0.00 & 1.500 & 1.500 & 0.00 & 28.7 \\
\hline 373.150 & 3.941 & 3.941 & 0.01 & 1.500 & 1.500 & -0.01 & 25.5 \\
\hline 398.150 & 4.317 & 4.318 & 0.02 & 1.500 & 1.500 & -0.02 & 23.0 \\
\hline 423.150 & 4.690 & 4.691 & 0.03 & 1.500 & 1.500 & -0.03 & 20.9 \\
\hline 448.150 & 5.060 & 5.062 & 0.03 & 1.500 & 1.499 & -0.03 & 19.2 \\
\hline 473.150 & 5.428 & 5.430 & 0.04 & 1.500 & 1.499 & -0.04 & 17.7 \\
\hline 498.150 & 5.793 & 5.796 & 0.05 & 1.500 & 1.499 & -0.05 & 16.5 \\
\hline 523.150 & 6.158 & 6.161 & 0.05 & 1.500 & 1.499 & -0.05 & 15.4 \\
\hline 548.150 & 6.522 & 6.525 & 0.05 & 1.500 & 1.499 & -0.05 & 14.5 \\
\hline
\end{tabular}


Table 6. PVT DATA (continued)

Data from Douslin and Harrison [9] (continued)

\begin{tabular}{|c|c|c|c|c|c|c|c|}
\hline $\begin{array}{l}\mathrm{T} \\
\mathrm{K}\end{array}$ & $\begin{array}{c}\mathrm{P}, \text { expt } \\
\mathrm{MPa}\end{array}$ & $\begin{array}{c}\mathrm{P}, \mathrm{calc} \\
\mathrm{MPa}\end{array}$ & $\begin{array}{c}\mathrm{dev} \\
8\end{array}$ & $\begin{array}{l}\rho, \text { expt } \\
\mathrm{mol} 1 \cdot \mathrm{dm}^{-3}\end{array}$ & $\begin{array}{l}\rho, \quad \text { calc } \\
\mathrm{mol} \cdot \mathrm{dm}^{-3}\end{array}$ & $\begin{array}{r}\mathrm{dev} \\
8\end{array}$ & wt \\
\hline 573.150 & 6.883 & 6.887 & 0.06 & 1.500 & 1.499 & -0.06 & 13.7 \\
\hline 598.150 & 7.244 & 7.248 & 0.06 & 1.500 & 1.499 & -0.06 & 12.9 \\
\hline 623.150 & 7.607 & 7.609 & 0.03 & 1.500 & 1.500 & -0.03 & 12.3 \\
\hline 298.150 & 3.329 & 3.329 & 0.00 & 2.000 & 2.000 & 0.01 & 34.2 \\
\hline 303.150 & 3.445 & 3.445 & 0.00 & 2.000 & 2.000 & 0.00 & 32.7 \\
\hline 323.150 & 3.898 & 3.898 & 0.01 & 2.000 & 2.000 & -0.01 & 27.9 \\
\hline 348.150 & 4.447 & 4.448 & 0.01 & 2.000 & 2.000 & -0.01 & 23.7 \\
\hline 373.150 & 4.985 & 4.986 & 0.02 & 2.000 & 2.000 & -0.02 & 20.6 \\
\hline 398.150 & 5.514 & 5.516 & 0.03 & 2.000 & 1.999 & -0.04 & 18.3 \\
\hline 423.150 & 6.037 & 6.039 & 0.05 & 2.000 & 1.999 & -0.05 & 16.4 \\
\hline 448.150 & 6.555 & 6.558 & 0.05 & 2.000 & 1.999 & -0.06 & 15.0 \\
\hline 473.150 & 7.070 & 7.074 & 0.06 & 2.000 & 1.999 & -0.06 & 13.7 \\
\hline 498.150 & 7.581 & 7.586 & 0.07 & 2.000 & 1.999 & -0.07 & 12.7 \\
\hline 523.150 & 8.090 & 8.096 & 0.08 & 2.000 & 1.998 & -0.08 & 11.8 \\
\hline 548.150 & 8.596 & 8.604 & 0.09 & 2.000 & 1.998 & -0.09 & 11.0 \\
\hline 573.150 & 9.101 & 9.110 & 0.10 & 2.000 & 1.998 & -0.10 & 10.3 \\
\hline 598.150 & 9.605 & 9.614 & 0.09 & 2.000 & 1.998 & -0.09 & 9.7 \\
\hline 623.150 & 10.111 & 10.117 & 0.06 & 2.000 & 1.999 & -0.06 & 9.2 \\
\hline 298.150 & 3.737 & 3.737 & 0.01 & 2.500 & 2.500 & -0.01 & 32.0 \\
\hline 303.150 & 3.892 & 3.892 & 0.01 & 2.500 & 2.499 & -0.03 & 30.3 \\
\hline 323.150 & 4.493 & 4.493 & 0.01 & 2.500 & 2.499 & -0.02 & 25.1 \\
\hline 348.150 & 5.217 & 5.218 & 0.03 & 2.500 & 2.499 & -0.04 & 20.7 \\
\hline 373.150 & 5.923 & 5.925 & 0.04 & 2.500 & 2.499 & -0.05 & 17.7 \\
\hline 398.150 & 6.618 & 6.621 & 0.05 & 2.500 & 2.499 & -0.06 & 15.5 \\
\hline 423.150 & 7.303 & 7.308 & 0.06 & 2.500 & 2.498 & -0.08 & 13.7 \\
\hline 448.150 & 7.982 & 7.988 & 0.07 & 2.500 & 2.498 & -0.08 & 12.4 \\
\hline 473.150 & 8.654 & 8.662 & 0.09 & 2.500 & 2.497 & -0.10 & 11.2 \\
\hline 498.150 & 9.323 & 9.333 & 0.10 & 2.500 & 2.497 & -0.11 & 10.3 \\
\hline 523.150 & 9.989 & 9.999 & 0.10 & 2.500 & 2.497 & -0.11 & 9.5 \\
\hline 548.150 & 10.652 & 10.663 & 0.10 & 2.500 & 2.497 & -0.11 & 8.9 \\
\hline 573.150 & 11.312 & 11.324 & 0.11 & 2.500 & 2.497 & -0.11 & 8.3 \\
\hline 598.150 & 11.970 & 11.983 & 0.10 & 2.500 & 2.497 & -0.10 & 7.8 \\
\hline 623.150 & 12.631 & 12.639 & 0.06 & 2.500 & 2.498 & -0.06 & 7.3 \\
\hline 298.150 & 4.017 & 4.018 & 0.01 & 3.000 & 2.999 & -0.02 & 31.0 \\
\hline 303.150 & 4.215 & 4.216 & 0.01 & 3.000 & 2.999 & -0.04 & 29.1 \\
\hline
\end{tabular}


Table 6. PVT DATA (continued)

Data from Douslin and Harrison [9] (continued)

\begin{tabular}{|c|c|c|c|c|c|c|c|}
\hline $\begin{array}{l}\mathrm{T} \\
\mathrm{K}\end{array}$ & $\begin{array}{l}P, \exp _{\mathrm{MPa}} \\
\text { exa }\end{array}$ & $\begin{array}{c}\mathrm{P}, \underset{\mathrm{MPa}}{\mathrm{calc}} \\
\end{array}$ & $\begin{array}{c}\mathrm{dev} \\
8\end{array}$ & $\begin{array}{l}\rho, \operatorname{expt} \\
\mathrm{mol} \cdot \mathrm{dm}^{-3}\end{array}$ & $\begin{array}{l}\rho, \text { calc } \\
\mathrm{mol} \cdot \mathrm{dm}^{-3}\end{array}$ & $\begin{array}{r}\text { dev } \\
8\end{array}$ & wt \\
\hline 323.150 & 4.975 & 4.976 & 0.02 & 3.000 & 2.999 & -0.04 & 23.4 \\
\hline 348.150 & 5.886 & 5.888 & 0.03 & 3.000 & 2.998 & -0.05 & 18.8 \\
\hline 373.150 & 6.773 & 6.777 & 0.05 & 3.000 & 2.998 & -0.07 & 15.7 \\
\hline 398.150 & 7.645 & 7.650 & 0.07 & 3.000 & 2.997 & -0.08 & 13.5 \\
\hline 423.150 & 8.504 & 8.512 & 0.09 & 3.000 & 2.997 & -0.11 & 11.9 \\
\hline 448.150 & 9.356 & 9.365 & 0.10 & 3.000 & 2.997 & -0.11 & 10.6 \\
\hline 473.150 & 10.201 & 10.212 & 0.11 & 3.000 & 2.996 & -0.12 & 9.6 \\
\hline 498.150 & 11.039 & 11.052 & 0.12 & 3.000 & 2.996 & -0.13 & 8.7 \\
\hline 523.150 & 11.874 & 11.888 & 0.12 & 3.000 & 2.996 & -0.13 & 8.0 \\
\hline 548.150 & 12.706 & 12.720 & 0.12 & 3.000 & 2.996 & -0.12 & 7.4 \\
\hline 573.150 & 13.533 & 13.549 & 0.12 & 3.000 & 2.996 & -0.12 & 6.9 \\
\hline 598.150 & 14.359 & 14.374 & 0.10 & 3.000 & 2.997 & -0.10 & 6.4 \\
\hline 623.150 & 15.188 & 15.196 & 0.05 & 3.000 & 2.998 & -0.05 & 6.1 \\
\hline 303.150 & 4.435 & 4.435 & 0.02 & 3.500 & 3.498 & -0.06 & 28.5 \\
\hline 323.150 & 5.364 & 5.365 & 0.03 & 3.500 & 3.498 & -0.06 & 22.3 \\
\hline 348.150 & 6.472 & 6.475 & 0.04 & 3.500 & 3.498 & -0.07 & 17.4 \\
\hline 373.150 & 7.551 & 7.556 & 0.06 & 3.500 & 3.497 & -0.09 & 14.3 \\
\hline 398.150 & 8.612 & 8.619 & 0.08 & 3.500 & 3.496 & -0.10 & 12.1 \\
\hline 423.150 & 9.659 & 9.669 & 0.10 & 3.500 & 3.496 & -0.13 & 10.5 \\
\hline 448.150 & 10.696 & 10.708 & 0.11 & 3.500 & 3.496 & -0.13 & 9.3 \\
\hline 473.150 & 11.726 & 11.739 & 0.11 & 3.500 & 3.496 & -0.13 & 8.3 \\
\hline 498.150 & 12.747 & 12.763 & 0.12 & 3.500 & 3.495 & -0.13 & 7.5 \\
\hline 523.150 & 13.764 & 13.781 & 0.12 & 3.500 & 3.496 & -0.13 & 6.9 \\
\hline 548.150 & 14.776 & 14.794 & 0.12 & 3.500 & 3.496 & -0.12 & 6.3 \\
\hline 573.150 & 15.785 & 15.803 & 0.11 & 3.500 & 3.496 & -0.11 & 5.9 \\
\hline 598.150 & 16.792 & 16.807 & 0.09 & 3.500 & 3.497 & -0.09 & 5.5 \\
\hline 623.150 & 17.803 & 17.808 & 0.03 & 3.500 & 3.499 & -0.03 & 5.1 \\
\hline 303.150 & 4.571 & 4.572 & 0.01 & 4.000 & 3.997 & -0.08 & 28.3 \\
\hline 323.150 & 5.677 & 5.678 & 0.02 & 4.000 & 3.998 & -0.05 & 21.5 \\
\hline 348.150 & 6.992 & 6.995 & 0.04 & 4.000 & 3.997 & -0.06 & 16.4 \\
\hline 373.150 & 8.274 & 8.279 & 0.07 & 4.000 & 3.996 & -0.10 & 13.1 \\
\hline 398.150 & 9.535 & 9.544 & 0.09 & 4.000 & 3.995 & -0.12 & 11.0 \\
\hline 423.150 & 10.782 & 10.794 & 0.11 & 4.000 & 3.995 & -0.14 & 9.4 \\
\hline 448.150 & 12.019 & 12.033 & 0.11 & 4.000 & 3.995 & -0.13 & 8.2 \\
\hline 473.150 & 13.246 & 13.262 & 0.12 & 4.000 & 3.995 & -0.13 & 7.3 \\
\hline
\end{tabular}


Table 6. PVT DATA (continued)

Data from Douslin and Harrison [9] (continued)

\begin{tabular}{|c|c|c|c|c|c|c|c|}
\hline $\begin{array}{l}\mathrm{T} \\
\mathrm{K}\end{array}$ & $\begin{array}{l}\mathrm{P}, \underset{\mathrm{MPa}}{\exp t} \\
\text { exp }\end{array}$ & $\begin{array}{c}\mathrm{P}, \mathrm{calc} \\
\mathrm{MPa}\end{array}$ & $\begin{array}{c}\mathrm{dev} \\
8\end{array}$ & $\begin{array}{l}\rho, \text { expt } \\
\mathrm{mol} \cdot \mathrm{dm}^{-3}\end{array}$ & $\begin{array}{l}\rho, \quad \text { calc } \\
\mathrm{mol} \cdot \mathrm{dm}^{-3}\end{array}$ & $\begin{array}{r}\mathrm{dev} \\
8\end{array}$ & wt \\
\hline 498.150 & 14.465 & 14.483 & 0.12 & 4.000 & 3.995 & -0.13 & 6.6 \\
\hline 523.150 & 15.679 & 15.697 & 0.11 & 4.000 & 3.995 & -0.11 & 6.0 \\
\hline 548.150 & 16.886 & 16.905 & 0.11 & 4.000 & 3.996 & -0.11 & 5.5 \\
\hline 573.150 & 18.090 & 18.108 & 0.10 & 4.000 & 3.996 & -0.10 & 5.0 \\
\hline 598.150 & 19.292 & 19.305 & 0.07 & 4.000 & 3.997 & -0.07 & 4.7 \\
\hline 623.150 & 20.499 & 20.499 & 0.00 & 4.000 & 4.000 & 0.00 & 4.4 \\
\hline 303.150 & 4.645 & 4.646 & 0.02 & 4.500 & 4.490 & -0.23 & 28.1 \\
\hline 323.150 & 5.930 & 5.931 & 0.02 & 4.500 & 4.497 & -0.06 & 20.9 \\
\hline 348.150 & 7.460 & 7.463 & 0.04 & 4.500 & 4.497 & -0.07 & 15.5 \\
\hline 373.150 & 8.956 & 8.962 & 0.07 & 4.500 & 4.495 & -0.10 & 12.2 \\
\hline 398.150 & 10.432 & 10.441 & 0.09 & 4.500 & 4.495 & -0.12 & 10.0 \\
\hline 423.150 & 11.892 & 11.905 & 0.11 & 4.500 & 4.494 & -0.13 & 8.5 \\
\hline 448.150 & 13.343 & 13.357 & 0.10 & 4.500 & 4.495 & -0.11 & 7.3 \\
\hline 473.150 & 14.782 & 14.799 & 0.11 & 4.500 & 4.495 & -0.12 & 6.5 \\
\hline 498.150 & 16.215 & 16.232 & 0.10 & 4.500 & 4.495 & -0.10 & 5.8 \\
\hline 523.150 & 17.640 & 17.656 & 0.09 & 4.500 & 4.496 & -0.09 & 5.2 \\
\hline 548.150 & 19.057 & 19.074 & 0.09 & 4.500 & 4.496 & -0.09 & 4.8 \\
\hline 573.150 & 20.471 & 20.486 & 0.08 & 4.500 & 4.497 & -0.07 & 4.4 \\
\hline 598.150 & 21.884 & 21.892 & 0.04 & 4.500 & 4.498 & -0.04 & 4.1 \\
\hline 623.150 & 23.303 & 23.293 & -0.04 & 4.500 & 4.502 & 0.04 & 3.8 \\
\hline 323.150 & 6.139 & 6.141 & 0.02 & 5.000 & 4.996 & -0.08 & 20.4 \\
\hline 348.150 & 7.892 & 7.895 & 0.04 & 5.000 & 4.997 & -0.07 & 14.7 \\
\hline 373.150 & 9.614 & 9.619 & 0.06 & 5.000 & 4.995 & -0.09 & 11.3 \\
\hline 398.150 & 11.318 & 11.327 & 0.08 & 5.000 & 4.995 & -0.10 & 9.1 \\
\hline 423.150 & 13.008 & 13.020 & 0.09 & 5.000 & 4.995 & -0.11 & 7.6 \\
\hline 448.150 & 14.687 & 14.700 & 0.09 & 5.000 & 4.995 & -0.10 & 6.6 \\
\hline 473.150 & 16.355 & 16.370 & 0.09 & 5.000 & 4.995 & -0.09 & 5.7 \\
\hline 498.150 & 18.015 & 18.031 & 0.09 & 5.000 & 4.996 & -0.08 & 5.1 \\
\hline 523.150 & 19.667 & 19.683 & 0.08 & 5.000 & 4.996 & -0.08 & 4.6 \\
\hline 548.150 & 21.312 & 21.327 & 0.07 & 5.000 & 4.997 & -0.07 & 4.2 \\
\hline 573.150 & 22.954 & 22.964 & 0.04 & 5.000 & 4.998 & -0.04 & 3.8 \\
\hline 598.150 & 24.595 & 24.594 & 0.00 & 5.000 & 5.000 & 0.00 & 3.5 \\
\hline 623.150 & 26.244 & 26.218 & -0.10 & 5.000 & 5.004 & 0.09 & 3.3 \\
\hline 323.150 & 6.318 & 6.320 & 0.02 & 5.500 & 5.495 & -0.09 & 19.9 \\
\hline 348.150 & 8.302 & 8.304 & 0.03 & 5.500 & 5.497 & -0.05 & 13.9 \\
\hline
\end{tabular}


Table 6. PVT DATA (continued)

Data from Douslin and Harrison [9] (continued)

\begin{tabular}{|c|c|c|c|c|c|c|c|}
\hline $\begin{array}{l}\mathrm{T} \\
\mathrm{K}\end{array}$ & $\mathrm{P},{ }_{\mathrm{MPa}}$ expt & $\begin{array}{c}\mathrm{P}, \mathrm{MPa} \\
\mathrm{MPa}\end{array}$ & $\begin{array}{c}\text { dev } \\
8\end{array}$ & $\begin{array}{l}\rho, \quad \operatorname{expt} \\
\mathrm{mol} \cdot \mathrm{dm}^{-3}\end{array}$ & $\begin{array}{l}\rho, \quad \text { calc } \\
\mathrm{mol} \cdot \mathrm{dm}^{-3}\end{array}$ & $\begin{array}{r}\text { dev } \\
8\end{array}$ & wt \\
\hline 373.150 & 10.264 & 10.268 & 0.04 & 5.500 & 5.496 & -0.06 & 10.5 \\
\hline 398.150 & 12.211 & 12.219 & 0.06 & 5.500 & 5.496 & -0.07 & 8.3 \\
\hline 423.150 & 14.147 & 14.157 & 0.07 & 5.500 & 5.496 & -0.08 & 6.9 \\
\hline 448.150 & 16.072 & 16.083 & 0.07 & 5.500 & 5.496 & -0.07 & 5.9 \\
\hline 473.150 & 17.986 & 17.999 & 0.07 & 5.500 & 5.496 & -0.07 & 5.1 \\
\hline 498.150 & 19.893 & 19.905 & 0.06 & 5.500 & 5.497 & -0.06 & 4.5 \\
\hline 523.150 & 21.790 & 21.801 & 0.05 & 5.500 & 5.497 & -0.05 & 4.1 \\
\hline 548.150 & 23.681 & 23.689 & 0.04 & 5.500 & 5.498 & -0.03 & 3.7 \\
\hline 573.150 & 25.569 & 25.569 & 0.00 & 5.500 & 5.500 & 0.00 & 3.4 \\
\hline 598.150 & 27.455 & 27.441 & -0.05 & 5.500 & 5.502 & 0.04 & 3.1 \\
\hline 623.150 & 29.353 & 29.307 & -0.16 & 5.500 & 5.507 & 0.13 & 2.9 \\
\hline 323.150 & 6.479 & 6.480 & 0.02 & 6.000 & 5.996 & -0.07 & 19.5 \\
\hline 348.150 & 8.705 & 8.706 & 0.01 & 6.000 & 5.998 & -0.03 & 13.2 \\
\hline 373.150 & 10.921 & 10.925 & 0.03 & 6.000 & 5.998 & $-C .04$ & 9.7 \\
\hline 398.150 & 13.131 & 13.136 & 0.04 & 6.000 & 5.997 & -0.04 & 7.6 \\
\hline 423.150 & 15.329 & 15.337 & 0.05 & 6.000 & 5.997 & -0.36 & 6.2 \\
\hline 448.150 & 17.520 & 17.529 & 0.05 & 6.000 & 5.997 & -0.05 & 5.3 \\
\hline 473.150 & 19.701 & 19.710 & 0.04 & 6.000 & 5.998 & -0.04 & 4.5 \\
\hline 498.150 & 21.875 & 21.880 & 0.02 & 6.000 & 5.999 & -0.02 & 4.0 \\
\hline 523.150 & 24.036 & 24.041 & 0.02 & 6.000 & 5.999 & -0.02 & 3.6 \\
\hline 548.150 & 26.194 & 26.192 & -0.01 & 6.000 & 6.000 & 0.00 & 3.2 \\
\hline 573.150 & 28.346 & 28.334 & -0.04 & 6.000 & 6.002 & 0.04 & 3.0 \\
\hline 598.150 & 30.500 & 30.468 & -0.10 & 6.000 & 6.005 & 0.08 & 2.7 \\
\hline 623.150 & 32.667 & 32.593 & -0.22 & 6.000 & 6.011 & 0.18 & 2.5 \\
\hline 323.150 & 6.633 & 6.634 & 0.00 & 6.500 & 6.499 & -0.01 & 18.9 \\
\hline 348.150 & 9.116 & 9.116 & 0.00 & 6.500 & 6.500 & 0.00 & 12.4 \\
\hline 373.150 & 11.606 & 11.609 & 0.03 & 6.500 & 6.498 & -0.03 & 8.9 \\
\hline 398.150 & 14.096 & 14.100 & 0.03 & 6.500 & 6.498 & -0.04 & 6.9 \\
\hline 423.150 & 16.580 & 16.586 & 0.04 & 6.500 & 6.498 & -0.04 & 5.6 \\
\hline 448.150 & 19.058 & 19.064 & 0.03 & 6.500 & 6.498 & -0.03 & 4.7 \\
\hline 473.150 & 21.527 & 21.531 & 0.02 & 6.500 & 6.499 & -0.02 & 4.0 \\
\hline 498.150 & 23.989 & 23.988 & 0.00 & 6.500 & 6.500 & 0.00 & 3.5 \\
\hline 523.150 & 26.437 & 26.434 & -0.01 & 6.500 & 6.501 & 0.01 & 3.1 \\
\hline 548.150 & 28.885 & 28.870 & -0.05 & 6.500 & 6.503 & 0.04 & 2.8 \\
\hline 573.150 & 31.324 & 31.296 & -0.09 & 6.500 & 6.505 & 0.07 & 2.6 \\
\hline
\end{tabular}


Table 6. PVT DATA (continued)

Dat: from Douslin and Harrison [9] (continued)

\begin{tabular}{|c|c|c|c|c|c|c|c|}
\hline $\begin{array}{l}\mathrm{T} \\
\mathrm{K}\end{array}$ & $\mathrm{P}, \underset{\mathrm{MPa}}{\operatorname{expt}}$ & $\underset{\mathrm{MPa}}{\mathrm{P}, \mathrm{calc}}$ & $\begin{array}{c}\text { dev } \\
8\end{array}$ & $\begin{array}{l}\rho, \text { expt } \\
\mathrm{mo} 1 \cdot \mathrm{dm}^{-3}\end{array}$ & $\begin{array}{l}\rho, \text { calc } \\
\mathrm{mol} \cdot \mathrm{dm}^{-3}\end{array}$ & $\begin{array}{r}\text { dev } \\
\frac{8}{8}\end{array}$ & $w t$ \\
\hline 598.150 & 33.768 & 33.712 & -0.17 & 6.500 & 6.508 & 0.13 & 2.4 \\
\hline 623.150 & 36.221 & 36.119 & -0.28 & 6.500 & 6.514 & 0.22 & 2.2 \\
\hline 323.150 & 6.792 & 6.791 & -0.01 & 7.000 & 7.003 & 0.04 & 18.3 \\
\hline 348.150 & 9.552 & 9.552 & 0.00 & 7.000 & 7.000 & 0.00 & 11.4 \\
\hline 373.150 & 12.337 & 12.341 & 0.03 & 7.000 & 6.997 & -0.04 & 8.0 \\
\hline 398.150 & 15.134 & 15.137 & 0.02 & 7.000 & 6.999 & -0.02 & 6.1 \\
\hline 423.150 & 17.927 & 17.931 & 0.02 & 7.000 & 6.999 & -0.02 & 4.9 \\
\hline 448.150 & 20.718 & 20.718 & 0.00 & 7.000 & 7.000 & 0.00 & 4.1 \\
\hline 473.150 & 23.498 & 23.495 & -0.01 & 7.000 & 7.001 & 0.01 & 3.5 \\
\hline 498.150 & 26.270 & 26.262 & -0.03 & 7.000 & 7.002 & 0.02 & 3.1 \\
\hline 523.150 & 29.033 & 29.018 & -0.05 & 7.000 & 7.003 & 0.04 & 2.8 \\
\hline 548.150 & 31.791 & 31.762 & -0.09 & 7.000 & 7.005 & 0.07 & 2.5 \\
\hline 573.150 & 34.542 & 34.495 & -0.14 & 7.000 & 7.007 & 0.10 & 2.3 \\
\hline 598.150 & 37.299 & 37.216 & -0.22 & 7.000 & 7.011 & 0.16 & 2.1 \\
\hline 623.150 & 40.073 & 39.928 & -0.36 & 7.000 & 7.018 & 0.26 & 1.9 \\
\hline 323.150 & 6.969 & 6.966 & -0.04 & 7.500 & 7.508 & 0.11 & 17.5 \\
\hline 348.150 & 10.030 & 10.033 & 0.03 & 7.500 & 7.497 & -0.03 & 10.4 \\
\hline 373.150 & 13.138 & 13.146 & 0.06 & 7.500 & 7.495 & -0.06 & 7.2 \\
\hline 398.150 & 16.269 & 16.273 & 0.02 & 7.500 & 7.498 & -0.02 & 5.4 \\
\hline 423.150 & 19.399 & 19.402 & 0.01 & 7.500 & 7.499 & -0.01 & 4.3 \\
\hline 448.150 & 22.530 & 22.525 & -0.02 & 7.500 & 7.501 & 0.02 & 3.6 \\
\hline 473.150 & 25.651 & 25.640 & -0.04 & 7.500 & 7.502 & 0.03 & 3.1 \\
\hline 498.150 & 28.765 & 28.743 & -0.07 & 7.500 & 7.504 & 0.05 & 2.7 \\
\hline 523.150 & 31.865 & 31.834 & -0.09 & 7.500 & 7.505 & 0.07 & 2.4 \\
\hline 548.150 & 34.957 & 34.913 & -0.13 & 7.500 & 7.507 & 0.09 & 2.2 \\
\hline 573.150 & 38.045 & 37.978 & -0.18 & 7.500 & 7.509 & 0.12 & 2.0 \\
\hline 323.150 & 7.178 & 7.174 & -0.05 & 8.000 & 8.007 & 0.09 & 16.2 \\
\hline 348.150 & 10.575 & 10.583 & 0.08 & 8.000 & 7.993 & -0.09 & 9.2 \\
\hline 373.150 & 14.042 & 14.053 & 0.08 & 8.000 & 7.994 & -0.07 & 6.3 \\
\hline 398.150 & 17.534 & 17.542 & 0.05 & 8.000 & 7.997 & -0.04 & 4.7 \\
\hline 423.150 & 21.033 & 21.036 & 0.01 & 8.000 & 7.999 & -0.01 & 3.8 \\
\hline 448.150 & 24.535 & 24.526 & -0.03 & 8.000 & 8.002 & 0.03 & 3.1 \\
\hline 473.150 & 28.034 & 28.008 & -0.09 & 8.000 & 8.005 & 0.07 & 2.7 \\
\hline 498.150 & 31.510 & 31.477 & -0.10 & 8.000 & 8.006 & 0.07 & 2.4 \\
\hline 523.150 & 34.978 & 34.933 & -0.13 & 8.000 & 8.007 & 0.09 & 2.1 \\
\hline
\end{tabular}


Table 6. PVT DATA (continued)

Data from Douslin and Harrison [9] (continued)

\begin{tabular}{|c|c|c|c|c|c|c|c|}
\hline $\begin{array}{l}\mathrm{T} \\
\mathrm{K}\end{array}$ & $\mathrm{P}, \underset{\mathrm{MPa}}{\exp t}$ & $\begin{array}{c}\mathrm{P}, \underset{\mathrm{MPa}}{\mathrm{c}} \text { calc } \\
\text {. }\end{array}$ & $\begin{array}{c}\mathrm{dev} \\
8\end{array}$ & $\begin{array}{l}\rho, \operatorname{expt} \\
\mathrm{mol} \cdot \mathrm{dm}^{-3}\end{array}$ & $\begin{array}{l}\rho, \text { calc } \\
\mathrm{mol} \cdot \mathrm{dm}^{-3}\end{array}$ & $\begin{array}{r}\text { dev } \\
8\end{array}$ & wt \\
\hline 548.150 & 38.441 & 38.374 & -0.17 & 8.000 & 8.009 & 0.11 & 1.9 \\
\hline 323.150 & 7.441 & 7.439 & -0.03 & 8.500 & 8.503 & 0.04 & 14.5 \\
\hline 348.150 & 11.215 & 11.231 & 0.14 & 8.500 & 8.489 & -0.13 & 8.0 \\
\hline 373.150 & 15.076 & 15.094 & 0.12 & 8.500 & 8.492 & -0.09 & 5.4 \\
\hline 398.150 & 18.971 & 18.981 & 0.06 & 8.500 & 8.497 & -0.04 & 4.1 \\
\hline 423.150 & 22.873 & 22.876 & 0.01 & 8.500 & 8.499 & -0.01 & 3.3 \\
\hline 448.150 & 26.782 & 26.767 & -0.06 & 8.500 & 8.503 & 0.04 & 2.7 \\
\hline 473.150 & 30.686 & 30.649 & -0.12 & 8.500 & 8.507 & 0.08 & 2.3 \\
\hline 498.150 & 34.563 & 34.517 & -0.13 & 8.500 & 8.507 & 0.08 & 2.0 \\
\hline 523.150 & 38.431 & 38.369 & -0.16 & 8.500 & 8.508 & 0.10 & 1.8 \\
\hline 323.150 & 7.786 & 7.789 & 0.05 & 9.000 & 8.995 & -0.05 & 12.3 \\
\hline 348.150 & 11.986 & 12.010 & 0.20 & 9.000 & 8.986 & -0.16 & 6.7 \\
\hline 373.150 & 16.286 & 16.308 & 0.14 & 9.000 & 8.991 & -0.10 & 4.6 \\
\hline 398.150 & 20.626 & 20.635 & 0.05 & 9.000 & 8.9 .77 & -0.03 & 3.4 \\
\hline 423.150 & 24.973 & 24.970 & -0.01 & 9.000 & 9.001 & 0.01 & 2.8 \\
\hline 448.150 & 29.323 & 29.301 & -0.07 & 9.000 & 9.004 & 0.04 & 2.3 \\
\hline 473.150 & 33.673 & 33.621 & -0.15 & 9.000 & 9.008 & 0.09 & 2.0 \\
\hline 498.150 & 37.985 & 37.924 & -0.16 & 9.000 & 9.008 & 0.09 & 1.7 \\
\hline 323.150 & 8.250 & 8.264 & 0.17 & 9.500 & 9.487 & -0.13 & 9.8 \\
\hline 348.150 & 12.932 & 12.963 & 0.24 & 9.500 & 9.485 & -0.15 & 5.5 \\
\hline 373.150 & 17.720 & 17.747 & 0.15 & 9.500 & 9.492 & -0.09 & 3.8 \\
\hline 398.150 & 22.551 & 22.560 & 0.04 & 9.500 & 9.498 & -0.02 & 2.9 \\
\hline 423.150 & 27.390 & 27.380 & -0.03 & 9.500 & 9.502 & 0.02 & 2.3 \\
\hline 448.150 & 32.233 & 32.194 & -0.12 & 9.500 & 9.506 & 0.07 & 2.0 \\
\hline 473.150 & 37.061 & 36.992 & -0.19 & 9.500 & 9.510 & 0.10 & 1.7 \\
\hline 323.150 & 8.884 & 8.909 & 0.29 & 10.000 & 9.983 & -0.17 & 7.5 \\
\hline 348.150 & 14.109 & 14.147 & 0.27 & 10.000 & 9.985 & -0.15 & 4.4 \\
\hline 373.150 & 19.442 & 19.470 & 0.14 & 10.000 & 9.993 & -0.07 & 3.1 \\
\hline 398.150 & 24.821 & 24.822 & 0.01 & 10.000 & 10.000 & 0.00 & 2.4 \\
\hline 423.150 & 30.198 & 30.177 & -0.07 & 10.000 & 10.003 & 0.03 & 1.9 \\
\hline 448.150 & 35.572 & 35.520 & -0.15 & 10.000 & 10.007 & 0.07 & 1.6 \\
\hline 323.150 & 9.748 & 9.787 & 0.40 & 10.500 & 10.481 & -0.18 & 5.6 \\
\hline 348.150 & 15.584 & 15.630 & 0.30 & 10.500 & 10.486 & -0.13 & 3.4 \\
\hline 373.150 & 21.524 & 21.555 & 0.14 & 10.500 & 10.493 & -0.06 & 2.5 \\
\hline 398.150 & 27.501 & 27.502 & 0.00 & 10.500 & 10.500 & 0.00 & 2.0 \\
\hline
\end{tabular}


Table 6. PVT DATA (continued)

Data from Douslin and Harrison [9] (continued)

\begin{tabular}{|c|c|c|c|c|c|c|c|}
\hline $\begin{array}{l}\mathrm{T} \\
\mathrm{K}\end{array}$ & $\begin{array}{c}\mathrm{P}, \operatorname{expt}_{\mathrm{MPa}} \\
\mathrm{Pa}\end{array}$ & $\begin{array}{c}\mathrm{P}, \text { calc } \\
\mathrm{MPa}\end{array}$ & $\begin{array}{c}\mathrm{dev} \\
8\end{array}$ & $\begin{array}{l}\rho, \text { expt } \\
\mathrm{mol} \cdot \mathrm{dm}^{-3}\end{array}$ & $\begin{array}{l}\rho, \quad \text { calc } \\
\mathrm{mol} \cdot \mathrm{dm}^{-3}\end{array}$ & $\begin{array}{r}\mathrm{dev} \\
8\end{array}$ & wt \\
\hline 423.150 & 33.473 & 33.445 & -0.08 & 10.500 & 10.504 & 0.04 & 1.6 \\
\hline 448.150 & 39.435 & 39.368 & -0.17 & 10.500 & 10.508 & 0.08 & 1.4 \\
\hline 305.370 & 4.956 & 4.958 & 0.02 & 9.001 & 8.994 & -0.08 & 0.0 \\
\hline 305.370 & $4 . y 01$ & 4.900 & 0.00 & 8.500 & 8.502 & 0.02 & 0.0 \\
\hline 305.370 & 4.881 & 4.881 & 0.00 & 8.000 & 7.989 & -0.14 & 0.0 \\
\hline 305.370 & 4.877 & 4.878 & 0.01 & 7.695 & 7.646 & -0.64 & 0.0 \\
\hline 305.370 & 4.876 & 4.877 & 0.01 & 7.500 & 7.375 & -1.67 & 0.0 \\
\hline 305.370 & 4.876 & 4.876 & 0.01 & 7.395 & 7.197 & -2.68 & 0.0 \\
\hline 305.370 & 4.876 & 4.876 & 0.00 & 7.195 & 6.887 & -4.29 & 0.0 \\
\hline 305.370 & 4.876 & 4.876 & 0.00 & 7.095 & 6.979 & -1.64 & 0.0 \\
\hline 305.370 & 4.876 & 4.876 & 0.00 & 7.000 & 6.774 & -3.23 & 0.0 \\
\hline 305.370 & 4.876 & 4.876 & 0.00 & 6.896 & 6.774 & -1.77 & 0.0 \\
\hline 305.370 & 4.876 & 4.876 & 0.00 & 6.796 & 6.690 & -1.56 & 0.0 \\
\hline 305.370 & 4.876 & 4.876 & 0.00 & 6.696 & 6.674 & -0.32 & 0.0 \\
\hline 305.370 & 4.876 & 4.876 & 0.00 & 6.596 & 6.594 & -0.03 & 0.0 \\
\hline 305.370 & 4.876 & 4.876 & 0.00 & 6.500 & 6.594 & 1.44 & 0.0 \\
\hline 305.370 & 4.876 & 4.876 & 0.00 & 6.396 & 6.439 & 0.66 & 0.0 \\
\hline 305.370 & 4.875 & 4.875 & 0.00 & 6.197 & 6.272 & 1.22 & 0.0 \\
\hline 305.370 & 4.874 & 4.874 & -0.01 & 6.000 & 6.049 & 0.81 & 0.0 \\
\hline 305.370 & 4.872 & 4.872 & -0.01 & 5.747 & 5.784 & 0.65 & 0.0 \\
\hline 305.370 & 4.867 & 4.867 & -0.01 & 5.500 & 5.528 & 0.51 & 0.0 \\
\hline 305.370 & 4.845 & 4.844 & -0.01 & 5.000 & 5.007 & 0.15 & 0.0 \\
\hline 305.370 & 8.883 & 8.970 & 0.98 & 12.001 & 11.976 & -0.21 & 0.0 \\
\hline 305.370 & 7.421 & 7.474 & 0.72 & 11.499 & 11.478 & -0.19 & 0.0 \\
\hline 305.370 & 6.408 & 6.447 & 0.62 & 11.000 & 10.976 & -0.22 & 0.0 \\
\hline 305.370 & 5.738 & 5.766 & 0.50 & 10.500 & 10.473 & -0.26 & 0.0 \\
\hline 305.370 & 5.323 & 5.338 & 0.28 & 10.000 & 9.977 & -0.23 & 0.0 \\
\hline 305.370 & 5.081 & 5.088 & 0.13 & 9.500 & 9.483 & -0.19 & 0.0 \\
\hline 305.370 & 4.794 & 4.794 & 0.00 & 4.500 & 4.500 & 0.01 & 0.0 \\
\hline 305.370 & 4.699 & 4.699 & 0.01 & 4.000 & 3.998 & -0.04 & 0.0 \\
\hline 305.370 & 4.541 & 4.542 & 0.02 & 3.500 & 3.498 & -0.05 & 0.0 \\
\hline 305.370 & 4.301 & 4.302 & 0.02 & 3.000 & 2.999 & -0.04 & 0.0 \\
\hline 305.370 & 3.960 & 3.960 & 0.01 & 2.500 & 2.500 & -0.02 & 0.0 \\
\hline 305.370 & 3.496 & 3.496 & 0.00 & 2.000 & 2.000 & 0.00 & 0.0 \\
\hline 305.370 & 2.888 & 2.888 & -0.01 & 1.500 & 1.500 & 0.01 & 0.0 \\
\hline
\end{tabular}


Table 6. PVT DATA (continued)

Data from Douslin and Harrison [9] (continued)

\begin{tabular}{|c|c|c|c|c|c|c|c|}
\hline $\begin{array}{l}\mathrm{T} \\
\mathrm{K}\end{array}$ & $\mathrm{P}, \underset{\mathrm{MPa}}{\exp t}$ & $\begin{array}{c}\mathrm{P}, \underset{\mathrm{MPa}}{\mathrm{calc}} \\
\text { cal }\end{array}$ & $\begin{array}{c}\text { dev } \\
8\end{array}$ & $\begin{array}{l}\rho, \operatorname{expt} \\
\mathrm{mol} \cdot \mathrm{dm}^{-3}\end{array}$ & $\begin{array}{l}\rho, \text { calc } \\
\mathrm{mol} \cdot \mathrm{dm}^{-3}\end{array}$ & $\begin{array}{r}\mathrm{dev} \\
8\end{array}$ & $w t$ \\
\hline 305.370 & 2.117 & 2.117 & -0.01 & 1.000 & 1.000 & 0.01 & 0.0 \\
\hline 305.370 & 1.663 & 1.663 & -0.02 & 0.750 & 0.750 & 0.02 & 0.0 \\
\hline 298.150 & 4.640 & 4.668 & 0.61 & 11.000 & 10.976 & -0.22 & 0.0 \\
\hline 323.150 & 10.920 & 10.974 & 0.49 & 11.000 & 10.980 & -0.18 & 0.0 \\
\hline 348.150 & 17.442 & 17.499 & 0.32 & 11.000 & 10.986 & -0.12 & 0.0 \\
\hline 373.150 & 24.060 & 24.092 & 0.13 & 11.000 & 10.994 & -0.05 & 0.0 \\
\hline 398.150 & 30.698 & 30.696 & -0.01 & 11.000 & 11.000 & 0.00 & 0.0 \\
\hline 423.150 & 37.323 & 37.284 & -0.10 & 11.000 & 11.005 & 0.04 & 0.0 \\
\hline 298.150 & 5.412 & 5.450 & 0.71 & 11.500 & 11.480 & -0.17 & 0.0 \\
\hline 323.150 & 12.498 & 12.569 & 0.57 & 11.500 & 11.481 & -0.17 & 0.0 \\
\hline 348.150 & 19.780 & 19.856 & 0.38 & 11.500 & 11.486 & -0.13 & 0.0 \\
\hline 373.150 & 27.147 & 27.189 & 0.15 & 11.500 & 11.494 & -0.05 & 0.0 \\
\hline 398.150 & 34.524 & 34.515 & -0.03 & 11.500 & 11.501 & 0.01 & 0.0 \\
\hline 298.150 & 6.614 & 6.675 & 0.92 & 12.000 & 11.979 & -0.17 & 0.0 \\
\hline 323.150 & 14.602 & 14.692 & 0.61 & 12.000 & 11.982 & -0.15 & 0.0 \\
\hline 348.150 & 22.722 & 22.823 & 0.44 & 12.000 & 11.985 & -0.13 & 0.0 \\
\hline 373.150 & 30.916 & 30.969 & 0.17 & 12.000 & 11.994 & -0.05 & 0.0 \\
\hline 398.150 & 39.114 & 39.084 & -0.08 & 12.000 & 12.003 & 0.03 & 0.0 \\
\hline 298.150 & 8.377 & 8.480 & 1.23 & 12.500 & 12.476 & -0.19 & 0.0 \\
\hline 323.150 & 17.352 & 17.481 & 0.75 & 12.500 & 12.479 & -0.16 & 0.0 \\
\hline 348.150 & 26.406 & 26.539 & 0.50 & 12.500 & 12.484 & -0.13 & 0.0 \\
\hline 373.150 & 35.507 & 35.573 & 0.18 & 12.500 & 12.494 & -0.05 & 0.0 \\
\hline 298.150 & 10.859 & 11.022 & 1.51 & 13.000 & 12.972 & -0.21 & 0.0 \\
\hline 323.150 & 20.886 & 21.094 & 1.00 & 13.000 & 12.974 & -0.20 & 0.0 \\
\hline 348.150 & 30.988 & 31.160 & 0.56 & 13.000 & 12.983 & -0.13 & 0.0 \\
\hline 273.150 & 3.158 & 3.280 & 3.86 & 13.500 & 13.477 & -0.17 & 0.0 \\
\hline 298.150 & 14.231 & 14.479 & 1.74 & 13.500 & 13.469 & -0.23 & 0.0 \\
\hline 323.150 & 25.415 & 25.707 & 1.15 & 13.500 & 13.472 & -0.21 & 0.0 \\
\hline 348.150 & 36.640 & 36.865 & 0.61 & 13.500 & 13.482 & -0.13 & 0.0 \\
\hline 273.150 & 6.278 & 6.501 & 3.54 & 14.000 & 13.970 & -0.21 & 0.0 \\
\hline 298.150 & 18.704 & 19.043 & 1.82 & 14.000 & 13.967 & -0.24 & 0.0 \\
\hline 323.150 & 31.174 & 31.515 & 1.10 & 14.000 & 13.973 & -0.19 & 0.0 \\
\hline 273.150 & 10.624 & 10.960 & 3.17 & 14.500 & 14.467 & -0.23 & 0.0 \\
\hline 298.150 & 24.511 & 24.932 & 1.72 & 14.500 & 14.468 & -0.22 & 0.0 \\
\hline 323.150 & 38.361 & 38.739 & 0.99 & 14.500 & 14.476 & -0.16 & 0.0 \\
\hline
\end{tabular}


Table 6. PVT DATA (continued)

Data from Douslin and Harrison [9] (continued)

\begin{tabular}{|c|c|c|c|c|c|c|c|}
\hline $\begin{array}{l}\mathrm{T} \\
\mathrm{K}\end{array}$ & $\mathrm{P}, \operatorname{MPa}^{\exp t}$ & $\begin{array}{c}\mathrm{P}, \underset{\mathrm{MPa}}{\mathrm{calc}} \\
\text {. }\end{array}$ & $\begin{array}{c}\text { dev } \\
\frac{8}{8}\end{array}$ & $\begin{array}{l}\rho, \quad \operatorname{expt} \\
\mathrm{mol} \cdot \mathrm{dm}^{-3}\end{array}$ & $\begin{array}{l}\rho, \quad \mathrm{calc} \\
\mathrm{mol} \cdot \mathrm{dm}^{-3}\end{array}$ & $\begin{array}{r}\text { dev } \\
8\end{array}$ & $w t$ \\
\hline 273.150 & 16.425 & 16.896 & 2.86 & 15.000 & 14.965 & -0.23 & 0.0 \\
\hline 298.150 & 31.856 & 32.387 & 1.67 & 15.000 & 14.968 & -0.21 & 0.0 \\
\hline 248.150 & 6.739 & 7.129 & 5.79 & 15.500 & 15.471 & -0.19 & 0.0 \\
\hline 273.150 & 24.035 & 24.569 & 2.22 & 15.500 & 15.469 & -0.20 & 0.0 \\
\hline 298.150 & 41.026 & 41.679 & 1.59 & 15.500 & 15.468 & -0.21 & 0.0 \\
\hline 248.150 & 14.466 & 15.001 & 3.70 & 16.000 & 15.970 & -0.19 & 0.0 \\
\hline 273.150 & 33.691 & 34.276 & 1.74 & 16.000 & 15.973 & -0.17 & 0.0 \\
\hline 243.150 & 1.307 & 2.134 & 63.29 & 15.382 & 15.312 & -0.46 & 0.0 \\
\hline 243.150 & 1.128 & 1.824 & 61.69 & 15.356 & 15.297 & -0.39 & 0.0 \\
\hline 248.150 & 1.176 & 1.176 & 0.00 & 0.700 & 0.700 & 0.00 & 0.0 \\
\hline 253.150 & 1.695 & 2.104 & 24.15 & 14.778 & 14.733 & -0.30 & 0.0 \\
\hline 253.150 & 1.586 & 2.002 & 26.26 & 14.767 & 14.721 & -0.31 & 0.0 \\
\hline 253.150 & 1.461 & 1.921 & 31.48 & 14.758 & 14.707 & -0.34 & 0.0 \\
\hline 253.150 & 1.418 & 1.420 & 0.11 & 0.862 & 0.861 & -0.15 & 0.0 \\
\hline 253.150 & 1.409 & 1.409 & 0.02 & 0.853 & 0.853 & -0.03 & 0.0 \\
\hline 253.150 & 1.399 & 1.399 & 0.00 & 0.845 & 0.845 & -0.01 & 0.0 \\
\hline 253.150 & 1.344 & 1.343 & -0.03 & 0.800 & 0.800 & 0.04 & 0.0 \\
\hline 253.150 & 1.279 & 1.279 & -0.03 & 0.750 & 0.750 & 0.04 & 0.0 \\
\hline 253.150 & 1.212 & 1.212 & -0.03 & 0.700 & 0.700 & 0.04 & 0.0 \\
\hline 263.150 & 2.056 & 2.303 & 12.03 & 14.120 & 14.085 & -0.25 & 0.0 \\
\hline 263.150 & 1.983 & 2.220 & 11.94 & 14.108 & 14.074 & -0.24 & 0.0 \\
\hline 263.150 & 1.909 & 2.137 & 11.96 & 14.096 & 14.063 & -0.24 & 0.0 \\
\hline 263.150 & 1.855 & 1.856 & 0.05 & 1.151 & 1.150 & -0.07 & 0.0 \\
\hline 263.150 & 1.852 & 1.853 & 0.03 & 1.148 & 1.147 & -0.04 & 0.0 \\
\hline 263.150 & 1.848 & 1.848 & 0.00 & 1.144 & 1.144 & 0.00 & 0.0 \\
\hline 263.150 & 1.829 & 1.829 & -0.02 & 1.126 & 1.126 & 0.04 & 0.0 \\
\hline 273.150 & 2.525 & 2.623 & 3.88 & 13.371 & 13.350 & -0.15 & 0.0 \\
\hline 273.150 & 2.472 & 2.571 & 4.03 & 13.360 & 13.339 & -0.16 & 0.0 \\
\hline 273.150 & 2.417 & 2.512 & 3.91 & 13.348 & 13.327 & -0.15 & 0.0 \\
\hline 273.150 & 2.381 & 2.381 & 0.03 & 1.532 & 1.531 & -0.05 & 0.0 \\
\hline 273.150 & 2.377 & 2.377 & 0.01 & 1.528 & 1.527 & -0.02 & 0.0 \\
\hline 273.150 & 2.353 & 2.352 & -0.03 & 1.500 & 1.501 & 0.06 & 0.0 \\
\hline 283.150 & 3.086 & 3.131 & 1.45 & 12.484 & 12.469 & -0.12 & 0.0 \\
\hline 283.150 & 3.058 & 3.095 & 1.22 & 12.472 & 12.459 & -0.10 & 0.0 \\
\hline 283.150 & 3.028 & 3.064 & 1.19 & 12.461 & 12.448 & -0.10 & 0.0 \\
\hline
\end{tabular}


Table 6. PVT DATA (continued)

Data from Dous1in and Harrison [9] (continued)

\begin{tabular}{|c|c|c|c|c|c|c|c|}
\hline $\begin{array}{l}\mathrm{T} \\
\mathrm{K}\end{array}$ & $\mathrm{P}, \underset{\mathrm{MPa}}{\operatorname{expt}}$ & $\underset{\mathrm{MPa}}{\mathrm{P}, \mathrm{calc}}$ & $\begin{array}{c}\mathrm{dev} \\
\frac{8}{8}\end{array}$ & $\begin{array}{l}\rho, \quad \operatorname{expt} \\
\mathrm{mol} 1 \cdot \mathrm{dm}^{-3}\end{array}$ & $\begin{array}{l}\rho, \quad \text { calc } \\
\mathrm{mol} \cdot \mathrm{dm}^{-3}\end{array}$ & $\begin{array}{r}\text { dev } \\
\&\end{array}$ & wt \\
\hline 283.150 & 3.013 & 3.014 & 0.03 & 2.063 & 2.062 & -0.06 & 0.0 \\
\hline 283.150 & 3.007 & 3.007 & 0.00 & 2.053 & 2.053 & 0.00 & 0.0 \\
\hline 283.150 & 3.005 & 3.005 & 0.00 & 2.050 & 2.050 & 0.01 & 0.0 \\
\hline 283.150 & 2.966 & 2.965 & -0.03 & 1.994 & 1.995 & 0.06 & 0.0 \\
\hline 293.150 & 3.782 & 3.802 & 0.53 & 11.312 & 11.297 & -0.14 & 0.0 \\
\hline 293.150 & 3.771 & 3.789 & 0.48 & 11.302 & 11.288 & -0.13 & 0.0 \\
\hline 293.150 & 3.755 & 3.756 & 0.04 & 2.849 & 2.846 & -0.11 & 0.0 \\
\hline 293.150 & 3.731 & 3.735 & 0.09 & 2.799 & 2.792 & -0.26 & 0.0 \\
\hline 298.150 & 4.218 & 4.238 & 0.45 & 10.540 & 10.511 & -0.27 & 0.0 \\
\hline 298.150 & 4.255 & 4.274 & 0.44 & 10.590 & 10.564 & -0.24 & 0.0 \\
\hline 298.150 & 4.176 & 4.178 & 0.04 & 3.449 & 3.443 & -0.16 & 0.0 \\
\hline 298.150 & 4.163 & 4.164 & 0.03 & 3.399 & 3.395 & -0.12 & 0.0 \\
\hline 302.150 & 4.608 & 4.616 & 0.17 & 9.738 & 9.715 & -0.24 & 0.0 \\
\hline 302.150 & 4.593 & 4.600 & 0.16 & 9.688 & 9.664 & -0.24 & 0.0 \\
\hline 302.150 & 4.578 & 4.585 & 0.16 & 9.639 & 9.613 & -0.27 & 0.0 \\
\hline 302.150 & 4.567 & 4.572 & 0.11 & 9.590 & 9.569 & -0.21 & 0.0 \\
\hline 302.150 & 4.557 & 4.559 & 0.04 & 4.298 & 4.281 & -0.37 & 0.0 \\
\hline 302.150 & 4.545 & 4.546 & 0.02 & 4.196 & 4.188 & -0.18 & 0.0 \\
\hline 302.150 & 4.530 & 4.531 & 0.02 & 4.098 & 4.091 & -0.16 & 0.0 \\
\hline 302.150 & 4.513 & 4.514 & 0.02 & 3.998 & 3.993 & -0.13 & 0.0 \\
\hline 303.150 & 4.676 & 4.678 & 0.05 & 9.342 & 9.330 & -0.13 & 0.0 \\
\hline 303.150 & 4.667 & 4.669 & 0.04 & 9.292 & 9.283 & -0.10 & 0.0 \\
\hline 303.150 & 4.661 & 4.662 & 0.02 & 9.252 & 9.246 & -0.07 & 0.0 \\
\hline 303.150 & 4.655 & 4.656 & 0.02 & 9.219 & 9.213 & -0.07 & 0.0 \\
\hline 303.150 & 4.654 & 4.655 & 0.03 & 4.606 & 4.588 & -0.41 & 0.0 \\
\hline 303.150 & 4.653 & 4.655 & 0.03 & 4.598 & 4.581 & -0.38 & 0.0 \\
\hline 303.150 & 4.649 & 4.650 & 0.03 & 4.548 & 4.535 & -0.29 & 0.0 \\
\hline 303.150 & 4.645 & 4.646 & 0.02 & 4.500 & 4.490 & -0.23 & 0.0 \\
\hline 304.150 & 4.764 & 4.764 & -0.02 & 8.893 & 8.901 & 0.09 & 0.0 \\
\hline 304.150 & 4.760 & 4.759 & -0.03 & 8.842 & 8.855 & 0.15 & 0.0 \\
\hline 304.150 & 4.756 & 4.754 & -0.03 & 8.794 & 8.811 & 0.19 & 0.0 \\
\hline 304.150 & 4.753 & 4.751 & -0.05 & 8.743 & 8.773 & 0.35 & 0.0 \\
\hline 304.150 & 4.751 & 4.752 & 0.02 & 4.998 & 4.981 & -0.34 & 0.0 \\
\hline 304.150 & 4.746 & 4.747 & 0.01 & 4.898 & 4.888 & -0.21 & 0.0 \\
\hline 304.150 & 4.740 & 4.740 & 0.01 & 4.798 & 4.792 & -0.13 & 0.0 \\
\hline
\end{tabular}


Table 6. PVT DATA (continued)

Data from Douslin and Harrison [9] (continued)

\begin{tabular}{|c|c|c|c|c|c|c|c|}
\hline $\begin{array}{l}\mathrm{T} \\
\mathrm{K}\end{array}$ & $\begin{array}{l}\mathrm{P}, \operatorname{MPa}_{\mathrm{MPp}} \\
\text { exp }\end{array}$ & $\begin{array}{c}\mathrm{P}, \underset{\mathrm{MPa}}{\mathrm{calc}} \\
\text {. }\end{array}$ & $\begin{array}{c}\text { dev } \\
\frac{8}{8}\end{array}$ & $\begin{array}{l}\rho, \quad \operatorname{expt} \\
\mathrm{mol} \cdot \mathrm{dm}^{-3}\end{array}$ & $\begin{array}{l}\rho, \quad \mathrm{calc} \\
\mathrm{mol} \cdot \mathrm{dm}^{-3}\end{array}$ & $\begin{array}{r}\text { dev } \\
8\end{array}$ & $w t$ \\
\hline 305.150 & 4.857 & 4.856 & -0.01 & 8.093 & 8.122 & 0.36 & 0.0 \\
\hline 305.150 & 4.855 & 4.854 & -0.01 & 7.994 & 8.024 & 0.38 & 0.0 \\
\hline 305.150 & 4.854 & 4.853 & -0.01 & 7.895 & 7.946 & 0.66 & 0.0 \\
\hline 305.150 & 4.854 & 4.853 & -0.02 & 7.844 & 7.930 & 1.10 & 0.0 \\
\hline 305.150 & 4.853 & 4.853 & 0.00 & 5.897 & 5.889 & -0.13 & 0.0 \\
\hline 305.150 & 4.853 & 4.853 & 0.00 & 5.847 & 5.865 & 0.30 & 0.0 \\
\hline 305.150 & 4.853 & 4.853 & 0.00 & 5.797 & 5.817 & 0.35 & 0.0 \\
\hline 305.150 & 4.852 & 4.852 & 0.00 & 5.747 & 5.762 & 0.25 & 0.0 \\
\hline 305.150 & 4.852 & 4.851 & 0.00 & 5.697 & 5.715 & 0.33 & 0.0 \\
\hline 305.150 & 4.851 & 4.851 & 0.00 & 5.647 & 5.661 & 0.25 & 0.0 \\
\hline 305.150 & 4.848 & 4.848 & -0.01 & 5.497 & 5.513 & 0.30 & 0.0 \\
\hline 305.150 & 4.839 & 4.838 & -0.01 & 5.198 & 5.208 & 0.20 & 0.0 \\
\hline 305.150 & 4.834 & 4.833 & -0.01 & 5.098 & 5.107 & 0.18 & 0.0 \\
\hline 305.150 & 4.828 & 4.828 & -0.01 & 4.998 & 5.003 & 0.10 & 0.0 \\
\hline 305.250 & 4.865 & 4.865 & 0.00 & 7.895 & 7.908 & 0.17 & 0.0 \\
\hline 305.250 & 4.865 & 4.864 & 0.00 & 7.794 & 7.822 & 0.35 & 0.0 \\
\hline 305.250 & 4.864 & 4.864 & 0.00 & 7.695 & 7.725 & 0.39 & 0.0 \\
\hline 305.250 & 4.863 & 4.863 & 0.00 & 6.096 & 6.110 & 0.22 & 0.0 \\
\hline 305.250 & 4.863 & 4.863 & 0.00 & 5.997 & 6.001 & 0.08 & 0.0 \\
\hline 305.250 & 4.863 & 4.862 & 0.00 & 5.897 & 5.916 & 0.32 & 0.0 \\
\hline 305.250 & 4.861 & 4.861 & 0.00 & 5.747 & 5.761 & 0.24 & 0.0 \\
\hline 305.250 & 4.857 & 4.856 & -0.01 & 5.497 & 5.514 & 0.31 & 0.0 \\
\hline 305.350 & 4.875 & 4.875 & 0.01 & 7.695 & 7.642 & -0.68 & 0.0 \\
\hline 305.350 & 4.874 & 4.874 & 0.00 & 7.495 & 7.418 & -1.02 & 0.0 \\
\hline 305.350 & 4.874 & 4.874 & 0.00 & 7.395 & 7.265 & -1.76 & 0.0 \\
\hline 305.350 & 4.874 & 4.874 & 0.00 & 7.195 & 7.178 & -0.24 & 0.0 \\
\hline 243.150 & 1.228 & 1.992 & 62.23 & 15.370 & 15.305 & -0.42 & 0.0 \\
\hline 305.350 & 4.874 & 4.874 & 0.00 & 7.095 & 6.866 & -3.23 & 0.0 \\
\hline 305.350 & 4.874 & 4.874 & 0.00 & 6.995 & 6.792 & -2.91 & 0.0 \\
\hline 305.350 & 4.874 & 4.874 & 0.00 & 6.896 & 6.672 & -3.24 & 0.0 \\
\hline 305.350 & 4.874 & 4.874 & 0.00 & 6.746 & 6.608 & -2.04 & 0.0 \\
\hline 305.350 & 4.874 & 4.874 & 0.00 & 6.496 & 6.590 & 1.44 & 0.0 \\
\hline 305.350 & 4.874 & 4.874 & 0.00 & 6.396 & 6.505 & 1.70 & 0.0 \\
\hline 305.350 & 4.874 & 4.873 & 0.00 & 6.296 & 6.383 & 1.38 & 0.0 \\
\hline 305.350 & 4.873 & 4.873 & 0.00 & 6.197 & 6.269 & 1.16 & 0.0 \\
\hline
\end{tabular}


Table 6. PVT DATA (continued)

Data from Douslin and Harrison [9] (continued)

\begin{tabular}{|c|c|c|c|c|c|c|c|}
\hline $\begin{array}{l}\mathrm{T} \\
\mathrm{K}\end{array}$ & $\mathrm{P}, \underset{\mathrm{MPa}}{\exp t}$ & $\begin{array}{c}\mathrm{P}, \underset{\mathrm{MPa}}{\mathrm{calc}} \\
\text { calc }\end{array}$ & $\begin{array}{c}\mathrm{dev} \\
8\end{array}$ & $\begin{array}{l}\rho, \operatorname{expt} \\
\mathrm{mol} \cdot \mathrm{dm}^{-3}\end{array}$ & $\begin{array}{l}\rho, \text { calc } \\
\mathrm{mol} \cdot \mathrm{dm}^{-3}\end{array}$ & $\begin{array}{r}\text { dev } \\
\%\end{array}$ & wt \\
\hline 305.350 & 4.873 & 4.873 & -0.01 & 6.097 & 6.162 & 1.08 & 0.0 \\
\hline 305.350 & 4.873 & 4.872 & -0.01 & 5.997 & 6.045 & 0.80 & 0.0 \\
\hline 305.350 & 4.872 & 4.871 & -0.01 & 5.897 & 5.946 & 0.84 & 0.0 \\
\hline 305.350 & 4.870 & 4.870 & -0.01 & 5.747 & 5.785 & 0.66 & 0.0 \\
\hline 305.390 & 4.880 & 4.880 & 0.01 & 7.695 & 7.636 & -0.76 & 0.0 \\
\hline 305.390 & 4.879 & 4.879 & 0.01 & 7.495 & 7.352 & -1.90 & 0.0 \\
\hline 305.390 & 4.878 & 4.879 & 0.01 & 7.395 & 7.228 & -2.25 & 0.0 \\
\hline 305.390 & 4.878 & 4.878 & 0.00 & 7.195 & 6.968 & -3.15 & 0.0 \\
\hline 305.390 & 4.878 & 4.878 & 0.00 & 6.996 & 6.830 & -2.37 & 0.0 \\
\hline 305.390 & 4.878 & 4.878 & 0.00 & 6.796 & 6.732 & -0.94 & 0.0 \\
\hline 305.390 & 4.878 & 4.878 & 0.00 & 6.596 & 6.596 & -0.01 & 0.0 \\
\hline 305.390 & 4.878 & 4.878 & 0.00 & 6.396 & 6.472 & 1.18 & 0.0 \\
\hline 305.390 & 4.877 & 4.877 & -0.01 & 6.197 & 6.272 & 1.22 & 0.0 \\
\hline 305.390 & 4.876 & 4.876 & -0.01 & 5.997 & 6.056 & 0.98 & 0.0 \\
\hline 305.390 & 4.874 & 4.873 & -0.01 & 5.747 & 5.789 & 0.73 & 0.0 \\
\hline 248.150 & 1.618 & 2.062 & 27.44 & 15.083 & 15.041 & -0.28 & 0.0 \\
\hline 248.150 & 1.350 & 1.869 & 38.38 & 15.065 & 15.015 & -0.33 & 0.0 \\
\hline 248.150 & 1.227 & 1.227 & 0.07 & 0.740 & 0.739 & -0.10 & 0.0 \\
\hline 248.150 & 1.493 & 1.965 & 31.58 & 15.074 & 15.029 & -0.30 & 0.0 \\
\hline
\end{tabular}

Pressure Calculation:

$\mathrm{AAD} \%=1.10$ BIAS $8=1.07$ RMS $8=6.20$

Density Calculation:

AAD2 $z=0.24 \quad$ BIAS $2 \%=-0.10 \quad$ RMS $2 \%=0.55$

Absolute Deviations:

Pressure Calculation:

$\mathrm{AAD}=0.04$ BIAS $=0.03$ RMS $=0.12 \mathrm{MPa}$

Density Calculation:

AAD2 $=0.017$ BIAS2 $=-0.008 \quad \mathrm{RMS} 2=0.040 \mathrm{~mol} \cdot \mathrm{dm}^{-3}$ 
Table 6. PVT DATA (continued)

Data from Douslin and Harrison [9] (continued)

Weighted Data:

Number of Points [9] 257

Pressure Calculation:

$\mathrm{AAD} \%=0.07 \quad$ BIAS $\%=0.02$ RMS $\%=0.09$

Density Calculation:

$\mathrm{AAD} 2 \%=0.06 \quad$ BIAS $2 \%=-0.03 \quad$ RMS $2 \%=0.07$

Absolute Deviations:

Pressure Calculation:

$\mathrm{AAD}=0.01$ BIAS $=0.00 \quad \mathrm{RMS}=0.02 \mathrm{MPa}$

Density Calculation:

AAD2 $=0.003 \quad$ BIAS2 $=-0.001 \quad$ RMS2 $=0.005 \mathrm{~mol} \cdot \mathrm{dm}^{-3}$

Data from Golovskiy et a1. [51]

\begin{tabular}{|c|c|c|c|c|c|c|c|}
\hline $\begin{array}{l}\mathrm{T} \\
\mathrm{K}\end{array}$ & $\begin{array}{l}\mathrm{P}, \operatorname{MPa}^{\exp t} \\
\text { a }\end{array}$ & $\begin{array}{c}\mathrm{P}, \mathrm{calc} \\
\mathrm{MPa}\end{array}$ & $\begin{array}{c}\text { dev } \\
\%\end{array}$ & $\begin{array}{l}\rho, \operatorname{expt} \\
\mathrm{mol} \cdot \mathrm{dm}^{-3}\end{array}$ & $\begin{array}{l}\rho, \quad \text { calc } \\
\mathrm{mol} \cdot \mathrm{dm}^{-3}\end{array}$ & $\begin{array}{r}\text { dev } \\
\frac{8}{8}\end{array}$ & wt \\
\hline 233.810 & 6.475 & 6.503 & 0.43 & 16.169 & 16.167 & -0.01 & 0.0 \\
\hline 243.990 & 14.130 & 14.667 & 3.80 & 16.162 & 16.133 & -0.18 & 0.0 \\
\hline 262.070 & 28.125 & 28.877 & 2.67 & 16.149 & 16.114 & -0.22 & 0.0 \\
\hline 227.550 & 1.971 & 2.288 & 16.10 & 16.225 & 16.206 & -0.12 & 0.0 \\
\hline 238.960 & 11.111 & 11.553 & 3.98 & 16.215 & 16.191 & -0.15 & 0.0 \\
\hline 255.290 & 24.252 & 24.583 & 1.37 & 16.202 & 16.186 & -0.10 & 0.0 \\
\hline 267.050 & 33.549 & 33.857 & 0.92 & 16.196 & 16.182 & -0.08 & 0.0 \\
\hline 212.910 & 3.550 & 4.040 & 13.81 & 17.010 & 16.988 & -0.13 & 0.0 \\
\hline 217.690 & 8.444 & 8.614 & 2.01 & 17.004 & 16.996 & -0.04 & 0.0 \\
\hline 228.700 & 18.809 & 19.203 & 2.09 & 16.997 & 16.981 & -0.09 & 0.0 \\
\hline 239.980 & 29.400 & 29.865 & 1.58 & 16.990 & 16.973 & -0.10 & 0.0 \\
\hline 254.750 & 42.698 & 43.420 & 1.69 & 16.977 & 16.952 & -0.15 & 0.0 \\
\hline 270.210 & 56.994 & 57.307 & 0.55 & 16.964 & 16.954 & -0.06 & 0.0 \\
\hline 205.610 & 4.335 & 4.937 & 13.89 & 17.369 & 17.345 & -0.14 & 0.0 \\
\hline 213.510 & 12.660 & 13.198 & 4.25 & 17.363 & 17.342 & -0.12 & 0.0 \\
\hline 222.780 & 21.781 & 22.767 & 4.53 & 17.356 & 17.321 & -0.20 & 0.0 \\
\hline 234.050 & 33.500 & 34.235 & 2.20 & 17.350 & 17.325 & -0.14 & 0.0 \\
\hline 241.440 & 41.188 & 41.570 & 0.93 & 17.343 & 17.331 & -0.07 & 0.0 \\
\hline 242.410 & 42.071 & 42.554 & 1.15 & 17.343 & 17.328 & -0.09 & 0.0 \\
\hline 250.920 & 50.955 & 50.911 & -0.09 & 17.336 & 17.338 & 0.01 & 0.0 \\
\hline
\end{tabular}


Table 6. PVT DATA (continued)

Data from Golovskiy et al. [51] (continued)

\begin{tabular}{|c|c|c|c|c|c|c|c|}
\hline $\begin{array}{l}\mathrm{T} \\
\mathrm{K}\end{array}$ & $\begin{array}{l}\mathrm{P}, \text { expt } \\
\mathrm{MPa}\end{array}$ & $\begin{array}{c}\mathrm{P}, \underset{\mathrm{MPa}}{\mathrm{calc}} \\
\text { a }\end{array}$ & $\begin{array}{c}\mathrm{dev} \\
\frac{\gamma}{8}\end{array}$ & $\begin{array}{l}\rho, \operatorname{expt} \\
\mathrm{mol} \cdot \cdot \mathrm{dm}^{-3}\end{array}$ & $\begin{array}{l}\rho, \quad \text { calc } \\
\mathrm{mol} \cdot \mathrm{dm}^{-3}\end{array}$ & $\begin{array}{r}\text { dev } \\
8\end{array}$ & wt \\
\hline 259.660 & 59.566 & 59.273 & -0.49 & 17.326 & 17.335 & 0.05 & 0.0 \\
\hline 195.790 & 3.815 & 4.573 & 19.87 & 17.772 & 17.745 & -0.15 & 0.0 \\
\hline 201.830 & 11.258 & 11.552 & 2.61 & 17.769 & 17.759 & -0.06 & 0.0 \\
\hline 209.250 & 19.594 & 19.931 & 1.72 & 17.762 & 17.751 & -0.06 & 0.0 \\
\hline 218.220 & 29.400 & 29.938 & 1.83 & 17.755 & 17.739 & -0.09 & 0.0 \\
\hline 227.930 & 40.080 & 40.600 & 1.30 & 17.749 & 17.733 & -0.09 & 0.0 \\
\hline 235.940 & 48.974 & 49.218 & 0.50 & 17.742 & 17.735 & -0.04 & 0.0 \\
\hline 245.390 & 59.222 & 59.155 & -0.11 & 17.732 & 17.734 & 0.01 & 0.0 \\
\hline 187.590 & 4.207 & 4.000 & -4.91 & 18.091 & 18.098 & 0.04 & 0.0 \\
\hline 195.040 & 13.278 & 13.163 & -0.87 & 18.084 & 18.088 & 0.02 & 0.0 \\
\hline 203.780 & 24.115 & 23.754 & -1.50 & 18.078 & 18.088 & 0.06 & 0.0 \\
\hline 217.990 & 40.982 & 40.596 & -0.94 & 18.068 & 18.078 & 0.06 & 0.0 \\
\hline 224.830 & 48.837 & 48.469 & -0.75 & 18.061 & 18.071 & 0.05 & 0.0 \\
\hline 233.610 & 58.899 & 58.503 & -0.67 & 18.055 & 18.064 & 0.05 & 0.0 \\
\hline 180.920 & 2.726 & 2.872 & 5.34 & 18.327 & 18.323 & -0.02 & 0.0 \\
\hline 187.490 & 11.346 & 11.394 & 0.42 & 18.321 & 18.319 & -0.01 & 0.0 \\
\hline 194.010 & 19.809 & 19.832 & 0.11 & 18.317 & 18.317 & 0.00 & 0.0 \\
\hline 202.280 & 30.146 & 30.270 & 0.41 & 18.311 & 18.307 & -0.02 & 0.0 \\
\hline 211.470 & 41.874 & 41.682 & -0.46 & 18.304 & 18.309 & 0.03 & 0.0 \\
\hline 216.960 & 48.602 & 48.289 & -0.64 & 18.297 & 18.305 & 0.04 & 0.0 \\
\hline 225.970 & 59.507 & 59.152 & -0.60 & 18.291 & 18.299 & 0.05 & 0.0 \\
\hline 172.850 & 5.168 & 5.323 & 3.00 & 18.716 & 18.712 & -0.02 & 0.0 \\
\hline 180.260 & 15.946 & 15.803 & -0.90 & 18.710 & 18.713 & 0.02 & 0.0 \\
\hline 187.640 & 25.968 & 26.037 & 0.27 & 18.703 & 18.701 & -0.01 & 0.0 \\
\hline 194.120 & 35.059 & 34.975 & -0.24 & 18.700 & 18.702 & 0.01 & 0.0 \\
\hline 200.730 & 43.914 & 43.818 & -0.22 & 18.693 & 18.695 & 0.01 & 0.0 \\
\hline 207.050 & 52.318 & 52.286 & -0.06 & 18.690 & 18.690 & 0.00 & 0.0 \\
\hline 212.300 & 59.213 & 59.215 & 0.00 & 18.686 & 18.686 & 0.00 & 0.0 \\
\hline 162.110 & 1.932 & 3.104 & 60.66 & 19.075 & 19.047 & -0.15 & 0.0 \\
\hline 167.580 & 10.650 & 11.635 & 9.25 & 19.072 & 19.049 & -0.12 & 0.0 \\
\hline 173.440 & 19.731 & 20.492 & 3.86 & 19.066 & 19.048 & -0.09 & 0.0 \\
\hline 179.530 & 29.165 & 29.702 & 1.84 & 19.062 & 19.050 & -0.06 & 0.0 \\
\hline 186.040 & 38.883 & 39.245 & 0.93 & 19.056 & 19.048 & -0.04 & 0.0 \\
\hline 192.640 & 48.366 & 48.924 & 1.15 & 19.052 & 19.041 & -0.06 & 0.0 \\
\hline 200.210 & 59.291 & 59.710 & 0.71 & 19.046 & 19.037 & -0.04 & 0.0 \\
\hline
\end{tabular}


Table 6. PVT DATA (continued)

Data from Golovskiy et al. [51] (continued)

\begin{tabular}{|c|c|c|c|c|c|c|c|}
\hline $\begin{array}{l}\mathrm{T} \\
\mathrm{K}\end{array}$ & $\begin{array}{l}\mathrm{P}, \mathrm{MPa}_{\mathrm{MPp}} \\
\text { ext }\end{array}$ & $\begin{array}{c}\mathrm{P}, \mathrm{calc} \\
\mathrm{MPa}\end{array}$ & $\begin{array}{c}\mathrm{dev} \\
8\end{array}$ & $\begin{array}{l}\rho, \operatorname{expt} \\
\mathrm{mol} \cdot \mathrm{dm}^{-3}\end{array}$ & $\begin{array}{l}\rho, \quad \text { calc } \\
\mathrm{mol} \cdot \mathrm{dm}^{-3}\end{array}$ & $\begin{array}{r}\mathrm{dev} \\
8\end{array}$ & wt \\
\hline 154.070 & 2.285 & 2.896 & 26.73 & 19.378 & 19.365 & -0.07 & 0.0 \\
\hline 160.390 & 10.856 & 13.341 & 22.89 & 19.371 & 19.318 & -0.28 & 0.0 \\
\hline 167.320 & 23.281 & 24.745 & 6.29 & 19.368 & 19.338 & -0.16 & 0.0 \\
\hline 172.200 & 31.754 & 32.427 & 2.12 & 19.361 & 19.348 & -0.07 & 0.0 \\
\hline 178.490 & 41.943 & 42.433 & 1.17 & 19.358 & 19.349 & -0.05 & 0.0 \\
\hline 184.890 & 52.064 & 52.278 & 0.41 & 19.352 & 19.347 & -0.02 & 0.0 \\
\hline 190.050 & 59.821 & 60.388 & 0.95 & 19.352 & 19.341 & -0.05 & 0.0 \\
\hline 144.570 & 1.922 & 3.636 & 89.19 & 19.751 & 19.716 & -0.17 & 0.0 \\
\hline 149.480 & 11.278 & 12.614 & 11.85 & 19.747 & 19.721 & -0.13 & 0.0 \\
\hline 155.380 & 22.104 & 23.241 & 5.14 & 19.744 & 19.723 & -0.11 & 0.0 \\
\hline 160.360 & 30.822 & 32.028 & 3.91 & 19.741 & 19.719 & -0.11 & 0.0 \\
\hline 165.540 & 39.854 & 41.034 & 2.96 & 19.737 & 19.716 & -0.11 & 0.0 \\
\hline 171.550 & 50.102 & 51.153 & 2.10 & 19.731 & 19.712 & -0.09 & 0.0 \\
\hline 176.280 & 58.340 & 59.105 & 1.31 & 19.727 & 19.714 & -0.07 & 0.0 \\
\hline 131.860 & 3.393 & 4.583 & 35.07 & 20.236 & 20.216 & -0.10 & 0.0 \\
\hline 135.790 & 11.680 & 12.646 & 8.27 & 20.233 & 20.216 & -0.08 & 0.0 \\
\hline 140.510 & 20.937 & 22.209 & 6.08 & 20.229 & 20.208 & -0.10 & 0.0 \\
\hline 145.550 & 30.871 & 32.252 & 4.47 & 20.226 & 20.204 & -0.11 & 0.0 \\
\hline 150.360 & 40.197 & 41.662 & 3.64 & 20.223 & 20.200 & -0.11 & 0.0 \\
\hline 155.360 & 49.788 & 51.292 & 3.02 & 20.219 & 20.196 & -0.12 & 0.0 \\
\hline 160.510 & 59.311 & 60.838 & 2.57 & 20.213 & 20.190 & -0.11 & 0.0 \\
\hline 121.300 & 4.227 & 6.239 & 47.59 & 20.645 & 20.614 & -0.15 & 0.0 \\
\hline 125.740 & 14.573 & 16.334 & 12.09 & 20.642 & 20.615 & -0.13 & 0.0 \\
\hline 129.620 & 23.301 & 25.204 & 8.17 & 20.642 & 20.614 & -0.14 & 0.0 \\
\hline 132.670 & 29.881 & 31.857 & 6.61 & 20.639 & 20.610 & -0.14 & 0.0 \\
\hline 136.070 & 37.363 & 39.202 & 4.92 & 20.635 & 20.609 & -0.13 & 0.0 \\
\hline 140.820 & 47.464 & 49.397 & 4.07 & 20.632 & 20.605 & -0.13 & 0.0 \\
\hline 146.370 & 59.173 & 61.126 & 3.30 & 20.629 & 20.602 & -0.13 & 0.0 \\
\hline 111.570 & 5.168 & 7.010 & 35.65 & 21.004 & 20.979 & -0.12 & 0.0 \\
\hline 114.920 & 13.376 & 15.308 & 14.44 & 21.001 & 20.974 & -0.13 & 0.0 \\
\hline 119.800 & 25.340 & 27.300 & 7.73 & 20.998 & 20.971 & -0.13 & 0.0 \\
\hline 122.930 & 33.264 & 34.774 & 4.54 & 20.994 & 20.974 & -0.10 & 0.0 \\
\hline 126.260 & 40.031 & 42.889 & 7.14 & 20.994 & 20.957 & -0.18 & 0.0 \\
\hline 129.570 & 48.739 & 50.593 & 3.80 & 20.991 & 20.967 & -0.11 & 0.0 \\
\hline 134.760 & 60.409 & 62.614 & 3.65 & 20.988 & 20.960 & -0.13 & 0.0 \\
\hline
\end{tabular}


Table 6. PVT DATA (continued)

Data from Golovskiy et al. [51] (continued)

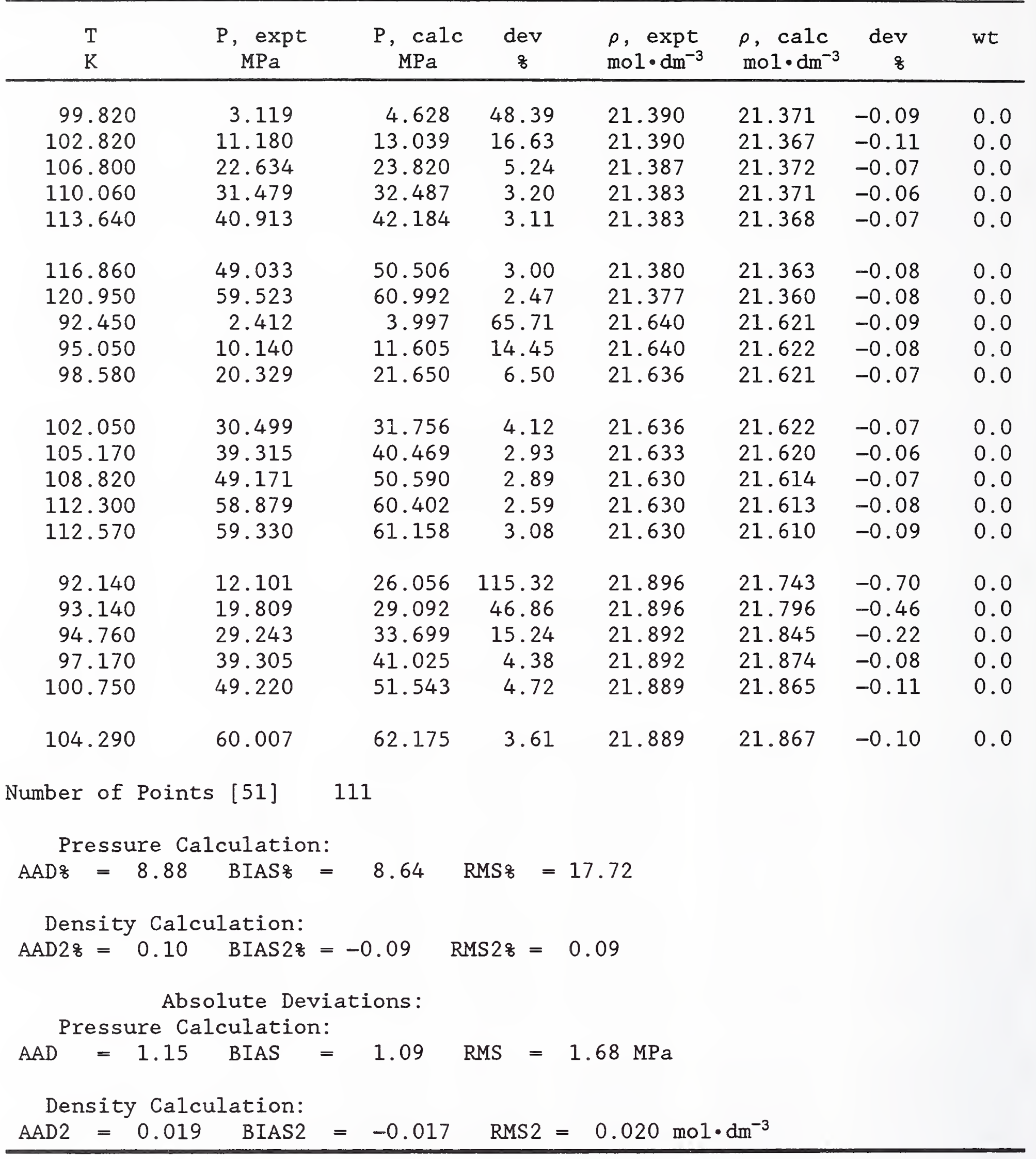


Table 6. PVT DATA (continued)

Data from Khazanova and Sominskaya [53]

\begin{tabular}{|c|c|c|c|c|c|c|c|}
\hline $\begin{array}{l}\mathrm{T} \\
\mathrm{K}\end{array}$ & $\mathrm{P}, \mathrm{MPa}_{\mathrm{MPp}}$ & $\begin{array}{c}\mathrm{P}, \underset{\mathrm{MPa}}{\text { calc }} \\
\text { cal }\end{array}$ & $\begin{array}{c}\mathrm{dev} \\
\frac{8}{8}\end{array}$ & $\begin{array}{l}\rho, \operatorname{expt} \\
\mathrm{mol} \cdot \mathrm{dm}^{-3}\end{array}$ & $\begin{array}{l}\rho, \quad \mathrm{calc} \\
\mathrm{mol} \cdot \mathrm{dm}^{-3}\end{array}$ & $\begin{array}{r}\text { dev } \\
8\end{array}$ & wt \\
\hline 299.300 & 5.467 & 5.441 & -0.49 & 11.326 & 11.341 & 0.13 & 0.0 \\
\hline 302.800 & 6.381 & 6.378 & -0.05 & 11.326 & 11.328 & 0.02 & 0.0 \\
\hline 305.390 & 7.082 & 7.077 & -0.06 & 11.326 & 11.328 & 0.02 & 0.0 \\
\hline 305.610 & 6.730 & 6.517 & -3.16 & 11.006 & 11.125 & 1.09 & 0.0 \\
\hline 303.390 & 6.144 & 5.964 & -2.94 & 11.006 & 11.116 & 1.00 & 0.0 \\
\hline 307.100 & 7.112 & 6.891 & -3.11 & 11.006 & 11.124 & 1.07 & 0.0 \\
\hline 309.360 & 7.434 & 7.460 & 0.35 & 11.006 & 10.992 & -0.12 & 0.0 \\
\hline 306.120 & 6.424 & 6.261 & -2.54 & 10.761 & 10.871 & 1.02 & 0.0 \\
\hline 308.520 & 7.004 & 6.829 & -2.50 & 10.761 & 10.869 & 1.00 & 0.0 \\
\hline 304.720 & 6.084 & 5.932 & -2.49 & 10.761 & 10.869 & 1.01 & 0.0 \\
\hline 303.430 & 5.774 & 5.631 & -2.47 & 10.761 & 10.870 & 1.01 & 0.0 \\
\hline 305.920 & 5.723 & 5.631 & -1.60 & 10.241 & 10.341 & 0.98 & 0.0 \\
\hline 303.240 & 5.150 & 5.083 & -1.31 & 10.241 & 10.329 & 0.86 & 0.0 \\
\hline 306.900 & 5.928 & 5.834 & -1.58 & 10.241 & 10.338 & 0.95 & 0.0 \\
\hline 308.520 & 6.273 & 6.171 & -1.63 & 10.241 & 10.338 & 0.95 & 0.0 \\
\hline 305.350 & 5.239 & 4.998 & -4.60 & 9.211 & 9.841 & 6.85 & 0.0 \\
\hline 303.250 & 4.842 & 4.670 & -3.55 & 9.211 & 9.817 & 6.59 & 0.0 \\
\hline 305.820 & 3.121 & 3.085 & -1.15 & 1.642 & 1.670 & 1.71 & 0.0 \\
\hline 304.520 & 3.098 & 3.062 & -1.16 & 1.642 & 1.671 & 1.74 & 0.0 \\
\hline 303.420 & 3.077 & 3.042 & -1.15 & 1.642 & 1.671 & 1.74 & 0.0 \\
\hline 308.820 & 3.176 & 3.139 & -1.19 & 1.642 & 1.671 & 1.75 & 0.0 \\
\hline 306.220 & 1.847 & 1.984 & 7.39 & 0.920 & 0.845 & -8.17 & 0.0 \\
\hline 303.970 & 1.829 & 1.963 & 7.36 & 0.920 & 0.845 & -8.16 & 0.0 \\
\hline 303.160 & 1.823 & 1.956 & 7.32 & 0.920 & 0.845 & -8.13 & 0.0 \\
\hline 308.320 & 1.868 & 2.003 & 7.19 & 0.920 & 0.847 & -7.93 & 0.0 \\
\hline 308.230 & 0.998 & 1.082 & 8.41 & 0.458 & 0.419 & -8.39 & 0.0 \\
\hline 305.560 & 0.989 & 1.071 & 8.30 & 0.458 & 0.420 & -8.30 & 0.0 \\
\hline 303.170 & 0.980 & 1.061 & 8.30 & 0.458 & 0.419 & -8.31 & 0.0 \\
\hline 303.600 & 0.982 & 1.063 & 8.26 & 0.458 & 0.420 & -8.27 & 0.0 \\
\hline 307.120 & 5.570 & 5.513 & -1.02 & 9.749 & 9.843 & 0.96 & 0.0 \\
\hline 308.220 & 5.774 & 5.715 & -1.03 & 9.749 & 9.840 & 0.93 & 0.0 \\
\hline 308.230 & 5.488 & 5.457 & -0.56 & 9.209 & 9.291 & 0.90 & 0.0 \\
\hline 306.990 & 5.288 & 5.258 & -0.57 & 9.209 & 9.303 & 1.02 & 0.0 \\
\hline 305.240 & 5.014 & 4.980 & -0.67 & 9.209 & 9.338 & 1.40 & 0.0 \\
\hline 306.290 & 4.935 & 4.961 & 0.51 & 6.106 & 5.320 & -12.87 & 0.0 \\
\hline
\end{tabular}


Table 6. PVT DATA (continued)

Data from Khazanova and Sominskaya [53] (continued)

\begin{tabular}{|c|c|c|c|c|c|c|c|}
\hline $\begin{array}{l}\mathrm{T} \\
\mathrm{K}\end{array}$ & $\begin{array}{l}\mathrm{P}, \operatorname{MPa}_{\mathrm{MPp}} \\
\text { exp }\end{array}$ & $\begin{array}{c}\mathrm{P}, \mathrm{MPa} \\
\mathrm{MPa}\end{array}$ & $\begin{array}{c}\mathrm{dev} \\
8\end{array}$ & $\begin{array}{l}\rho, \operatorname{expt} \\
\mathrm{mol} 1 \cdot \mathrm{dm}^{-3}\end{array}$ & $\begin{array}{l}\rho, \quad \text { calc } \\
\mathrm{mol} \cdot \mathrm{dm}^{-3}\end{array}$ & $\begin{array}{r}\text { dev } \\
\frac{8}{8}\end{array}$ & wt \\
\hline 309.320 & 5.234 & 5.243 & 0.16 & 6.106 & 5.982 & -2.03 & 0.0 \\
\hline 312.650 & 5.564 & 5.551 & -0.24 & 6.106 & 6.218 & 1.85 & 0.0 \\
\hline 316.970 & 5.997 & 5.948 & -0.83 & 6.106 & 6.366 & 4.26 & 0.0 \\
\hline 305.790 & 4.865 & 4.900 & 0.74 & 5.452 & 4.862 & -10.82 & 0.0 \\
\hline 309.380 & 5.167 & 5.197 & 0.59 & 5.452 & 5.173 & -5.13 & 0.0 \\
\hline 313.620 & 5.505 & 5.542 & 0.67 & 5.452 & 5.246 & -3.78 & 0.0 \\
\hline 316.360 & 5.720 & 5.762 & 0.75 & 5.452 & 5.264 & -3.46 & 0.0 \\
\hline 308.590 & 5.102 & 5.132 & 0.59 & 5.452 & 5.137 & -5.79 & 0.0 \\
\hline 305.730 & 4.744 & 4.763 & 0.41 & 4.190 & 4.101 & -2.11 & 0.0 \\
\hline 308.560 & 4.908 & 4.933 & 0.50 & 4.190 & 4.099 & -2.18 & 0.0 \\
\hline 308.820 & 2.530 & 2.495 & -1.37 & 1.204 & 1.226 & 1.79 & 0.0 \\
\hline 304.920 & 2.481 & 2.447 & -1.37 & 1.204 & 1.226 & 1.81 & 0.0 \\
\hline 306.320 & 2.500 & 2.465 & -1.40 & 1.204 & 1.226 & 1.85 & 0.0 \\
\hline 303.470 & 2.464 & 2.429 & -1.41 & 1.204 & 1.227 & 1.87 & 0.0 \\
\hline 308.250 & 1.723 & 1.697 & -1.51 & 0.757 & 0.770 & 1.78 & 0.0 \\
\hline 304.890 & 1.699 & 1.672 & -1.58 & 0.757 & 0.771 & 1.88 & 0.0 \\
\hline 303.370 & 1.688 & 1.661 & -1.59 & 0.757 & 0.771 & 1.88 & 0.0 \\
\hline 306.280 & 1.690 & 1.682 & -0.46 & 0.757 & 0.761 & 0.54 & 0.0 \\
\hline 303.560 & 0.487 & 0.537 & 10.12 & 0.221 & 0.200 & -9.54 & 0.0 \\
\hline 303.260 & 0.487 & 0.536 & 10.00 & 0.221 & 0.200 & -9.44 & 0.0 \\
\hline 305.710 & 0.492 & 0.541 & 9.84 & 0.221 & 0.201 & -9.29 & 0.0 \\
\hline 308.760 & 0.499 & 0.547 & 9.67 & 0.221 & 0.201 & -9.14 & 0.0 \\
\hline 316.070 & 5.345 & 5.378 & 0.63 & 4.202 & 4.118 & -1.98 & 0.0 \\
\hline 312.520 & 5.141 & 5.171 & 0.57 & 4.202 & 4.115 & -2.07 & 0.0 \\
\hline 312.890 & 4.438 & 4.494 & 1.26 & 2.866 & 2.794 & -2.53 & 0.0 \\
\hline 306.840 & 4.229 & 4.275 & 1.09 & 2.866 & 2.798 & -2.40 & 0.0 \\
\hline 309.770 & 4.333 & 4.382 & 1.13 & 2.866 & 2.798 & -2.38 & 0.0 \\
\hline 318.010 & 4.353 & 4.676 & 7.42 & 2.866 & 2.512 & -12.37 & 0.0 \\
\hline 303.020 & 4.311 & 4.287 & -0.56 & 3.153 & 3.205 & 1.64 & 0.0 \\
\hline 304.570 & 4.377 & 4.352 & -0.57 & 3.153 & 3.204 & 1.62 & 0.0 \\
\hline 306.250 & 4.449 & 4.422 & -0.61 & 3.153 & 3.206 & 1.67 & 0.0 \\
\hline 308.470 & 4.540 & 4.513 & -0.59 & 3.153 & 3.201 & 1.53 & 0.0 \\
\hline 308.500 & 3.891 & 3.855 & -0.92 & 2.278 & 2.316 & 1.66 & 0.0 \\
\hline 303.140 & 3.740 & 3.710 & -0.81 & 2.278 & 2.312 & 1.52 & 0.0 \\
\hline 304.840 & 3.790 & 3.756 & -0.89 & 2.278 & 2.315 & 1.65 & 0.0 \\
\hline
\end{tabular}


Table 6. PVT DATA (continued)

Data from Khazanova and Sominskaya [53] (continued)

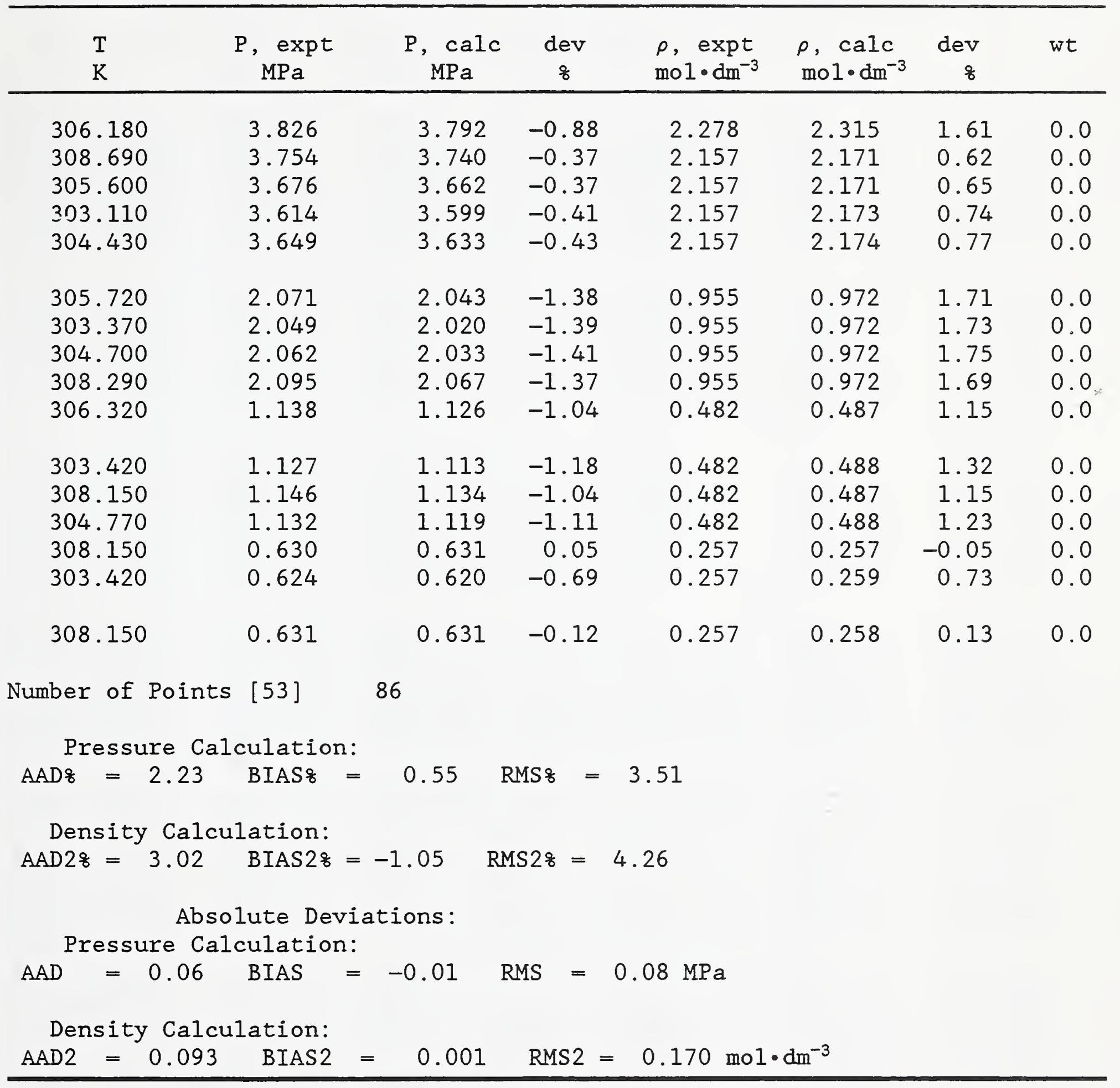


Table 6. PVT DATA (continued)

Data from Law [54]

\begin{tabular}{|c|c|c|c|c|c|c|c|}
\hline $\begin{array}{l}\mathrm{T} \\
\mathrm{K}\end{array}$ & $\begin{array}{l}\mathrm{P}, \operatorname{expt} \\
\mathrm{MPa}\end{array}$ & $\begin{array}{c}\mathrm{P}, \underset{\mathrm{MPa}}{\text { calc }} \\
\text {. }\end{array}$ & $\begin{array}{c}\mathrm{dev} \\
\frac{8}{8}\end{array}$ & $\begin{array}{l}\rho, \text { expt } \\
\mathrm{mol} \cdot \mathrm{dm}^{-3}\end{array}$ & $\begin{array}{l}\rho, \text { calc } \\
\mathrm{mol} \cdot \mathrm{dm}^{-3}\end{array}$ & $\begin{array}{r}\text { dev } \\
8\end{array}$ & wt \\
\hline 300.000 & 31.099 & 31.211 & 0.36 & 14.859 & 14.852 & -0.05 & 0.0 \\
\hline 300.000 & 33.967 & 34.092 & 0.37 & 15.033 & 15.026 & -0.05 & 0.0 \\
\hline 300.000 & 27.644 & 27.722 & 0.28 & 14.627 & 14.622 & -0.04 & 0.0 \\
\hline 300.000 & 24.190 & 24.219 & 0.12 & 14.367 & 14.364 & -0.02 & 0.0 \\
\hline 300.000 & 20.047 & 20.008 & -0.19 & 14.004 & 14.007 & 0.03 & 0.0 \\
\hline 300.000 & 15.693 & 15.583 & -0.70 & 13.533 & 13.546 & 0.10 & 0.0 \\
\hline 300.000 & 11.557 & 11.368 & -1.64 & 12.934 & 12.965 & 0.25 & 0.0 \\
\hline 300.000 & 6.808 & 6.575 & -3.42 & 11.760 & 11.847 & 0.74 & 0.0 \\
\hline 300.000 & 4.644 & 4.447 & -4.23 & 10.266 & 10.552 & 2.78 & 0.0 \\
\hline 320.000 & 33.987 & 34.136 & 0.44 & 14.309 & 14.299 & -0.07 & 0.0 \\
\hline 320.000 & 30.094 & 30.161 & 0.22 & 14.017 & 14.011 & -0.04 & 0.0 \\
\hline 320.000 & 25.064 & 25.041 & -0.09 & 13.573 & 13.575 & 0.02 & 0.0 \\
\hline 320.000 & 20.061 & 19.972 & -0.44 & 13.019 & 13.030 & 0.09 & 0.0 \\
\hline 320.000 & 15.058 & 14.913 & -0.96 & 12.250 & 12.277 & 0.22 & 0.0 \\
\hline 320.000 & 9.331 & 9.161 & -1.82 & 10.552 & 10.638 & 0.81 & 0.0 \\
\hline 320.000 & 7.418 & 7.260 & -2.13 & 8.985 & 9.197 & 2.37 & 0.0 \\
\hline 320.000 & 6.493 & 6.392 & -1.55 & 6.782 & 7.170 & 5.72 & 0.0 \\
\hline 320.000 & 6.010 & 5.944 & -1.10 & 5.086 & 5.301 & 4.22 & 0.0 \\
\hline 320.000 & 5.504 & 5.456 & -0.86 & 3.900 & 3.991 & 2.34 & 0.0 \\
\hline 280.000 & 34.309 & 34.644 & 0.98 & 15.776 & 15.760 & -0.10 & 0.0 \\
\hline 280.000 & 29.588 & 29.915 & 1.11 & 15.534 & 15.516 & -0.11 & 0.0 \\
\hline 280.000 & 24.994 & 25.347 & 1.41 & 15.272 & 15.250 & -0.14 & 0.0 \\
\hline 280.000 & 19.648 & 19.922 & 1.40 & 14.912 & 14.892 & -0.13 & 0.0 \\
\hline 280.000 & 14.435 & 14.653 & 1.51 & 14.487 & 14.467 & -0.14 & 0.0 \\
\hline 280.000 & 10.192 & 10.313 & 1.19 & 14.044 & 14.030 & -0.10 & 0.0 \\
\hline 280.000 & 7.078 & 7.128 & 0.71 & 13.625 & 13.617 & -0.06 & 0.0 \\
\hline 280.000 & 4.292 & 4.239 & -1.24 & 13.105 & 13.116 & 0.09 & 0.0 \\
\hline 260.000 & 34.311 & 34.484 & 0.50 & 16.468 & 16.461 & -0.04 & 0.0 \\
\hline 260.000 & 29.843 & 30.045 & 0.68 & 16.278 & 16.269 & -0.06 & 0.0 \\
\hline 260.000 & 24.822 & 25.063 & 0.97 & 16.044 & 16.032 & -0.08 & 0.0 \\
\hline 260.000 & 19.982 & 20.259 & 1.39 & 15.790 & 15.775 & -0.10 & 0.0 \\
\hline 260.000 & 14.978 & 15.257 & 1.86 & 15.488 & 15.470 & -0.12 & 0.0 \\
\hline 260.000 & 10.165 & 10.429 & 2.59 & 15.144 & 15.124 & -0.14 & 0.0 \\
\hline 260.000 & 4.967 & 5.156 & 3.80 & 14.673 & 14.653 & -0.13 & 0.0 \\
\hline 260.000 & 2.023 & 2.120 & 4.78 & 14.321 & 14.309 & -0.09 & 0.0 \\
\hline
\end{tabular}


Table 6. PVT DATA (continued)

Data from Law [54] (continued)

\begin{tabular}{|c|c|c|c|c|c|c|c|}
\hline $\begin{array}{l}\mathrm{T} \\
\mathrm{K}\end{array}$ & $\mathrm{P}, \underset{\mathrm{MPa}}{\exp t}$ & $\underset{\mathrm{MPa}}{\mathrm{P}, \mathrm{calc}}$ & $\begin{array}{c}\text { dev } \\
\frac{8}{8}\end{array}$ & $\begin{array}{l}\rho, \operatorname{expt} \\
\mathrm{mol} \cdot \mathrm{dm}^{-3}\end{array}$ & $\begin{array}{l}\rho, \quad \mathrm{calc} \\
\mathrm{mol} \cdot \mathrm{dm}^{-3}\end{array}$ & $\begin{array}{r}\text { dev } \\
q\end{array}$ & wt \\
\hline 240.000 & 34.314 & 34.603 & 0.84 & 17.160 & 17.150 & -0.06 & 0.0 \\
\hline 240.000 & 29.617 & 29.822 & 0.69 & 16.988 & 16.980 & -0.05 & 0.0 \\
\hline 240.000 & 24.939 & 25.178 & 0.96 & 16.808 & 16.798 & -0.06 & 0.0 \\
\hline 240.000 & 20.007 & 20.354 & 1.74 & 16.603 & 16.588 & -0.09 & 0.0 \\
\hline 240.000 & 14.825 & 15.238 & 2.78 & 16.362 & 16.341 & -0.13 & 0.0 \\
\hline 240.000 & 9.974 & 10.342 & 3.69 & 16.101 & 16.080 & -0.13 & 0.0 \\
\hline 240.000 & 5.027 & 5. 391 & 7.25 & 15.794 & 15.770 & -0.16 & 0.0 \\
\hline 240.000 & 2.026 & 2.370 & 16.98 & 15.578 & 15.551 & -0.17 & 0.0 \\
\hline 240.000 & 1.137 & 1.452 & 27.72 & 15.506 & 15.480 & -0.16 & 0.0 \\
\hline 350.000 & 34.311 & 34.302 & -0.03 & 13.220 & 13.221 & 0.01 & 0.0 \\
\hline 350.000 & 30.076 & 30.086 & 0.03 & 12.819 & 12.818 & -0.01 & 0.0 \\
\hline 350.000 & 25.349 & 25.329 & -0.08 & 12.265 & 12.268 & 0.02 & 0.0 \\
\hline 350.000 & 19.830 & 19.775 & -0.28 & 11.381 & 11.392 & 0.10 & 0.0 \\
\hline 350.000 & 15.694 & 15.638 & -0.35 & 10.371 & 10.388 & 0.17 & 0.0 \\
\hline 350.000 & 12.930 & 12.881 & -0.38 & 9.293 & 9.317 & 0.26 & 0.0 \\
\hline 350.000 & 10.860 & 10.810 & -0.46 & 7.977 & 8.017 & 0.50 & 0.0 \\
\hline 350.000 & 9.482 & 9.437 & -0.47 & 6.654 & 6.703 & 0.75 & 0.0 \\
\hline 350.000 & 8.381 & 8.344 & -0.44 & 5.374 & 5.418 & 0.81 & 0.0 \\
\hline 350.000 & 7.275 & 7.251 & -0.34 & 4.160 & 4.186 & 0.61 & 0.0 \\
\hline 350.000 & 6.034 & 6.009 & -0.41 & 3.043 & 3.063 & 0.64 & 0.0 \\
\hline 350.000 & 4.655 & 4.630 & -0.53 & 2.086 & 2.101 & 0.73 & 0.0 \\
\hline
\end{tabular}

Number of Points [54] 56

Pressure Calculation:

$\mathrm{AAD} ;=2.03$ BIAS $8=1.16$ RMS $8=4.57$

Density Calculation:

$\mathrm{AAD} 2 \%=0.49 \quad$ BIAS $2 \%=0.38 \quad$ RMS $2 \%=1.08$

Absolute Deviations:

Pressure Calculation:

$\mathrm{AAD}=0.16$ BIAS $=0.09$ RMS $=0.18 \mathrm{MPa}$

Density Calculation:

AAD2 $=0.038$ BIAS2 $=0.023 \quad$ RMS2 $=0.077 \mathrm{~mol} \cdot \mathrm{dm}^{-3}$ 
Table 6. PVT DATA (continued)

Data from Michels et al. [47]

\begin{tabular}{|c|c|c|c|c|c|c|c|}
\hline $\begin{array}{l}\mathrm{T} \\
\mathrm{K}\end{array}$ & $\begin{array}{c}\mathrm{P}, \operatorname{expt} \\
\mathrm{MPa}\end{array}$ & $\begin{array}{c}\mathrm{P}, \underset{\mathrm{MPa}}{\mathrm{calc}} \\
\text { cal }\end{array}$ & $\begin{array}{c}\mathrm{dev} \\
\frac{8}{8}\end{array}$ & $\begin{array}{l}\rho, \operatorname{expt} \\
\mathrm{mol} \cdot \mathrm{dm}^{-3}\end{array}$ & $\begin{array}{l}\rho, \text { calc } \\
\mathrm{mol} \cdot \mathrm{dm}^{-3}\end{array}$ & $\begin{array}{r}\mathrm{dev} \\
8\end{array}$ & wt \\
\hline 273.150 & 1.587 & 1.586 & -0.09 & 0.854 & 0.855 & 0.12 & 46.2 \\
\hline 298.142 & 1.798 & 1.797 & -0.04 & 0.854 & 0.854 & 0.04 & 39.9 \\
\hline 323.140 & 2.004 & 2.003 & -0.02 & 0.854 & 0.854 & 0.02 & 35.2 \\
\hline 348.143 & 2.206 & 2.206 & -0.01 & 0.854 & 0.854 & 0.02 & 31.6 \\
\hline 373.150 & 2.407 & 2.407 & 0.01 & 0.854 & 0.854 & -0.01 & 28.7 \\
\hline 398.160 & 2.605 & 2.606 & 0.02 & 0.854 & 0.854 & -0.02 & 26.3 \\
\hline 423.170 & 2.803 & 2.804 & 0.02 & 0.854 & 0.854 & -0.02 & 24.3 \\
\hline 273.150 & 1.880 & 1.878 & -0.12 & 1.067 & 1.069 & 0.16 & 40.2 \\
\hline 298.142 & 2.154 & 2.153 & -0.04 & 1.067 & 1.068 & 0.05 & 34.0 \\
\hline 323.140 & 2.420 & 2.419 & -0.03 & 1.067 & 1.068 & 0.03 & 29.7 \\
\hline 348.143 & 2.681 & 2.681 & -0.02 & 1.067 & 1.067 & 0.02 & 26.4 \\
\hline 373.150 & 2.939 & 2.938 & 0.00 & 1.067 & 1.067 & 0.00 & 23.8 \\
\hline 398.160 & 3.193 & 3.194 & 0.02 & 1.067 & 1.067 & -0.02 & 21.7 \\
\hline 423.170 & 3.447 & 3.447 & 0.01 & 1.067 & 1.067 & -0.01 & 19.9 \\
\hline 273.150 & 2.135 & 2.132 & -0.15 & 1.281 & 1.284 & 0.23 & 36.5 \\
\hline 298.142 & 2.477 & 2.475 & -0.06 & 1.281 & 1.282 & 0.08 & 30.3 \\
\hline 323.140 & 2.807 & 2.806 & -0.03 & 1.281 & 1.282 & 0.04 & 26.0 \\
\hline 348.143 & 3.129 & 3.128 & -0.03 & 1.281 & 1.282 & 0.03 & 22.9 \\
\hline 373.150 & 3.446 & 3.446 & 0.00 & 1.281 & 1.281 & 0.00 & 20.5 \\
\hline 398.160 & 3.760 & 3.760 & 0.01 & 1.281 & 1.281 & -0.02 & 18.6 \\
\hline 423.170 & 4.071 & 4.072 & 0.02 & 1.281 & 1.281 & -0.02 & 17.0 \\
\hline 273.150 & 2.344 & 2.340 & -0.17 & 1.487 & 1.491 & 0.28 & 34.3 \\
\hline 298.142 & 2.756 & 2.755 & -0.06 & 1.487 & 1.488 & 0.08 & 27.8 \\
\hline 323.140 & 3.151 & 3.150 & -0.04 & 1.487 & 1.488 & 0.05 & 23.6 \\
\hline 348.143 & 3.535 & 3.534 & -0.03 & 1.487 & 1.488 & 0.04 & 20.5 \\
\hline 373.150 & 3.913 & 3.912 & -0.01 & 1.487 & 1.487 & 0.01 & 18.2 \\
\hline 398.160 & 4.285 & 4.285 & 0.02 & 1.487 & 1.487 & -0.02 & 16.4 \\
\hline 423.170 & 4.654 & 4.655 & 0.02 & 1.487 & 1.487 & -0.02 & 15.0 \\
\hline 298.142 & 2.940 & 2.938 & -0.05 & 1.635 & 1.637 & 0.08 & 26.5 \\
\hline 323.140 & 3.383 & 3.382 & -0.04 & 1.635 & 1.636 & 0.05 & 22.2 \\
\hline 348.143 & 3.814 & 3.813 & -0.04 & 1.635 & 1.636 & 0.05 & 19.2 \\
\hline 373.150 & 4.236 & 4.235 & -0.01 & 1.635 & 1.636 & 0.01 & 17.0 \\
\hline 398.160 & 4.652 & 4.653 & 0.01 & 1.635 & 1.635 & -0.01 & 15.2 \\
\hline 423.170 & 5.065 & 5.065 & 0.02 & 1.635 & 1.635 & -0.02 & 13.8 \\
\hline 298.142 & 3.019 & 3.017 & -0.06 & 1.703 & 1.705 & 0.09 & 26.0 \\
\hline
\end{tabular}


Table 6. PVT DATA (continued)

Data from Michels et al. [47] (continued)

\begin{tabular}{|c|c|c|c|c|c|c|c|}
\hline $\begin{array}{l}\mathrm{T} \\
\mathrm{K}\end{array}$ & $\begin{array}{c}\mathrm{P}, \operatorname{MPa}_{\mathrm{MPa}} \\
\text { expt }\end{array}$ & $\begin{array}{c}\mathrm{P}, \mathrm{calc} \\
\mathrm{MPa}\end{array}$ & $\begin{array}{c}\text { dev } \\
8\end{array}$ & $\begin{array}{l}\rho, \operatorname{expt} \\
\mathrm{mol} \cdot \mathrm{dm}^{-3}\end{array}$ & $\begin{array}{l}\rho, \text { calc } \\
\mathrm{mol} \cdot \mathrm{dm}^{-3}\end{array}$ & $\begin{array}{r}\mathrm{dev} \\
\frac{8}{8}\end{array}$ & wt \\
\hline 323.140 & 3.485 & 3.483 & -0.04 & 1.703 & 1.704 & 0.05 & 21.7 \\
\hline 348.143 & 3.937 & 3.936 & -0.04 & 1.703 & 1.704 & 0.05 & 18.7 \\
\hline 373.150 & 4.380 & 4.379 & -0.01 & 1.703 & 1.703 & 0.01 & 16.5 \\
\hline 398.160 & 4.816 & 4.817 & 0.02 & 1.703 & 1.703 & -0.03 & 14.7 \\
\hline 423.170 & 5.249 & 5.250 & 0.02 & 1.703 & 1.703 & -0.02 & 13.3 \\
\hline 298.142 & 3.248 & 3.246 & -0.06 & 1.916 & 1.918 & 0.10 & 24.7 \\
\hline 323.140 & 3.787 & 3.786 & -0.03 & 1.916 & 1.917 & 0.05 & 20.3 \\
\hline 348.143 & 4.309 & 4.308 & -0.03 & 1.916 & 1.917 & 0.04 & 17.3 \\
\hline 373.150 & 4.819 & 4.819 & 0.00 & 1.916 & 1.916 & 0.00 & 15.1 \\
\hline 398.160 & 5.321 & 5.323 & 0.03 & 1.916 & 1.916 & -0.03 & 13.4 \\
\hline 423.170 & 5.819 & 5.821 & 0.03 & 1.916 & 1.916 & -0.03 & 12.1 \\
\hline 298.142 & 3.306 & 3.305 & -0.04 & 1.975 & 1.977 & 0.07 & 24.4 \\
\hline 323.140 & 3.866 & 3.865 & -0.03 & 1.975 & 1.976 & 0.05 & 20.0 \\
\hline 348.143 & 4.408 & 4.407 & -0.03 & 1.975 & 1.976 & 0.04 & 17.0 \\
\hline 373.150 & 4.938 & 4.937 & -0.02 & 1.975 & 1.976 & 0.02 & 14.8 \\
\hline 398.160 & 5.458 & 5.459 & 0.02 & 1.975 & 1.975 & -0.02 & 13.1 \\
\hline 423.170 & 5.974 & 5.975 & 0.03 & 1.975 & 1.975 & -0.03 & 11.8 \\
\hline 298.142 & 3.443 & 3.441 & -0.05 & 2.122 & 2.124 & 0.10 & 23.8 \\
\hline 323.140 & 4.056 & 4.054 & -0.03 & 2.122 & 2.123 & 0.05 & 19.2 \\
\hline 348.143 & 4.647 & 4.646 & -0.03 & 2.122 & 2.123 & 0.04 & 16.2 \\
\hline 373.150 & 5.224 & 5.224 & 0.00 & 2.122 & 2.122 & 0.00 & 14.1 \\
\hline 398.160 & 5.791 & 5.793 & 0.03 & 2.122 & 2.121 & -0.03 & 12.4 \\
\hline 423.170 & 6.354 & 6.355 & 0.03 & 2.122 & 2.121 & -0.03 & 11.1 \\
\hline 298.142 & 3.618 & 3.616 & -0.05 & 2.334 & 2.336 & 0.10 & 23.1 \\
\hline 323.140 & 4.310 & 4.309 & -0.03 & 2.334 & 2.335 & 0.05 & 18.4 \\
\hline 348.143 & 4.976 & 4.974 & -0.03 & 2.334 & 2.335 & 0.04 & 15.3 \\
\hline 373.150 & 5.624 & 5.624 & 0.00 & 2.334 & 2.334 & 0.00 & 13.2 \\
\hline 398.160 & 6.261 & 6.263 & 0.03 & 2.334 & 2.333 & -0.04 & 11.6 \\
\hline 423.170 & 6.892 & 6.895 & 0.04 & 2.334 & 2.333 & -0.05 & 10.3 \\
\hline 298.142 & 3.673 & 3.672 & -0.03 & 2.408 & 2.410 & 0.07 & 22.9 \\
\hline 323.140 & 4.394 & 4.393 & -0.02 & 2.408 & 2.409 & 0.03 & 18.1 \\
\hline 348.143 & 5.086 & 5.085 & -0.02 & 2.408 & 2.409 & 0.03 & 15.0 \\
\hline 373.150 & 5.760 & 5.760 & 0.00 & 2.408 & 2.408 & 0.00 & 12.9 \\
\hline 398.160 & 6.422 & 6.425 & 0.04 & 2.408 & 2.407 & -0.05 & 11.3 \\
\hline 423.170 & 7.078 & 7.081 & 0.05 & 2.408 & 2.407 & -0.06 & 10.1 \\
\hline
\end{tabular}


Table 6. PVT DATA (continued)

Data from Michels et al. [47] (continued)

\begin{tabular}{|c|c|c|c|c|c|c|c|}
\hline $\begin{array}{l}\mathrm{T} \\
\mathrm{K}\end{array}$ & $\begin{array}{l}\mathrm{P}, \text { expt } \\
\mathrm{MPa}\end{array}$ & $\begin{array}{c}\mathrm{P}, \mathrm{MPa} \\
\mathrm{Malc}\end{array}$ & $\begin{array}{c}\text { dev } \\
8\end{array}$ & $\begin{array}{l}\rho, \operatorname{expt} \\
\mathrm{mol} \cdot \mathrm{dm}^{-3}\end{array}$ & $\begin{array}{l}\rho, \quad \text { calc } \\
\mathrm{mol} \cdot \mathrm{dm}^{-3}\end{array}$ & $\begin{array}{r}\text { dev } \\
8\end{array}$ & wt \\
\hline 298.142 & 3.990 & 3.989 & -0.02 & 2.939 & 2.941 & 0.05 & 22.1 \\
\hline 323.140 & 4.923 & 4.923 & 0.00 & 2.939 & 2.940 & 0.01 & 16.7 \\
\hline 348.143 & 5.813 & 5.811 & -0.02 & 2.939 & 2.940 & 0.03 & 13.5 \\
\hline 373.150 & 6.677 & 6.678 & 0.01 & 2.939 & 2.939 & -0.02 & 11.3 \\
\hline 398.160 & 7.525 & 7.529 & 0.05 & 2.939 & 2.937 & -0.06 & 9.8 \\
\hline 423.170 & 8.364 & 8.369 & 0.07 & 2.939 & 2.937 & -0.08 & 8.6 \\
\hline 323.140 & 5.420 & 5.421 & 0.00 & 3.582 & 3.582 & -0.01 & 15.7 \\
\hline 348.143 & 6.565 & 6.564 & -0.01 & 3.582 & 3.583 & 0.02 & 12.2 \\
\hline 373.150 & 7.677 & 7.678 & 0.02 & 3.582 & 3.581 & -0.02 & 10.0 \\
\hline 398.160 & 8.768 & 8.774 & 0.06 & 3.582 & 3.579 & -0.08 & 8.4 \\
\hline 423.170 & 9.848 & 9.856 & 0.08 & 3.582 & 3.579 & -0.10 & 7.3 \\
\hline 323.140 & 5.897 & 5.898 & 0.01 & 4.429 & 4.428 & -0.02 & 0.0 \\
\hline 348.143 & 7.400 & 7.398 & -0.02 & 4.429 & 4.431 & 0.04 & 0.0 \\
\hline 373.150 & 8.866 & 8.866 & 0.01 & 4.429 & 4.428 & -0.02 & 0.0 \\
\hline 398.160 & 10.310 & 10.315 & 0.05 & 4.429 & 4.426 & -0.07 & 0.0 \\
\hline 423.170 & 11.740 & 11.748 & 0.07 & 4.429 & 4.425 & -0.08 & 0.0 \\
\hline 323.140 & 6.300 & 6.299 & -0.01 & 5.440 & 5.442 & 0.03 & 0.0 \\
\hline 348.143 & 8.260 & 8.255 & -0.05 & 5.440 & 5.445 & 0.09 & 0.0 \\
\hline 373.150 & 10.193 & 10.191 & -0.03 & 5.440 & 5.442 & 0.04 & 0.0 \\
\hline 398.160 & 12.110 & 12.112 & 0.02 & 5.440 & 5.439 & -0.02 & 0.0 \\
\hline 423.170 & 14.017 & 14.020 & 0.03 & 5.440 & 5.439 & -0.03 & 0.0 \\
\hline 323.140 & 6.691 & 6.689 & -0.03 & 6.682 & 6.689 & 0.11 & 0.0 \\
\hline 348.143 & 9.279 & 9.270 & -0.10 & 6.682 & 6.693 & 0.16 & 0.0 \\
\hline 373.150 & 11.877 & 11.868 & -0.07 & 6.682 & 6.688 & 0.09 & 0.0 \\
\hline 398.160 & 14.473 & 14.469 & -0.03 & 6.682 & 6.684 & 0.03 & 0.0 \\
\hline 423.170 & 17.071 & 17.065 & -0.04 & 6.682 & 6.684 & 0.03 & 0.0 \\
\hline 323.140 & 7.278 & 7.272 & -0.09 & 8.202 & 8.216 & 0.16 & 0.0 \\
\hline 348.143 & 10.835 & 10.831 & -0.03 & 8.202 & 8.205 & 0.03 & 0.0 \\
\hline 373.150 & 14.460 & 14.456 & -0.03 & 8.202 & 8.205 & 0.03 & 0.0 \\
\hline 398.160 & 18.110 & 18.103 & -0.04 & 8.202 & 8.205 & 0.03 & 0.0 \\
\hline 423.170 & 21.766 & 21.756 & -0.04 & 8.202 & 8.205 & 0.03 & 0.0 \\
\hline
\end{tabular}


Table 6. PVT DATA (continued)

Data from Michels et al. [47] (continued)

Number of Points [47] 101

Pressure Calculation:

$\mathrm{AAD} \%=0.03 \quad$ BIAS $8=-0.01 \quad \mathrm{RMS} \%=0.04$

Density Calculation:

$\mathrm{AAD} 2 \%=0.05 \quad$ BIAS $2 \%=0.02 \quad \mathrm{RMS} 2 \%=0.06$

Absolute Deviations:

Pressure Calculation:

$\mathrm{AAD}=0.00$ BIAS $=0.00 \quad \mathrm{RMS}=0.00 \mathrm{MPa}$

Density Calculation:

$\mathrm{AAD} 2=0.001$ BIAS2 $=0.001 \quad \mathrm{RMS} 2=0.002 \mathrm{~mol} \cdot \mathrm{dm}^{-3}$

Weighted Data:

Number of Points [47] 81

Pressure Calculation:

$\mathrm{AAD}$ \% $=0.03$ BIAS\& $=-0.01$ RMS $8=0.04$

Density Calculation:

$\mathrm{AAD} 28=0.84 \quad$ BIAS28 $=0.02 \quad$ RMS $2 \%=0.06$

Absolute Deviations:

Pressure Calculation:

$\mathrm{AAD}=0.00$ BIAS $=0.00 \quad \mathrm{RMS}=0.00 \mathrm{MPa}$

Density Calculation:

AAD2 $=0.001$ BIAS2 $=0.000 \quad \operatorname{RMS} 2=0.001 \mathrm{~mol} \cdot \mathrm{dm}^{-3}$

Data from Miniovich and Sorina [12]

\begin{tabular}{ccccccrr}
\hline $\mathrm{T}$ & $\mathrm{P}, \operatorname{expt}$ & $\mathrm{P}, \mathrm{calc}$ \\
$\mathrm{MPa}$ & $\mathrm{MPa}$ & $\begin{array}{c}\mathrm{dev} \\
\text { s }\end{array}$ & $\begin{array}{c}\rho, \operatorname{expt} \\
\mathrm{mol} \cdot \mathrm{dm}^{-3}\end{array}$ & $\begin{array}{c}\rho, \mathrm{calc} \\
\mathrm{mol} \cdot \mathrm{dm}^{-3}\end{array}$ & $\begin{array}{r}\mathrm{dev} \\
8\end{array}$ & wt \\
\hline 303.036 & 4.648 & 4.647 & -0.02 & 9.271 & 9.278 & 0.07 & 0.0 \\
303.530 & 4.724 & 4.725 & 0.02 & 9.271 & 9.267 & -0.05 & 0.0 \\
304.072 & 4.810 & 4.810 & 0.00 & 9.271 & 9.272 & 0.01 & 0.0 \\
304.525 & 4.881 & 4.882 & 0.01 & 9.271 & 9.270 & -0.02 & 0.0 \\
303.887 & 4.728 & 4.726 & -0.05 & 8.894 & 8.917 & 0.27 & 0.0
\end{tabular}


Table 6. PVT DATA (continued)

Data from Miniovich and Sorina [12] (continued)

\begin{tabular}{|c|c|c|c|c|c|c|c|}
\hline $\begin{array}{l}\mathrm{T} \\
\mathrm{K}\end{array}$ & $\begin{array}{l}\mathrm{P}, \operatorname{MPa}_{\mathrm{MPp}} \\
\text { exp }\end{array}$ & $\underset{\mathrm{MPa}}{\mathrm{P}, \mathrm{calc}^{-}}$ & $\begin{array}{c}\text { dev } \\
8\end{array}$ & $\begin{array}{l}\rho, \text { expt } \\
\mathrm{mol} \cdot \mathrm{dm}^{-3}\end{array}$ & $\begin{array}{l}\rho, \quad \operatorname{calc} \\
\mathrm{mol} \cdot \mathrm{dm}^{-3}\end{array}$ & $\begin{array}{r}\text { dev } \\
8\end{array}$ & wt \\
\hline 303.922 & 4.734 & 4.731 & -0.06 & 8.894 & 8.925 & 0.35 & 0.0 \\
\hline 304.340 & 4.792 & 4.791 & -0.03 & 8.894 & 8.907 & 0.15 & 0.0 \\
\hline 304.820 & 4.863 & 4.860 & -0.05 & 8.894 & 8.913 & 0.21 & 0.0 \\
\hline 305.102 & 4.904 & 4.901 & -0.05 & 8.894 & 8.911 & 0.19 & 0.0 \\
\hline 304.595 & 4.798 & 4.797 & -0.03 & 8.488 & 8.524 & 0.42 & 0.0 \\
\hline 304.705 & 4.813 & 4.811 & -0.05 & 8.488 & 8.534 & 0.54 & 0.0 \\
\hline 305.090 & 4.864 & 4.862 & -0.02 & 8.488 & 8.508 & 0.23 & 0.0 \\
\hline 305.550 & 4.924 & 4.924 & -0.01 & 8.488 & 8.496 & 0.10 & 0.0 \\
\hline 305.060 & 4.847 & 4.844 & -0.05 & 8.052 & 8.180 & 1.59 & 0.0 \\
\hline 305.272 & 4.871 & 4.870 & -0.02 & 8.052 & 8.096 & 0.55 & 0.0 \\
\hline 305.575 & 4.907 & 4.907 & 0.00 & 8.052 & 8.050 & -0.02 & 0.0 \\
\hline 305.272 & 4.868 & 4.866 & -0.03 & 7.623 & 7.872 & 3.26 & 0.0 \\
\hline 305.470 & 4.889 & 4.889 & -0.01 & 7.623 & 7.711 & 1.15 & 0.0 \\
\hline 305.672 & 4.911 & 4.912 & 0.03 & 7.623 & 7.481 & -1.86 & 0.0 \\
\hline 305.345 & 4.875 & 4.874 & -0.02 & 7.257 & 7.685 & 5.90 & 0.0 \\
\hline 305.645 & 4.906 & 4.906 & 0.00 & 7.257 & 7.217 & -0.55 & 0.0 \\
\hline 305.960 & 4.940 & 4.941 & 0.02 & 7.257 & 7.142 & -1.59 & 0.0 \\
\hline 305.360 & 4.876 & 4.875 & -0.02 & 6.998 & 7.604 & 8.65 & 0.0 \\
\hline 305.657 & 4.906 & 4.906 & 0.00 & 6.998 & 7.026 & 0.39 & 0.0 \\
\hline 305.990 & 4.941 & 4.942 & 0.02 & 6.998 & 6.906 & -1.32 & 0.0 \\
\hline 305.560 & 4.896 & 4.896 & -0.01 & 6.844 & 6.960 & 1.69 & 0.0 \\
\hline 305.910 & 4.931 & 4.932 & 0.01 & 6.844 & 6.759 & -1.24 & 0.0 \\
\hline 306.020 & 4.943 & 4.943 & 0.02 & 6.844 & 6.761 & -1.22 & 0.0 \\
\hline 305.360 & 4.876 & 4.875 & -0.02 & 6.702 & 7.555 & 12.74 & 0.0 \\
\hline 305.672 & 4.907 & 4.907 & -0.01 & 6.702 & 6.852 & 2.25 & 0.0 \\
\hline 306.043 & 4.945 & 4.944 & -0.02 & 6.702 & 6.786 & 1.26 & 0.0 \\
\hline 305.380 & 4.878 & 4.877 & -0.03 & 6.505 & 7.537 & 15.85 & 0.0 \\
\hline 305.690 & 4.908 & 4.907 & -0.02 & 6.505 & 6.671 & 2.55 & 0.0 \\
\hline 306.078 & 4.947 & 4.946 & -0.02 & 6.505 & 6.597 & 1.41 & 0.0 \\
\hline 305.335 & 4.872 & 4.872 & 0.00 & 6.317 & 6.260 & -0.90 & 0.0 \\
\hline 305.722 & 4.909 & 4.909 & 0.01 & 6.317 & 6.274 & -0.68 & 0.0 \\
\hline 306.110 & 4.947 & 4.947 & 0.00 & 6.317 & 6.326 & 0.15 & 0.0 \\
\hline 305.280 & 4.867 & 4.866 & -0.01 & 6.059 & 6.194 & 2.22 & 0.0 \\
\hline 305.752 & 4.911 & 4.910 & -0.02 & 6.059 & 6.130 & 1.18 & 0.0 \\
\hline 306.135 & 4.947 & 4.946 & -0.02 & 6.059 & 6.120 & 1.01 & 0.0 \\
\hline
\end{tabular}


Table 6. PVT DATA (continued)

Data from Miniovich and Sorina [12] (continued)

\begin{tabular}{|c|c|c|c|c|c|c|c|}
\hline $\begin{array}{l}\mathrm{T} \\
\mathrm{K}\end{array}$ & $\begin{array}{l}\mathrm{P}, \operatorname{MPa}_{\mathrm{MPp}} \\
\text { exp }\end{array}$ & $\begin{array}{c}\mathrm{P}, \mathrm{calc} \\
\mathrm{MPa}\end{array}$ & $\begin{array}{c}\mathrm{dev} \\
8\end{array}$ & $\begin{array}{l}\rho, \operatorname{expt} \\
\mathrm{mol} \cdot \mathrm{dm}^{-3}\end{array}$ & $\begin{array}{l}\rho, \text { calc } \\
\mathrm{mol} \cdot \mathrm{dm}^{-3}\end{array}$ & $\begin{array}{r}\mathrm{dev} \\
\frac{8}{8}\end{array}$ & wt \\
\hline 305.045 & 4.842 & 4.842 & -0.02 & 5.653 & 5.716 & 1.13 & 0.0 \\
\hline 305.760 & 4.904 & 4.904 & -0.01 & 5.653 & 5.665 & 0.22 & 0.0 \\
\hline 306.188 & 4.942 & 4.941 & -0.02 & 5.653 & 5.691 & 0.68 & 0.0 \\
\hline 304.640 & 4.801 & 4.800 & -0.01 & 5.277 & 5.287 & 0.19 & 0.0 \\
\hline 304.660 & 4.803 & 4.802 & -0.02 & 5.277 & 5.309 & 0.59 & 0.0 \\
\hline 305.340 & 4.859 & 4.857 & -0.04 & 5.277 & 5.322 & 0.84 & 0.0 \\
\hline 306.025 & 4.917 & 4.912 & -0.11 & 5.277 & 5.387 & 2.08 & 0.0 \\
\hline 303.945 & 4.731 & 4.730 & -0.01 & 4.873 & 4.883 & 0.20 & 0.0 \\
\hline 304.001 & 4.736 & 4.734 & -0.03 & 4.873 & 4.900 & 0.55 & 0.0 \\
\hline 304.537 & 4.775 & 4.774 & -0.03 & 4.873 & 4.893 & 0.40 & 0.0 \\
\hline 305.287 & 4.830 & 4.829 & -0.03 & 4.873 & 4.891 & 0.36 & 0.0 \\
\hline 305.960 & 4.880 & 4.878 & -0.04 & 4.873 & 4.892 & 0.39 & 0.0 \\
\hline 306.620 & 4.928 & 4.926 & -0.04 & 4.873 & 4.892 & 0.39 & 0.0 \\
\hline 302.910 & 4.630 & 4.625 & -0.11 & 4.455 & 4.506 & 1.15 & 0.0 \\
\hline 303.380 & 4.660 & 4.656 & -0.07 & 4.455 & 4.487 & 0.72 & 0.0 \\
\hline 304.035 & 4.703 & 4.700 & -0.07 & 4.455 & 4.482 & 0.61 & 0.0 \\
\hline 304.599 & 4.739 & 4.737 & -0.04 & 4.455 & 4.471 & 0.36 & 0.0 \\
\hline 305.000 & 4.766 & 4.763 & -0.05 & 4.455 & 4.471 & 0.36 & 0.0 \\
\hline 305.440 & 4.795 & 4.792 & -0.06 & 4.455 & 4.475 & 0.45 & 0.0 \\
\hline 306.074 & 4.836 & 4.833 & -0.05 & 4.455 & 4.469 & 0.31 & 0.0 \\
\hline 306.522 & 4.865 & 4.863 & -0.04 & 4.455 & 4.467 & 0.27 & 0.0 \\
\hline 307.025 & 4.898 & 4.895 & -0.05 & 4.455 & 4.469 & 0.32 & 0.0 \\
\hline 307.475 & 4.928 & 4.925 & -0.06 & 4.455 & 4.472 & 0.39 & 0.0 \\
\hline
\end{tabular}

Number of Points [12]

63

Pressure Calculation:

$\mathrm{AAD} \%=0.03 \quad \mathrm{BIAS} \%=-0.03 \quad \mathrm{RMS} \%=0.03$

Density Calculation:

$\mathrm{AAD} 2 \%=1.41 \quad$ BIAS2 $8=1.11 \quad$ RMS $2 \%=2.85$

Absolute Deviations:

Pressure Calculation:

$\mathrm{AAD}=0.00 \mathrm{BIAS}=0.00 \quad \mathrm{RMS}=0.00 \mathrm{MPa}$

Density Calculation:

AAD2 $=0.093$ BIAS2 $=0.072 \quad$ RMS2 $=0.191 \mathrm{~mol} \cdot \mathrm{dm}^{-3}$ 
Table 6. PVT DATA (continued)

Data from Pal et al. [13]

Data slightly adjusted after personal communication with author.

\begin{tabular}{|c|c|c|c|c|c|c|c|}
\hline $\begin{array}{l}\mathrm{T} \\
\mathrm{K}\end{array}$ & $P,{ }_{\mathrm{MPa}}^{\exp t}$ & $\begin{array}{c}\mathrm{P}, \underset{\mathrm{MPa}}{\text { calc }} \\
\text {. }\end{array}$ & $\begin{array}{c}\text { dev } \\
8\end{array}$ & $\begin{array}{l}\rho, \operatorname{expt} \\
\mathrm{mol} \cdot \mathrm{dm}^{-3}\end{array}$ & $\begin{array}{l}\rho, \quad \mathrm{calc} \\
\mathrm{mol} \cdot \mathrm{dm}^{-3}\end{array}$ & $\begin{array}{r}\text { dev } \\
8\end{array}$ & wt \\
\hline 263.847 & 1.799 & 1.816 & 0.92 & 1.106 & 1.091 & -1.33 & 0.0 \\
\hline 267.267 & 1.846 & 1.857 & 0.59 & 1.106 & 1.096 & -0.84 & 0.0 \\
\hline 270.457 & 1.885 & 1.894 & 0.50 & 1.105 & 1.098 & -0.70 & 0.0 \\
\hline 273.094 & 1.916 & 1.925 & 0.47 & 1.105 & 1.098 & -0.65 & 0.0 \\
\hline 276.946 & 1.962 & 1.970 & 0.40 & 1.105 & 1.099 & -0.55 & 0.0 \\
\hline 281.362 & 2.014 & 2.021 & 0.34 & 1.105 & 1.100 & -0.45 & 0.0 \\
\hline 288.868 & 2.101 & 2.107 & 0.28 & 1.104 & 1.100 & -0.37 & 0.0 \\
\hline 298.954 & 2.214 & 2.220 & 0.26 & 1.104 & 1.100 & -0.33 & 0.0 \\
\hline 306.232 & 2.295 & 2.300 & 0.22 & 1.103 & 1.100 & -0.28 & 0.0 \\
\hline 315.928 & 2.402 & 2.406 & 0.20 & 1.103 & 1.100 & -0.25 & 0.0 \\
\hline 326.185 & 2.514 & 2.518 & 0.17 & 1.102 & 1.100 & -0.20 & 0.0 \\
\hline 332.655 & 2.584 & 2.587 & 0.15 & 1.102 & 1.100 & -0.18 & 0.0 \\
\hline 343.612 & 2.701 & 2.705 & 0.13 & 1.101 & 1.099 & -0.16 & 0.0 \\
\hline 290.936 & 3.548 & 3.559 & 0.32 & 2.597 & 2.575 & -0.85 & 0.0 \\
\hline 292.700 & 3.614 & 3.619 & 0.14 & 2.596 & 2.587 & -0.35 & 0.0 \\
\hline 294.967 & 3.691 & 3.695 & 0.11 & 2.596 & 2.589 & -0.26 & 0.0 \\
\hline 297.293 & 3.768 & 3.772 & 0.10 & 2.596 & 2.589 & -0.24 & 0.0 \\
\hline 299.402 & 3.838 & 3.841 & 0.07 & 2.595 & 2.591 & -0.17 & 0.0 \\
\hline 300.717 & 3.880 & 3.884 & 0.11 & 2.595 & 2.589 & -0.24 & 0.0 \\
\hline 306.169 & 4.055 & 4.059 & 0.09 & 2.594 & 2.589 & -0.19 & 0.0 \\
\hline 311.528 & 4.226 & 4.229 & 0.09 & 2.594 & 2.589 & -0.16 & 0.0 \\
\hline 317.621 & 4.416 & 4.419 & 0.08 & 2.593 & 2.589 & -0.14 & 0.0 \\
\hline 325.244 & 4.651 & 4.654 & 0.08 & 2.592 & 2.588 & -0.13 & 0.0 \\
\hline 333.158 & 4.891 & 4.895 & 0.08 & 2.590 & 2.587 & -0.12 & 0.0 \\
\hline 343.446 & 5.199 & 5.203 & 0.08 & 2.589 & 2.586 & -0.13 & 0.0 \\
\hline 305.270 & 4.786 & 4.800 & 0.28 & 4.594 & 4.492 & -2.22 & 0.0 \\
\hline 306.575 & 4.875 & 4.888 & 0.27 & 4.594 & 4.507 & -1.89 & 0.0 \\
\hline 306.998 & 4.904 & 4.917 & 0.26 & 4.594 & 4.515 & -1.72 & 0.0 \\
\hline 308.355 & 4.993 & 5.008 & 0.29 & 4.594 & 4.516 & -1.70 & 0.0 \\
\hline 309.475 & 5.067 & 5.083 & 0.31 & 4.593 & 4.518 & -1.65 & 0.0 \\
\hline 310.226 & 5.117 & 5.133 & 0.31 & 4.593 & 4.521 & -1.57 & 0.0 \\
\hline 311.461 & 5.197 & 5.214 & 0.33 & 4.593 & 4.522 & -1.54 & 0.0 \\
\hline 314.528 & 5.396 & 5.416 & 0.37 & 4.592 & 4.525 & -1.46 & 0.0 \\
\hline 316.957 & 5.552 & 5.574 & 0.40 & 4.591 & 4.527 & -1.41 & 0.0 \\
\hline 326.208 & 6.137 & 6.166 & 0.48 & 4.589 & 4.530 & -1.27 & 0.0 \\
\hline
\end{tabular}


Table 6. PVT DATA (continued)

Data from Pal et al. [13] (continued)

\begin{tabular}{|c|c|c|c|c|c|c|c|}
\hline $\begin{array}{l}\mathrm{T} \\
\mathrm{K}\end{array}$ & $\begin{array}{l}\mathrm{P}, \operatorname{expt} \\
\mathrm{MPa}\end{array}$ & $\begin{array}{c}\mathrm{P}, \underset{\mathrm{MPa}}{\mathrm{calc}} \\
\text { cal }\end{array}$ & $\begin{array}{c}\mathrm{dev} \\
\frac{8}{8}\end{array}$ & $\begin{array}{l}\rho, \operatorname{expt} \\
\mathrm{mol} \cdot \mathrm{dm}^{-3}\end{array}$ & $\begin{array}{l}\rho, \quad \text { calc } \\
\mathrm{mol} \cdot \mathrm{dm}^{-3}\end{array}$ & $\begin{array}{r}\text { dev } \\
8\end{array}$ & wt \\
\hline 329.914 & 6.365 & 6.401 & 0.57 & 4.588 & 4.524 & -1.39 & 0.0 \\
\hline$\because 33.690$ & 6.622 & 6.638 & 0.24 & 4.587 & 4.561 & -0.56 & 0.0 \\
\hline 305.232 & 4.830 & 4.851 & 0.43 & 5.359 & 4.945 & -7.74 & 0.0 \\
\hline 306.165 & 4.917 & 4.927 & 0.22 & 5.359 & 5.171 & -3.52 & 0.0 \\
\hline 307.343 & 5.014 & 5.023 & 0.17 & 5.359 & 5.242 & -2.18 & 0.0 \\
\hline 308.378 & 5.097 & 5.107 & 0.19 & 5.359 & 5.254 & -1.95 & 0.0 \\
\hline 310.753 & 5.289 & 5.297 & 0.15 & 5.358 & 5.295 & -1.17 & 0.0 \\
\hline 315.523 & 5.666 & 5.675 & 0.16 & 5.356 & 5.314 & -0.80 & 0.0 \\
\hline 320.133 & 6.025 & 6.036 & 0.18 & 5.355 & 5.318 & -0.70 & 0.0 \\
\hline 324.789 & 6.385 & 6.397 & 0.19 & 5.354 & 5.321 & -0.61 & 0.0 \\
\hline 329.529 & 6.749 & 6.762 & 0.19 & 5.352 & 5.324 & -0.53 & 0.0 \\
\hline 334.774 & 7.150 & 7.163 & 0.19 & 5.351 & 5.327 & -0.45 & 0.0 \\
\hline 342.584 & 7.745 & 7.758 & 0.17 & 5.348 & 5.330 & -0.35 & 0.0 \\
\hline 305.380 & 4.868 & 4.876 & 0.16 & 6.137 & 5.527 & -9.93 & 0.0 \\
\hline 305.932 & 4.920 & 4.928 & 0.15 & 6.137 & 5.711 & -6.94 & 0.0 \\
\hline 306.528 & 4.976 & 4.984 & 0.15 & 6.137 & 5.829 & -5.02 & 0.0 \\
\hline 307.927 & 5.109 & 5.115 & 0.12 & 6.136 & 6.001 & -2.21 & 0.0 \\
\hline 309.803 & 5.285 & 5.290 & 0.10 & 6.135 & 6.066 & -1.13 & 0.0 \\
\hline 314.618 & 5.733 & 5.736 & 0.05 & 6.134 & 6.114 & -0.32 & 0.0 \\
\hline 320.295 & 6.255 & 6.259 & 0.06 & 6.132 & 6.117 & -0.25 & 0.0 \\
\hline 325.427 & 6.724 & 6.730 & 0.08 & 6.130 & 6.115 & -0.25 & 0.0 \\
\hline 330.799 & 7.215 & 7.221 & 0.08 & 6.128 & 6.115 & -0.21 & 0.0 \\
\hline 336.699 & 7.755 & 7.760 & 0.07 & 6.126 & 6.117 & -0.15 & 0.0 \\
\hline 343.543 & 8.378 & 8.385 & 0.08 & 6.124 & 6.114 & -0.15 & 0.0 \\
\hline 305.423 & 4.879 & 4.881 & 0.05 & 6.789 & 6.006 & -11.54 & 0.0 \\
\hline 305.743 & 4.903 & 4.914 & 0.22 & 6.789 & 5.699 & -16.05 & 0.0 \\
\hline 306.184 & 4.946 & 4.960 & 0.28 & 6.789 & 5.862 & -13.65 & 0.0 \\
\hline 306.693 & 4.998 & 5.012 & 0.29 & 6.788 & 6.082 & -10.41 & 0.0 \\
\hline 307.188 & 5.050 & 5.063 & 0.26 & 6.788 & 6.284 & -7.44 & 0.0 \\
\hline 309.162 & 5.258 & 5.267 & 0.18 & 6.787 & 6.617 & -2.52 & 0.0 \\
\hline 312.231 & 5.567 & 5.585 & 0.32 & 6.787 & 6.616 & -2.51 & 0.0 \\
\hline 317.552 & 6.119 & 6.137 & 0.30 & 6.785 & 6.693 & -1.35 & 0.0 \\
\hline 322.347 & 6.620 & 6.637 & 0.26 & 6.783 & 6.724 & -0.86 & 0.0 \\
\hline 327.148 & 7.123 & 7.140 & 0.23 & 6.781 & 6.739 & -0.62 & 0.0 \\
\hline 333.270 & 7.768 & 7.782 & 0.19 & 6.778 & 6.751 & -0.40 & 0.0 \\
\hline
\end{tabular}


Table 6. PVT DATA (continued)

Data from Pal et al. [13] (continued)

\begin{tabular}{|c|c|c|c|c|c|c|c|}
\hline $\begin{array}{l}\mathrm{T} \\
\mathrm{K}\end{array}$ & $P$, expt & $\begin{array}{c}\mathrm{P}, \underset{\mathrm{MPa}}{\mathrm{calc}} \\
\text { cal }\end{array}$ & $\begin{array}{c}\mathrm{dev} \\
8\end{array}$ & $\begin{array}{l}\rho, \text { expt } \\
\mathrm{mol} \cdot \mathrm{dm}^{-3}\end{array}$ & $\begin{array}{l}\rho, \text { calc } \\
\mathrm{mol} \cdot \mathrm{dm}^{-3}\end{array}$ & $\begin{array}{r}\text { dev } \\
\&\end{array}$ & wt \\
\hline 343.351 & 8.827 & 8.843 & 0.19 & 6.775 & 6.753 & -0.32 & 0.0 \\
\hline 305.633 & 4.899 & 4.916 & 0.35 & 8.102 & 6.081 & -24.94 & 0.0 \\
\hline 306.319 & 4.982 & 5.002 & 0.39 & 8.102 & 7.371 & -9.03 & 0.0 \\
\hline 307.031 & 5.081 & 5.091 & 0.20 & 8.101 & 7.916 & -2.29 & 0.0 \\
\hline 308.123 & 5.216 & 5.229 & 0.25 & 8.101 & 7.938 & -2.01 & 0.0 \\
\hline 311.913 & 5.697 & 5.717 & 0.36 & 8.099 & 7.981 & -1.46 & 0.0 \\
\hline 316.998 & 6.365 & 6.389 & 0.37 & 8.097 & 8.020 & -0.95 & 0.0 \\
\hline 322.404 & 7.090 & 7.117 & 0.38 & 8.094 & 8.035 & -0.73 & 0.0 \\
\hline 327.708 & 7.808 & 7.842 & 0.44 & 8.092 & 8.036 & -0.69 & 0.0 \\
\hline 333.451 & 8.594 & 8.634 & 0.48 & 8.089 & 8.036 & -0.65 & 0.0 \\
\hline 339.014 & 9.365 & 9.407 & 0.44 & 8.086 & 8.042 & -0.55 & 0.0 \\
\hline 343.093 & 9.933 & 9.975 & 0.43 & 8.084 & 8.045 & -0.49 & 0.0 \\
\hline 305.015 & 4.843 & 4.879 & 0.74 & 8.810 & 8.246 & -6.41 & 0.0 \\
\hline 306.220 & 5.013 & 5.052 & 0.77 & 8.810 & 8.482 & -3.73 & 0.0 \\
\hline 307.783 & 5.233 & 5.279 & 0.88 & 8.809 & 8.541 & -3.04 & 0.0 \\
\hline 309.310 & 5.478 & 5.504 & 0.47 & 8.808 & 8.699 & -1.24 & 0.0 \\
\hline 310.839 & 5.706 & 5.733 & 0.47 & 8.807 & 8.716 & -1.04 & 0.0 \\
\hline 314.681 & 6.275 & 6.316 & 0.66 & 8.805 & 8.707 & -1.12 & 0.0 \\
\hline 319.840 & 7.067 & 7.117 & 0.69 & 8.803 & 8.720 & -0.94 & 0.0 \\
\hline 325.591 & 7.972 & 8.024 & 0.66 & 8.800 & 8.733 & -0.76 & 0.0 \\
\hline 331.302 & 8.879 & 8.937 & 0.65 & 8.797 & 8.739 & -0.66 & 0.0 \\
\hline 337.309 & 9.843 & 9.904 & 0.62 & 8.794 & 8.742 & -0.58 & 0.0 \\
\hline 342.720 & 10.714 & 10.780 & 0.62 & 8.791 & 8.743 & -0.55 & 0.0 \\
\hline 303.430 & 4.803 & 4.823 & 0.40 & 9.689 & 9.635 & -0.55 & 0.0 \\
\hline 303.752 & 4.862 & 4.879 & 0.36 & 9.689 & 9.642 & -0.49 & 0.0 \\
\hline 303.962 & 4.898 & 4.916 & 0.36 & 9.689 & 9.642 & -0.48 & 0.0 \\
\hline 304.336 & 4.948 & 4.982 & 0.67 & 9.688 & 9.603 & -0.88 & 0.0 \\
\hline 305.899 & 5.227 & 5.259 & 0.62 & 9.687 & 9.618 & -0.72 & 0.0 \\
\hline 308.437 & 5.681 & 5.716 & 0.61 & 9.686 & 9.626 & -0.62 & 0.0 \\
\hline 313.081 & 6.535 & 6.569 & 0.52 & 9.683 & 9.640 & -0.45 & 0.0 \\
\hline 318.048 & 7.473 & 7.501 & 0.37 & 9.681 & 9.653 & -0.29 & 0.0 \\
\hline 322.924 & 8.395 & 8.428 & 0.39 & 9.678 & 9.651 & -0.28 & 0.0 \\
\hline 327.991 & 9.361 & 9.401 & 0.43 & 9.675 & 9.647 & -0.29 & 0.0 \\
\hline 334.413 & 10.581 & 10.643 & 0.59 & 9.671 & 9.635 & -0.38 & 0.0 \\
\hline 342.647 & 12.202 & 12.247 & 0.36 & 9.667 & 9.645 & -0.23 & 0.0 \\
\hline
\end{tabular}


Table 6. PVT DATA (continued)

Daca from $\mathrm{Pal}$ et al. [13] (continued)

\begin{tabular}{|c|c|c|c|c|c|c|c|}
\hline $\begin{array}{l}T \\
K\end{array}$ & $\begin{array}{l}\mathrm{P}, \operatorname{MPp}_{\mathrm{MP}} \\
\text { expt }\end{array}$ & $\underset{\mathrm{MPa}}{\mathrm{P}, \mathrm{calc}}$ & $\begin{array}{c}\mathrm{dev} \\
8\end{array}$ & $\begin{array}{l}\rho, \text { expt } \\
\mathrm{mol} \cdot \mathrm{dm}^{-3}\end{array}$ & $\begin{array}{l}\rho, \quad \text { calc } \\
\mathrm{mol} \cdot \mathrm{dm}^{-3}\end{array}$ & $\begin{array}{r}\mathrm{dev} \\
\%\end{array}$ & $w t$ \\
\hline 293.336 & 3.898 & 3.937 & 1.01 & 11.376 & 11.348 & -0.25 & 0.0 \\
\hline 293.674 & 3.988 & 4.026 & 0.97 & 11.376 & 11.349 & -0.24 & 0.0 \\
\hline 294.103 & 4.098 & 4.140 & 1.01 & 11.376 & 11.347 & -0.25 & 0.0 \\
\hline 294.883 & 4.304 & 4.345 & 0.97 & 11.375 & 11.347 & -0.25 & 0.0 \\
\hline 295.416 & 4.444 & 4.487 & 0.97 & 11.375 & 11.347 & -0.25 & 0.0 \\
\hline 295.792 & 4.542 & 4.586 & 0.97 & 11.375 & 11.346 & -0.25 & 0.0 \\
\hline 296.034 & 4.606 & 4.651 & 0.98 & 11.375 & 11.346 & -0.25 & 0.0 \\
\hline 298.624 & 5.295 & 5.342 & 0.90 & 11.373 & 11.346 & -0.24 & 0.0 \\
\hline 301.993 & 6.195 & 6.249 & 0.87 & 11.371 & 11.344 & -0.24 & 0.0 \\
\hline 304.995 & 7.009 & 7.063 & 0.77 & 11.369 & 11.344 & -0.22 & 0.0 \\
\hline 309.657 & 8.281 & 8.335 & 0.65 & 11.365 & 11.344 & -0.19 & 0.0 \\
\hline 314.468 & 9.599 & 9.659 & 0.62 & 11.362 & 11.341 & -0.19 & 0.0 \\
\hline 319.264 & 10.933 & 10.984 & 0.47 & 11.359 & 11.343 & -0.14 & 0.0 \\
\hline 324.667 & 12.442 & 12.484 & 0.34 & 11.355 & 11.343 & -0.11 & 0.0 \\
\hline 328.588 & 13.530 & 13.575 & 0.33 & 11.353 & 11.341 & -0.11 & 0.0 \\
\hline 335.200 & 15.371 & 15.420 & 0.32 & 11.348 & 11.336 & -0.11 & 0.0 \\
\hline 341.335 & 17.025 & 17.135 & 0.65 & 11.344 & 11.320 & -0.22 & 0.0 \\
\hline 277.504 & 2.735 & 2.752 & 0.62 & 12.989 & 12.985 & -0.03 & 0.0 \\
\hline 277.925 & 2.896 & 2.916 & 0.70 & 12.989 & 12.983 & -0.04 & 0.0 \\
\hline 278.136 & 2.968 & 2.999 & 1.04 & 12.989 & 12.981 & -0.06 & 0.0 \\
\hline 278.777 & 3.220 & 3.249 & 0.88 & 12.988 & 12.981 & -0.05 & 0.0 \\
\hline 279.387 & 3.454 & 3.487 & 0.98 & 12.988 & 12.979 & -0.06 & 0.0 \\
\hline 279.902 & 3.655 & 3.689 & 0.92 & 12.987 & 12.979 & -0.06 & 0.0 \\
\hline 282.317 & 4.572 & 4.633 & 1.35 & 12.985 & 12.971 & -0.11 & 0.0 \\
\hline 284.543 & 5.454 & 5.506 & 0.96 & 12.984 & 12.972 & -0.09 & 0.0 \\
\hline 286.727 & 6.307 & 6.363 & 0.88 & 12.982 & 12.970 & -0.09 & 0.0 \\
\hline 290.618 & 7.830 & 7.893 & 0.81 & 12.979 & 12.966 & -0.10 & 0.0 \\
\hline 297.190 & 10.410 & 10.484 & 0.72 & 12.974 & 12.961 & -0.10 & 0.0 \\
\hline 304.683 & 13.370 & 13.440 & 0.52 & 12.968 & 12.957 & -0.08 & 0.0 \\
\hline 312.625 & 16.492 & 16.575 & 0.50 & 12.962 & 12.950 & -0.09 & 0.0 \\
\hline 320.832 & 19.726 & 19.805 & 0.40 & 12.955 & 12.945 & -0.08 & 0.0 \\
\hline 329.753 & 23.259 & 23.309 & 0.21 & 12.948 & 12.942 & -0.05 & 0.0 \\
\hline 339.989 & 27.248 & 27.315 & 0.25 & 12.940 & 12.933 & -0.06 & 0.0 \\
\hline 268.389 & 2.184 & 2.304 & 5.52 & 13.716 & 13.695 & -0.15 & 0.0 \\
\hline 268.613 & 2.287 & 2.407 & 5.26 & 13.716 & 13.695 & -0.15 & 0.0 \\
\hline
\end{tabular}


Table 6. PVT DATA (continued)

Data from Pal et al. [13] (continued)

\begin{tabular}{|c|c|c|c|c|c|c|c|}
\hline $\begin{array}{l}\mathrm{T} \\
\mathrm{K}\end{array}$ & $\mathrm{P}, \underset{\mathrm{MPa}}{\operatorname{expt}}$ & $\begin{array}{c}\mathrm{P}, \mathrm{MPa} \\
\mathrm{MPa}\end{array}$ & $\begin{array}{c}\mathrm{dev} \\
\frac{8}{8}\end{array}$ & $\begin{array}{l}\rho, \operatorname{expt} \\
\mathrm{mol} 1 \cdot \mathrm{dm}^{-3}\end{array}$ & $\begin{array}{l}\rho, \text { calc } \\
\mathrm{mol} \cdot \mathrm{dm}^{-3}\end{array}$ & $\begin{array}{r}\mathrm{dev} \\
8\end{array}$ & wt \\
\hline 268.944 & 2.439 & 2.560 & 4.94 & 13.715 & 13.694 & -0.15 & 0.0 \\
\hline 269.313 & 2.604 & 2.732 & 4.95 & 13.715 & 13.693 & -0.16 & 0.0 \\
\hline 269.861 & 2.857 & 2.985 & 4.49 & 13.715 & 13.693 & -0.16 & 0.0 \\
\hline 270.539 & 3.164 & 3.298 & 4.22 & 13.714 & 13.691 & -0.16 & 0.0 \\
\hline 271.359 & 3.541 & 3.678 & 3.86 & 13.713 & 13.691 & -0.17 & 0.0 \\
\hline 272.788 & 4.196 & 4.341 & 3.45 & 13.712 & 13.689 & -0.17 & 0.0 \\
\hline 277.605 & 6.405 & 6.573 & 2.62 & 13.708 & 13.683 & -0.18 & 0.0 \\
\hline 281.789 & 8.338 & 8.511 & 2.08 & 13.705 & 13.680 & -0.18 & 0.0 \\
\hline 286.317 & 10.423 & 10.610 & 1.79 & 13.701 & 13.676 & -0.18 & 0.0 \\
\hline 290.328 & 12.271 & 12.467 & 1.59 & 13.698 & 13.673 & -0.18 & 0.0 \\
\hline 296.212 & 14.987 & 15.186 & 1.33 & 13.693 & 13.669 & -0.17 & 0.0 \\
\hline 302.145 & 17.713 & 17.922 & 1.18 & 13.688 & 13.665 & -0.17 & 0.0 \\
\hline 310.715 & 21.650 & 21.862 & 0.98 & 13.681 & 13.660 & -0.15 & 0.0 \\
\hline 319.093 & 25.508 & 25.693 & 0.72 & 13.674 & 13.657 & -0.13 & 0.0 \\
\hline 334.225 & 32.389 & 32.558 & 0.52 & 13.661 & 13.647 & -0.10 & 0.0 \\
\hline 256.613 & 1.904 & 2.130 & 11.85 & 14.555 & 14.528 & -0.18 & 0.0 \\
\hline 257.326 & 2.290 & 2.530 & 10.47 & 14.554 & 14.526 & -0.19 & 0.0 \\
\hline 257.911 & 2.611 & 2.860 & 9.51 & 14.554 & 14.525 & -0.20 & 0.0 \\
\hline 259.065 & 3.256 & 3.507 & 7.71 & 14.553 & 14.524 & -0.20 & 0.0 \\
\hline 263.450 & 5.691 & 5.962 & 4.76 & 14.549 & 14.520 & -0.20 & 0.0 \\
\hline 268.631 & 8.553 & 8.858 & 3.57 & 14.544 & 14.513 & -0.21 & 0.0 \\
\hline 273.496 & 11.269 & 11.567 & 2.64 & 14.539 & 14.511 & -0.19 & 0.0 \\
\hline 283.116 & 16.588 & 16.902 & 1.90 & 14.531 & 14.504 & -0.19 & 0.0 \\
\hline 291.287 & 21.078 & 21.404 & 1.55 & 14.523 & 14.497 & -0.18 & 0.0 \\
\hline 298.372 & 24.959 & 25.283 & 1.30 & 14.517 & 14.493 & -0.17 & 0.0 \\
\hline 307.254 & 29.794 & 30.112 & 1.07 & 14.509 & 14.487 & -0.15 & 0.0 \\
\hline 319.529 & 36.406 & 36.722 & 0.87 & 14.498 & 14.478 & -0.14 & 0.4 \\
\hline 248.290 & 1.422 & 1.801 & 26.64 & 15.050 & 15.013 & -0.24 & 0.0 \\
\hline 249.192 & 2.002 & 2.366 & 18.15 & 15.049 & 15.014 & -0.23 & 0.0 \\
\hline 250.248 & 2.656 & 3.028 & 13.98 & 15.048 & 15.013 & -0.23 & 0.0 \\
\hline 253.374 & 4.599 & 4.984 & 8.37 & 15.045 & 15.010 & -0.23 & 0.0 \\
\hline 258.654 & 7.873 & 8.280 & 5.17 & 15.040 & 15.005 & -0.23 & 0.0 \\
\hline 265.858 & 12.335 & 12.755 & 3.41 & 15.033 & 15.000 & -0.22 & 0.0 \\
\hline 272.972 & 16.704 & 17.145 & 2.64 & 15.027 & 14.994 & -0.22 & 0.0 \\
\hline 279.035 & 20.408 & 20.864 & 2.23 & 15.021 & 14.989 & -0.21 & 0.0 \\
\hline
\end{tabular}


Table 6. PVT DATA (continued)

Daia from Pal et al. [13] (continued)

\begin{tabular}{|c|c|c|c|c|c|c|c|}
\hline $\begin{array}{l}\mathrm{T} \\
\mathrm{K}\end{array}$ & $\mathrm{P}, \underset{\mathrm{MPa}}{\exp t}$ & $\underset{\mathrm{MPa}}{\mathrm{P}, \underset{c}{\mathrm{c}}}$ & $\begin{array}{c}\mathrm{dev} \\
\frac{8}{8}\end{array}$ & $\begin{array}{l}\rho, \operatorname{expt} \\
\mathrm{mol} \cdot \mathrm{dm}^{-3}\end{array}$ & $\begin{array}{l}\rho, \quad \mathrm{calc} \\
\mathrm{mol} \cdot \mathrm{dm}^{-3}\end{array}$ & $\begin{array}{r}\mathrm{dev} \\
8\end{array}$ & wt \\
\hline 235.189 & 24.149 & 24.611 & 1.91 & 15.015 & 14.984 & -0.20 & 0.0 \\
\hline 294.840 & 29.996 & 30.447 & 1.50 & 15.006 & 14.978 & -0.19 & 0.0 \\
\hline 304.470 & 35.755 & 36.211 & 1.28 & 14.997 & 14.971 & -0.18 & 0.4 \\
\hline 316.744 & 43.238 & 43.471 & 0.54 & 14.985 & 14.973 & -0.08 & 0.3 \\
\hline 240.739 & 1.010 & 1.344 & 33.10 & 15.455 & 15.427 & -0.18 & 0.0 \\
\hline 240.885 & 1.107 & 1.446 & 30.72 & 15.455 & 15.427 & -0.18 & 0.0 \\
\hline 241.249 & 1.364 & 1.697 & 24.43 & 15.455 & 15.427 & -0.18 & 0.0 \\
\hline 241.891 & 1.803 & 2.138 & 18.58 & 15.454 & 15.427 & -0.18 & 0.0 \\
\hline 243.148 & 2.660 & 2.999 & 12.76 & 15.453 & 15.425 & -0.18 & 0.0 \\
\hline 246.601 & 4.982 & 5.365 & 7.69 & 15.449 & 15.420 & -0.19 & 0.0 \\
\hline 251.930 & 8.627 & 8.998 & 4.30 & 15.444 & 15.417 & -0.18 & 0.0 \\
\hline 257.186 & 12.201 & 12.566 & 2.99 & 15.439 & 15.414 & -0.16 & 0.0 \\
\hline 261.745 & 15.281 & 15.644 & 2.37 & 15.435 & 15.411 & -0.16 & 0.0 \\
\hline 267.242 & 18.986 & 19.328 & 1.80 & 15.429 & 15.407 & -0.14 & 0.0 \\
\hline 273.834 & 23.374 & 23.723 & 1.49 & 15.423 & 15.402 & -0.14 & 0.0 \\
\hline 282.799 & 29.309 & 29.645 & 1.15 & 15.414 & 15.395 & -0.12 & 0.0 \\
\hline 290.022 & 34.031 & 34.371 & 1.00 & 15.407 & 15.389 & -0.12 & 0.0 \\
\hline 298.219 & 39.368 & 39.687 & 0.81 & 15.399 & 15.383 & -0.10 & 0.3 \\
\hline 309.559 & 46.679 & 46.960 & 0.60 & 15.388 & 15.375 & -0.09 & 0.3 \\
\hline 230.051 & 0.806 & 1.325 & 64.39 & 16.037 & 16.003 & -0.21 & 0.0 \\
\hline 230.294 & 0.996 & 1.514 & 51.93 & 16.037 & 16.002 & -0.21 & 0.0 \\
\hline 230.807 & 1.397 & 1.918 & 37.30 & 16.036 & 16.002 & -0.21 & 0.0 \\
\hline 232.572 & 2.784 & 3.295 & 18.34 & 16.034 & 16.001 & -0.20 & 0.0 \\
\hline 234.160 & 4.001 & 4.532 & 13.30 & 16.033 & 15.999 & -0.21 & 0.0 \\
\hline 235.255 & 4.850 & 5.387 & 11.07 & 16.032 & 15.998 & -0.21 & 0.0 \\
\hline 242.791 & 10.658 & 11.221 & 5.28 & 16.024 & 15.991 & -0.21 & 0.0 \\
\hline 249.107 & 15.504 & 16.060 & 3.59 & 16.017 & 15.986 & -0.19 & 0.0 \\
\hline 254.888 & 19.880 & 20.455 & 2.89 & 16.011 & 15.981 & -0.19 & 0.0 \\
\hline 262.164 & 25.381 & 25.942 & 2.21 & 16.004 & 15.976 & -0.18 & 0.0 \\
\hline 271.236 & 32.092 & 32.703 & 1.90 & 15.995 & 15.966 & -0.18 & 0.0 \\
\hline 279.282 & 38.044 & 38.629 & 1.54 & 15.986 & 15.960 & -0.16 & 0.3 \\
\hline 286.940 & 43.652 & 44.210 & 1.28 & 15.978 & 15.954 & -0.15 & 0.3 \\
\hline 294.543 & 49.179 & 49.704 & 1.07 & 15.971 & 15.949 & -0.14 & 0.3 \\
\hline 301.025 & 53.742 & 54.343 & 1.12 & 15.964 & 15.940 & -0.15 & 0.2 \\
\hline 222.875 & 0.762 & 1.692 & 121.90 & 16.423 & 16.370 & -0.33 & 0.0 \\
\hline
\end{tabular}


Table 6. PVT DATA (continued)

Data from $\mathrm{Pal}$ et al. [13] (continued)

\begin{tabular}{|c|c|c|c|c|c|c|c|}
\hline $\begin{array}{l}\mathrm{T} \\
\mathrm{K}\end{array}$ & $\mathrm{P}, \underset{\mathrm{MPa}}{\exp t}$ & $\begin{array}{c}\mathrm{P}, \underset{\mathrm{MPa}}{\mathrm{calc}} \\
\text { cal }\end{array}$ & $\begin{array}{c}\mathrm{dev} \\
8\end{array}$ & $\begin{array}{l}\rho, \operatorname{expt} \\
\mathrm{mol} \cdot \mathrm{dm}^{-3}\end{array}$ & $\begin{array}{l}\rho, \quad \mathrm{calc} \\
\mathrm{mol} \cdot \mathrm{dm}^{-3}\end{array}$ & $\begin{array}{r}\text { dev } \\
8\end{array}$ & wt \\
\hline 223.264 & 1.083 & 2.025 & 86.97 & 16.423 & 16.369 & -0.33 & 0.0 \\
\hline 223.573 & 1.346 & 2.288 & 69.96 & 16.423 & 16.369 & -0.33 & 0.0 \\
\hline 225.014 & 2.579 & 3.518 & 36.44 & 16.421 & 16.368 & -0.32 & 0.0 \\
\hline 230.291 & 7.010 & 7.985 & 13.90 & 16.416 & 16.363 & -0.32 & 0.0 \\
\hline 237.396 & 12.950 & 13.941 & 7.66 & 16.408 & 16.358 & -0.31 & 0.0 \\
\hline 252.785 & 25.571 & 26.618 & 4.09 & 16.391 & 16.344 & -0.29 & 0.0 \\
\hline 259.848 & 31.261 & 32.345 & 3.47 & 16.384 & 16.337 & -0.29 & 0.0 \\
\hline 267.602 & 37.448 & 38.557 & 2.96 & 16.376 & 16.330 & -0.28 & 0.2 \\
\hline 275.649 & 43.890 & 44.930 & 2.37 & 16.367 & 16.326 & -0.25 & 0.2 \\
\hline 282.466 & 49.233 & 50.283 & 2.13 & 16.360 & 16.321 & -0.24 & 0.2 \\
\hline 293.185 & 57.574 & 58.591 & 1.77 & 16.349 & 16.312 & -0.22 & 0.2 \\
\hline 215.247 & 0.682 & 0.956 & 40.16 & 16.754 & 16.740 & -0.08 & 0.0 \\
\hline 215.504 & 0.932 & 1.191 & 27.77 & 16.754 & 16.741 & -0.08 & 0.0 \\
\hline 215.892 & 1.278 & 1.549 & 21.27 & 16.754 & 16.740 & -0.08 & 0.0 \\
\hline 216.369 & 1.726 & 1.984 & 14.98 & 16.753 & 16.740 & -0.08 & 0.0 \\
\hline 216.832 & 2.151 & 2.412 & 12.13 & 16.753 & 16.739 & -0.08 & 0.0 \\
\hline 217.058 & 2.347 & 2.617 & 11.52 & 16.752 & 16.739 & -0.08 & 0.0 \\
\hline 220.809 & 5.784 & 6.037 & 4.37 & 16.748 & 16.736 & -0.07 & 0.0 \\
\hline 226.802 & 11.252 & 11.460 & 1.85 & 16.741 & 16.732 & -0.06 & 0.0 \\
\hline 234.917 & 18.554 & 18.724 & 0.92 & 16.733 & 16.725 & -0.04 & 0.0 \\
\hline 243.045 & 25.738 & 25.893 & 0.60 & 16.724 & 16.717 & -0.04 & 0.0 \\
\hline 250.885 & 32.618 & 32.718 & 0.31 & 16.715 & 16.711 & -0.02 & 0.0 \\
\hline 260.378 & 40.837 & 40.873 & 0.09 & 16.705 & 16.703 & -0.01 & 0.2 \\
\hline 269.927 & 49.045 & 48.950 & -0.19 & 16.694 & 16.697 & 0.02 & 0.2 \\
\hline 278.130 & 55.970 & 55.802 & -0.30 & 16.685 & 16.691 & 0.03 & 0.2 \\
\hline 284.744 & 61.514 & 61.276 & -0.39 & 16.678 & 16.686 & 0.05 & 0.2 \\
\hline 293.608 & 68.881 & 68.528 & -0.51 & 16.668 & 16.679 & 0.07 & 0.2 \\
\hline 208.453 & 0.812 & 2.121 & 161.39 & 17.125 & 17.066 & -0.34 & 0.0 \\
\hline 209.053 & 1.409 & 2.719 & 93.00 & 17.125 & 17.066 & -0.34 & 0.0 \\
\hline 213.403 & 5.704 & 7.025 & 23.16 & 17.120 & 17.063 & -0.33 & 0.0 \\
\hline 218.566 & 10.754 & 12.087 & 12.40 & 17.114 & 17.058 & -0.32 & 0.0 \\
\hline 227.034 & 18.957 & 20.287 & 7.02 & 17.104 & 17.052 & -0.30 & 0.0 \\
\hline 233.700 & 25.300 & 26.651 & 5.34 & 17.096 & 17.046 & -0.30 & 0.0 \\
\hline 241.770 & 32.899 & 34.261 & 4.14 & 17.087 & 17.039 & -0.28 & 0.0 \\
\hline 251.549 & 42.028 & 43.341 & 3.12 & 17.076 & 17.032 & -0.26 & 0.2 \\
\hline
\end{tabular}


Table 6. PVT DATA (continued)

Data from $\mathrm{Pal}$ et al. [13] (continued)

\begin{tabular}{|c|c|c|c|c|c|c|c|}
\hline $\begin{array}{l}\mathrm{I} \\
\mathrm{K}\end{array}$ & $\begin{array}{c}\mathrm{P}, \operatorname{expt} \\
\mathrm{MPa}\end{array}$ & $\begin{array}{c}\mathrm{P}, \mathrm{calc} \\
\mathrm{MPa}\end{array}$ & $\begin{array}{c}\mathrm{dev} \\
8\end{array}$ & $\begin{array}{l}\rho, \text { expt } \\
\mathrm{mol} \cdot \mathrm{dm}^{-3}\end{array}$ & $\begin{array}{l}\rho, \quad \text { calc } \\
\mathrm{mol} \cdot \mathrm{dm}^{-3}\end{array}$ & $\begin{array}{r}\mathrm{dev} \\
8\end{array}$ & wt \\
\hline 260.957 & 50.705 & 51.936 & 2.43 & 17.066 & 17.026 & -0.23 & 0.2 \\
\hline 268.486 & 57.440 & 58.719 & 2.23 & 17.057 & 17.018 & -0.23 & 0.2 \\
\hline 280.494 & 68.304 & 69.396 & 1.60 & 17.044 & 17.012 & -0.19 & 0.2 \\
\hline 198.356 & 0.713 & 1.012 & 41.93 & 17.529 & 17.518 & -0.07 & 0.0 \\
\hline 199.230 & 1.659 & 1.965 & 18.45 & 17.528 & 17.516 & -0.07 & 0.0 \\
\hline 200.946 & 3.544 & 3.831 & 8.11 & 17.526 & 17.515 & -0.06 & 0.0 \\
\hline 204.756 & 7.681 & 7.943 & 3.40 & 17.522 & 17.512 & -0.06 & 0.0 \\
\hline 210.931 & 14.342 & 14.541 & 1.39 & 17.514 & 17.507 & -0.04 & 0.0 \\
\hline 220.998 & 25.028 & 25.116 & 0.35 & 17.503 & 17.500 & -0.02 & 0.0 \\
\hline 227.537 & 31.801 & 31.875 & 0.23 & 17.495 & 17.492 & -0.01 & 0.0 \\
\hline 235.067 & 39.592 & 39.560 & -0.08 & 17.486 & 17.487 & 0.01 & 0.2 \\
\hline 242.758 & 47.394 & 47.294 & -0.21 & 17.477 & 17.480 & 0.02 & 0.2 \\
\hline 249.071 & 53.746 & 53.555 & -0.36 & 17.470 & 17.475 & 0.03 & 0.2 \\
\hline 256.389 & 61.108 & 60.748 & -0.59 & 17.461 & 17.471 & 0.06 & 0.2 \\
\hline 263.603 & 68.221 & 67.739 & -0.71 & 17.453 & 17.466 & 0.07 & 0.2 \\
\hline 188.907 & 0.673 & 1.156 & 71.89 & 17.942 & 17.925 & -0.09 & 0.0 \\
\hline 189.331 & 1.176 & 1.669 & 41.87 & 17.942 & 17.925 & -0.09 & 0.0 \\
\hline 189.746 & 1.684 & 2.159 & 28.21 & 17.941 & 17.925 & -0.09 & 0.0 \\
\hline 192.590 & 5.087 & 5.536 & 8.82 & 17.937 & 17.922 & -0.08 & 0.0 \\
\hline 198.660 & 12.300 & 12.680 & 3.09 & 17.930 & 17.918 & -0.07 & 0.0 \\
\hline 205.509 & 20.264 & 20.608 & 1.70 & 17.921 & 17.911 & -0.06 & 0.0 \\
\hline 213.217 & 29.113 & 29.397 & 0.97 & 17.912 & 17.904 & -0.05 & 0.0 \\
\hline 220.733 & 37.667 & 37.820 & 0.41 & 17.903 & 17.898 & -0.02 & 0.2 \\
\hline 228.543 & 46.431 & 46.448 & 0.04 & 17.893 & 17.893 & 0.00 & 0.1 \\
\hline 238.949 & 58.019 & 57.723 & -0.51 & 17.880 & 17.888 & 0.04 & 0.1 \\
\hline 176.719 & 0.347 & 1.138 & 227.86 & 18.446 & 18.423 & -0.13 & 0.0 \\
\hline 177.382 & 1.231 & 2.032 & 65.05 & 18.445 & 18.422 & -0.13 & 0.0 \\
\hline 178.429 & 2.659 & 3.419 & 28.59 & 18.444 & 18.421 & -0.12 & 0.0 \\
\hline 179.691 & 4.342 & 5.096 & 17.37 & 18.442 & 18.420 & -0.12 & 0.0 \\
\hline 182.793 & 8.481 & 9.198 & 8.46 & 18.438 & 18.418 & -0.11 & 0.0 \\
\hline 188.263 & 15.673 & 16.351 & 4.32 & 18.431 & 18.412 & -0.10 & 0.0 \\
\hline 195.148 & 24.580 & 25.203 & 2.53 & 18.422 & 18.406 & -0.09 & 0.0 \\
\hline 201.949 & 33.279 & 33.806 & 1.59 & 18.413 & 18.400 & -0.07 & 0.0 \\
\hline 208.686 & 41.821 & 42.190 & 0.88 & 18.405 & 18.396 & -0.05 & 0.1 \\
\hline 215.452 & 50.282 & 50.484 & 0.40 & 18.396 & 18.391 & -0.03 & 0.1 \\
\hline
\end{tabular}


Table 6. PVT DATA (continued)

Data from Pal et al. [13] (continued)

\begin{tabular}{|c|c|c|c|c|c|c|c|}
\hline $\begin{array}{l}\mathrm{T} \\
\mathrm{K}\end{array}$ & $\begin{array}{c}\mathrm{P} \text {, expt } \\
\mathrm{MPa}\end{array}$ & $\begin{array}{c}\mathrm{P}, \underset{\mathrm{MPa}}{\text { calc }} \\
\text {. }\end{array}$ & $\begin{array}{c}\mathrm{dev} \\
8\end{array}$ & $\begin{array}{l}\rho, \operatorname{expt} \\
\mathrm{mol} \cdot \mathrm{dm}^{-3}\end{array}$ & $\begin{array}{l}\rho, \text { calc } \\
\mathrm{mol} \cdot \mathrm{dm}^{-3}\end{array}$ & $\begin{array}{r}\mathrm{dev} \\
8\end{array}$ & wt \\
\hline 222.008 & 58.443 & 58.394 & -0.08 & 18.387 & 18.389 & 0.01 & 0.1 \\
\hline 227.030 & 64.631 & 64.394 & -0.37 & 18.381 & 18.386 & 0.03 & 0.1 \\
\hline 229.121 & 67.237 & 66.874 & -0.54 & 18.378 & 18.387 & 0.04 & 0.1 \\
\hline 168.032 & 1.000 & 2.254 & 125.34 & 18.824 & 18.791 & -0.17 & 0.0 \\
\hline 168.479 & 1.659 & 2.902 & 74.88 & 18.823 & 18.791 & -0.17 & 0.0 \\
\hline 169.437 & 3.051 & 4.291 & 40.63 & 18.822 & 18.790 & -0.17 & 0.0 \\
\hline 171.067 & 5.423 & 6.656 & 22.73 & 18.820 & 18.788 & -0.17 & 0.0 \\
\hline 173.679 & 9.221 & 10.403 & 12.82 & 18.816 & 18.786 & -0.16 & 0.0 \\
\hline 177.673 & 14.928 & 16.088 & 7.77 & 18.811 & 18.782 & -0.15 & 0.0 \\
\hline 183.136 & 22.604 & 23.757 & 5.10 & 18.803 & 18.776 & -0.15 & 0.0 \\
\hline 188.844 & 30.544 & 31.647 & 3.61 & 18.796 & 18.770 & -0.14 & 0.0 \\
\hline 195.448 & 39.684 & 40.622 & 2.37 & 18.787 & 18.766 & -0.11 & 0.1 \\
\hline 201.251 & 47.685 & 48.397 & 1.49 & 18.779 & 18.764 & -0.08 & 0.1 \\
\hline 206.784 & 55.063 & 55.710 & 1.18 & 18.772 & 18.758 & -0.07 & 0.1 \\
\hline 212.460 & 62.851 & 63.108 & 0.41 & 18.764 & 18.759 & -0.03 & 0.1 \\
\hline 217.337 & 69.284 & 69.404 & 0.17 & 18.758 & 18.755 & -0.01 & 0.1 \\
\hline 157.201 & 0.522 & 1.491 & 185.68 & 19.227 & 19.204 & -0.12 & 0.0 \\
\hline 158.496 & 2.616 & 3.551 & 35.73 & 19.225 & 19.203 & -0.11 & 0.0 \\
\hline 159.577 & 4.283 & 5.263 & 22.90 & 19.223 & 19.200 & -0.12 & 0.0 \\
\hline 160.786 & 6.293 & 7.180 & 14.09 & 19.221 & 19.201 & -0.11 & 0.0 \\
\hline 163.656 & 10.866 & 11.697 & 7.64 & 19.217 & 19.199 & -0.10 & 0.0 \\
\hline 167.103 & 16.135 & 17.072 & 5.80 & 19.213 & 19.192 & -0.11 & 0.0 \\
\hline 172.270 & 24.169 & 25.005 & 3.46 & 19.205 & 19.187 & -0.09 & 0.0 \\
\hline 177.321 & 31.853 & 32.648 & 2.49 & 19.198 & 19.182 & -0.09 & 0.0 \\
\hline 183.160 & 40.762 & 41.332 & 1.40 & 19.190 & 19.178 & -0.06 & 0.1 \\
\hline 188.652 & 49.035 & 49.381 & 0.70 & 19.182 & 19.175 & -0.04 & 0.1 \\
\hline 194.552 & 57.854 & 57.910 & 0.10 & 19.174 & 19.173 & -0.01 & 0.1 \\
\hline 199.115 & 64.770 & 64.394 & -0.58 & 19.168 & 19.175 & 0.04 & 0.1 \\
\hline 202.417 & 69.454 & 69.068 & -0.56 & 19.163 & 19.171 & 0.04 & 0.1 \\
\hline
\end{tabular}

Number of Points [13] 309

Pressure Calculation:

AAD $8=8.38$ BIAS $8=8.34$ RMS $\%=24.68$

Density Calculation:

AAD2 $8=0.78 \quad$ BIAS2\% $=-0.78 \quad$ RMS $2 \%=2.32$ 
Table 6. PVT DATA (continued)

Data from Pal et al. [13] (continued)

Ahsolute Deviations:

Pressure Calculation:

$$
\mathrm{AAD}=0.30 \mathrm{BIAS}=0.27 \mathrm{RMS}=0.38 \mathrm{MPa}
$$

Density Calculation:

$$
\text { AAD2 }=0.061 \text { BIAS2 }=-0.060 \quad \mathrm{RMS} 2=0.169 \mathrm{~mol} \cdot \mathrm{dm}^{-3}
$$

Weighted Data:

Number of Points [13] 45

Pressure Calculation:

$\mathrm{AAD} \&=0.96 \quad \mathrm{BIAS} \%=0.70 \quad \mathrm{RMS} \%=1.05$

Density Calculation:

$\mathrm{AAD} 2 \%=0.09 \quad \mathrm{BIAS} 2 \%=-0.07 \quad \mathrm{RMS} 2 \%=0.10$

Absolute Deviations:

Pressure Calculation:

$\mathrm{AAD}=0.47 \mathrm{BIAS}=0.30 \mathrm{RMS}=0.51 \mathrm{MPa}$

Density Calculation:

$\mathrm{AAD2}=0.015$ BIAS2 $=-0.011 \quad \mathrm{RMS} 2=0.017 \mathrm{~mol} \cdot \mathrm{dm}^{-3}$

Data from Parrish [55]

\begin{tabular}{ccccccrr}
\hline $\begin{array}{c}\mathrm{T} \\
\mathrm{K}\end{array}$ & $\begin{array}{c}\mathrm{P}, \text { expt } \\
\mathrm{MPa}\end{array}$ & $\begin{array}{c}\mathrm{P}, \mathrm{calc} \\
\mathrm{MPa}\end{array}$ & $\begin{array}{c}\mathrm{dev} \\
8\end{array}$ & $\begin{array}{c}\rho, \text { expt } \\
\mathrm{mol} \cdot \mathrm{dm}^{-3}\end{array}$ & $\begin{array}{r}\rho, \mathrm{calc} \\
\mathrm{mol} \cdot \mathrm{dm}^{-3}\end{array}$ & $\begin{array}{r}\mathrm{dev} \\
8\end{array}$ & wt \\
\hline 299.850 & 5.520 & 5.535 & 0.28 & 11.297 & 11.288 & -0.08 & 4.9 \\
299.850 & 6.890 & 6.919 & 0.42 & 11.902 & 11.892 & -0.09 & 2.8 \\
310.950 & 6.890 & 6.911 & 0.30 & 10.429 & 10.413 & -0.15 & 6.5 \\
310.950 & 8.270 & 8.293 & 0.28 & 11.204 & 11.194 & -0.09 & 3.6 \\
310.950 & 9.650 & 9.682 & 0.33 & 11.703 & 11.693 & -0.08 & 2.5 \\
322.050 & 5.520 & 5.498 & -0.40 & 3.788 & 3.825 & 0.98 & 16.8 \\
322.050 & 6.890 & 6.885 & -0.07 & 7.645 & 7.658 & 0.16 & 13.4 \\
322.050 & 8.270 & 8.291 & 0.26 & 9.704 & 9.686 & -0.18 & 7.0 \\
322.050 & 9.650 & 9.699 & 0.51 & 10.582 & 10.558 & -0.23 & 4.2
\end{tabular}


Table 6. PVT DATA (continued)

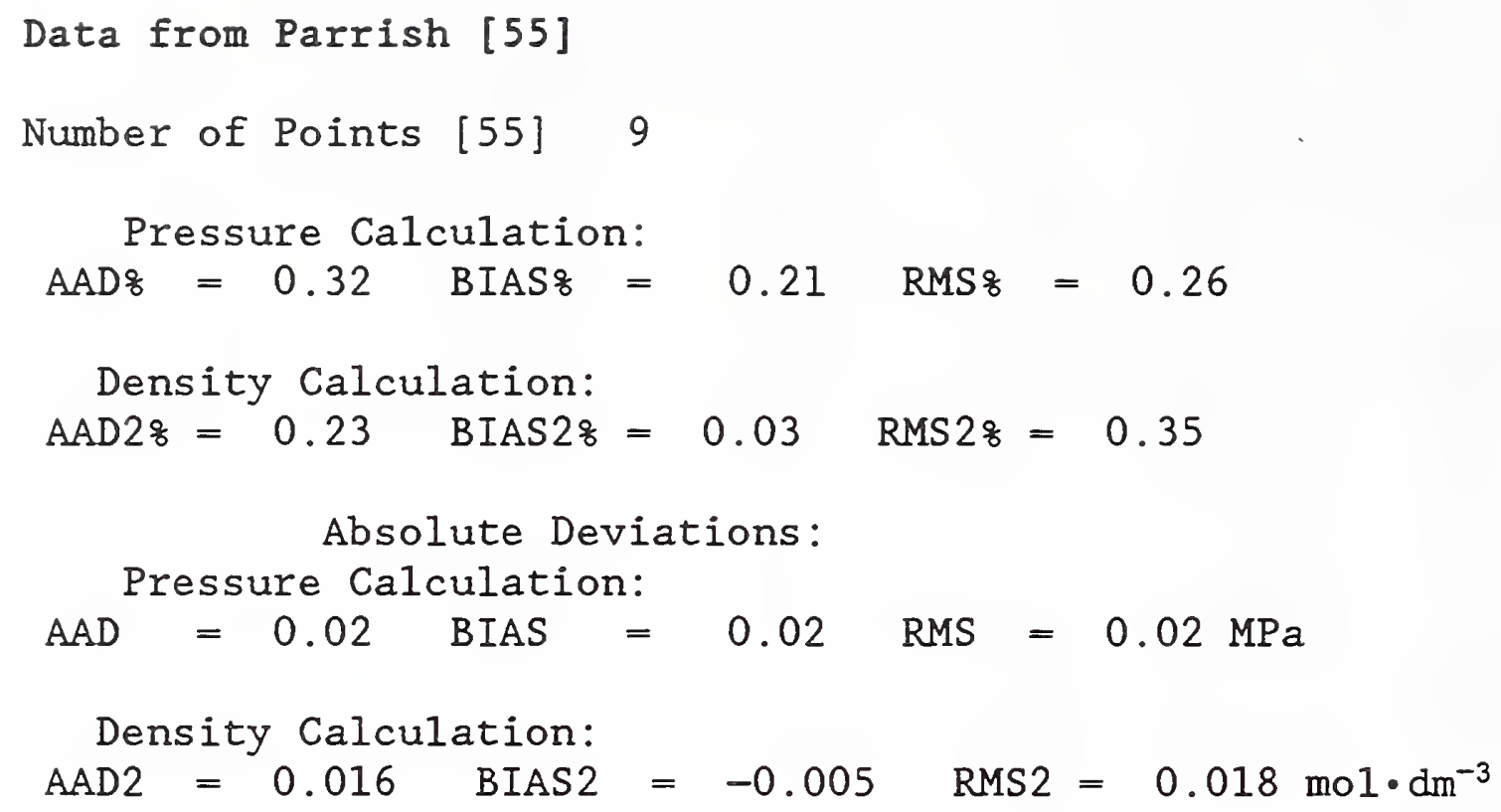

Weighted Data:

Number of Points [55] 9

Pressure Calculation: $\mathrm{AAD} \%=0.32$ BIAS $=0.21 \quad$ RMS $\%=0.26$

Density Calculation: $\mathrm{AAD} 28=0.23 \quad$ BIAS2 $8=0.03 \quad$ RMS28 $=0.35$

Absolute Deviations:

Pressure Calculation:

$\mathrm{AAD}=0.02$ BIAS $=0.02$ RMS $=0.02 \mathrm{MPa}$

Density Calculation:

AAD2 $=0.016$ BIAS2 $=-0.005 \quad$ RMS2 $=0.018 \mathrm{~mol} \cdot \mathrm{dm}^{-3}$

Data from Reamer et al. [42]

\begin{tabular}{cccccccc}
\hline $\begin{array}{c}\mathrm{T} \\
\mathrm{K}\end{array}$ & $\begin{array}{c}\mathrm{P}, \text { expt } \\
\mathrm{MPa}\end{array}$ & $\begin{array}{c}\mathrm{P}, \mathrm{calc} \\
\mathrm{MPa}\end{array}$ & $\begin{array}{c}\mathrm{dev} \\
\text { o }\end{array}$ & $\begin{array}{c}\rho, \text { expt } \\
\mathrm{mol} \cdot \mathrm{dm}^{-3}\end{array}$ & $\begin{array}{c}\rho, \mathrm{calc} \\
\mathrm{mol} \cdot \mathrm{dm}^{-3}\end{array}$ & $\begin{array}{c}\mathrm{dev} \\
\text { o }\end{array}$ & wt \\
\hline 310.928 & 4.826 & 4.816 & -0.22 & 3.525 & 3.547 & 0.63 & 0.0 \\
310.928 & 5.171 & 5.158 & -0.25 & 4.502 & 4.558 & 1.24 & 0.0 \\
310.928 & 5.343 & 5.331 & -0.23 & 5.518 & 5.623 & 1.91 & 0.0 \\
310.928 & 5.516 & 5.492 & -0.44 & 7.270 & 7.517 & 3.41 & 0.0 \\
310.928 & 5.654 & 5.636 & -0.32 & 8.363 & 8.451 & 1.05 & 0.0
\end{tabular}


Table 6. PVT DATA (continued)

Data from Reamer et al. [42] (continued)

\begin{tabular}{|c|c|c|c|c|c|c|c|}
\hline $\begin{array}{l}\mathrm{T} \\
\mathrm{K}\end{array}$ & $\begin{array}{l}\mathrm{P}, \text { expt } \\
\mathrm{MPa}\end{array}$ & $\begin{array}{c}\mathrm{P}, \mathrm{calc} \\
\mathrm{MPa}\end{array}$ & $\begin{array}{c}\mathrm{dev} \\
8\end{array}$ & $\begin{array}{l}\rho, \quad \operatorname{expt} \\
\mathrm{mol} \cdot \mathrm{dm}^{-3}\end{array}$ & $\begin{array}{l}\rho, \text { calc } \\
\mathrm{mol} \cdot \mathrm{dm}^{-3}\end{array}$ & $\begin{array}{r}\text { dev } \\
8\end{array}$ & $w t$ \\
\hline 310.928 & 5.792 & 5.759 & -0.57 & 8.848 & 8.945 & 1.10 & 0.0 \\
\hline 310.928 & 5.929 & 5.889 & -0.68 & 9.189 & 9.276 & 0.94 & 0.0 \\
\hline 310.928 & 6.067 & 6.018 & -0.81 & 9.445 & 9.527 & 0.87 & 0.0 \\
\hline 310.928 & 6.205 & 6.154 & -0.83 & 9.659 & 9.732 & 0.75 & 0.0 \\
\hline 310.928 & 6.343 & 6.291 & -0.82 & 9.842 & 9.905 & 0.63 & 0.0 \\
\hline 310.928 & 6.481 & 6.421 & -0.93 & 9.992 & 10.055 & 0.63 & 0.0 \\
\hline 310.928 & 6.619 & 6.558 & -0.92 & 10.132 & 10.189 & 0.57 & 0.0 \\
\hline 310.928 & 6.757 & 6.695 & -0.91 & 10.258 & 10.310 & 0.51 & 0.0 \\
\hline 344.261 & 7.584 & 7.567 & -0.23 & 4.925 & 4.948 & 0.46 & 0.0 \\
\hline 344.261 & 8.274 & 8.245 & -0.35 & 5.841 & 5.880 & 0.68 & 0.0 \\
\hline 344.261 & 8.963 & 8.922 & -0.46 & 6.752 & 6.805 & 0.79 & 0.0 \\
\hline 344.261 & 9.653 & 9.605 & -0.50 & 7.558 & 7.609 & 0.67 & 0.0 \\
\hline 344.261 & 10.342 & 10.302 & -0.38 & 8.229 & 8.263 & 0.41 & 0.0 \\
\hline 344.261 & 11.032 & 10.963 & -0.63 & 8.743 & 8.792 & 0.55 & 0.0 \\
\hline 344.261 & 11.721 & 11.633 & -0.76 & 9.175 & 9.227 & 0.56 & 0.0 \\
\hline 344.261 & 12.411 & 12.324 & -0.70 & 9.550 & 9.593 & 0.45 & 0.0 \\
\hline 344.261 & 13.100 & 13.009 & -0.70 & 9.868 & 9.907 & 0.40 & 0.0 \\
\hline 310.928 & 1.379 & 1.375 & -0.26 & 0.589 & 0.591 & 0.29 & 0.0 \\
\hline 344.261 & 1.379 & 1.378 & -0.10 & 0.516 & 0.517 & 0.11 & 0.0 \\
\hline 377.594 & 1.379 & 1.379 & -0.03 & 0.462 & 0.462 & 0.03 & 0.0 \\
\hline 410.928 & 1.379 & 1.379 & -0.01 & 0.419 & 0.419 & 0.01 & 0.0 \\
\hline 444.261 & 1.379 & 1.379 & -0.02 & 0.384 & 0.384 & 0.02 & 0.0 \\
\hline 477.594 & 1.379 & 1.379 & -0.03 & 0.354 & 0.355 & 0.03 & 0.0 \\
\hline 510.928 & 1.379 & 1.378 & -0.07 & 0.329 & 0.330 & 0.07 & 0.0 \\
\hline 310.928 & 2.758 & 2.748 & -0.34 & 1.347 & 1.353 & 0.46 & 0.0 \\
\hline 344.261 & 2.758 & 2.754 & -0.16 & 1.121 & 1.123 & 0.19 & 0.0 \\
\hline 377.594 & 2.758 & 2.756 & -0.07 & 0.976 & 0.977 & 0.08 & 0.0 \\
\hline 410.928 & 2.758 & 2.757 & -0.01 & 0.871 & 0.871 & 0.02 & 0.0 \\
\hline 444.261 & 2.758 & 2.757 & -0.04 & 0.789 & 0.790 & 0.04 & 0.0 \\
\hline 477.594 & 2.758 & 2.756 & -0.06 & 0.723 & 0.724 & 0.06 & 0.0 \\
\hline 510.928 & 2.758 & 2.754 & -0.13 & 0.668 & 0.669 & 0.14 & 0.0 \\
\hline 310.928 & 4.137 & 4.126 & -0.27 & 2.496 & 2.509 & 0.51 & 0.0 \\
\hline 344.261 & 4.137 & 4.129 & -0.20 & 1.857 & 1.862 & 0.26 & 0.0 \\
\hline 377.594 & 4.137 & 4.131 & -0.14 & 1.555 & 1.557 & 0.17 & 0.0 \\
\hline 410.928 & 4.137 & 4.136 & -0.03 & 1.360 & 1.361 & 0.03 & 0.0 \\
\hline
\end{tabular}


Table 6. PVT DATA (continued)

Data from Reamer et al. [42] (continued)

\begin{tabular}{|c|c|c|c|c|c|c|c|}
\hline $\begin{array}{l}\mathrm{T} \\
\mathrm{K}\end{array}$ & $\mathrm{P},{ }_{\mathrm{MPa}} \operatorname{expt}^{-}$ & $\underset{\mathrm{MPa}}{\mathrm{P}, \mathrm{calc}}$ & $\begin{array}{c}\mathrm{dev} \\
8\end{array}$ & $\begin{array}{l}\rho, \operatorname{expt} \\
\mathrm{mol} \cdot \mathrm{dm}^{-3}\end{array}$ & $\begin{array}{l}\rho, \quad \mathrm{calc} \\
\mathrm{mol} \cdot \mathrm{dm}^{-3}\end{array}$ & $\begin{array}{r}\mathrm{dev} \\
8\end{array}$ & wt \\
\hline 444.261 & 4.137 & 4.134 & -0.08 & 1.217 & 1.218 & 0.09 & 0.0 \\
\hline 477.594 & 4.137 & 4.133 & -0.10 & 1.107 & 1.108 & 0.10 & 0.0 \\
\hline 510.928 & 4.137 & 4.129 & -0.19 & 1.017 & 1.019 & 0.20 & 0.0 \\
\hline 310.928 & 5.516 & 5.492 & -0.44 & 7.270 & 7.517 & 3.41 & 0.0 \\
\hline 344.261 & 5.516 & 5.505 & -0.20 & 2.802 & 2.810 & 0.31 & 0.0 \\
\hline 377.594 & 5.516 & 5.505 & -0.19 & 2.213 & 2.219 & 0.25 & 0.0 \\
\hline 410.928 & 5.516 & 5.512 & -0.07 & 1.890 & 1.892 & 0.08 & 0.0 \\
\hline 444.261 & 5.516 & 5.510 & -0.11 & 1.668 & 1.671 & 0.13 & 0.0 \\
\hline 477.594 & 5.516 & 5.509 & -0.13 & 1.504 & 1.506 & 0.14 & 0.0 \\
\hline 510.928 & 5.516 & 5.502 & -0.24 & 1.373 & 1.377 & 0.26 & 0.0 \\
\hline 310.928 & 6.895 & 6.835 & -0.87 & 10.374 & 10.421 & 0.45 & 0.0 \\
\hline 344.261 & 6.895 & 6.879 & -0.22 & 4.096 & 4.113 & 0.42 & 0.0 \\
\hline 377.594 & 6.895 & 6.882 & -0.18 & 2.970 & 2.977 & 0.24 & 0.0 \\
\hline 410.928 & 6.895 & 6.887 & -0.11 & 2.463 & 2.466 & 0.14 & 0.0 \\
\hline 444.261 & 6.895 & 6.885 & -0.15 & 2.143 & 2.146 & 0.17 & 0.0 \\
\hline 477.594 & 6.895 & 6.883 & -0.18 & 1.914 & 1.918 & 0.19 & 0.0 \\
\hline 510.928 & 6.895 & 6.875 & -0.29 & 1.738 & 1.743 & 0.31 & 0.0 \\
\hline 310.928 & 8.618 & 8.475 & -1.67 & 11.282 & 11.339 & 0.51 & 0.0 \\
\hline 344.261 & 8.618 & 8.590 & -0.33 & 6.314 & 6.352 & 0.61 & 0.0 \\
\hline 377.594 & 8.618 & 8.601 & -0.20 & 4.065 & 4.077 & 0.29 & 0.0 \\
\hline 410.928 & 8.618 & 8.603 & -0.18 & 3.237 & 3.245 & 0.22 & 0.0 \\
\hline 444.261 & 8.618 & 8.602 & -0.19 & 2.765 & 2.770 & 0.21 & 0.0 \\
\hline 477.594 & 8.618 & 8.600 & -0.22 & 2.443 & 2.449 & 0.24 & 0.0 \\
\hline 510.928 & 8.618 & 8.592 & -0.31 & 2.203 & 2.210 & 0.33 & 0.0 \\
\hline 310.928 & 10.342 & 10.044 & -2.88 & 11.812 & 11.894 & 0.70 & 0.0 \\
\hline 344.261 & 10.342 & 10.302 & -0.38 & 8.229 & 8.263 & 0.41 & 0.0 \\
\hline 377.594 & 10.342 & 10.308 & -0.33 & 5.278 & 5.302 & 0.47 & 0.0 \\
\hline 410.928 & 10.342 & 10.324 & -0.18 & 4.069 & 4.078 & 0.23 & 0.0 \\
\hline 444.261 & 10.342 & 10.324 & -0.17 & 3.415 & 3.421 & 0.20 & 0.0 \\
\hline 477.594 & 10.342 & 10.318 & -0.23 & 2.986 & 2.994 & 0.26 & 0.0 \\
\hline 510.928 & 10.342 & 10.309 & -0.32 & 2.675 & 2.685 & 0.34 & 0.0 \\
\hline 310.928 & 12.066 & 11.674 & -3.25 & 12.218 & 12.302 & 0.69 & 0.0 \\
\hline 344.261 & 12.066 & 11.973 & -0.77 & 9.368 & 9.417 & 0.53 & 0.0 \\
\hline 377.594 & 12.066 & 12.008 & -0.48 & 6.471 & 6.509 & 0.59 & 0.0 \\
\hline 410.928 & 12.066 & 12.037 & -0.24 & 4.922 & 4.936 & 0.29 & 0.0 \\
\hline
\end{tabular}


Table 6. PVT DATA (continued)

Data from Reamer et al. [42] (continued)

\begin{tabular}{|c|c|c|c|c|c|c|c|}
\hline $\begin{array}{l}\mathrm{T} \\
\mathrm{K}\end{array}$ & $P$, expt $_{\mathrm{MPa}}$ & $\underset{\mathrm{MPa}}{\mathrm{P}, \mathrm{calc}}$ & $\begin{array}{c}\text { dev } \\
8\end{array}$ & $\begin{array}{l}\rho, \operatorname{expt} \\
\mathrm{mol} \cdot \mathrm{dm}^{-3}\end{array}$ & $\begin{array}{l}\rho, \quad \mathrm{calc} \\
\mathrm{mol} \cdot \mathrm{dm}^{-3}\end{array}$ & $\begin{array}{r}\text { dev } \\
\frac{8}{8}\end{array}$ & wt \\
\hline 444.261 & 12.066 & 12.042 & -0.20 & 4.078 & 4.087 & 0.23 & 0.0 \\
\hline 477.594 & 12.066 & 12.036 & -0.24 & 3.537 & 3.546 & 0.27 & 0.0 \\
\hline 510.928 & 12.066 & 12.035 & -0.26 & 3.154 & 3.162 & 0.27 & 0.0 \\
\hline 310.928 & 13.790 & 13.326 & -3.36 & 12.548 & 12.630 & 0.65 & 0.0 \\
\hline 344.261 & 13.790 & 13.670 & -0.86 & 10.136 & 10.181 & 0.44 & 0.0 \\
\hline 377.594 & 13.790 & 13.729 & -0.44 & 7.515 & 7.548 & 0.44 & 0.0 \\
\hline 410.928 & 13.790 & 13.747 & -0.31 & 5.758 & 5.778 & 0.35 & 0.0 \\
\hline 444.261 & 13.790 & 13.755 & -0.25 & 4.739 & 4.753 & 0.28 & 0.0 \\
\hline 477.594 & 13.790 & 13.756 & -0.24 & 4.088 & 4.099 & 0.26 & 0.0 \\
\hline 510.928 & 13.790 & 13.763 & -0.19 & 3.632 & 3.640 & 0.20 & 0.0 \\
\hline 310.928 & 15.513 & 15.035 & -3.08 & 12.833 & 12.906 & 0.56 & 0.0 \\
\hline 344.261 & 15.513 & 15.256 & -1.66 & 10.667 & 10.742 & 0.70 & 0.0 \\
\hline 377.594 & 15.513 & 15.442 & -0.46 & 8.354 & 8.385 & 0.37 & 0.0 \\
\hline 410.928 & 15.513 & 15.440 & -0.47 & 6.529 & 6.561 & 0.49 & 0.0 \\
\hline 444.261 & 15.513 & 15.456 & -0.37 & 5.380 & 5.401 & 0.39 & 0.0 \\
\hline 477.594 & 15.513 & 15.469 & -0.29 & 4.630 & 4.644 & 0.30 & 0.0 \\
\hline 510.928 & 15.513 & 15.487 & -0.17 & 4.104 & 4.111 & 0.17 & 0.0 \\
\hline 310.928 & 17.237 & 16.699 & -3.12 & 13.074 & 13.145 & 0.55 & 0.0 \\
\hline 344.261 & 17.237 & 16.877 & -2.09 & 11.098 & 11.183 & 0.76 & 0.0 \\
\hline 377.594 & 17.237 & 17.149 & -0.51 & 9.026 & 9.057 & 0.34 & 0.0 \\
\hline 410.928 & 17.237 & 17.148 & -0.52 & 7.229 & 7.263 & 0.47 & 0.0 \\
\hline 444.261 & 17.237 & 17.165 & -0.42 & 5.992 & 6.017 & 0.41 & 0.0 \\
\hline 477.594 & 17.237 & 17.180 & -0.33 & 5.156 & 5.173 & 0.33 & 0.0 \\
\hline 510.928 & 17.237 & 17.209 & -0.16 & 4.566 & 4.573 & 0.16 & 0.0 \\
\hline 310.928 & 18.961 & 18.365 & -3.14 & 13.287 & 13.358 & 0.53 & 0.0 \\
\hline 344.261 & 18.961 & 18.541 & -2.21 & 11.465 & 11.548 & 0.73 & 0.0 \\
\hline 377.594 & 18.961 & 18.820 & -0.74 & 9.565 & 9.606 & 0.43 & 0.0 \\
\hline 410.928 & 18.961 & 18.862 & -0.52 & 7.846 & 7.879 & 0.42 & 0.0 \\
\hline 444.261 & 18.961 & 18.859 & -0.53 & 6.558 & 6.591 & 0.49 & 0.0 \\
\hline 477.594 & 18.961 & 18.879 & -0.43 & 5.657 & 5.680 & 0.41 & 0.0 \\
\hline 510.928 & 18.961 & 18.928 & -0.17 & 5.013 & 5.022 & 0.16 & 0.0 \\
\hline 310.928 & 20.684 & 20.010 & -3.26 & 13.477 & 13.550 & 0.54 & 0.0 \\
\hline 344.261 & 20.684 & 20.230 & -2.20 & 11.781 & 11.859 & 0.66 & 0.0 \\
\hline 377.594 & 20.684 & 20.478 & -1.00 & 10.014 & 10.066 & 0.51 & 0.0 \\
\hline 410.928 & 20.684 & 20.583 & -0.49 & 8.387 & 8.417 & 0.35 & 0.0 \\
\hline
\end{tabular}


Table 6. PVT DATA (continued)

Data from Reamer et al. [42] (continued)

\begin{tabular}{|c|c|c|c|c|c|c|c|}
\hline $\begin{array}{l}\mathrm{T} \\
\mathrm{K}\end{array}$ & $\begin{array}{c}\mathrm{P}, \operatorname{expt}_{\mathrm{MPa}} \\
\text { exp }\end{array}$ & $\begin{array}{c}\mathrm{P}, \mathrm{MPa}^{\mathrm{c}} \\
\text { calc }\end{array}$ & $\begin{array}{c}\text { dev } \\
\frac{8}{8}\end{array}$ & $\begin{array}{l}\rho, \quad \operatorname{expt} \\
\mathrm{mol} \cdot \mathrm{dm}^{-3}\end{array}$ & $\begin{array}{l}\rho, \quad \mathrm{calc} \\
\mathrm{mol} \cdot \mathrm{dm}^{-3}\end{array}$ & $\begin{array}{r}\mathrm{dev} \\
8\end{array}$ & wt \\
\hline 444.261 & 20.684 & 20.559 & -0.61 & 7.081 & 7.118 & 0.52 & 0.0 \\
\hline 477.594 & 20.684 & 20.580 & -0.50 & 6.133 & 6.161 & 0.46 & 0.0 \\
\hline 510.928 & 20.684 & 20.649 & -0.17 & 5.445 & 5.453 & 0.16 & 0.0 \\
\hline 310.928 & 24.132 & 23.377 & -3.13 & 13.817 & 13.886 & 0.50 & 0.0 \\
\hline 344.261 & 24.132 & 23.712 & -1.74 & 12.315 & 12.371 & 0.46 & 0.0 \\
\hline 377.594 & 24.132 & 23.813 & -1.32 & 10.738 & 10.798 & 0.56 & 0.0 \\
\hline 410.928 & 24.132 & 23.916 & -0.89 & 9.255 & 9.304 & 0.53 & 0.0 \\
\hline 444.261 & 24.132 & 24.002 & -0.54 & 8.004 & 8.036 & 0.39 & 0.0 \\
\hline 477.594 & 24.132 & 23.983 & -0.61 & 6.999 & 7.034 & 0.50 & 0.0 \\
\hline 510.928 & 24.132 & 24.062 & -0.29 & 6.243 & 6.259 & 0.25 & 0.0 \\
\hline 310.928 & 27.579 & 26.656 & -3.35 & 14.102 & 14.176 & 0.52 & 0.0 \\
\hline 344.261 & 27.579 & 27.152 & -1.55 & 12.740 & 12.788 & 0.37 & 0.0 \\
\hline 377.594 & 27.579 & 27.155 & -1.54 & 11.304 & 11.368 & 0.56 & 0.0 \\
\hline 410.928 & 27.579 & 27.279 & -1.09 & 9.948 & 10.003 & 0.55 & 0.0 \\
\hline 444.261 & 27.579 & 27.366 & -0.77 & 8.752 & 8.795 & 0.49 & 0.0 \\
\hline 477.594 & 27.579 & 27.397 & -0.66 & 7.754 & 7.791 & 0.48 & 0.0 \\
\hline 510.928 & 27.579 & 27.447 & -0.48 & 6.955 & 6.982 & 0.38 & 0.0 \\
\hline 310.928 & 31.026 & 29.918 & -3.57 & 14.353 & 14.432 & 0.55 & 0.0 \\
\hline 344.261 & 31.026 & 30.443 & -1.88 & 13.083 & 13.139 & 0.43 & 0.0 \\
\hline 377.594 & 31.026 & 30.499 & -1.70 & 11.768 & 11.834 & 0.56 & 0.0 \\
\hline 410.928 & 31.026 & 30.618 & -1.32 & 10.512 & 10.574 & 0.59 & 0.0 \\
\hline 444.261 & 31.026 & 30.733 & -0.94 & 9.380 & 9.430 & 0.53 & 0.0 \\
\hline 477.594 & 31.026 & 30.799 & -0.73 & 8.405 & 8.445 & 0.48 & 0.0 \\
\hline 510.928 & 31.026 & 30.846 & -0.58 & 7.593 & 7.625 & 0.42 & 0.0 \\
\hline 310.928 & 34.474 & 33.022 & -4.21 & 14.568 & 14.662 & 0.65 & 0.0 \\
\hline 344.261 & 34.474 & 33.656 & -2.37 & 13.376 & 13.445 & 0.52 & 0.0 \\
\hline 377.594 & 34.474 & 33.842 & -1.83 & 12.160 & 12.228 & 0.56 & 0.0 \\
\hline 410.928 & 34.474 & 33.964 & -1.48 & 10.987 & 11.053 & 0.60 & 0.0 \\
\hline 444.261 & 34.474 & 34.069 & -1.17 & 9.910 & 9.969 & 0.60 & 0.0 \\
\hline 477.594 & 34.474 & 34.220 & -0.74 & 8.974 & 9.013 & 0.44 & 0.0 \\
\hline 510.928 & 34.474 & 34.215 & -0.75 & 8.155 & 8.196 & 0.50 & 0.0 \\
\hline 310.928 & 41.368 & 39.058 & -5.58 & 14.936 & 15.063 & 0.85 & 0.0 \\
\hline 344.261 & 41.368 & 39.988 & -3.34 & 13.866 & 13.961 & 0.69 & 0.0 \\
\hline 377.594 & 41.368 & 40.606 & -1.84 & 12.809 & 12.873 & 0.50 & 0.0 \\
\hline 410.928 & 41.368 & 40.700 & -1.62 & 11.758 & 11.824 & 0.57 & 0.0 \\
\hline
\end{tabular}


Table 6. PVT DATA (continued)

Data from Reamer et al. [42] (continued)

\begin{tabular}{|c|c|c|c|c|c|c|c|}
\hline $\begin{array}{l}\mathrm{T} \\
\mathrm{K}\end{array}$ & $\begin{array}{l}\mathrm{P}, \operatorname{expt} \\
\mathrm{MPa}\end{array}$ & $\begin{array}{c}\mathrm{P}, \mathrm{calc} \\
\mathrm{MPa}\end{array}$ & $\begin{array}{c}\text { dev } \\
8\end{array}$ & $\begin{array}{l}\rho, \quad \operatorname{expt} \\
\mathrm{mol} \cdot \mathrm{dm}^{-3}\end{array}$ & $\begin{array}{l}\rho, \quad \mathrm{calc} \\
\mathrm{mol} \cdot \mathrm{dm}^{-3}\end{array}$ & $\begin{array}{r}\text { dev } \\
q\end{array}$ & wt \\
\hline 444.261 & 41.368 & 40.836 & -1.29 & 10.782 & 10.842 & 0.55 & 0.0 \\
\hline 477.594 & 41.368 & 40.993 & -0.91 & 9.905 & 9.950 & 0.46 & 0.0 \\
\hline 510.928 & 41.368 & 41.187 & -0.44 & 9.137 & 9.160 & 0.25 & 0.0 \\
\hline 310.928 & 48.263 & 45.586 & -5.55 & 15.279 & 15.407 & 0.84 & 0.0 \\
\hline 344.261 & 48.263 & 46.700 & -3.24 & 14.298 & 14.389 & 0.64 & 0.0 \\
\hline 377.594 & 48.263 & 47.115 & -2.38 & 13.312 & 13.392 & 0.60 & 0.0 \\
\hline 410.928 & 48.263 & 47.222 & -2.16 & 12.349 & 12.433 & 0.68 & 0.0 \\
\hline 444.261 & 48.263 & 47.468 & -1.65 & 11.458 & 11.530 & 0.63 & 0.0 \\
\hline 477.594 & $1: 8.263$ & 47.627 & -1.32 & 10.634 & 10.696 & 0.59 & 0.0 \\
\hline 510.928 & 48.263 & 47.852 & -0.85 & 9.899 & 9.942 & 0.43 & 0.0 \\
\hline 310.928 & 55.158 & 52.286 & -5.21 & 15.588 & 15.709 & 0.78 & 0.0 \\
\hline 344.261 & 55.158 & 53.504 & -3.00 & 14.672 & 14.756 & 0.57 & 0.0 \\
\hline 377.594 & 55.158 & 53.467 & -3.07 & 13.727 & 13.828 & 0.73 & 0.0 \\
\hline 410.928 & 55.158 & 53.698 & -2.65 & 12.837 & 12.937 & 0.78 & 0.0 \\
\hline 444.261 & 55.158 & 54.076 & -1.96 & 12.013 & 12.095 & 0.68 & 0.0 \\
\hline 477.594 & 55.158 & 54.221 & -1.70 & 11.234 & 11.311 & 0.69 & 0.0 \\
\hline 510.928 & 55.158 & 54.435 & -1.31 & 10.529 & 10.592 & 0.60 & 0.0 \\
\hline 310.928 & 62.053 & 59.060 & -4.82 & 15.866 & 15.980 & 0.72 & 0.0 \\
\hline 344.261 & 62.053 & 60.285 & -2.85 & 14.999 & 15.078 & 0.53 & 0.0 \\
\hline 377.594 & 62.053 & 60.092 & -3.16 & 14.102 & 14.204 & 0.72 & 0.0 \\
\hline 410.928 & 62.053 & 60.228 & -2.94 & 13.259 & 13.367 & 0.81 & 0.0 \\
\hline 444.261 & 62.053 & 60.617 & -2.31 & 12.480 & 12.574 & 0.75 & 0.0 \\
\hline 477.594 & 62.053 & 60.777 & -2.06 & 11.742 & 11.832 & 0.77 & 0.0 \\
\hline 510.928 & 62.053 & 61.131 & -1.49 & 11.076 & 11.146 & 0.63 & 0.0 \\
\hline 310.928 & 68.948 & 66.328 & -3.80 & 16.135 & 16.225 & 0.56 & 0.0 \\
\hline 344.261 & 68.948 & 66.687 & -3.28 & 15.275 & 15.366 & 0.60 & 0.0 \\
\hline 377.594 & 68.948 & 66.826 & -3.08 & 14.438 & 14.536 & 0.68 & 0.0 \\
\hline 410.928 & 68.948 & 66.909 & -2.96 & 13.636 & 13.742 & 0.78 & 0.0 \\
\hline 444.261 & 68.948 & 67.100 & -2.68 & 12.884 & 12.990 & 0.82 & 0.0 \\
\hline 477.594 & 68.948 & 67.358 & -2.30 & 12.185 & 12.284 & 0.81 & 0.0 \\
\hline 510.928 & 68.948 & 67.827 & -1.63 & 11.552 & 11.626 & 0.64 & 0.0 \\
\hline
\end{tabular}


Table 6. PVT DATA (continued)

Data from Reamer et al. [42] (continued)

Number of Points [42] 176

Pressure Calculation:

$\mathrm{AAD} ;=1.13$ BIAS $8=-1.13$ RMS $8=1.22$

Density Calculation:

$\mathrm{AAD} 2 \%=0.50 \quad$ BIAS2 $8=0.50 \quad$ RMS $2 \%=0.40$

Absolute Deviations:

Pressure Calculation:

$\mathrm{AAD}=0.41$ BIAS $=-0.41$ RMS $=0.65 \mathrm{MPa}$

Density Calculation:

AAD2 $=0.047$ BIAS2 $=0.047$ RMS2 $=0.040 \mathrm{~mol} \cdot \mathrm{dm}^{-3}$

Data from Sengers [56]

Data were calculated from a scaled equation of state.

\begin{tabular}{|c|c|c|c|c|c|c|c|}
\hline $\begin{array}{l}\mathrm{T} \\
\mathrm{K}\end{array}$ & $\begin{array}{l}\mathrm{P}, \operatorname{expt} \\
\mathrm{MPa}\end{array}$ & $\begin{array}{c}\mathrm{P}, \mathrm{calc} \\
\mathrm{MPa}\end{array}$ & $\begin{array}{c}\text { dev } \\
\frac{8}{8}\end{array}$ & $\begin{array}{l}\rho, \operatorname{expt} \\
\mathrm{mol} \cdot \mathrm{dm}^{-3}\end{array}$ & $\begin{array}{l}\rho, \text { calc } \\
\mathrm{mol} \cdot \mathrm{dm}^{-3}\end{array}$ & $\begin{array}{r}\text { dev } \\
8\end{array}$ & wt \\
\hline 304.000 & 4.735 & 4.736 & 0.02 & 4.900 & 4.883 & -0.34 & 105.4 \\
\hline 304.000 & 4.754 & 4.754 & -0.01 & 9.000 & 9.002 & 0.02 & 102.2 \\
\hline 306.000 & 4.893 & 4.892 & -0.02 & 5.000 & 5.011 & 0.23 & 101.8 \\
\hline 306.000 & 4.933 & 4.932 & -0.02 & 6.000 & 6.074 & 1.23 & 101.3 \\
\hline 306.000 & 4.938 & 4.938 & -0.01 & 6.500 & 6.537 & 0.57 & 101.2 \\
\hline 306.000 & 4.941 & 4.942 & 0.02 & 6.900 & 6.811 & -1.28 & 101.1 \\
\hline 306.000 & 4.946 & 4.948 & 0.04 & 7.500 & 7.336 & -2.18 & 101.0 \\
\hline 306.000 & 4.957 & 4.958 & 0.03 & 8.000 & 7.944 & -0.70 & 100.6 \\
\hline 306.000 & 5.051 & 5.052 & 0.02 & 9.000 & 8.994 & -0.06 & 93.3 \\
\hline 310.000 & 5.191 & 5.190 & -0.02 & 5.000 & 5.007 & 0.13 & 95.3 \\
\hline 310.000 & 5.298 & 5.298 & -0.01 & 6.000 & 6.009 & 0.15 & 93.9 \\
\hline 310.000 & 5.333 & 5.334 & 0.01 & 6.500 & 6.491 & -0.13 & 93.4 \\
\hline 310.000 & 5.359 & 5.361 & 0.04 & 6.900 & 6.870 & -0.44 & 92.9 \\
\hline 310.000 & 5.403 & 5.406 & 0.05 & 7.500 & 7.466 & -0.45 & 91.8 \\
\hline 310.000 & 5.455 & 5.456 & 0.02 & 8.000 & 7.990 & -0.12 & 90.1 \\
\hline 310.000 & 5.666 & 5.666 & 0.01 & 9.000 & 8.999 & -0.01 & 77.6 \\
\hline 315.000 & 5.556 & 5.556 & 0.00 & 5.000 & 5.001 & 0.02 & 87.9 \\
\hline 315.000 & 5.749 & 5.750 & 0.02 & 6.000 & 5.994 & -0.10 & 85.7 \\
\hline 315.000 & 5.827 & 5.828 & 0.02 & 6.500 & 6.492 & -0.13 & 84.5 \\
\hline 315.000 & 5.888 & 5.890 & 0.02 & 6.900 & 6.891 & -0.13 & 83.5 \\
\hline
\end{tabular}


Table 6. PVT DATA (continued)

Data from Sengers [56] (continued)

\begin{tabular}{ccccccrr}
\hline $\begin{array}{c}\mathrm{T} \\
\mathrm{K}\end{array}$ & $\begin{array}{c}\mathrm{P}, \text { expt } \\
\mathrm{MPa}\end{array}$ & $\begin{array}{c}\mathrm{P}, \mathrm{calc} \\
\mathrm{MPa}\end{array}$ & $\begin{array}{c}\mathrm{dev} \\
8\end{array}$ & $\begin{array}{c}\rho, \text { expt } \\
\mathrm{mol} \cdot \mathrm{dm}^{-3}\end{array}$ & $\begin{array}{r}\rho, \mathrm{calc} \\
\mathrm{mol} \cdot \mathrm{dm}^{-3}\end{array}$ & $\begin{array}{r}\mathrm{dev} \\
8\end{array}$ & wt \\
\hline 315.000 & 5.991 & 5.991 & 0.00 & 7.500 & 7.500 & -0.01 & 81.2 \\
315.000 & 6.101 & 6.098 & -0.04 & 8.000 & 8.010 & 0.13 & 78.0 \\
315.000 & 6.458 & 6.459 & 0.02 & 9.000 & 8.998 & -0.02 & 62.7 \\
325.000 & 6.273 & 6.272 & 0.00 & 5.000 & 5.000 & 0.01 & 75.6 \\
325.000 & 6.643 & 6.646 & 0.03 & 6.000 & 5.993 & -0.11 & 71.8 \\
325.000 & 6.816 & 6.817 & 0.01 & 6.500 & 6.498 & -0.03 & 69.7 \\
325.000 & 6.958 & 6.957 & -0.01 & 6.900 & 6.902 & 0.03 & 67.6 \\
325.000 & 7.193 & 7.189 & -0.05 & 7.500 & 7.508 & 0.11 & 63.6 \\
325.000 & 7.426 & 7.422 & -0.06 & 8.000 & 8.009 & 0.11 & 58.8 \\
325.000 & 8.085 & 8.096 & 0.13 & 9.000 & 8.988 & -0.14 & 44.2
\end{tabular}

Number of Points [56] 30

Pressure Calculation:

$\mathrm{AAD} \%=0.03 \quad \mathrm{BIAS} \%=0.01 \quad \mathrm{RMS} \%=0.03$

Density Calculation:

$\mathrm{AAD} 2 \%=0.30 \quad \mathrm{BIAS} 2 \%=-0.12 \quad \mathrm{RMS} 2 \%=0.55$

Absolute Deviations:

Pressure Calculation:

$\mathrm{AAD}=0.00 \mathrm{BIAS}=0.00 \mathrm{RMS}=0.00 \mathrm{MPa}$

Density Calculation:

AAD2 $=0.021$ BIAS2 $=-0.010$ RMS2 $=0.039 \mathrm{~mol} \cdot \mathrm{dm}^{-3}$

Weighted Data:

Number of Points [56] 30

Pressure Calculation:

$\mathrm{AAD} \delta=0.03$ BIAS $8=0.01$ RMS $8=0.03$

Density Calculation:

$\mathrm{AAD} 2 \%=0.30 \quad \mathrm{BIAS} 2 \%=-0.12 \quad \mathrm{RMS} 2 \%=0.55$ 
Table 6. PVT DATA (continued)

Data from Sengers [56] (continued)

Absolute Deviations:

Pressure Calculation:

$\mathrm{AAD}=0.00 \mathrm{BIAS}=0.00 \quad \mathrm{RMS}=0.00 \mathrm{MPa}$

Density Calculation:

$\mathrm{AAD2}=0.021$ BIAS2 $=-0.010$ RMS2 $=0.039 \mathrm{mo} 1 \cdot \mathrm{dm}^{-3}$

Data from Straty and Tsumura [19]

\begin{tabular}{|c|c|c|c|c|c|c|c|}
\hline $\begin{array}{l}\mathrm{T} \\
\mathrm{K}\end{array}$ & $\begin{array}{c}\mathrm{P}, \operatorname{expt} \\
\mathrm{MPa}\end{array}$ & $\begin{array}{c}\mathrm{P}, \mathrm{calc} \\
\mathrm{MPa}\end{array}$ & $\begin{array}{c}\mathrm{dev} \\
8\end{array}$ & $\begin{array}{l}\rho, \operatorname{expt} \\
\mathrm{mol} \cdot \mathrm{dm}^{-3}\end{array}$ & $\begin{array}{l}\rho, \quad \mathrm{calc} \\
\mathrm{mol} \cdot \mathrm{dm}^{-3}\end{array}$ & $\begin{array}{r}\mathrm{dev} \\
\frac{8}{8}\end{array}$ & wt \\
\hline 288.000 & 3.055 & 3.051 & -0.13 & 1.955 & 1.959 & 0.24 & 43.4 \\
\hline 296.000 & 3.237 & 3.233 & -0.12 & 1.952 & 1.956 & 0.21 & 40.1 \\
\hline 304.000 & 3.416 & 3.411 & -0.14 & 1.950 & 1.954 & 0.22 & 37.4 \\
\hline 312.000 & 3.591 & 3.585 & -0.15 & 1.947 & 1.952 & 0.24 & 35.1 \\
\hline 320.000 & 3.763 & 3.756 & -0.18 & 1.945 & 1.950 & 0.26 & 33.1 \\
\hline 296.000 & 3.881 & 3.879 & -0.04 & 2.880 & 2.884 & 0.13 & 36.6 \\
\hline 300.000 & 4.031 & 4.028 & -0.07 & 2.875 & 2.881 & 0.18 & 34.8 \\
\hline 304.000 & 4.178 & 4.176 & -0.07 & 2.873 & 2.878 & 0.17 & 33.1 \\
\hline 308.000 & 4.324 & 4.321 & -0.07 & 2.871 & 2.875 & 0.16 & 31.7 \\
\hline 312.000 & 4.467 & 4.464 & -0.07 & 2.869 & 2.873 & 0.16 & 30.3 \\
\hline 316.000 & 4.608 & 4.605 & -0.08 & 2.866 & 2.871 & 0.16 & 29.1 \\
\hline 320.000 & 4.748 & 4.744 & -0.09 & 2.864 & 2.869 & 0.17 & 28.0 \\
\hline 306.000 & 4.944 & 4.938 & -0.11 & 6.533 & 7.151 & 9.45 & 0.0 \\
\hline 308.000 & 5.143 & 5.137 & -0.12 & 6.530 & 6.691 & 2.47 & 0.0 \\
\hline 312.000 & 5.540 & 5.534 & -0.11 & 6.522 & 6.583 & 0.94 & 0.0 \\
\hline 316.000 & 5.936 & 5.929 & -0.11 & 6.513 & 6.552 & 0.60 & 0.0 \\
\hline 320.000 & 6.331 & 6.323 & -0.13 & 6.503 & 6.536 & 0.50 & 0.0 \\
\hline 304.000 & 4.772 & 4.767 & -0.10 & 9.097 & 9.128 & 0.34 & 30.1 \\
\hline 306.000 & 5.076 & 5.071 & -0.08 & 9.092 & 9.111 & 0.21 & 27.4 \\
\hline 308.000 & 5.387 & 5.381 & -0.12 & 9.086 & 9.107 & 0.23 & 24.9 \\
\hline 312.000 & 6.021 & 6.011 & -0.16 & 9.072 & 9.094 & 0.24 & 20.8 \\
\hline 316.000 & 6.661 & 6.650 & -0.16 & 9.054 & 9.072 & 0.20 & 17.7 \\
\hline 320.000 & 7.306 & 7.293 & -0.18 & 9.032 & 9.050 & 0.20 & 15.4 \\
\hline 304.000 & 4.967 & 4.965 & -0.05 & 9.788 & 9.794 & 0.06 & 22.5 \\
\hline 308.000 & 5.697 & 5.690 & -0.12 & 9.774 & 9.785 & 0.11 & 17.8 \\
\hline
\end{tabular}


Table 6. PVT DATA (continued)

Data from Straty and Tsumura [19] (continued)

\begin{tabular}{|c|c|c|c|c|c|c|c|}
\hline $\begin{array}{l}\mathrm{T} \\
\mathrm{K}\end{array}$ & $\begin{array}{l}\mathrm{P} \text {, expt } \\
\mathrm{MPa}\end{array}$ & $\begin{array}{c}\mathrm{P}, \mathrm{calc} \\
\mathrm{MPa}\end{array}$ & $\begin{array}{c}\text { dev } \\
\frac{8}{8}\end{array}$ & $\begin{array}{l}\rho, \text { expt } \\
\mathrm{mol} \cdot \mathrm{dm}^{-3}\end{array}$ & $\begin{array}{l}\rho, \quad \text { calc } \\
\mathrm{mol} \cdot \mathrm{dm}^{-3}\end{array}$ & $\begin{array}{r}\text { dev } \\
8\end{array}$ & wt \\
\hline 312.000 & 6.433 & 6.425 & -0.12 & 9.756 & 9.766 & 0.10 & 14.8 \\
\hline 316.000 & 7.173 & 7.162 & -0.15 & 9.732 & 9.743 & 0.11 & 12.7 \\
\hline 320.000 & 7.917 & 7.904 & -0.17 & 9.710 & 9.721 & 0.12 & 11.1 \\
\hline 296.000 & 4.396 & 4.402 & 0.13 & 11.211 & 11.207 & -0.04 & 9.5 \\
\hline 300.000 & 5.397 & 5.405 & 0.15 & 11.196 & 11.191 & -0.04 & 8.0 \\
\hline 304.000 & 6.395 & 6.401 & 0.10 & 11.173 & 11.169 & -0.03 & 7.0 \\
\hline 308.000 & 7.378 & 7.381 & 0.04 & 11.141 & 11.140 & -0.01 & 6.3 \\
\hline 312.000 & 8.374 & 8.368 & -0.08 & 11.116 & 11.119 & 0.03 & 5.7 \\
\hline 316.000 & 9.388 & 9.373 & -0.16 & 11.099 & 11.105 & 0.05 & 5.2 \\
\hline 320.000 & 10.414 & 10.389 & -0.23 & 11.087 & 11.096 & 0.08 & 4.7 \\
\hline 292.000 & 3.811 & 3.792 & -0.50 & 11.522 & 11.534 & 0.11 & 8.3 \\
\hline 296.000 & 4.869 & 4.864 & -0.08 & 11.505 & 11.508 & 0.02 & 7.0 \\
\hline 300.000 & 5.948 & 5.935 & -0.23 & 11.485 & 11.492 & 0.06 & 6.1 \\
\hline 304.000 & 6.998 & 6.982 & -0.23 & 11.455 & 11.462 & 0.06 & 5.5 \\
\hline 308.000 & 8.035 & 8.024 & -0.13 & 11.424 & 11.429 & 0.04 & 5.0 \\
\hline 312.000 & 9.111 & 9.087 & -0.26 & 11.404 & 11.413 & 0.08 & 4.6 \\
\hline 316.000 & 10.198 & 10.167 & -0.31 & 11.390 & 11.401 & 0.09 & 4.2 \\
\hline 320.000 & 11.248 & 11.256 & 0.07 & 11.380 & 11.377 & -0.02 & 3.9 \\
\hline 288.000 & 3.971 & 3.962 & -0.23 & 12.194 & 12.197 & 0.03 & 4.7 \\
\hline 292.000 & 5.216 & 5.209 & -0.13 & 12.173 & 12.176 & 0.02 & 4.2 \\
\hline 296.000 & 6.439 & 6.433 & -0.09 & 12.146 & 12.148 & 0.02 & 3.9 \\
\hline 300.000 & 7.628 & 7.616 & -0.16 & 12.109 & 12.113 & 0.03 & 3.6 \\
\hline 304.000 & 8.847 & 8.823 & -0.28 & 12.082 & 12.089 & 0.06 & 3.3 \\
\hline 308.000 & 10.094 & 10.061 & -0.32 & 12.066 & 12.074 & 0.07 & 3.1 \\
\hline 312.000 & 11.357 & 11.314 & -0.38 & 12.054 & 12.064 & 0.08 & 2.9 \\
\hline 316.000 & 12.624 & 12.575 & -0.39 & 12.044 & 12.055 & 0.09 & 2.7 \\
\hline 320.000 & 13.901 & 13.839 & -0.45 & 12.036 & 12.049 & 0.11 & 2.6 \\
\hline 280.000 & 2.976 & 2.963 & -0.42 & 12.787 & 12.791 & 0.03 & 0.0 \\
\hline 284.000 & 4.398 & 4.392 & -0.15 & 12.768 & 12.770 & 0.01 & 0.0 \\
\hline 288.000 & 5.803 & 5.799 & -0.07 & 12.745 & 12.746 & 0.01 & 2.8 \\
\hline 292.000 & 7.140 & 7.133 & -0.09 & 12.707 & 12.709 & 0.01 & 2.7 \\
\hline 296.000 & 8.482 & 8.464 & -0.20 & 12.672 & 12.676 & 0.03 & 2.5 \\
\hline 300.000 & 9.884 & 9.852 & -0.33 & 12.652 & 12.659 & 0.05 & 2.4 \\
\hline 304.000 & 11.309 & 11.265 & -0.38 & 12.638 & 12.647 & 0.07 & 2.2 \\
\hline 308.000 & 12.742 & 12.690 & -0.41 & 12.627 & 12.637 & 0.07 & 2.1 \\
\hline
\end{tabular}


Table 6. PVT DATA (continued)

Data from Straty and Tsumura [19] (continued)

\begin{tabular}{|c|c|c|c|c|c|c|c|}
\hline $\begin{array}{l}\mathrm{T} \\
\mathrm{K}\end{array}$ & $\begin{array}{c}P, \operatorname{expt}_{\mathrm{MPa}} \\
\end{array}$ & $\begin{array}{c}\mathrm{P}, \mathrm{calc} \\
\mathrm{MPa}\end{array}$ & $\begin{array}{c}\mathrm{dev} \\
8\end{array}$ & $\begin{array}{l}\rho, \operatorname{expt} \\
\mathrm{mol} \cdot \mathrm{dm}^{-3}\end{array}$ & $\begin{array}{l}\rho, \quad \mathrm{calc} \\
\mathrm{mol} \cdot \mathrm{dm}^{-3}\end{array}$ & $\begin{array}{r}\text { dev } \\
8\end{array}$ & wt \\
\hline 312.000 & 14.182 & 14.119 & -0.44 & 12.618 & 12.629 & 0.09 & 2.0 \\
\hline 315.000 & 15.263 & 15.193 & -0.46 & 12.612 & 12.624 & 0.09 & 1.9 \\
\hline 320.000 & 17.066 & 16.983 & -0.49 & 12.602 & 12.615 & 0.10 & 1.8 \\
\hline 276.000 & 3.268 & 3.286 & 0.55 & 13.256 & 13.252 & -0.03 & 0.0 \\
\hline 280.000 & 4.843 & 4.857 & 0.28 & 13.234 & 13.231 & -0.02 & 0.0 \\
\hline 284.000 & 6.360 & 6.373 & 0.22 & 13.203 & 13.201 & -0.02 & 2.1 \\
\hline 288.000 & 7.797 & 7.800 & 0.04 & 13.160 & 13.159 & 0.00 & 2.0 \\
\hline 292.000 & 9.311 & 9.292 & -0.20 & 13.131 & 13.134 & 0.02 & 1.9 \\
\hline 296.000 & 10.870 & 10.842 & -0.26 & 13.115 & 13.119 & 0.03 & 1.8 \\
\hline 300.000 & 12.449 & 12.411 & -0.31 & 13.102 & 13.108 & 0.05 & 1.7 \\
\hline 300.000 & 12.449 & 12.411 & -0.31 & 13.102 & 13.108 & 0.05 & 1.7 \\
\hline 304.000 & 14.036 & 13.988 & -0.35 & 13.092 & 13.099 & 0.05 & 1.7 \\
\hline 308.000 & 15.626 & 15.568 & -0.37 & 13.083 & 13.091 & 0.06 & 1.6 \\
\hline 312.000 & 17.218 & 17.149 & -0.40 & 13.075 & 13.084 & 0.07 & 1.5 \\
\hline 316.000 & 18.804 & 18.729 & -0.40 & 13.068 & 13.077 & 0.07 & 1.4 \\
\hline 320.000 & 20.401 & 20.308 & -0.46 & 13.060 & 13.072 & 0.09 & 1.4 \\
\hline 272.000 & 3.626 & 3.714 & 2.44 & 13.670 & 13.655 & -0.11 & 0.0 \\
\hline 276.000 & 5.327 & 5.407 & 1.51 & 13.644 & 13.631 & -0.09 & 0.0 \\
\hline 280.000 & 6.919 & 6.994 & 1.08 & 13.604 & 13.593 & -0.08 & 1.7 \\
\hline 284.000 & 8.488 & 8.542 & 0.63 & 13.563 & 13.555 & -0.06 & 1.6 \\
\hline 288.000 & 10.166 & 10.199 & 0.32 & 13.540 & 13.535 & -0.03 & 1.6 \\
\hline 292.000 & 11.877 & 11.901 & 0.20 & 13.525 & 13.522 & -0.02 & 1.5 \\
\hline 296.000 & 13.604 & 13.619 & 0.11 & 13.514 & 13.512 & -0.01 & 1.4 \\
\hline 300.000 & 15.338 & 15.343 & 0.04 & 13.504 & 13.503 & -0.01 & 1.3 \\
\hline 304.000 & 17.094 & 17.068 & -0.15 & 13.495 & 13.498 & 0.02 & 1.3 \\
\hline 308.000 & 18.831 & 18.793 & -0.20 & 13.487 & 13.491 & 0.03 & 1.2 \\
\hline 312.000 & 20.567 & 20.515 & -0.25 & 13.480 & 13.485 & 0.04 & 1.2 \\
\hline 316.000 & 22.300 & 22.235 & -0.29 & 13.472 & 13.479 & 0.05 & 1.1 \\
\hline 320.000 & 24.032 & 23.950 & -0.34 & 13.465 & 13.474 & 0.06 & 1.1 \\
\hline 268.000 & 3.055 & 3.138 & 2.72 & 13.884 & 13.871 & -0.09 & 0.0 \\
\hline 272.000 & 4.851 & 4.921 & 1.45 & 13.859 & 13.849 & -0.08 & 0.0 \\
\hline 276.000 & 6.546 & 6.610 & 0.99 & 13.824 & 13.815 & -0.07 & 1.5 \\
\hline 280.000 & 8.153 & 8.192 & 0.49 & 13.778 & 13.772 & -0.04 & 1.5 \\
\hline 284.000 & 9.884 & 9.898 & 0.14 & 13.751 & 13.749 & -0.01 & 1.4 \\
\hline 288.000 & 11.668 & 11.670 & 0.01 & 13.735 & 13.735 & 0.00 & 1.4 \\
\hline
\end{tabular}


Table 6. PVT DATA (continued)

Data from Straty and Tsumura [19] (continued)

\begin{tabular}{|c|c|c|c|c|c|c|c|}
\hline $\begin{array}{l}\mathrm{T} \\
\mathrm{K}\end{array}$ & $\begin{array}{l}\mathrm{P}, \operatorname{expt} \\
\mathrm{MPa}\end{array}$ & $\begin{array}{c}\mathrm{P}, \underset{\mathrm{MPa}}{\mathrm{calc}} \\
\text { a }\end{array}$ & $\begin{array}{c}\text { dev } \\
8\end{array}$ & $\begin{array}{l}\rho, \operatorname{expt} \\
\mathrm{mol} \cdot \mathrm{dm}^{-3}\end{array}$ & $\begin{array}{l}\rho, \quad \mathrm{calc} \\
\mathrm{mol} \cdot \mathrm{dm}^{-3}\end{array}$ & $\begin{array}{r}\text { dev } \\
\%\end{array}$ & $w t$ \\
\hline 292.000 & 13.469 & 13.462 & -0.05 & 13.723 & 13.724 & 0.01 & 1.3 \\
\hline 296.000 & 15.277 & 15.262 & -0.10 & 13.713 & 13.715 & 0.01 & 1.2 \\
\hline 300.000 & 17.091 & 17.064 & -0.15 & 13.704 & 13.707 & 0.02 & 1.2 \\
\hline 304.000 & 18.901 & 18.865 & -0.19 & 13.696 & 13.699 & 0.03 & 1.1 \\
\hline 308.000 & 20.713 & 20.664 & -0.24 & 13.688 & 13.693 & 0.04 & 1.1 \\
\hline 312.000 & 22.524 & 22.460 & -0.29 & 13.681 & 13.687 & 0.05 & 1.1 \\
\hline 316.000 & 24.331 & 24.251 & -0.33 & 13.673 & 13.681 & 0.06 & 1.0 \\
\hline 320.000 & 26.130 & 26.037 & -0.36 & 13.667 & 13.675 & 0.06 & 1.0 \\
\hline 264.000 & 2.404 & 2.490 & 3.59 & 14.085 & 14.072 & -0.09 & 0.0 \\
\hline 268.000 & 4.265 & 4.366 & 2.37 & 14.062 & 14.048 & -0.10 & 0.0 \\
\hline 272.000 & 6.068 & 6.166 & 1.61 & 14.032 & 14.019 & -0.09 & 1.4 \\
\hline 276.000 & 7.720 & 7.798 & 1.02 & 13.983 & 13.973 & -0.07 & 1.4 \\
\hline 280.000 & 9.489 & 9.531 & 0.45 & 13.951 & 13.946 & -0.04 & 1.3 \\
\hline 284.000 & 11.339 & 11.364 & 0.22 & 13.933 & 13.930 & -0.02 & 1.3 \\
\hline 288.000 & 13.206 & 13.228 & 0.16 & 13.920 & 13.918 & -0.02 & 1.2 \\
\hline 292.000 & 15.086 & 15.102 & 0.10 & 13.909 & 13.908 & -0.01 & 1.2 \\
\hline 296.000 & 16.974 & 16.978 & 0.03 & 13.900 & 13.900 & 0.00 & 1.1 \\
\hline 300.000 & 18.862 & 18.854 & -0.04 & 13.892 & 13.892 & 0.01 & 1.1 \\
\hline 304.000 & 20.745 & 20.728 & -0.08 & 13.884 & 13.885 & 0.01 & 1.0 \\
\hline 308.000 & 22.630 & 22.598 & -0.14 & 13.876 & 13.879 & 0.02 & 1.0 \\
\hline 312.000 & 24.512 & 24.463 & -0.20 & 13.869 & 13.873 & 0.03 & 1.0 \\
\hline 316.000 & 26.389 & 26.323 & -0.25 & 13.862 & 13.868 & 0.04 & 0.9 \\
\hline 320.000 & 28.259 & 28.177 & -0.29 & 13.855 & 13.862 & 0.05 & 0.9 \\
\hline 260.000 & 2.686 & 2.852 & 6.19 & 14.414 & 14.394 & -0.14 & 0.0 \\
\hline 264.000 & 4.677 & 4.839 & 3.48 & 14.389 & 14.370 & -0.13 & 0.0 \\
\hline 268.000 & 6.547 & 6.695 & 2.27 & 14.351 & 14.335 & -0.12 & 1.2 \\
\hline 272.000 & 8.294 & 8.414 & 1.45 & 14.302 & 14.289 & -0.09 & 1.2 \\
\hline 276.000 & 10.221 & 10.310 & 0.87 & 14.275 & 14.266 & -0.07 & 1.1 \\
\hline 280.000 & 12.214 & 12.288 & 0.61 & 14.259 & 14.252 & -0.05 & 1.1 \\
\hline 284.000 & 14.226 & 14.289 & 0.45 & 14.247 & 14.241 & -0.04 & 1.0 \\
\hline 288.000 & 16.244 & 16.299 & 0.34 & 14.237 & 14.232 & -0.04 & 1.0 \\
\hline 292.000 & 18.264 & 18.310 & 0.25 & 14.228 & 14.223 & -0.03 & 1.0 \\
\hline 296.000 & 20.285 & 20.319 & 0.17 & 14.219 & 14.216 & -0.02 & 0.9 \\
\hline 300.000 & 22.302 & 22.324 & 0.10 & 14.211 & 14.209 & -0.01 & 0.9 \\
\hline 304.000 & 24.314 & 24.324 & 0.04 & 14.204 & 14.203 & -0.01 & 0.9 \\
\hline
\end{tabular}


Table 6. PVT DATA (continued)

Data from Straty and Tsumura [19] (continued)

\begin{tabular}{|c|c|c|c|c|c|c|c|}
\hline $\begin{array}{l}\mathrm{T} \\
\mathrm{K}\end{array}$ & $\begin{array}{l}\mathrm{P}, \operatorname{MPa}_{\mathrm{MPp}} \\
\text { exp }\end{array}$ & $\begin{array}{c}\mathrm{P}, \mathrm{MPa} \\
\mathrm{MPa}\end{array}$ & $\begin{array}{c}\text { dev } \\
\&\end{array}$ & $\begin{array}{l}\rho, \quad \operatorname{expt} \\
\mathrm{mol} \cdot \mathrm{dm}^{-3}\end{array}$ & $\begin{array}{l}\rho, \quad \operatorname{calc} \\
\mathrm{mol} \cdot \mathrm{dm}^{-3}\end{array}$ & $\begin{array}{r}\text { dev } \\
8\end{array}$ & wt \\
\hline 308.000 & 26.325 & 26.318 & -0.02 & 14.196 & 14.197 & 0.00 & 0.8 \\
\hline 312.000 & 28.329 & 28.306 & -0.08 & 14.189 & 14.191 & 0.01 & 0.8 \\
\hline 316.000 & 30.324 & 30.286 & -0.12 & 14.182 & 14.185 & 0.02 & 0.8 \\
\hline 320.000 & 32.317 & 32.260 & -0.18 & 14.176 & 14.180 & 0.03 & 0.8 \\
\hline 252.000 & 1.593 & 1.728 & 8.48 & 14.811 & 14.797 & -0.10 & 0.0 \\
\hline 256.000 & 3.706 & 3.936 & 6.19 & 14.790 & 14.767 & -0.16 & 0.0 \\
\hline 260.000 & 5.799 & 5.995 & 3.38 & 14.757 & 14.738 & -0.13 & 1.0 \\
\hline 264.000 & 7.656 & 7.820 & 2.14 & 14.705 & 14.689 & -0.11 & 1.0 \\
\hline 268.000 & 9.668 & 9.783 & 1.19 & 14.669 & 14.658 & -0.07 & 1.0 \\
\hline 272.000 & 11.808 & 11.904 & 0.81 & 14.650 & 14.641 & -0.06 & 0.9 \\
\hline 276.000 & 13.984 & 14.067 & 0.60 & 14.636 & 14.629 & -0.05 & 0.9 \\
\hline 280.000 & 16.165 & 16.243 & 0.48 & 14.625 & 14.619 & -0.04 & 0.9 \\
\hline 284.000 & 18.356 & 18.422 & 0.36 & 14.615 & 14.610 & -0.04 & 0.8 \\
\hline 288.000 & 20.548 & 20.600 & 0.25 & 14.607 & 14.602 & -0.03 & 0.8 \\
\hline 292.000 & 22.739 & 22.772 & 0.14 & 14.598 & 14.596 & -0.02 & 0.8 \\
\hline 296.000 & 24.876 & 24.940 & 0.26 & 14.591 & 14.586 & -0.03 & 0.8 \\
\hline 300.000 & 27.093 & 27.099 & 0.02 & 14.583 & 14.583 & 0.00 & 0.7 \\
\hline 304.000 & 29.263 & 29.252 & -0.04 & 14.576 & 14.576 & 0.01 & 0.7 \\
\hline 308.000 & 31.426 & 31.395 & -0.10 & 14.569 & 14.571 & 0.01 & 0.7 \\
\hline 312.000 & 33.582 & 33.531 & -0.15 & 14.562 & 14.565 & 0.02 & 0.7 \\
\hline 248.000 & 1.738 & 1.913 & 10.07 & 15.078 & 15.061 & -0.11 & 0.0 \\
\hline 252.000 & 3.975 & 4.228 & 6.37 & 15.056 & 15.032 & -0.15 & 0.0 \\
\hline 256.000 & 6.140 & 6.346 & 3.36 & 15.018 & 15.000 & -0.12 & 0.9 \\
\hline 260.000 & 8.064 & 8.225 & 2.00 & 14.963 & 14.949 & -0.09 & 0.9 \\
\hline 264.000 & 10.229 & 10.340 & 1.08 & 14.931 & 14.922 & -0.06 & 0.8 \\
\hline 268.000 & 12.503 & 12.594 & 0.72 & 14.914 & 14.906 & -0.05 & 0.8 \\
\hline 272.000 & 14.806 & 14.885 & 0.53 & 14.901 & 14.895 & -0.04 & 0.8 \\
\hline 276.000 & 17.120 & 17.187 & 0.39 & 14.890 & 14.885 & -0.03 & 0.8 \\
\hline 280.000 & 19.438 & 19.491 & 0.27 & 14.880 & 14.876 & -0.03 & 0.7 \\
\hline 284.000 & 21.750 & 21.791 & 0.19 & 14.871 & 14.869 & -0.02 & 0.7 \\
\hline 288.000 & 24.058 & 24.086 & 0.12 & 14.863 & 14.861 & -0.01 & 0.7 \\
\hline 292.000 & 26.364 & 26.373 & 0.03 & 14.855 & 14.855 & 0.00 & 0.7 \\
\hline 296.000 & 28.648 & 28.653 & 0.02 & 14.848 & 14.847 & 0.00 & 0.7 \\
\hline 300.000 & 30.950 & 30.923 & -0.09 & 14.840 & 14.842 & 0.01 & 0.6 \\
\hline 304.000 & 33.231 & 33.184 & -0.14 & 14.833 & 14.836 & 0.02 & 0.6 \\
\hline
\end{tabular}


Table 6. PVT DATA (continued)

Data from Straty and Tsumura [19] (continued)

\begin{tabular}{|c|c|c|c|c|c|c|c|}
\hline $\begin{array}{l}\mathrm{T} \\
\mathrm{K}\end{array}$ & $\mathrm{P}, \operatorname{MPa}_{\mathrm{MPa}}$ & $\begin{array}{c}\mathrm{P}, \underset{\mathrm{MPa}}{\mathrm{calc}} \\
\text { cal }\end{array}$ & $\begin{array}{c}\mathrm{dev} \\
8\end{array}$ & $\begin{array}{l}\rho, \operatorname{expt} \\
\mathrm{mol} \cdot \mathrm{dm}^{-3}\end{array}$ & $\begin{array}{l}\rho, \quad \text { calc } \\
\mathrm{mol} \cdot \mathrm{dm}^{-3}\end{array}$ & $\begin{array}{r}\text { dev } \\
\frac{8}{8}\end{array}$ & wt \\
\hline 308.000 & 35.502 & 35.436 & -0.19 & 14.826 & 14.830 & 0.03 & 0.6 \\
\hline 240.000 & 1.255 & 1.567 & 24.87 & 15.515 & 15.490 & -0.16 & 0.0 \\
\hline 244.000 & 3.637 & 4.039 & 11.05 & 15.487 & 15.456 & -0.20 & 0.0 \\
\hline 248.000 & 5.992 & 6.341 & 5.81 & 15.449 & 15.423 & -0.17 & 0.7 \\
\hline 252.000 & 8.032 & 8.333 & 3.74 & 15.392 & 15.369 & -0.15 & 0.7 \\
\hline 256.000 & 10.374 & 10.610 & 2.28 & 15.358 & 15.341 & -0.11 & 0.7 \\
\hline 260.000 & 12.853 & 13.068 & 1.67 & 15.340 & 15.325 & -0.10 & 0.7 \\
\hline 264.000 & 15.369 & 15.569 & 1.30 & 15.327 & 15.313 & -0.09 & 0.7 \\
\hline 268.000 & 17.894 & 18.084 & 1.06 & 15.316 & 15.303 & -0.08 & 0.6 \\
\hline 272.000 & 20.423 & 20.600 & 0.87 & 15.306 & 15.294 & -0.07 & 0.6 \\
\hline 276.000 & 22.946 & 23.112 & 0.72 & 15.297 & 15.286 & -0.07 & 0.6 \\
\hline 280.000 & 25.463 & 25.617 & 0.60 & 15.288 & 15.279 & -0.06 & 0.6 \\
\hline 284.000 & 27.973 & 28.113 & 0.50 & 15.280 & 15.272 & -0.05 & 0.6 \\
\hline 288.000 & 30.472 & 30.599 & 0.42 & 15.273 & 15.265 & -0.05 & 0.6 \\
\hline 292.000 & 32.967 & 33.074 & 0.32 & 15.265 & 15.259 & -0.04 & 0.5 \\
\hline 296.000 & 35.448 & 35.538 & 0.25 & 15.258 & 15.253 & -0.03 & 0.5 \\
\hline 240.000 & 2.202 & 2.481 & 12.68 & 15.586 & 15.565 & -0.14 & 0.0 \\
\hline 244.000 & 4.670 & 4.955 & 6.10 & 15.556 & 15.535 & -0.14 & 0.0 \\
\hline 248.000 & 6.891 & 7.130 & 3.47 & 15.508 & 15.490 & -0.11 & 0.7 \\
\hline 252.000 & 9.055 & 9.224 & 1.86 & 15.457 & 15.445 & -0.08 & 0.7 \\
\hline 256.000 & 11.532 & 11.658 & 1.09 & 15.433 & 15.424 & -0.06 & 0.7 \\
\hline 260.000 & 14.078 & 14.188 & 0.78 & 15.417 & 15.410 & -0.05 & 0.7 \\
\hline 264.000 & 16.647 & 16.743 & 0.58 & 15.405 & 15.399 & -0.04 & 0.6 \\
\hline 268.000 & 19.231 & 19.304 & 0.38 & 15.395 & 15.390 & -0.03 & 0.6 \\
\hline 272.000 & 21.804 & 21.863 & 0.27 & 15.385 & 15.382 & -0.02 & 0.6 \\
\hline 276.000 & 24.384 & 24.417 & 0.13 & 15.377 & 15.375 & -0.01 & 0.6 \\
\hline 280.000 & 26.947 & 26.962 & 0.05 & 15.368 & 15.367 & -0.01 & 0.6 \\
\hline 284.000 & 29.497 & 29.497 & 0.00 & 15.360 & 15.360 & 0.00 & 0.6 \\
\hline 288.000 & 32.045 & 32.021 & -0.07 & 15.353 & 15.354 & 0.01 & 0.5 \\
\hline 232.000 & 1.267 & 1.558 & 23.00 & 15.950 & 15.930 & -0.12 & 0.0 \\
\hline 236.000 & 3.851 & 4.228 & 9.79 & 15.920 & 15.895 & -0.16 & 0.0 \\
\hline 240.000 & 6.337 & 6.639 & 4.77 & 15.877 & 15.857 & -0.12 & 0.6 \\
\hline 244.000 & 8.536 & 8.754 & 2.56 & 15.817 & 15.803 & -0.09 & 0.6 \\
\hline 248.000 & 11.135 & 11.294 & 1.43 & 15.788 & 15.778 & -0.06 & 0.6 \\
\hline 252.000 & 13.865 & 13.996 & 0.94 & 15.771 & 15.763 & -0.05 & 0.6 \\
\hline
\end{tabular}


Table 6. PVT DATA (continued)

Data from Straty and Tsumura [19] (continued)

\begin{tabular}{|c|c|c|c|c|c|c|c|}
\hline $\begin{array}{l}\mathrm{T} \\
\mathrm{K}\end{array}$ & $\mathrm{P}, \underset{\mathrm{MPa}}{\operatorname{expt}}$ & $\begin{array}{c}\mathrm{P}, \mathrm{calc} \\
\mathrm{MPa}\end{array}$ & $\begin{array}{c}\text { dev } \\
\frac{8}{8}\end{array}$ & $\begin{array}{l}\rho, \quad \operatorname{expt} \\
\mathrm{mol} \cdot \mathrm{dm}^{-3}\end{array}$ & $\begin{array}{l}\rho, \quad \mathrm{calc} \\
\mathrm{mol} \cdot \mathrm{dm}^{-3}\end{array}$ & $\begin{array}{r}\mathrm{dev} \\
\frac{8}{8}\end{array}$ & wt \\
\hline 256.000 & 16.625 & 16.738 & 0.68 & 15.758 & 15.751 & -0.04 & 0.6 \\
\hline 260.000 & 19.395 & 19.492 & 0.50 & 15.747 & 15.741 & -0.04 & 0.5 \\
\hline 264.000 & 22.162 & 22.245 & 0.38 & 15.737 & 15.732 & -0.03 & 0.5 \\
\hline 268.000 & 24.927 & 24.990 & 0.25 & 15.728 & 15.724 & -0.02 & 0.5 \\
\hline 272.000 & 27.678 & 27.727 & 0.18 & 15.719 & 15.717 & -0.02 & 0.5 \\
\hline 276.000 & 30.417 & 30.453 & 0.12 & 15.711 & 15.709 & -0.01 & 0.5 \\
\hline 280.000 & 33.152 & 33.166 & 0.04 & 15.703 & 15.703 & 0.00 & 0.5 \\
\hline 284.000 & 35.872 & 35.867 & -0.02 & 15.696 & 15.696 & 0.00 & 0.5 \\
\hline 228.000 & 1.855 & 2.204 & 18.79 & 16.198 & 16.176 & -0.13 & 0.0 \\
\hline 232.000 & 4.583 & 4.984 & 8.76 & 16.167 & 16.143 & -0.15 & 0.0 \\
\hline 236.000 & 7.005 & 7.353 & 4.97 & 16.114 & 16.094 & -0.13 & 0.5 \\
\hline 240.000 & 9.421 & 9.670 & 2.65 & 16.062 & 16.048 & -0.09 & 0.5 \\
\hline 244.000 & 12.225 & 12.427 & 1.66 & 16.038 & 16.027 & -0.07 & 0.5 \\
\hline 248.000 & 15.113 & 15.296 & 1.21 & 16.023 & 16.013 & -0.06 & 0.5 \\
\hline 252.000 & 18.030 & 18.194 & 0.91 & 16.011 & 16.002 & -0.06 & 0.5 \\
\hline 256.000 & 20.949 & 21.098 & 0.71 & 16.000 & 15.992 & -0.05 & 0.5 \\
\hline 260.000 & 23.866 & 23.999 & 0.55 & 15.990 & 15.983 & -0.04 & 0.5 \\
\hline 264.000 & 26.772 & 26.891 & 0.44 & 15.981 & 15.975 & -0.04 & 0.5 \\
\hline 268.000 & 29.668 & 29.771 & 0.35 & 15.973 & 15.968 & -0.03 & 0.5 \\
\hline 272.000 & 32.554 & 32.639 & 0.26 & 15.964 & 15.960 & -0.03 & 0.4 \\
\hline 276.000 & 35.431 & 35.493 & 0.18 & 15.956 & 15.954 & -0.02 & 0.4 \\
\hline 220.000 & 1.430 & 1.745 & 22.03 & 16.567 & 16.550 & -0.10 & 0.0 \\
\hline 224.000 & 4.332 & 4.745 & 9.53 & 16.536 & 16.514 & -0.13 & 0.0 \\
\hline 228.000 & 6.923 & 7.274 & 5.07 & 16.483 & 16.465 & -0.11 & 0.5 \\
\hline 232.000 & 9.458 & 9.697 & 2.52 & 16.428 & 16.416 & -0.08 & 0.5 \\
\hline 236.000 & 12.475 & 12.654 & 1.43 & 16.403 & 16.394 & -0.06 & 0.5 \\
\hline 240.000 & 15.597 & 15.745 & 0.94 & 16.387 & 16.380 & -0.04 & 0.5 \\
\hline 244.000 & 18.742 & 18.870 & 0.68 & 16.375 & 16.369 & -0.04 & 0.4 \\
\hline 248.000 & 21.890 & 22.003 & 0.51 & 16.364 & 16.359 & -0.03 & 0.4 \\
\hline 252.000 & 25.039 & 25.131 & 0.37 & 16.354 & 16.350 & -0.03 & 0.4 \\
\hline 256.000 & 28.172 & 28.249 & 0.27 & 16.345 & 16.341 & -0.02 & 0.4 \\
\hline 260.000 & 31.294 & 31.354 & 0.19 & 16.336 & 16.333 & -0.02 & 0.4 \\
\hline 264.000 & 34.399 & 34.444 & 0.13 & 16.328 & 16.326 & -0.01 & 0.4 \\
\hline 216.000 & 2.104 & 2.532 & 20.33 & 16.798 & 16.776 & -0.13 & 0.0 \\
\hline 220.000 & 5.125 & 5.556 & 8.41 & 16.761 & 16.740 & -0.13 & 0.0 \\
\hline
\end{tabular}


Table 6. PVT DATA (continued)

Data from Straty and Tsumura [19] (continued)

\begin{tabular}{|c|c|c|c|c|c|c|c|}
\hline $\begin{array}{l}\mathrm{T} \\
\mathrm{K}\end{array}$ & $\begin{array}{l}\mathrm{P}, \underset{\mathrm{MPa}}{\operatorname{expt}} \\
\text { exa }\end{array}$ & $\begin{array}{c}\mathrm{P}, \mathrm{calc} \\
\mathrm{MPa}\end{array}$ & $\begin{array}{c}\text { dev } \\
\frac{8}{8}\end{array}$ & $\begin{array}{l}\rho, \operatorname{expt} \\
\mathrm{mol} \cdot \cdot \mathrm{dm}^{-3}\end{array}$ & $\begin{array}{l}\rho, \quad \text { calc } \\
\mathrm{mol} \cdot \mathrm{dm}^{-3}\end{array}$ & $\begin{array}{r}\text { dev } \\
8\end{array}$ & wt \\
\hline 224.000 & 7.612 & 7.970 & 4.70 & 16.698 & 16.681 & -0.10 & 0.4 \\
\hline 228.000 & 10.476 & 10.703 & 2.16 & 16.654 & 16.643 & -0.07 & 0.4 \\
\hline 232.000 & 13.690 & 13.870 & 1.31 & 16.633 & 16.625 & -0.05 & 0.4 \\
\hline 236.000 & 16.976 & 17.132 & 0.92 & 16.618 & 16.611 & -0.04 & 0.4 \\
\hline 240.000 & 20.279 & 20.419 & 0.69 & 16.606 & 16.600 & -0.04 & 0.4 \\
\hline 244.000 & 23.587 & 23.698 & 0.47 & 16.595 & 16.590 & -0.03 & 0.4 \\
\hline 248.000 & 26.880 & 26.989 & 0.41 & 16.586 & 16.581 & -0.03 & 0.4 \\
\hline 252.000 & 30.162 & 30.257 & 0.32 & 16.576 & 16.573 & -0.02 & 0.4 \\
\hline 256.000 & 33.425 & 33.511 & 0.25 & 16.568 & 16.564 & -0.02 & 0.4 \\
\hline 212.000 & 3.427 & 3.962 & 15.60 & 17.048 & 17.024 & -0.14 & 0.0 \\
\hline 216.000 & 6.405 & 6.821 & 6.49 & 16.998 & 16.979 & -0.11 & 0.0 \\
\hline 220.000 & 8.998 & 9.274 & 3.06 & 16.933 & 16.921 & -0.07 & 0.4 \\
\hline 224.000 & 12.271 & 12.462 & 1.55 & 16.904 & 16.896 & -0.05 & 0.4 \\
\hline 228.000 & 15.716 & 15.871 & 0.98 & 16.887 & 16.880 & -0.04 & 0.4 \\
\hline 232.000 & 19.203 & 19.333 & 0.68 & 16.874 & 16.868 & -0.03 & 0.4 \\
\hline 236.000 & 22.690 & 22.807 & 0.51 & 16.862 & 16.857 & -0.03 & 0.4 \\
\hline 240.000 & 26.177 & 26.276 & 0.38 & 16.852 & 16.848 & -0.02 & 0.4 \\
\hline 244.000 & 29.648 & 29.733 & 0.29 & 16.842 & 16.839 & -0.02 & 0.3 \\
\hline 248.000 & 33.104 & 33.174 & 0.21 & 16.833 & 16.830 & -0.02 & 0.3 \\
\hline 200.000 & 0.680 & 0.710 & 4.39 & 17.445 & 17.444 & -0.01 & 0.0 \\
\hline 204.000 & 3.776 & 4.240 & 12.29 & 17.411 & 17.393 & -0.11 & 0.0 \\
\hline 208.000 & 6.803 & 7.097 & 4.31 & 17.354 & 17.342 & -0.07 & 0.3 \\
\hline 212.000 & 9.657 & 9.794 & 1.41 & 17.293 & 17.287 & -0.03 & 0.3 \\
\hline 216.000 & 13.235 & 13.296 & 0.46 & 17.267 & 17.264 & -0.01 & 0.3 \\
\hline 220.000 & 16.967 & 16.990 & 0.14 & 17.250 & 17.249 & -0.01 & 0.3 \\
\hline 224.000 & 20.726 & 20.732 & 0.03 & 17.237 & 17.236 & 0.00 & 0.3 \\
\hline 228.000 & 24.489 & 24.481 & -0.03 & 17.225 & 17.225 & 0.00 & 0.3 \\
\hline 232.000 & 28.240 & 28.223 & -0.06 & 17.215 & 17.215 & 0.00 & 0.3 \\
\hline 236.000 & 31.981 & 31.950 & -0.10 & 17.205 & 17.206 & 0.01 & 0.3 \\
\hline 240.000 & 35.705 & 35.657 & -0.13 & 17.196 & 17.198 & 0.01 & 0.3 \\
\hline 196.000 & 0.494 & 0.663 & 34.03 & 17.619 & 17.613 & -0.04 & 0.0 \\
\hline 200.000 & 3.574 & 4.209 & 17.79 & 17.581 & 17.557 & -0.14 & 0.0 \\
\hline 204.000 & 6.742 & 7.152 & 6.07 & 17.524 & 17.508 & -0.09 & 0.3 \\
\hline 208.000 & 9.662 & 9.881 & 2.27 & 17.461 & 17.453 & -0.05 & 0.3 \\
\hline 212.000 & 13.358 & 13.495 & 1.02 & 17.434 & 17.429 & -0.03 & 0.3 \\
\hline
\end{tabular}


Table 6. PVT DATA (continued)

Data from Straty and Tsumura [19] (continued)

\begin{tabular}{|c|c|c|c|c|c|c|c|}
\hline $\begin{array}{l}\mathrm{T} \\
\mathrm{K}\end{array}$ & $\begin{array}{c}\mathrm{P}, \mathrm{MPa}_{\mathrm{MPpt}} \\
\text { exa }\end{array}$ & $\begin{array}{c}\mathrm{P}, \mathrm{MPa} \\
\mathrm{Malc}\end{array}$ & $\begin{array}{c}\mathrm{dev} \\
8\end{array}$ & $\begin{array}{l}\rho, \operatorname{expt} \\
\mathrm{mol} \cdot \mathrm{dm}^{-3}\end{array}$ & $\begin{array}{l}\rho, \quad \mathrm{calc} c \\
\mathrm{~mol} \cdot \mathrm{dm}^{-3}\end{array}$ & $\begin{array}{r}\text { dev } \\
\&\end{array}$ & wt \\
\hline 216.000 & 17.223 & 17.318 & 0.55 & 17.417 & 17.414 & -0.02 & 0.3 \\
\hline 220.000 & 21.111 & 21.191 & 0.38 & 17.404 & 17.401 & -0.02 & 0.3 \\
\hline 224.000 & 25.013 & 25.073 & 0.24 & 17.392 & 17.390 & -0.01 & 0.3 \\
\hline 228.000 & 28.895 & 28.947 & 0.18 & 17.382 & 17.380 & -0.01 & 0.3 \\
\hline 232.000 & 32.752 & 32.805 & 0.16 & 17.372 & 17.370 & -0.01 & 0.3 \\
\hline 192.000 & 0.987 & 1.299 & 31.72 & 17.815 & 17.804 & -0.06 & 0.0 \\
\hline 196.000 & 4.453 & 4.904 & 10.13 & 17.775 & 17.759 & -0.09 & 0.0 \\
\hline 200.000 & 7.393 & 7.721 & 4.43 & 17.709 & 17.698 & -0.07 & 0.3 \\
\hline 204.000 & 10.698 & 10.834 & 1.27 & 17.657 & 17.653 & -0.03 & 0.3 \\
\hline 208.000 & 14.625 & 14.695 & 0.48 & 17.634 & 17.632 & -0.01 & 0.3 \\
\hline 212.000 & 18.667 & 18.707 & 0.22 & 17.618 & 17.617 & -0.01 & 0.3 \\
\hline 216.000 & 22.737 & 22.756 & 0.08 & 17.606 & 17.605 & 0.00 & 0.3 \\
\hline 220.000 & 26.805 & 26.807 & 0.01 & 17.594 & 17.594 & 0.00 & 0.3 \\
\hline 224.000 & 30.857 & 30.846 & -0.03 & 17.584 & 17.584 & 0.00 & 0.3 \\
\hline 228.000 & 34.886 & 34.867 & -0.05 & $17.5 \% \div$ & 17.575 & 0.00 & 0.3 \\
\hline 188.000 & 1.982 & 2.377 & 19.95 & 18.021 & 18.008 & -0.07 & 0.0 \\
\hline 192.000 & 5.596 & 5.995 & 7.13 & 17.976 & 17.963 & -0.07 & 0.0 \\
\hline 196.000 & 8.433 & 8.655 & 2.63 & 17.903 & 17.896 & -0.04 & 0.3 \\
\hline 200.000 & 12.237 & 12.311 & 0.60 & 17.866 & 17.863 & -0.01 & 0.3 \\
\hline 204.000 & 16.408 & 16.433 & 0.15 & 17.846 & 17.845 & 0.00 & 0.3 \\
\hline 208.000 & 20.655 & 20.653 & -0.01 & 17.831 & 17.831 & 0.00 & 0.3 \\
\hline 212.000 & 24.909 & 24.895 & -0.06 & 17.819 & 17.819 & 0.00 & 0.3 \\
\hline 216.000 & 29.154 & 29.132 & -0.08 & 17.808 & 17.808 & 0.00 & 0.3 \\
\hline 220.000 & 33.347 & 33.354 & 0.02 & 17.797 & 17.797 & 0.00 & 0.3 \\
\hline 180.000 & 0.525 & 0.583 & 11.20 & 18.295 & 18.293 & -0.01 & 0.0 \\
\hline 184.000 & 3.972 & 4.528 & 13.98 & 18.253 & 18.236 & -0.09 & 0.0 \\
\hline 188.000 & 7.206 & 7.527 & 4.45 & 18.186 & 18.176 & -0.05 & 0.3 \\
\hline 192.000 & 10.667 & 10.774 & 1.01 & 18.128 & 18.125 & -0.02 & 0.3 \\
\hline 196.000 & 14.966 & 14.999 & 0.22 & 18.104 & 18.103 & -0.01 & 0.3 \\
\hline 200.000 & 19.418 & 19.420 & 0.01 & 18.087 & 18.087 & 0.00 & 0.2 \\
\hline 204.000 & 23.905 & 23.887 & -0.08 & 18.074 & 18.074 & 0.00 & 0.2 \\
\hline 208.000 & 28.379 & 28.357 & -0.08 & 18.062 & 18.063 & 0.00 & 0.2 \\
\hline 212.000 & 32.842 & 32.813 & -0.09 & 18.051 & 18.052 & 0.00 & 0.2 \\
\hline 176.000 & 1.107 & 1.594 & 43.95 & 18.488 & 18.474 & -0.08 & 0.0 \\
\hline 180.000 & 4.958 & 5.529 & 11.51 & 18.442 & 18.426 & -0.09 & 0.0 \\
\hline
\end{tabular}


Table 6. PVT DATA (continued)

Data from Straty and Tsumura [19] (continued)

\begin{tabular}{|c|c|c|c|c|c|c|c|}
\hline $\begin{array}{l}\mathrm{T} \\
\mathrm{K}\end{array}$ & 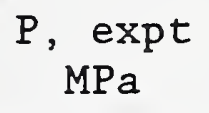 & $\begin{array}{c}\mathrm{P}, \underset{\mathrm{MPa}}{\mathrm{calc}} \\
\text { a }\end{array}$ & $\begin{array}{c}\mathrm{dev} \\
\frac{8}{8}\end{array}$ & $\begin{array}{l}\rho, \text { expt } \\
\mathrm{mol} \cdot \cdot \mathrm{dm}^{-3}\end{array}$ & $\begin{array}{l}\rho, \quad \text { calc } \\
\mathrm{mol} \cdot \mathrm{dm}^{-3}\end{array}$ & $\begin{array}{r}\mathrm{dev} \\
8\end{array}$ & wt \\
\hline 184.000 & 7.979 & 8.391 & 5.15 & 18.368 & 18.356 & -0.07 & 0.2 \\
\hline 188.000 & 11.972 & 12.159 & 1.56 & 18.323 & 18.318 & -0.03 & 0.2 \\
\hline 192.000 & 16.519 & 16.654 & 0.82 & 18.302 & 18.298 & -0.02 & 0.2 \\
\hline 196.000 & 21.159 & 21.290 & 0.62 & 18.286 & 18.282 & -0.02 & 0.2 \\
\hline 200.000 & 25.852 & 25.954 & 0.40 & 18.273 & 18.270 & -0.02 & 0.2 \\
\hline 204.000 & 30.230 & 30.634 & 1.33 & 18.262 & 18.251 & -0.06 & 0.2 \\
\hline 208.000 & 35.087 & 35.263 & 0.50 & 18.251 & 18.246 & -0.03 & 0.2 \\
\hline 172.000 & 1.245 & 1.646 & 32.18 & 18.650 & 18.639 & -0.06 & 0.0 \\
\hline 176.000 & 5.209 & 5.615 & 7.79 & 18.602 & 18.590 & -0.06 & 0.0 \\
\hline 180.000 & 8.278 & 8.461 & 2.21 & 18.525 & 18.520 & -0.03 & 0.2 \\
\hline 184.000 & 12.481 & 12.478 & -0.02 & 18.483 & 18.484 & 0.00 & 0.2 \\
\hline 188.000 & 17.206 & 17.152 & -0.31 & 18.462 & 18.464 & 0.01 & 0.2 \\
\hline 192.000 & 22.033 & 21.956 & -0.35 & 18.447 & 18.449 & 0.01 & 0.2 \\
\hline 196.000 & 26.867 & 26.788 & -0.29 & 18.434 & 18.436 & 0.01 & 0.2 \\
\hline 200.000 & 31.694 & 31.613 & -0.26 & 18.422 & 18.425 & 0.01 & 0.2 \\
\hline 204.000 & 36.490 & 36.416 & -0.20 & 18.412 & 18.414 & 0.01 & 0.2 \\
\hline 168.000 & 1.730 & 1.957 & 13.11 & 18.817 & 18.811 & -0.03 & 0.0 \\
\hline 172.000 & 5.761 & 6.036 & 4.78 & 18.768 & 18.761 & -0.04 & 0.0 \\
\hline 176.000 & 8.876 & 8.934 & 0.66 & 18.691 & 18.689 & -0.01 & 0.2 \\
\hline 180.000 & 13.300 & 13.310 & 0.08 & 18.655 & 18.655 & 0.00 & 0.2 \\
\hline 184.000 & 18.265 & 18.189 & -0.42 & 18.635 & 18.637 & 0.01 & 0.2 \\
\hline 188.000 & 23.327 & 23.180 & -0.63 & 18.620 & 18.623 & 0.02 & 0.2 \\
\hline 192.000 & 28.346 & 28.196 & -0.53 & 18.607 & 18.611 & 0.02 & 0.2 \\
\hline 196.000 & 33.368 & 33.200 & -0.50 & 18.595 & 18.599 & 0.02 & 0.2 \\
\hline 162.000 & 2.082 & 2.577 & 23.81 & 19.067 & 19.055 & -0.06 & 0.0 \\
\hline 164.000 & 4.258 & 4.760 & 11.78 & 19.043 & 19.031 & -0.06 & 0.0 \\
\hline 166.000 & 6.191 & 6.590 & 6.45 & 19.011 & 19.001 & -0.05 & 0.2 \\
\hline 168.000 & 7.644 & 7.946 & 3.95 & 18.968 & 18.961 & -0.04 & 0.2 \\
\hline 170.000 & 9.532 & 9.649 & 1.23 & 18.935 & 18.932 & -0.02 & 0.2 \\
\hline 172.000 & 11.878 & 11.910 & 0.28 & 18.916 & 18.915 & 0.00 & 0.2 \\
\hline 176.000 & 16.991 & 16.962 & -0.17 & 18.892 & 18.893 & 0.00 & 0.2 \\
\hline 180.000 & 22.263 & 22.210 & -0.24 & 18.876 & 18.877 & 0.01 & 0.2 \\
\hline 184.000 & 27.548 & 27.502 & -0.17 & 18.862 & 18.863 & 0.01 & 0.2 \\
\hline 188.000 & 32.834 & 32.788 & -0.14 & 18.850 & 18.851 & 0.01 & 0.2 \\
\hline 152.000 & 1.208 & 1.409 & 16.66 & 19.424 & 19.420 & -0.02 & 0.0 \\
\hline
\end{tabular}


Table 6. PVT DATA (continued)

Data from Straty and Tsumura [19] (continued)

\begin{tabular}{|c|c|c|c|c|c|c|c|}
\hline $\begin{array}{l}\mathrm{T} \\
\mathrm{K}\end{array}$ & $\begin{array}{l}\mathrm{P}, \mathrm{MPa}_{\mathrm{MPpt}} \\
\text { exp }\end{array}$ & $\begin{array}{c}\mathrm{P}, \mathrm{calc} \\
\mathrm{MPa}\end{array}$ & $\begin{array}{c}\mathrm{dev} \\
8\end{array}$ & $\begin{array}{l}\rho, \text { expt } \\
\mathrm{mol} \cdot \mathrm{dm}^{-3}\end{array}$ & $\begin{array}{l}\rho, \quad \mathrm{calc} \\
\mathrm{mol} \cdot \mathrm{dm}^{-3}\end{array}$ & $\begin{array}{r}\mathrm{dev} \\
8\end{array}$ & wt \\
\hline 156.000 & 5.598 & 5.785 & 3.35 & 19.369 & 19.365 & -0.02 & 0.0 \\
\hline 160.000 & 8.963 & 8.838 & -1.40 & 19.288 & 19.290 & 0.01 & 0.2 \\
\hline 164.000 & 14.047 & 13.738 & -2.20 & 19.250 & 19.257 & 0.04 & 0.2 \\
\hline 168.000 & 19.651 & 19.295 & -1.81 & 19.230 & 19.237 & 0.04 & 0.2 \\
\hline 172.000 & 25.340 & 24.980 & -1.42 & 19.214 & 19.222 & 0.04 & 0.2 \\
\hline 176.000 & 31.037 & 30.686 & -1.13 & 19.201 & 19.208 & 0.04 & 0.2 \\
\hline 180.000 & 36.709 & 36.373 & -0.92 & 19.189 & 19.196 & 0.04 & 0.2 \\
\hline 144.000 & 0.939 & 1.126 & 20.00 & 19.722 & 19.718 & -0.02 & 0.0 \\
\hline 148.000 & 5.449 & 5.837 & 7.12 & 19.667 & 19.660 & -0.04 & 0.0 \\
\hline 152.000 & 8.924 & 8.970 & 0.52 & 19.584 & 19.583 & 0.00 & 0.2 \\
\hline 156.000 & 14.285 & 14.136 & -1.04 & 19.545 & 19.548 & 0.02 & 0.2 \\
\hline 160.000 & 20.232 & 20.056 & -0.87 & 19.524 & 19.528 & 0.02 & 0.2 \\
\hline 164.000 & 26.288 & 26.120 & -0.64 & 19.508 & 19.511 & 0.02 & 0.2 \\
\hline 168.000 & 32.344 & 32.205 & -0.43 & 19.494 & 19.497 & 0.01 & 0.1 \\
\hline 172.000 & 38.372 & 38.267 & -0.27 & 19.482 & 19.484 & 0.01 & 0.1 \\
\hline 136.000 & 1.369 & 1.555 & 13.56 & 20.030 & 20.026 & -0.02 & 0.0 \\
\hline 138.000 & 3.783 & 4.408 & 16.53 & 20.008 & 19.997 & -0.06 & 0.0 \\
\hline 140.000 & 6.057 & 6.414 & 5.89 & 19.972 & 19.965 & -0.03 & 0.1 \\
\hline 142.000 & 7.656 & 7.859 & 2.66 & 19.926 & 19.922 & -0.02 & 0.1 \\
\hline 144.000 & 9.846 & 9.789 & -0.58 & 19.890 & 19.891 & 0.01 & 0.1 \\
\hline 148.000 & 15.772 & 15.587 & -1.17 & 19.856 & 19.859 & 0.02 & 0.1 \\
\hline 152.000 & 22.175 & 21.987 & -0.85 & 19.836 & 19.839 & 0.02 & 0.1 \\
\hline 156.000 & 28.659 & 28.504 & -0.54 & 19.820 & 19.823 & 0.01 & 0.1 \\
\hline 160.000 & 35.147 & 35.028 & -0.34 & 19.806 & 19.808 & 0.01 & 0.1 \\
\hline 128.000 & 0.671 & 0.718 & 6.99 & 20.312 & 20.311 & 0.00 & 0.0 \\
\hline 130.000 & 2.929 & 3.603 & 23.04 & 20.287 & 20.276 & -0.06 & 0.0 \\
\hline 132.000 & 5.498 & 5.917 & 7.62 & 20.254 & 20.247 & -0.04 & 0.1 \\
\hline 134.000 & 7.237 & 7.512 & 3.80 & 20.209 & 20.204 & -0.02 & 0.1 \\
\hline 136.000 & 9.249 & 9.261 & 0.13 & 20.167 & 20.167 & 0.00 & 0.1 \\
\hline 138.000 & 12.124 & 12.007 & -0.96 & 20.144 & 20.146 & 0.01 & 0.1 \\
\hline 140.000 & 15.338 & 15.194 & -0.94 & 20.129 & 20.131 & 0.01 & 0.1 \\
\hline 142.000 & 18.702 & 18.541 & -0.86 & 20.117 & 20.120 & 0.01 & 0.1 \\
\hline 144.000 & 22.109 & 21.964 & -0.66 & 20.107 & 20.110 & 0.01 & 0.1 \\
\hline 146.000 & 25.532 & 25.421 & -0.43 & 20.099 & 20.100 & 0.01 & 0.1 \\
\hline 148.000 & 28.968 & 28.891 & -0.26 & 20.091 & 20.092 & 0.01 & 0.1 \\
\hline
\end{tabular}


Table 6. PVT DATA (continued)

Data from Straty and Tsumura [19] (continued)

\begin{tabular}{|c|c|c|c|c|c|c|c|}
\hline $\begin{array}{l}\mathrm{T} \\
\mathrm{K}\end{array}$ & $\begin{array}{l}\mathrm{P}, \operatorname{MPa}_{\mathrm{MPa}} \\
\text { expt }\end{array}$ & $\begin{array}{c}\mathrm{P}, \mathrm{calc} \\
\mathrm{MPa}\end{array}$ & $\begin{array}{c}\text { dev } \\
8\end{array}$ & $\begin{array}{l}\rho, \operatorname{expt} \\
\mathrm{mol} \cdot \mathrm{dm}^{-3}\end{array}$ & $\begin{array}{l}\rho, \quad \text { calc } \\
\mathrm{mol} \cdot \mathrm{dm}^{-3}\end{array}$ & $\begin{array}{r}\mathrm{dev} \\
8\end{array}$ & wt \\
\hline 150.000 & 32.409 & 32.362 & -0.15 & 20.083 & 20.084 & 0.00 & 0.1 \\
\hline 152.000 & 35.831 & 35.830 & 0.00 & 20.077 & 20.077 & 0.00 & 0.1 \\
\hline 120.000 & 1.275 & 1.601 & 25.62 & 20.621 & 20.616 & -0.02 & 0.0 \\
\hline 122.000 & 3.866 & 4.455 & 15.22 & 20.592 & 20.583 & -0.04 & 0.0 \\
\hline 124.000 & 6.244 & 6.529 & 4.57 & 20.553 & 20.548 & -0.02 & 0.1 \\
\hline 126.000 & 7.924 & 8.019 & 1.20 & 20.504 & 20.503 & -0.01 & 0.1 \\
\hline 128.000 & 10.479 & 10.296 & -1.74 & 20.470 & 20.472 & 0.01 & 0.1 \\
\hline 130.000 & 13.750 & 13.490 & -1.89 & 20.450 & 20.454 & 0.02 & 0.1 \\
\hline 132.000 & 17.276 & 17.006 & -1.57 & 20.436 & 20.440 & 0.02 & 0.1 \\
\hline 134.000 & 20.890 & 20.652 & -1.14 & 20.425 & 20.429 & 0.02 & 0.1 \\
\hline 136.000 & 24.552 & 24.354 & -0.81 & 20.416 & 20.419 & 0.02 & 0.1 \\
\hline 138.000 & 28.231 & 28.082 & -0.53 & 20.407 & 20.410 & 0.01 & 0.1 \\
\hline 140.000 & 31.909 & 31.817 & -0.29 & 20.400 & 20.401 & 0.01 & 0.1 \\
\hline 142.000 & 35.582 & 35.549 & -0.09 & 20.392 & 20.393 & 0.00 & 0.1 \\
\hline 116.000 & 1.964 & 2.189 & 11.49 & 20.776 & 20.772 & -0.02 & 0.0 \\
\hline 120.000 & 6.813 & 6.913 & 1.47 & 20.702 & 20.701 & -0.01 & 0.0 \\
\hline 124.000 & 11.683 & 11.303 & -3.25 & 20.627 & 20.632 & 0.03 & 0.1 \\
\hline 128.000 & 18.891 & 18.496 & -2.09 & 20.597 & 20.603 & 0.03 & 0.1 \\
\hline 132.000 & 26.464 & 26.180 & -1.07 & 20.577 & 20.581 & 0.02 & 0.1 \\
\hline 136.000 & 34.068 & 33.949 & -0.35 & 20.561 & 20.563 & 0.01 & 0.1 \\
\hline 112.000 & 1.191 & 1.488 & 24.89 & 20.911 & 20.907 & -0.02 & 0.0 \\
\hline 114.000 & 3.874 & 4.504 & 16.26 & 20.882 & 20.873 & -0.04 & 0.0 \\
\hline 116.000 & 6.316 & 6.606 & 4.60 & 20.841 & 20.836 & -0.02 & 0.1 \\
\hline 118.000 & 8.048 & 8.126 & 0.98 & 20.791 & 20.790 & -0.01 & 0.1 \\
\hline 120.000 & 10.802 & 10.592 & -1.94 & 20.757 & 20.760 & 0.01 & 0.1 \\
\hline 122.000 & 14.300 & 14.035 & -1.85 & 20.737 & 20.741 & 0.02 & 0.1 \\
\hline 124.000 & 18.049 & 17.814 & -1.30 & 20.724 & 20.727 & 0.02 & 0.1 \\
\hline 126.000 & 21.955 & 21.717 & -1.09 & 20.712 & 20.716 & 0.02 & 0.1 \\
\hline 128.000 & 25.862 & 25.688 & -0.67 & 20.703 & 20.705 & 0.01 & 0.1 \\
\hline 130.000 & 29.781 & 29.683 & -0.33 & 20.694 & 20.696 & 0.01 & 0.1 \\
\hline 132.000 & 33.696 & 33.684 & -0.04 & 20.687 & 20.687 & 0.00 & 0.1 \\
\hline 108.000 & 1.823 & 2.144 & 17.61 & 21.065 & 21.061 & -0.02 & 0.1 \\
\hline 110.000 & 4.710 & 5.099 & 8.25 & 21.034 & 21.029 & -0.03 & 0.1 \\
\hline 112.000 & 6.842 & 7.058 & 3.16 & 20.990 & 20.987 & -0.01 & 0.1 \\
\hline 114.000 & 8.815 & 8.709 & -1.20 & 20.942 & 20.943 & 0.01 & 0.1 \\
\hline
\end{tabular}


Table 6. PVT DATA (continued)

Data from Straty and Tsumura [19] (continued)

\begin{tabular}{|c|c|c|c|c|c|c|c|}
\hline $\begin{array}{l}\mathrm{T} \\
\mathrm{K}\end{array}$ & $\begin{array}{l}\mathrm{P}, \operatorname{MPa}_{\mathrm{MPa}} \\
\text { expt }\end{array}$ & $\begin{array}{c}\mathrm{P}, \mathrm{calc} \\
\mathrm{MPa}\end{array}$ & $\begin{array}{c}\text { dev } \\
8\end{array}$ & $\begin{array}{l}\rho, \operatorname{expt} \\
\mathrm{mol} \cdot \mathrm{dm}^{-3}\end{array}$ & $\begin{array}{l}\rho, \text { calc } \\
\mathrm{mol} \cdot \mathrm{dm}^{-3}\end{array}$ & $\begin{array}{r}\text { dev } \\
8\end{array}$ & wt \\
\hline 116.000 & 12.003 & 11.690 & -2.61 & 20.913 & 20.918 & 0.02 & 0.1 \\
\hline 118.000 & 15.749 & 15.417 & -2.11 & 20.896 & 20.901 & 0.02 & 0.1 \\
\hline 120.000 & 19.692 & 19.404 & -1.46 & 20.883 & 20.887 & 0.02 & 0.1 \\
\hline 122.000 & 23.719 & 23.498 & -0.93 & 20.873 & 20.876 & 0.01 & 0.1 \\
\hline 124.000 & 27.761 & 27.642 & -0.43 & 20.863 & 20.865 & 0.01 & 0.1 \\
\hline 126.000 & 31.841 & 31.800 & -0.13 & 20.855 & 20.855 & 0.00 & 0.1 \\
\hline 128.000 & 35.903 & 35.961 & 0.16 & 20.847 & 20.846 & 0.00 & 0.1 \\
\hline 104.000 & 1.183 & 1.330 & 12.45 & 21.198 & 21.196 & -0.01 & 0.0 \\
\hline 108.000 & 6.451 & 6.517 & 1.02 & 21.125 & 21.124 & 0.00 & 0.0 \\
\hline 112.000 & 11.285 & 10.793 & -4.36 & 21.041 & 21.048 & 0.03 & 0.1 \\
\hline 116.000 & 19.066 & 18.587 & -2.51 & 21.008 & 21.015 & 0.03 & 0.1 \\
\hline 118.000 & 23.202 & 22.786 & -1.79 & 20.997 & 21.003 & 0.03 & 0.1 \\
\hline 120.000 & 27.359 & 27.048 & -1.14 & 20.988 & 20.992 & 0.02 & 0.1 \\
\hline 122.000 & 31.531 & 31.335 & -0.62 & 20.979 & 20.982 & 0.01 & 0.1 \\
\hline 100.000 & 2.250 & 1.452 & -35.45 & 21.343 & 21.354 & 0.05 & 0.0 \\
\hline 102.000 & 4.953 & 4.636 & -6.40 & 21.313 & 21.317 & 0.02 & 0.0 \\
\hline 104.000 & 7.026 & 6.514 & -7.28 & 21.266 & 21.273 & 0.03 & 0.1 \\
\hline 106.000 & 9.185 & 8.319 & -9.43 & 21.219 & 21.230 & 0.05 & 0.1 \\
\hline 108.000 & 12.655 & 11.622 & -8.16 & 21.192 & 21.206 & 0.06 & 0.1 \\
\hline 110.000 & 16.821 & 15.612 & -7.19 & 21.175 & 21.191 & 0.07 & 0.1 \\
\hline 112.000 & 20.989 & 19.914 & -5.12 & 21.162 & 21.176 & 0.07 & 0.1 \\
\hline 114.000 & 25.323 & 24.305 & -4.02 & 21.152 & 21.165 & 0.06 & 0.1 \\
\hline 116.000 & 29.525 & 28.768 & -2.56 & 21.143 & 21.152 & 0.05 & 0.1 \\
\hline 100.000 & 1.799 & 1.769 & -1.69 & 21.348 & 21.348 & 0.00 & 0.0 \\
\hline 102.000 & 4.796 & 4.797 & 0.02 & 21.315 & 21.315 & 0.00 & 0.0 \\
\hline 104.000 & 6.958 & 6.675 & -4.07 & 21.268 & 21.272 & 0.02 & 0.1 \\
\hline 106.000 & 9.057 & 8.443 & -6.78 & 21.221 & 21.229 & 0.04 & 0.1 \\
\hline 108.000 & 12.481 & 11.689 & -6.34 & 21.193 & 21.203 & 0.05 & 0.1 \\
\hline 110.000 & 16.477 & 15.696 & -4.73 & 21.176 & 21.186 & 0.05 & 0.1 \\
\hline 112.000 & 20.690 & 19.969 & -3.48 & 21.163 & 21.172 & 0.04 & 0.1 \\
\hline 114.000 & 24.971 & 24.360 & -2.45 & 21.152 & 21.160 & 0.04 & 0.1 \\
\hline 116.000 & 29.282 & 28.801 & -1.64 & 21.143 & 21.149 & 0.03 & 0.1 \\
\hline 94.000 & 3.587 & 5.117 & 42.66 & 21.599 & 21.581 & -0.08 & 0.0 \\
\hline 96.000 & 6.309 & 7.223 & 14.49 & 21.555 & 21.544 & -0.05 & 0.0 \\
\hline 98.000 & 8.185 & 8.648 & 5.65 & 21.503 & 21.498 & -0.03 & 0.1 \\
\hline
\end{tabular}


Table 6. PVT DATA (continued)

Data from Straty and Tsumura [19] (continued)

\begin{tabular}{|c|c|c|c|c|c|c|c|}
\hline $\begin{array}{l}\mathrm{T} \\
\mathrm{K}\end{array}$ & $\mathrm{P}, \operatorname{MPa}_{\mathrm{MPa}}$ & $\begin{array}{c}\mathrm{P}, \mathrm{calc} \\
\mathrm{MPa}\end{array}$ & $\begin{array}{c}\mathrm{dev} \\
8\end{array}$ & $\begin{array}{l}\rho, \quad \operatorname{expt} \\
\operatorname{mol} 1 \cdot \mathrm{dm}^{-3}\end{array}$ & $\begin{array}{l}\rho, \quad c a l c \\
\mathrm{~mol} \cdot \mathrm{dm}^{-3}\end{array}$ & $\begin{array}{r}\text { dev } \\
8\end{array}$ & wt \\
\hline 100.000 & 11.375 & 11.428 & 0.46 & 21.468 & 21.467 & 0.00 & 0.1 \\
\hline 102.000 & 15.489 & 15.448 & -0.26 & 21.448 & 21.448 & 0.00 & 0.1 \\
\hline 104.000 & 19.910 & 19.894 & -0.08 & 21.433 & 21.434 & 0.00 & 0.1 \\
\hline 106.000 & 24.433 & 24.519 & 0.35 & 21.422 & 21.421 & 0.00 & 0.1 \\
\hline 108.000 & 29.005 & 29.223 & 0.75 & 21.412 & 21.409 & -0.01 & 0.1 \\
\hline 110.000 & 33.581 & 33.965 & 1.14 & 21.403 & 21.398 & -0.02 & 0.1 \\
\hline 93.000 & 8.787 & 9.283 & 5.64 & 21.682 & 21.677 & -0.03 & 0.0 \\
\hline 94.000 & 10.498 & 10.617 & 1.14 & 21.664 & 21.662 & -0.01 & 0.0 \\
\hline 96.000 & 14.622 & 14.504 & -0.80 & 21.641 & 21.642 & 0.01 & 0.1 \\
\hline 98.000 & 19.173 & 19.007 & -0.87 & 21.625 & 21.627 & 0.01 & 0.1 \\
\hline 100.000 & 23.869 & 23.763 & -0.45 & 21.613 & 21.614 & 0.01 & 0.1 \\
\hline 102.000 & 28.538 & 28.652 & 0.40 & 21.603 & 21.601 & -0.01 & 0.1 \\
\hline 104.000 & 33.196 & 33.601 & 1.22 & 21.594 & 21.589 & -0.02 & 0.1 \\
\hline 272.000 & 2.084 & 2.074 & -0.52 & 1.243 & 1.253 & 0.79 & 0.0 \\
\hline 276.000 & 2.138 & 2.127 & -0.50 & 1.242 & 1.251 & 0.74 & 0.0 \\
\hline 280.000 & 2.190 & 2.179 & -0.50 & 1.241 & 1.250 & 0.72 & 0.0 \\
\hline 284.000 & 2.243 & 2.231 & -0.51 & 1.240 & 1.249 & 0.72 & 0.0 \\
\hline 288.000 & 2.295 & 2.283 & -0.54 & 1.239 & 1.248 & 0.75 & 0.0 \\
\hline 292.000 & 2.347 & 2.335 & -0.49 & 1.239 & 1.247 & 0.68 & 0.0 \\
\hline 296.000 & 2.399 & 2.385 & -0.55 & 1.238 & 1.247 & 0.75 & 0.0 \\
\hline 300.000 & 2.449 & 2.436 & -0.56 & 1.237 & 1.246 & 0.75 & 0.0 \\
\hline 304.000 & 2.500 & 2.487 & -0.52 & 1.237 & 1.245 & 0.69 & 0.0 \\
\hline 308.000 & 2.550 & 2.536 & -0.54 & 1.236 & 1.245 & 0.71 & 0.0 \\
\hline 312.000 & 2.600 & 2.585 & -0.56 & 1.235 & 1.244 & 0.72 & 0.0 \\
\hline 316.000 & 2.649 & 2.635 & -0.52 & 1.235 & 1.243 & 0.66 & 0.0 \\
\hline 320.000 & 2.698 & 2.684 & -0.53 & 1.234 & 1.242 & 0.68 & 0.0 \\
\hline 304.000 & 4.683 & 4.680 & -0.06 & 4.325 & 4.344 & 0.44 & 0.0 \\
\hline 306.000 & 4.809 & 4.806 & -0.07 & 4.323 & 4.341 & 0.43 & 0.0 \\
\hline 308.000 & 4.934 & 4.930 & -0.07 & 4.321 & 4.337 & 0.38 & 0.0 \\
\hline 312.000 & 5.179 & 5.175 & -0.08 & 4.316 & 4.330 & 0.31 & 0.0 \\
\hline 316.000 & 5.420 & 5.416 & -0.07 & 4.312 & 4.322 & 0.24 & 0.0 \\
\hline 320.000 & 5.657 & 5.653 & -0.08 & 4.307 & 4.317 & 0.23 & 0.0 \\
\hline
\end{tabular}


Table 6. PVT DATA (continued)

Data from Straty and Tsumura [19] (continued)

Number of Points [19] 477

Pressure Calculation:

$\mathrm{AAD} \%=2.73 \quad \mathrm{BIAS} \%=1.84$ RMS $8=6.23$

Density Calculation:

$\mathrm{AAD} 2 \%=0.10 \quad$ BIAS $28=0.05 \quad \operatorname{RMS} 2 \%=0.47$

Absolute Deviations:

Pressure Calculation:

$\mathrm{AAD}=0.15$ BIAS $=0.04 \quad \mathrm{RMS}=0.25 \mathrm{MPa}$

Density Calculation:

$\mathrm{AAD2}=0.009$ BIAS2 $=0.000 \mathrm{RMS} 2=0.031 \mathrm{~mol} \cdot \mathrm{dm}^{-3}$

Weighted Data:

Number of Points [19] 381

Pressure Calculation:

$\mathrm{AAD} \%=1.07 \quad \mathrm{BIAS} \%=0.23 \quad \mathrm{RMS} \%=2.07$

Density Calculation:

$\mathrm{AAD} 28=0.04 \quad$ BIAS2\% $=0.00 \quad$ RMS $2 \%=0.06$

Absolute Deviations:

Pressure Calculation:

$\mathrm{AAD}=0.13$ BIAS $=-0.01$ RMS $=0.22 \mathrm{MPa}$

Density Calculation:

AAD2 $=0.006$ BIAS2 $=-0.001 \quad$ RMS2 $=0.007 \mathrm{~mol} \cdot \mathrm{dm}^{-3}$

Data from Tomlinson [29]

\begin{tabular}{ccccccrr}
\hline $\mathrm{T}$ & $\mathrm{P}$, expt \\
$\mathrm{MPa}$ & $\begin{array}{c}\mathrm{P}, \mathrm{calc} \\
\mathrm{MPa}\end{array}$ & $\begin{array}{c}\mathrm{dev} \\
8\end{array}$ & $\begin{array}{c}\rho, \text { expt } \\
\mathrm{mol} \cdot \mathrm{dm}^{-3}\end{array}$ & $\begin{array}{c}\rho, \mathrm{calc} \\
\mathrm{mol} \cdot \mathrm{dm}^{-3}\end{array}$ & $\begin{array}{r}\mathrm{dev} \\
8\end{array}$ & wt \\
\hline 305.372 & 4.648 & 4.651 & 0.07 & 3.821 & 3.810 & -0.29 & 0.0 \\
313.706 & 5.086 & 5.088 & 0.03 & 3.817 & 3.813 & -0.10 & 0.0 \\
322.039 & 5.515 & 5.513 & -0.04 & 3.814 & 3.818 & 0.10 & 0.0 \\
305.372 & 4.835 & 4.845 & 0.19 & 5.002 & 4.877 & -2.51 & 0.0 \\
310.928 & 5.250 & 5.258 & 0.16 & 4.998 & 4.949 & -0.99 & 0.0
\end{tabular}


Table 6. PVT DATA (continued)

Data from Tomlinson [29] (continued)

\begin{tabular}{|c|c|c|c|c|c|c|c|}
\hline $\begin{array}{l}\mathrm{T} \\
\mathrm{K}\end{array}$ & $\begin{array}{l}\mathrm{P}, \underset{\mathrm{MPa}}{\exp t} \\
\mathrm{~Pa}\end{array}$ & $\underset{\mathrm{MPa}}{\mathrm{P}, \underset{\mathrm{Calc}}{ }}$ & $\begin{array}{c}\mathrm{dev} \\
8\end{array}$ & $\begin{array}{l}\rho, \operatorname{expt} \\
\mathrm{mol} \cdot \mathrm{dm}^{-3}\end{array}$ & $\begin{array}{l}\rho, \text { calc } \\
\mathrm{mol} \cdot \mathrm{dm}^{-3}\end{array}$ & $\begin{array}{r}\text { dev } \\
8\end{array}$ & wt \\
\hline 316.483 & 5.659 & 5.662 & 0.06 & 4.996 & 4.983 & -0.26 & 0.0 \\
\hline 322.039 & 6.066 & 6.059 & -0.11 & 4.993 & 5.011 & 0.37 & 0.0 \\
\hline 306.483 & 4.976 & 4.991 & 0.31 & 6.828 & 5.978 & -12.46 & 0.0 \\
\hline 316.483 & 6.016 & 6.033 & 0.28 & 6.821 & 6.728 & -1.37 & 0.0 \\
\hline 324.817 & 6.895 & 6.907 & 0.17 & 6.815 & 6.781 & -0.50 & 0.0 \\
\hline 305.372 & 4.932 & 4.958 & 0.54 & 9.003 & 8.827 & -1.95 & 0.0 \\
\hline 309.261 & 5.534 & 5.551 & 0.29 & 8.998 & 8.945 & -0.60 & 0.0 \\
\hline 313.706 & 6.229 & 6.248 & 0.32 & 8.994 & 8.951 & -0.48 & 0.0 \\
\hline 322.039 & 7.585 & 7.595 & 0.13 & 8.985 & 8.972 & -0.14 & 0.0 \\
\hline 302.594 & 4.659 & 4.695 & 0.78 & 9.742 & 9.631 & -1.13 & 0.0 \\
\hline 305.372 & 5.154 & 5.189 & 0.68 & 9.737 & 9.663 & -0.77 & 0.0 \\
\hline 313.706 & 6.688 & 6.723 & 0.52 & 9.727 & 9.686 & -0.42 & 0.0 \\
\hline 322.039 & 8.275 & 8.307 & 0.38 & 9.718 & 9.692 & -0.27 & 0.0 \\
\hline 298.150 & 4.279 & 4.320 & 0.95 & 10.650 & 10.597 & -0.50 & 0.0 \\
\hline 299.817 & 4.635 & 4.685 & 1.08 & 10.646 & 10.589 & -0.54 & 0.0 \\
\hline 303.589 & 5.469 & 5.523 & 1.00 & 10.640 & 10.590 & -0.47 & 0.0 \\
\hline 310.928 & 7.135 & 7.190 & 0.77 & 10.629 & 10.593 & -0.34 & 0.0 \\
\hline 322.039 & 9.711 & 9.768 & 0.59 & 10.616 & 10.589 & -0.25 & 0.0 \\
\hline 298.150 & 4.485 & 4.532 & 1.04 & 10.879 & 10.834 & -0.42 & 0.0 \\
\hline 305.372 & 6.193 & 6.237 & 0.71 & 10.867 & 10.837 & -0.27 & 0.0 \\
\hline 313.706 & 8.205 & 8.250 & 0.55 & 10.856 & 10.833 & -0.21 & 0.0 \\
\hline 322.039 & 10.256 & 10.292 & 0.35 & 10.846 & 10.831 & -0.14 & 0.0 \\
\hline 295.928 & 4.393 & 4.424 & 0.69 & 11.241 & 11.219 & -0.20 & 0.0 \\
\hline 296.483 & 4.539 & 4.565 & 0.57 & 11.239 & 11.221 & -0.16 & 0.0 \\
\hline 298.150 & 4.969 & 4.990 & 0.43 & 11.236 & 11.222 & -0.12 & 0.0 \\
\hline 299.817 & 5.389 & 5.418 & 0.54 & 11.233 & 11.215 & -0.16 & 0.0 \\
\hline 302.594 & 6.112 & 6.136 & 0.39 & 11.228 & 11.215 & -0.12 & 0.0 \\
\hline 310.928 & 8.292 & 8.316 & 0.30 & 11.216 & 11.206 & -0.09 & 0.0 \\
\hline 316.483 & 9.763 & 9.786 & 0.24 & 11.209 & 11.201 & -0.08 & 0.0 \\
\hline 322.039 & 11.258 & 11.260 & 0.01 & 11.201 & 11.201 & 0.00 & 0.0 \\
\hline 293.150 & 4.548 & 4.574 & 0.57 & 11.773 & 11.761 & -0.11 & 0.0 \\
\hline 298.150 & 5.998 & 6.031 & 0.55 & 11.763 & 11.750 & -0.11 & 0.0 \\
\hline 306.483 & 8.449 & 8.490 & 0.49 & 11.750 & 11.736 & -0.12 & 0.0 \\
\hline 314.261 & 10.770 & 10.805 & 0.33 & 11.739 & 11.729 & -0.08 & 0.0 \\
\hline 322.039 & 13.108 & 13.132 & 0.18 & 11.730 & 11.724 & -0.05 & 0.0 \\
\hline
\end{tabular}


Table 6. PVT DATA (continued)

Data from Tomlinson [29] (continued)

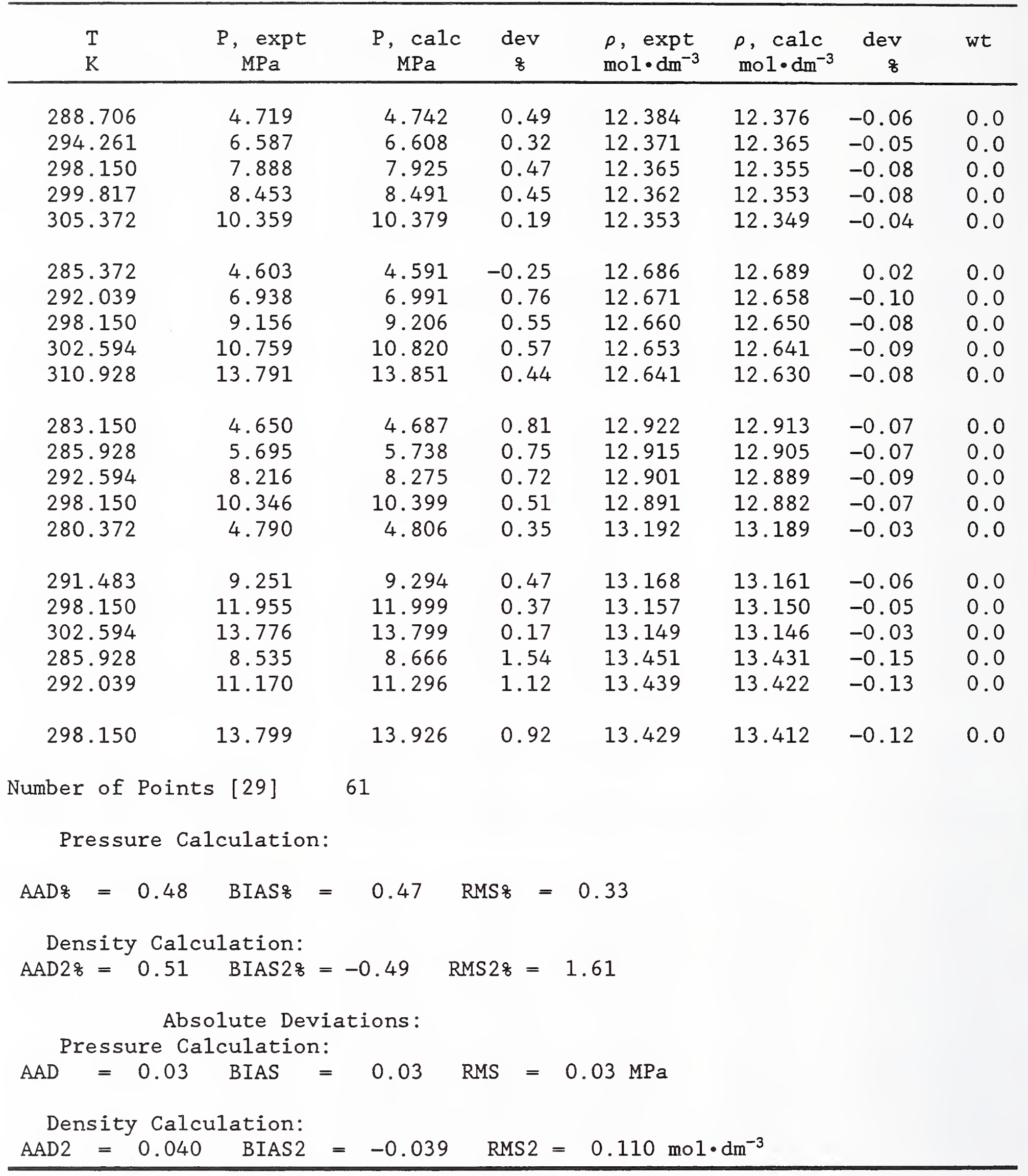


Table 6. PVT DATA (continued)

Data from Wallace et al. [57]

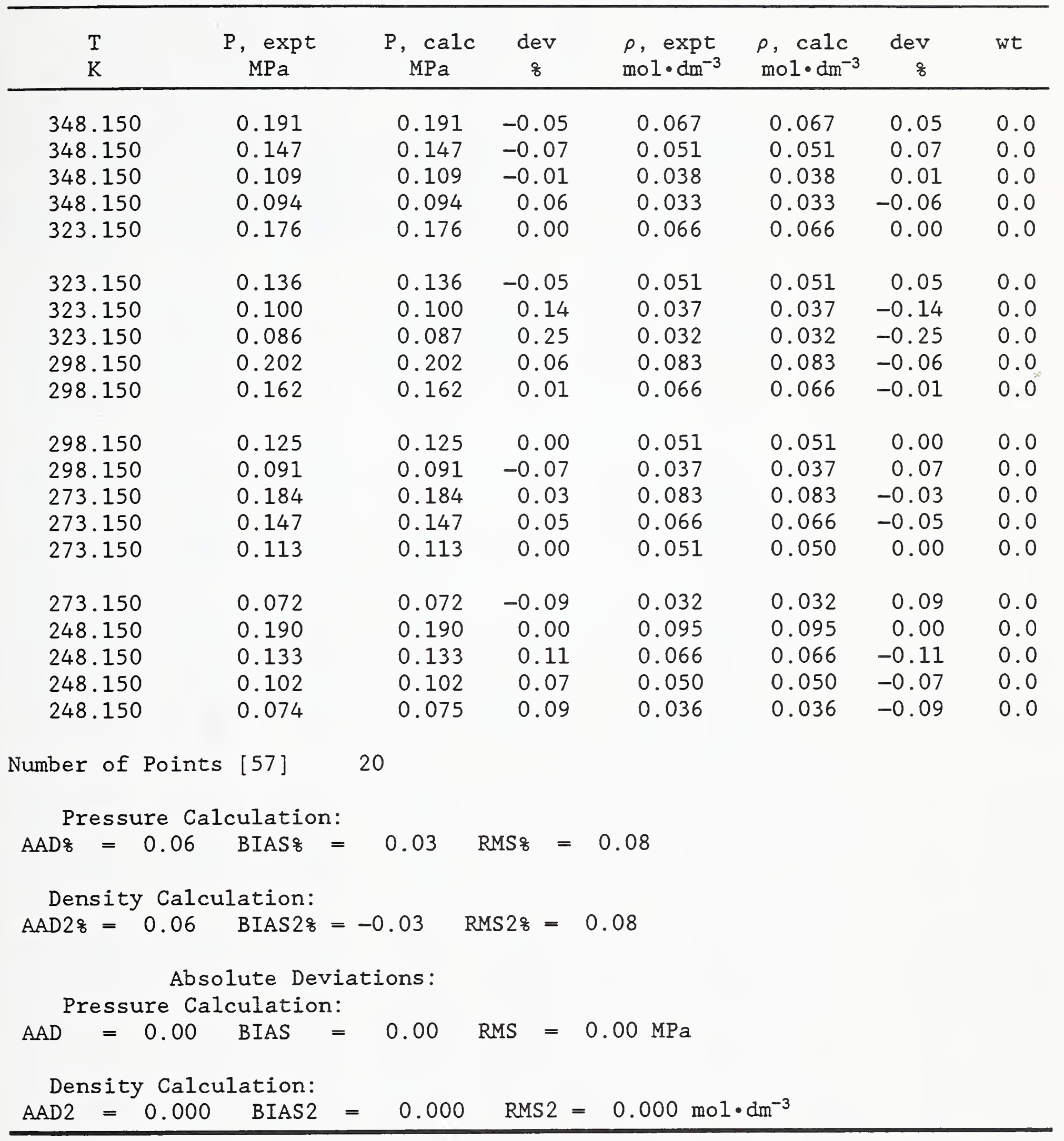


Table 6. PVT DATA (continued)

\begin{tabular}{|c|c|c|c|c|c|c|c|}
\hline \multirow[t]{4}{*}{ Overall Results: } & $\mathrm{N}=2112$ & AAD? & $=2.84$ & BIAS 8 & $=2.30$ & RMS 8 & $=11.57$ \\
\hline & & $\mathrm{AAD} 28$ & $=0.483$ & BIAS $2 \%$ & $=-0.113$ & RMS28 & $=1.509$ \\
\hline & & $A A D$ & $=0.19$ & BIAS & $=0.08$ & RMS & $=0.55 \mathrm{MPa}$ \\
\hline & & AAD2 & $=0.030$ & BIAS2 & $=-0.006$ & RMS2 & $=0.094 \mathrm{~mol} \cdot \mathrm{dm}^{-3}$ \\
\hline \multirow[t]{4}{*}{ Weighted Data: } & $\mathrm{N}=803$ & $\mathrm{AAD} \&$ & $=0.59$ & BIAS 8 & $=0.16$ & RMS \% & $=1.46$ \\
\hline & & $\operatorname{AAD} 28$ & $=0.064$ & BIAS $2 \%$ & $=-0.014$ & RMS 28 & $=0.135$ \\
\hline & & $A A D$ & $=0.09$ & BIAS & $=0.01$ & RMS & $=0.21 \mathrm{MPa}$ \\
\hline & & $\mathrm{AAD} 2$ & $=0.006$ & BIAS2 & $=-0.002$ & RMS 2 & $=0.011 \mathrm{~mol} \cdot \mathrm{dm}^{-3}$ \\
\hline
\end{tabular}


Table 7. ISOCHORIC SPECIFIC HEAT CAPACITY

Data from Roder [58]

The densities are from the tabulation of [58].

\begin{tabular}{|c|c|c|c|c|c|c|c|c|c|}
\hline $\begin{array}{l}\mathrm{T} \\
\mathrm{K}\end{array}$ & $\begin{array}{l}\mathrm{P}, \underset{\mathrm{MPa}}{\text { expt }} \\
\mathrm{MPa}\end{array}$ & $\begin{array}{c}\rho \\
\mathrm{mol} \cdot \mathrm{dm}^{-3}\end{array}$ & $\begin{array}{c}\mathrm{C}_{\mathrm{v}}, \text { expt } \\
\mathrm{J} \cdot \mathrm{mol}^{-}\end{array}$ & $\begin{array}{l}\mathrm{C}_{\mathrm{v}}, \mathrm{calc} \\
-\mathrm{K}^{-1}\end{array}$ & $\begin{array}{l}\text { dev } \\
8\end{array}$ & $\begin{array}{l}\operatorname{dev} 2 \\
8\end{array}$ & $\begin{array}{l}\operatorname{dev} 3 \\
8\end{array}$ & $\begin{array}{l}\operatorname{dev} 4 \\
8\end{array}$ & wt \\
\hline 288.868 & 2.7144 & 1.585 & 49.301 & 49.262 & -0.08 & -0.08 & 0.00 & 0.00 & 1.030 \\
\hline 292.068 & 2.7708 & 1.585 & 49.209 & 49.237 & 0.06 & 0.05 & -0.02 & 0.01 & 1.032 \\
\hline 295.282 & 2.8271 & 1.585 & 48.278 & 49.266 & 2.05 & 2.04 & -0.03 & 0.02 & 1.052 \\
\hline 299.364 & 2.8980 & 1.585 & 48.874 & 49.366 & 1.01 & 1.00 & -0.04 & 0.03 & 1.040 \\
\hline 304.333 & 2.9836 & 1.584 & 49.322 & 49.563 & 0.49 & 0.49 & 0.01 & 0.00 & 1.030 \\
\hline 309.311 & 3.0687 & 1.584 & 49.104 & 49.831 & 1.48 & 1.48 & 0.00 & 0.00 & 1.035 \\
\hline 314.289 & 3.1531 & 1.583 & 49.849 & 50.149 & 0.60 & 0.61 & 0.05 & -0.03 & 1.020 \\
\hline 319.287 & 3.2372 & 1.583 & 50.205 & 50.514 & 0.61 & 0.62 & 0.03 & -0.02 & 1.013 \\
\hline 324.315 & 3.3213 & 1.582 & 50.175 & 50.913 & 1.47 & 1.48 & 0.08 & -0 & 1.013 \\
\hline 329.076 & 3.4004 & 1.582 & 51.109 & 51.320 & 0.41 & 0.42 & 0.06 & -0.04 & 0.995 \\
\hline 302.904 & 4.3292 & 3.276 & 56.641 & 18 & 0.84 & 0.65 & -0.59 & 0.19 & 0.897 \\
\hline 307.501 & 4.5274 & 3.275 & 55.600 & 56.149 & 0.99 & 0.82 & -0.63 & 0.23 & 0.914 \\
\hline 312.122 & 4.7242 & 3.274 & 55.111 & 55.510 & 0.72 & 0.59 & -0.61 & 0.25 & 0.922 \\
\hline 316.773 & 4.9200 & 3.272 & 54.334 & 55. & 1.44 & 1.34 & -0.53 & 24 & 0.936 \\
\hline 321.425 & 5.1138 & 3.271 & 53.926 & 54.914 & 1.83 & 1.76 & -0.49 & 0.23 & 0.943 \\
\hline 326.113 & 5.3073 & 3.270 & 907 & 5 & 1.76 & 1.70 & -0.44 & 0.22 & 0.943 \\
\hline 298.669 & 4.1439 & 3.276 & 60.310 & 58.403 & -3.16 & -3.32 & -0.42 & 0.11 & 0.000 \\
\hline 308.116 & 4.9833 & 4.611 & 62.026 & 62.256 & 0.37 & -0.12 & -1.41 & 0.23 & 0.819 \\
\hline 312.344 & 5.2602 & 4.609 & 59.590 & 60. & 1. & 0. & -1.41 & 0 & 0.853 \\
\hline 316.397 & 5.5228 & 4.607 & 58.605 & 59.155 & 0.94 & 0.65 & -1.31 & 0.36 & 0.867 \\
\hline 320.349 & 5.7766 & 4.604 & 57.298 & 285 & 1.72 & 1.51 & -1.17 & 0.37 & 0.887 \\
\hline 324.259 & 6.0260 & 4.603 & 56.954 & 57.693 & 1.30 & 1.14 & -1.07 & 0.38 & 0.893 \\
\hline 328.210 & 6.2766 & 4.601 & 56.924 & 57.297 & 0.66 & 0.54 & -0.95 & 0.37 & 0.893 \\
\hline 313.190 & 5.5670 & 5.884 & 62.976 & 63.533 & 0.88 & 0.81 & -0.50 & 0.07 & 0.807 \\
\hline 317.960 & 5.9863 & 5.881 & 60.780 & 61.211 & 0.71 & 0.68 & -0.28 & 0.06 & 0.836 \\
\hline 322.792 & 6.4088 & 5.878 & 59.470 & & 0.32 & 0.31 & -0.13 & & 0.855 \\
\hline 327.675 & 6.8341 & 5.876 & 58.705 & 58.659 & -0.08 & -0.08 & -0.04 & 0.01 & 0.866 \\
\hline 308.511 & 5.1530 & 5.887 & 68.850 & 66.900 & -2.83 & -2 & -0.74 & 0. & 0.000 \\
\hline 317.440 & 6.4742 & 7.882 & 61.255 & 59.830 & -2.33 & -3.52 & 3.72 & -1.43 & 0.000 \\
\hline 320.690 & 6.9054 & 7.880 & 60.542 & 58.838 & -2.81 & -3.68 & 3.34 & -1.53 & 0.000 \\
\hline 321.099 & 6.9598 & 7.879 & 60.201 & 58.736 & -2.43 & -3.28 & 3.31 & -1.54 & 0.000 \\
\hline 324.541 & 7.4171 & 7.877 & 59.881 & 58.023 & -3.10 & -3.71 & 2.96 & -1.58 & 0.000 \\
\hline 328.165 & 7.8992 & 7.875 & 59.490 & 57.524 & -3.31 & -3.74 & 2.64 & -1.58 & 0.000 \\
\hline 307.853 & 5.2078 & 7.891 & 67.130 & 65.297 & -2.73 & -5.32 & 4.52 & -0.54 & 0.000 \\
\hline 311.011 & 5.6238 & 7.888 & 63.750 & 62.959 & -1.24 & -3.40 & 4.49 & -0.97 & 0.000 \\
\hline
\end{tabular}


Table 7. ISOCHORIC SPECIFIC HEAT CAPACITY (continued)

Data from Roder [58] (continued)

\begin{tabular}{|c|c|c|c|c|c|c|c|c|c|}
\hline $\begin{array}{l}\mathrm{T} \\
\mathrm{K}\end{array}$ & $\begin{array}{c}P, \text { expt } \\
\mathrm{MPa}\end{array}$ & $\stackrel{\rho}{\mathrm{mol} \cdot \mathrm{dm}^{-3}}$ & $\begin{array}{c}\mathrm{C}_{\mathrm{v}}, \operatorname{expt} \\
\mathrm{J} \cdot \mathrm{mol}^{-}\end{array}$ & $\mathrm{C}_{\mathrm{v}}$, calc & $\begin{array}{l}\text { dev } \\
8\end{array}$ & $\begin{array}{l}\text { dev2 } \\
8\end{array}$ & $\begin{array}{l}\operatorname{dev} 3 \\
8\end{array}$ & $\begin{array}{l}\text { dev4 } \\
8\end{array}$ & wt \\
\hline 314.211 & 6.0466 & 7.884 & 62.200 & 61 & 1.65 & -3.27 & 4.13 & -1.26 & .000 \\
\hline 306.311 & 5.1849 & 9.177 & 80 & & 8 & -2.20 & 1.63 & -0.85 & .000 \\
\hline 306.501 & 5.2172 & 9.177 & 59.724 & 59 & -0.75 & -2.08 & 1.67 & -0.89 & .851 \\
\hline 309.793 & 5.7778 & 9.173 & 58.441 & 58.114 & -0.56 & $-1,84$ & 2.01 & -1.40 & 0.870 \\
\hline 313.122 & 6.3472 & 9.169 & 57.701 & 57.268 & -0.75 & -1.79 & 2.05 & -1.68 & 0.881 \\
\hline 316.478 & 6.9235 & 9.166 & 57.342 & 5 & -1.17 & -1.97 & 1.97 & -1.81 & .886 \\
\hline 326.629 & 8.6765 & 9.158 & 56. & & -1 & -2.11 & 1.58 & -1.78 & 893 \\
\hline 309.399 & 5.7106 & 9.173 & 58.489 & 58 & -0.43 & -1.74 & 2.00 & -1.36 & .869 \\
\hline 312.513 & 6.2429 & 9.169 & 57.769 & 57.4 & -0.63 & -1.72 & 2.07 & -1.65 & .880 \\
\hline 315.643 & 6.7799 & 9.166 & 57.355 & 56.8 & -0.97 & -1.82 & 2.01 & -1.80 & .886 \\
\hline 318.812 & 7.3256 & 9.164 & 35 & & -1.16 & -1 & 1.90 & -1.8 & 891 \\
\hline 321.990 & 7.8740 & 9.161 & & & -1 & -1.99 & 1.78 & -1.85 & 893 \\
\hline 325.186 & 8.4267 & 9.159 & 56.765 & & -1.45 & -1.83 & 1.64 & -1.81 & .896 \\
\hline 328.408 & 8.9849 & 9.156 & 56.845 & 55. & -1.70 & -1.99 & 1.52 & -1.75 & .894 \\
\hline 297.554 & 4.6182 & 11.049 & 53.786 & & -1.70 & -1.85 & 0.26 & -0.78 & .945 \\
\hline .530 & 5.3719 & 11.044 & & & . & -1 & 8 & -1 & \\
\hline 303.509 & 6.1267 & 11.039 & 53. & & -1 & -1.46 & .45 & -1.32 & 950 \\
\hline 306.501 & 6.8874 & 11.035 & 53.590 & & -1.32 & -1.49 & 0.47 & -1.39 & .948 \\
\hline 309.501 & 7.6523 & 11.031 & 53.581 & & -1.14 & -1.28 & 0.48 & -1.41 & .949 \\
\hline 312.511 & 8.4210 & 11.028 & 53.545 & 53.094 & -0.84 & -0.97 & 0.47 & -1.36 & .949 \\
\hline 315.531 & 9.1928 & 11.025 & & & -0 & -0 & .46 & -1 & 947 \\
\hline 318.570 & 9.9703 & 11.021 & 53.846 & & -0.78 & -0.87 & 0.44 & -1.24 & .944 \\
\hline 321.607 & 10.7480 & 11.018 & 54.035 & & -0.76 & -0.84 & 0.42 & -1.16 & .941 \\
\hline 324.656 & 11.5291 & 11.015 & 54.138 & 53.839 & -0.55 & -0.61 & 0.39 & -1.07 & 0.939 \\
\hline 328.087 & 12.4082 & 11.012 & 54.442 & 54.101 & -0.63 & -0.67 & 0.35 & -0.96 & 0.934 \\
\hline 290.820 & 4.3794 & 11.976 & 51. & & -1.84 & -1.92 & .21 & -1.36 & 982 \\
\hline 295.076 & 5.6995 & 11.968 & 51.643 & 50. & -1.26 & -1.33 & 0.24 & -1.39 & .984 \\
\hline 291.384 & 4.5546 & 11.975 & 51.622 & 50.812 & -1.57 & -1.65 & 0.22 & -1.37 & 0.984 \\
\hline 295.658 & 5.8805 & 11.967 & 51.733 & 51.026 & -1.37 & -1.44 & 0.25 & -1.38 & 0.982 \\
\hline 299.925 & 7.2048 & 11.959 & 51.983 & 51.283 & -1.35 & -1.41 & 0.26 & -1.31 & 0.978 \\
\hline 304.195 & 8.5348 & 11.954 & 52.142 & 51.573 & -1.09 & -1.14 & 0.24 & -1.14 & 0.975 \\
\hline 308.490 & 9.8732 & 11.948 & 52.374 & 51.896 & -0.91 & -0.95 & 0.23 & -1.02 & 0.970 \\
\hline 312.666 & 11.1743 & 11.943 & 52.654 & 52.235 & -0.80 & -0.82 & 0.20 & -0.87 & 0.965 \\
\hline 316.715 & 12.4352 & 11.938 & 52.990 & 52.585 & -0.76 & -0.79 & 0.18 & -0.74 & 0.959 \\
\hline 320.765 & 13.6959 & 11.933 & 53.330 & 52.952 & -0.71 & -0.73 & 0.16 & -0.62 & 0.953 \\
\hline
\end{tabular}


Table 7. ISOCHORIC SPECIFIC HEAT CAPACITY (continued)

Data from Roder [58] (continued)

\begin{tabular}{|c|c|c|c|c|c|c|c|c|c|}
\hline $\begin{array}{l}\mathrm{T} \\
\mathrm{K}\end{array}$ & $\begin{array}{c}\mathrm{P}, \operatorname{expt} \\
\mathrm{MPa}\end{array}$ & $\stackrel{\rho}{\mathrm{mol} \cdot \mathrm{dm}^{-3}}$ & $\begin{array}{r}\mathrm{C}_{\mathrm{v}}, \operatorname{expt} \\
\mathrm{J} \cdot \mathrm{mol}^{-}\end{array}$ & $\mathrm{C}_{\mathrm{v}}, \mathrm{calc}$ & $\begin{array}{l}\text { dev } \\
\frac{8}{8}\end{array}$ & $\begin{array}{l}\text { dev2 } \\
\text { \% }\end{array}$ & $\begin{array}{l}\operatorname{dev} 3 \\
8\end{array}$ & $\begin{array}{l}\operatorname{dev} 4 \\
8\end{array}$ & $w t$ \\
\hline 324.828 & 14.9594 & 11.928 & 53.623 & 53.335 & -0.54 & -0.55 & 0.13 & -0.51 & 0.948 \\
\hline 328. & 16.2269 & 11.923 & 54.030 & & -0.55 & -0.56 & 0.11 & -0.41 & .941 \\
\hline 280.688 & 3.8940 & 12.950 & 49.949 & 49.014 & -1.87 & -1.89 & 0.09 & -1.29 & .017 \\
\hline 284.835 & 5.4845 & 12.941 & 49.928 & 49.322 & -1.21 & -1.23 & 0.10 & -1.02 & 1.017 \\
\hline 288.976 & 7.0695 & 12.932 & 50.221 & 49.653 & -1.13 & -1.15 & 0.09 & -0.82 & .012 \\
\hline 293.117 & 8.6596 & 12.925 & 50.473 & 34 & -0.93 & -0.94 & 0.08 & -0.64 & .007 \\
\hline 293.453 & 8.7885 & 12.925 & 50. & & -1.12 & -1.13 & 8 & -0.60 & .004 \\
\hline 297.602 & 10.3808 & 12.918 & 50.830 & 50 & -0.84 & -0.84 & 0.07 & -0.47 & .000 \\
\hline 301.740 & 11.9663 & 12.912 & 51.196 & 50.790 & -0.79 & -0.80 & 0.05 & -0.32 & 0.993 \\
\hline 310.014 & 15.1274 & 12.900 & 51.711 & 51.604 & -0.21 & -0.21 & 0.02 & -0.09 & 0.983 \\
\hline 314.163 & 16.7073 & 12. & 52.222 & 0 & -0.37 & -0.37 & 0.00 & 0.0 & .973 \\
\hline 318.308 & 18.2826 & 12.888 & 59 & 52 & -0.41 & -0.41 & 2 & 0.09 & .965 \\
\hline 322.453 & 19.8541 & 12.882 & 53.170 & 52 & -0.49 & -0.49 & -0.03 & 0.15 & .956 \\
\hline 326.617 & 21.4289 & 12.876 & 53.564 & 53.363 & -0.38 & -0.38 & -0.04 & 0.21 & 0.949 \\
\hline 266.868 & 3.3507 & 14.004 & 48.300 & 47.303 & -2.06 & -2.06 & -0.03 & 0.92 & 0.000 \\
\hline 270.918 & 5.2993 & 13.994 & 285 & & -1.30 & -1.30 & & 0.7 & .052 \\
\hline 274.958 & 7.2348 & 13.983 & 05 & & -1.20 & -1.20 & -0 . & 0.56 & 1.045 \\
\hline 278.996 & 9.1760 & 13.976 & 48.893 & & -1.00 & -1.00 & -0.05 & 0.66 & 1.039 \\
\hline 283.030 & 11.1121 & 13.968 & 49.180 & 48. & -0.78 & -0.78 & -0.06 & 0.63 & 1.033 \\
\hline & 13.0367 & 13.961 & 49.570 & 49.198 & -0.75 & -0.75 & -0.07 & 0.67 & 1.025 \\
\hline 068 & 14.9507 & 13.954 & 95 & & -0.77 & -0.7 & -0 & 0. & 1.016 \\
\hline 295.074 & 16.8537 & 13.947 & 50.337 & 50 & -0.61 & -0.61 & -0.09 & 0.73 & 1.009 \\
\hline 299.072 & 18.7465 & 13.940 & 50.718 & 50.4 & -0.51 & -0.52 & -0.10 & 0.74 & 1.002 \\
\hline 303.068 & 20.6313 & 13.933 & 51.123 & 50.8 & -0.45 & -0.46 & -0.11 & 0.76 & 0.994 \\
\hline 307.053 & 22.5042 & 13.926 & 51.579 & 51.331 & -0.48 & -0.49 & -0.11 & 0.76 & 0.985 \\
\hline 311.509 & 24.5909 & 13.5 & 996 & 5 & -0.32 & -0.33 & -0.12 & 0.75 & .978 \\
\hline 315.497 & 26.4506 & 13.911 & 52.444 & 52.281 & -0.31 & -0.32 & -0.12 & 0.74 & 0.969 \\
\hline 319.489 & 28.3058 & 13.904 & 52.843 & 52.737 & -0.20 & -0.21 & -0.12 & 0.73 & 0.962 \\
\hline 323.481 & 30.1545 & 13.897 & 53.299 & 53.197 & -0.19 & -0.20 & -0.13 & 0.72 & 0.954 \\
\hline 327.478 & 31.9986 & 13.890 & 53.800 & 53.661 & -0.26 & -0.27 & -0.13 & 0.70 & 0.945 \\
\hline 258.973 & 4.1721 & 14.644 & 47.198 & 46 & -1.46 & -1.46 & -0 . & 2.70 & 1.075 \\
\hline 265.038 & 7.5076 & 14.628 & 47.568 & 47.073 & -1.04 & -1.04 & -0.09 & 1.81 & 1.067 \\
\hline 271.085 & 10.8449 & 14.616 & 48.168 & 47.664 & -1.05 & -1.05 & -0.10 & 1.50 & 1.054 \\
\hline 277.10 & 14.1445 & 14.604 & 48.608 & 48.274 & -0.69 & -0.70 & -0.11 & 1.34 & 1.045 \\
\hline 283.089 & 17.4014 & 14.592 & 49.118 & 48.899 & -0.45 & -0.46 & -0.12 & 1.23 & 1.034 \\
\hline
\end{tabular}


Table 7. ISOCHORIC SPECIFIC HEAT CAPACITY (continued)

Data from Roder [58] (continued)

\begin{tabular}{|c|c|c|c|c|c|c|c|c|c|}
\hline $\begin{array}{l}\mathrm{T} \\
\mathrm{K}\end{array}$ & $\begin{array}{c}\mathrm{P}, \operatorname{expt} \\
\mathrm{MPa}\end{array}$ & $\begin{array}{c}\rho \\
\mathrm{mol}\end{array} \mathrm{dm}^{-3}$ & $\begin{array}{r}\mathrm{C}_{\mathrm{v}}, \operatorname{expt} \\
\mathrm{J} \cdot \mathrm{mo} 1^{-}\end{array}$ & $\mathrm{C}_{\mathrm{v}}$, calc & $\begin{array}{l}\text { dev } \\
8\end{array}$ & $\begin{array}{l}\text { dev2 } \\
8\end{array}$ & $\begin{array}{l}\text { dev3 } \\
8\end{array}$ & $\begin{array}{l}\operatorname{dev} 4 \\
8\end{array}$ & wt \\
\hline 289.038 & 20.6173 & 14.580 & 49.761 & & 0.45 & 0.40 & -0.13 & 1.14 & .021 \\
\hline 290.026 & 21.1489 & 14.578 & 49.844 & & o. & -0.41 & -0.13 & 1.13 & 019 \\
\hline 295.968 & 24.3355 & 14.567 & 50.481 & & -0.36 & -0.38 & -0.14 & 1.10 & .007 \\
\hline 301.879 & 27.4845 & 14.555 & 51.124 & & -0.32 & -0.34 & -0 . & 1.02 & 0.994 \\
\hline 307.773 & 30.6037 & 14.543 & 51.821 & 51.627 & -0.37 & -0.39 & -0.14 & 0.94 & 0.981 \\
\hline 313.655 & 33.6958 & 14.532 & 52.457 & $J 4$ & -0.29 & -0.31 & -0 . & 0.70 & .909 \\
\hline 257.577 & 3.3934 & 14.647 & 47.195 & & -1 & -1.72 & -0 . & 19 & 075 \\
\hline 260.618 & 5.0545 & 14.637 & 47.302 & & -1 & -1 & -0 . & .34 & .073 \\
\hline 263.680 & 6.7547 & 14.631 & 47.384 & 46 & -0.93 & -0 . & -0 . & 1.97 & .071 \\
\hline 266.736 & 8.4464 & 14.624 & 47.736 & 36 & -1.05 & -1.05 & -0.09 & 1.63 & 1.064 \\
\hline 269. & .1279 & 14.6 & 47. & & -0 & -0 & -0 & .4 & 059 \\
\hline 272.823 & 11.7994 & 14.612 & 48.204 & & -0 & -0 & -0 & .40 & 053 \\
\hline 275.855 & 13.4608 & 14.606 & 48.482 & & -0 & -0 & -0 & .33 & 047 \\
\hline 258.449 & 7.9440 & 15.039 & 47.151 & & -1.34 & -1.35 & -0 . & 2.46 & 1.076 \\
\hline 262.425 & 10.3308 & 15.030 & 47.459 & & $-1 \quad 1$ & $\begin{array}{ll}-1 & 17\end{array}$ & -0 . & 2.09 & 1.070 \\
\hline $26 c$ & 8.8885 & 15. & 6 & & -1 & -1 & -0 & . & 075 \\
\hline 264. & 11.2775 & 15 . & 47.518 & & . & -0 & -0 & 4 & 068 \\
\hline 267.971 & 13.6450 & 15.018 & 47.972 & & -1 & -1.07 & -0 . & .79 & .058 \\
\hline 271.915 & 15.9882 & 15.009 & 48.292 & & -0.8 & -0.89 & -0 . & 1.60 & .051 \\
\hline 275.845 & 18.3111 & 15.001 & 48.655 & & -0.7 & -0.79 & -0 & 1.53 & 1.044 \\
\hline 250.496 & 3.1815 & 15. & 30 & & 2 & -2 & -0 & .2 & 00 \\
\hline 254.472 & 5.5686 & 15.051 & 46.780 & & -1 & -1 . & -0 . & 3.29 & 000 \\
\hline 256.033 & 6.5052 & 15.046 & 46.910 & & -1.3 & -1.33 & -0.11 & 2.80 & 0.000 \\
\hline 279.768 & 20.6179 & 14.993 & 49.048 & & -0.7 & -0 & -0.14 & 1.46 & 1.036 \\
\hline 283.679 & 22.9060 & 14.984 & 49.408 & 49.120 & -0.5 & -0.60 & -0.14 & 1.34 & 1.028 \\
\hline 287.579 & 25.1761 & 14. & 49.746 & & -0 . & -0 & -0 & 1.29 & 021 \\
\hline 291.462 & 27.4251 & 14.968 & 50.272 & & -0.5 & -0 . & -0.15 & 1.23 & 1.011 \\
\hline 295.34 & 29.6660 & 14.959 & 50.623 & & -0.4 & -0 & -0.14 & 1.13 & 1.004 \\
\hline 300.025 & 32.3465 & 14.949 & 51.179 & & -0.4 & -0.50 & -0.14 & 1.05 & 0.993 \\
\hline 231.182 & 3.6030 & 16.140 & 45.310 & 44.453 & -1.89 & -1.91 & -0.10 & 7.45 & 1.119 \\
\hline 234.160 & 5.8108 & 16.129 & 45.163 & & -0.98 & -1.01 & -0 . & . & .123 \\
\hline 237.158 & 8.0795 & 16.121 & 45.529 & 44.997 & -1.1 & -1.2 & -0.12 & 3.98 & 1.114 \\
\hline 240.148 & 10.3326 & 16.113 & 45.792 & 45.2 & -1.12 & -1.15 & -0.12 & 3.28 & 1.108 \\
\hline 243.126 & 12.5647 & 16.105 & 46.060 & 45.561 & -1.08 & -1.11 & -0.12 & 2.81 & 1.10: \\
\hline 244.528 & 13.6123 & 16.101 & 46.079 & 45.696 & -0.83 & -0.86 & -0.12 & 2.60 & 1.101 \\
\hline
\end{tabular}


Table 7. ISOCHORIC SPECIFIC HEAT CAPACITY (continued)

Data from Roder [58] (continued)

\begin{tabular}{|c|c|c|c|c|c|c|c|c|c|}
\hline $\begin{array}{l}\mathrm{T} \\
\mathrm{K}\end{array}$ & $\mathrm{P}, \underset{\mathrm{MPa}}{\exp t}$ & $\begin{array}{c}\rho \\
\mathrm{mol}\end{array} \mathrm{dm}^{-3}$ & $\begin{array}{c}\mathrm{C}_{\mathrm{v}}, \operatorname{expt} \\
\mathrm{J} \cdot \mathrm{mol}\end{array}$ & $\mathrm{C}_{\mathrm{v}}, \mathrm{calc}$ & $\begin{array}{l}\text { dev } \\
8\end{array}$ & $\begin{array}{l}\text { dev2 } \\
8\end{array}$ & $\begin{array}{l}\operatorname{dev} 3 \\
8\end{array}$ & $\begin{array}{l}\operatorname{dev} 4 \\
8\end{array}$ & wt \\
\hline 250.439 & 17.9966 & 16.086 & 46.681 & 46.275 & -0.87 & -0.90 & -0.13 & 2.17 & .087 \\
\hline 256.303 & 22.3017 & 16.071 & 47.337 & 46.864 & -1.00 & -1.03 & -0.13 & 1.87 & .072 \\
\hline 262.126 & 26.5329 & 16.056 & 47.918 & 47.460 & -0.96 & -0.99 & -0.13 & 1.64 & .059 \\
\hline 267.928 & 30.7076 & 16.041 & 48.534 & 48.065 & -0.97 & -1.00 & -0.13 & 1.45 & 1.046 \\
\hline 272.246 & 33.7881 & 16.029 & 48.910 & 48.521 & -0.79 & -0.83 & -0.12 & 1.27 & 1.038 \\
\hline 214.213 & 2.9576 & 16.914 & 44.887 & 4 & -2.93 & -2.96 & -0.08 & 9. & .128 \\
\hline 218.081 & 6.3375 & 16.898 & 44.881 & & -2.19 & -2.22 & -0.08 & 4. & .129 \\
\hline 221.935 & 9.6893 & 16.884 & 44.972 & 44.227 & -1.66 & -1.69 & -0.09 & 3.46 & 1.127 \\
\hline 225.787 & 13.0630 & 16.872 & 45.159 & 44.567 & -1.31 & -1.35 & -0.09 & 2.75 & $1.12 ?$ \\
\hline 228.421 & 15.3573 & 16.864 & 45.308 & 44.804 & -1.11 & -1.15 & -0.10 & 2.44 & 1.119 \\
\hline 232.253 & 18.6722 & 16.852 & 03 & & -1.21 & -1.24 & -0 . & & .109 \\
\hline 236.060 & 21.9407 & 16.841 & 45.982 & & -1.04 & -1.07 & -0 & 1. & .103 \\
\hline 239.848 & 25.1666 & 16.830 & 46.364 & 45 & -1.08 & -1.12 & -0.10 & 1.68 & 1.094 \\
\hline 243.612 & 28.3476 & 16.818 & 46.753 & 46.220 & -1.14 & -1.17 & -0.10 & 1.44 & 1.085 \\
\hline 247.355 & 31.4871 & 16.807 & 47.074 & & -1.05 & -1.08 & -0.10 & & 1.078 \\
\hline 18 & 2.6 & 17.990 & 44.545 & & -3 & -3 & -0 . & & .135 \\
\hline 192.360 & 6.1935 & 17.974 & 44.210 & & -2.58 & -2.60 & -0.03 & & .144 \\
\hline 195.613 & 9.6990 & 17.959 & 44.088 & 43.296 & -1.80 & -1.82 & -0.03 & 1 . & 1.147 \\
\hline 198.035 & 12.3416 & 17.950 & 44.273 & 43.471 & -1.81 & -1.83 & -0.04 & 1.71 & 1.143 \\
\hline 201.281 & 15.8641 & 17.938 & 44.497 & 43.710 & -1.77 & -1.79 & -0.04 & 1.44 & 1.137 \\
\hline 204.510 & 19.3399 & 17.927 & 06 & & -1.68 & -1.71 & -0 & & .132 \\
\hline 207.717 & 22.7643 & 17.915 & 44.937 & 44.199 & -1.64 & -1.67 & -0.04 & 1 . & 1.127 \\
\hline 210.907 & 26.1421 & 17.903 & 45.208 & 44.447 & -1.68 & -1.70 & -0 . & & 1.120 \\
\hline 214.081 & 29.4749 & 17.892 & 45.460 & 44.700 & -1.67 & -1.69 & -0.04 & 0.88 & 1.114 \\
\hline 217.237 & 32.7623 & 17.881 & 45.744 & 44.955 & -1.73 & -1.75 & -0.04 & 0.80 & 1.107 \\
\hline 168.242 & 5.8435 & 18.908 & 186 & & -2.64 & -2.64 & 0.00 & 0 & 1.141 \\
\hline 166.260 & 3.2642 & 18.919 & 44.154 & 42.935 & -2.76 & -2.76 & 0.00 & & 1.142 \\
\hline 169.533 & 7.5377 & 18.901 & 44.209 & 43.076 & -2.56 & -2.56 & 0.00 & 0.27 & 1.141 \\
\hline 172.795 & 11.7968 & 18.885 & 44.170 & 43.230 & -2.13 & -2.13 & -0.01 & 0.43 & 1.143 \\
\hline 176.058 & 16.0901 & 18.871 & 44.269 & 43.396 & -1.97 & -1.98 & -0.01 & 0.43 & 1.140 \\
\hline 179.304 & 20.3225 & 18.857 & 44.433 & & -1.95 & -1.95 & -0 . & & 1.137 \\
\hline 182.531 & 24.4871 & 18.843 & 44.625 & 43.746 & -1.97 & -1.98 & -0.01 & 0.21 & 1.132 \\
\hline 185.736 & 28.5823 & 18.830 & 44.825 & 43.930 & -2.00 & -2.00 & -0.01 & 0.21 & 1.127 \\
\hline 188.925 & 32.6166 & 18.816 & 45.120 & 44.118 & -2.22 & -2.22 & 0.00 & 0.05 & 1.120 \\
\hline 174.677 & 14.2771 & 18.877 & 44.830 & 43.325 & -3.36 & -3.36 & -0.01 & 0.48 & 0.000 \\
\hline
\end{tabular}


Table 7. ISOCHORIC SPECIFIC HEAT CAPACITY (continued)

Data from Roder [58] (continued)

\begin{tabular}{|c|c|c|c|c|c|c|c|c|c|}
\hline $\begin{array}{l}\mathrm{T} \\
\mathrm{K}\end{array}$ & $\begin{array}{c}P, \text { expt } \\
\mathrm{MPa}\end{array}$ & $\begin{array}{c}\rho \\
\mathrm{mol} \cdot \mathrm{dm}^{-3}\end{array}$ & $\begin{array}{c}\mathrm{C}_{\mathrm{v}}, \operatorname{expt} \\
\mathrm{J} \cdot \mathrm{mol}\end{array}$ & $\begin{array}{c}\mathrm{C}_{\mathrm{v}}, \text { calc } \\
-1 \cdot \mathrm{K}^{-1}\end{array}$ & $\begin{array}{l}\text { dev } \\
8\end{array}$ & $\begin{array}{l}\operatorname{dev} 2 \\
8\end{array}$ & $\begin{array}{l}\operatorname{dev} 3 \\
8\end{array}$ & $\begin{array}{l}\operatorname{dev} 4 \\
8\end{array}$ & wt \\
\hline 166.750 & 3.8855 & 18.916 & 44.640 & 42.955 & -3.78 & -3.78 & 0.00 & 0.42 & .000 \\
\hline 170.023 & 8.1541 & 18.898 & 44.520 & 43.098 & -3.20 & -3.20 & 0.00 & 0.34 & .000 \\
\hline 173.301 & 12.4645 & 18.883 & 44.480 & 43.255 & -2.75 & -2.76 & -0.01 & 0.50 & .000 \\
\hline 176.572 & 16.7633 & 18.868 & 44.500 & 43.421 & -2.42 & -2.43 & 0.00 & 0.23 & 0.000 \\
\hline 179.822 & 20.9940 & 18.855 & 44.710 & 43.596 & -2.49 & -2.50 & -0.01 & 0.36 & 0.000 \\
\hline 183.059 & 25.1643 & 18.841 & 44.810 & 43.776 & -2.31 & -2.31 & -0.01 & 0.24 & .000 \\
\hline 186.280 & 29.2726 & 18.827 & 45.010 & & -2.33 & -2.33 & 0.00 & 0.10 & .000 \\
\hline 145.929 & 1.6026 & 19.657 & 44.784 & 43.688 & -2.45 & -2.44 & 0.01 & -5.19 & 1.122 \\
\hline 148.039 & 4.7507 & 19.643 & 44.886 & 43.705 & -2.63 & -2.63 & 0.00 & -0.98 & 1.120 \\
\hline 148.064 & 4.7897 & 19.643 & 44.975 & 43.705 & -2.82 & -2.82 & 0.00 & -0.83 & 1.118 \\
\hline 151.130 & 9.4205 & 19.624 & 44.405 & & -1.49 & -1.48 & & -0.48 & .133 \\
\hline 151.151 & 9.4530 & 19.624 & 44.387 & 45 & -1.45 & -1.44 & 0.00 & -0.42 & 1.133 \\
\hline 154.270 & 14.2807 & 19.609 & 44.484 & 43.808 & -1.52 & -1.52 & 0.00 & -0.18 & 1.132 \\
\hline 157.366 & 19.0182 & 19.594 & 44.659 & 43.881 & -1.74 & -1.74 & 0.00 & -0.19 & 1.128 \\
\hline 160.437 & 23.6640 & 19.579 & 44.835 & 43.965 & -1.94 & -1.93 & 0.01 & -0.29 & 1.124 \\
\hline 163.485 & 28.2233 & 19.565 & 44. & & -1.94 & -1.94 & & -0.24 & 1.122 \\
\hline 165.894 & 31.7892 & 19.553 & 45.097 & 44.139 & -2.12 & -2.11 & 0.01 & -0.39 & 1.118 \\
\hline 148.027 & 4.7424 & 19.643 & 44.778 & 43.704 & -2.40 & -2.39 & 0.01 & -1.27 & 1.123 \\
\hline 151.152 & 9.4813 & 19.624 & 44.516 & 43.745 & -1.73 & -1.73 & 0.01 & -0.70 & 1.130 \\
\hline 154.267 & 14.2771 & 19.609 & 44.552 & 43.808 & -1.67 & -1.67 & 0.00 & -0.20 & 1.130 \\
\hline 157.364 & 19.0097 & 19.594 & 54 & & -1.73 & -1.73 & & -0.16 & 128 \\
\hline 133.939 & 11.0190 & 20.270 & 45.146 & & -0.22 & -0.21 & 0.00 & -0.44 & 1.110 \\
\hline 136.801 & 16.1127 & 20.254 & 45.230 & 45.000 & -0.51 & -0.50 & 0.01 & -0.58 & 1.109 \\
\hline 139.647 & 21.1154 & 20.239 & 45.247 & 44.969 & -0.61 & -0.60 & 0.01 & -0.48 & 1.109 \\
\hline 142.469 & 26.0146 & 20.224 & 45.398 & 44.951 & -0.98 & -0.97 & 0.01 & -0.52 & 1.106 \\
\hline 145.270 & 30.8177 & 20.209 & 45.462 & 44.946 & -1.14 & -1.12 & 2 & -0.61 & 1.105 \\
\hline 112.401 & 7.4628 & 20.981 & 46.067 & 47.358 & 2.80 & 2.80 & 0.00 & -0.05 & 2.161 \\
\hline 114.288 & 11.4471 & 20.969 & 46.203 & 47.272 & 2.31 & 2.32 & 0.00 & -0.54 & 2.156 \\
\hline 116.167 & 15.3828 & 20.957 & 46.210 & 47.186 & 2.11 & 2.12 & 0.01 & -0.90 & 2.158 \\
\hline 118.324 & 19.8576 & 20.944 & 46.282 & 47.090 & 1.75 & 1.76 & 0.01 & -0.93 & 2.156 \\
\hline 120.762 & 24.8511 & 20.930 & 46.410 & 46.986 & 1.24 & 1.26 & & -0.77 & 2.152 \\
\hline 123.178 & 29.7379 & 20.915 & 46.427 & 46.884 & 0.98 & 1.01 & 0.02 & -1.00 & 2.153 \\
\hline 110.494 & 3.6415 & 20.996 & 44.790 & 47.451 & 5.94 & 5.94 & 0.00 & 0.21 & 0.000 \\
\hline 131.088 & 6.0623 & 20.289 & 45.990 & 45.119 & -1.89 & -1.89 & 0.00 & -0.42 & 0.000 \\
\hline
\end{tabular}


Table 7. ISOCHORIC SPECIFIC HEAT CAPACITY (continued)

\section{Data from Roder [58] (continued)}

Number of Points [58] 209

Using temperature and density:

$\mathrm{AAD} \%=1.32$ BIAS $8=-0.93$ RMS $8=1.27$

Using temperature and pressure:

$\mathrm{AAD} 2 \%=1.43 \quad$ BIAS $2 \%=-1.06 \quad$ RMS $2 \%=1.35$

Absolute Deviations:

Using temperature and density:

$\mathrm{AAD}=0.66 \quad \mathrm{BIAS}=-0.46 \mathrm{RMS}=0.63 \mathrm{~J} \cdot \mathrm{mol}^{-1} \cdot \mathrm{K}^{-1}$

Using temperature and pressure:

$\mathrm{AAD} 2=0.72 \quad \mathrm{BIAS} 2=-0.53 \quad \mathrm{RMS} 2=0.71 \mathrm{~J} \cdot \mathrm{mol}^{-1} \cdot \mathrm{K}^{-1}$

Weighted Data:

Number of Points [58] 184

Using temperature and density:

$\mathrm{AAD} \%=1.16$ BIAS $8=-0.78$ RMS $8=1.09$

Using temperature and pressure:

$\mathrm{AAD} 2 \%=1.22 \quad$ BIAS $2 \%=-0.86 \quad$ RMS $2 \%=1.10$

Absolute Deviations:

Using temperature and density:

$\mathrm{AAD}=0.56$ BIAS $=-0.36 \mathrm{RMS}=0.52 \mathrm{~J} \cdot \mathrm{mol}^{-1} \cdot \mathrm{K}^{-1}$

Using temperature and pressure:

$\mathrm{AAD2}=0.60$ BIAS2 $=-0.41 \quad \mathrm{RMS} 2=0.54 \mathrm{~J} \cdot \mathrm{mol}^{-1} \cdot \mathrm{K}^{-1}$

Data from Sengers [56]

These data were calculated from the scaled equation of state of [56].

\begin{tabular}{|c|c|c|c|c|c|c|c|c|c|}
\hline $\begin{array}{l}\mathrm{T} \\
\mathrm{K}\end{array}$ & $\mathrm{P}, \underset{\mathrm{MPa}}{\exp t}$ & $\underset{\mathrm{mol}}{\rho} \mathrm{dm}^{-3}$ & $\begin{array}{l}\mathrm{C}_{\mathrm{v}}, \operatorname{expt} \\
\mathrm{J} \cdot \mathrm{mol}^{-1}\end{array}$ & $\begin{array}{l}\mathrm{C}_{\mathrm{v}} \text {, calc } \\
1 \cdot \mathrm{K}^{-1}\end{array}$ & $\begin{array}{l}\text { dev } \\
\frac{8}{8}\end{array}$ & $\begin{array}{l}\text { dev2 } \\
\frac{8}{8}\end{array}$ & $\begin{array}{l}\operatorname{dev} 3 \\
\frac{8}{8}\end{array}$ & $\begin{array}{l}\text { dev4 } \\
8\end{array}$ & wt \\
\hline 304.000 & 4.7347 & 4.900 & 73.000 & 66.516 & -8.88 & -9.02 & -0.34 & 0.02 & 0.348 \\
\hline 304.000 & 4.7541 & 9.000 & 63.954 & 61.623 & -3.64 & -3.67 & 0.02 & -0.01 & 0.397 \\
\hline 306.000 & 4.8928 & 5.000 & 68.563 & 65.496 & -4.47 & -4.39 & 0.23 & -0.02 & 0.371 \\
\hline 306.000 & 4.9332 & 6.000 & 79.978 & 69.632 & -12.94 & -12.70 & 1.23 & -0.02 & 0.318 \\
\hline 306.000 & 4.9385 & 6.500 & 84.432 & 70.487 & -16.52 & -16.49 & 0.57 & -0.01 & 0.301 \\
\hline
\end{tabular}


Table 7. ISOCHORIC SPECIFIC HEAT CAPACITY (continued)

Data from Sengers [56] (continued)

\begin{tabular}{|c|c|c|c|c|c|c|c|c|c|}
\hline $\begin{array}{l}\mathrm{T} \\
\mathrm{K}\end{array}$ & $\begin{array}{c}\mathrm{P}, \underset{\mathrm{MPa}}{\exp t} \\
\end{array}$ & $\stackrel{\rho}{\rho \mathrm{mol} \cdot \mathrm{dm}^{-3}}$ & $\begin{array}{c}\mathrm{C}_{\mathrm{v}}, \operatorname{expt} \\
\mathrm{J} \cdot \mathrm{mol}^{-}\end{array}$ & $\mathrm{C}_{\mathrm{v}}$, calc & $\begin{array}{l}\mathrm{dev} \\
\frac{g}{8}\end{array}$ & $\begin{array}{l}\operatorname{dev} 2 \\
8\end{array}$ & $\begin{array}{l}\operatorname{dev} 3 \\
8\end{array}$ & $\begin{array}{l}\text { dev4 } \\
8\end{array}$ & wt \\
\hline 306.000 & 4.9410 & 6.900 & 84.532 & 70.366 & 16.76 & -16.65 & -1.28 & 0.02 & .301 \\
\hline 306.000 & 4.9460 & 7.500 & 78.528 & & -12.39 & -11.65 & -2.18 & 0.04 & .324 \\
\hline 306.000 & 4.9565 & 8.000 & 71.456 & 66.428 & -7.04 & -6.62 & -0.70 & 0.03 & 0.356 \\
\hline 306.000 & 5.0510 & 9.000 & 61.270 & 60.495 & -1.27 & -1.21 & -0.06 & 0.02 & 0.415 \\
\hline 310.000 & 5.1910 & 5.000 & 63.745 & & -1.29 & -1.25 & 0.13 & & 0.399 \\
\hline 310. & 5.2983 & 6.000 & 31 & & -1.81 & -1.79 & 0 . & 1 & 379 \\
\hline 310. & 5.3334 & 6.500 & 67.639 & & -1.76 & -1.77 & -0.13 & & 0.376 \\
\hline 310.000 & 5.3593 & 6.900 & 67.274 & 66.271 & -1.49 & -1.44 & -0.44 & & 0.378 \\
\hline 310.000 & 5.4035 & 7.500 & 65.575 & 65.001 & -0.88 & -0.72 & -0.45 & & 0.388 \\
\hline 310.000 & 5.4553 & 8.000 & 63.478 & 63.192 & -0.45 & -0.39 & -0.12 & 2 & 0.400 \\
\hline & 5.6658 & 0 & & & -0 . & -0.52 & -0.01 & & .430 \\
\hline 315.0 & 5.5560 & 5.000 & 61.205 & 60. & -0.86 & -0.86 & 0.02 & & 0.415 \\
\hline 315.000 & 5.7488 & 6.000 & 62.916 & 62. & -0.37 & -0.38 & -0.10 & & 0.404 \\
\hline 315.000 & 5.8268 & 6.500 & 63.053 & 62.963 & -0.14 & -0.14 & -0.13 & 0 . & 0.403 \\
\hline 315.000 & 5.8884 & 6.900 & 62.778 & 62.761 & -0.03 & -0.01 & -0.13 & 0.02 & 0.405 \\
\hline 315.000 & 5.9914 & 7.500 & 06 & 6 & -0.04 & -0.04 & -0.01 & & .411 \\
\hline 315.000 & 6.1006 & 8.000 & 60.640 & 60.476 & -0.27 & -0.32 & 0.13 & -0 & 0.419 \\
\hline 315.000 & 6.4576 & 9.000 & 58.035 & 57.396 & -1.10 & -1.09 & -0.02 & & 0.438 \\
\hline & .2726 & & & & -1.47 & -1.47 & 0.01 & 0 . & 0.430 \\
\hline 325.000 & 6.6434 & 6.000 & 59.953 & 59.205 & -1.25 & -1.25 & -0.11 & 0.03 & 0.424 \\
\hline 325.000 & 6.8161 & 6.500 & & & -1.23 & -1.23 & -0.03 & & 0.424 \\
\hline 325.000 & 6.9579 & 6.900 & 59.825 & 59.062 & -1.28 & -1.28 & 0.03 & -0.01 & 0.425 \\
\hline 325.000 & 7.1928 & 7.500 & 59.332 & & -1.46 & -1.48 & 0.11 & -0.05 & 0.428 \\
\hline 325.000 & 7.4265 & 8.000 & 58.749 & & -1.68 & -1.70 & 0.11 & -0.06 & 0.433 \\
\hline 325.000 & 8.0855 & 9.000 & 57.397 & 56.189 & -2.10 & -2.07 & -0.14 & 0.13 & 0.443 \\
\hline
\end{tabular}

Number of Points [56] 30

Using temperature and density:

$\mathrm{AAD} ;=3.51 \quad \mathrm{BIAS} \%=-3.51 \quad \mathrm{RMS} \%=4.82$

Using temperature and pressure:

$A A D 2 \%=3.45 \quad$ BIAS $2 \%=-3.45 \quad$ RMS $2 \%=4.75$ 
Table 7. ISOCHORIC SPECIFIC HEAT CAPACITY (continued)

Data from Sengers [56] (continued)

Absolute Deviations:

Using temperature and density:

$\mathrm{AAD}=2.64$ BIAS $=-2.64 \mathrm{RMS}=4.01 \mathrm{~J} \cdot \mathrm{mol}^{-1} \cdot \mathrm{K}^{-1}$

Using temperature and pressure:

$\mathrm{AAD2}=2.59$ BIAS2 $=-2.59 \quad \mathrm{RMS} 2=3.95 \mathrm{~J} \cdot \mathrm{mol}^{-1} \cdot \mathrm{K}^{-1}$

Weighted Data:

Number of Points [88] 30

Using temperature and density:

$\mathrm{AAD} \%=3.51$ BIAS $\%=-3.51$ RMS $\%=4.82$

Using temperature and pressure:

$A A D 2 \%=3.45 \quad$ BIAS2 $8=-3.45 \quad$ RMS2 $z=4.75$

Absolute Deviations:

Using temperature and density:

$\mathrm{AAD}=2.64 \quad \mathrm{BIAS}=-2.64 \mathrm{RMS}=4.01 \mathrm{~J} \cdot \mathrm{mol}^{-1} \cdot \mathrm{K}^{-1}$

Using temperature and pressure:

$\mathrm{AAD} 2=2.59 \quad \mathrm{BIAS} 2=-2.59 \quad \mathrm{RMS} 2=3.95 \mathrm{~J} \cdot \mathrm{mol}^{-1} \cdot \mathrm{K}^{-1}$

Overall Results: $N=239$

$\begin{array}{llll}\mathrm{AAD} z=1.60 & \text { BIAS } 8=-1.26 & \mathrm{RMS} z=2.25 \\ \mathrm{AAD} 2 \%=1.69 & \text { BIAS2 } 8=-1.36 & \mathrm{RMS} 2 \%=2.25 \\ \mathrm{AAD}=0.90 & \text { BIAS }=-0.73 & \mathrm{RMS}=1.70 \mathrm{~J} \cdot \mathrm{mol}^{-1} \cdot \mathrm{K}^{-1} \\ \mathrm{AAD} 2=0.96 & \text { BIAS2 }=-0.79 & \mathrm{RMS} 2=1.69 \mathrm{~J} \cdot \mathrm{mol}^{-1} \cdot \mathrm{K}^{-1}\end{array}$

Weighted Data: $\quad N=214$

$\begin{array}{llll}\mathrm{AAD} z=1.49 & \text { BIAS } 8=-1.16 & \mathrm{RMS} z=2.28 \\ \mathrm{AAD} 2 \%=1.53 & \text { BIAS2 } 8=-1.22 & \mathrm{RMS} 2 \%=2.24 \\ \mathrm{AAD}=0.85 & \text { BIAS }=-0.68 & \mathrm{RMS}=1.76 \mathrm{~J} \cdot \mathrm{mol}^{-1} \cdot \mathrm{K}^{-1} \\ \mathrm{AAD2}=0.88 & \text { BIAS2 }=-0.72 & \mathrm{RMS} 2=1.74 \mathrm{~J} \cdot \mathrm{mol}^{-1} \cdot \mathrm{K}^{-1}\end{array}$


Table 8. ISOBARIC SPECIFIC HEAT CAPACITY

Data from Bier et al. [34]

Data extrapolated to zero pressure have been included in table 4.

\begin{tabular}{|c|c|c|c|c|c|c|}
\hline $\begin{array}{l}\mathrm{T} \\
\mathrm{K}\end{array}$ & $\begin{array}{c}\mathrm{P} \\
\mathrm{MPa}\end{array}$ & $\begin{array}{c}\rho \\
\mathrm{mol} \cdot \mathrm{dm}^{-3}\end{array}$ & $\begin{array}{c}C_{p}, \quad \operatorname{expt} \\
J \cdot \mathrm{mol}^{-1} \cdot \mathrm{K}^{-1}\end{array}$ & $\begin{array}{r}\mathrm{C}_{\mathrm{p}}, \quad \mathrm{calc} \\
\mathrm{J} \cdot \mathrm{mol} 1^{-1} \cdot \mathrm{K}^{-1}\end{array}$ & $\begin{array}{c}\mathrm{dev} \\
8\end{array}$ & wt \\
\hline 283.150 & 0.100 & 0.04 & 50.877 & 51.120 & 0.48 & 1.27 \\
\hline 298.150 & 0.100 & 0.04 & 52.772 & 52.845 & 0.14 & 1.22 \\
\hline 305.350 & 0.100 & 0.04 & 53.554 & 53.699 & 0.27 & 1.20 \\
\hline 323.150 & 0.100 & 0.04 & 55.749 & 55.867 & 0.21 & 1.16 \\
\hline 348.150 & 0.100 & 0.03 & 58.365 & 59.008 & 1.10 & 1.11 \\
\hline 373.150 & 0.100 & 0.03 & 61.372 & 62.208 & 1.36 & 1.05 \\
\hline 398.150 & 0.100 & 0.03 & 64.709 & 65.422 & 1.10 & 1.00 \\
\hline 423.150 & 0.100 & 0.03 & 67.897 & 68.614 & 1.06 & 0.95 \\
\hline 473.150 & 0.100 & 0.03 & 74.181 & 74.842 & 0.89 & 0.87 \\
\hline 283.150 & 0.200 & 0.09 & 51.298 & 51.591 & 0.57 & 1.26 \\
\hline 298.150 & 0.200 & 0.08 & 53.133 & 53.239 & 0.20 & 1.21 \\
\hline 305.350 & 0.200 & 0.08 & 53.944 & 54.062 & 0.22 & 1.20 \\
\hline 323.150 & 0.200 & 0.08 & 55.989 & 56.167 & 0.32 & 1.15 \\
\hline 348.150 & 0.200 & 0.07 & 58.575 & 59.243 & 1.14 & 1.10 \\
\hline 373.150 & 0.200 & 0.06 & 61.582 & 62.398 & 1.33 & 1.05 \\
\hline 398.150 & 0.200 & 0.06 & 64.860 & 65.579 & 1.11 & 1.00 \\
\hline 423.150 & 0.200 & 0.06 & 68.017 & 68.745 & 1.07 & 0.95 \\
\hline 473.150 & 0.200 & 0.05 & 74.271 & 74.938 & 0.90 & 0.87 \\
\hline 283.150 & 0.500 & 0.22 & 52.802 & 53.155 & 0.67 & 1.22 \\
\hline 298.150 & 0.500 & 0.21 & 54.426 & 54.522 & 0.18 & 1.19 \\
\hline 305.350 & 0.500 & 0.20 & 55.087 & 55.236 & 0.27 & 1.17 \\
\hline 323.150 & 0.500 & 0.19 & 56.951 & 57.124 & 0.30 & 1.13 \\
\hline 348.150 & 0.500 & 0.18 & 59.327 & 59.984 & 1.11 & 1.09 \\
\hline 373.150 & 0.500 & 0.16 & 62.214 & 62.989 & 1.25 & 1.04 \\
\hline 398.150 & 0.500 & 0.15 & 65.311 & 66.062 & 1.15 & 0.99 \\
\hline 423.150 & 0.500 & 0.14 & 68.408 & 69.148 & 1.08 & 0.94 \\
\hline 473.150 & 0.500 & 0.13 & 74.632 & 75.232 & 0.80 & 0.87 \\
\hline 283.150 & 1.000 & 0.47 & 56.019 & 56.440 & 0.75 & 1.15 \\
\hline 298.150 & 1.000 & 0.44 & 56.951 & 57.088 & 0.24 & 1.13 \\
\hline 305.350 & 1.000 & 0.42 & 57.252 & 57.542 & 0.51 & 1.13 \\
\hline 323.150 & 1.000 & 0.40 & 58.786 & 58.941 & 0.26 & 1.10 \\
\hline 348.150 & 1.000 & 0.36 & 60.680 & 61.346 & 1.10 & 1.06 \\
\hline 373.150 & 1.000 & 0.33 & 63.356 & 64.051 & 1.10 & 1.02 \\
\hline 398.150 & 1.000 & 0.31 & 66.093 & 66.915 & 1.24 & 0.98 \\
\hline 423.150 & 1.000 & 0.29 & 69.220 & 69.851 & 0.91 & 0.93 \\
\hline
\end{tabular}


Table 8. ISOBARIC SPECIFIC HEAT CAPACITY (continued)

Data from Bier et al. [34] (continued)

\begin{tabular}{|c|c|c|c|c|c|c|}
\hline $\begin{array}{l}\mathrm{T} \\
\mathrm{K}\end{array}$ & $\begin{array}{c}\mathrm{P} \\
\mathrm{MPa}\end{array}$ & $\stackrel{\rho}{\mathrm{mol} \cdot \mathrm{dm}^{-3}}$ & $\begin{array}{c}C_{p}, \quad \operatorname{expt} \\
\mathrm{J} \cdot \mathrm{mol} 1^{-1} \cdot \mathrm{K}^{-1}\end{array}$ & $\begin{array}{r}\mathrm{C}_{\mathrm{p}}, \quad \mathrm{calc} \\
\mathrm{J} \cdot \mathrm{mol}^{-1} \cdot \mathrm{K}^{-1}\end{array}$ & $\begin{array}{c}\text { dev } \\
8\end{array}$ & wt \\
\hline 473.150 & 1.000 & 0.26 & 75.174 & 75.735 & 0.75 & 0.86 \\
\hline 283.150 & 2.000 & 1.07 & 67.446 & 68.265 & 1.21 & 0.96 \\
\hline 298.150 & 2.000 & 0.97 & 64.679 & 64.905 & 0.35 & 1.00 \\
\hline 305.350 & 2.000 & 0.93 & 63.988 & 64.207 & 0.34 & 1.01 \\
\hline 323.150 & 2.000 & 0.85 & 63.537 & 63.731 & 0.31 & 1.02 \\
\hline 348.150 & 2.000 & 0.76 & 63.988 & 64.659 & 1.05 & 1.01 \\
\hline 373.150 & 2.000 & 0.70 & 65.912 & 66.506 & 0.90 & 0.98 \\
\hline 398.150 & 2.000 & 0.64 & 68.047 & 68.820 & 1.14 & 0.95 \\
\hline 423.150 & 2.000 & 0.60 & 70.783 & 71.380 & 0.84 & 0.91 \\
\hline 473.150 & 2.000 & 0.52 & 76.256 & 76.794 & 0.71 & 0.85 \\
\hline 298.150 & 3.000 & 1.69 & 81.638 & 81.980 & 0.42 & 0.79 \\
\hline 305.350 & 3.000 & 1.58 & 76.857 & 76.858 & 0.00 & 0.84 \\
\hline 323.150 & 3.000 & 1.40 & 70.844 & 71.125 & 0.40 & 0.91 \\
\hline 348.150 & 3.000 & 1.22 & 68.498 & 69.068 & 0.83 & 0.94 \\
\hline 373.150 & 3.000 & 1.09 & 68.919 & 69.511 & 0.86 & 0.94 \\
\hline 398.150 & 3.000 & 1.00 & 70.272 & 71.030 & 1.08 & 0.92 \\
\hline 423.150 & 3.000 & 0.92 & 72.527 & 73.090 & 0.78 & 0.89 \\
\hline 473.150 & 3.000 & 0.80 & 77.308 & 77.924 & 0.80 & 0.84 \\
\hline 323.150 & 3.200 & 1.52 & 72.978 & 73.118 & 0.19 & 0.88 \\
\hline 473.150 & 3.500 & 0.94 & 77.940 & 78.515 & 0.74 & 0.83 \\
\hline 373.150 & 3.600 & 1.35 & 70.783 & 71.644 & 1.22 & 0.91 \\
\hline 298.150 & 3.900 & 2.76 & 153.564 & 145.911 & -4.98 & 0.42 \\
\hline 305.350 & 4.000 & 2.55 & 114.685 & 113.037 & -1.44 & 0.56 \\
\hline 323.150 & 4.000 & 2.08 & 84.615 & 84.104 & -0.60 & 0.76 \\
\hline 348.150 & 4.000 & 1.74 & 74.783 & 75.158 & 0.50 & 0.86 \\
\hline 373.150 & 4.000 & 1.53 & 72.407 & 73.229 & 1.14 & 0.89 \\
\hline 398.150 & 4.000 & 1.37 & 72.828 & 73.598 & 1.06 & 0.89 \\
\hline 423.150 & 4.000 & 1.26 & 74.452 & 74.996 & 0.73 & 0.87 \\
\hline 473.150 & 4.000 & 1.08 & 78.541 & 79.124 & 0.74 & 0.82 \\
\hline 305.350 & 4.600 & 3.66 & 276.428 & 231.009 & -16.43 & 0.00 \\
\hline 307.150 & 4.880 & 4.33 & 401.607 & 375.105 & -6.60 & 0.00 \\
\hline 313.150 & 4.880 & 3.45 & 157.985 & 156.226 & -1.11 & 0.00 \\
\hline 316.150 & 4.880 & 3.23 & 134.410 & 133.141 & -0.94 & 0.48 \\
\hline 323.150 & 4.880 & 2.89 & 108.069 & 107.514 & -0.51 & 0.60 \\
\hline 348.150 & 4.880 & 2.27 & 82.059 & 82.686 & 0.76 & 0.79 \\
\hline
\end{tabular}


Table 8. ISOBARIC SPECIFIC HEAT CAPACITY (continued)

Data from Bier et al. [34] (continued)

\begin{tabular}{|c|c|c|c|c|c|c|}
\hline $\begin{array}{l}\mathrm{T} \\
\mathrm{K}\end{array}$ & $\begin{array}{c}\mathrm{P} \\
\mathrm{MPa}\end{array}$ & $\stackrel{\rho}{\mathrm{mol} \cdot \mathrm{dm}^{-3}}$ & $\begin{array}{c}C_{p}, \operatorname{expt} \\
J \cdot \mathrm{mol}^{-1} \cdot \mathrm{K}^{-1}\end{array}$ & $\begin{array}{r}\mathrm{C}_{\mathrm{p}}, \mathrm{calc}^{\mathrm{calc}} \\
\mathrm{J} \cdot \mathrm{mol}^{-1} \cdot \mathrm{K}^{-1}\end{array}$ & $\begin{array}{l}\mathrm{dev} \\
8\end{array}$ & wt \\
\hline 373.150 & 4.880 & 1.95 & 76.587 & 77.254 & 0.87 & 0.84 \\
\hline 398.150 & 4.880 & 1.73 & 75.354 & 76.194 & 1.11 & 0.86 \\
\hline 423.150 & 4.880 & 1.57 & 76.106 & 76.846 & 0.97 & 0.85 \\
\hline 473.150 & 4.880 & 1.34 & 79.564 & 80.235 & 0.84 & 0.81 \\
\hline 305.350 & 5.200 & 9.77 & 273.842 & 265.777 & -2.95 & 0.24 \\
\hline 305.350 & 6.000 & 10.70 & 162.615 & 163.743 & 0.69 & 0.40 \\
\hline 309.150 & 6.000 & 9.91 & 204.292 & 206.650 & 1.15 & 0.32 \\
\hline 313.150 & 6.000 & 8.57 & 341.859 & 346.649 & 1.40 & 0.19 \\
\hline 314.650 & 6.000 & 7.76 & 427.527 & 453.251 & 6.02 & 0.15 \\
\hline 316.150 & 6.000 & 6.83 & 458.318 & 482.826 & 5.35 & 0.14 \\
\hline 317.150 & 6.000 & 6.29 & 429.541 & 435.906 & 1.48 & 0.15 \\
\hline 319.150 & 6.000 & 5.51 & 326.343 & 321.170 & -1.59 & 0.20 \\
\hline 323.150 & 6.000 & 4.65 & 206.757 & 203.897 & -1.38 & 0.31 \\
\hline 348.150 & 6.000 & 3.09 & 96.403 & 97.012 & 0.63 & 0.67 \\
\hline 373.150 & 6.000 & 2.54 & 82.992 & 83.652 & 0.80 & 0.78 \\
\hline 398.150 & 6.000 & 2.21 & 79.293 & 80.001 & 0.89 & 0.81 \\
\hline 423.150 & 6.000 & 1.98 & 78.722 & 79.439 & 0.91 & 0.82 \\
\hline 473.150 & 6.000 & 1.67 & 81.067 & 81.719 & 0.80 & 0.80 \\
\hline 323.150 & 6.300 & 5.44 & 258.958 & 259.961 & 0.39 & 0.25 \\
\hline 323.150 & 6.600 & 6.39 & 296.575 & 302.408 & 1.97 & 0.22 \\
\hline 323.150 & 6.800 & 7.03 & 288.065 & 299.435 & 3.95 & 0.22 \\
\hline 305.350 & 7.000 & 11.30 & 132.997 & 134.008 & 0.76 & 0.49 \\
\hline 313.150 & 7.000 & 10.12 & 168.599 & 169.568 & 0.57 & 0.38 \\
\hline 316.150 & 7.000 & 9.52 & 198.007 & 194.701 & -1.67 & 0.33 \\
\hline 320.150 & 7.000 & 8.51 & 243.231 & 245.202 & 0.81 & 0.27 \\
\hline 323.150 & 7.000 & 7.59 & 272.699 & 276.469 & 1.38 & 0.24 \\
\hline 325.150 & 7.000 & 6.97 & 266.655 & 274.122 & 2.80 & 0.24 \\
\hline 329.150 & 7.000 & 5.96 & 225.400 & 228.663 & 1.45 & 0.29 \\
\hline 339.150 & 7.000 & 4.61 & 141.176 & 144.588 & 2.42 & 0.46 \\
\hline 348.150 & 7.000 & 4.01 & 115.376 & 116.310 & 0.81 & 0.56 \\
\hline 373.150 & 7.000 & 3.14 & 90.148 & 90.754 & 0.67 & 0.72 \\
\hline 398.150 & 7.000 & 2.68 & 82.962 & 83.891 & 1.12 & 0.78 \\
\hline 423.150 & 7.000 & 2.38 & 81.127 & 81.970 & 1.04 & 0.80 \\
\hline 473.150 & 7.000 & 1.98 & 82.360 & 83.100 & 0.90 & 0.78 \\
\hline 305.350 & 8.000 & 11.70 & 120.608 & 120.476 & -0.11 & 0.53 \\
\hline
\end{tabular}


Table 8. ISOBARIC SPECIFIC HEAT CAPACITY (continued)

Data from Bier et al. [34] (continued)

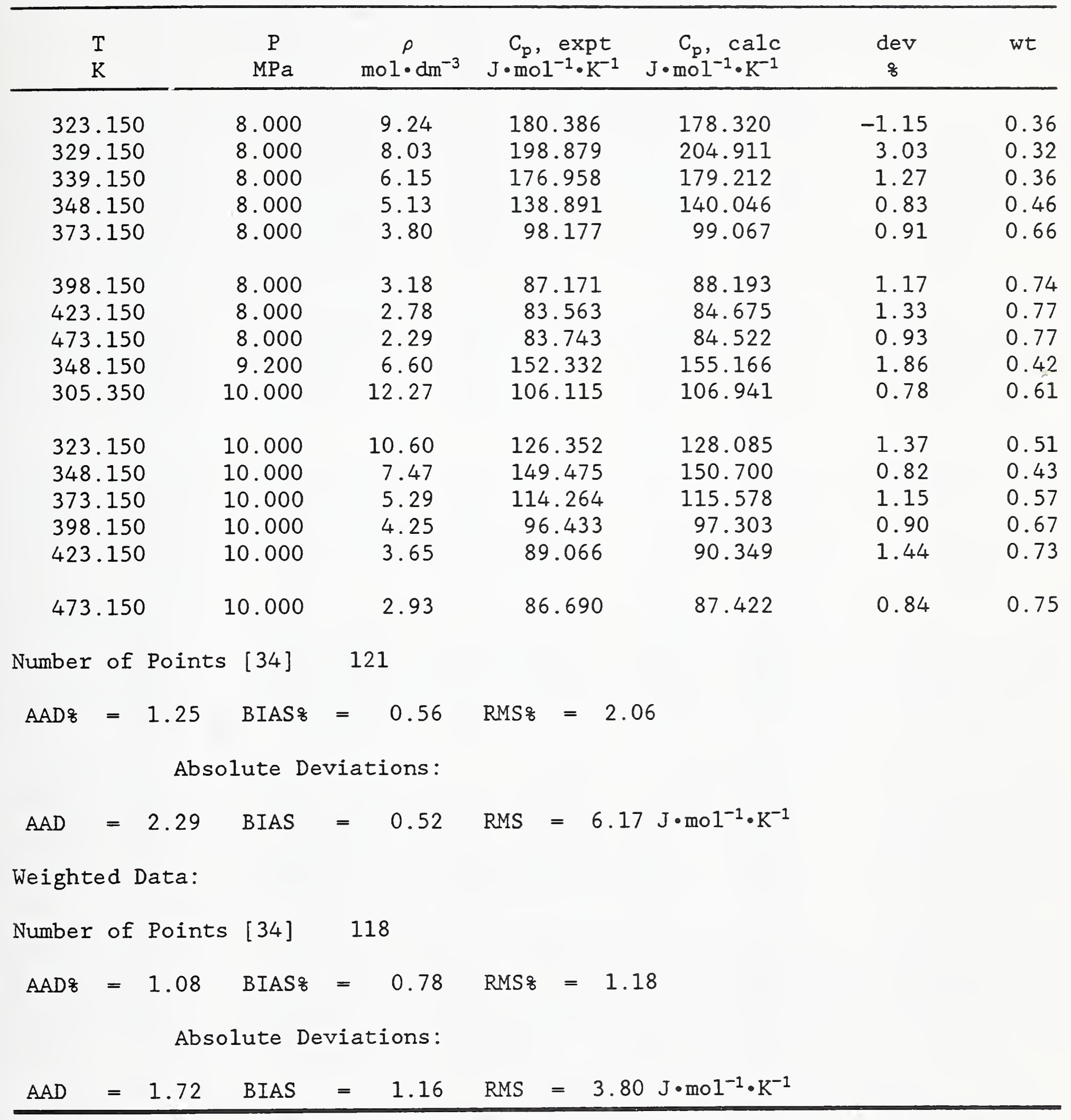


Table 8. ISOBARIC SPECIFIC HEAT CAPACITY (continued)

Data from Furtado [35]

Data extrapolated to zero pressure have been included in table 4.

\begin{tabular}{|c|c|c|c|c|c|c|}
\hline $\begin{array}{l}\mathrm{T} \\
\mathrm{K}\end{array}$ & $\begin{array}{c}\mathrm{P} \\
\mathrm{MPa}\end{array}$ & $\stackrel{\rho}{\mathrm{mol} \cdot \mathrm{dm}^{-3}}$ & $\begin{array}{c}C_{p}, \operatorname{expt} \\
J \cdot \mathrm{mol}^{-1} \cdot \mathrm{K}^{-1}\end{array}$ & $\begin{array}{r}\mathrm{C}_{\mathrm{p}}, \quad \mathrm{calc} \\
\mathrm{J} \cdot \mathrm{mol}^{-1} \cdot \mathrm{K}^{-1}\end{array}$ & $\begin{array}{c}\mathrm{dev} \\
8\end{array}$ & wt \\
\hline 99.817 & 1.724 & 21.35 & 68.451 & 70.000 & 2.26 & 18.35 \\
\hline 110.928 & 1.724 & 20.95 & 68.694 & 70.743 & 2.98 & 18.39 \\
\hline 118.372 & 1.724 & 20.68 & 68.826 & 70.513 & 2.45 & 18.41 \\
\hline 122.039 & 1.724 & 20.55 & 68.938 & 70.355 & 2.06 & 18.40 \\
\hline 133.150 & 1.724 & 20.14 & 69.200 & 69.974 & 1.12 & 18.39 \\
\hline 144.261 & 1.724 & 19.72 & 69.819 & 69.918 & 0.14 & 18.27 \\
\hline 155.372 & 1.724 & 19.30 & 70.700 & 70.234 & -0.66 & 18.08 \\
\hline 166.483 & 1.724 & 18.87 & 71.468 & 70.898 & -0.80 & 17.92 \\
\hline 177.594 & 1.724 & 18.43 & 72.462 & 71.882 & -0.80 & 17.69 \\
\hline 186.872 & 1.724 & 18.05 & 73.343 & 72.940 & -0.55 & 17.50 \\
\hline 188.706 & 1.724 & 17.97 & 73.474 & 73.175 & -0.41 & 17.47 \\
\hline 199.817 & 1.724 & 17.49 & 74.992 & 74.788 & -0.27 & 17.13 \\
\hline 210.928 & 1.724 & 16.99 & 76.754 & 76.770 & 0.02 & 16.75 \\
\hline 222.039 & 1.724 & 16.47 & 78.891 & 79.216 & 0.41 & 16.31 \\
\hline 233.150 & 1.724 & 15.90 & 82.021 & 82.305 & 0.35 & 15.70 \\
\hline 241.761 & 1.724 & 15.43 & 85.301 & 85.354 & 0.06 & 15.10 \\
\hline 244.261 & 1.724 & 15.28 & 85.938 & 86.388 & 0.52 & 14.99 \\
\hline 249.817 & 1.724 & 14.95 & 89.331 & 89.013 & -0.36 & 14.42 \\
\hline 252.594 & 1.724 & 14.77 & 91.336 & 90.537 & -0.88 & 14.11 \\
\hline 255.372 & 1.724 & 14.59 & 94.110 & 92.242 & -1.99 & 13.69 \\
\hline 258.150 & 1.724 & 14.40 & 98.759 & 94.172 & -4.64 & 0.00 \\
\hline 260.928 & 1.724 & 1.05 & 74.355 & 74.605 & 0.34 & 17.33 \\
\hline 262.594 & 1.724 & 1.04 & 72.724 & 72.927 & 0.28 & 17.72 \\
\hline 265.372 & 1.724 & 1.01 & 70.213 & 70.656 & 0.63 & 18.36 \\
\hline 266.483 & 1.724 & 1.00 & 69.444 & 69.895 & 0.65 & 18.56 \\
\hline 277.594 & 1.724 & 0.92 & 65.171 & 65.109 & -0.09 & 19.79 \\
\hline 282.706 & 1.724 & 0.89 & 64.046 & 63.939 & -0.17 & 20.14 \\
\hline 288.706 & 1.724 & 0.86 & 63.278 & 63.023 & -0.40 & 20.39 \\
\hline 299.817 & 1.724 & 0.80 & 62.397 & 62.157 & -0.38 & 20.68 \\
\hline 305.261 & 1.724 & 0.78 & 62.153 & 61.996 & -0.25 & 20.76 \\
\hline 310.928 & 1.724 & 0.76 & 62.022 & 61.959 & -0.10 & 20.81 \\
\hline 322.039 & 1.724 & 0.72 & 62.284 & 62.176 & -0.17 & 20.73 \\
\hline 324.817 & 1.724 & 0.71 & 62.397 & 62.279 & -0.19 & 20.69 \\
\hline 333.150 & 1.724 & 0.69 & 62.772 & 62.675 & -0.15 & 20.57 \\
\hline 344.261 & 1.724 & 0.66 & 63.652 & 63.372 & -0.44 & 20.29 \\
\hline
\end{tabular}


Table 8. ISOBARIC SPECIFIC HEAT CAPACITY (continued)

Data from Furtado [35] (continued)

\begin{tabular}{|c|c|c|c|c|c|c|}
\hline $\begin{array}{l}\mathrm{T} \\
\mathrm{K}\end{array}$ & $\begin{array}{c}\mathrm{P} \\
\mathrm{MPa}\end{array}$ & $\stackrel{\rho}{\mathrm{mol} \cdot \mathrm{dm}^{-3}}$ & $\begin{array}{c}C_{p}, \quad \operatorname{expt} \\
\mathrm{J} \cdot \mathrm{mol}^{-1} \cdot \mathrm{K}^{-1}\end{array}$ & $\begin{array}{r}\mathrm{C}_{\mathrm{p}}, \mathrm{calc}^{\mathrm{calc}} \\
\mathrm{J} \cdot \mathrm{mol}^{-1} \cdot \mathrm{K}^{-1}\end{array}$ & $\begin{array}{l}\text { dev } \\
8\end{array}$ & wt \\
\hline 355.372 & 1.724 & 0.63 & 64.533 & 64.215 & -0.49 & 20.01 \\
\hline 366.483 & 1.724 & 0.61 & 65.302 & 65.166 & -0.21 & 19.78 \\
\hline 366.817 & 1.724 & 0.61 & 65.414 & 65.196 & -0.33 & 19.75 \\
\hline 377.594 & 1.724 & 0.59 & 66.183 & 66.200 & 0.03 & 19.52 \\
\hline 277.594 & 2.827 & 13.00 & 115.365 & 113.353 & -1.74 & 11.18 \\
\hline 278.706 & 2.827 & 12.89 & 116.134 & 115.851 & -0.24 & 11.10 \\
\hline 279.817 & 2.827 & 12.77 & 118.645 & 118.684 & 0.03 & 10.87 \\
\hline 233.150 & 3.447 & 16.01 & 81.777 & 81.180 & -0.73 & 15.74 \\
\hline 241.761 & 3.447 & 15.56 & 84.420 & 83.812 & -0.72 & 15.26 \\
\hline 244.261 & 3.447 & 15.43 & 85.301 & 84.684 & -0.72 & 15.10 \\
\hline 255.372 & 3.447 & 14.78 & 89.200 & 89.398 & 0.22 & 14.45 \\
\hline 266.483 & 3.447 & 14.04 & 96.884 & 96.382 & -0.52 & 13.31 \\
\hline 277.594 & 3.447 & 13.15 & 109.705 & 108.860 & -0.77 & 11.75 \\
\hline 282.706 & 3.447 & 12.64 & 119.264 & 119.504 & 0.20 & 10.81 \\
\hline 285.928 & 3.447 & 12.26 & 128.823 & 130.320 & 1.16 & 10.01 \\
\hline 287.594 & 3.447 & 12.03 & 136.883 & 138.412 & 1.12 & 9.42 \\
\hline 288.706 & 3.447 & 11.87 & 142.806 & 145.437 & 1.84 & 9.03 \\
\hline 290.372 & 3.447 & 2.43 & 131.597 & 135.639 & 3.07 & 0.00 \\
\hline 291.483 & 3.447 & 2.37 & 122.282 & 126.846 & 3.73 & 0.00 \\
\hline 292.594 & 3.447 & 2.32 & 115.628 & 119.929 & 3.72 & 0.00 \\
\hline 294.261 & 3.447 & 2.26 & 107.193 & 111.905 & 4.40 & 0.00 \\
\hline 297.039 & 3.447 & 2.16 & 98.515 & 102.442 & 3.99 & 0.00 \\
\hline 299.817 & 3.447 & 2.08 & 98.384 & 95.883 & -2.54 & 0.00 \\
\hline 305.261 & 3.447 & 1.96 & 87.063 & 87.522 & 0.53 & 14.82 \\
\hline 310.928 & 3.447 & 1.85 & 82.283 & 82.181 & -0.12 & 15.68 \\
\hline 322.039 & 3.447 & 1.69 & 76.248 & 76.318 & 0.09 & 16.93 \\
\hline 324.817 & 3.447 & 1.66 & 75.611 & 75.390 & -0.29 & 17.07 \\
\hline 333.150 & 3.447 & 1.57 & 73.718 & 73.368 & -0.47 & 17.51 \\
\hline 344.261 & 3.447 & 1.47 & 72.218 & 71.853 & -0.51 & 17.88 \\
\hline 355.372 & 3.447 & 1.39 & 71.468 & 71.164 & -0.43 & 18.07 \\
\hline 366.483 & 3.447 & 1.32 & 71.468 & 70.998 & -0.66 & 18.07 \\
\hline 366.817 & 3.447 & 1.32 & 71.468 & 70.999 & -0.66 & 18.07 \\
\hline 282.706 & 4.137 & 12.83 & 111.598 & 112.962 & 1.22 & 11.56 \\
\hline 288.706 & 4.137 & 12.17 & 129.591 & 128.775 & -0.63 & 9.95 \\
\hline 294.261 & 4.137 & 11.35 & 161.661 & 163.215 & 0.96 & 0.00 \\
\hline
\end{tabular}


Table 8. ISOBARIC SPECIFIC HEAT CAPACITY (continued)

Data from Furtado [35] (continued)

\begin{tabular}{|c|c|c|c|c|c|c|}
\hline $\begin{array}{l}\mathrm{T} \\
\mathrm{K}\end{array}$ & $\begin{array}{c}\mathrm{P} \\
\mathrm{MPa}\end{array}$ & $\begin{array}{c}\rho \\
\mathrm{mol} \cdot \mathrm{dm}^{-3}\end{array}$ & $\begin{array}{c}C_{p}, \quad \operatorname{expt} \\
J \cdot m o 1^{-1} \cdot K^{-1}\end{array}$ & $\begin{array}{r}\mathrm{C}_{\mathrm{p}}, \mathrm{calc} \\
\mathrm{J} \cdot \mathrm{mol}^{-1} \cdot \mathrm{K}^{-1}\end{array}$ & $\begin{array}{l}\mathrm{dev} \\
\frac{8}{8}\end{array}$ & wt \\
\hline 295.928 & 4.137 & 11.01 & 179.280 & 186.430 & 3.99 & 0.00 \\
\hline 297.039 & 4.137 & 10.73 & 195.006 & 212.731 & 9.09 & 0.00 \\
\hline 298.706 & 4.137 & 3.24 & 220.797 & 213.515 & -3.30 & 0.00 \\
\hline 299.817 & 4.137 & 3.12 & 189.983 & 183.009 & -3.67 & 0.00 \\
\hline 299.817 & 4.137 & 3.12 & 189.851 & 183.009 & -3.60 & 0.00 \\
\hline 300.650 & 4.137 & 3.04 & 168.596 & 167.607 & -0.59 & 0.00 \\
\hline 301.206 & 4.137 & 3.00 & 157.763 & 159.463 & 1.08 & 0.00 \\
\hline 302.594 & 4.137 & 2.90 & 137.895 & 143.930 & 4.38 & 0.00 \\
\hline 305.261 & 4.137 & 2.74 & 122.413 & 124.965 & 2.08 & 0.00 \\
\hline 310.928 & 4.137 & 2.51 & 104.682 & 104.283 & -0.38 & 0.00 \\
\hline 322.039 & 4.137 & 2.21 & 89.200 & 87.649 & -1.74 & 14.47 \\
\hline 324.817 & 4.137 & 2.16 & 86.932 & 85.372 & -1.79 & 14.85 \\
\hline 298.428 & 4.668 & 10.94 & 174.875 & 177.215 & 1.34 & 0.00 \\
\hline 299.817 & 4.668 & 10.63 & 198.792 & 200.852 & 1.04 & 0.00 \\
\hline 300.650 & 4.668 & 10.40 & 221.434 & 222.710 & 0.58 & 0.00 \\
\hline 301.206 & 4.668 & 10.23 & 240.309 & 243.530 & 1.34 & 0.00 \\
\hline 302.039 & 4.668 & 9.92 & 279.313 & 294.684 & 5.50 & 0.00 \\
\hline 302.594 & 4.668 & 9.66 & 320.830 & 362.143 & 12.88 & 0.00 \\
\hline 302.872 & 4.668 & 9.49 & 352.281 & 423.859 & 20.32 & 0.00 \\
\hline 303.150 & 4.668 & 9.29 & 401.351 & 538.763 & 34.24 & 0.00 \\
\hline 303.983 & 4.668 & 4.25 & 425.249 & 464.788 & 9.30 & 0.00 \\
\hline 304.261 & 4.668 & 4.15 & 352.281 & 401.223 & 13.89 & 0.00 \\
\hline 305.261 & 4.668 & 3.90 & 299.444 & 286.082 & -4.46 & 0.00 \\
\hline 305.372 & 4.668 & 3.88 & 293.146 & 278.404 & -5.03 & 0.00 \\
\hline 305.928 & 4.668 & 3.78 & 261.695 & 247.358 & -5.48 & 0.00 \\
\hline 306.761 & 4.668 & 3.65 & 223.946 & 215.605 & -3.72 & 0.00 \\
\hline 308.150 & 4.668 & 3.49 & 184.941 & 182.545 & -1.30 & 0.00 \\
\hline 309.539 & 4.668 & 3.35 & 162.299 & 161.738 & -0.35 & 0.00 \\
\hline 310.928 & 4.668 & 3.24 & 148.466 & 147.287 & -0.79 & 0.00 \\
\hline 310.928 & 4.668 & 3.24 & 148.841 & 147.287 & -1.04 & 0.00 \\
\hline 313.706 & 4.668 & 3.07 & 130.847 & 128.345 & -1.91 & 0.00 \\
\hline 316.483 & 4.668 & 2.92 & 120.276 & 116.382 & -3.24 & 10.73 \\
\hline 322.039 & 4.668 & 2.71 & 119.020 & 102.019 & -14.28 & 0.00 \\
\hline 324.817 & 4.668 & 2.62 & 97.878 & 97.372 & -0.52 & 13.19 \\
\hline 282.706 & 4.916 & 13.01 & 106.069 & 107,749 & 1.58 & 12.16 \\
\hline
\end{tabular}


Table 8. ISOBARIC SPECIFIC HEAT CAPACITY (continued)

Data from Furtado [35] (continued)

\begin{tabular}{|c|c|c|c|c|c|c|}
\hline $\begin{array}{l}\mathrm{T} \\
\mathrm{K}\end{array}$ & $\begin{array}{c}\mathrm{P} \\
\mathrm{MPa}\end{array}$ & $\begin{array}{c}\rho \\
\mathrm{mol} \cdot \mathrm{dm}^{-3}\end{array}$ & $\begin{array}{c}C_{p}, \quad \operatorname{expt} \\
J \cdot m o 1^{-1} \cdot K^{-1}\end{array}$ & $\begin{array}{r}\mathrm{C}_{\mathrm{p}}, \mathrm{calc}^{\mathrm{c}} \\
\mathrm{J} \cdot \mathrm{mol} 1^{-1} \cdot \mathrm{K}^{-1}\end{array}$ & $\begin{array}{l}\mathrm{dev} \\
8\end{array}$ & wt \\
\hline 283.150 & 4.916 & 12.97 & 106.687 & 108.377 & 1.58 & 12.09 \\
\hline 288.706 & 4.916 & 12.44 & 119.151 & 118.397 & -0.63 & 10.83 \\
\hline 294.261 & 4.916 & 11.78 & 135.496 & 135.755 & 0.19 & 0.00 \\
\hline 297.039 & 4.916 & 11.37 & 149.085 & 150.704 & 1.09 & 0.00 \\
\hline 298.261 & 4.916 & 11.17 & 156.638 & 159.972 & 2.13 & 0.00 \\
\hline 299.817 & 4.916 & 10.87 & 177.893 & 175.987 & -1.07 & 0.00 \\
\hline 300.928 & 4.916 & 10.63 & 193.131 & 192.271 & -0.45 & 0.00 \\
\hline 302.039 & 4.916 & 10.35 & 217.404 & 216.130 & -0.59 & 0.00 \\
\hline 302.594 & 4.916 & 10.19 & 231.237 & 233.099 & 0.81 & 0.00 \\
\hline 303.150 & 4.916 & 10.01 & 246.475 & 256.079 & 3.90 & 0.00 \\
\hline 303.706 & 4.916 & 9.80 & 274.403 & 289.517 & 5.51 & 0.00 \\
\hline 304.261 & 4.916 & 9.55 & 566.162 & 344.108 & -39.22 & 0.00 \\
\hline 304.817 & 4.916 & 9.22 & 691.967 & 455.469 & -34.18 & 0.00 \\
\hline 305.261 & 4.916 & 8.83 & 1006.499 & 708.886 & -29.57 & 0.00 \\
\hline 305.261 & 4.916 & 8.83 & 1132.323 & 708.886 & -37.40 & 0.00 \\
\hline 306.483 & 4.916 & 4.87 & 553.585 & 726.302 & 31.20 & 0.00 \\
\hline 307.039 & 4.916 & 4.57 & 488.152 & 480.476 & -1.57 & 0.00 \\
\hline 307.594 & 4.916 & 4.37 & 375.560 & 375.203 & -0.10 & 0.00 \\
\hline 308.150 & 4.916 & 4.22 & 332.395 & 315.085 & -5.21 & 0.00 \\
\hline 309.261 & 4.916 & 3.99 & 261.826 & 247.744 & -5.38 & 0.00 \\
\hline 310.928 & 4.916 & 3.75 & 200.048 & 196.828 & -1.61 & 0.00 \\
\hline 313.706 & 4.916 & 3.47 & 158.138 & 155.823 & -1.46 & 0.00 \\
\hline 316.483 & 4.916 & 3.27 & 135.496 & 134.367 & -0.83 & 9.53 \\
\hline 322.039 & 4.916 & 2.98 & 108.824 & 111.862 & 2.79 & 11.86 \\
\hline 324.817 & 4.916 & 2.87 & 101.908 & 105.194 & 3.22 & 12.67 \\
\hline 327.594 & 4.916 & 2.77 & 102.545 & 100.124 & -2.36 & 12.59 \\
\hline 333.150 & 4.916 & 2.61 & 95.366 & 92.952 & -2.53 & 13.54 \\
\hline 322.039 & 5.171 & 3.30 & 125.805 & 125.444 & -0.29 & 10.26 \\
\hline 324.817 & 5.171 & 3.15 & 116.883 & 115.501 & -1.18 & 11.04 \\
\hline 333.150 & 5.171 & 2.84 & 99.265 & 98.534 & -0.74 & 13.01 \\
\hline 344.261 & 5.171 & 2.55 & 88.825 & 88.036 & -0.89 & 14.54 \\
\hline 355.372 & 5.171 & 2.34 & 83.033 & 82.757 & -0.33 & 15.56 \\
\hline 366.483 & 5.171 & 2.18 & 79.266 & 79.852 & 0.74 & 16.30 \\
\hline 366.817 & 5.171 & 2.17 & 79.134 & 79.788 & 0.83 & 16.32 \\
\hline 377.594 & 5.171 & 2.04 & 76.754 & 78.240 & 1.94 & 16.83 \\
\hline
\end{tabular}


Table 8. ISOBARIC SPECIFIC HEAT CAPACITY (continued)

Data from Furtado [35] (continued)

\begin{tabular}{|c|c|c|c|c|c|c|}
\hline $\begin{array}{l}\mathrm{T} \\
\mathrm{K}\end{array}$ & $\begin{array}{c}\mathrm{P} \\
\mathrm{MPa}\end{array}$ & $\stackrel{\rho}{\mathrm{mol} \cdot \mathrm{dm}^{-3}}$ & $\begin{array}{c}C_{p}, \operatorname{expt} \\
\mathrm{J} \cdot \mathrm{mol}^{-1} \cdot \mathrm{K}^{-1}\end{array}$ & $\begin{array}{r}\mathrm{C}_{\mathrm{p}}, \quad \mathrm{calc} \\
\mathrm{J} \cdot \mathrm{mo}^{-1} \cdot \mathrm{K}^{-1}\end{array}$ & $\begin{array}{c}\mathrm{dev} \\
\frac{8}{8}\end{array}$ & wt \\
\hline 310.372 & 5.647 & 8.75 & 408.886 & 384.757 & -5.90 & 3.16 \\
\hline 310.650 & 5.647 & 8.59 & 444.124 & 419.568 & -5.53 & 2.91 \\
\hline 310.928 & 5.647 & 8.42 & 483.129 & 461.106 & -4.56 & 2.67 \\
\hline 311.206 & 5.647 & 8.23 & 530.924 & 509.575 & -4.02 & 2.43 \\
\hline 311.483 & 5.647 & 8.01 & 577.483 & 563.150 & -2.48 & 2.23 \\
\hline 311.761 & 5.647 & 7.78 & 626.553 & 617.416 & -1.46 & 2.06 \\
\hline 312.039 & 5.647 & 7.53 & 666.813 & 664.009 & -0.42 & 1.94 \\
\hline 312.150 & 5.647 & 7.42 & 680.646 & 678.428 & -0.33 & 1.90 \\
\hline 312.261 & 5.647 & 7.32 & 689.455 & 689.753 & 0.04 & 1.87 \\
\hline 312.428 & 5.647 & 7.16 & 693.223 & 700.368 & 1.03 & 1.86 \\
\hline 312.594 & 5.647 & 7.00 & 694.479 & 703.048 & 1.23 & 1.86 \\
\hline 312.706 & 5.647 & 6.90 & 693.223 & 700.590 & 1.06 & 1.86 \\
\hline 312.817 & 5.647 & 6.80 & 691.967 & 695.040 & 0.44 & 1.87 \\
\hline 312.928 & 5.647 & 6.70 & 679.390 & 686.714 & 1.08 & 1.90 \\
\hline 313.150 & 5.647 & 6.51 & 644.153 & 663.239 & 2.96 & 2.00 \\
\hline 313.428 & 5.647 & 6.29 & 595.101 & 625.100 & 5.04 & 2.17 \\
\hline 313.706 & 5.647 & 6.10 & 552.310 & 582.501 & 5.47 & 2.34 \\
\hline 313.983 & 5.647 & 5.92 & 514.580 & 539.840 & 4.91 & 2.51 \\
\hline 314.261 & 5.647 & 5.77 & 485.640 & 499.394 & 2.83 & 2.66 \\
\hline 110.928 & 6.895 & 21.03 & 68.563 & 70.542 & 2.89 & 18.42 \\
\hline 118.372 & 6.895 & 20.76 & 68.694 & 70.297 & 2.33 & 18.44 \\
\hline 122.039 & 6.895 & 20.63 & 68.826 & 70.126 & 1.89 & 18.43 \\
\hline 133.150 & 6.895 & 20.23 & 69.069 & 69.688 & 0.90 & 18.42 \\
\hline 144.261 & 6.895 & 19.83 & 69.707 & 69.555 & -0.22 & 18.30 \\
\hline 155.372 & 6.895 & 19.42 & 70.456 & 69.774 & -0.97 & 18.14 \\
\hline 166.483 & 6.895 & 19.00 & 71.206 & 70.317 & -1.25 & 17.98 \\
\hline 177.594 & 6.895 & 18.57 & 71.843 & 71.146 & -0.97 & 17.85 \\
\hline 186.872 & 6.895 & 18.21 & 72.462 & 72.040 & -0.58 & 17.71 \\
\hline 188.706 & 6.895 & 18.14 & 73.230 & 72.237 & -1.36 & 17.53 \\
\hline 199.817 & 6.895 & 17.69 & 73.980 & 73.581 & -0.54 & 17.37 \\
\hline 210.928 & 6.895 & 17.22 & 75.367 & 75.189 & -0.24 & 17.06 \\
\hline 222.039 & 6.895 & 16.73 & 76.998 & 77.094 & 0.13 & 16.71 \\
\hline 233.150 & 6.895 & 16.22 & 79.266 & 79.359 & 0.12 & 16.24 \\
\hline 241.761 & 6.895 & 15.81 & 81.140 & 81.425 & 0.35 & 15.87 \\
\hline 244.261 & 6.895 & 15.68 & 82.415 & 82.088 & -0.40 & 15.63 \\
\hline
\end{tabular}


Table 8. ISOBARIC SPECIFIC HEAT CAPACITY (continued)

Data from Furtado [35] (continued)

\begin{tabular}{|c|c|c|c|c|c|c|}
\hline $\begin{array}{l}\mathrm{T} \\
\mathrm{K}\end{array}$ & $\begin{array}{c}\mathrm{P} \\
\mathrm{MPa}\end{array}$ & $\stackrel{\rho}{\mathrm{mol}} \cdot \mathrm{dm}^{-3}$ & $\begin{array}{c}C_{p}, \quad \operatorname{expt} \\
J \cdot \mathrm{mol}^{-1} \cdot \mathrm{K}^{-1}\end{array}$ & $\begin{array}{r}\mathrm{C}_{\mathrm{p}},{ }^{\mathrm{calc}} \\
\mathrm{J} \cdot \mathrm{mol}^{-1} \cdot \mathrm{K}^{-1}\end{array}$ & $\begin{array}{l}\mathrm{dev} \\
8\end{array}$ & wt \\
\hline 255.372 & 6.895 & 15.10 & 85.676 & 85.465 & -0.25 & 15.04 \\
\hline 266.483 & 6.895 & 14.47 & 89.462 & 89.820 & 0.40 & 14.41 \\
\hline 277.594 & 6.895 & 13.76 & 95.741 & 95.819 & 0.08 & 13.47 \\
\hline 282.706 & 6.895 & 13.39 & 98.890 & 99.489 & 0.61 & 13.04 \\
\hline 288.706 & 6.895 & 12.93 & 104.925 & 104.987 & 0.06 & 12.29 \\
\hline 299.817 & 6.895 & 11.90 & 111.842 & 121.514 & 8.65 & 11.54 \\
\hline 305.261 & 6.895 & 11.26 & 137.145 & 135.726 & -1.03 & 9.41 \\
\hline 310.928 & 6.895 & 10.42 & 158.531 & 160.391 & 1.17 & 8.14 \\
\hline 314.261 & 6.895 & 9.80 & 176.394 & 184.806 & 4.77 & 7.32 \\
\hline 316.483 & 6.895 & 9.31 & 200.160 & 208.818 & 4.33 & 6.45 \\
\hline 317.594 & 6.895 & 9.03 & 222.690 & 223.893 & 0.54 & 5.80 \\
\hline 319.261 & 6.895 & 8.56 & 254.272 & 249.719 & -1.79 & 5.08 \\
\hline 320.372 & 6.895 & 8.22 & 270.616 & 267.042 & -1.32 & 4.77 \\
\hline 320.928 & 6.895 & 8.04 & 277.795 & 274.756 & -1.09 & 4.65 \\
\hline 322.039 & 6.895 & 7.67 & 284.974 & 286.155 & 0.41 & 4.53 \\
\hline 322.706 & 6.895 & 7.45 & 285.592 & 289.482 & 1.36 & 4.52 \\
\hline 323.706 & 6.895 & 7.12 & 285.218 & 288.823 & 1.26 & 4.53 \\
\hline 324.817 & 6.895 & 6.78 & 281.825 & 280.858 & -0.34 & 4.58 \\
\hline 324.817 & 6.895 & 6.78 & 282.200 & 280.858 & -0.48 & 4.57 \\
\hline 325.372 & 6.895 & 6.62 & 277.158 & 274.703 & -0.89 & 4.66 \\
\hline 326.483 & 6.895 & 6.31 & 258.302 & 259.775 & 0.57 & 5.00 \\
\hline 327.594 & 6.895 & 6.04 & 240.927 & 243.422 & 1.04 & 5.36 \\
\hline 330.372 & 6.895 & 5.50 & 200.554 & 205.666 & 2.55 & 6.44 \\
\hline 333.150 & 6.895 & 5.08 & 176.506 & 177.273 & 0.43 & 7.31 \\
\hline 335.928 & 6.895 & 4.76 & 158.906 & 156.965 & -1.22 & 8.13 \\
\hline 338.706 & 6.895 & 4.51 & 143.686 & 142.236 & -1.01 & 8.99 \\
\hline 344.261 & 6.895 & 4.11 & 123.294 & 122.839 & -0.37 & 10.47 \\
\hline 355.372 & 6.895 & 3.59 & 104.419 & 103.016 & -1.34 & 12.37 \\
\hline 366.483 & 6.895 & 3.24 & 94.485 & 93.454 & -1.09 & 13.67 \\
\hline 366.817 & 6.895 & 3.23 & 94.242 & 93.248 & -1.05 & 13.71 \\
\hline 282.706 & 8.618 & 13.66 & 94.860 & 95.084 & 0.24 & 13.60 \\
\hline 288.706 & 8.618 & 13.25 & 98.890 & 98.839 & -0.05 & 13.04 \\
\hline 299.817 & 8.618 & 12.39 & 108.205 & 108.388 & 0.17 & 11.92 \\
\hline 305.261 & 8.618 & 11.91 & 114.616 & 114.963 & 0.30 & 11.26 \\
\hline 310.928 & 8.618 & 11.34 & 123.425 & 123.825 & 0.32 & 10.46 \\
\hline
\end{tabular}


Table 8. ISOBARIC SPECIFIC HEAT CAPACITY (cont:inued)

Data from Furtado [35] (continued)

\begin{tabular}{|c|c|c|c|c|c|c|}
\hline $\begin{array}{l}\mathrm{T} \\
\mathrm{K}\end{array}$ & $\begin{array}{c}\mathrm{P} \\
\mathrm{MPa}\end{array}$ & $\stackrel{\rho}{\mathrm{mol} \cdot \mathrm{dm}^{-3}}$ & $\begin{array}{c}C_{p}, \quad \operatorname{expt} \\
\mathrm{J} \cdot \mathrm{mol}^{-1} \cdot \mathrm{K}^{-1}\end{array}$ & 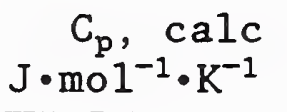 & $\begin{array}{l}\text { dev } \\
8\end{array}$ & wt \\
\hline 316.483 & 8.618 & 10.70 & 134.615 & 135.283 & 0.50 & 9.59 \\
\hline 322.039 & 8.618 & 9.96 & 149.965 & 150.444 & 0.32 & 8.61 \\
\hline 324.817 & 8.618 & 9.54 & 160.162 & 159.443 & -0.45 & 8.06 \\
\hline 327.594 & 8.618 & 9.09 & 168.203 & 168.747 & 0.32 & 7.68 \\
\hline 330.372 & 8.618 & 8.61 & 176.131 & 177.027 & 0.51 & 7.33 \\
\hline 333.150 & 8.618 & 8.12 & 180.536 & 182.443 & 1.06 & 7.15 \\
\hline 334.817 & 8.618 & 7.83 & 181.173 & 183.672 & 1.38 & 7.13 \\
\hline 337.039 & 8.618 & 7.44 & 180.292 & 182.695 & 1.33 & 7.16 \\
\hline 338.706 & 8.618 & 7.17 & 177.649 & 180.131 & 1.40 & 7.27 \\
\hline 341.483 & 8.618 & 6.74 & 170.227 & 173.186 & 1.74 & 7.59 \\
\hline 344.261 & 8.618 & 6.35 & 161.793 & 164.321 & 1.56 & 7.98 \\
\hline 349.817 & 8.618 & 5.72 & 148.466 & 146.134 & -1.57 & 8.70 \\
\hline 355.372 & 8.618 & 5.23 & 133.996 & 131.199 & -2.09 & 9.64 \\
\hline 366.483 & 8.618 & 4.54 & 114.241 & 111.748 & -2.18 & 11.31 \\
\hline 366.817 & 8.618 & 4.53 & 113.978 & 111.324 & -2.33 & 11.33 \\
\hline 377.594 & 8.618 & 4.08 & 101.664 & 100.887 & -0.76 & 12.71 \\
\hline 241.761 & 10.342 & 16.02 & 79.641 & 79.646 & 0.01 & 16.17 \\
\hline 244.261 & 10.342 & 15.90 & 80.147 & 80.181 & 0.04 & 16.07 \\
\hline 255.372 & 10.342 & 15.37 & 82.789 & 82.816 & 0.03 & 15.56 \\
\hline 266.483 & 10.342 & 14.80 & 85.938 & 85.970 & 0.04 & 15.00 \\
\hline 277.594 & 10.342 & 14.19 & 89.837 & 89.844 & 0.01 & 14.35 \\
\hline 282.706 & 10.342 & 13.89 & 91.842 & 91.953 & 0.12 & 14.04 \\
\hline 288.706 & 10.342 & 13.52 & 94.729 & 94.774 & 0.05 & 13.62 \\
\hline 299.817 & 10.342 & 12.76 & 101.664 & 101.322 & -0.34 & 12.69 \\
\hline 305.261 & 10.342 & 12.35 & 105.675 & 105.370 & -0.29 & 12.21 \\
\hline 310.928 & 10.342 & 11.89 & 110.342 & 110.345 & 0.00 & 11.70 \\
\hline 316.483 & 10.342 & 11.40 & 115.628 & 116.102 & 0.41 & 11.16 \\
\hline 322.039 & 10.342 & 10.87 & 122.169 & 122.821 & 0.53 & 10.57 \\
\hline 324.817 & 10.342 & 10.58 & 126.443 & 126.535 & 0.07 & 10.21 \\
\hline 327.594 & 10.342 & 10.28 & 129.966 & 130.436 & 0.36 & 9.93 \\
\hline 333.150 & 10.342 & 9.64 & 137.895 & 138.348 & 0.33 & 9.36 \\
\hline 338.706 & 10.342 & 8.96 & 144.811 & 144.874 & 0.04 & 8.92 \\
\hline 344.261 & 10.342 & 8.26 & 150.846 & 147.807 & -2.01 & 8.56 \\
\hline 344.928 & 10.342 & 8.18 & 151.727 & 147.853 & -2.55 & 8.51 \\
\hline 345.928 & 10.342 & 8.06 & 151.484 & 147.797 & -2.43 & 8.53 \\
\hline
\end{tabular}


Table 8. ISOBARIC SPECIFIC HEAT CAPACITY (continued)

Data from Furtado [35] (continued)

\begin{tabular}{|c|c|c|c|c|c|c|}
\hline $\begin{array}{l}\mathrm{T} \\
\mathrm{K}\end{array}$ & $\begin{array}{c}\mathrm{P} \\
\mathrm{MPa}\end{array}$ & $\stackrel{\rho}{\mathrm{mol} \cdot \mathrm{dm}^{-3}}$ & $\begin{array}{c}C_{p}, \operatorname{expt} \\
\mathrm{J} \cdot \mathrm{mol}^{-1} \cdot \mathrm{K}^{-1}\end{array}$ & $\begin{array}{r}\mathrm{C}_{\mathrm{p}},{ }^{\mathrm{calc}} \\
\mathrm{J} \cdot \mathrm{mol}^{-1} \cdot \mathrm{K}^{-1}\end{array}$ & $\begin{array}{c}\mathrm{dev} \\
\frac{8}{8}\end{array}$ & wt \\
\hline 348.150 & 10.342 & 7.79 & 151.109 & 147.147 & -2.62 & 8.55 \\
\hline 349.817 & 10.342 & 7.59 & 149.216 & 146.216 & -2.01 & 8.66 \\
\hline 352.594 & 10.342 & 7.28 & 144.811 & 143.925 & -0.61 & 8.92 \\
\hline 355.372 & 10.342 & 6.99 & 140.912 & 140.894 & -0.01 & 9.17 \\
\hline 360.928 & 10.342 & 6.46 & 132.347 & 133.557 & 0.91 & 9.76 \\
\hline 366.483 & 10.342 & 6.01 & 126.817 & 125.894 & -0.73 & 10.19 \\
\hline 366.817 & 10.342 & 5.98 & 126.443 & 125.448 & -0.79 & 10.22 \\
\hline 377.594 & 10.342 & 5.30 & 116.752 & 112.944 & -3.26 & 11.07 \\
\hline 324.817 & 12.066 & 11.22 & 113.735 & 112.861 & -0.77 & 11.35 \\
\hline 333.150 & 12.066 & 10.48 & 120.276 & 119.676 & -0.50 & 10.73 \\
\hline 338.706 & 12.066 & 9.96 & 124.681 & 124.094 & -0.47 & 10.36 \\
\hline 344.261 & 12.066 & 9.42 & 129.704 & 127.726 & -1.53 & 9.96 \\
\hline 347.039 & 12.066 & 9.14 & 131.597 & 129.023 & -1.96 & 9.81 \\
\hline 349.817 & 12.066 & 8.87 & 132.740 & 129.884 & -2.15 & 9.73 \\
\hline 351.761 & 12.066 & 8.68 & 133.115 & 130.210 & -2.18 & 9.70 \\
\hline 352.317 & 12.066 & 8.62 & 133.228 & 130.261 & -2.23 & 9.69 \\
\hline 355.372 & 12.066 & 8.33 & 132.347 & 130.215 & -1.61 & 9.76 \\
\hline 358.150 & 12.066 & 8.07 & 130.341 & 129.715 & -0.48 & 9.91 \\
\hline 360.928 & 12.066 & 7.81 & 128.336 & 128.824 & 0.38 & 10.06 \\
\hline 366.483 & 12.066 & 7.33 & 124.043 & 126.072 & 1.64 & 10.41 \\
\hline 366.817 & 12.066 & 7.31 & 123.800 & 125.873 & 1.67 & 10.43 \\
\hline 377.594 & 12.066 & 6.51 & 117.258 & 118.365 & 0.94 & 11.02 \\
\hline 110.928 & 13.790 & 21.12 & 68.188 & 70.300 & 3.10 & 18.52 \\
\hline 118.372 & 13.790 & 20.86 & 68.451 & 70.042 & 2.32 & 18.51 \\
\hline 122.039 & 13.790 & 20.73 & 68.563 & 69.857 & 1.89 & 18.50 \\
\hline 133.150 & 13.790 & 20.34 & 68.938 & 69.356 & 0.61 & 18.46 \\
\hline 144.261 & 13.790 & 19.95 & 69.444 & 69.138 & -0.44 & 18.37 \\
\hline 155.372 & 13.790 & 19.56 & 70.081 & 69.251 & -1.18 & 18.24 \\
\hline 166.483 & 13.790 & 19.16 & 70.588 & 69.667 & -1.30 & 18.14 \\
\hline 177.594 & 13.790 & 18.76 & 71.094 & 70.340 & -1.06 & 18.03 \\
\hline 186.872 & 13.790 & 18.41 & 71.581 & 71.075 & -0.71 & 17.93 \\
\hline 188.706 & 13.790 & 18.34 & 71.712 & 71.237 & -0.66 & 17.90 \\
\hline 199.817 & 13.790 & 17.92 & 72.724 & 72.336 & -0.53 & 17.67 \\
\hline 210.928 & 13.790 & 17.49 & 73.980 & 73.625 & -0.48 & 17.38 \\
\hline 222.039 & 13.790 & 17.04 & 75.367 & 75.107 & -0.34 & 17.07 \\
\hline
\end{tabular}


Table 8. ISOBARIC SPECIFIC HEAT CAPACITY (continued)

Data from Furtado [35] (continued)

\begin{tabular}{|c|c|c|c|c|c|c|}
\hline $\begin{array}{l}\mathrm{T} \\
\mathrm{K}\end{array}$ & $\begin{array}{c}\mathrm{P} \\
\mathrm{MPa}\end{array}$ & $\stackrel{\rho}{\mathrm{mol} \cdot \mathrm{dm}^{-3}}$ & $\begin{array}{c}C_{p}, \operatorname{expt} \\
\mathrm{J} \cdot \mathrm{mol}^{-1} \cdot \mathrm{K}^{-1}\end{array}$ & $\begin{array}{r}\mathrm{C}_{\mathrm{p}}, \mathrm{calc}^{\mathrm{c}} \\
\mathrm{J} \cdot \mathrm{mol}^{-1} \cdot \mathrm{K}^{-1}\end{array}$ & $\begin{array}{c}\text { dev } \\
\frac{8}{8}\end{array}$ & wt \\
\hline 233.150 & 13.790 & 16.58 & 77.129 & 76.794 & -0.43 & 16.69 \\
\hline 241.761 & 13.790 & 16.21 & 78.628 & 78.255 & -0.47 & 16.38 \\
\hline 244.261 & 13.790 & 16.10 & 79.003 & 78.707 & -0.37 & 16.30 \\
\hline 255.372 & 13.790 & 15.60 & 81.140 & 80.881 & -0.32 & 15.88 \\
\hline 266.483 & 13.790 & 15.08 & 83.408 & 83.367 & -0.05 & 15.46 \\
\hline 277.594 & 13.790 & 14.53 & 86.313 & 85.236 & -0.09 & 14.94 \\
\hline 282.706 & 13.790 & 14.27 & 87.700 & 87.710 & 0.01 & 14.71 \\
\hline 288.706 & 13.790 & 13.94 & 89.706 & 89.585 & -0.13 & 14.38 \\
\hline 299.817 & 13.790 & 13.31 & 92.967 & 93.539 & 0.61 & 13.88 \\
\hline 305.261 & 13.790 & 12.99 & 94.860 & 95.737 & 0.92 & 13.60 \\
\hline 310.928 & 13.790 & 12.63 & 97.878 & 98.226 & 0.36 & 13.19 \\
\hline 322.039 & 13.790 & 11.88 & 103.801 & 103.700 & -0.10 & 12.44 \\
\hline 324.817 & 13.790 & 11.69 & 105.431 & 105.178 & -0.24 & 12.24 \\
\hline 333.150 & 13.790 & 11.06 & 110.455 & 109.769 & -0.62 & 11.69 \\
\hline 344.261 & 13.790 & 10.18 & 116.621 & 115.636 & -0.84 & 11.07 \\
\hline 355.372 & 13.790 & 9.26 & 120.276 & 119.450 & -0.69 & 10.74 \\
\hline 366.483 & 13.790 & 8.36 & 120.651 & 119.675 & -0.81 & 10.71 \\
\hline 366.817 & 13.790 & 8.34 & 118.383 & 119.628 & 1.05 & 10.91 \\
\hline 377.594 & 13.790 & 7.55 & 112.610 & 116.826 & 3.74 & 11.47 \\
\hline
\end{tabular}

Number of Points [35] 299

$\mathrm{AAD} ;=2.22 \quad \mathrm{BIAS} \%=-0.11$ RMS $8=5.59$

Absolute Deviations:

$\mathrm{AAD}=8.32 \quad \mathrm{BIAS}=-2.27 \quad \mathrm{RMS}=38.37 \mathrm{~J} \cdot \mathrm{mol}^{-1} \cdot \mathrm{K}^{-1}$

Weighted Data:

Number of Points [35] 241

$\mathrm{AAD} \%=1.09 \quad \mathrm{BIAS} \%=-0.02$ RMS $\%=1.63$

Absolute Deviations:

$\mathrm{AAD}=2.19$ BIAS $=0.12 \quad \mathrm{RMS}=5.15 \mathrm{~J} \cdot \mathrm{mol}^{-1} \cdot \mathrm{K}^{-1}$ 
Table 8. ISOBARIC SPECIFIC HEAT CAPACITY (continued)

Data from Miyazaki et al. [59]

\begin{tabular}{|c|c|c|c|c|c|c|}
\hline $\begin{array}{l}\mathrm{T} \\
\mathrm{K}\end{array}$ & $\begin{array}{c}\mathrm{P} \\
\mathrm{MPa}\end{array}$ & $\stackrel{\rho}{\mathrm{mol} \cdot \mathrm{dm}^{-3}}$ & $\begin{array}{c}C_{p}, \operatorname{expt} \\
\mathrm{J} \cdot \mathrm{mo} 1^{-1} \cdot \mathrm{K}^{-1}\end{array}$ & $\begin{array}{c}\mathrm{C}_{\mathrm{p}}, \quad \mathrm{calc} \\
\mathrm{J} \cdot \mathrm{mo1^{-1 }} \cdot \mathrm{K}^{-1}\end{array}$ & $\begin{array}{c}\mathrm{dev} \\
\frac{8}{8}\end{array}$ & wt \\
\hline 298.150 & 4.473 & 10.82 & 217.702 & 191.188 & -12.18 & 0.00 \\
\hline 303.150 & 4.473 & 3.61 & 278.142 & 246.560 & -11.35 & 0.00 \\
\hline 308.150 & 4.473 & 3.11 & 148.543 & 144.723 & -2.57 & 0.00 \\
\hline 313.150 & 4.473 & 2.83 & 119.376 & 116.386 & -2.50 & 0.00 \\
\hline 318.150 & 4.473 & 2.63 & 105.243 & 102.582 & -2.53 & 0.00 \\
\hline 323.150 & 4.473 & 2.48 & 97.123 & 94.384 & -2.82 & 0.00 \\
\hline 298.150 & 5.167 & 11.35 & 152.151 & 148.856 & -2.17 & 0.00 \\
\hline 303.150 & 5.167 & 10.37 & 213.793 & 203.509 & -4.81 & 0.00 \\
\hline 308.150 & 5.167 & 6.90 & 1533.539 & 1851.331 & 20.72 & 0.00 \\
\hline 313.150 & 5.167 & 4.09 & 231.534 & 222.399 & -3.95 & 0.00 \\
\hline 318.150 & 5.167 & 3.55 & 157.864 & 147.546 & -6.54 & 0.00 \\
\hline 323.150 & 5.167 & 3.23 & 121.780 & 120.825 & -0.78 & 0.00 \\
\hline 298.150 & 5.514 & 11.53 & 143.431 & 138.885 & -3.17 & 0.00 \\
\hline 303.150 & 5.514 & 10.72 & 177.410 & 171.804 & -3.16 & 0.00 \\
\hline 308.150 & 5.514 & 9.39 & 333.770 & 290.290 & -13.03 & 0.00 \\
\hline 313.150 & 5.514 & 5.52 & 490.131 & 503.995 & 2.83 & 0.00 \\
\hline 318.150 & 5.514 & 4.24 & 222.814 & 201.370 & -9.62 & 0.00 \\
\hline 323.150 & 5.514 & 3.72 & 147.340 & 144.594 & -1.86 & 0.00 \\
\hline 298.150 & 6.203 & 11.83 & 132.005 & 126.231 & -4.37 & 0.00 \\
\hline 303.150 & 6.203 & 11.19 & 145.837 & 143.568 & -1.56 & 0.00 \\
\hline 308.150 & 6.203 & 10.35 & 183.724 & 175.799 & -4.31 & 0.00 \\
\hline 313.150 & 6.203 & 9.09 & 291.974 & 262.642 & -10.05 & 0.00 \\
\hline 318.150 & 6.203 & 6.80 & 411.951 & 411.102 & -0.21 & 0.00 \\
\hline 323.150 & 6.203 & 5.17 & 240.250 & 240.972 & 0.30 & 0.00 \\
\hline 298.150 & 13.001 & 13.30 & 97.124 & 94.188 & -3.02 & 0.00 \\
\hline 303.150 & 13.001 & 13.00 & 97.244 & 96.374 & -0.90 & 0.00 \\
\hline 308.150 & 13.001 & 12.67 & 102.537 & 98.760 & -3.68 & 0.00 \\
\hline 313.150 & 13.001 & 12.34 & 105.544 & 101.359 & -3.96 & 0.00 \\
\hline 318.150 & 13.001 & 11.98 & 108.250 & 104.174 & -3.77 & 0.00 \\
\hline 323.150 & 13.001 & 11.61 & 111.860 & 107.192 & -4.17 & 0.00 \\
\hline 307.150 & 5.167 & 8.69 & 703.624 & 592.019 & -15.86 & 0.00 \\
\hline 307.550 & 5.167 & 8.23 & 1169.700 & 928.052 & -20.66 & 0.00 \\
\hline 307.950 & 5.167 & 7.43 & 1900.386 & 1684.962 & -11.34 & 0.00 \\
\hline 308.550 & 5.167 & 6.03 & 932.151 & 1369.314 & 46.90 & 0.00 \\
\hline 309.150 & 5.167 & 5.34 & 439.013 & 748.959 & 70.60 & 0.00 \\
\hline
\end{tabular}


Table 8. ISOBARIC SPECIFIC HEAT CAPACITY (continued)

Data from Miyazaki et al. [59] (continued)

\begin{tabular}{cccccrr}
\hline $\mathrm{T}$ & $\begin{array}{c}\mathrm{P} \\
\mathrm{MPa}\end{array}$ & $\begin{array}{c}\rho \\
\mathrm{mol} \cdot \mathrm{dm}^{-3}\end{array}$ & $\begin{array}{c}\mathrm{C}_{\mathrm{p}}, \operatorname{expt} \\
\mathrm{J} \cdot \mathrm{mol} \mathbf{l}^{-1} \cdot \mathrm{K}^{-1}\end{array}$ & $\begin{array}{c}\mathrm{C}_{\mathrm{p}}, \mathrm{calc} \\
\mathrm{J} \cdot \mathrm{mol} \mathbf{l}^{-1} \cdot \mathrm{K}^{-1}\end{array}$ & $\begin{array}{r}\mathrm{dev} \\
\text { o }\end{array}$ & wt \\
\hline 309.150 & 5.514 & 8.93 & 439.013 & 374.520 & -14.69 & 0.00 \\
310.150 & 5.514 & 8.27 & 565.305 & 562.179 & -0.55 & 0.00 \\
311.250 & 5.514 & 7.13 & 869.006 & 843.507 & -2.93 & 0.00 \\
312.150 & 5.514 & 6.19 & 685.582 & 722.845 & 5.44 & 0.00 \\
315.150 & 5.514 & 4.79 & 339.784 & 301.005 & -11.41 & 0.00 \\
315.150 & 6.203 & 8.31 & 363.840 & 344.229 & -5.39 & 0.00 \\
316.550 & 6.203 & 7.62 & 408.944 & 404.779 & -1.02 & 0.00 \\
317.450 & 6.203 & 7.15 & 420.972 & 419.082 & -0.45 & 0.00 \\
318.150 & 6.203 & 6.80 & 414.958 & 411.102 & -0.93 & 0.00 \\
320.150 & 6.203 & 5.96 & 342.791 & 338.457 & -1.26 & 0.00
\end{tabular}

Number of Points [59] 45

$\mathrm{AAD} \%=7.92 \quad \mathrm{BIAS} \%=-1.39 \quad \mathrm{RMS} \%=14.64$

Absolute Deviations:

$\mathrm{AAD}=46.67 \quad \mathrm{BIAS}=2.97 \mathrm{RMS}=107.38 \mathrm{~J} \cdot \mathrm{mol}^{-1} \cdot \mathrm{K}^{-1}$

Overall Results: $N=465$

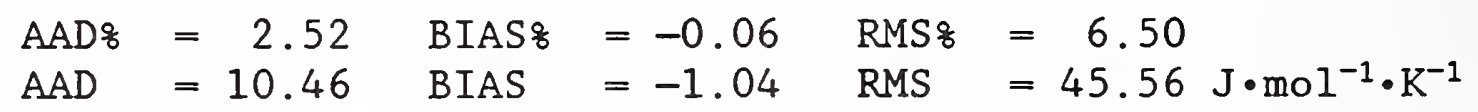

Weighted Data: $\quad N=359$

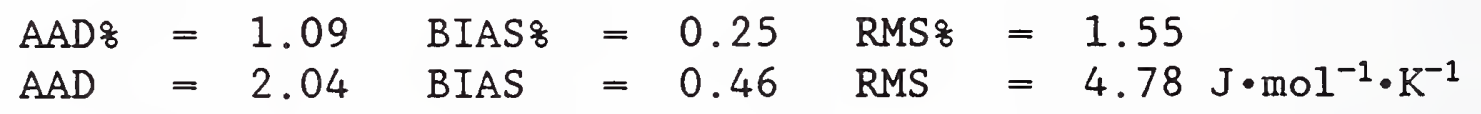


Table 9. SPECIFIC HEAT CAPACITY OF THE SATURATED LIQUID

Data from Roder [58]

\begin{tabular}{|c|c|c|c|c|c|}
\hline $\begin{array}{l}\mathrm{T} \\
\mathrm{K}\end{array}$ & $\stackrel{\rho}{\mathrm{mol} \cdot \mathrm{dm}^{-3}}$ & $\begin{array}{r}\mathrm{C}_{\sigma \mathrm{L}}, \operatorname{expt} \\
\mathrm{J} \cdot \mathrm{mol}^{-1} \cdot \mathrm{K}^{-1}\end{array}$ & $\begin{array}{r}\mathrm{C}_{\sigma \mathrm{L}}, \mathrm{calc} c \\
\mathrm{~J} \cdot \mathrm{mol}^{-1} \cdot \mathrm{K}^{-1}\end{array}$ & $\begin{array}{l}\text { dev } \\
8\end{array}$ & wt \\
\hline 93.712 & 21.547 & 68.270 & 68.482 & 0.31 & 0.0058 \\
\hline 97.045 & 21.429 & 68.300 & 69.515 & 1.78 & 0.0055 \\
\hline 98.095 & 21.391 & 68.440 & 69.750 & 1.91 & 0.0054 \\
\hline 100.322 & 21.311 & 68.450 & 70.135 & 2.46 & 0.0052 \\
\hline 101.482 & 21.270 & 68.440 & 70.283 & 2.69 & 0.0052 \\
\hline 103.565 & 21.195 & 68.460 & 70.478 & 2.95 & 0.0050 \\
\hline 104.828 & 21.149 & 68.530 & 70.559 & 2.96 & 0.0049 \\
\hline 106.788 & 21.079 & 68.350 & 70.638 & 3.35 & 0.0048 \\
\hline 108.142 & 21.030 & 68.600 & 70.667 & 3.01 & 0.0047 \\
\hline In8. 287 & 21.024 & 68.600 & 70.669 & 3.02 & 0.0047 \\
\hline 110.531 & 20.943 & 68.660 & 70.677 & 2.94 & 0.0046 \\
\hline 110.862 & 20.931 & 68.720 & 70.675 & 2.84 & 0.0045 \\
\hline 113.180 & 20.847 & 68.730 & 70.644 & 2.78 & 0.0044 \\
\hline 115.018 & 20.780 & 68.710 & 70.603 & 2.75 & 0.0043 \\
\hline 115.760 & 20.753 & 68.820 & 70.584 & 2.56 & 0.0043 \\
\hline 118.003 & 20.670 & 68.840 & 70.518 & 2.44 & 0.0041 \\
\hline 119.454 & 20.617 & 68.810 & 70.473 & 2.42 & 0.0041 \\
\hline 120.599 & 20.575 & 68.930 & 70.436 & 2.19 & 0.0040 \\
\hline 122.751 & 20.496 & 68.970 & 70.368 & 2.03 & 0.0039 \\
\hline 123.782 & 20.458 & 68.950 & 70.337 & 2.01 & 0.0039 \\
\hline 124.252 & 20.440 & 68.990 & 70.323 & 1.93 & 0.0039 \\
\hline 127.423 & 20.323 & 69.060 & 70.236 & 1.70 & 0.0037 \\
\hline 129.007 & 20.264 & 69.220 & 70.198 & 1.41 & 0.0036 \\
\hline 132.037 & 20.151 & 69.290 & 70.141 & 1.23 & 0.0035 \\
\hline 133.712 & 20.088 & 69.390 & 70.119 & 1.05 & 0.0035 \\
\hline 136.389 & 19.988 & 69.500 & 70.097 & 0.86 & 0.0034 \\
\hline 138.362 & 19.914 & 69.620 & 70.093 & 0.68 & 0.0033 \\
\hline 141.365 & 19.800 & 69.640 & 70.105 & 0.67 & 0.0032 \\
\hline 142.426 & 19.760 & 69.790 & 70.115 & 0.47 & 0.0032 \\
\hline 142.604 & 19.753 & 69.780 & 70.117 & 0.48 & 0.0031 \\
\hline 146.274 & 19.613 & 69.980 & 70.177 & 0.28 & 0.0030 \\
\hline 149.205 & 19.501 & 70.130 & 70.250 & 0.17 & 0.0029 \\
\hline 151.131 & 19.426 & 70.220 & 70.311 & 0.13 & 0.0029 \\
\hline 153.786 & 19.324 & 70.370 & 70.410 & 0.06 & 0.0028 \\
\hline 155.932 & 19.240 & 70.570 & 70.503 & -0.10 & 0.0028 \\
\hline
\end{tabular}


Table 9. SPECIFIC HEAT CAPACITY OF THE SATURATED LIQUID (continued)

Data from Roder [58] (continued)

\begin{tabular}{|c|c|c|c|c|c|}
\hline $\begin{array}{l}\mathrm{T} \\
\mathrm{K}\end{array}$ & $\stackrel{\rho}{\mathrm{mol} \cdot \mathrm{dm}^{-3}}$ & $\begin{array}{r}C_{\sigma \mathrm{L}}, \operatorname{expt} \\
\mathrm{J} \cdot \mathrm{mol}^{-1} \cdot \mathrm{K}^{-1}\end{array}$ & $\begin{array}{r}\mathrm{C}_{\sigma \mathrm{L}}, \mathrm{calc} c \\
\mathrm{~J} \cdot \mathrm{mol}^{-1} \cdot \mathrm{K}^{-1}\end{array}$ & $\begin{array}{l}\text { dev } \\
\frac{8}{8}\end{array}$ & wt \\
\hline 158.321 & 19.147 & 70.760 & 70.620 & -0.20 & 0.0027 \\
\hline 160.684 & 19.054 & 70.840 & 70.750 & -0.13 & 0.0026 \\
\hline 160.830 & 19.049 & 70.870 & 70.758 & -0.16 & 0.0026 \\
\hline 161.771 & 19.011 & 71.030 & 70.814 & -0.30 & 0.0026 \\
\hline 162.290 & 18.991 & 70.950 & 70.846 & -0.15 & 0.0013 \\
\hline 162.575 & 18.980 & 70.960 & 70.863 & -0.14 & 0.0013 \\
\hline 165.384 & 18.868 & 71.220 & 71.048 & -0.24 & 0.0013 \\
\hline 165.464 & 18.865 & 71.230 & 71.054 & -0.25 & 0.0013 \\
\hline 170.038 & 18.682 & 71.720 & 71.395 & -0.45 & 0.0012 \\
\hline 170.061 & 18.681 & 71.690 & 71.397 & -0.41 & 0.0012 \\
\hline 172.446 & 18.585 & 71.910 & 71.595 & -0.44 & 0.0012 \\
\hline 174.613 & 18.497 & 72.080 & 71.786 & -0.41 & 0.0012 \\
\hline 177.535 & 18.378 & 72.340 & 72.061 & -0.39 & 0.0011 \\
\hline 179.126 & 18.312 & 72.520 & 72.219 & -0.41 & 0.0011 \\
\hline 182.564 & 18.170 & 72.900 & 72.582 & -0.44 & 0.0011 \\
\hline 183.603 & 18.127 & 73.000 & 72.696 & -0.42 & 0.0011 \\
\hline 186.415 & 18.009 & 73.420 & 73.020 & -0.54 & 0.0010 \\
\hline 187.540 & 17.961 & 73.460 & 73.155 & -0.41 & 0.0010 \\
\hline 187.745 & 17.953 & 73.370 & 73.180 & -0.26 & 0.0010 \\
\hline 190.995 & 17.814 & 73.940 & 73.589 & -0.47 & 0.0010 \\
\hline 192.556 & 17.747 & 74.060 & 73.794 & -0.36 & 0.0010 \\
\hline 195.533 & 17.619 & 74.490 & 74.203 & -0.38 & 0.0010 \\
\hline 197.332 & 17.540 & 74.750 & 74.462 & -0.39 & 0.0010 \\
\hline 200.029 & 17.422 & 75.140 & 74.865 & -0.37 & 0.0009 \\
\hline 202.057 & 17.332 & 75.400 & 75.181 & -0.29 & 0.0009 \\
\hline 202.127 & 17.328 & 75.450 & 75.192 & -0.34 & 0.0009 \\
\hline 204.490 & 17.222 & 75.900 & 75.576 & -0.43 & 0.0009 \\
\hline 204.866 & 17.206 & 75.760 & 75.638 & -0.16 & 0.0009 \\
\hline 206.723 & 17.121 & 76.150 & 75.953 & -0.26 & 0.0009 \\
\hline 208.850 & 17.024 & 76.430 & 76.326 & -0.14 & 0.0009 \\
\hline 209.372 & 17.000 & 76.560 & 76.420 & -0.18 & 0.0009 \\
\hline 211.363 & 16.908 & 76.970 & 76.785 & -0.24 & 0.0008 \\
\hline 213.835 & 16.793 & 77.410 & 77.256 & -0.20 & 0.0008 \\
\hline 215.978 & 16.691 & 77.870 & 77.681 & -0.24 & 0.0008 \\
\hline 218.259 & 16.582 & 78.370 & 78.151 & -0.28 & 0.0008 \\
\hline
\end{tabular}


Table 9. SPECIFIC HEAT CAPACITY OF THE SATURATED LIQUID (continued)

Data from Roder [58] (continued)

\begin{tabular}{|c|c|c|c|c|c|}
\hline $\begin{array}{l}\mathrm{T} \\
\mathrm{K}\end{array}$ & $\stackrel{\rho}{\mathrm{mol} \cdot \mathrm{dm}^{-3}}$ & $\begin{array}{c}C_{\sigma \mathrm{L}}, \underset{\operatorname{expt}}{\mathrm{J} \cdot \mathrm{mol}^{-1} \cdot \mathrm{K}^{-1}}\end{array}$ & $\begin{array}{c}\mathrm{C}_{\sigma \mathrm{L}}, \quad \text { calc } \\
\mathrm{J} \cdot \mathrm{mol}^{-1} \cdot \mathrm{K}^{-1}\end{array}$ & $\begin{array}{c}\text { dev } \\
8\end{array}$ & wt \\
\hline 220.536 & 16.472 & 78.870 & 78.640 & -0.29 & 0.0008 \\
\hline 222.647 & 16.369 & 79.130 & 79.110 & -0.02 & 0.0008 \\
\hline 225.058 & 16.249 & 79.790 & 79.670 & -0.15 & 0.0008 \\
\hline 229.541 & 16.023 & 80.790 & 80.778 & -0.02 & 0.0007 \\
\hline 231.507 & 15.921 & 81.410 & 81.293 & -0.14 & 0.0006 \\
\hline 233.988 & 15.791 & 81.970 & 81.972 & 0.00 & 0.0006 \\
\hline 236.091 & 15.679 & 82.600 & 82.573 & -0.03 & 0.0005 \\
\hline 238.894 & 15.527 & 83.410 & 83.415 & 0.01 & 0.0005 \\
\hline 240.641 & 15.431 & 84.060 & 83.965 & -0.11 & 0.0005 \\
\hline$? 43.704$ & 15.259 & 84.900 & 84.979 & 0.09 & 0.0005 \\
\hline 244.814 & 15.196 & 85.260 & 85.364 & 0.12 & 0.0005 \\
\hline 247.726 & 15.027 & 86.410 & 86.419 & 0.01 & 0.0005 \\
\hline 248.469 & 14.983 & 86.560 & 86.700 & 0.16 & 0.0005 \\
\hline 252.414 & 14.745 & 88.290 & 88.279 & -0.01 & 0.0005 \\
\hline 257.059 & 14.453 & 90.390 & 90.356 & -0.04 & 0.0004 \\
\hline 260.020 & 14.260 & 91.870 & 91.828 & -0.05 & 0.0004 \\
\hline 260.744 & 14.211 & 92.040 & 92.208 & 0.18 & 0.0004 \\
\hline 261.383 & 14.168 & 92.320 & 92.550 & 0.25 & 0.0004 \\
\hline 264.754 & 13.935 & 94.600 & 94.476 & -0.13 & 0.0004 \\
\hline 269.456 & 13.592 & 97.750 & 97.569 & -0.19 & 0.0004 \\
\hline 274.128 & 13.227 & 101.370 & 101.264 & -0.10 & 0.0004 \\
\hline 274.528 & 13.194 & 101.810 & 101.617 & -0.19 & 0.0004 \\
\hline 279.261 & 12.789 & 106.680 & 106.364 & -0.30 & 0.0003 \\
\hline 283.620 & 12.379 & 112.750 & 112.012 & -0.65 & 0.0003 \\
\hline 283.946 & 12.346 & 112.850 & 112.501 & -0.31 & 0.0003 \\
\hline 288.238 & 11.889 & 121.720 & 120.219 & -1.23 & 0.0002 \\
\hline 291.779 & 11.461 & 130.470 & 129.277 & -0.91 & 0.0002 \\
\hline 292.479 & 11.368 & 133.410 & 131.523 & -1.41 & 0.0002 \\
\hline 292.539 & 11.360 & 133.540 & 131.724 & -1.36 & 0.0002 \\
\hline 295.236 & 10.974 & 146.320 & 142.709 & -2.47 & 0.0002 \\
\hline 296.550 & 10.763 & 151.710 & 149.978 & -1.14 & 0.0002 \\
\hline 296.661 & 10.745 & 152.770 & 150.674 & -1.37 & 0.0002 \\
\hline 298.389 & 10.435 & 167.240 & 163.795 & -2.06 & 0.0002 \\
\hline 300.295 & 10.034 & 187.540 & 186.437 & -0.59 & 0.0002 \\
\hline 300.550 & 9.974 & 191.340 & 190.574 & -0.40 & 0.0001 \\
\hline 301.473 & 9.737 & 212.290 & 209.246 & -1.43 & 0.0001 \\
\hline
\end{tabular}




$$
\begin{aligned}
& \text { Data from Roder [58] (continued) } \\
& \text { Number of Points [58] } 106 \\
& \mathrm{AAD} \%=0.84 \quad \mathrm{BIAS} \%=0.31 \text { RMS\% }=1.23 \\
& \text { Absolute Deviations: } \\
& \mathrm{AAD}=0.71 \text { BIAS }=0.09 \text { RMS }=1.08 \mathrm{~J} \cdot \mathrm{mol}^{-1} \cdot \mathrm{K}^{-1}
\end{aligned}
$$

Weighted Data:

Number of Points [58] 106

\begin{tabular}{|c|c|c|c|c|c|}
\hline $\begin{array}{l}\mathrm{T} \\
\mathrm{K}\end{array}$ & $\begin{array}{c}\rho \\
\mathrm{mol} \cdot \mathrm{dm}^{-3}\end{array}$ & $\begin{array}{c}\mathrm{C}_{\sigma \mathrm{L}}, \operatorname{expt} \\
\mathrm{J} \cdot \mathrm{mol}^{-1} \cdot \mathrm{K}^{-1}\end{array}$ & $\begin{array}{c}\mathrm{C}_{\sigma \mathrm{L}}, \quad \mathrm{calc} \\
\mathrm{J} \cdot \mathrm{mol}^{-1} \cdot \mathrm{K}^{-1}\end{array}$ & $\begin{array}{l}\text { dev } \\
8\end{array}$ & wt \\
\hline 96.770 & 21.438 & 68.460 & 69.447 & 1.44 & 0.0000 \\
\hline 96.820 & 21.437 & 68.760 & 69.459 & 1.02 & 0.0000 \\
\hline 98.060 & 21.392 & 68.550 & 69.742 & 1.74 & 0.0000 \\
\hline 101.540 & 21.268 & 68.710 & 70.290 & 2.30 & 0.0000 \\
\hline 107.080 & 21.068 & 68.630 & 70.646 & 2.94 & 0.0000 \\
\hline 108.650 & 21.011 & 68.550 & 70.673 & 3.10 & 0.0000 \\
\hline 115.740 & 20.753 & 68.670 & 70.584 & 2.79 & 0.0000 \\
\hline 116.190 & 20.737 & 68.450 & 70.572 & 3.10 & 0.0000 \\
\hline 122.700 & 20.498 & 69.130 & 70.370 & 1.79 & 0.0000 \\
\hline 123.600 & 20.464 & 69.170 & 70.342 & 1.69 & 0.0000 \\
\hline 128.080 & 20.299 & 69.550 & 70.220 & 0.96 & 0.0000 \\
\hline 128.490 & 20.283 & 69.510 & 70.210 & 1.01 & 0.0000 \\
\hline 132.650 & 20.128 & 69.840 & 70.132 & 0.42 & 0.0000 \\
\hline 138.000 & 19.927 & 69.890 & 70.093 & 0.29 & 0.0000 \\
\hline 138.180 & 19.920 & 70.010 & 70.092 & 0.12 & 0.0000 \\
\hline 138.310 & 19.916 & 69.840 & 70.092 & 0.36 & 0.0000 \\
\hline 142.430 & 19.760 & 70.100 & 70.115 & 0.02 & 0.0000 \\
\hline 143.360 & 19.724 & 70.050 & 70.127 & 0.11 & 0.0000 \\
\hline 151.750 & 19.403 & 69.970 & 70.332 & 0.52 & 0.0000 \\
\hline 152.600 & 19.370 & 70.140 & 70.363 & 0.32 & 0.0000 \\
\hline
\end{tabular}

$$
\mathrm{AAD} \%=0.84 \quad \text { BIAS } 8=0.31 \quad \text { RMS } \%=1.23
$$

Absolute Deviations:

$\mathrm{AAD}=0.71$ BIAS $=0.09 \mathrm{RMS}=1.08 \mathrm{~J} \cdot \mathrm{mol}^{-1} \cdot \mathrm{K}^{-1}$

Data from Wiebe et a1. [60] 
Table 9. SPECIFIC HEAT CAPACITY OF THE SATURATED LIQUID (continued)

Data from Wiebe et al. [60] (continued)

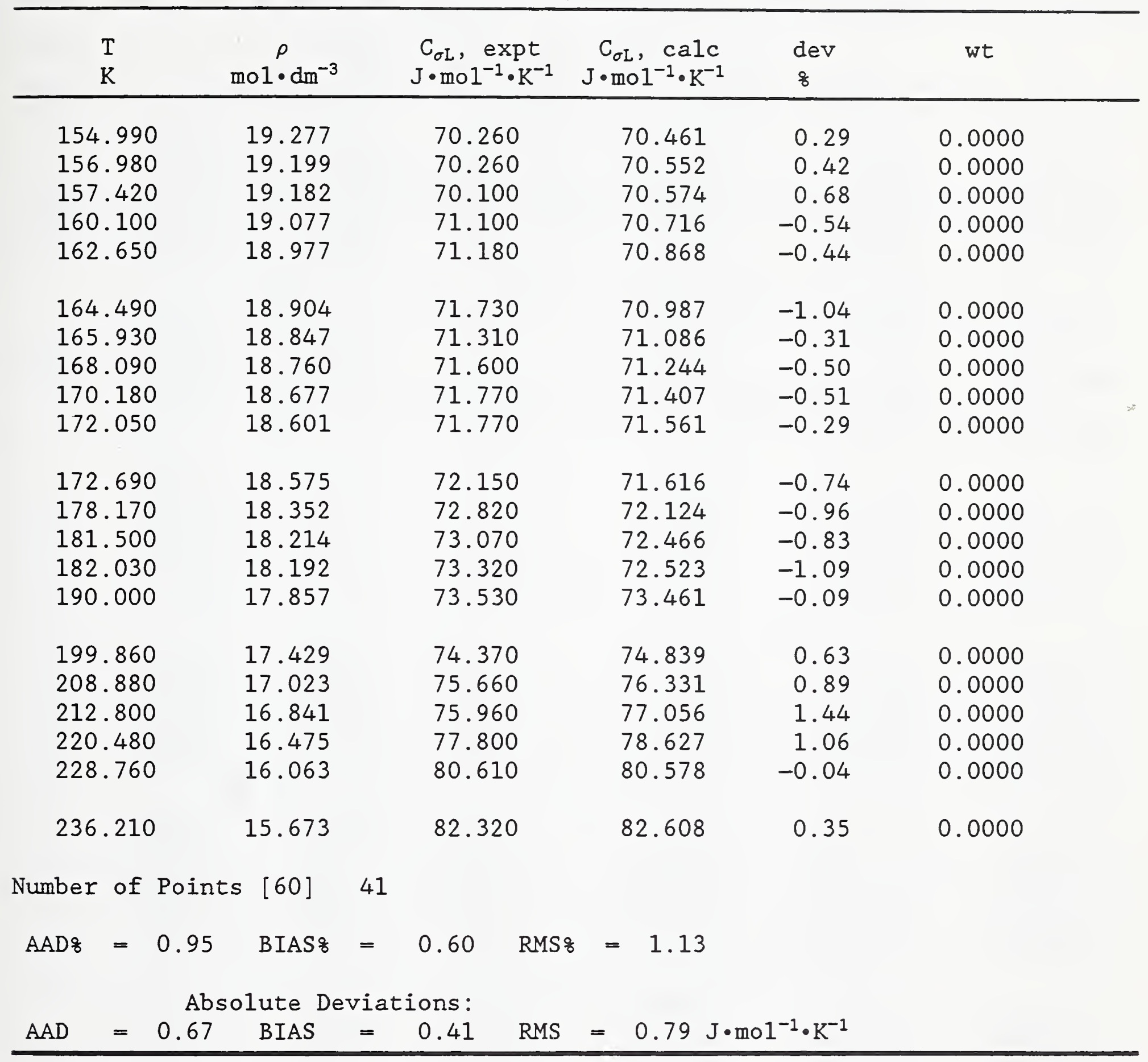

Data from Witt and Kemp [61]

\begin{tabular}{cccccc}
$\mathrm{T}$ & $\begin{array}{c}\rho \\
\mathrm{K}\end{array}$ & $\begin{array}{c}C_{\sigma \mathrm{L}}, \operatorname{expt} \\
\mathrm{Jol} \cdot \mathrm{dm}^{-3} \cdot \mathrm{K}^{-1}\end{array}$ & $\begin{array}{c}C_{\sigma \mathrm{L}}, \mathrm{calc} \\
\mathrm{J} \cdot \mathrm{mol} 1^{-1} \cdot \mathrm{K}^{-1}\end{array}$ & $\begin{array}{c}\text { dev } \\
\text { o }\end{array}$ & wt \\
\hline 91.590 & 21.623 & 68.300 & 67.539 & -1.11 & 0.0000 \\
92.970 & 21.574 & 68.460 & 68.181 & -0.41 & 0.0000 \\
94.940 & 21.504 & 68.380 & 68.919 & 0.79 & 0.0000 \\
96.600 & 21.445 & 68.250 & 69.403 & 1.69 & 0.0000 \\
98.230 & 21.386 & 68.880 & 69.777 & 1.30 & 0.0000
\end{tabular}


Table 9. SPECIFIC HEAT CAPACITY OF THE SATURATED LIQUID (continued)

Data from Witt and Kemp [61] (continued)

\begin{tabular}{|c|c|c|c|c|c|}
\hline $\begin{array}{l}\mathrm{T} \\
\mathrm{K}\end{array}$ & $\stackrel{\rho}{\mathrm{mol} \cdot \mathrm{dm}^{-3}}$ & $\begin{array}{c}C_{\sigma \mathrm{L}}, \underset{\mathrm{J} \cdot \mathrm{mo} 1^{-1} \cdot \mathrm{K}^{-1}}{\operatorname{expt}}\end{array}$ & $\begin{array}{r}\mathrm{C}_{\sigma \mathrm{L}}, \quad \text { calc } \\
\mathrm{J} \cdot \mathrm{mol}^{-1} \cdot \mathrm{K}^{-1}\end{array}$ & $\begin{array}{l}\text { dev } \\
\frac{8}{8}\end{array}$ & wt \\
\hline 98.890 & 21.363 & 68.380 & 69.903 & 2.23 & 0.0000 \\
\hline 100.490 & 21.305 & 68.340 & 70.158 & 2.66 & 0.0000 \\
\hline 104.050 & 21.177 & 68.590 & 70.512 & 2.80 & 0.0000 \\
\hline 106.670 & 21.083 & 68.880 & 70.635 & 2.55 & 0.0000 \\
\hline 109.240 & 20.990 & 68.920 & 70.677 & 2.55 & 0.0000 \\
\hline 111.670 & 20.902 & 69.130 & 70.667 & 2.22 & 0.0000 \\
\hline 114.200 & 20.809 & 69.010 & 70.623 & 2.34 & 0.0000 \\
\hline 116.240 & 20.735 & 69.170 & 70.570 & 2.02 & 0.0000 \\
\hline 119.330 & 20.622 & 69.300 & 70.477 & 1.70 & 0.0000 \\
\hline 122.720 & 20.497 & 69.430 & 70.369 & 1.35 & 0.0000 \\
\hline 125.960 & 20.377 & 69.590 & 70.274 & 0.98 & 0.0000 \\
\hline 129.470 & 20.247 & 69.550 & 70.188 & 0.92 & 0.0000 \\
\hline 134.490 & 20.059 & 69.640 & 70.111 & 0.68 & 0.0000 \\
\hline 138.720 & 19.900 & 69.760 & 70.093 & 0.48 & 0.0000 \\
\hline 142.830 & 19.744 & 69.890 & 70.120 & 0.33 & 0.0000 \\
\hline 145.970 & 19.625 & 70.050 & 70.171 & 0.17 & 0.0000 \\
\hline 149.800 & 19.478 & 70.310 & 70.268 & -0.06 & 0.0000 \\
\hline 153.580 & 19.332 & 70.770 & 70.401 & -0.52 & 0.0000 \\
\hline 157.950 & 19.162 & 70.770 & 70.601 & -0.24 & 0.0000 \\
\hline 162.820 & 18.970 & 71.020 & 70.879 & -0.20 & 0.0000 \\
\hline 167.430 & 18.787 & 71.520 & 71.194 & -0.46 & 0.0000 \\
\hline 172.020 & 18.602 & 71.560 & 71.559 & 0.00 & 0.0000 \\
\hline 176.540 & 18.419 & 72.060 & 71.965 & -0.13 & 0.0000 \\
\hline 180.880 & 18.240 & 72.110 & 72.401 & 0.40 & 0.0000 \\
\hline 236.210 & 15.673 & 82.257 & 82.608 & 0.43 & 0.0000 \\
\hline 244.610 & 15.208 & 83.889 & 85.292 & 1.67 & 0.0000 \\
\hline 252.530 & 14.738 & 87.069 & 88.328 & 1.45 & 0.0000 \\
\hline 258.220 & 14.378 & 88.366 & 90.918 & 2.89 & 0.0000 \\
\hline 265.250 & 13.900 & 92.257 & 94.778 & 2.73 & 0.0000 \\
\hline 273.060 & 13.313 & 98.031 & 100.353 & 2.37 & 0.0000 \\
\hline 278.070 & 12.895 & 101.253 & 105.058 & 3.76 & 0.0000 \\
\hline 284.070 & 12.334 & 109.035 & 112.691 & 3.35 & 0.0000 \\
\hline 291.270 & 11.526 & 122.591 & 127.757 & 4.21 & 0.0000 \\
\hline 294.850 & 11.033 & 135.687 & 140.859 & 3.81 & 0.0000 \\
\hline
\end{tabular}


Table 9. SPECIFIC HEAT CAPACITY OF THE SATURATED LIQUID (continued)

Data from Witt and Kemp [61] (continued)

Number of Points [61] 39

$\mathrm{AAD} \%=1.54 \quad \mathrm{BIAS} \%=1.38 \quad \mathrm{RMS} \%=1.36$

Absolute Deviations:

$\mathrm{AAD}=1.31 \mathrm{BIAS}=1.20 \mathrm{RMS}=1.40 \mathrm{~J} \cdot \mathrm{mol}^{-1} \cdot \mathrm{K}^{-1}$

Overall Results: $\mathrm{N}=186$

$\mathrm{AAD} \&=1.01 \quad$ BIAS $8=0.60 \quad$ RMS $8=1.31$

$\mathrm{AAD}=0.83 \quad \mathrm{BIAS}=0.39 \mathrm{RMS}=1.18 \mathrm{~J} \cdot \mathrm{mol}^{-1} \cdot \mathrm{K}^{-1}$

Weighted Data: $\quad N=106$

$\mathrm{AAD} \%=0.84 \quad$ BIAS $\%=0.31 \quad$ RMS $8=1.23$

$\mathrm{AAD}=0.71 \quad \mathrm{BIAS}=0.09$ RMS $=1.08 \mathrm{~J} \cdot \mathrm{mol}^{-1} \cdot \mathrm{K}^{-1}$ 
Table 10. SOUND SPEED IN THE SINGLE-PHASE FLUID

Data from Terres et al. [62]

\begin{tabular}{|c|c|c|c|c|c|c|c|c|c|}
\hline $\begin{array}{l}\mathrm{T} \\
\mathrm{K}\end{array}$ & $\begin{array}{c}\mathrm{P} \\
\mathrm{MPa}\end{array}$ & $\stackrel{\rho}{\mathrm{mol} \cdot \mathrm{dm}^{-3}}$ & $\begin{array}{l}W, \operatorname{expt} \\
\mathrm{m} \cdot \mathrm{s}^{-1}\end{array}$ & $\begin{array}{l}\mathrm{W}, \mathrm{calc} \\
\mathrm{m} \cdot \mathrm{s}^{-1}\end{array}$ & $\begin{array}{c}\text { dev } \\
\frac{8}{b}\end{array}$ & \multicolumn{2}{|c|}{$\begin{array}{c}\partial \mathrm{P} /\left.\partial \rho\right|_{\mathrm{T}} \\
\mathrm{MPa} \cdot \mathrm{dm}^{3} \cdot \mathrm{mol}^{-1}\end{array}$} & $\begin{array}{c}\operatorname{dev} 2 \\
8\end{array}$ & wt \\
\hline 292.65 & 0.098 & 0.04 & 308.8 & 308.8 & -0.01 & 2.40 & 2.40 & -0.01 & 5.88 \\
\hline 292.65 & 0.490 & 0.21 & 302.9 & 301.8 & -0.35 & 2.26 & 2.24 & -0.71 & 2.71 \\
\hline 292.65 & 0.981 & 0.44 & 294.8 & 292.6 & -0.76 & 2.07 & 2.04 & -1.51 & 1.89 \\
\hline 292.65 & 1.471 & 0.69 & 285.2 & 282.6 & -0.93 & 1.85 & 1.82 & -1.84 & 1.50 \\
\hline 292.65 & 1.961 & 0.98 & 274.5 & 271.6 & -1.06 & 1.62 & 1.59 & -2.12 & 1.27 \\
\hline 292.65 & 2.452 & 1.32 & 263.0 & 259.3 & -1.42 & 1.37 & 1.33 & -2.82 & 1.11 \\
\hline 292.65 & 2.942 & 1.73 & 249.0 & 245.0 & -1.62 & 1.08 & 1.05 & -3.21 & 0.98 \\
\hline 292.65 & 3.432 & 2.30 & 232.0 & 227.0 & -2.17 & 0.73 & 0.70 & -4.29 & 0.88 \\
\hline 323.15 & 0.098 & 0.04 & 324.0 & 322.8 & -0.36 & 2.68 & 2.66 & -0.71 & 10.53 \\
\hline 323.15 & 0.981 & 0.39 & 312.3 & 310.7 & -0.51 & 2.40 & 2.37 & -1.02 & 2.12 \\
\hline 323.15 & 1.961 & 0.83 & 298.5 & 296.2 & -0.77 & 2.07 & 2.04 & -1.53 & 1.46 \\
\hline 323.15 & 2.942 & 1.36 & 282.5 & 280.5 & -0.71 & 1.71 & 1.69 & -1.42 & 1.16 \\
\hline 323.15 & 3.432 & 1.67 & 274.4 & 272.1 & -0.84 & 1.53 & 1.50 & -1.68 & 1.06 \\
\hline 323.15 & 3.923 & 2.02 & 266.0 & 263.3 & -1.01 & 1.33 & 1.31 & -2.01 & 0.98 \\
\hline 323.15 & 4.413 & 2.43 & 257.8 & 254.2 & -1.41 & 1.14 & 1.11 & -2.81 & 0.92 \\
\hline 323.15 & 4.903 & 2.92 & 249.5 & 244.7 & -1.93 & 0.93 & 0.90 & -3.82 & 0.86 \\
\hline 323.15 & 5.394 & 3.54 & 241.2 & 235.2 & -2.50 & 0.72 & 0.68 & -4.94 & 0.81 \\
\hline 323.15 & 5.884 & 4.40 & 233.0 & 226.6 & -2.74 & 0.50 & 0.48 & -5.40 & 0.77 \\
\hline 323.15 & 6.374 & 5.66 & 227.0 & 223.2 & -1.69 & 0.34 & 0.32 & -3.36 & 0.75 \\
\hline 323.15 & 6.865 & 7.22 & 235.0 & 238.3 & 1.42 & 0.34 & 0.34 & 2.86 & 0.75 \\
\hline 323.15 & 7.845 & 9.07 & 296.0 & 306.0 & 3.38 & 0.79 & 0.84 & 6.88 & 0.83 \\
\hline 323.15 & 8.826 & 9.94 & 347.0 & 360.9 & 4.02 & 1.34 & 1.45 & 8.19 & 0.98 \\
\hline 323.15 & 9.807 & 10.51 & 396.0 & 404.9 & 2.25 & 1.96 & 2.05 & 4.55 & 1.34 \\
\hline 348.15 & 0.098 & 0.03 & 334.2 & 333.8 & -0.12 & 2.88 & 2.87 & -0.25 & 8.28 \\
\hline 348.15 & 0.981 & 0.36 & 325.8 & 324.0 & -0.54 & 2.66 & 2.63 & -1.08 & 2.35 \\
\hline 348.15 & 1.961 & 0.75 & 314.4 & 312.8 & -0.50 & 2.38 & 2.36 & -1.00 & 1.60 \\
\hline 348.15 & 2.942 & 1.19 & 303.2 & 301.3 & -0.62 & 2.11 & 2.08 & -1.24 & 1.29 \\
\hline 348.15 & 3.923 & 1.70 & 292.2 & 289.7 & -0.87 & 1.84 & 1.80 & -1.73 & 1.11 \\
\hline 348.15 & 4.903 & 2.29 & 281.2 & 278.2 & -1.07 & 1.56 & 1.52 & -2.13 & 0.99 \\
\hline 348.15 & 5.884 & 3.00 & 270.2 & 267.7 & -0.94 & 1.28 & 1.25 & -1.86 & 0.90 \\
\hline 348.15 & 6.865 & 3.87 & 261.8 & 259.8 & -0.76 & 1.03 & 1.01 & -1.51 & 0.84 \\
\hline 348.15 & 7.355 & 4.38 & 259.8 & 257.9 & -0.74 & 0.93 & 0.91 & -1.47 & 0.81 \\
\hline 348.15 & 7.845 & 4.94 & 260.0 & 258.1 & -0.72 & 0.85 & 0.84. & -1.43 & 0.80 \\
\hline 348.15 & 8.336 & 5.54 & 265.0 & 261.3 & -1.38 & 0.83 & 0.81 & -2.75 & 0.79 \\
\hline 348.15 & 8.826 & 6.15 & 272.0 & 268.1 & -1.42 & 0.84 & 0.81 & -2.83 & 0.80 \\
\hline
\end{tabular}


Table 10. SOUND SPEED IN THE SINGLE-PHASE FLUID (continued)

Data from Terres et al. [62] (continued)

\begin{tabular}{|c|c|c|c|c|c|c|c|c|c|}
\hline $\begin{array}{l}\mathrm{T} \\
\mathrm{K}\end{array}$ & $\begin{array}{c}\mathrm{P} \\
\mathrm{MPa}\end{array}$ & $\stackrel{\rho}{\mathrm{mol} \cdot \mathrm{dm}^{-3}}$ & $\mathrm{~W}, \operatorname{expt}_{\mathrm{m} \cdot \mathrm{s}^{-1}}$ & $\begin{array}{l}\mathrm{W}, \mathrm{calc} \\
\mathrm{m} \cdot \mathrm{s}^{-1}\end{array}$ & $\begin{array}{c}\text { dev } \\
\frac{8}{8}\end{array}$ & $\begin{array}{l}\mathrm{MPa} \\
\operatorname{expt}\end{array}$ & $\begin{array}{l}\left.\rho\right|_{\mathrm{T}} \\
\mathrm{mol}^{-1} \\
\mathrm{calc}\end{array}$ & $\begin{array}{r}\text { dev2 } \\
\frac{8}{8}\end{array}$ & wt \\
\hline 348.15 & 9.316 & 6.73 & 280.0 & 278.5 & -0.52 & 0.88 & 0.87 & -1.04 & 0.80 \\
\hline 348.15 & 9.807 & 7.27 & 291.0 & 291.8 & 0.27 & 0.96 & 0.97 & 0.54 & 0.82 \\
\hline 348.15 & 10.297 & 7.75 & 304.0 & 306.8 & 0.90 & 1.08 & 1.10 & 1.82 & 0.85 \\
\hline 348.15 & 10.787 & 8.17 & 319.0 & 322.4 & 1.08 & 1.23 & 1.26 & 2.17 & 0.89 \\
\hline 348.15 & 11.768 & 8.86 & 348.0 & 353.9 & 1.71 & 1.57 & 1.62 & 3.44 & 0.99 \\
\hline 373.15 & 0.098 & 0.03 & 345.1 & 344.3 & -0.23 & 3.09 & 3.08 & -0.46 & 12.67 \\
\hline 373.15 & 0.981 & 0.33 & 337.9 & 336.4 & -0.45 & 2.90 & 2.88 & -0.89 & 2.57 \\
\hline 373.15 & 1.961 & 0.68 & 329.5 & 327.6 & -0.59 & 2.69 & 2.65 & -1.18 & 1.77 \\
\hline 373.15 & 2.942 & 1.07 & 321.0 & 318.8 & -0.70 & 2.46 & 2.43 & -1.39 & 1.43 \\
\hline 373.15 & 3.923 & 1.49 & 312.5 & 310.2 & -0.73 & 2.24 & 2.21 & -1.46 & 1.23 \\
\hline 373.15 & 4.903 & 1.96 & 304.1 & 302.1 & -0.65 & 2.02 & 2.00 & -1.30 & 1.10 \\
\hline 373.15 & 5.884 & 2.48 & 297.0 & 294.9 & -0.72 & 1.82 & 1.79 & -1.43 & 1.01 \\
\hline 373.15 & 6.865 & 3.05 & 281.0 & 289.0 & 2.85 & 1.52 & 1.61 & 5.79 & 0.91 \\
\hline 373.15 & 7.845 & 3.70 & 286.8 & 285.4 & -0.49 & 1.47 & 1.46 & -0.97 & 0.89 \\
\hline 373.15 & 8.826 & 4.40 & 285.5 & 285.1 & -0.15 & 1.35 & 1.35 & -0.30 & 0.86 \\
\hline 373.15 & 9.807 & 5.14 & 289.5 & 289.2 & -0.12 & 1.30 & 1.30 & -0.23 & 0.85 \\
\hline 373.15 & 10.787 & 5.90 & 299.2 & 298.5 & -0.24 & 1.33 & 1.32 & -0.48 & 0.86 \\
\hline 373.15 & 11.768 & 6.61 & 313.0 & 312.8 & -0.06 & 1.43 & 1.43 & -0.12 & 0.88 \\
\hline 398.15 & 0.098 & 0.03 & 355.0 & 354.5 & -0.15 & 3.30 & 3.29 & -0.30 & 11.83 \\
\hline 398.15 & 0.981 & 0.31 & 349.1 & 348.0 & -0.31 & 3.14 & 3.12 & -0.62 & 2.76 \\
\hline 398.15 & 1.961 & 0.63 & 342.5 & 341.0 & -0.45 & 2.96 & 2.93 & -0.90 & 1.93 \\
\hline 398.15 & 2.942 & 0.97 & 336.0 & 334.1 & -0.56 & 2.78 & 2.75 & -1.12 & 1.57 \\
\hline 398.15 & 3.923 & 1.34 & 329.5 & 327.6 & -0.58 & 2.60 & 2.57 & -1.15 & 1.35 \\
\hline 398.15 & 4.903 & 1.74 & 323.0 & 321.6 & -0.43 & 2.42 & 2.40 & -0.86 & 1.21 \\
\hline 398.15 & 5.884 & 2.16 & 317.2 & 316.3 & -0.28 & 2.25 & 2.24 & -0.55 & 1.11 \\
\hline 398.15 & 6.865 & 2.62 & 313.0 & 312.0 & -0.31 & 2.11 & 2.09 & -0.62 & 1.04 \\
\hline 398.15 & 7.845 & 3.10 & 310.7 & 309.0 & -0.54 & 1.99 & 1.97 & -1.07 & 0.99 \\
\hline 398.15 & 8.826 & 3.61 & 309.3 & 307.7 & -0.51 & 1.89 & 1.87 & -1.01 & 0.96 \\
\hline 398.15 & 9.807 & 4.15 & 309.9 & 308.5 & -0.45 & 1.82 & 1.80 & -0.90 & 0.94 \\
\hline 398.15 & 10.787 & 4.70 & 312.8 & 311.7 & -0.35 & 1.78 & 1.77 & -0.70 & 0.92 \\
\hline 398.15 & 11.768 & 5.25 & 318.2 & 317.6 & -0.20 & 1.79 & 1.78 & -0.39 & 0.93 \\
\hline 423.15 & 0.098 & 0.03 & 365.2 & 364.3 & -0.24 & 3.52 & 3.50 & -0.47 & 67.43 \\
\hline 423.15 & 0.981 & 0.29 & 360.8 & 359.1 & -0.48 & 3.39 & 3.36 & -0.96 & 3.17 \\
\hline 423.15 & 1.961 & 0.58 & 355.7 & 353.4 & -0.65 & 3.24 & 3.20 & -1.29 & 2.16 \\
\hline 423.15 & 2.942 & 0.90 & 350.7 & 348.0 & -0.77 & 3.09 & 3.04 & -1.53 & 1.75 \\
\hline
\end{tabular}


Table 10. SOUND SPEED IN THE SINGLE-PHASE FLUID (continued)

Data from Terres et al. [62] (continued)

\begin{tabular}{|c|c|c|c|c|c|c|c|c|c|}
\hline $\begin{array}{l}\mathrm{T} \\
\mathrm{K}\end{array}$ & $\begin{array}{c}\mathrm{P} \\
\mathrm{MPa}\end{array}$ & $\stackrel{\rho}{\mathrm{mol} \cdot \mathrm{dm}^{-3}}$ & $\begin{array}{l}W, \operatorname{expt} \\
\mathrm{m} \cdot \mathrm{s}^{-1}\end{array}$ & $\begin{array}{l}\mathrm{W}, \mathrm{calc} \\
\mathrm{m} \cdot \mathrm{s}^{-1}\end{array}$ & $\begin{array}{l}\text { dev } \\
8\end{array}$ & $\begin{array}{l}\mathrm{MPa} \\
\operatorname{expt}\end{array}$ & $\begin{array}{l}\left.\rho\right|_{\mathrm{T}} \\
\mathrm{mol}^{-1} \\
\mathrm{calc}^{-1}\end{array}$ & $\begin{array}{c}\text { dev2 } \\
8\end{array}$ & wt \\
\hline 423.15 & 3.923 & 1.23 & 345.8 & 343.0 & -0.82 & 2.94 & 2.90 & -1.63 & 1.51 \\
\hline 423.15 & 4.903 & 1.58 & 340.8 & 338.4 & -0.69 & 2.80 & 2.76 & -1.38 & 1.35 \\
\hline 423.15 & 5.884 & 1.94 & 336.3 & 334.5 & -0.53 & 2.66 & 2.63 & -1.06 & 1.23 \\
\hline 423.15 & 6.865 & 2.32 & 332.7 & 331.3 & -0.41 & 2.54 & 2.51 & -0.82 & 1.15 \\
\hline 423.15 & 7.845 & 2.72 & 330.2 & 329.1 & -0.34 & 2.43 & 2.41 & -0.67 & 1.10 \\
\hline 423.15 & 8.826 & 3.13 & 328.9 & 327.9 & -0.30 & 2.35 & 2.33 & -0.59 & 1.06 \\
\hline 423.15 & 9.807 & 3.56 & 329.1 & 328.0 & -0.32 & 2.28 & 2.27 & -0.64 & 1.03 \\
\hline 423.15 & 10.787 & 4.00 & 330.9 & 329.6 & -0.40 & 2.25 & 2.23 & -0.80 & 1.01 \\
\hline 423.15 & 11.768 & 4.44 & 334.3 & 332.7 & -0.49 & 2.24 & 2.22 & -0.97 & 1.01 \\
\hline 448.15 & 0.098 & 0.03 & 375.4 & 373.9 & -0.39 & 3.74 & 3.71 & -0.78 & 9.24 \\
\hline 448.15 & 0.981 & 0.27 & 371.4 & 369.6 & -0.48 & 3.62 & 3.59 & -0.95 & 3.54 \\
\hline 448.15 & 1.961 & 0.55 & 366.8 & 365.1 & -0.47 & 3.49 & 3.45 & -0.94 & 2.33 \\
\hline 448.15 & 2.942 & 0.84 & 362.6 & 360.8 & -0.49 & 3.36 & 3.32 & -0.98 & 1.88 \\
\hline 448.15 & 3.923 & 1.14 & 358.2 & 356.9 & -0.36 & 3.23 & 3.20 & -0.71 & 1.62 \\
\hline 448.15 & 4.903 & 1.45 & 354.4 & 353.5 & -0.26 & 3.11 & 3.09 & -0.53 & 1.45 \\
\hline 448.15 & 5.884 & 1.77 & 351.2 & 350.5 & -0.19 & 3.00 & 2.99 & -0.38 & 1.34 \\
\hline 448.15 & 6.865 & 2.11 & 348.8 & 348.2 & -0.17 & 2.90 & 2.89 & -0.33 & 1.26 \\
\hline 448.15 & 7.845 & 2.45 & 347.2 & 346.6 & -0.17 & 2.82 & 2.81 & -0.34 & 1.20 \\
\hline 448.15 & 8.826 & 2.80 & 346.8 & 345.8 & -0.29 & 2.76 & 2.75 & -0.58 & 1.16 \\
\hline 448.15 & 9.807 & 3.16 & 347.5 & 345.9 & -0.47 & 2.72 & 2.69 & -0.94 & 1.14 \\
\hline 448.15 & 10.787 & 3.53 & 348.9 & 346.9 & -0.57 & 2.69 & 2.66 & -1.15 & 1.12 \\
\hline 448.15 & 11.768 & 3.90 & 350.9 & 349.0 & -0.55 & 2.67 & 2.64 & -1.10 & 1.11 \\
\hline
\end{tabular}

Number of Points [62] 92

Calculation of sound speed:

$\mathrm{AAD} \&=0.79 \quad \mathrm{BIAS} \%=-0.40$ RMS $8=1.00$

Calculation of $\partial \mathrm{P} /\left.\partial \rho\right|_{\mathrm{T}}$ :

AAD2\% $=1.57 \quad$ BIAS2\% $=-0.79 \quad$ RMS $2 \%=2.00$

Absolute Deviations:

Calculation of sound speed:

$\mathrm{AAD}=2.35$ BIAS $=-1.11$ RMS $=2.98 \mathrm{~m} \cdot \mathrm{s}^{-1}$

Calculation of $\partial \mathrm{P} /\left.\partial \rho\right|_{\mathrm{T}}$ :

AAD2 $=0.026$ BIAS2 $=-0.016 \mathrm{RMS} 2=0.027 \mathrm{MPa} \cdot \mathrm{dm}^{3} \cdot \mathrm{mol}^{-1}$ 
Table 10. SOUND SPEED IN THE SINGLE-PHASE FLUID (continued)

Data from Terres et al. [62] (continued)

Weighted Data:

Number of Points [62] 92

Calculation of sound speed:

$\mathrm{AAD} \%=0.79 \quad \mathrm{BIAS} \%=-0.40$ RMS $\%=1.00$

Calculation of $\partial P /\left.\partial \rho\right|_{I}$ :

$\mathrm{AAD} 2 \%=1.57 \quad \mathrm{BIAS} 2 \%=-0.79 \quad$ RMS $2 \%=2.00$

Absolute Deviations:

Calculation of sound speed:

$\mathrm{AAD}=2.35$ BIAS $=-1.11$ RMS $=2.98 \mathrm{~m} \cdot \mathrm{s}^{-1}$

Calculation of $\partial P /\left.\partial \rho\right|_{T}$ :

$\mathrm{AAD2}=0.026 \quad$ BIAS2 $=-0.016 \mathrm{RMS} 2=0.027 \mathrm{MPa} \cdot \mathrm{dm}^{3} \cdot \mathrm{mol}^{-1}$

Data from Tsumura and Straty [63]

$\begin{array}{lcccccccc}\mathrm{T} & \mathrm{P} & \rho & \mathrm{W}, \operatorname{expt} & \mathrm{W}, \mathrm{calc} & \mathrm{dev} & \partial \mathrm{P} / \partial \rho \mid \mathrm{I} & \operatorname{dev} 2 & \text { wt } \\ \mathrm{K} & \mathrm{MPa} & \mathrm{mol} \cdot \mathrm{dm}^{-3} & \mathrm{~m} \cdot \mathrm{s}^{-1} & \mathrm{~m}^{-1} \mathrm{~s}^{-1} & \& & \mathrm{MPa} \cdot \mathrm{dm}^{3} \cdot \mathrm{mol}^{-1} & \& & \end{array}$ expt calc

$\begin{array}{rrrrrrrrrrr}100.00 & 31.949 & 21.70 & 2051.3 & 2068.1 & 0.82 & 89.29 & 90.76 & 1.65 & 0.12 \\ 100.00 & 28.495 & 21.67 & 2040.0 & 2054.9 & 0.73 & 88.07 & 89.36 & 1.46 & 0.12 \\ 100.00 & 25.196 & 21.63 & 2028.8 & 2042.1 & 0.65 & 86.88 & 88.02 & 1.31 & 0.12 \\ 100.00 & 21.728 & 21.59 & 2017.3 & 2028.4 & 0.55 & 85.66 & 86.61 & 1.11 & 0.12 \\ 100.00 & 18.427 & 21.55 & 2005.7 & 2015.3 & 0.48 & 84.45 & 85.25 & 0.96 & 0.12 \\ 100.00 & 14.981 & 21.51 & 1993.6 & 2001.3 & 0.39 & 83.19 & 83.83 & 0.78 & 0.13 \\ 100.00 & 11.414 & 21.47 & 1981.0 & 1986.7 & 0.29 & 81.89 & 82.36 & 0.58 & 0.13 \\ 100.00 & 7.962 & 21.43 & 1968.4 & 1972.3 & 0.20 & 80.60 & 80.92 & 0.40 & 0.13 \\ 100.00 & 4.122 & 21.38 & 1954.2 & 1956.1 & 0.10 & 79.16 & 79.31 & 0.19 & 0.13 \\ 120.00 & 36.342 & 21.11 & 1943.2 & 1951.8 & 0.44 & 77.77 & 78.46 & 0.89 & 0.13 \\ 120.00 & 28.584 & 21.01 & 1914.2 & 1920.6 & 0.33 & 74.84 & 75.34 & 0.67 & 0.13 \\ 120.00 & 18.912 & 20.88 & 1876.1 & 1880.1 & 0.21 & 71.07 & 71.38 & 0.43 & 0.14 \\ 120.00 & 14.963 & 20.82 & 1860.0 & 1863.1 & 0.17 & 69.51 & 69.75 & 0.33 & 0.14 \\ 120.00 & 8.105 & 20.72 & 1831.0 & 1832.8 & 0.10 & 66.75 & 66.88 & 0.19 & 0.14 \\ 140.00 & 35.565 & 20.46 & 1819.4 & 1820.2 & 0.04 & 66.31 & 66.37 & 0.08 & 0.14 \\ 140.00 & 14.708 & 20.12 & 1725.2 & 1723.6 & -0.10 & 57.85 & 57.74 & -0.19 & 0.15 \\ 140.00 & 7.800 & 20.00 & 1690.7 & 1688.9 & -0.11 & 54.89 & 54.78 & -0.21 & 0.16 \\ 140.00 & 28.434 & 20.35 & 1788.1 & 1788.3 & 0.01 & 63.45 & 63.46 & 0.03 & 0.14 \\ 140.00 & 21.457 & 20.23 & 1756.7 & 1756.0 & -0.04 & 60.62 & 60.58 & -0.08 & 0.15 \\ 160.00 & 35.328 & 19.81 & 1698.2 & 1694.8 & -0.20 & 56.56 & 56.33 & -0.40 & 0.15\end{array}$


Table 10. SOUND SPEED IN THE SINGLE-PHASE FLUID (continued)

Data from Tsumura and Straty [63] (continued)

\begin{tabular}{|c|c|c|c|c|c|c|c|c|c|}
\hline $\begin{array}{l}\mathrm{T} \\
\mathrm{K}\end{array}$ & $\begin{array}{c}\mathrm{P} \\
\mathrm{MPa}\end{array}$ & $\stackrel{\rho}{\mathrm{mol} \cdot \mathrm{dm}^{-3}}$ & $W, \operatorname{expt}$ & $\begin{array}{c}\mathrm{W}, \mathrm{calc} \\
\mathrm{m} \cdot \mathrm{s}^{-1}\end{array}$ & $\begin{array}{c}\mathrm{dev} \\
8\end{array}$ & \multicolumn{2}{|c|}{$\begin{array}{c}\partial \mathrm{P} /\left.\partial \rho\right|_{\mathrm{T}} \\
\mathrm{MPa} \cdot \mathrm{dm}^{3} \cdot \mathrm{mol}^{-1}\end{array}$} & $\begin{array}{c}\text { dev2 } \\
8\end{array}$ & wt \\
\hline 60.00 & 8.145 & 19.68 & 1662.8 & 1658.8 & -0.24 & 53.62 & 53.36 & -0.48 & .16 \\
\hline 160.00 & 1.806 & 19.56 & 1629.9 & 1625.7 & 0.26 & 50.95 & 50.69 & -0.52 & .16 \\
\hline 160.00 & 14.846 & 19.42 & 1591.7 & 1587.5 & -0.26 & 47.94 & 47.69 & -0.52 & 0.17 \\
\hline 160.00 & 8.013 & 19.27 & 1551.5 & 1548.0 & -0.23 & 44.88 & 44.68 & -0.45 & 0.17 \\
\hline 180.00 & 35.697 & 19.17 & 1583.4 & 1576.6 & -0.43 & 48.45 & 48.03 & -0.86 & 0.17 \\
\hline 80.00 & 28.615 & 19.02 & 1543.8 & 1536.9 & -0.45 & 45.47 & 45.06 & -0.89 & .17 \\
\hline 180.00 & 21.600 & 18.86 & 1502.0 & 1495.3 & -0.45 & 42.42 & 42.04 & -0.89 & 0.18 \\
\hline 180.00 & 14.716 & 18.69 & 1457.9 & 1451.8 & -0.42 & 39.33 & 39.00 & -0.83 & 0.19 \\
\hline 180.00 & 7.879 & 18.51 & 1410.3 & 1405.5 & -0.34 & 36.12 & 35.88 & -0.68 & 0.19 \\
\hline 180.00 & 11.517 & 18.61 & 1435.6 & 1430.6 & -0.35 & 37.82 & 37.55 & -0.70 & 0.19 \\
\hline 00.00 & .190 & 17.93 & 1321.0 & 1314.8 & -0.47 & 31.64 & 31.35 & -0.93 & .21 \\
\hline 200.00 & 36.755 & 18.55 & 1475.4 & 146 & -0.54 & 41.72 & 41.26 & -1.08 & .18 \\
\hline 200.00 & 32.140 & 18.44 & 1446.9 & 1438.9 & -0.55 & 39.75 & 39.31 & -1.10 & 0.19 \\
\hline 200.00 & 28.520 & 18.34 & 1423.6 & 1415.8 & -0.55 & 38.18 & 37.76 & -1.10 & 0.19 \\
\hline 200.00 & 25.381 & 18.26 & 1402.5 & 1395.0 & -0.53 & 36.78 & 36.39 & -1.07 & 0.19 \\
\hline 200.00 & 21.834 & 18.16 & 77.9 & 13 & -0.52 & 35.19 & 34.82 & -1.04 & 0.20 \\
\hline 200.00 & 18.326 & 18.05 & 1352.4 & 1345.7 & -0.49 & 33.58 & 33.25 & -0.99 & 0.20 \\
\hline 200.00 & 14.831 & 17.95 & 1325.7 & 1319.7 & -0.45 & 31.93 & 31.64 & -0.90 & 0.21 \\
\hline 200.00 & 11.523 & 17.84 & 1299.1 & 1294.0 & -0.40 & 30.33 & 30.10 & -0.79 & 0.21 \\
\hline 200.00 & 7.881 & 17.72 & 1268.2 & 1264.1 & -0.32 & 28.53 & 28.35 & -0.64 & 0.22 \\
\hline 200.00 & 4.934 & 17.61 & 1241.6 & 123 & -0.24 & 27.03 & 26.90 & -0.48 & 0.23 \\
\hline 220.00 & 15.513 & 17.19 & 1200.8 & 1195.0 & -0.48 & 25.85 & 25.60 & -0.96 & 0.23 \\
\hline 220.00 & 35.977 & 17.87 & 1361.0 & 1352.0 & -0.66 & 35.23 & 34.77 & -1.32 & 0.20 \\
\hline 220.00 & 28.535 & 17.65 & 1308.1 & 1299.5 & -0.65 & 31.97 & 31.55 & -1.30 & 0.21 \\
\hline 220.00 & 21.679 & 17.42 & 1254.4 & 1246.9 & -0.60 & 28.82 & 28.48 & -1.19 & 0.22 \\
\hline 220.00 & 14.782 & 17.16 & 1194.2 & 1188.5 & -0.48 & 25.49 & 25.25 & -0.95 & 0.23 \\
\hline 220.00 & 7.875 & 16.87 & 1125.4 & 1122.4 & -0.27 & 21.95 & 21.84 & -0.53 & 0.25 \\
\hline 220.00 & 10.057 & 16.97 & 1147.8 & 1144.3 & -0.30 & 23.08 & 22.94 & -0.61 & 0.25 \\
\hline 220.00 & 10.029 & 16.97 & 1147.5 & 1144.0 & -0.30 & 23.06 & 22.92 & -0.60 & 0.25 \\
\hline 240.00 & 34.279 & 17.15 & 1242.2 & 1233.7 & -0.69 & 29.16 & 28.76 & -1.37 & 0.22 \\
\hline 240.00 & 28.538 & 16.94 & 1195.8 & 1188.0 & -0.65 & 26.58 & 26.24 & -1.30 & 0.23 \\
\hline 240.00 & 21.670 & 16.66 & 1134.4 & 1128.1 & -0.56 & 23.36 & 23.10 & -1.11 & 0.25 \\
\hline 240.00 & 14.733 & 16.34 & 1063.4 & 1059.4 & -0.37 & 19.90 & 19.75 & -0.74 & 0.27 \\
\hline 240.00 & 7.867 & 15.95 & 980.1 & 979.5 & -0.06 & 16.21 & 16.19 & -0.13 & 0.30 \\
\hline 240.00 & 3.559 & 15.67 & 917.3 & 919.7 & 0.26 & 13.69 & 13.77 & 0.52 & 0.33 \\
\hline
\end{tabular}


Table 10. SOUND SPEED IN THE SINGLE-PHASE FLUID (continued)

Data from Tsumura and Straty [63] (continued)

\begin{tabular}{|c|c|c|c|c|c|c|c|c|c|}
\hline $\begin{array}{l}\mathrm{T} \\
\mathrm{K}\end{array}$ & $\begin{array}{c}\mathrm{P} \\
\mathrm{MPa}\end{array}$ & $\stackrel{\rho}{\mathrm{n} .01 \cdot \mathrm{dm}^{-3}}$ & $\mathrm{~W}, \underset{\mathrm{m} \cdot \mathrm{s}^{-1}}{\operatorname{expt}}$ & $\begin{array}{c}\mathrm{W}, \mathrm{calc} \\
\mathrm{m} \cdot \mathrm{s}^{-1}\end{array}$ & $\begin{array}{c}\mathrm{dev} \\
8\end{array}$ & $\begin{array}{r}\partial \mathrm{P} \\
\mathrm{MPa} \cdot \mathrm{dm} \\
\text { expt }\end{array}$ & $\begin{array}{l}\left.\rho\right|_{\mathrm{T}} \\
\mathrm{mol}^{-1} \\
\mathrm{calc}\end{array}$ & $\begin{array}{l}\operatorname{dev} 2 \\
8\end{array}$ & wt \\
\hline 260.00 & 22.529 & 15.91 & 1028.1 & 1022.8 & -0.51 & 19.12 & 18.93 & -1.02 & 0.28 \\
\hline 260.00 & 34.541 & 16.47 & 1142.5 & 1134.9 & -0.66 & 24.69 & 24.37 & -1.32 & 0.24 \\
\hline 260.00 & 31.987 & 16.36 & 1120.1 & 1113.0 & -0.64 & 23.54 & 23.25 & -1.27 & 0.25 \\
\hline 260.00 & $28 .<78$ & 16.21 & 1087.8 & 1081.3 & -0.60 & 21.94 & 21.68 & -1.19 & 0.26 \\
\hline 260.00 & 25.289 & 16.05 & 1056.6 & 1050.8 & -0.55 & 20.44 & 20.22 & -1.09 & 0.27 \\
\hline 260.00 & 21.770 & 15.87 & 1019.5 & 1014.8 & -0.46 & 18.74 & 18.56 & -0.91 & 0.28 \\
\hline 260.00 & 17.581 & 15.63 & 971.2 & 968.1 & -0.32 & 16.63 & 16.52 & -0.64 & 0.30 \\
\hline 260.00 & 14.835 & 15.46 & 936.3 & 934.4 & -0.20 & 15.18 & 15.12 & -0.40 & 0.32 \\
\hline 260.00 & 11.364 & 15.22 & 887.6 & 887.6 & 0.00 & 13.28 & 13.28 & -0.01 & 0.34 \\
\hline 260.00 & 7.801 & 14.93 & 830.2 & 832.4 & 0.26 & 11.21 & 11.27 & 0.53 & 0.38 \\
\hline 260.00 & 5.134 & 14.67 & 780.0 & 784.3 & 0.55 & 9.55 & 9.66 & 1.11 & 0.42 \\
\hline 280.00 & 34.921 & 15.79 & 1050.9 & 1044.1 & -0.64 & 20.97 & 20.70 & -1.29 & 0.26 \\
\hline 280.00 & 28.585 & 15.46 & 987.7 & 982.4 & -0.54 & 18.09 & 17.89 & -1.08 & 0.29 \\
\hline 280.00 & 21.654 & 15.03 & 907.7 & 904.5 & -0.35 & 14.74 & 14.64 & -0.70 & 0.32 \\
\hline 280.00 & $14.75 ?$ & 14.50 & 809.9 & 809.7 & -0.03 & 11.12 & 11.12 & -0.05 & 0.39 \\
\hline 280.00 & 7.824 & 13.73 & 676.0 & 679.6 & 0.53 & 7.00 & 7.08 & 1.05 & 0.53 \\
\hline 280.00 & 36.829 & 15.88 & 1068.5 & 1061.4 & -0.66 & 21.82 & 21.53 & -1.32 & 0.26 \\
\hline 280.00 & 32.035 & 15.65 & 1023.2 & 1016.9 & -0.62 & 19.68 & 19.44 & -1.23 & 0.27 \\
\hline 280.00 & 28.535 & 15.46 & 987.4 & 981.9 & -0.56 & 18.07 & 17.87 & -1.12 & 0.29 \\
\hline 280.00 & 25.322 & 15.27 & 952.0 & 947.3 & -0.49 & 16.55 & 16.38 & -0.98 & 0.30 \\
\hline 280.00 & 21.684 & 15.04 & 908.2 & 904.9 & -0.37 & 14.76 & 14.65 & -0.73 & 0.32 \\
\hline 280.00 & 18.345 & 14.79 & 863.9 & 861.8 & -0.24 & 13.05 & 12.99 & -0.48 & 0.35 \\
\hline 280.00 & 14.738 & 14.49 & 809.9 & 809.5 & -0.05 & 11.12 & 11.11 & -0.11 & 0.39 \\
\hline 280.00 & 11.310 & 14.16 & 750.2 & 751.5 & 0.18 & 9.17 & 9.20 & 0.35 & 0.44 \\
\hline 280.00 & 7.804 & 13.72 & 675.7 & 679.1 & 0.50 & 7.00 & 7.07 & 1.00 & 0.53 \\
\hline 280.00 & 4.998 & 13.26 & 598.0 & 603.6 & 0.94 & 5.04 & 5.14 & 1.90 & 0.69 \\
\hline 300.00 & 35.788 & 15.13 & 971.1 & 965.8 & -0.55 & 18.05 & 17.86 & -1.09 & 0.29 \\
\hline 300.00 & 28.536 & 14.68 & 892.6 & 888.8 & -0.42 & 14.78 & 14.65 & -0.85 & 0.33 \\
\hline 300.00 & 25.137 & 14.44 & 850.7 & 847.9 & -0.32 & 13.17 & 13.08 & -0.65 & 0.35 \\
\hline 300.00 & 21.672 & 14.15 & 803.6 & 801.9 & -0.22 & 11.47 & 11.42 & -0.43 & 0.38 \\
\hline 300.00 & 9.545 & 12.59 & 566.1 & 567.7 & 0.28 & 4.69 & 4.72 & 0.55 & 0.77 \\
\hline 300.00 & 9.547 & 12.59 & 566.2 & 567.7 & 0.27 & 4.69 & 4.72 & 0.54 & 0.77 \\
\hline 300.00 & 7.823 & 12.17 & 509.8 & 511.4 & 0.31 & 3.52 & 3.54 & 0.62 & 1.13 \\
\hline 300.00 & 7.000 & 11.91 & 477.2 & 478.6 & 0.29 & 2.91 & 2.93 & 0.59 & 1.77 \\
\hline 300.00 & 30.764 & 14.83 & 918.0 & 913.8 & -0.46 & 15.80 & 15.66 & -0.92 & 0.00 \\
\hline
\end{tabular}


Table 10. SOUND SPEED IN THE SINGLE-PHASE FLUID (continued)

Data from Tsumura and Straty [63] (continued)

\begin{tabular}{|c|c|c|c|c|c|c|c|c|c|}
\hline $\begin{array}{l}\mathrm{T} \\
\mathrm{K}\end{array}$ & $\begin{array}{c}\mathrm{P} \\
\mathrm{MPa}\end{array}$ & $\begin{array}{c}\rho \\
\mathrm{mol} \cdot \mathrm{dm}^{-3}\end{array}$ & $W, \operatorname{mexp}^{-1}$ & $\begin{array}{c}\mathrm{W}, \mathrm{calc} \\
\mathrm{m} \cdot \mathrm{s}^{-1}\end{array}$ & $\begin{array}{c}\mathrm{dev} \\
8\end{array}$ & $\begin{array}{r}\partial \mathrm{P} \\
\mathrm{MPa} \cdot \mathrm{dn} \\
\text { expt }\end{array}$ & $\begin{array}{l}\left.\rho\right|_{T} \\
\text { mol }^{-1} \\
\text { calc }\end{array}$ & $\begin{array}{c}\text { dev2 } \\
8\end{array}$ & wt \\
\hline 300.00 & 28.502 & 14.68 & 892.0 & 888.4 & -0.40 & 14.76 & 14.64 & -0.80 & 0.00 \\
\hline 300.00 & 21.711 & 14.16 & 804.1 & 802.4 & -0.21 & 11.49 & 11.44 & -0.42 & 0.00 \\
\hline 300.00 & 18.200 & 13.83 & 750.2 & 749.7 & -0.07 & 9.69 & 9.67 & -0.14 & 0.00 \\
\hline 300.00 & 14.841 & 13.44 & 690.4 & 690.8 & 0.06 & 7.87 & 7.88 & 0.13 & 0.00 \\
\hline 300.00 & 13.268 & 13.23 & 658.2 & 659.3 & 0.16 & 6.97 & 6.99 & 0.32 & 0.00 \\
\hline 300.00 & 11.337 & 12.93 & 614.0 & 615.5 & 0.24 & 5.82 & 5.85 & 0.48 & 0.00 \\
\hline 300.00 & 11.304 & 12.92 & 613.3 & 614.7 & 0.22 & 5.80 & 5.83 & 0.44 & 0.00 \\
\hline 300.00 & 8.753 & 12.41 & 541.8 & 543.4 & 0.30 & 4.16 & 4.19 & 0.59 & 0.00 \\
\hline 300.00 & 7.928 & 12.20 & 513.6 & 515.2 & 0.32 & 3.59 & 3.61 & 0.63 & 0.00 \\
\hline 300.00 & 7.821 & 12.17 & 509.8 & 511.3 & 0.29 & 3.52 & 3.54 & 0.59 & 0.00 \\
\hline 300.00 & 7.815 & 12.17 & 509.6 & $5 i 1.1$ & 0.29 & 3.51 & 3.53 & 0.58 & 0.00 \\
\hline 300.00 & 7.036 & 11.93 & 478.7 & 480.2 & 0.30 & 2.94 & 2.96 & 0.61 & 0.00 \\
\hline 300.00 & 6.177 & 11.60 & 438.3 & 439.5 & 0.27 & 2.26 & 2.28 & 0.54 & 0.00 \\
\hline 300.00 & 5.735 & 11.38 & 413.2 & 414.4 & 0.28 & 1.89 & 1.90 & 0.56 & 0.00 \\
\hline 300.00 & 5.565 & 11.29 & 402.6 & 403.6 & 0.24 & 1.74 & 1.75 & 0.48 & 0.00 \\
\hline 300.00 & 4.855 & 10.78 & 346.2 & 346.8 & 0.18 & 1.05 & 1.06 & 0.36 & 0.00 \\
\hline 300.00 & 4.610 & 10.51 & 318.4 & 318.8 & 0.12 & 0.78 & 0.78 & 0.25 & 0.00 \\
\hline 300.00 & 4.584 & 10.48 & 314.9 & 315.3 & 0.13 & 0.74 & 0.75 & 0.26 & 0.00 \\
\hline 305.33 & 24.830 & 14.19 & 821.6 & 819.3 & -0.28 & 12.25 & 12.18 & -0.57 & 0.00 \\
\hline 305.33 & 21.127 & 13.86 & 768.9 & 767.7 & -0.16 & 10.43 & 10.39 & -0.32 & 0.00 \\
\hline 305.33 & 18.210 & 13.56 & 722.0 & 721.6 & -0.06 & 8.93 & 8.92 & -0.12 & 0.00 \\
\hline 305.33 & 14.694 & 13.12 & 656.1 & 656.6 & 0.08 & 7.02 & 7.03 & 0.15 & 0.00 \\
\hline 305.33 & 11.461 & 12.58 & 581.3 & 582.3 & 0.16 & 5.13 & 5.15 & 0.33 & 0.00 \\
\hline 305.33 & 11.324 & 12.55 & 577.8 & 578.6 & 0.15 & 5.05 & 5.07 & 0.29 & 0.00 \\
\hline 305.33 & 10.156 & 12.31 & 544.8 & 545.7 & 0.17 & 4.32 & 4.33 & 0.33 & 0.00 \\
\hline 305.33 & 8.269 & 11.79 & 480.6 & 481.3 & 0.15 & 3.05 & 3.06 & 0.31 & 0.00 \\
\hline 305.33 & 7.711 & 11.60 & 457.8 & 458.3 & 0.10 & 2.65 & 2.66 & 0.20 & 0.00 \\
\hline 305.33 & 7.427 & 11.49 & 445.1 & 445.5 & 0.08 & 2.44 & 2.45 & 0.17 & 0.00 \\
\hline 305.33 & 6.085 & 10.77 & 369.2 & 369.4 & 0.04 & 1.37 & 1.37 & 0.09 & 0.00 \\
\hline 305.33 & 5.701 & 10.45 & 338.7 & 338.6 & -0.02 & 1.03 & 1.03 & -0.04 & 0.00 \\
\hline 305.33 & 5.260 & 9.89 & 289.9 & 290.0 & 0.03 & 0.58 & 0.58 & 0.06 & 0.00 \\
\hline 305.33 & 5.222 & 9.82 & 284.4 & 284.5 & 0.03 & 0.53 & 0.53 & 0.06 & 0.00 \\
\hline 305.33 & 5.088 & 9.52 & 261.7 & 261.7 & 0.01 & J.37 & 0.37 & 0.02 & 0.00 \\
\hline 305.33 & 5.052 & 9.42 & 254.2 & 254.3 & 0.04 & 0.32 & 0.32 & 0.09 & 0.00 \\
\hline 305.33 & 5.023 & 9.32 & 247.5 & 247.7 & 0.09 & 0.28 & 0.28 & 0.18 & 0.00 \\
\hline
\end{tabular}


Table 10. SOUND SPEED IN THE SINGLE-PHASE FLUID (continued)

Data from Tsumura and Straty [63] (continued)

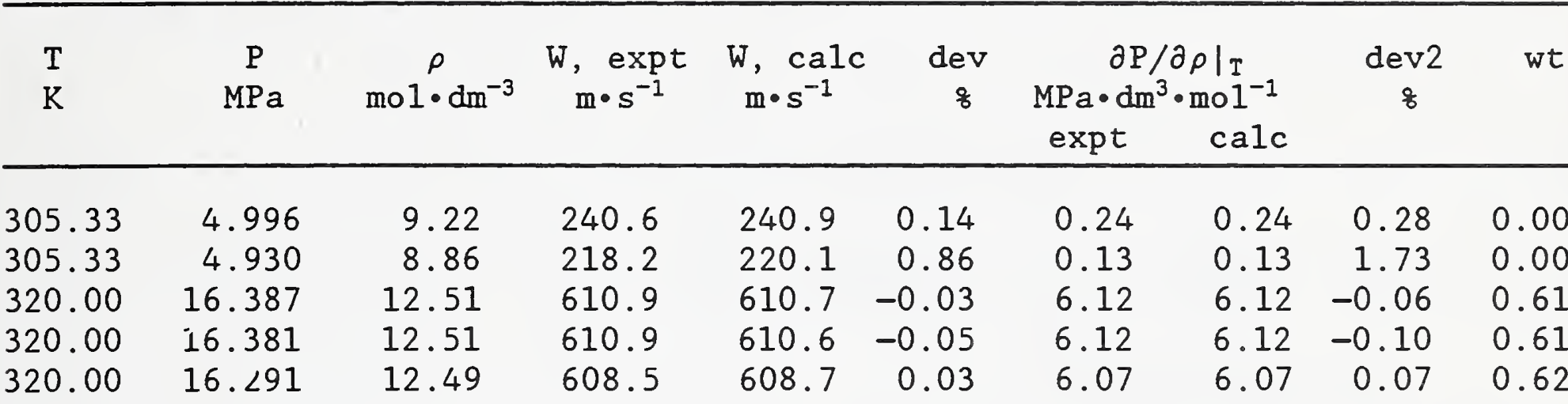

$\begin{array}{rrrrrrrrrr}320.00 & 34.482 & 14.33 & 877.1 & 873.1 & -0.46 & 14.75 & 14.61 & -0.91 & 0.33 \\ 320.00 & 27.495 & 13.80 & 792.8 & 790.3 & -0.31 & 11.61 & 11.54 & -0.63 & 0.38 \\ 320.00 & 21.649 & 13.22 & 707.5 & 706.4 & -0.15 & 8.82 & 8.79 & -0.31 & 0.46 \\ 320.00 & 14.707 & 12.21 & 573.8 & 573.4 & -0.07 & 5.22 & 5.21 & -0.14 & 0.71 \\ 320.00 & 11.258 & 11.38 & 479.3 & 478.6 & -0.15 & 3.24 & 3.23 & -0.30 & 1.50 \\ & & & & & & & & & \\ 320.00 & 9.969 & 10.92 & 433.6 & 432.9 & -0.15 & 2.45 & 2.44 & -0.31 & 2.48 \\ 320.00 & 8.588 & 10.22 & 372.5 & 372.6 & 0.04 & 1.57 & 1.57 & 0.07 & 1.09 \\ 320.00 & 7.802 & 9.62 & 328.1 & 329.1 & 0.30 & 1.05 & 1.05 & 0.60 & 0.90 \\ 320.00 & 7.348 & 9.11 & 297.2 & 298.3 & 0.38 & 0.74 & 0.75 & 0.75 & 0.83 \\ 320.00 & 31.610 & 14.13 & 844.1 & 840.8 & -0.39 & 13.47 & 13.37 & -0.77 & 0.35 \\ & & & & & & & & & \\ 323.15 & 33.170 & 14.12 & 850.1 & 846.7 & -0.40 & 13.78 & 13.67 & -0.81 & 0.34 \\ 323.15 & 28.536 & 13.76 & 793.5 & 791.0 & -0.31 & 11.71 & 11.64 & -0.62 & 0.38 \\ 323.15 & 25.114 & 13.44 & 746.6 & 744.9 & -0.22 & 10.12 & 10.08 & -0.45 & 0.42 \\ 323.15 & 21.665 & 13.07 & 693.5 & 692.6 & -0.14 & 8.46 & 8.44 & -0.27 & 0.48 \\ 323.15 & 17.118 & 12.44 & 611.3 & 609.9 & -0.23 & 6.19 & 6.16 & -0.45 & 0.00 \\ 323.15 & 14.694 & 12.00 & 556.5 & 556.0 & -0.09 & 4.87 & 4.86 & -0.18 & 0.77 \\ 323.15 & 13.346 & 11.70 & 521.9 & 521.5 & -0.08 & 4.12 & 4.12 & -0.15 & 0.00 \\ 323.15 & 10.176 & 10.68 & 419.9 & 419.7 & -0.06 & 2.27 & 2.27 & -0.12 & 0.00 \\ 323.15 & 10.034 & 10.62 & 414.6 & 414.1 & -0.12 & 2.19 & 2.18 & -0.25 & 1.62 \\ 323.15 & 9.077 & 10.11 & 372.8 & 373.0 & 0.05 & 1.60 & 1.60 & 0.10 & 1.10 \\ 323.15 & 9.061 & 10.10 & 371.7 & 372.2 & 0.15 & 1.59 & 1.59 & 0.29 & 0.00 \\ 323.15 & 8.035 & 9.28 & 316.2 & 317.8 & 0.52 & 0.95 & 0.96 & 1.04 & 0.00 \\ 323.15 & 7.049 & 7.71 & 250.7 & 250.2 & -0.21 & 0.41 & 0.41 & -0.42 & 0.00 \\ 323.15 & 6.707 & 6.74 & 231.8 & 230.5 & -0.56 & 0.32 & 0.31 & -1.11 & 0.00 \\ 323.15 & 6.323 & 5.51 & 223.5 & 223.0 & -0.24 & 0.34 & 0.33 & -0.48 & 0.00\end{array}$

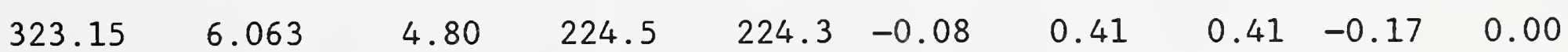


Table 10. SOUND SPEED IN THE SINGLE-PHASE FLUID (continued)

Data from Tsumura and Straty [63] (continued)

Number of Points [63] 156

Calculation of sound speed:

$\mathrm{AAD} \%=0.31 \quad \mathrm{BIAS} \%=-0.09 \quad \mathrm{RMS} \%=0.36$

Calculation of $\partial \mathrm{P} /\left.\partial \rho\right|_{\mathrm{I}}$ :

$\mathrm{AAD} 2 \%=0.62 \quad$ BIAS $2 \%=-0.18 \quad$ RMS $2 \%=0.72$

Absolute Deviations:

Calculation of sound speed:

$\mathrm{AAD}=3.29$ BIAS $=-1.13$ RMS $=4.38 \mathrm{~m} \cdot \mathrm{s}^{-1}$

Calculation of $\partial \mathrm{P} /\left.\partial \rho\right|_{\mathrm{T}}$ :

AAD2 $=0.162 \quad$ BIAS2 $=-0.033 \mathrm{RMS} 2=0.283 \mathrm{MPa} \cdot \mathrm{dm}^{3} \cdot \mathrm{mol}^{-1}$

Weighted Data:

Number of Points [63] 109

Calculation of sound speed:

$\mathrm{AAD} \%=0.36 \quad \mathrm{BIAS} \%=-0.16$ RMS $\%=0.38$

Calculation of $\partial \mathrm{P} /\left.\partial \rho\right|_{\mathrm{T}}$ :

$\mathrm{AAD} 2 \%=0.71 \quad$ BIAS2\% $=-0.32 \quad \operatorname{RMS} 2 \%=0.76$

Absolute Deviations:

Calculation of sound speed:

$\mathrm{AAD}=4.29$ BIAS $=-1.70 \quad$ RMS $=5.07 \mathrm{~m} \cdot \mathrm{s}^{-1}$

Calculation of $\partial \mathrm{P} /\left.\partial \rho\right|_{\mathrm{T}}$ :

AAD2 $=0.225 \quad$ BIAS2 $=-0.045 \quad \mathrm{RMS} 2=0.337 \mathrm{MPa} \cdot \mathrm{dm}^{3} \cdot \mathrm{mol}^{-1}$

Overall Results: $\mathrm{N}=248$

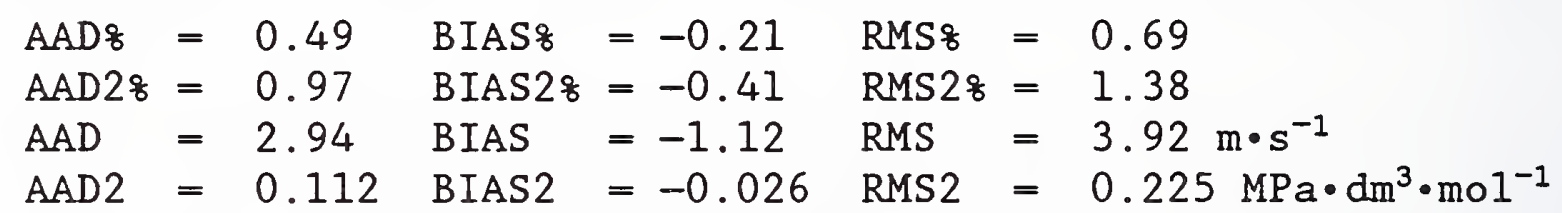

Weighted Data: $\quad N=201$

\begin{tabular}{|c|c|c|c|c|c|}
\hline $\mathrm{AAD}$ \% & 0.55 & BIAS \& & $=-0.27$ & RMS \% & 0.74 \\
\hline AA & 1.11 & BIAS 28 & -0.53 & RMS 28 & 1.48 \\
\hline$A A D$ & 3.40 & BIAS & $=-1.43$ & RMS & $4.26 \mathrm{~m} \cdot \mathrm{s}^{-1}$ \\
\hline $\mathrm{AD} 2$ & 0.134 & BIAS2 & $=-0.032$ & RMS2 & $0.249 \mathrm{MPa} \cdot \mathrm{dm}^{3} \cdot \mathrm{mol}^{-1}$ \\
\hline
\end{tabular}


Table 11. SOUND SPEED IN THE SATURATED LIQUID

Data from Poole and Aziz [64]

\begin{tabular}{|c|c|c|c|c|c|c|c|c|c|}
\hline \multirow[t]{2}{*}{$\begin{array}{l}\mathrm{T} \\
\mathrm{K}\end{array}$} & \multirow[t]{2}{*}{$\begin{array}{r}\mathrm{P}_{\sigma} \\
\mathrm{MPa}\end{array}$} & \multirow[t]{2}{*}{$\begin{array}{l}\rho_{\sigma \mathrm{L}} \\
\mathrm{mol} \cdot \mathrm{dm}^{-3}\end{array}$} & \multirow[t]{2}{*}{$\begin{array}{c}\mathrm{W}_{\sigma \mathrm{L}}, \operatorname{expt} \\
\mathrm{m} \cdot \mathrm{s}^{-1}\end{array}$} & \multirow[t]{2}{*}{$\begin{array}{l}\mathrm{W}_{\sigma \mathrm{L}}, \mathrm{calc} \\
\mathrm{m} \cdot \mathrm{s}^{-1}\end{array}$} & \multirow[t]{2}{*}{$\begin{array}{c}\mathrm{dev} \\
8\end{array}$} & \multicolumn{2}{|c|}{$\begin{array}{c}\partial \mathrm{P} /\left.\partial \rho\right|_{\mathrm{T}} \\
\mathrm{MPa} \cdot \mathrm{dm}^{3} \cdot \mathrm{mol}^{-1}\end{array}$} & \multirow[t]{2}{*}{$\begin{array}{r}\operatorname{dev} 2 \\
8\end{array}$} & \multirow[t]{2}{*}{ wt } \\
\hline & & & & & & $\exp t$ & calc & & \\
\hline 92.95 & $0.2 E-5$ & 21.57 & 1977.3 & 1981.9 & 0.23 & 82.62 & 83.00 & 0.46 & 0.00 \\
\hline 98.72 & $0.8 E-5$ & 21.37 & 1933.6 & 1946.7 & 0.68 & 77.49 & 78.55 & 1.36 & 0.00 \\
\hline 100.15 & $0.1 E-4$ & 21.32 & 1921.9 & 1937.3 & 0.80 & 76.24 & 77.46 & 1.60 & 0.00 \\
\hline 103.15 & $0.2 E-4$ & 21.21 & 1904.5 & 1916.8 & 0.65 & 74.24 & 75.21 & 1.30 & 0.00 \\
\hline 108.30 & $0.6 z-4$ & 21.02 & 1867.2 & 1880.5 & 0.71 & 70.44 & 71.44 & 1.43 & 0.00 \\
\hline 111.52 & $0.1 E-3$ & 20.91 & 1844.4 & 1857.3 & 0.70 & 68.20 & 69.16 & 1.40 & 0.00 \\
\hline 114.94 & $0.2 \mathrm{E}-3$ & 20.78 & 1818.0 & 1832.5 & 0.80 & 65.75 & 66.80 & 1.60 & 0.00 \\
\hline 121.01 & $0.4 E-3$ & 20.56 & 1774.6 & 1788.1 & 0.76 & 61.83 & 62.77 & 1.53 & 0.00 \\
\hline 127.25 & $0.9 E-3$ & 20.33 & 1730.4 & 1742.2 & 0.68 & 58.04 & 58.83 & 1.37 & 0.00 \\
\hline 132.32 & $0.2 E-2$ & 20.14 & 1693.8 & 1704.7 & 0.64 & 55.05 & 55.76 & 1.29 & 0.00 \\
\hline 134.09 & $0.2 \mathrm{E}-2$ & 20.07 & 1682.6 & 1691.6 & 0.53 & 54.15 & 54.73 & 1.07 & 0.00 \\
\hline 139.91 & $0.4 \mathrm{E}-2$ & 19.85 & 1639.7 & 1648.4 & 0.53 & 50.87 & 51.41 & 1.07 & 0.00 \\
\hline 147.12 & $0.8 \mathrm{E}-2$ & 19.58 & 1587.7 & 1594.8 & 0.44 & 47.08 & 47.50 & 0.89 & 0.00 \\
\hline 147.82 & $0.8 \mathrm{E}-2$ & 19.55 & 1583.4 & 1589.5 & 0.39 & 46.77 & 47.13 & 0.78 & 0.00 \\
\hline 154.05 & 0.014 & 19.31 & 1536.7 & 1543.1 & 0.41 & 43.59 & 43.95 & 0.83 & 0.00 \\
\hline 159.77 & 0.021 & 19.09 & 1494.7 & 1500.2 & 0.37 & 40.85 & 41.15 & 0.74 & 0.00 \\
\hline 165.52 & 0.032 & 18.87 & 1453.2 & 1457.0 & 0.26 & 38.26 & 38.46 & 0.53 & 0.00 \\
\hline 168.94 & 0.040 & 18.73 & 1430.5 & 1431.2 & 0.05 & 36.87 & 36.91 & 0.10 & 0.00 \\
\hline 174.26 & 0.056 & 18.51 & 1391.7 & 1391.1 & -0.04 & 34.61 & 34.58 & -0.08 & 0.00 \\
\hline 177.14 & 0.067 & 18.40 & 1368.6 & 1369.4 & 0.06 & 33.32 & 33.36 & 0.11 & 0.00 \\
\hline 180.46 & 0.081 & 18.26 & 1343.2 & 1344.2 & 0.08 & 31.93 & 31.98 & 0.15 & 0.00 \\
\hline 183.08 & 0.094 & 18.15 & 1325.4 & 1324.3 & -0.08 & 30.96 & 30.91 & -0.16 & 0.00 \\
\hline 184.57 & 0.101 & 18.09 & 1312.9 & 1313.0 & 0.00 & 30.31 & 30.32 & 0.01 & 0.00 \\
\hline 188.83 & 0.127 & 17.91 & 1280.3 & 1280.5 & 0.01 & 28.64 & 28.65 & 0.03 & 0.00 \\
\hline 198.78 & 0.206 & 17.48 & 1202.2 & 1204.2 & 0.17 & 24.86 & 24.94 & 0.34 & 0.00 \\
\hline
\end{tabular}

Number of Points [64] 25

Calculation of sound speed:

$\mathrm{AAD} z=0.40 \quad$ BIAS $8=0.39$ RMS $8=0.29$

Calculation of $\partial P /\left.\partial \rho\right|_{T}$ :

AAD2 $8=0.81 \quad$ BIAS2 $8=0.79 \quad$ RMS2 $8=0.58$ 
Table 11. SOUND SPEED IN THE SATURATED IIQUID (ccintinued)

Data from Poole and Aziz [64] (continued)

Absolute Deviations:

Calculation of sound speed :

$\mathrm{AAD}=7.01 \quad \mathrm{BIAS}=6.88$ RMS $=5.39 \mathrm{~m} \cdot \mathrm{s}^{-1}$

Calculation of $\partial \mathrm{P} /\left.\partial \rho\right|_{\mathrm{T}}$ :

AAD2 $=0.486 \quad$ BIAS2 $=0.480 \quad$ RMS2 $=0.410 \mathrm{MPa} \cdot \mathrm{drn}^{3} \cdot \mathrm{mol}^{-1}$

Data from Tsumura and Straty [63]

These data were adjusted slightly as in [20].

\begin{tabular}{|c|c|c|c|c|c|c|c|c|c|}
\hline \multirow[t]{2}{*}{$\begin{array}{l}\mathrm{T} \\
\mathrm{K}\end{array}$} & \multirow[t]{2}{*}{$\begin{array}{r}\mathrm{P}_{\sigma} \\
\mathrm{MPa}\end{array}$} & \multirow[t]{2}{*}{$\begin{array}{c}\rho_{\sigma \mathrm{L}} \\
\mathrm{mol} \cdot \mathrm{dm}^{-3}\end{array}$} & \multirow[t]{2}{*}{$\begin{array}{c}\mathrm{W}_{\sigma \mathrm{L}}, \operatorname{expt} \\
\mathrm{m} \cdot \mathrm{s}^{-1}\end{array}$} & \multirow[t]{2}{*}{$\begin{array}{l}\mathrm{W}_{\sigma \mathrm{L}}, \mathrm{calc} \\
\mathrm{m} \cdot \mathrm{s}^{-1}\end{array}$} & \multirow[t]{2}{*}{$\begin{array}{c}\mathrm{dev} \\
8\end{array}$} & \multicolumn{2}{|c|}{$\begin{array}{c}\partial \mathrm{P} /\left.\partial \rho\right|_{\mathrm{T}} \\
\mathrm{MPa} \cdot \mathrm{dm}^{3} \cdot \mathrm{mol}^{-1}\end{array}$} & \multirow[t]{2}{*}{$\begin{array}{r}\operatorname{dev} 2 \\
8\end{array}$} & \multirow[t]{2}{*}{ wt } \\
\hline & & & & & & $x p$ & calc & & \\
\hline 91.00 & $0.1 E-5$ & 21.64 & 2002.6 & 1992.0 & -0.53 & 85.43 & 84.52 & -1.06 & 0.42 \\
\hline 95.00 & $0.4 \mathrm{E}-5$ & 21.50 & 1974.1 & 1970.1 & -0.20 & 81.74 & 81.42 & -0.40 & 0.41 \\
\hline 100.00 & $0.1 E-4$ & 21.32 & 1938.7 & 1938.3 & -0.02 & 77.61 & 77.58 & -0.04 & 0.40 \\
\hline 105.00 & $0.3 E-4$ & 21.14 & 1902.8 & 1903.9 & 0.06 & 73.75 & 73.84 & 0.11 & 0.39 \\
\hline 110.00 & $0.7 E-4$ & 20.96 & 1867.4 & 1868.3 & 0.05 & 70.17 & 70.23 & 0.09 & 0.38 \\
\hline 115.00 & $0.2 E-3$ & 20.78 & 1831.2 & 183 & 0.05 & 66.70 & 66.76 & 0.09 & 0.37 \\
\hline 120.00 & $0.4 \mathrm{E}-3$ & 20.60 & 1795.0 & 1795.5 & 0.03 & 63.39 & 63.43 & 0.05 & 0.37 \\
\hline 125.00 & $0.7 \mathrm{E}-3$ & 20.41 & 1759.0 & 1758.7 & -0.01 & 60.24 & 60.23 & -0.03 & 0.36 \\
\hline 130.00 & $0.1 E-2$ & 20.23 & 1722.8 & 1721.8 & -0.06 & 57.21 & 57.15 & -0.11 & 0.36 \\
\hline 135.00 & $0.2 E-2$ & 20.04 & 1685.9 & 1684.8 & -0.06 & 54.26 & 54.20 & -0.12 & 0.35 \\
\hline 140.00 & $0.4 E-2$ & 19.85 & 1649.4 & 164 & -0.10 & 51.46 & 51.36 & -0.20 & 0.35 \\
\hline 145.00 & $0.6 \mathrm{E}-2$ & 19.66 & 1612.7 & 1610.6 & -0.13 & 48.76 & 48.63 & -0.26 & 0.35 \\
\hline 150.00 & $0.1 \mathrm{E}-1$ & 19.47 & 1575.5 & 1573.3 & -0.14 & 46.13 & 46.00 & -0.28 & 0.35 \\
\hline 155.00 & 0.015 & 19.28 & 1538.3 & 1535.9 & -0.15 & 43.61 & 43.47 & -0.31 & 0.35 \\
\hline 160.00 & 0.021 & 19.08 & 1501.1 & 1498.5 & -0.17 & 41.18 & 41.04 & -0.35 & 0.35 \\
\hline 165.00 & 0.031 & 18.89 & 1463.6 & 1460.9 & -0.18 & 38.84 & 38.70 & -0.36 & 0.35 \\
\hline 170.00 & 0.043 & 18.69 & 1426.0 & 1423.3 & -0.19 & 36.58 & 36.44 & -0.38 & 0.35 \\
\hline 175.00 & 0.059 & 18.48 & 1388.1 & 1385.5 & -0.18 & 34.39 & 34.26 & -0.37 & 0.35 \\
\hline 180.00 & 0.079 & 18.28 & 1350.3 & 1347.7 & -0.19 & 32.29 & 32.17 & -0.39 & 0.35 \\
\hline 185.00 & 0.104 & 18.07 & 1312.1 & 1309.7 & -0.18 & 30.26 & 30.15 & -0.37 & 0.35 \\
\hline 190.00 & 0.135 & 17.86 & 1273.6 & 1271.6 & -0.16 & 28.29 & 28.20 & -0.32 & 0.36 \\
\hline 195.00 & 0.172 & 17.64 & 1235.0 & 1233.3 & -0.14 & 26.39 & 26.32 & -0.28 & 0.36 \\
\hline 200.00 & 0.217 & 17.42 & 1196.0 & 1194.8 & -0.10 & 24.55 & 24.50 & -0.19 & 0.36 \\
\hline 205.00 & 0.271 & 17.20 & 1156.9 & 1156.2 & -0.06 & 22.78 & 22.76 & -0.12 & 0.37 \\
\hline 210.00 & 0.334 & 16.97 & 1117.4 & 1117.4 & 0.00 & 21.07 & 21.07 & 0.00 & 0.38 \\
\hline
\end{tabular}


Table 11. SOUND SPEED IN THE SATURATED LIQUID (continued)

Data from Tsumura and Straty [63] (continued)

\begin{tabular}{|c|c|c|c|c|c|c|c|c|c|}
\hline $\begin{array}{l}\mathrm{T} \\
\mathrm{K}\end{array}$ & $\begin{array}{r}\mathrm{P}_{\sigma} \\
\mathrm{MPa}\end{array}$ & $\begin{array}{c}\rho_{\sigma \mathrm{L}} \\
\mathrm{mol} \cdot \mathrm{dm}^{-3}\end{array}$ & $\begin{array}{c}W_{\sigma L}, \operatorname{expt} \\
\mathrm{m} \cdot \mathrm{s}^{-1}\end{array}$ & $\begin{array}{l}\mathrm{W}_{\sigma \mathrm{L}}, \mathrm{calc} \mathrm{c} \\
\mathrm{m} \cdot \mathrm{s}^{-1}\end{array}$ & $\begin{array}{c}\mathrm{dev} \\
8\end{array}$ & $\begin{array}{r}\partial \mathrm{P} / \\
\mathrm{MPa} \cdot \mathrm{dn} \\
\operatorname{expt}\end{array}$ & $\begin{array}{l}\text { II } \\
\text { mol-1 } \\
\text { calc }\end{array}$ & $\begin{array}{r}\operatorname{dev} 2 \\
8\end{array}$ & $w t$ \\
\hline 215.00 & 0.407 & 16.74 & 1077.7 & 1078.3 & 0.06 & 19.43 & 19.45 & 0.11 & 0.39 \\
\hline 220.00 & 0.492 & 16.50 & 1037.7 & 1039.0 & 0.12 & 17.84 & 17.89 & 0.25 & 0.40 \\
\hline 225.00 & 0.590 & 16.25 & 997.2 & 999.4 & 0.22 & 16.31 & 16.38 & 0.43 & 0.41 \\
\hline 230.00 & 0.701 & 16.00 & 956.5 & 959.4 & 0.30 & 14.85 & 14.93 & 0.60 & 0.42 \\
\hline 235.00 & 0.826 & 15.74 & 915.2 & 919.0 & 0.42 & 13.43 & 13.54 & 0.84 & 0.44 \\
\hline 240.00 & 0.967 & 15.47 & 873.6 & 878.2 & 0.53 & 12.07 & 12.20 & 1.06 & 0.46 \\
\hline 245.00 & 1.125 & 15.19 & 831.3 & 836.9 & 0.68 & 10.77 & 10.92 & 1.35 & 0.48 \\
\hline 250.00 & 1.301 & 14.89 & 788.5 & 795.0 & 0.82 & 9.52 & 9.68 & 1.65 & 0.51 \\
\hline 255.00 & 1.497 & 14.59 & 745.0 & 752.4 & 0.99 & 8.33 & 8.50 & 1.98 & 0.55 \\
\hline 260.00 & 1.712 & 14.27 & 700.7 & 708.9 & 1.16 & 7.20 & 7.37 & 2.34 & 0.60 \\
\hline 265.00 & 1.950 & 13.93 & 655.4 & 664.3 & 1.36 & 6.12 & 6.29 & 2.73 & 0.67 \\
\hline 270.00 & 2.211 & 13.56 & 609.0 & 618.4 & 1.55 & 5.10 & 5.26 & 3.12 & 0.76 \\
\hline 275.00 & 2.496 & 13.17 & 561.1 & 570.8 & 1.73 & 4.14 & 4.29 & 3.50 & 0.93 \\
\hline 280.00 & 2.808 & 12.74 & 511.5 & 520.9 & 1.84 & 3.24 & 3.36 & 3.72 & 1.30 \\
\hline 285.00 & 3.147 & 12.26 & 459.4 & 467.9 & 1.84 & 2.41 & 2.49 & 3.72 & 6.51 \\
\hline 290.00 & 3.517 & 11.70 & 404.2 & 410.5 & 1.55 & 1.65 & 1.70 & 3.13 & 1.38 \\
\hline 295.00 & 3.919 & 11.01 & 343.9 & 347.3 & 1.00 & 0.98 & 1.00 & 2.01 & 0.97 \\
\hline 295.40 & 3.952 & 10.95 & 339.0 & 342.0 & 0.89 & 0.93 & 0.95 & 1.80 & 0.96 \\
\hline 296.00 & 4.003 & 10.85 & 331.1 & 333.9 & 0.84 & 0.86 & 0.87 & 1.69 & 0.93 \\
\hline 297.00 & 4.089 & 10.68 & 317.8 & 320.1 & 0.73 & 0.74 & 0.76 & 1.47 & 0.89 \\
\hline 298.00 & 4.177 & 10.50 & 304.0 & 306.0 & 0.67 & 0.63 & 0.64 & 1.35 & 0.86 \\
\hline 299.00 & 4.266 & 10.30 & 289.6 & 291.6 & 0.69 & 0.53 & 0.53 & 1.39 & 0.83 \\
\hline 300.00 & 4.357 & 10.08 & 274.8 & 276.8 & 0.71 & 0.43 & 0.43 & 1.43 & 0.00 \\
\hline 301.00 & 4.449 & 9.85 & 259.1 & 261.4 & 0.91 & 0.33 & 0.34 & 1.82 & 0.00 \\
\hline 302.00 & 4.543 & 9.58 & 241.9 & 245.5 & 1.50 & 0.24 & 0.25 & 3.02 & 0.00 \\
\hline 303.00 & 4.639 & 9.25 & 223.2 & 228.8 & 2.49 & 0.16 & 0.16 & 5.04 & 0.00 \\
\hline
\end{tabular}

Number of Points [63] 51

Calculation of sound speed:

$\mathrm{AAD} \&=0.56 \quad \mathrm{BIAS} \%=0.45 \quad \mathrm{RMS} 8=0.70$

Calculation of $\partial \mathrm{P} /\left.\partial \rho\right|_{\mathrm{I}}$ :

$\mathrm{AAD} 28=1.13 \quad$ BIAS2 $8=0.90 \quad$ RMS $28=1.40$ 
Data from Tsumura and Straty [63] (continued)

Absolute Deviations:

Calculation of sound speed:

$\mathrm{AAD}=3.42$ BIAS $=1.59$ RMS $=4.15 \mathrm{~m} \cdot \mathrm{s}^{-1}$

Calculation of $\partial \mathrm{P} /\left.\partial \rho\right|_{\mathrm{T}}$ :

AAD2 $=0.100$ BIAS2 $=-0.015$ RMS2 $=0.164 \mathrm{MPa} \cdot \mathrm{dm}^{3} \cdot \mathrm{mol}^{-1}$

Weighted Data:

Number of Points [63] 47

Calculation of sound speed:

$\mathrm{AAD} \%=0.49 \quad \mathrm{BIAS} \%=0.37$ RMS $\%=0.63$

Calculation of $\partial \mathrm{P} /\left.\partial \rho\right|_{\mathrm{T}}$ :

AAD2 $8=0.99 \quad$ BIAS $2 \%=0.74 \quad$ RMS $2 \%=1.28$

Absolute Deviations:

Calculation of sound speed:

$\mathrm{AAD}=3.42$ BIAS $=1.43$ RMS $=4.26 \mathrm{~m} \cdot \mathrm{s}^{-1}$

Calculation of $\partial \mathrm{P} /\left.\partial \rho\right|_{\mathrm{T}}$ :

AAD2 $=0.108$ BIAS2 $=-0.016 \mathrm{RMS} 2=0.171 \mathrm{MPa} \cdot \mathrm{dm}^{3} \cdot \mathrm{mol}^{-1}$

Data from Vangee1 [65]

These unpublished data were tabulated in [20].

\begin{tabular}{|c|c|c|c|c|c|c|c|c|c|}
\hline \multirow[t]{2}{*}{$\begin{array}{l}\mathrm{T} \\
\mathrm{K}\end{array}$} & \multirow[t]{2}{*}{$\begin{array}{r}\mathrm{P}_{\sigma} \\
\mathrm{MPa}\end{array}$} & \multirow[t]{2}{*}{$\stackrel{\rho_{\sigma \mathrm{L}}}{\mathrm{mol} \cdot \mathrm{dm}^{-3}}$} & \multirow[t]{2}{*}{$\begin{array}{c}\mathrm{W}_{\sigma \mathrm{L}}, \operatorname{expt} \\
\mathrm{m} \cdot \mathrm{s}^{-1}\end{array}$} & \multirow[t]{2}{*}{$\begin{array}{l}\mathrm{W}_{\sigma \mathrm{L}}, \mathrm{calc} \\
\mathrm{m} \cdot \mathrm{s}^{-1}\end{array}$} & \multirow[t]{2}{*}{$\begin{array}{c}\mathrm{dev} \\
8\end{array}$} & \multicolumn{2}{|c|}{$\begin{array}{c}\partial \mathrm{P} /\left.\partial \rho\right|_{\mathrm{T}} \\
\mathrm{MPa} \cdot \mathrm{dm}^{3} \cdot \mathrm{mol}^{-1}\end{array}$} & \multirow[t]{2}{*}{$\begin{array}{r}\operatorname{dev} 2 \\
8\end{array}$} & \multirow[t]{2}{*}{ wt } \\
\hline & & & & & & expt & calc & & \\
\hline 95.50 & $0.4 E-5$ & 21.49 & 1963.0 & 1967.1 & 0.21 & 80.69 & 81.03 & 0.42 & 0.00 \\
\hline 99.70 & $0.1 \mathrm{E}-4$ & 21.34 & 1934.0 & 1940.3 & 0.32 & 77.30 & 77.80 & 0.65 & 0.00 \\
\hline 104.87 & $0.3 E-4$ & 21.15 & 1898.5 & 1904.8 & 0.33 & 73.45 & 73.93 & 0.67 & 0.00 \\
\hline 109.80 & $0.7 E-4$ & 20.97 & 1862.0 & 1869.7 & 0.41 & 69.79 & 70.37 & 0.83 & 0.00 \\
\hline 114.97 & $0.2 E-3$ & 20.78 & 1826.2 & 1832.3 & 0.33 & 66.34 & 66.78 & 0.67 & 0.00 \\
\hline 115.01 & $0.2 E-3$ & 20.78 & 1827.0 & 1832.0 & 0.27 & 66.39 & 66.75 & 0.55 & 0.00 \\
\hline 119.70 & $0.3 E-3$ & 20.61 & 1795.0 & 1797.7 & 0.15 & 63.43 & 63.62 & 0.30 & 0.00 \\
\hline 125.49 & $0.7 \mathrm{E}-3$ & 20.39 & 1752.8 & 1755.1 & 0.13 & 59.76 & 59.92 & 0.27 & 0.00 \\
\hline 130.11 & $0.1 \mathrm{E}-2$ & 20.22 & 1721.0 & 1721.0 & 0.00 & 57.08 & 57.08 & 0.00 & 0.00 \\
\hline 134.89 & $0.2 E-2$ & 20.04 & 1685.0 & 1685.7 & 0.04 & 54.22 & 54.26 & 0.08 & 0.00 \\
\hline
\end{tabular}


Table 11. SOUND SPEED IN THE SATURATED LIQUID (continued)

Data from Vangeel [65] (continued)

\begin{tabular}{|c|c|c|c|c|c|c|c|c|c|}
\hline \multirow[t]{2}{*}{$\begin{array}{l}\mathrm{T} \\
\mathrm{K}\end{array}$} & \multirow[t]{2}{*}{$\begin{array}{r}\mathrm{P}_{\sigma} \\
\mathrm{MPa}\end{array}$} & \multirow[t]{2}{*}{$\begin{array}{c}\rho_{\sigma \mathrm{L}} \\
\mathrm{mol} \cdot \mathrm{dm}^{-3}\end{array}$} & \multirow[t]{2}{*}{$\begin{array}{l}W_{\sigma L}, \operatorname{expt} \\
m \cdot s^{-1}\end{array}$} & \multirow[t]{2}{*}{$\begin{array}{l}\mathrm{W}_{\sigma \mathrm{L}}, \mathrm{calc} \\
\mathrm{m} \cdot \mathrm{s}^{-1}\end{array}$} & \multirow[t]{2}{*}{$\begin{array}{c}\text { dev } \\
8\end{array}$} & \multicolumn{2}{|c|}{$\begin{array}{c}\partial \mathrm{P} /\left.\partial \rho\right|_{\mathrm{T}} \\
\mathrm{MPa} \cdot \mathrm{dm}^{3} \cdot \mathrm{mol}^{-1}\end{array}$} & \multirow[t]{2}{*}{$\begin{array}{r}\text { dev2 } \\
8\end{array}$} & \multirow[t]{2}{*}{ wt } \\
\hline & & & & & & expt & calc & & \\
\hline 139.47 & $0.4 E-2$ & 19.87 & 1652.1 & 1651.7 & -0.02 & 51.68 & 51.65 & -0.05 & 0.00 \\
\hline 145.08 & $0.6 E-2$ & 19.66 & 1608.0 & 1610.0 & 0.12 & 48.47 & 48.59 & 0.25 & 0.00 \\
\hline 151.28 & 0.011 & 19.42 & 1565.7 & 1563.7 & -0.13 & 45.46 & 45.35 & -0.25 & 0.00 \\
\hline 155.94 & 0.016 & 19.24 & 1531.2 & 1528.9 & -0.15 & 43.14 & 43.01 & -0.30 & 0.00 \\
\hline 161.25 & 0.024 & 19.03 & 1491.2 & 1489.1 & -0.14 & 40.56 & 40.45 & -0.28 & 0.00 \\
\hline 161.26 & 0.024 & 19.03 & 1491.4 & 1489.0 & -0.16 & 40.57 & 40.44 & -0.32 & 0.00 \\
\hline 164.86 & 0.030 & 18.89 & 1464.0 & 1462.0 & -0.14 & 38.87 & 38.76 & -0.27 & 0.00 \\
\hline 164.89 & 0.030 & 18.89 & 1463.1 & 1461.8 & -0.09 & 38.82 & 38.75 & -0.18 & 0.00 \\
\hline 169.84 & 0.042 & 18.69 & 1426.5 & 1424.5 & -0.14 & 36.61 & 36.51 & -0.28 & 0.00 \\
\hline 174.59 & 0.057 & 18.50 & 1391.0 & 1388.6 & -0.17 & 34.56 & 34.44 & -0.34 & 0.00 \\
\hline 179.85 & 0.078 & 18.29 & 1350.9 & 1348.8 & -0.15 & 32.33 & 32.23 & -0.31 & 0.00 \\
\hline 184.67 & 0.102 & 18.09 & 1316.2 & 1312.2 & -0.30 & 30.46 & 30.28 & -0.61 & 0.00 \\
\hline 189.71 & 0.133 & 17.87 & 1276.1 & 1273.8 & -0.18 & 28.41 & 28.31 & -0.36 & 0.00 \\
\hline 195.09 & 0.173 & 17.64 & 1234.3 & 1232.6 & -0.14 & 26.36 & 26.28 & -0.27 & 0.00 \\
\hline 199.64 & 0.214 & 17.44 & 1199.1 & 1197.6 & -0.12 & 24.69 & 24.63 & -0.25 & 0.00 \\
\hline 204.42 & 0.264 & 17.23 & 1160.2 & 1160.7 & 0.04 & 22.94 & 22.96 & 0.09 & 0.00 \\
\hline 210.31 & 0.338 & 16.96 & 1115.1 & 1115.0 & -0.01 & 20.98 & 20.97 & -0.02 & 0.00 \\
\hline 214.56 & 0.401 & 16.76 & 1081.5 & 1081.8 & 0.02 & 19.58 & 19.59 & 0.05 & 0.00 \\
\hline 220.10 & 0.494 & 16.49 & 1036.4 & 1038.2 & 0.17 & 17.80 & 17.86 & 0.35 & 0.00 \\
\hline 224.96 & 0.589 & 16.25 & 996.8 & 999.7 & 0.29 & 16.30 & 16.40 & 0.58 & 0.00 \\
\hline 229.93 & 0.699 & 16.00 & 958.1 & 959.9 & 0.19 & 14.90 & 14.95 & 0.39 & 0.00 \\
\hline 234.91 & 0.824 & 15.74 & 916.4 & 919.8 & 0.37 & 13.47 & 13.57 & 0.73 & 0.00 \\
\hline 239.90 & 0.964 & 15.47 & 875.3 & 879.1 & 0.43 & 12.12 & 12.23 & 0.86 & 0.00 \\
\hline 244.96 & 1.124 & 15.19 & 831.4 & 837.2 & 0.70 & 10.77 & 10.93 & 1.41 & 0.00 \\
\hline 244.75 & 1.117 & 15.20 & 832.5 & 839.0 & 0.78 & 10.81 & 10.98 & 1.57 & 0.00 \\
\hline 250.22 & 1.310 & 14.88 & 787.7 & 793.1 & 0.69 & 9.50 & 9.63 & 1.38 & 0.00 \\
\hline 255.11 & 1.501 & 14.58 & 744.6 & 751.4 & 0.91 & 8.32 & 8.47 & 1.84 & 0.00 \\
\hline 259.93 & 1.709 & 14.27 & 700.0 & 709.5 & 1.35 & 7.19 & 7.39 & 2.72 & 0.00 \\
\hline 264.97 & 1.949 & 13.93 & 655.5 & 664.6 & 1.38 & 6.13 & 6.30 & 2.79 & 0.00 \\
\hline 269.92 & 2.206 & 13.57 & 609.7 & 619.2 & 1.55 & 5.12 & 5.28 & 3.13 & 0.00 \\
\hline 274.17 & 2.447 & 13.24 & 569.6 & 578.9 & 1.63 & 4.30 & 4.44 & 3.28 & 0.00 \\
\hline 280.05 & 2.811 & 12.74 & 509.4 & 520.4 & 2.16 & 3.21 & 3.35 & 4.37 & 0.00 \\
\hline 285.15 & 3.158 & 12.24 & 459.0 & 466.2 & 1.57 & 2.39 & 2.47 & 3.17 & 0.00 \\
\hline 288.57 & 3.408 & 11.87 & 423.8 & 427.4 & 0.85 & 1.89 & 1.92 & 1.71 & 0.00 \\
\hline
\end{tabular}


Table 11. SOUND SPEED IN THE SATURATED LIQUID (continued)

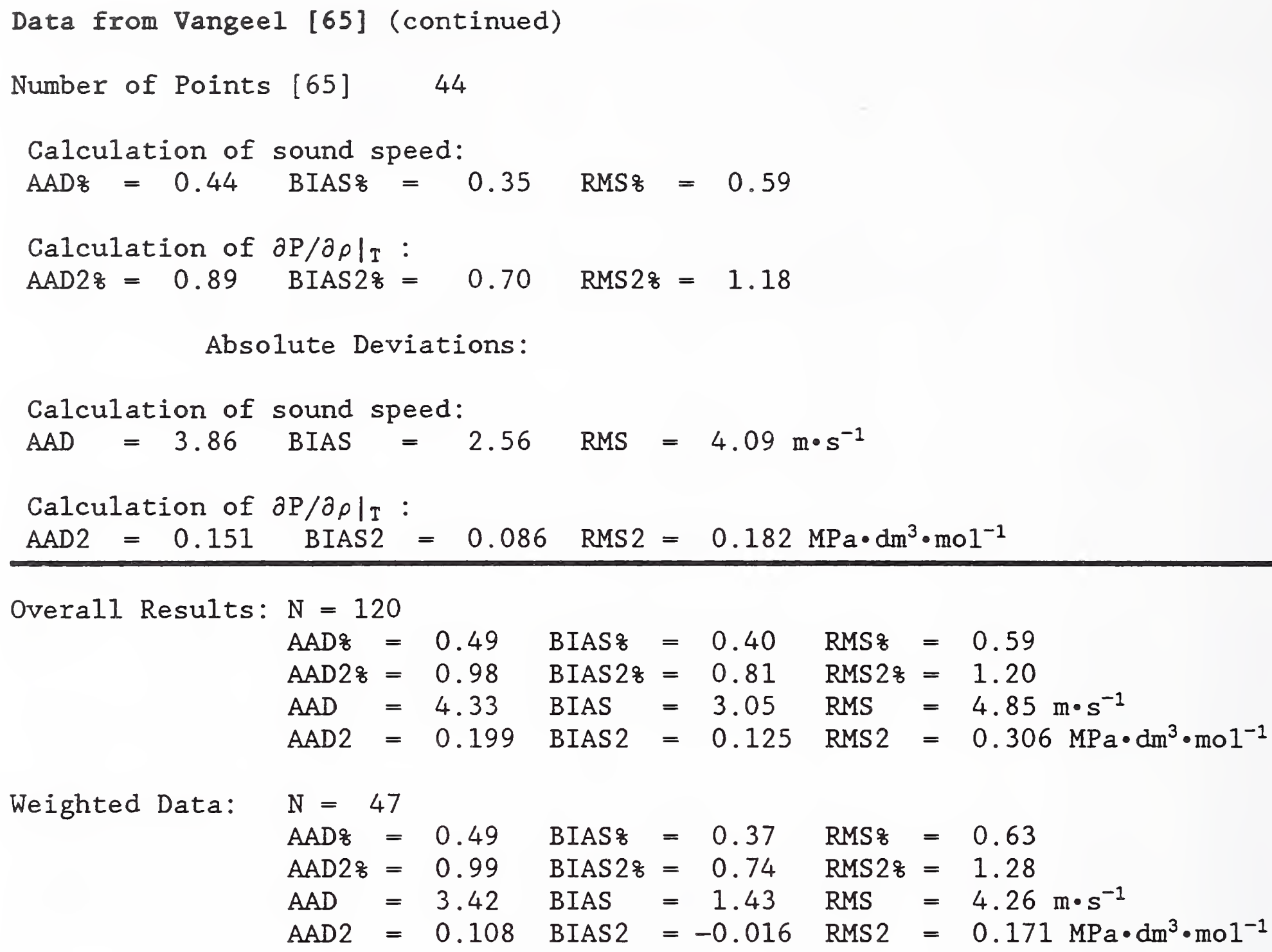


Table 12 gives comparisons for the dilute gas viscosity and table 13 is for the dilute gas thermal conductivity. In both tables, we list the pressure and the value of the transport property as tabulated in the experimental paper. Under the headings $\eta_{0}$, expt and $\lambda_{0}$, expt we give values adjusted to zero pressure according to our correlation of the transport property surfaces [1,2]. This adjustment is done by calculating the excess property (the difference between the total transport property and its dilute gas limit) at the experimental temperature and pressure and subtracting this value from the experimental measurement of the transport property at that state point. The experimental measurements are typically done at $0.1 \mathrm{MPa}$ or $0.101325 \mathrm{MPa}$ ( 1 atm), and the correction to zero pressure is seen to be extremely small for all of the tabulated data. When the experimental paper reports values which have already been extrapolated to zero pressure, these data are used in our comparisons, the pressure is indicated as $0 \mathrm{MPa}$, and the columns for the experimental quantity and the adjusted experimental quantity are identical.

The weights refer to the linear problem associated with the evaluation of coefficients for the dilute gas transport properties. In one instance, as indicated in table 13, the adjusted values used to determine the final correlation for the dilute gas were based on a preliminary version of the surface.

All data with pressures at or below $0.1 \mathrm{MPa}$ are included in these tables rather than with the data at elevated pressures in Sec. 6. We have included overall statistics which eliminate both very recently acquired data and some older data in order to agree with the data selection in the comparisons of [1]. 
Table 12. ZERO DENSITY VISCOSITY

Data from Abe et al. [66]

\begin{tabular}{cccccrc}
$\mathrm{T}$ & $\begin{array}{c}\mathrm{P} \\
\mathrm{MPa}\end{array}$ & $\begin{array}{c}\eta_{\text {expt }} \\
\mu \mathrm{Pa} \cdot \mathrm{s}\end{array}$ & $\begin{array}{c}\eta_{0} \text { expt } \\
\mu \mathrm{Pa} \cdot \mathrm{s}\end{array}$ & $\begin{array}{c}\eta_{0}, \text { calc } \\
\mu \mathrm{Pa} \cdot \mathrm{s}\end{array}$ & $\begin{array}{c}\text { Dev } \\
8\end{array}$ & wt \\
\hline 298.150 & 0.10 & 9.25 & 9.23 & 9.33 & 1.13 & 0.00 \\
333.150 & 0.10 & 0.370 & 10.35 & 10.34 & -0.04 & 0.00 \\
373.150 & 0.10 & 11.52 & 11.50 & 11.46 & -0.31 & 0.00 \\
418.150 & 0.10 & 12.74 & 12.72 & 12.68 & -0.35 & 0.00 \\
468.150 & 0.10 & 14.07 & 14.05 & 13.97 & -0.61 & 0.00
\end{tabular}

Number of Points [66] 5

\begin{tabular}{|c|c|c|c|c|c|}
\hline & 49 & 8 & -0.04 & RMS \& & .61 \\
\hline T & 0.05 & BIAS & $=-0.01$ & RMS & $=0.06$ \\
\hline
\end{tabular}

Data from Adzumi [67]

\begin{tabular}{|c|c|c|c|c|c|c|c|c|c|c|}
\hline \multicolumn{2}{|l|}{$\begin{array}{l}\mathrm{T} \\
\mathrm{K} \\
\end{array}$} & \multicolumn{2}{|c|}{$\begin{array}{c}\mathrm{P} \\
\mathrm{MPa}\end{array}$} & \multicolumn{2}{|c|}{$\begin{array}{c}\eta, \text { expt } \\
\mu \mathrm{Pa} \cdot \mathrm{s}\end{array}$} & \multicolumn{2}{|c|}{$\begin{array}{c}\eta_{0}, \text { expt } \\
\mu \mathrm{Pa} \cdot \mathrm{s}\end{array}$} & $\begin{array}{c}\eta_{0}, \quad \mathrm{calc} \\
\mu \mathrm{Pa} \cdot \mathrm{s}\end{array}$ & $\begin{array}{l}\text { Dev } \\
\frac{8}{8}\end{array}$ & wt \\
\hline \multicolumn{2}{|c|}{293.150} & \multicolumn{2}{|c|}{0.00} & \multicolumn{2}{|c|}{9.28} & \multicolumn{2}{|l|}{9.28} & 9.19 & -1.02 & 0.00 \\
\hline 303.1 & 150 & \multicolumn{2}{|c|}{0.00} & \multicolumn{2}{|c|}{9.66} & \multicolumn{2}{|l|}{9.66} & 9.48 & -1.88 & 0.00 \\
\hline 313.1 & 150 & \multicolumn{2}{|c|}{0.00} & \multicolumn{2}{|c|}{9.91} & \multicolumn{2}{|l|}{9.91} & 9.77 & -1.42 & 0.00 \\
\hline 323.1 & 150 & \multicolumn{2}{|c|}{0.00} & \multicolumn{2}{|c|}{10.16} & \multicolumn{2}{|l|}{10.16} & 10.06 & -1.00 & 0.00 \\
\hline 333.1 & 150 & \multicolumn{2}{|c|}{0.00} & \multicolumn{2}{|c|}{10.51} & \multicolumn{2}{|l|}{10.51} & 10.34 & -1.58 & 0.00 \\
\hline 343.1 & 150 & \multicolumn{2}{|c|}{0.00} & \multicolumn{2}{|c|}{10.80} & \multicolumn{2}{|l|}{10.80} & 10.63 & -1.60 & 0.00 \\
\hline 353.1 & 150 & \multicolumn{2}{|c|}{0.00} & \multicolumn{2}{|c|}{11.09} & \multicolumn{2}{|l|}{11.09} & 10.91 & -1.63 & 0.00 \\
\hline 363.1 & 150 & \multicolumn{2}{|c|}{0.00} & \multicolumn{2}{|c|}{11.43} & \multicolumn{2}{|l|}{11.43} & 11.19 & -2.12 & 0.00 \\
\hline 373.1 & 150 & \multicolumn{2}{|c|}{0.00} & \multicolumn{2}{|c|}{11.71} & \multicolumn{2}{|l|}{11.71} & 11.46 & -2.11 & 0.00 \\
\hline Number $\circ$ & of $F$ & ints & 67] & 9 & & & & & & \\
\hline $\mathrm{AAD} ;$ & $=$ & 1.60 & BIAS 8 & $=$ & -1.60 & RMS \& & $=$ & 0.38 & & \\
\hline$A A D$ & $=$ & 0.17 & BIAS & $=$ & -0.17 & RMS & $=$ & $0.05 \mu \mathrm{Pa} \cdot \mathrm{s}$ & & \\
\hline
\end{tabular}


Table 12. ZERO DENSITY VISCOSITY (continued)

Data from Carmichael et a1. [68]

\begin{tabular}{ccccccc}
\hline $\mathrm{T}$ & $\mathrm{P}$ & $\begin{array}{c}\eta \text { expt } \\
\mu \mathrm{Pa} \cdot \mathrm{s}\end{array}$ & $\begin{array}{c}\eta_{0}, \begin{array}{c}\text { expt } \\
\mu \mathrm{Pa} \cdot \mathrm{s}\end{array} \\
\mathrm{MPa}\end{array}$ & $\begin{array}{c}\eta_{0}, \begin{array}{c}\text { calc } \\
\mu \mathrm{Pa} \cdot \mathrm{s}\end{array} \\
\text { ( }\end{array}$ & $\begin{array}{c}\text { Dev } \\
8\end{array}$ & wt \\
\hline 305.372 & 0.10 & 9.56 & 9.54 & 9.54 & 0.08 & 0.00 \\
305.372 & 0.10 & 9.55 & 9.53 & 9.54 & 0.13 & 0.00 \\
305.372 & 0.10 & 9.53 & 9.51 & 9.54 & 0.33 & 0.00
\end{tabular}

Number of Points [68] 3

$\mathrm{AAD} \&=0.18 \quad \mathrm{BIAS} 8=0.18 \quad \mathrm{RMS} 8=0.11$
$\mathrm{AAD}=0.02 \quad \mathrm{BIAS}=0.02 \quad \mathrm{RMS}=0.01 \mu \mathrm{Pa} \cdot \mathrm{S}$

Data from Craven et al. [69]

\begin{tabular}{|c|c|c|c|c|c|c|c|c|}
\hline $\begin{array}{l}\mathrm{T} \\
\mathrm{K}\end{array}$ & \multicolumn{2}{|c|}{$\begin{array}{c}\mathrm{P} \\
\mathrm{MPa}\end{array}$} & \multicolumn{2}{|c|}{$\begin{array}{c}\eta, \text { expt } \\
\mu \mathrm{Pa} \cdot \mathrm{s}\end{array}$} & $\begin{array}{c}\eta_{0}, \text { expt } \\
\mu \mathrm{Pa} \cdot \mathrm{s}\end{array}$ & $\begin{array}{c}\eta_{0}, \quad \text { calc } \\
\mu \mathrm{Pa} \cdot \mathrm{s}\end{array}$ & $\begin{array}{c}\text { Dev } \\
\frac{8}{8}\end{array}$ & wt \\
\hline 308.150 & & & 9.4 & & 9.47 & 9.62 & 1.63 & 0.00 \\
\hline 323.150 & & & 9.8 & & 9.86 & 10.06 & 2.01 & 0.00 \\
\hline 338.150 & & & 10.3 & & 10.33 & 10.49 & 1.51 & 0.00 \\
\hline 350.950 & & & 10.7 & & 10.78 & 10.85 & 0.62 & 0.00 \\
\hline Number of 1 & ints & 59] & 4 & & & & & \\
\hline $\mathrm{AAD}$ 웅 $=$ & 1.44 & BIAS 8 & $=$ & 1.44 & RMS 웅 & 0.51 & & \\
\hline$A A D=$ & 0.14 & BIAS & $=$ & 0.14 & RMS & $0.05 \mu \mathrm{Pa} \cdot \mathrm{s}$ & & \\
\hline
\end{tabular}

Data from De Rocco and Halford [70]

\begin{tabular}{ccccccc}
\hline $\mathrm{T}$ & $\begin{array}{c}\mathrm{P} \\
\mathrm{MPa}\end{array}$ & $\begin{array}{c}\eta \text { expt } \\
\mu \mathrm{Pa} \cdot \mathrm{s}\end{array}$ & $\begin{array}{c}\eta_{0}, \operatorname{expt} \\
\mu \mathrm{Pa} \cdot \mathrm{s}\end{array}$ & $\begin{array}{c}\eta_{0}, \mathrm{calc} \\
\mu \mathrm{Pa} \cdot \mathrm{s}\end{array}$ & $\begin{array}{c}\text { Dev } \\
8\end{array}$ & wt \\
\hline 250.100 & 0.00 & 7.95 & 7.95 & 7.90 & -0.68 & 0.00 \\
273.160 & 0.00 & 8.69 & 8.69 & 8.59 & -1.13 & 0.00 \\
292.700 & 0.00 & 9.30 & 9.30 & 9.17 & -1.38 & 0.00 \\
300.700 & 0.00 & 9.50 & 9.50 & 9.41 & -0.98 & 0.00 \\
308.200 & 0.00 & 9.70 & 9.70 & 9.63 & -0.76 & 0.00 \\
320.700 & 0.00 & 10.10 & 10.10 & 9.99 & -1.11 & 0.00 \\
328.700 & 0.00 & 10.35 & 10.35 & 10.22 & -1.28 & 0.00 \\
337.300 & 0.00 & 10.64 & 10.64 & 10.46 & -1.67 & 0.00 \\
351.100 & 0.00 & 11.01 & 11.01 & 10.85 & -1.44 & 0.00 \\
363.100 & 0.00 & 11.27 & 11.27 & 11.19 & -0.75 & 0.00 \\
373.200 & 0.00 & 11.55 & 11.55 & 11.46 & -0.74 & 0.00 \\
387.900 & 0.00 & 12.04 & 12.04 & 11.87 & -1.44 & 0.00
\end{tabular}


Table 12. ZERO DENSITY VISCOSITY (continued)

Data from De Rocco and Halford [70] (continued)

\begin{tabular}{|c|c|c|c|c|c|c|c|}
\hline $\begin{array}{l}\mathrm{T} \\
\mathrm{K}\end{array}$ & & $\begin{array}{c}\mathrm{P} \\
\mathrm{MPa}\end{array}$ & $\begin{array}{c}\eta, \text { expt } \\
\mu \mathrm{Pa} \cdot \mathrm{s}\end{array}$ & $\begin{array}{c}\eta_{0}, \operatorname{expt} \\
\mu \mathrm{Pa} \cdot \mathrm{s}\end{array}$ & $\begin{array}{c}\eta_{0}, \quad \mathrm{calc} \\
\mu \mathrm{Pa} \cdot \mathrm{s}\end{array}$ & $\begin{array}{c}\text { Dev } \\
\frac{8}{8}\end{array}$ & wt \\
\hline 394.40 & & 0.00 & 12.30 & 12.30 & 12.04 & -2.10 & 0.00 \\
\hline 407.20 & & 0.00 & 12.54 & 12.54 & 12.38 & -1.24 & 0.00 \\
\hline 424.40 & & 0.00 & 12.89 & 12.89 & 12.84 & -0.39 & 0.00 \\
\hline 439.90 & & 0.00 & 13.41 & 13.41 & 13.24 & -1.24 & 0.00 \\
\hline 451.60 & & 0.00 & 13.72 & 13.72 & 13.54 & -1.28 & 0.00 \\
\hline 459.40 & & 0.00 & 13.90 & 13.90 & 13.74 & -1.12 & 0.00 \\
\hline 473.16 & & 0.00 & 14.18 & 14.18 & 14.09 & -0.62 & 0.00 \\
\hline Number of & $=$ Points & $s \quad[70]$ & 19 & & & & \\
\hline$A A D ?$ & $=1.12$ & BIAS $\%$ & $=-1.12$ & RMS 8 \% $=$ & 0.40 & & \\
\hline$A A D$ & $=0.13$ & BIAS & $=-0.13$ & RMS & $0.05 \mu \mathrm{Pa} \cdot \mathrm{s}$ & & \\
\hline
\end{tabular}

Data from Eakin et al. [71]

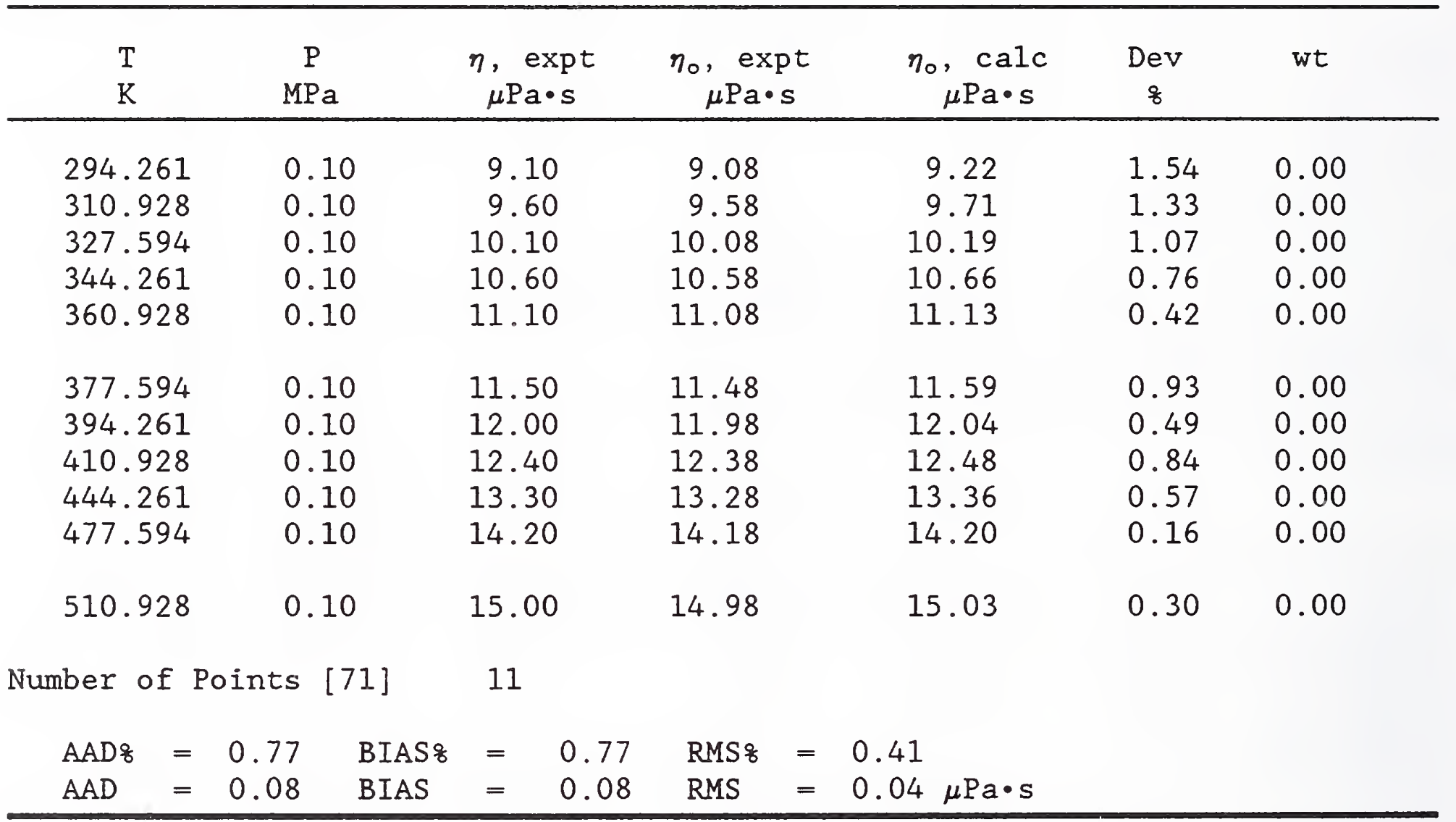


Table 12. ZERO DENSITY VISCOSITY (continued)

Data from Fleeter et al. [72]

\begin{tabular}{|c|c|c|c|c|c|c|}
\hline $\begin{array}{l}\mathrm{T} \\
\mathrm{K}\end{array}$ & $\begin{array}{c}\mathrm{P} \\
\mathrm{MPa}\end{array}$ & $\begin{array}{c}\eta, \text { expt } \\
\mu \mathrm{Pa} \cdot \mathrm{s}\end{array}$ & $\begin{array}{c}\eta_{0}, \operatorname{expt} \\
\mu \mathrm{Pa} \cdot \mathrm{s}\end{array}$ & $\begin{array}{c}\eta_{0}, \quad \mathrm{calc} \\
\mu \mathrm{Pa} \cdot \mathrm{s}\end{array}$ & $\begin{array}{c}\text { Dev } \\
\%\end{array}$ & wt \\
\hline 300.650 & 0.00 & 9.32 & 9.32 & 9.40 & 0.88 & 1.50 \\
\hline
\end{tabular}

Number of Points [72] 1

$$
\begin{aligned}
& \mathrm{AAD} \%=0.88 \quad \mathrm{BIAS} \%=0.88 \quad \mathrm{RMS} \%=0.00 \\
& \mathrm{AAD}=0.08 \quad \mathrm{BIAS}=0.08 \mathrm{RMS}=0.00 \mu \mathrm{Pa} \cdot \mathrm{s}
\end{aligned}
$$

Weighted Data:

Number of Points [72] 1

$$
\begin{aligned}
& \mathrm{AAD} \%=0.88 \quad \text { BIAS } 8=0.88 \quad \mathrm{RMS} \%=0.00 \\
& \mathrm{AAD}=0.08 \mathrm{BIAS}=0.08 \mathrm{RMS}=0.00 \mu \mathrm{Pa} \cdot \mathrm{s}
\end{aligned}
$$

Data from Iwasaki et a1. [73]

\begin{tabular}{ccccccc}
\hline $\mathrm{T}$ & $\mathrm{P}$ & $\begin{array}{c}\eta, \text { expt } \\
\mathrm{KPa} \cdot \mathrm{s}\end{array}$ & $\begin{array}{c}\eta_{0}, \operatorname{expt} \\
\mu \mathrm{Pa} \cdot \mathrm{s}\end{array}$ & $\begin{array}{c}\eta_{0}, \text { calc } \\
\mu \mathrm{Pa} \cdot \mathrm{s}\end{array}$ & $\begin{array}{c}\text { Dev } \\
\text { o }\end{array}$ & wt \\
\hline 298.150 & 0.10 & 9.26 & 9.24 & 9.33 & 1.02 & 0.00 \\
298.150 & 0.10 & 9.26 & 9.24 & 9.33 & 1.02 & 0.00 \\
305.650 & 0.10 & 9.46 & 9.44 & 9.55 & 1.20 & 0.00 \\
305.650 & 0.10 & 9.46 & 9.44 & 9.55 & 1.21 & 0.00 \\
305.850 & 0.10 & 9.46 & 9.44 & 9.56 & 1.26 & 0.00 \\
305.850 & 0.10 & 9.47 & 9.45 & 9.56 & 1.16 & 0.00 \\
306.150 & 0.10 & 9.50 & 9.48 & 9.57 & 0.93 & 0.00 \\
306.150 & 0.10 & 9.46 & 9.44 & 9.57 & 1.36 & 0.00 \\
306.450 & 0.10 & 9.48 & 9.46 & 9.57 & 1.24 & 0.00 \\
306.450 & 0.10 & 9.50 & 9.48 & 9.57 & 1.02 & 0.00 \\
308.150 & 0.10 & 9.54 & 9.52 & 9.62 & 1.12 & 0.00 \\
308.150 & 0.10 & 9.54 & 9.52 & 9.62 & 1.12 & 0.00 \\
323.150 & 0.10 & 9.99 & 9.97 & 10.06 & 0.90 & 0.00 \\
348.150 & 0.10 & 10.69 & 10.67 & 10.77 & 0.94 & 0.00 \\
348.150 & 0.10 & 10.69 & 10.67 & 10.77 & 0.94 & 0.00 \\
348.150 & 0.00 & 10.70 & 10.70 & 10.77 & 0.66 & 0.00
\end{tabular}


Table 12. ZERO DENSITY VISCOSITY (continued)

Data from Iwasaki et al. [73] (continued)

Number of Points [73] 16

$\mathrm{AAD} \%=1.07$ BIAS $\%=1.07$ RMS $\%=0.17$

$\mathrm{AAD}=0.10 \mathrm{BIAS}=0.10 \mathrm{RMS}=0.01 \mu \mathrm{Pa} \cdot \mathrm{s}$

Data from Kestin et al. [74]

\begin{tabular}{|c|c|c|c|c|c|c|}
\hline $\begin{array}{l}\mathrm{T} \\
\mathrm{K}\end{array}$ & $\begin{array}{c}\mathrm{P} \\
\mathrm{MPa}\end{array}$ & $\begin{array}{c}\eta, \text { expt } \\
\mu \mathrm{Pa} \cdot \mathrm{s}\end{array}$ & $\begin{array}{c}\eta_{0}, \operatorname{expt} \\
\mu \mathrm{Pa} \cdot \mathrm{s}\end{array}$ & $\begin{array}{c}\eta_{0}, \quad \text { calc } \\
\mu \mathrm{Pa} \cdot \mathrm{s}\end{array}$ & $\begin{array}{c}\text { Dev } \\
8\end{array}$ & wt \\
\hline 96.350 & 0.00 & 9.19 & 9.17 & 9.28 & 1.20 & 0.00 \\
\hline
\end{tabular}

Number of Points [74] 2

$\begin{array}{ll}\mathrm{AAD} \% & =1.13 \quad \mathrm{BIAS} \%=1.13 \quad \mathrm{RMS} \%=0.07 \\ \mathrm{AAD}=0.10 \quad \mathrm{BIAS}=0.10 \mathrm{RMS}=0.01 \mu \mathrm{Pa} \cdot \mathrm{S}\end{array}$

Weighted Data:

Number of Points [74] 1
$\mathrm{AAD}$ \% $=1.05 \quad$ BIAS 8
1.05 RMS $=0.00$
$\mathrm{AAD}=0.10 \mathrm{BIAS}=0.10 \mathrm{RMS}=0.00 \mu \mathrm{Pa} \cdot \mathrm{s}$

Data from Kestin et al. [75]

\begin{tabular}{cccccrc}
\hline $\mathrm{T}$ & $\begin{array}{c}\eta_{\text {, expt }} \\
\mathrm{MPa} \cdot \mathrm{s}\end{array}$ & $\begin{array}{c}\eta_{0}, \operatorname{expt} \\
\mu \mathrm{Pa} \cdot \mathrm{s}\end{array}$ & $\begin{array}{c}\eta_{0}, \text { calc } \\
\mu \mathrm{Pa} \cdot \mathrm{s}\end{array}$ & $\begin{array}{c}\text { Dev } \\
\text { 8 }\end{array}$ & wt \\
\hline 301.370 & 0.10 & 9.34 & 9.32 & 9.43 & 1.13 & 0.00 \\
334.250 & 0.10 & 10.40 & 10.38 & 10.38 & -0.04 & 1.00 \\
377.430 & 0.10 & 11.68 & 11.66 & 11.58 & -0.71 & 1.00 \\
421.190 & 0.10 & 12.93 & 12.91 & 12.76 & -1.17 & 1.00 \\
476.820 & 0.10 & 14.38 & 14.36 & 14.18 & -1.23 & 1.00
\end{tabular}

Number of Point [75] 5
$\mathrm{AAD} 8=0.86$
BIAS $8=-0.40$
RMS $\%=C .88$
$\mathrm{AAD}=0.10 \quad \mathrm{BIAS}=-0.06$
RMS $=0.10 \mu \mathrm{Pa} \cdot \mathrm{S}$ 
Table 12. ZERO DENSITY VISCOSITY (continued)

Data from Kestin et al. [75] (continued)

Weighted Data:

Number of Points [75] 4

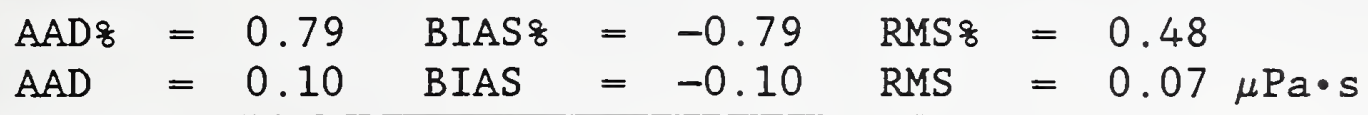

Data from Lambert et a1. [76]

\begin{tabular}{cccccrc}
\hline $\mathrm{T}$ & $\mathrm{P}$ & $\begin{array}{c}\eta \text { expt } \\
\mu \mathrm{Pa} \cdot \mathrm{s}\end{array}$ & $\begin{array}{c}\eta_{0}, \text { expt } \\
\mu \mathrm{Pa} \cdot \mathrm{s}\end{array}$ & $\begin{array}{c}\eta_{0}, \text { calc } \\
\mu \mathrm{Pa} \cdot \mathrm{s}\end{array}$ & $\begin{array}{c}\text { Dev } \\
\text { 8 }\end{array}$ & wt \\
\hline 308.150 & 0.10 & 9.61 & 9.59 & 9.62 & 0.38 & 0.00 \\
323.150 & 0.10 & 10.06 & 10.04 & 10.06 & 0.20 & 0.00 \\
338.150 & 0.10 & 10.53 & 10.51 & 10.49 & -0.21 & 0.00 \\
351.150 & 0.10 & 10.96 & 10.94 & 10.85 & -0.78 & 0.00
\end{tabular}

Number of Points [76] 4
$\mathrm{AAD} \%=0.39$
BIAS \& $=-0.10$
RMS $8=0.45$
$\mathrm{AAD}=0.04$
BIAS $=-0.01$
$\mathrm{RMS}=0.05 \mu \mathrm{Pa} \cdot \mathrm{s}$

Data from Trautz et al. [77]

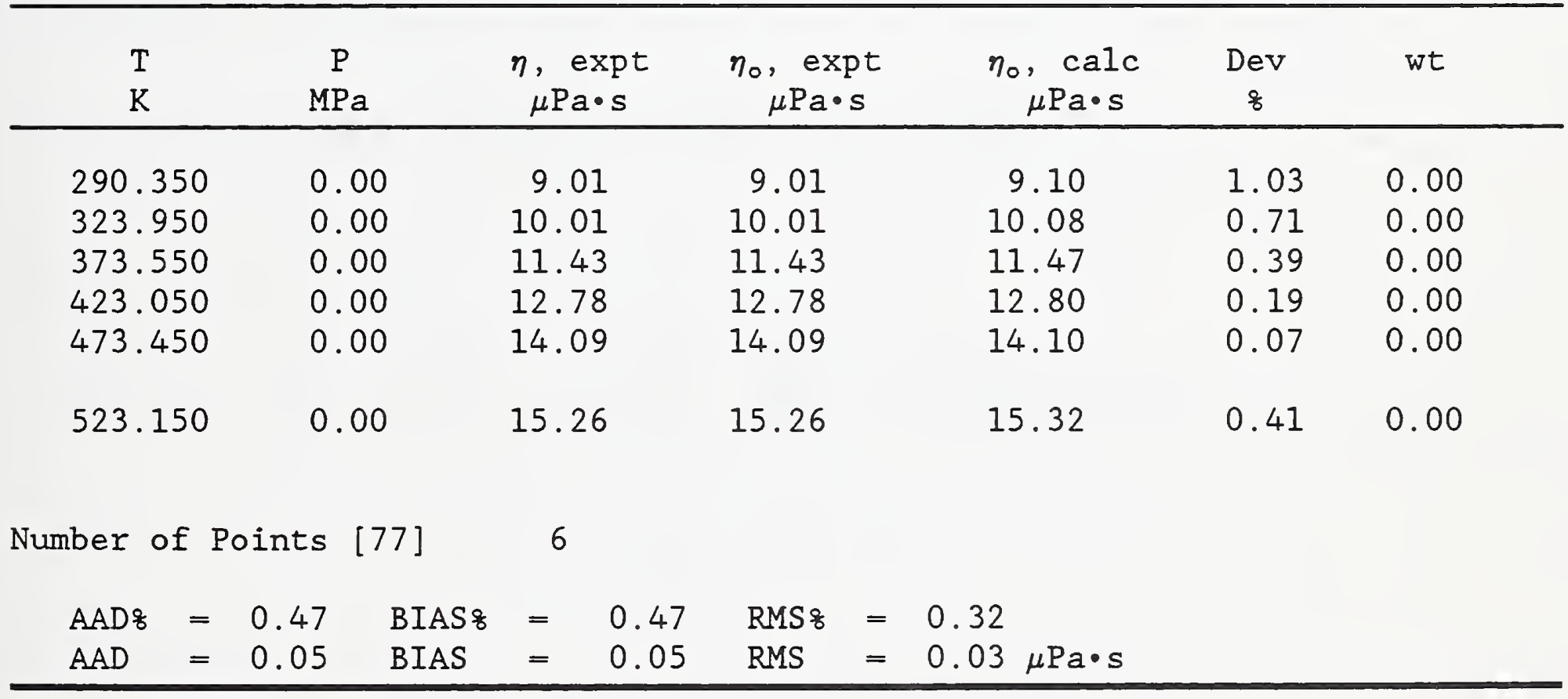


Table 12. ZERO DENSITY VISCOSITY (continued)

Data from Voge1 [78]

\begin{tabular}{cccccrc}
$\mathrm{T}$ & $\mathrm{P}$ & $\begin{array}{c}\eta \text { expt } \\
\mathrm{K}\end{array}$ & $\mathrm{MPa} \cdot \eta_{0}$ expt & $\begin{array}{c}\eta_{0}, \text { calc } \\
\mu \mathrm{Pa} \cdot \mathrm{s}\end{array}$ & $\begin{array}{c}\text { Dev } \\
\text { o }\end{array}$ & wt \\
\hline 194.600 & 0.10 & 6.40 & 6.38 & 6.18 & -3.14 & 0.00 \\
273.100 & 0.10 & 8.55 & 8.53 & 8.59 & 0.72 & 0.00
\end{tabular}

Number of Points [78] 2

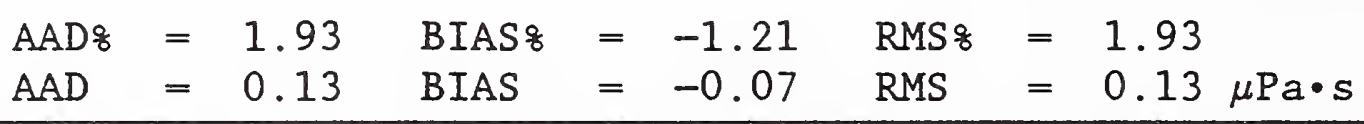

Overall Results: $\mathrm{N}=87$ AAD $8=0.98$ BIAS $8=-0.03$ RMS $8=1.13$

$\mathrm{AAD}=0.10 \quad \mathrm{BIAS}=-0.01 \mathrm{RMS}=0.12 \mu \mathrm{Pa} \cdot \mathrm{s}$

Weighted Data: $\quad \mathrm{N}=6$ AAD $8=0.85$ BIAS $8=-0.20 \quad$ RMS $8=0.92$

$\mathrm{AAD}=0.10 \quad \mathrm{BIAS}=-0.04 \quad \mathrm{RMS}=0.11 \mu \mathrm{Pa} \cdot \mathrm{s}$

Adzumi and Vogel excluded:

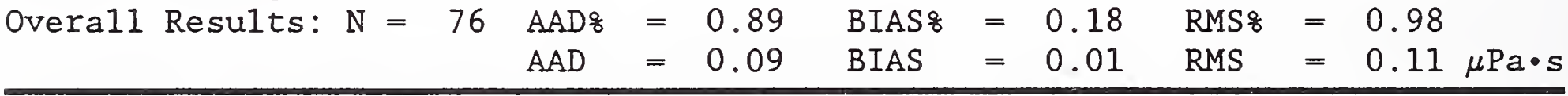


Table 13. ZERO DENSITY THERMAL CONDUCTIVITY

Data from Clifford et al. [79]

\begin{tabular}{|c|c|c|c|c|c|c|c|c|c|}
\hline $\begin{array}{l}\mathrm{T} \\
\mathrm{K}\end{array}$ & $\begin{array}{c}\mathrm{P} \\
\mathrm{MPa}\end{array}$ & $\begin{array}{l}\lambda, \\
\mathrm{mW} \cdot \mathrm{r}\end{array}$ & $\exp _{-1 \cdot 1}$ & & $\begin{array}{l}\lambda_{0}, \operatorname{expt} \\
\mathrm{mW} \cdot \mathrm{m}^{-1} \cdot \mathrm{K}^{-}\end{array}$ & & $\begin{array}{r}\lambda_{0}, \quad \text { calc } \\
\mathrm{mW} \cdot \mathrm{m}^{-1} \cdot \mathrm{K}^{-1}\end{array}$ & $\begin{array}{c}\text { Dev } \\
\&\end{array}$ & wt \\
\hline 303.00 & 0.00 & & .20 & & 22.20 & & 21.51 & -3.095 & 0.0 \\
\hline Number of & Points & 79] & 1 & & & & & & \\
\hline$A A D \&$ & $=3.09$ & BIAS 8 & $=$ & -3.09 & RMS \& & $=$ & 0.00 & & \\
\hline$A A D$ & $=0.69$ & BIAS & $=$ & -0.69 & RMS & $=$ & $0.00 \mathrm{~mW} \cdot \mathrm{m}^{-1}$ & -1 & \\
\hline
\end{tabular}

Data from Craven et al. [69]

\begin{tabular}{|c|c|c|c|c|c|c|c|c|c|}
\hline $\begin{array}{l}\mathrm{T} \\
\mathrm{K}\end{array}$ & $\underset{\mathrm{MPa}}{\mathrm{P}}$ & \multicolumn{3}{|c|}{$\begin{array}{c}\lambda, \operatorname{expt} \\
\mathrm{mW} \cdot \mathrm{m}^{-1} \cdot \mathrm{K}^{-1}\end{array}$} & \multicolumn{2}{|c|}{$\begin{array}{r}\lambda_{0}, \operatorname{expt} \\
\mathrm{mW} \cdot \mathrm{m}^{-1} \cdot \mathrm{K}^{-1} \\
\end{array}$} & $\begin{array}{r}\lambda_{0}, \text { calc } \\
\mathrm{mW} \cdot \mathrm{m}^{-1} \cdot \mathrm{K}^{-1}\end{array}$ & $\begin{array}{c}\text { Dev } \\
8\end{array}$ & wt \\
\hline 339.15 & 0.00 & \multicolumn{3}{|c|}{27.20} & \multicolumn{2}{|l|}{27.20} & 26.48 & -2.657 & 0.0 \\
\hline Number of & Points & \multicolumn{3}{|c|}{1} & & & & & \\
\hline $\mathrm{AAD} \%$ & $=2.66$ & BIAS 8 & $=$ & -2.66 & RMS 8 & $=$ & \multicolumn{2}{|l|}{0.00} & \\
\hline $\mathrm{AAD}$ & $=0.72$ & BIAS & $=$ & -0.72 & RMS & $=$ & \multicolumn{2}{|c|}{$0.00 \mathrm{~mW} \cdot \mathrm{m}^{-1} \cdot \mathrm{K}^{-1}$} & \\
\hline
\end{tabular}

Data from Fleeter et al. [72]

\begin{tabular}{|c|c|c|c|c|c|c|c|c|c|}
\hline $\begin{array}{l}\mathrm{T} \\
\mathrm{K}\end{array}$ & $\begin{array}{c}\mathrm{P} \\
\mathrm{MPa}\end{array}$ & \multicolumn{3}{|c|}{$\begin{array}{c}\lambda, \operatorname{expt} \\
\mathrm{mW} \cdot \mathrm{m}^{-1} \cdot \mathrm{K}^{-1}\end{array}$} & \multicolumn{2}{|c|}{$\begin{array}{r}\lambda_{0}, \operatorname{expt} \\
\mathrm{mW} \cdot \mathrm{m}^{-1} \cdot \mathrm{K}^{-1} \\
\end{array}$} & $\begin{array}{c}\lambda_{0}, \quad \operatorname{calc} \\
\mathrm{mW} \cdot \mathrm{m}^{-1} \cdot \mathrm{K}^{-1}\end{array}$ & $\begin{array}{c}\text { Dev } \\
\frac{8}{8}\end{array}$ & wt \\
\hline 300.65 & 0.00 & & .92 & & 21.92 & & 21.21 & -3.242 & 0.0 \\
\hline Number of & E Points & 72] & 1 & & & & & & \\
\hline$A A D \&$ & $=3.24$ & BIAS 8 & $=$ & -3.24 & RMS क & $=$ & 0.00 & & \\
\hline$A A D$ & $=0.71$ & BIAS & $=$ & -0.71 & RMS & $=$ & $0.00 \mathrm{~mW} \cdot \mathrm{m}^{-1}$ & $\mathrm{~K}^{-1}$ & \\
\hline
\end{tabular}


Data from Gilmore et al. [80]

\begin{tabular}{|c|c|c|c|c|c|c|c|c|c|}
\hline $\begin{array}{l}\mathrm{T} \\
\mathrm{K}\end{array}$ & $\begin{array}{c}\mathrm{P} \\
\mathrm{MPa}\end{array}$ & \multicolumn{2}{|c|}{$\begin{array}{c}\lambda, \underset{\mathrm{mW}}{\operatorname{expt} \mathrm{m}^{-1} \cdot \mathrm{K}^{-1}}\end{array}$} & \multicolumn{3}{|c|}{$\begin{array}{r}\lambda_{0}, \operatorname{expt} \\
\mathrm{mW} \cdot \mathrm{m}^{-1} \cdot \mathrm{K}^{-1}\end{array}$} & $\begin{array}{r}\lambda_{0}, \quad \text { calc } \\
\mathrm{mW} \cdot \mathrm{m}^{-1} \cdot \mathrm{K}^{-1}\end{array}$ & $\begin{array}{c}\text { Dev } \\
\frac{8}{8}\end{array}$ & wt \\
\hline \multirow{2}{*}{$\begin{array}{l}348.15 \\
348.15\end{array}$} & 0.10 & \multicolumn{2}{|c|}{27.57} & \multicolumn{3}{|c|}{27.51} & 27.80 & 1.051 & 0.0 \\
\hline & 0.10 & \multicolumn{2}{|c|}{27.74} & \multicolumn{3}{|c|}{27.67} & 27.80 & 0.440 & 0.0 \\
\hline \multicolumn{4}{|c|}{ Number of Points [80] } & \multicolumn{3}{|c|}{2} & & & \\
\hline $\mathrm{AAD} ;$ & 0.75 & BIAS \% & $=$ & 0.75 & RMS \% & $=$ & 0.31 & & \\
\hline $\mathrm{AAD}$ & $=0.21$ & BIAS & $=$ & 0.21 & RMS & $=$ & $0.08 \mathrm{~mW} \cdot \mathrm{m}^{-1}$ & & \\
\hline
\end{tabular}

Data from Keyes [81]

\begin{tabular}{ccccccc}
\hline $\mathrm{T}$ & $\begin{array}{c}\mathrm{P} \\
\mathrm{MPa}\end{array}$ & $\begin{array}{c}\lambda, \operatorname{expt} \\
\mathrm{mW} \cdot \mathrm{m}^{-1} \cdot \mathrm{K}^{-1}\end{array}$ & $\begin{array}{c}\lambda_{0}, \operatorname{expt} \\
\mathrm{mW} \cdot \mathrm{m}^{-1} \cdot \mathrm{K}^{-1}\end{array}$ & $\begin{array}{c}\lambda_{0}, \mathrm{calc} \\
\mathrm{mW} \cdot \mathrm{m}^{-1} \cdot \mathrm{K}^{-1}\end{array}$ & $\begin{array}{c}\text { Dev } \\
\text { of }\end{array}$ & wt \\
\hline 522.35 & 0.10 & 58.12 & 58.09 & 58.04 & -0.079 & 0.0 \\
325.05 & 0.00 & 25.06 & 25.06 & 24.48 & -2.337 & 0.0
\end{tabular}

Number of Points [81]

2

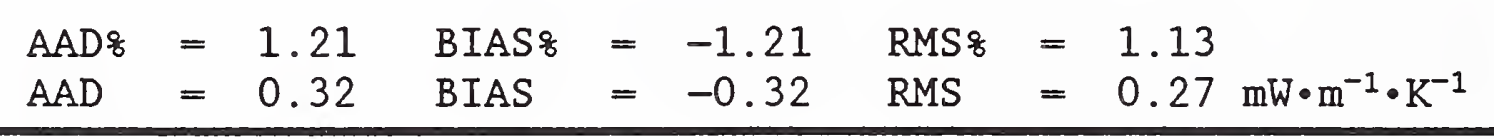

Data from Le Neindre et a1. [82]

\begin{tabular}{|c|c|c|c|c|c|c|c|c|c|}
\hline $\begin{array}{l}\mathrm{T} \\
\mathrm{K}\end{array}$ & $\begin{array}{c}\mathrm{P} \\
\mathrm{MPa}\end{array}$ & \multicolumn{2}{|c|}{$\begin{array}{c}\lambda, \underset{\mathrm{mW} \cdot \mathrm{m}^{-1} \cdot \mathrm{K}^{-1}}{\operatorname{expt}}\end{array}$} & \multicolumn{3}{|c|}{$\begin{array}{r}\lambda_{\circ}, \quad \operatorname{expt} \\
\mathrm{mW} \cdot \mathrm{m}^{-1} \cdot \mathrm{K}^{-1}\end{array}$} & $\begin{array}{r}\lambda_{0}, \quad \text { calc } \\
\mathrm{mW} \cdot \mathrm{m}^{-1} \cdot \mathrm{K}^{-1}\end{array}$ & $\begin{array}{c}\text { Dev } \\
\text { \& }\end{array}$ & wt \\
\hline 308.15 & 0.10 & \multicolumn{2}{|c|}{22.60} & \multicolumn{3}{|c|}{22.52} & 22.19 & -1.463 & 0.0 \\
\hline 343.15 & 0.10 & \multicolumn{2}{|c|}{27.40} & \multicolumn{3}{|c|}{27.33} & 27.06 & -1.002 & 0.0 \\
\hline 406.15 & 0.10 & \multicolumn{2}{|c|}{36.80} & \multicolumn{3}{|c|}{36.75} & 36.98 & 0.615 & 0.0 \\
\hline 483.15 & 0.10 & \multicolumn{2}{|c|}{50.60} & \multicolumn{3}{|c|}{50.56} & 50.63 & 0.126 & 0.0 \\
\hline 571.15 & 0.10 & \multicolumn{2}{|c|}{65.80} & \multicolumn{3}{|c|}{65.77} & 67.58 & 2.744 & 0.0 \\
\hline 649.15 & 0.10 & \multicolumn{2}{|c|}{80.70} & \multicolumn{3}{|c|}{80.68} & 83.36 & 3.323 & 0.0 \\
\hline 724.15 & 0.10 & \multicolumn{2}{|c|}{94.40} & \multicolumn{3}{|c|}{94.38} & 98.98 & 4.873 & 0.0 \\
\hline \multicolumn{4}{|c|}{ Number of Points [82] } & \multicolumn{3}{|c|}{7} & & & \\
\hline $\mathrm{AAD} ;$ & $=2.02$ & BIAS 8 & $=$ & 1.32 & RMS 웅 & $=$ & 2.19 & & \\
\hline $\mathrm{AAD}$ & $=1.43$ & BIAS & $=$ & 1.25 & RMS & $=$ & $1.73 \mathrm{~mW} \cdot \mathrm{m}$ & -1 & \\
\hline
\end{tabular}


Table 13. ZERO DENSITY THERMAL CONDUCTIVITY (continued)

Data from Leng et a1. [83]

\begin{tabular}{|c|c|c|c|c|c|c|c|c|c|}
\hline $\begin{array}{l}\mathrm{T} \\
\mathrm{K}\end{array}$ & $\begin{array}{c}\mathrm{P} \\
\mathrm{MPa}\end{array}$ & $\begin{array}{c}\lambda, \\
\mathrm{mW} \cdot 1\end{array}$ & $\begin{array}{l}\exp t \\
-1 \cdot \mathrm{K}\end{array}$ & & $\begin{array}{r}\lambda_{0}, \quad \operatorname{expt} \\
\mathrm{nW} \cdot \mathrm{m}^{-1} \cdot \mathrm{K}^{-}\end{array}$ & & $\begin{array}{r}\lambda_{0}, \quad c a l c \\
\mathrm{~mW} \cdot \mathrm{m}^{-1} \cdot \mathrm{K}^{-1}\end{array}$ & $\begin{array}{c}\text { Dev } \\
q\end{array}$ & wt \\
\hline 340.93 & 0.10 & & .52 & & 27.45 & & 26.74 & -2.600 & 0.0 \\
\hline Number of & oints & 33 ] & & & 1 & & & & \\
\hline $\mathrm{AAD} \&=$ & 2.60 & BIAS 8 & $=$ & -2.60 & RMS 앙 & $=$ & 0.00 & & \\
\hline $\mathrm{AAD}$ & 0.71 & BIAS & $=$ & -0.71 & RMS & $=$ & $0.00 \mathrm{~mW} \cdot \mathrm{m}^{-1}$ & $r^{-1}$ & \\
\hline
\end{tabular}

Data from Lenoir et al. [84]

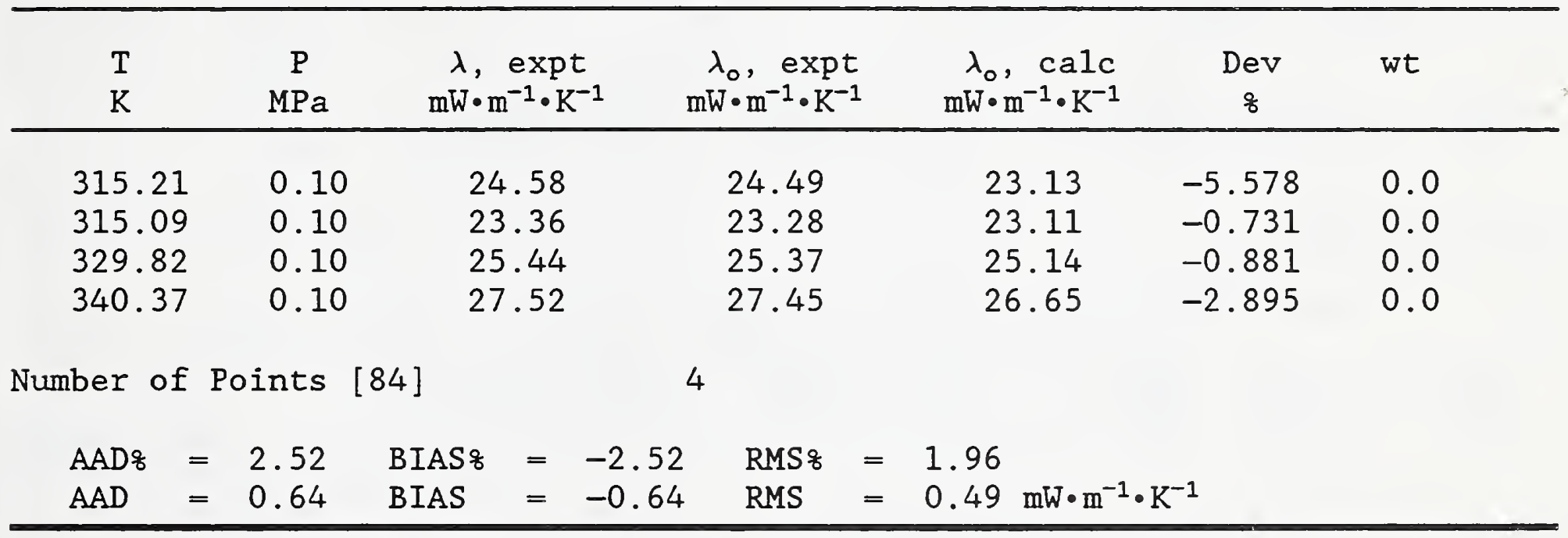

Data from Millat et al. [85]

\begin{tabular}{|c|c|c|c|c|c|c|c|c|c|}
\hline $\begin{array}{l}\mathrm{T} \\
\mathrm{K}\end{array}$ & $\begin{array}{c}\mathrm{P} \\
\mathrm{MPa}\end{array}$ & \multicolumn{3}{|c|}{$\begin{array}{c}\lambda, \quad \operatorname{expt} \\
\mathrm{mW} \cdot \mathrm{m}^{-1} \cdot \mathrm{K}^{-1}\end{array}$} & \multicolumn{2}{|c|}{$\begin{array}{r}\lambda_{0}, \quad \operatorname{expt} \\
\mathrm{mW} \cdot \mathrm{m}^{-1} \cdot \mathrm{K}^{-1}\end{array}$} & $\begin{array}{r}\lambda_{0}, \quad \text { calc } \\
\mathrm{mW} \cdot \mathrm{m}^{-1} \cdot \mathrm{K}^{-1}\end{array}$ & $\begin{array}{l}\text { Dev } \\
8\end{array}$ & wt \\
\hline 308.15 & 0.00 & \multicolumn{3}{|c|}{22.87} & 22.87 & & 22.19 & -2.988 & 0.0 \\
\hline 331.65 & 0.00 & \multicolumn{3}{|c|}{26.14} & 26.14 & & 25.40 & -2.819 & 0.0 \\
\hline 380.15 & 0.00 & \multicolumn{3}{|c|}{34.01} & 34.01 & & 32.72 & -3.784 & 0.0 \\
\hline 425.65 & 0.00 & \multicolumn{3}{|c|}{42.29} & 42.29 & & 40.30 & -4.708 & 0.0 \\
\hline Number of & E Points & 85 ] & 4 & & & & & & \\
\hline $\mathrm{AAD}$ 웅 & $=3.57$ & BIAS 8 & $=-3$ & 3.57 & RMS क्ष & $=$ & 0.75 & & \\
\hline $\mathrm{AAD}$ & $=1.17$ & BIAS & $=-1$ & 1.17 & RMS & $=$ & $0.53 \mathrm{~mW} \cdot \mathrm{m}^{-1}$ & $x^{-1}$ & \\
\hline
\end{tabular}


Table 13. ZERO DENSITY THERMAL CONDUCTIVITY (continued)

Data from Prasad et al. [86]

\begin{tabular}{ccccccc}
$\mathrm{T}$ & $\begin{array}{c}\mathrm{P} \\
\mathrm{K}\end{array}$ & $\begin{array}{c}\lambda, \operatorname{expt} \\
\mathrm{mW} \cdot \mathrm{m}^{-1} \cdot \mathrm{K}^{-1}\end{array}$ & $\begin{array}{c}\lambda_{0}, \operatorname{expt} \\
\mathrm{mW} \cdot \mathrm{m}^{-1} \cdot \mathrm{K}^{-1}\end{array}$ & $\begin{array}{c}\lambda_{0}, \mathrm{calc} \\
\mathrm{mW} \cdot \mathrm{m}^{-1} \cdot \mathrm{K}^{-1}\end{array}$ & $\begin{array}{c}\text { Dev } \\
8\end{array}$ & wt \\
\hline 295.00 & 0.10 & 20.28 & 20.19 & 20.49 & 1.498 & 0.0 \\
315.00 & 0.10 & 22.97 & 22.89 & 23.10 & 0.923 & 1.0 \\
350.00 & 0.10 & 27.94 & 27.88 & 28.07 & 0.700 & 1.0 \\
400.00 & 0.10 & 35.74 & 35.69 & 35.95 & 0.737 & 1.0 \\
498.00 & 0.10 & 53.53 & 53.50 & 53.41 & -0.171 & 1.0 \\
& & & & & & \\
600.00 & 0.10 & 73.91 & 73.89 & 73.35 & -0.727 & 1.0
\end{tabular}

Number of Points [86] 6

$\mathrm{AAD} 8=0.79 \quad \mathrm{BIAS} 8=0.49 \mathrm{RMS} z=0.73$
$\mathrm{AAD}=0.27$ BIAS $=0.06 \mathrm{RMS}=0.29 \mathrm{~mW} \cdot \mathrm{m}^{-1} \cdot \mathrm{K}^{-1}$

Weighted Data:

Number of Points [86] 5

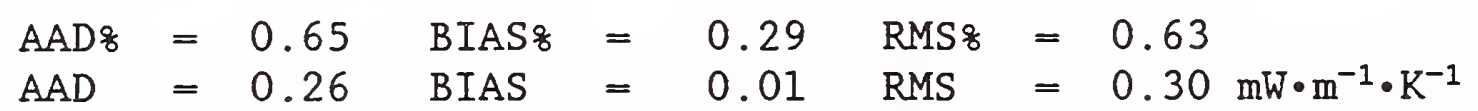

Original adjustment to zero pressure:

Number of Points [86] 6

$$
\begin{aligned}
& \mathrm{AAD}=0.91 \quad \mathrm{BIAS} 8=0.60 \mathrm{RMS} z=0.84 \\
& \mathrm{AAD}=0.30 \mathrm{BIAS}=0.08 \mathrm{RMS}=0.32 \mathrm{~mW} \cdot \mathrm{m}^{-1} \cdot \mathrm{K}^{-1}
\end{aligned}
$$

Weighted Data:

Number of Points [86] 5

$$
\begin{aligned}
& \mathrm{AAD}=0.73 \quad \text { BIAS } 8=0.36 \quad \mathrm{RMS} 8=0.71 \\
& \mathrm{AAD}=0.28 \text { BIAS }=0.02 \mathrm{RMS}=0.32 \mathrm{~mW} \cdot \mathrm{m}^{-1} \cdot \mathrm{K}^{-1}
\end{aligned}
$$


Table 13. ZERO DENSITY THERMAL CONDUCTIVITY (continued)

Data from Roder et a1. [87]

\begin{tabular}{|c|c|c|c|c|c|c|c|c|c|}
\hline $\begin{array}{l}\mathrm{T} \\
\mathrm{K}\end{array}$ & $\begin{array}{c}\mathrm{P} \\
\mathrm{MPa}\end{array}$ & \multicolumn{2}{|c|}{$\begin{array}{c}\lambda, \underset{\exp t}{\mathrm{~mW} \cdot \mathrm{m}^{-1} \cdot \mathrm{K}^{-1}}\end{array}$} & \multicolumn{2}{|r|}{$\begin{array}{r}\lambda_{0}, \operatorname{expt} \\
\mathrm{mW} \cdot \mathrm{m}^{-1} \cdot \mathrm{K}^{-1}\end{array}$} & \multicolumn{2}{|r|}{$\begin{array}{r}\lambda_{0}, \quad \text { calc } \\
\mathrm{mW} \cdot \mathrm{m}^{-1} \cdot \mathrm{K}^{-1}\end{array}$} & $\begin{array}{c}\text { Dev } \\
z\end{array}$ & wt \\
\hline 225.00 & 0.00 & \multicolumn{2}{|c|}{13.12} & \multicolumn{2}{|r|}{13.12} & \multicolumn{2}{|r|}{12.75} & -2.839 & 0.0 \\
\hline 235.00 & 0.00 & \multicolumn{2}{|c|}{14.00} & \multicolumn{2}{|r|}{14.00} & & 13.72 & -1.986 & 0.0 \\
\hline 245.00 & 0.00 & \multicolumn{2}{|c|}{14.94} & \multicolumn{3}{|c|}{14.94} & 14.74 & -1.345 & 1.0 \\
\hline 255.00 & 0.00 & \multicolumn{2}{|c|}{15.90} & \multicolumn{3}{|c|}{15.90} & 15.80 & -0.630 & 1.0 \\
\hline 265.00 & 0.00 & \multicolumn{2}{|c|}{17.08} & \multicolumn{3}{|c|}{17.08} & 16.90 & -1.025 & 1.0 \\
\hline 275.00 & 0.00 & \multicolumn{2}{|c|}{18.14} & \multicolumn{3}{|c|}{18.14} & 18.05 & -0.469 & 1.0 \\
\hline 285.00 & 0.00 & \multicolumn{2}{|c|}{19.34} & \multicolumn{2}{|r|}{19.34} & & 19.25 & -0.466 & 1.0 \\
\hline 295.00 & 0.00 & \multicolumn{2}{|c|}{20.57} & & 20.57 & & 20.49 & -0.391 & 1.0 \\
\hline 305.00 & 0.00 & \multicolumn{2}{|c|}{22.03} & \multicolumn{3}{|c|}{22.03} & 21.77 & -1.166 & 0.0 \\
\hline 312.00 & 0.00 & \multicolumn{2}{|c|}{22.59} & \multicolumn{3}{|c|}{22.59} & 22.70 & 0.476 & 1.0 \\
\hline 325.00 & 0.00 & \multicolumn{2}{|c|}{24.72} & \multirow{2}{*}{\multicolumn{3}{|c|}{24.72}} & 24.47 & -1.013 & 0.0 \\
\hline & & & 11 & & & & & & \\
\hline & & & & & & & & & \\
\hline AAD & $\begin{array}{l}=1.0 \% \\
=0.18\end{array}$ & $\begin{array}{l}\text { BIAS? } \\
\text { BIAS }\end{array}$ & $\begin{array}{l}= \\
=\end{array}$ & $\begin{array}{l}-0.99 \\
-0.16\end{array}$ & $\begin{array}{l}\text { RMS क } \\
\text { RMS }\end{array}$ & $\begin{array}{l}= \\
=\end{array}$ & $\begin{array}{l}0.84 \\
0.12 \mathrm{~mW} \cdot \mathrm{m}^{-1}\end{array}$ & $\cdot \mathrm{K}^{-1}$ & \\
\hline Weighted & Data: & & & & & & & & \\
\hline Number of & Points & [87] & 7 & & & & & & \\
\hline $\begin{array}{l}\mathrm{AAD} \% \\
\mathrm{AAD}\end{array}$ & $=0.69$ & BIAS \% & $\begin{array}{l}= \\
=\end{array}$ & $\begin{array}{l}-0.55 \\
-0.09\end{array}$ & $\begin{array}{l}\text { RMS \% } \\
\text { RMS }\end{array}$ & $\begin{array}{l}= \\
=\end{array}$ & $\begin{array}{l}0.53 \\
0.09 \mathrm{~mW} \cdot \mathrm{m}^{-1}\end{array}$ & $\cdot \mathrm{K}^{-1}$ & \\
\hline $\mathrm{AAD}$ & $=0.12$ & BIAS & $=$ & & & & & & \\
\hline
\end{tabular}


Table 13. ZERO DENSITY THERMAL CONDUCTIVITY (continued)

Data from Tufeu et al. [88]

\begin{tabular}{|c|c|c|c|c|c|c|c|c|c|}
\hline $\begin{array}{l}\mathrm{T} \\
\mathrm{K}\end{array}$ & $\begin{array}{c}\mathrm{P} \\
\mathrm{MPa}\end{array}$ & \multicolumn{3}{|c|}{$\begin{array}{c}\lambda, \quad \operatorname{expt} \\
\mathrm{mW} \cdot \mathrm{m}^{-1} \cdot \mathrm{K}^{-1}\end{array}$} & \multicolumn{2}{|c|}{$\begin{array}{r}\lambda_{0}, \quad \operatorname{expt} \\
\mathrm{mW} \cdot \mathrm{m}^{-1} \cdot \mathrm{K}^{-1}\end{array}$} & $\begin{array}{r}\lambda_{0}, \quad \text { calc } \\
\mathrm{mW} \cdot \mathrm{m}^{-1} \cdot \mathrm{K}^{-1}\end{array}$ & $\begin{array}{c}\text { Dev } \\
\text { \& }\end{array}$ & wt \\
\hline 307.05 & 0.00 & \multicolumn{3}{|c|}{22.40} & 22.40 & & 22.04 & -1.600 & 0.0 \\
\hline 307.80 & 0.00 & \multicolumn{3}{|c|}{22.50} & 22.50 & & 22.14 & -1.598 & 0.0 \\
\hline 311.80 & 0.00 & \multicolumn{3}{|c|}{23.00} & 23.00 & & 22.67 & -1.431 & 0.0 \\
\hline 313.35 & 0.00 & \multicolumn{3}{|c|}{23.20} & 23.20 & & 22.88 & -1.387 & 0.0 \\
\hline 322.75 & 0.00 & \multicolumn{3}{|c|}{24.40} & 24.40 & & 24.16 & -0.993 & 0.0 \\
\hline 434.15 & 0.00 & \multicolumn{3}{|c|}{41.20} & 41.20 & & 41.78 & 1.403 & 0.0 \\
\hline 500.30 & 0.00 & \multicolumn{3}{|c|}{52.00} & 52.00 & & 53.84 & 3.537 & 0.0 \\
\hline Number of & Points & 88] & 7 & & & & & & \\
\hline $\mathrm{AAD} \&$ & 1.71 & BIAS $q$ & $=$ & -0.30 & RMS $\frac{\gamma}{\gamma}$ & $=$ & 1.85 & & \\
\hline$A A D$ & $=0.58$ & BIAS & $=$ & 0.12 & RMS & $=$ & $0.77 \mathrm{~mW} \cdot \mathrm{m}^{-1}$ & -1 & \\
\hline
\end{tabular}

Data from Yakush et al. [89]

\begin{tabular}{|c|c|c|c|c|c|c|c|c|c|}
\hline $\begin{array}{l}\mathrm{T} \\
\mathrm{K}\end{array}$ & $\begin{array}{c}\mathrm{P} \\
\mathrm{MPa}\end{array}$ & \multicolumn{2}{|c|}{$\begin{array}{c}\lambda, \operatorname{expt} \\
\mathrm{mW} \cdot \mathrm{m}^{-1} \cdot \mathrm{K}^{-1}\end{array}$} & \multicolumn{3}{|c|}{$\begin{array}{r}\lambda_{0}, \quad \operatorname{expt} \\
\mathrm{mW} \cdot \mathrm{m}^{-1} \cdot \mathrm{K}^{-1}\end{array}$} & $\begin{array}{r}\lambda_{0}, \quad \text { calc } \\
\mathrm{mW} \cdot \mathrm{m}^{-1} \cdot \mathrm{K}^{-1}\end{array}$ & $\begin{array}{c}\text { Dev } \\
8\end{array}$ & wt \\
\hline 318.80 & 0.00 & \multicolumn{2}{|c|}{24.90} & \multicolumn{3}{|c|}{24.90} & 23.62 & -5.158 & 0.0 \\
\hline 327.54 & 0.00 & \multicolumn{2}{|c|}{25.80} & & 25.80 & & 24.82 & -3.783 & 0.0 \\
\hline 344.24 & 0.00 & \multicolumn{2}{|c|}{27.50} & & 27.50 & & 27.22 & -1.023 & 0.0 \\
\hline 379.60 & 0.00 & \multicolumn{2}{|c|}{32.20} & & 32.20 & & 32.64 & 1.352 & 0.0 \\
\hline 462.40 & 0.00 & \multicolumn{2}{|c|}{46.00} & & 46.00 & & 46.82 & 1.778 & 0.0 \\
\hline 580.50 & 0.00 & \multicolumn{2}{|c|}{67.20} & & & & 69.44 & 3.332 & 0.0 \\
\hline 590.20 & 0.00 & \multicolumn{2}{|c|}{67.50} & \multicolumn{3}{|c|}{67.50} & 71.38 & 5.748 & 0.0 \\
\hline Number of & Points & $89]$ & 7 & & & & & & \\
\hline $\mathrm{AAD} ;$ & $=3.17$ & BIAS \& & $=$ & 0.32 & RMS 8 & $=$ & 3.59 & & \\
\hline $\mathrm{AAD}$ & $=1.42$ & BIAS & $=$ & 0.69 & $\mathrm{RMS}$ & $=$ & $1.70 \mathrm{~mW} \cdot \mathrm{m}^{-1}$ & 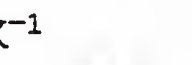 & \\
\hline
\end{tabular}


Table 13. ZERO DENSITY THERMAL CONDUCTIVITY (continued)

Overall Results:

$$
\begin{aligned}
& \mathrm{N}=54 \quad \mathrm{AAD} \%=1.94 \quad \mathrm{BIAS} \%=-0.66 \quad \mathrm{RMS} \%=2.34 \\
& \mathrm{AAD}=0.72 \text { BIAS }=0.05 \text { RMS }=1.16 \mathrm{~mW} \cdot \mathrm{m}^{-1} \cdot \mathrm{K}^{-1}
\end{aligned}
$$

Overall Results (excluding Gilmore, Keyes, Leng, Lenoir):

$$
\begin{aligned}
& \mathrm{N}=45 \quad \mathrm{AAD} \%=1.96 \quad \text { BIAS\% } 8=-0.48 \quad \mathrm{RMS} \%=2.38 \\
& \mathrm{AAD}=0.76 \quad \text { BIAS }=0.14 \text { RMS }=1.23 \mathrm{~mW} \cdot \mathrm{m}^{-1} \cdot \mathrm{K}^{-1}
\end{aligned}
$$

Weighted Data:

$$
\begin{aligned}
& \mathrm{N}=12 \quad \mathrm{AAD} \%=0.67 \quad \text { BIAS\& }=-0.20 \quad \mathrm{RMS} \%=0.71 \\
& \mathrm{AAD}=0.18 \quad \text { BIAS }=-0.05 \quad \mathrm{RMS}=0.21 \mathrm{~mW} \cdot \mathrm{m}^{-1} \cdot \mathrm{K}^{-1}
\end{aligned}
$$


6. Transport Properties at Elevated Pressures

Table 14 gives comparisons and weights for the viscosity at elevated pressures, and table 15 is for the thermal conductivity at elevated pressures. Unless otherwise noted, the entries given under the density column are values calculated from our equation of state and the experimentally measured temperatures and pressures. The calculated values of the transport properties represent evaluations of our correlating equations also at the experimental temperature and pressure-that is, using the densities given in these tables. The exceptions occur when the experimental pressures were not available and only densities were tabulated in the experimental papers. The percentage deviations given in the tables correspond to these calculations based on experimental pressures.

For each experimental source, we have also given summary statistics based on calculation of the transport property using the densities tabulated in the source papers. These statistics are omitted, and an indicative note is given, when tabulated densities were not given in the experimental paper. Except in the critical region, the deviations and the statistics based on experimental pressures and densities are quite similar.

Data corresponding to pressures at or below $0.1 \mathrm{MPa}$ are included in Sec. 5 and are excluded from the tables and statistics of this section. (A few exceptions occur when low density data were weighted in the determination of the coefficients of the transport correlations.) Again we have included overall statistics which correspond to the data selection of [1]. 
Table 14. VISCOSITY AT ELEVATED PRESSURES

Data from Baron et a1. [90]

\begin{tabular}{|c|c|c|c|c|c|c|}
\hline $\begin{array}{l}\mathrm{T} \\
\mathrm{K}\end{array}$ & $\begin{array}{c}\mathrm{P} \\
\mathrm{MPa}\end{array}$ & $\stackrel{\rho}{\mathrm{mol} \cdot \mathrm{dm}^{-3}}$ & $\begin{array}{c}\eta, \text { calc } \\
\mu \mathrm{Pa} \cdot \mathrm{s}\end{array}$ & $\begin{array}{c}\eta, \quad \operatorname{expt} \\
\mu \mathrm{Pa} \cdot \mathrm{s}\end{array}$ & $\begin{array}{c}\text { Dev } \\
\frac{8}{8}\end{array}$ & wt \\
\hline 324.817 & 0.690 & 0.266 & 10.271 & 10.000 & 2.714 & 0.000 \\
\hline 324.817 & 3.447 & 1.659 & 11.548 & 11.200 & 3.103 & 0.000 \\
\hline 324.817 & 6.895 & 6.779 & 22.168 & 22.800 & -2.773 & 0.000 \\
\hline 324.817 & 13.790 & 11.685 & 45.554 & 45.200 & 0.784 & 0.000 \\
\hline 324.817 & 20.684 & 12.865 & 55.334 & 54.700 & 1.159 & 0.000 \\
\hline 324.817 & 27.579 & 13.605 & 62.993 & 62.000 & 1.602 & 0.000 \\
\hline 324.817 & 34.474 & 14.157 & 69.744 & 67.800 & 2.867 & 0.000 \\
\hline 324.817 & 41.368 & 14.604 & 75.997 & 73.000 & 4.105 & 0.000 \\
\hline 324.817 & 48.263 & 14.981 & 81.946 & 78.000 & 5.060 & 0.000 \\
\hline 324.817 & 55.158 & 15.309 & 87.703 & 82.200 & 6.695 & 0.000 \\
\hline 352.594 & 0.690 & 0.243 & 11.053 & 10.800 & 2.341 & 0.000 \\
\hline 352.594 & 3.447 & 1.409 & 12.106 & 11.800 & 2.593 & 0.000 \\
\hline 352.594 & 6.895 & 3.697 & 15.507 & 15.600 & -0.595 & 0.000 \\
\hline 352.594 & 13.790 & 9.491 & 33.479 & 34.600 & -3.240 & 0.000 \\
\hline 352.594 & 20.684 & 11.413 & 44.527 & 45.400 & -1.923 & 0.000 \\
\hline 352.594 & 27.579 & 12.433 & 52.416 & 53.300 & -1.658 & 0.000 \\
\hline 352.594 & 34.474 & 13.140 & 59.080 & 59.800 & -1.203 & 0.000 \\
\hline 352.594 & 41.368 & 13.687 & 65.110 & 65.400 & -0.443 & 0.000 \\
\hline 352.594 & 48.263 & 14.137 & 70.764 & 70.400 & 0.518 & 0.000 \\
\hline 352.594 & 55.158 & 14.521 & 76.179 & 74.700 & 1.980 & 0.000 \\
\hline 380.372 & 0.690 & 0.223 & 11.815 & 11.700 & 0.984 & 0.000 \\
\hline 380.372 & 3.447 & 1.244 & 12.735 & 12.600 & 1.074 & 0.000 \\
\hline 380.372 & 6.895 & 2.922 & 15.002 & 15.100 & -0.646 & 0.000 \\
\hline 380.372 & 13.790 & 7.361 & 25.789 & 26.000 & -0.810 & 0.000 \\
\hline 380.372 & 20.684 & 9.918 & 36.494 & 37.200 & -1.898 & 0.000 \\
\hline 380.372 & 27.579 & 11.251 & 44.373 & 45.200 & -1.829 & 0.000 \\
\hline 380.372 & 34.474 & 12.128 & 50.889 & 51.900 & -1.948 & 0.000 \\
\hline 380.372 & 41.368 & 12.784 & 56.690 & 57.400 & -1.237 & 0.000 \\
\hline 380.372 & 48.263 & 13.311 & 62.067 & 62.200 & -0.214 & 0.000 \\
\hline 380.372 & 55.158 & 13.752 & 67.174 & 66.400 & 1.166 & 0.000 \\
\hline 408.150 & 0.690 & 0.207 & 12.558 & 12.700 & -1.114 & 0.000 \\
\hline 408.150 & 3.447 & 1.122 & 13.387 & 13.400 & -0.097 & 0.000 \\
\hline 408.150 & 6.895 & 2.499 & 15.154 & 15.000 & 1.030 & 0.000 \\
\hline 408.150 & 13.790 & 5.890 & 22.202 & 22.500 & -1.324 & 0.000 \\
\hline 408.150 & 20.684 & 8.542 & 31.045 & 31.000 & 0.145 & 0.000 \\
\hline 408.150 & 27.579 & 10.112 & 38.437 & 38.100 & 0.883 & 0.000 \\
\hline
\end{tabular}


Table 14. VISCOSITY AT ELEVATED PRESSURES (continued)

Data from Baron et a1. [90] (continued)

\begin{tabular}{|c|c|c|c|c|c|c|}
\hline $\begin{array}{l}\mathrm{T} \\
\mathrm{K}\end{array}$ & $\begin{array}{c}\mathrm{P} \\
\mathrm{MPa}\end{array}$ & $\stackrel{\rho}{\mathrm{mol} \cdot \mathrm{dm}^{-3}}$ & $\begin{array}{c}\eta, \text { calc } \\
\mu \mathrm{Pa} \cdot \mathrm{s}\end{array}$ & $\begin{array}{c}\eta, \operatorname{expt} \\
\mu \mathrm{Pa} \cdot \mathrm{s}\end{array}$ & $\begin{array}{c}\text { Dev } \\
\frac{8}{8}\end{array}$ & wt \\
\hline 408.150 & 34.474 & 11.148 & 44.661 & 44.000 & 1.502 & 0.000 \\
\hline 408.150 & 41.368 & 11.909 & 50.186 & 49.200 & 2.005 & 0.000 \\
\hline 408.150 & 48.263 & 12.511 & 55.281 & 53.700 & 2.944 & 0.000 \\
\hline 408.150 & 55.158 & 13.009 & 60.096 & 57.800 & 3.972 & 0.000 \\
\hline
\end{tabular}

Comparisons based on experimental pressures:

Number of Points [90] 40

$$
\begin{aligned}
& \mathrm{AAD} \%=1.85 \text { BIAS } 8=0.71 \text { RMS } \%=2.19 \\
& \mathrm{AAD}=0.88 \text { BIAS }=0.44 \mathrm{RMS}=1.34 \mu \mathrm{Pa} \cdot \mathrm{s}
\end{aligned}
$$

Comparisons based on experimental densities:

Number of Points [90] 40

\begin{tabular}{|c|c|c|c|c|c|c|}
\hline $\begin{array}{l}\mathrm{T} \\
\mathrm{K}\end{array}$ & $\begin{array}{c}\mathrm{P} \\
\mathrm{MPa}\end{array}$ & $\stackrel{\rho}{\mathrm{mol} \cdot \mathrm{dm}^{-3}}$ & $\begin{array}{c}\eta, \text { calc } \\
\mu \mathrm{Pa} \cdot \mathrm{s}\end{array}$ & $\begin{array}{c}\eta, \text { expt } \\
\mu \mathrm{Pa} \cdot \mathrm{s}\end{array}$ & $\begin{array}{c}\text { Dev } \\
8\end{array}$ & wt \\
\hline 299.817 & 8.932 & 12.467 & 50.998 & 51.503 & -0.981 & 1.000 \\
\hline 299.817 & 8.932 & 12.467 & 50.998 & 51.371 & -0.727 & 1.000 \\
\hline 299.817 & 8.925 & 12.466 & 50.983 & 50.560 & 0.836 & 1.000 \\
\hline 299.817 & 7.121 & 11.974 & 46.998 & 46.816 & 0.388 & 1.000 \\
\hline 299.817 & 7.134 & 11.978 & 47.031 & 46.495 & 1.152 & 1.000 \\
\hline 299.817 & 7.134 & 11.978 & 47.031 & 46.395 & 1.370 & 1.000 \\
\hline 299.817 & 5.792 & 11.440 & 43.118 & 42.598 & 1.221 & 1.000 \\
\hline 299.817 & 5.809 & 11.448 & 43.175 & 42.694 & 1.128 & 1.000 \\
\hline 299.817 & 4.384 & 10.216 & 35.622 & 35.244 & 1.073 & 1.000 \\
\hline 299.817 & 4.384 & 10.216 & 35.622 & 35.158 & 1.321 & 1.000 \\
\hline 299.817 & 35.817 & 15.138 & 83.345 & 83.362 & -0.020 & 1.000 \\
\hline 299.817 & 35.817 & 15.138 & 83.345 & 82.907 & 0.528 & 1.000 \\
\hline 299.817 & 35.772 & 15.136 & 83.302 & 82.802 & 0.604 & 1.000 \\
\hline 299.817 & 31.459 & 14.881 & 79.116 & 78.938 & 0.225 & 1.000 \\
\hline 299.817 & 31.459 & 14.881 & 79.116 & 79.091 & 0.031 & 1.000 \\
\hline 299.817 & 27.843 & 14.643 & 75.472 & 75.390 & 0.109 & 1.000 \\
\hline
\end{tabular}

$$
\begin{aligned}
& \mathrm{AAD} \%=1.73 \quad \text { BIAS\& }=0.39 \text { RMS \& }=2.07 \\
& \mathrm{AAD}=0.80 \mathrm{BIAS}=0.23 \mathrm{RMS}=1.22 \mu \mathrm{Pa} \cdot \mathrm{s}
\end{aligned}
$$

Data from Carmichael et al. [68] 
Table 14. VISCOSITY AT ELEVATED PRESSURES (continued)

Data from Carmichael et al. [68] (continued)

\begin{tabular}{|c|c|c|c|c|c|c|}
\hline $\begin{array}{l}\mathrm{T} \\
\mathrm{K}\end{array}$ & $\begin{array}{c}\mathrm{P} \\
\mathrm{MPa}\end{array}$ & $\stackrel{\rho}{\mathrm{mol} \cdot \mathrm{dm}^{-3}}$ & $\begin{array}{c}\eta, \text { calc } \\
\mu \mathrm{Pa} \cdot \mathrm{s}\end{array}$ & $\begin{array}{c}\eta, \text { expt } \\
\mu \mathrm{Pa} \cdot \mathrm{s}\end{array}$ & $\begin{array}{c}\text { Dev } \\
8\end{array}$ & wt \\
\hline 299.817 & 27.830 & 14.642 & 75.459 & 75.371 & 0.116 & 1.000 \\
\hline 299.817 & 21.110 & 14.113 & 68.203 & 68.347 & -0.210 & 1.000 \\
\hline 299.817 & 21.112 & 14.114 & 68.206 & 68.505 & -0.437 & 1.000 \\
\hline 299.817 & 14.812 & 13.448 & 60.404 & 60.553 & -0.247 & 1.000 \\
\hline 299.817 & 14.805 & 13.447 & 60.394 & 60.548 & -0.254 & 1.000 \\
\hline 299.817 & 14.805 & 13.447 & 60.394 & 60.557 & -0.269 & 1.000 \\
\hline 299.817 & 10.663 & 12.822 & 54.150 & 54.544 & -0.722 & 1.000 \\
\hline 299.817 & 10.686 & 12.826 & 54.189 & 54.486 & -0.546 & 1.000 \\
\hline 299.817 & 7.232 & 12.009 & 47.274 & 47.381 & -0.225 & 1.000 \\
\hline 299.817 & 7.232 & 12.009 & 47.274 & 47.357 & -0.174 & 1.000 \\
\hline 299.817 & 3.913 & 2.686 & 12.114 & 11.955 & 1.331 & 1.000 \\
\hline 299.817 & 3.913 & 2.686 & 12.114 & 12.008 & 0.884 & 1.000 \\
\hline 299.817 & 3.913 & 2.688 & 12.116 & 11.946 & 1.421 & 1.000 \\
\hline 299.817 & 3.485 & 2.124 & 11.329 & 11.204 & 1.112 & 1.000 \\
\hline 299.817 & 3.485 & 2.124 & 11.329 & 11.204 & 1.112 & 1.000 \\
\hline 299.817 & 1.796 & 0.845 & 9.961 & 9.844 & 1.186 & 1.000 \\
\hline 299.817 & 1.798 & 0.846 & 9.961 & 9.825 & 1.388 & 1.000 \\
\hline 299.817 & 0.190 & 0.077 & 9.423 & 9.413 & 0.108 & 1.000 \\
\hline 299.817 & 0.190 & 0.077 & 9.423 & 9.399 & 0.257 & 1.000 \\
\hline 305.372 & $0.972 \mathrm{E}-01$ & 0.039 & 9.565 & 9.557 & 0.079 & 1.000 \\
\hline 305.372 & $0.972 E-01$ & 0.039 & 9.565 & 9.552 & 0.131 & 1.000 \\
\hline 305.372 & $0.979 E-01$ & 0.039 & 9.565 & 9.533 & 0.332 & 1.000 \\
\hline 305.372 & 8.773 & 11.944 & 46.914 & 46.897 & 0.036 & 1.000 \\
\hline 305.372 & 8.778 & 11.945 & 46.926 & 47.032 & -0.225 & 1.000 \\
\hline 305.372 & 8.778 & 11.945 & 46.926 & 47.017 & -0.193 & 1.000 \\
\hline 305.372 & 7.168 & 11.370 & 42.785 & 42.837 & -0.122 & 1.000 \\
\hline 305.372 & 7.175 & 11.373 & 42.804 & 42.861 & -0.134 & 1.000 \\
\hline 305.372 & 6.129 & 10.793 & 39.085 & 39.021 & 0.164 & 1.000 \\
\hline 305.372 & 6.132 & 10.795 & 39.097 & 39.093 & 0.010 & 1.000 \\
\hline 305.372 & 5.219 & 9.798 & 33.578 & 33.764 & -0.551 & 1.000 \\
\hline 305.372 & 5.230 & 9.819 & 33.684 & 33.740 & -0.166 & 1.000 \\
\hline 305.372 & 4.113 & 2.704 & 12.321 & 12.319 & 0.018 & 1.000 \\
\hline 305.372 & 4.113 & 2.704 & 12.321 & 12.286 & 0.287 & 1.000 \\
\hline 305.372 & 4.113 & 2.704 & 12.321 & 12.334 & -0.104 & 1.000 \\
\hline 305.372 & 3.429 & 1.938 & 11.271 & 11.208 & 0.561 & 1.000 \\
\hline 305.372 & 3.429 & 1.938 & 11.271 & 11.275 & -0.036 & 1.000 \\
\hline
\end{tabular}


Table 14. VISCOSITY AT ELEVATED PRESSURES (continued)

Data from Carmichael et al. [68] (continued)

\begin{tabular}{|c|c|c|c|c|c|c|}
\hline $\begin{array}{l}\mathrm{T} \\
\mathrm{K}\end{array}$ & $\begin{array}{c}\mathrm{P} \\
\mathrm{MPa}\end{array}$ & $\stackrel{\rho}{\mathrm{mol}} \mathrm{dm}^{-3}$ & $\begin{array}{c}\eta, \text { calc } \\
\mu \mathrm{Pa} \cdot \mathrm{s}\end{array}$ & $\begin{array}{c}\eta, \operatorname{expt} \\
\mu \mathrm{Pa} \cdot \mathrm{s}\end{array}$ & $\begin{array}{c}\text { Dev } \\
8\end{array}$ & wt \\
\hline 305.372 & 3.429 & 1.938 & 11.271 & 11.223 & 0.427 & 1.000 \\
\hline 305.372 & 1.746 & 0.794 & 10.088 & 10.026 & 0.618 & 1.000 \\
\hline 305.372 & 1.746 & 0.794 & 10.088 & 10.150 & -0.611 & 1.000 \\
\hline 305.372 & 1.744 & 0.793 & 10.087 & 10.031 & 0.559 & 1.000 \\
\hline 305.372 & 4.862 & 5.330 & 17.522 & 18.342 & -4.468 & 0.000 \\
\hline 305.372 & 4.862 & 5.330 & 17.522 & 18.453 & -5.043 & 0.000 \\
\hline 305.372 & 4.862 & 5.330 & 17.522 & 18.333 & -4.421 & 0.000 \\
\hline 305.372 & 4.135 & 2.736 & 12.369 & 12.698 & -2.589 & 1.000 \\
\hline 305.372 & 4.142 & 2.745 & 12.383 & 12.678 & -2.327 & 1.000 \\
\hline 305.372 & 4.145 & 2.750 & 12.391 & 12.654 & -2.082 & 1.000 \\
\hline 310.928 & 35.183 & 14.706 & 76.893 & 76.415 & 0.625 & 1.000 \\
\hline 310.928 & 35.094 & 14.701 & 76.808 & 76.252 & 0.730 & 1.000 \\
\hline 310.928 & 35.052 & 14.698 & 76.769 & 76.314 & 0.596 & 1.000 \\
\hline 310.928 & 27.779 & 14.192 & 69.624 & 71.239 & -2.267 & 1.000 \\
\hline 310.928 & 27.772 & 14.191 & 69.617 & 71.354 & -2.434 & 1.000 \\
\hline 310.928 & 20.919 & 13.574 & 62.146 & 62.453 & -0.492 & 1.000 \\
\hline 310.928 & 20.899 & 13.572 & 62.122 & 62.453 & -0.530 & 1.000 \\
\hline 310.928 & 13.981 & 12.663 & 53.021 & 53.419 & -0.745 & 1.000 \\
\hline 310.928 & 13.981 & 12.663 & 53.021 & 53.419 & -0.745 & 1.000 \\
\hline 310.928 & 6.974 & 10.480 & 37.395 & 37.872 & -1.258 & 1.000 \\
\hline 310.928 & 6.974 & 10.480 & 37.395 & 37.652 & -0.681 & 1.000 \\
\hline 310.928 & 6.152 & 9.657 & 33.031 & 33.271 & -0.722 & 1.000 \\
\hline 310.928 & 6.152 & 9.657 & 33.031 & 33.281 & -0.752 & 1.000 \\
\hline 310.928 & 5.261 & 5.018 & 16.951 & 17.323 & -2.146 & 1.000 \\
\hline 310.928 & 5.271 & 5.077 & 17.092 & 17.198 & -0.619 & 1.000 \\
\hline 310.928 & 5.273 & 5.091 & 17.123 & 17.136 & -0.075 & 1.000 \\
\hline 310.928 & 4.329 & 2.740 & 12.556 & 12.396 & 1.290 & 1.000 \\
\hline 310.928 & 4.333 & 2.746 & 12.566 & 12.391 & 1.409 & 1.000 \\
\hline 310.928 & 3.483 & 1.881 & 11.378 & 11.242 & 1.209 & 1.000 \\
\hline 310.928 & 3.484 & 1.882 & 11.379 & 11.237 & 1.260 & 1.000 \\
\hline 310.928 & 1.712 & 0.755 & 10.224 & 10.102 & 1.210 & 1.000 \\
\hline 310.928 & 1.713 & 0.755 & 10.225 & 10.079 & 1.446 & 1.000 \\
\hline 310.928 & 0.154 & 0.060 & 9.739 & 9.734 & 0.050 & 1.000 \\
\hline 310.928 & 0.154 & 0.060 & 9.739 & 9.719 & 0.204 & 1.000 \\
\hline 310.928 & 4.960 & 3.859 & 14.481 & 14.469 & 0.083 & 1.000 \\
\hline 310.928 & 4.960 & 3.859 & 14.481 & 14.900 & -2.812 & 1.000 \\
\hline
\end{tabular}


Table 14. VISCOSITY AT ELEVATED PRESSURES (continued)

Data from Carmichael et al. [68] (continued)

\begin{tabular}{|c|c|c|c|c|c|c|}
\hline $\begin{array}{l}\mathrm{T} \\
\mathrm{K}\end{array}$ & $\begin{array}{c}\mathrm{P} \\
\mathrm{MPa}\end{array}$ & $\begin{array}{c}\rho \\
\mathrm{mol} \cdot \mathrm{dm}^{-3}\end{array}$ & $\begin{array}{c}\eta, \text { calc } \\
\mu \mathrm{Pa} \cdot \mathrm{s}\end{array}$ & $\begin{array}{c}\eta, \text { expt } \\
\mu \mathrm{Pa} \cdot \mathrm{s}\end{array}$ & $\begin{array}{c}\text { Dev } \\
8\end{array}$ & wt \\
\hline $\begin{array}{l}310.928 \\
310.928\end{array}$ & $\begin{array}{l}4.898 \\
4.904\end{array}$ & $\begin{array}{l}3.706 \\
3.721\end{array}$ & $\begin{array}{l}14.191 \\
14.218\end{array}$ & $\begin{array}{l}14.254 \\
14.297\end{array}$ & $\begin{array}{l}-0.443 \\
-0.551\end{array}$ & $\begin{array}{l}1.000 \\
1.000\end{array}$ \\
\hline $\begin{array}{l}310.928 \\
310.928 \\
310.928 \\
310.928 \\
310.928\end{array}$ & $\begin{array}{l}4.561 \\
4.561 \\
4.564 \\
5.940 \\
5.926\end{array}$ & $\begin{array}{l}3.068 \\
3.068 \\
3.073 \\
9.297 \\
9.269\end{array}$ & $\begin{array}{l}13.075 \\
13.075 \\
13.082 \\
31.310 \\
31.180\end{array}$ & $\begin{array}{l}13.176 \\
13.138 \\
13.066 \\
30.441 \\
30.470\end{array}$ & $\begin{array}{r}-0.768 \\
-0.481 \\
0.121 \\
2.855 \\
2.330\end{array}$ & $\begin{array}{l}1.000 \\
1.000 \\
1.000 \\
1.000 \\
1.000\end{array}$ \\
\hline $\begin{array}{l}310.928 \\
310.928 \\
310.928 \\
310.928 \\
310.928\end{array}$ & $\begin{array}{l}5.920 \\
5.222 \\
5.222 \\
5.229 \\
5.229\end{array}$ & $\begin{array}{l}9.256 \\
4.798 \\
4.798 \\
4.834 \\
4.834\end{array}$ & $\begin{array}{l}31.120 \\
16.444 \\
16.444 \\
16.526 \\
16.526\end{array}$ & $\begin{array}{l}30.480 \\
16.715 \\
16.288 \\
16.357 \\
16.346\end{array}$ & $\begin{array}{r}2.101 \\
-1.620 \\
0.959 \\
1.035 \\
1.103\end{array}$ & $\begin{array}{l}1.000 \\
1.000 \\
1.000 \\
1.000 \\
1.000\end{array}$ \\
\hline $\begin{array}{l}310.928 \\
310.928 \\
310.928 \\
310.928 \\
310.928\end{array}$ & $\begin{array}{l}4.142 \\
4.142 \\
4.142 \\
4.142 \\
4.364\end{array}$ & $\begin{array}{l}2.514 \\
2.514 \\
2.514 \\
2.514 \\
2.786\end{array}$ & $\begin{array}{l}12.221 \\
12.221 \\
12.221 \\
12.221 \\
12.626\end{array}$ & $\begin{array}{l}12.195 \\
12.180 \\
12.477 \\
12.472 \\
12.516\end{array}$ & $\begin{array}{r}0.215 \\
0.339 \\
-2.050 \\
-2.011 \\
0.881\end{array}$ & $\begin{array}{l}1.000 \\
1.000 \\
1.000 \\
1.000 \\
1.000\end{array}$ \\
\hline $\begin{array}{l}310.928 \\
327.594 \\
327.594 \\
327.594 \\
327.594\end{array}$ & $\begin{array}{r}4.151 \\
14.551 \\
14.551 \\
14.351 \\
14.351\end{array}$ & $\begin{array}{r}2.525 \\
11.667 \\
11.667 \\
11.620 \\
11.620\end{array}$ & $\begin{array}{l}12.237 \\
45.510 \\
45.510 \\
45.174 \\
45.174\end{array}$ & $\begin{array}{l}12.295 \\
45.538 \\
45.547 \\
45.356 \\
45.504\end{array}$ & $\begin{array}{l}-0.471 \\
-0.062 \\
-0.081 \\
-0.401 \\
-0.725\end{array}$ & $\begin{array}{l}1.000 \\
1.000 \\
1.000 \\
1.000 \\
1.000\end{array}$ \\
\hline $\begin{array}{l}327.594 \\
327.594 \\
327.594 \\
327.594 \\
327.594\end{array}$ & $\begin{array}{l}8.643 \\
8.643 \\
8.814 \\
8.814 \\
7.599\end{array}$ & $\begin{array}{l}9.114 \\
9.114 \\
9.272 \\
9.272 \\
7.689\end{array}$ & $\begin{array}{l}30.966 \\
30.966 \\
31.684 \\
31.684 \\
25.275\end{array}$ & $\begin{array}{l}31.610 \\
31.662 \\
31.476 \\
31.533 \\
25.151\end{array}$ & $\begin{array}{r}-2.039 \\
-2.200 \\
0.662 \\
0.480 \\
0.493\end{array}$ & $\begin{array}{l}1.000 \\
1.000 \\
1.000 \\
1.000 \\
1.000\end{array}$ \\
\hline $\begin{array}{l}327.594 \\
327.594 \\
327.594 \\
327.594 \\
327.594\end{array}$ & $\begin{array}{l}7.606 \\
7.606 \\
6.935 \\
6.935 \\
6.983\end{array}$ & $\begin{array}{l}7.701 \\
7.701 \\
6.146 \\
6.146 \\
6.270\end{array}$ & $\begin{array}{l}25.317 \\
25.317 \\
20.392 \\
20.392 \\
20.740\end{array}$ & $\begin{array}{l}24.993 \\
25.012 \\
20.267 \\
20.191 \\
20.238\end{array}$ & $\begin{array}{l}1.298 \\
1.221 \\
0.614 \\
0.993 \\
2.481\end{array}$ & $\begin{array}{l}1.000 \\
1.000 \\
1.000 \\
1.000 \\
1.000\end{array}$ \\
\hline $\begin{array}{l}327.594 \\
327.594 \\
327.594 \\
327.594\end{array}$ & $\begin{array}{l}6.223 \\
6.211 \\
6.212 \\
5.521\end{array}$ & $\begin{array}{l}4.530 \\
4.506 \\
4.508 \\
3.444\end{array}$ & $\begin{array}{l}16.390 \\
16.340 \\
16.343 \\
14.253\end{array}$ & $\begin{array}{l}16.490 \\
16.432 \\
16.451 \\
14.493\end{array}$ & $\begin{array}{l}-0.606 \\
-0.561 \\
-0.658 \\
-1.655\end{array}$ & $\begin{array}{l}1.000 \\
1.000 \\
1.000 \\
1.000\end{array}$ \\
\hline
\end{tabular}


Table 14. VISCOSITY AT ELEVATED PRESSURES (continued)

Data from Carmichael et al. [68] (continued)

\begin{tabular}{|c|c|c|c|c|c|c|}
\hline $\begin{array}{l}\mathrm{T} \\
\mathrm{K}\end{array}$ & $\begin{array}{c}\mathrm{P} \\
\mathrm{MPa}\end{array}$ & $\begin{array}{c}\rho \\
\mathrm{mol} \cdot \mathrm{dm}^{-3}\end{array}$ & $\begin{array}{c}\eta, \text { calc } \\
\mu \mathrm{Pa} \cdot \mathrm{s}\end{array}$ & $\begin{array}{c}\eta, \text { expt } \\
\mu \mathrm{Pa} \cdot \mathrm{s}\end{array}$ & $\begin{array}{c}\text { Dev } \\
\%\end{array}$ & wt \\
\hline 327.594 & 5.521 & 3.444 & 14.253 & 14.445 & -1.329 & 1.000 \\
\hline 327.594 & 5.524 & 3.447 & 14.259 & 14.503 & -1.680 & 1.000 \\
\hline 327.594 & 4.819 & 2.678 & 12.995 & 13.114 & -0.908 & 1.000 \\
\hline 327.594 & 4.822 & 2.680 & 12.998 & 13.143 & -1.104 & 1.000 \\
\hline 327.594 & 4.822 & 2.680 & 12.998 & 13.100 & -0.779 & 1.000 \\
\hline 344.261 & 35.231 & 13.508 & 62.685 & 62.425 & 0.417 & 1.000 \\
\hline 344.261 & 35.194 & 13.505 & 62.651 & 62.453 & 0.317 & 1.000 \\
\hline 344.261 & 35.161 & 13.502 & 62.621 & 62.530 & 0.146 & 1.000 \\
\hline 344.261 & 26.950 & 12.717 & 54.645 & 54.687 & -0.077 & 1.000 \\
\hline 344.261 & 26.951 & 12.717 & 54.647 & 54.673 & -0.048 & 1.000 \\
\hline 344.261 & 20.617 & 11.847 & 47.384 & 47.295 & 0.189 & 1.000 \\
\hline 344.261 & 20.599 & 11.844 & 47.361 & 47.324 & 0.077 & 1.000 \\
\hline 344.261 & 20.589 & 11.843 & 47.348 & 47.300 & 0.102 & 1.000 \\
\hline 344.261 & 14.138 & 10.307 & 37.416 & 37.681 & -0.702 & 1.000 \\
\hline 344.261 & 14.144 & 10.309 & 37.427 & 37.542 & -0.306 & 1.000 \\
\hline 344.261 & 23.742 & 12.319 & 51.144 & 51.135 & 0.018 & 1.000 \\
\hline 344.261 & 23.742 & 12.319 & 51.144 & 51.178 & -0.066 & 1.000 \\
\hline 344.261 & 23.742 & 12.319 & 51.144 & 51.120 & 0.047 & 1.000 \\
\hline 344.261 & 23.742 & 12.319 & 51.144 & 51.116 & 0.055 & 1.000 \\
\hline 344.261 & 17.824 & 11.315 & 43.578 & 43.517 & 0.140 & 1.000 \\
\hline 344.261 & 17.824 & 11.315 & 43.578 & 43.503 & 0.172 & 1.000 \\
\hline 344.261 & 17.828 & 11.316 & 43.585 & 43.484 & 0.232 & 1.000 \\
\hline 344.261 & 13.997 & 10.257 & 37.139 & 37.226 & -0.233 & 1.000 \\
\hline 344.261 & 14.000 & 10.258 & 37.146 & 37.121 & 0.068 & 1.000 \\
\hline 344.261 & 14.032 & 10.270 & 37.209 & 37.231 & -0.060 & 1.000 \\
\hline 344.261 & 10.531 & 8.419 & 28.538 & 28.574 & -0.128 & 1.000 \\
\hline 344.261 & 10.531 & 8.419 & 28.538 & 28.402 & 0.477 & 1.000 \\
\hline 344.261 & 10.531 & 8.419 & 28.538 & 28.349 & 0.665 & 1.000 \\
\hline 344.261 & 7.212 & 4.481 & 16.820 & 16.825 & -0.033 & 1.000 \\
\hline 344.261 & 7.212 & 4.481 & 16.820 & 16.734 & 0.511 & 1.000 \\
\hline 344.261 & 7.222 & 4.494 & 16.846 & 16.691 & 0.931 & 1.000 \\
\hline 344.261 & 5.361 & 2.690 & 13.536 & 13.416 & 0.891 & 1.000 \\
\hline 344.261 & 5.361 & 2.690 & 13.536 & 13.430 & 0.786 & 1.000 \\
\hline 344.261 & 5.361 & 2.690 & 13.536 & 13.444 & 0.681 & 1.000 \\
\hline 344.261 & 3.580 & 1.544 & 12.007 & 12.022 & -0.121 & 1.000 \\
\hline 344.261 & 3.580 & 1.544 & 12.007 & 11.931 & 0.640 & 1.000 \\
\hline
\end{tabular}


Table 14. VISCOSITY AT ELEVATED PRESSURES (continued)

Data from Carmichael et al. [68] (continued)

\begin{tabular}{|c|c|c|c|c|c|c|}
\hline $\begin{array}{l}\mathrm{T} \\
\mathrm{K}\end{array}$ & $\begin{array}{c}\mathrm{P} \\
\mathrm{MPa}\end{array}$ & $\begin{array}{c}\rho \\
\mathrm{mol}\end{array} \mathrm{dm}^{-3}$ & $\begin{array}{c}\eta, \text { calc } \\
\mu \mathrm{Pa} \cdot \mathrm{s}\end{array}$ & $\begin{array}{c}\eta, \exp t \\
\mu \mathrm{Pa} \cdot \mathrm{s}\end{array}$ & $\begin{array}{c}\text { Dev } \\
\frac{8}{8}\end{array}$ & wt \\
\hline 344.261 & 3.541 & 1.522 & 11.984 & 11.917 & 0.558 & 1.000 \\
\hline 344.261 & 1.924 & 0.744 & 11.206 & 11.132 & 0.662 & 1.000 \\
\hline 344.261 & 1.924 & 0.744 & 11.206 & 11.127 & 0.707 & 1.000 \\
\hline 344.261 & 0.175 & 0.062 & 10.697 & 10.725 & -0.262 & 1.000 \\
\hline 344.261 & 0.176 & 0.062 & 10.697 & 10.725 & -0.259 & 1.000 \\
\hline 377.594 & 32.034 & 11.956 & 49.403 & 48.999 & 0.825 & 1.000 \\
\hline 377.594 & 32.034 & 11.956 & 49.403 & 48.980 & 0.864 & 1.000 \\
\hline 377.594 & 32.059 & 11.959 & 49.427 & 49.047 & 0.774 & 1.000 \\
\hline 377.594 & 26.847 & 11.257 & 44.320 & 44.240 & 0.180 & 1.000 \\
\hline 377.594 & 26.875 & 11.262 & 44.349 & 44.322 & 0.062 & 1.000 \\
\hline 377.594 & 21.010 & 10.144 & 37.598 & 37.561 & 0.098 & 1.000 \\
\hline 377.594 & 21.020 & 10.147 & 37.611 & 37.484 & 0.339 & 1.000 \\
\hline 377.594 & 14.495 & 7.913 & 27.646 & 27.439 & 0.754 & 1.000 \\
\hline 377.594 & 14.498 & 7.915 & 27.652 & 27.361 & 1.064 & 1.000 \\
\hline 377.594 & 8.909 & 4.277 & 17.428 & 17.433 & -0.026 & 1.000 \\
\hline 377.594 & 8.918 & 4.284 & 17.442 & 17.260 & 1.052 & 1.000 \\
\hline 377.594 & 4.457 & 1.703 & 13.182 & 12.985 & 1.520 & 1.000 \\
\hline 377.594 & 4.457 & 1.703 & 13.182 & 13.004 & 1.372 & 1.000 \\
\hline 377.594 & 0.233 & 0.075 & 11.635 & 11.697 & -0.533 & 1.000 \\
\hline 377.594 & 0.233 & 0.075 & 11.635 & 11.659 & -0.209 & 1.000 \\
\hline 410.928 & 21.304 & 8.594 & 31.343 & 31.275 & 0.217 & 1.000 \\
\hline 410.928 & 21.300 & 8.593 & 31.338 & 31.174 & 0.527 & 1.000 \\
\hline 410.928 & 21.278 & 8.586 & 31.313 & 31.557 & -0.774 & 1.000 \\
\hline 410.928 & 15.368 & 6.498 & 24.005 & 24.035 & -0.126 & 1.000 \\
\hline 410.928 & 15.368 & 6.498 & 24.005 & 24.030 & -0.105 & 1.000 \\
\hline 410.928 & 10.583 & 4.197 & 18.266 & 18.400 & -0.727 & 1.000 \\
\hline 410.928 & 10.585 & 4.198 & 18.268 & 18.318 & -0.271 & 1.000 \\
\hline 410.928 & 5.743 & 1.983 & 14.499 & 14.555 & -0.386 & 1.000 \\
\hline 410.928 & 5.750 & 1.986 & 14.503 & 14.526 & -0.158 & 1.000 \\
\hline 410.928 & 0.701 & 0.209 & 12.634 & 12.779 & -1.132 & 1.000 \\
\hline 410.928 & 0.701 & 0.209 & 12.634 & 12.750 & -0.907 & 1.000 \\
\hline 410.928 & 36.031 & 11.246 & 45.420 & 45.217 & 0.449 & 1.000 \\
\hline 410.928 & 36.021 & 11.244 & 45.412 & 45.065 & 0.769 & 1.000 \\
\hline 410.928 & 36.021 & 11.244 & 45.412 & 45.035 & 0.836 & 1.000 \\
\hline 410.928 & 27.775 & 10.039 & 38.133 & 37.513 & 1.653 & 1.000 \\
\hline 410.928 & 27.775 & 10.039 & 38.133 & 38.911 & -1.999 & 1.000 \\
\hline
\end{tabular}


Table 14. VISCOSITY AT ELEVATED PRESSURES (continued)

Data from Carmichael et al. [68] (continued)

\begin{tabular}{|c|c|c|c|c|c|c|}
\hline $\begin{array}{l}\mathrm{T} \\
\mathrm{K}\end{array}$ & $\underset{\mathrm{MPa}}{\mathrm{P}}$ & $\begin{array}{c}\rho \\
\mathrm{mol} \cdot \mathrm{dm}^{-3}\end{array}$ & $\begin{array}{c}\eta, \text { calc } \\
\mu \mathrm{Pa} \cdot \mathrm{s}\end{array}$ & $\begin{array}{c}\eta, \operatorname{expt} \\
\mu \mathrm{Pa} \cdot \mathrm{s}\end{array}$ & $\begin{array}{c}\text { Dev } \\
\frac{\gamma}{\delta}\end{array}$ & wt \\
\hline 410.928 & 27.775 & 10.039 & 38.133 & 37.772 & 0.956 & 1.000 \\
\hline 444.261 & 33.375 & 9.806 & 38.005 & 37.762 & 0.643 & 1.000 \\
\hline 444.261 & 33.360 & 9.804 & 37.993 & 37.738 & 0.676 & 1.000 \\
\hline 444.261 & 27.964 & 8.872 & 33.569 & 33.357 & 0.634 & 1.000 \\
\hline 444.261 & 27.955 & 8.870 & 33.561 & 33.410 & 0.452 & 1.000 \\
\hline 444.261 & 20.780 & 7.146 & 27.032 & 26.764 & 1.000 & 0.000 \\
\hline 444.261 & 20.780 & 7.146 & 27.032 & 26.822 & 0.782 & 1.000 \\
\hline 444.261 & 14.051 & 4.853 & 20.675 & 20.875 & -0.957 & 1.000 \\
\hline 444.261 & 14.051 & 4.853 & 20.675 & 20.866 & -0.915 & 1.000 \\
\hline 444.261 & 7.151 & 2.237 & 15.781 & 15.771 & 0.066 & 1.000 \\
\hline 444.261 & 7.155 & 2.238 & 15.784 & 15.824 & -0.254 & 1.000 \\
\hline 444.261 & 0.674 & 0.185 & 13.494 & 13.650 & -1.140 & 1.000 \\
\hline 444.261 & 0.674 & 0.185 & 13.494 & 13.646 & -1.111 & 1.000 \\
\hline 477.594 & 34.291 & 8.985 & 35.108 & 34.966 & 0.406 & 1.000 \\
\hline 477.594 & 34.260 & 8.980 & 35.086 & 35.162 & -0.215 & 1.000 \\
\hline 477.594 & 34.260 & 8.980 & 35.086 & 34.837 & 0.716 & 1.000 \\
\hline 477.594 & 28.024 & 7.881 & 30.597 & 30.216 & 1.260 & 1.000 \\
\hline 477.594 & 28.003 & 7.877 & 30.581 & 30.446 & 0.443 & 1.000 \\
\hline 477.594 & 27.997 & 7.876 & 30.576 & 30.571 & 0.017 & 1.000 \\
\hline 477.594 & 7.119 & 1.986 & 16.324 & 16.518 & -1.177 & 1.000 \\
\hline 477.594 & 7.121 & 1.987 & 16.324 & 16.537 & -1.287 & 1.000 \\
\hline 477.594 & 0.677 & 0.172 & 14.336 & 14.656 & -2.183 & 1.000 \\
\hline 477.594 & 0.678 & 0.173 & 14.336 & 14.637 & -2.054 & 1.000 \\
\hline 477.594 & 0.678 & 0.173 & 14.336 & 14.646 & -2.114 & 1.000 \\
\hline 477.594 & 21.044 & 6.258 & 25.271 & 25.314 & -0.168 & 1.000 \\
\hline 477.594 & 21.044 & 6.258 & 25.271 & 25.347 & -0.298 & 1.000 \\
\hline 477.594 & 21.044 & 6.258 & 25.271 & 25.467 & -0.768 & 1.000 \\
\hline 477.594 & 14.255 & 4.247 & 20.275 & 20.808 & -2.560 & 1.000 \\
\hline 477.594 & 14.255 & 4.247 & 20.275 & 20.918 & -3.072 & 1.000 \\
\hline 477.594 & 14.238 & 4.242 & 20.264 & 20.846 & -2.793 & 1.000 \\
\hline
\end{tabular}


Table 14. VISCOSITY AT ELEVATED PRESSURES (continued)

Data from Carmichael et al. [68] (continued)

Comparisons based on experimental pressures:

Number of Points [68] 226

$$
\begin{array}{ll}
\mathrm{AAD} \& & =0.81 \quad \mathrm{BIAS} \%=-0.07 \quad \mathrm{RMS} \%=1.14 \\
\mathrm{AAD}=0.21 & \mathrm{BIAS}=-0.01 \quad \mathrm{RMS}=0.31 \mu \mathrm{Pa} \cdot \mathrm{s}
\end{array}
$$

Weighted Data:

Number of Points [68] 222

$$
\begin{aligned}
& \mathrm{AAD} \&=0.76 \quad \text { BIAS } 8=-0.02 \quad \text { RMS } 8=1.01 \\
& \mathrm{AAD}=0.20 \quad \mathrm{BIAS}=0.00 \mathrm{RMS}=0.30 \mu \mathrm{Pa} \cdot \mathrm{s}
\end{aligned}
$$

\begin{tabular}{|c|c|c|c|c|c|c|}
\hline $\begin{array}{l}\mathrm{T} \\
\mathrm{K}\end{array}$ & $\begin{array}{c}\mathrm{P} \\
\mathrm{MPa}\end{array}$ & $\stackrel{\rho}{\mathrm{mol} \cdot \mathrm{dm}^{-3}}$ & $\begin{array}{c}\eta, \text { calc } \\
\mu \mathrm{Pa} \cdot \mathrm{s}\end{array}$ & $\begin{array}{c}\eta, \text { expt } \\
\mu \mathrm{Pa} \cdot \mathrm{s}\end{array}$ & $\begin{array}{c}\text { Dev } \\
\frac{q}{\delta}\end{array}$ & wt \\
\hline 320.000 & 31.029 & 14.084 & 68.589 & 68.720 & -0.191 & 1.000 \\
\hline 320.000 & 21.378 & 13.188 & 58.335 & 58.570 & -0.402 & 1.000 \\
\hline 320.000 & 14.102 & 12.090 & 48.477 & 48.960 & -0.986 & 1.000 \\
\hline 320.000 & 13.699 & 12.006 & 47.815 & 48.540 & -1.494 & 1.000 \\
\hline 320.000 & 10.253 & 11.035 & 40.999 & 41.740 & -1.775 & 1.000 \\
\hline 320.000 & 10.099 & 10.975 & 40.620 & 41.050 & -1.048 & 1.000 \\
\hline 320.000 & 8.785 & 10.344 & 36.888 & 37.460 & -1.527 & 1.000 \\
\hline 320.000 & 8.363 & 10.073 & 35.412 & 35.640 & -0.640 & 1.000 \\
\hline 320.000 & 7.319 & 9.068 & 30.534 & 30.950 & -1.343 & 1.000 \\
\hline 320.000 & 6.937 & 8.427 & 27.828 & 27.930 & -0.364 & 1.000 \\
\hline 320.000 & 6.818 & 8.161 & 26.786 & 26.960 & -0.647 & 1.000 \\
\hline 320.000 & 6.718 & 7.901 & 25.808 & 26.040 & -0.891 & 1.000 \\
\hline 320.000 & 6.456 & 7.032 & 22.815 & 22.930 & -0.500 & 1.000 \\
\hline 320.000 & 6.366 & 6.677 & 21.702 & 21.950 & -1.130 & 1.000 \\
\hline 320.000 & 6.153 & 5.820 & 19.256 & 18.990 & 1.403 & 1.000 \\
\hline 320.000 & 6.064 & 5.491 & 18.400 & 18.430 & -0.165 & 1.000 \\
\hline 320.000 & 5.778 & 4.612 & 16.323 & 16.090 & 1.450 & 1.000 \\
\hline 320.000 & 5.443 & 3.875 & 14.807 & 14.710 & 0.658 & 1.000 \\
\hline 320.000 & 5.248 & 3.541 & 14.183 & 13.900 & 2.039 & 1.000 \\
\hline 320.000 & 4.759 & 2.881 & 13.067 & 12.960 & 0.825 & 1.000 \\
\hline
\end{tabular}

Densities are not tabulated in [68]

Data from Diller et al. [91] 
Table 14. VISCOSITY AT ELEVATED PRESSURES (continued)

Data from Diller et al. [91] (continued)

\begin{tabular}{|c|c|c|c|c|c|c|}
\hline $\begin{array}{l}\mathrm{T} \\
\mathrm{K}\end{array}$ & $\begin{array}{c}\mathrm{P} \\
\mathrm{MPa}\end{array}$ & $\stackrel{\rho}{\mathrm{mol} \cdot \mathrm{dm}^{-3}}$ & $\begin{array}{c}\eta, \text { calc } \\
\mu \mathrm{Pa} \cdot \mathrm{s}\end{array}$ & $\begin{array}{c}\eta, \text { expt } \\
\mu \mathrm{Pa} \cdot \mathrm{s}\end{array}$ & $\begin{array}{c}\text { Dev } \\
8\end{array}$ & wt \\
\hline 320.000 & 4.671 & 2.781 & 12.911 & 12.880 & 0.242 & 1.000 \\
\hline 320.000 & 3.902 & 2.059 & 11.887 & 11.960 & -0.608 & 0.000 \\
\hline 320.000 & 3.873 & 2.036 & 11.858 & 12.010 & -1.265 & 0.000 \\
\hline 320.000 & 3.448 & 1.719 & 11.467 & 11.550 & -0.715 & 0.000 \\
\hline 320.000 & 3.078 & 1.472 & 11.189 & 11.540 & -3.044 & 0.000 \\
\hline 320.000 & 2.115 & 0.924 & 10.644 & 10.870 & -2.081 & 0.000 \\
\hline 320.000 & 2.056 & 0.894 & 10.617 & 10.990 & -3.395 & 0.000 \\
\hline 320.000 & 1.107 & 0.447 & 10.257 & 10.480 & -2.131 & 0.000 \\
\hline 290.000 & 3.399 & 2.365 & 11.330 & 11.120 & 1.889 & 0.000 \\
\hline 290.000 & 3.213 & 2.097 & 10.974 & 10.760 & 1.991 & 0.000 \\
\hline 290.000 & 2.609 & 1.476 & 10.250 & 10.350 & -0.968 & 0.000 \\
\hline 290.000 & 2.516 & 1.399 & 10.170 & 10.280 & -1.073 & 0.000 \\
\hline 290.000 & 1.924 & 0.975 & 9.767 & 9.920 & -1.539 & 0.000 \\
\hline 290.000 & 1.799 & 0.896 & 9.700 & 9.940 & -2.416 & 0.000 \\
\hline 290.000 & 1.238 & 0.577 & 9.449 & 9.720 & -2.786 & 0.000 \\
\hline 290.000 & 1.132 & 0.521 & 9.409 & 9.550 & -1.472 & 0.000 \\
\hline 290.000 & 1.068 & 0.489 & 9.387 & 9.640 & -2.628 & 0.000 \\
\hline 290.000 & 1.019 & 0.464 & 9.370 & 9.640 & -2.806 & 0.000 \\
\hline 290.000 & 0.603 & 0.264 & 9.240 & 9.410 & -1.809 & 0.000 \\
\hline 290.000 & 30.343 & 15.183 & 83.670 & 83.310 & 0.432 & 1.000 \\
\hline 290.000 & 27.327 & 14.998 & 80.585 & 80.380 & 0.255 & 1.000 \\
\hline 290.000 & 23.904 & 14.767 & 76.959 & 76.590 & 0.482 & 1.000 \\
\hline 290.000 & 20.466 & 14.506 & 73.143 & 72.650 & 0.679 & 1.000 \\
\hline 290.000 & 17.691 & 14.268 & 69.889 & 69.490 & 0.574 & 1.000 \\
\hline 290.000 & 14.881 & 13.992 & 66.375 & 66.330 & 0.068 & 1.000 \\
\hline 290.000 & 11.206 & 13.554 & 61.280 & 61.160 & 0.195 & 1.000 \\
\hline 290.000 & 9.268 & 13.267 & 58.235 & 57.800 & 0.752 & 1.000 \\
\hline 290.000 & 7.266 & 12.902 & 54.649 & 54.600 & 0.090 & 1.000 \\
\hline 290.000 & 5.411 & 12.451 & 50.615 & 50.520 & 0.189 & 1.000 \\
\hline 290.000 & 3.517 & 11.698 & 44.705 & 44.460 & 0.550 & 1.000 \\
\hline 250.000 & 27.148 & 16.518 & 110.150 & 109.880 & 0.246 & 1.000 \\
\hline 250.000 & 24.209 & 16.388 & 106.922 & 106.650 & 0.255 & 1.000 \\
\hline 250.000 & 23.703 & 16.365 & 106.362 & 106.410 & -0.045 & 1.000 \\
\hline 250.000 & 20.658 & 16.220 & 102.949 & 104.510 & -1.493 & 1.000 \\
\hline 250.000 & 17.052 & 16.033 & 98.808 & 98.970 & -0.163 & 1.000 \\
\hline 250.000 & 15.159 & 15.928 & 96.580 & 97.250 & -0.689 & 1.000 \\
\hline
\end{tabular}


Table 14. VISCOSITY AT ELEVATED PRESSURES (continued)

Data from Diller et al. [91] (continued)

\begin{tabular}{|c|c|c|c|c|c|c|}
\hline $\begin{array}{l}\mathrm{T} \\
\mathrm{K}\end{array}$ & $\begin{array}{c}\mathrm{P} \\
\mathrm{MPa}\end{array}$ & $\begin{array}{c}\rho \\
\mathrm{mol}\end{array} \mathrm{dm}^{-3}$ & $\begin{array}{c}\eta, \text { calc } \\
\mu \mathrm{Pa} \cdot \mathrm{s}\end{array}$ & $\begin{array}{c}\eta, \text { expt } \\
\mu \mathrm{Pa} \cdot \mathrm{s}\end{array}$ & $\begin{array}{c}\text { Dev } \\
q\end{array}$ & wt \\
\hline 250.000 & 11.712 & 15.721 & 92.401 & 93.930 & -1.628 & 1.000 \\
\hline 250.000 & 9.124 & 15.549 & 89.131 & 89.750 & -0.690 & 1.000 \\
\hline 250.000 & 6.297 & 15.340 & 85.384 & 85.900 & -0.600 & 1.000 \\
\hline 250.000 & 3.427 & 15.099 & 81.327 & 81.870 & -0.663 & 1.000 \\
\hline 250.000 & 1.302 & 14.894 & 78.088 & 78.490 & -0.512 & 1.000 \\
\hline 200.000 & 29.623 & 18.371 & 177.052 & 176.630 & 0.239 & 1.000 \\
\hline 200.000 & 26.170 & 18.279 & 172.540 & 173.040 & -0.289 & 1.000 \\
\hline 200.000 & 22.714 & 18.183 & 168.033 & 167.750 & 0.169 & 1.000 \\
\hline 200.000 & 19.396 & 18.087 & 163.705 & 164.380 & -0.411 & 1.000 \\
\hline 200.000 & 15.918 & 17.981 & 159.161 & 160.740 & -0.982 & 1.000 \\
\hline 200.000 & 14.590 & 17.939 & 157.421 & 158.330 & -0.574 & 1.000 \\
\hline 200.000 & 11.165 & 17.828 & 152.920 & 154.050 & -0.734 & 1.000 \\
\hline 200.000 & 7.644 & 17.707 & 148.258 & 149.530 & -0.851 & 1.000 \\
\hline 200.000 & 5.233 & 17.619 & 145.036 & 145.450 & -0.284 & 0.000 \\
\hline 200.000 & 2.562 & 17.518 & 141.434 & 141.270 & 0.116 & 1.000 \\
\hline 200.000 & 0.300 & 17.428 & 138.347 & 138.490 & -0.103 & 1.000 \\
\hline 150.000 & 25.183 & 19.961 & 320.235 & 321.210 & -0.304 & 1.000 \\
\hline 150.000 & 22.913 & 19.920 & 315.522 & 318.010 & -0.782 & 1.000 \\
\hline 150.000 & 20.043 & 19.868 & 309.634 & 312.740 & -0.993 & 1.000 \\
\hline 150.000 & 17.035 & 19.813 & 303.542 & 301.330 & 0.734 & 1.000 \\
\hline 150.000 & 14.344 & 19.762 & 298.159 & 301.240 & -1.023 & 1.000 \\
\hline 150.000 & 11.566 & 19.708 & 292.661 & 294.580 & -0.652 & 1.000 \\
\hline 150.000 & 8.756 & 19.653 & 287.158 & 289.330 & -0.751 & 1.000 \\
\hline 150.000 & 6.030 & 19.597 & 281.873 & 284.720 & -1.000 & 1.000 \\
\hline 150.000 & 2.665 & 19.527 & 275.416 & 278.590 & -1.139 & 1.000 \\
\hline 130.000 & 31.980 & 20.727 & 482.779 & 478.710 & 0.850 & 1.000 \\
\hline 130.000 & 28.123 & 20.672 & 470.458 & 473.890 & -0.724 & 1.000 \\
\hline 130.000 & 24.916 & 20.625 & 460.477 & 457.020 & 0.756 & 1.000 \\
\hline 130.000 & 21.442 & 20.573 & 449.919 & 448.880 & 0.231 & 1.000 \\
\hline 130.000 & 18.283 & 20.525 & 440.536 & 440.960 & -0.096 & 1.000 \\
\hline 130.000 & 14.451 & 20.465 & 429.415 & 435.490 & -1.395 & 1.000 \\
\hline 130.000 & 11.069 & 20.411 & 419.825 & 424.650 & -1.136 & 1.000 \\
\hline 130.000 & 7.878 & 20.359 & 410.955 & 413.910 & -0.714 & 1.000 \\
\hline 130.000 & 5.243 & 20.315 & 403.759 & 405.110 & -0.333 & 1.000 \\
\hline 130.000 & 2.041 & 20.261 & 395.161 & 400.100 & -1.234 & 1.000 \\
\hline 120.000 & 30.380 & 21.032 & 602.752 & 599.520 & 0.539 & 1.000 \\
\hline
\end{tabular}


Table 14. VISCOSITY AT ELEVATED PRESSURES (continued)

Data from Diller et al. [91] (continued)

\begin{tabular}{|c|c|c|c|c|c|c|}
\hline $\begin{array}{l}\mathrm{T} \\
\mathrm{K}\end{array}$ & $\begin{array}{c}\mathrm{P} \\
\mathrm{MPa}\end{array}$ & $\begin{array}{c}\rho \\
\mathrm{mol}\end{array} \mathrm{dm}^{-3}$ & $\begin{array}{c}\eta, \text { calc } \\
\mu \mathrm{Pa} \cdot \mathrm{s}\end{array}$ & $\begin{array}{c}\eta, \text { expt } \\
\mu \mathrm{Pa} \cdot \mathrm{s}\end{array}$ & $\begin{array}{c}\text { Dev } \\
\%\end{array}$ & wt \\
\hline 120.000 & 26.917 & 20.986 & 588.190 & 587.430 & 0.129 & 1.000 \\
\hline 120.000 & 23.229 & 20.936 & 573.162 & 571.400 & 0.308 & 1.000 \\
\hline 120.000 & 19.750 & 20.888 & 559.414 & 556.980 & 0.437 & 1.000 \\
\hline 120.000 & 18.395 & 20.869 & 554.168 & 552.650 & 0.275 & 1.000 \\
\hline 120.000 & 14.810 & 20.818 & 540.560 & 536.030 & 0.845 & 1.000 \\
\hline 120.000 & 11.301 & 20.767 & 527.610 & 528.450 & -0.159 & 1.000 \\
\hline 120.000 & 7.815 & 20.716 & 515.087 & 512.010 & 0.601 & 1.000 \\
\hline 120.000 & 4.982 & 20.673 & 505.150 & 501.320 & 0.764 & 1.000 \\
\hline 120.000 & 2.057 & 20.628 & 495.099 & 492.610 & 0.505 & 1.000 \\
\hline 110.000 & 32.108 & 21.381 & 809.781 & 788.920 & 2.644 & 0.000 \\
\hline 110.000 & 28.650 & 21.339 & 788.299 & 785.820 & 0.316 & 1.000 \\
\hline 110.000 & 25.260 & 21.297 & 768.026 & 771.740 & -0.481 & 1.000 \\
\hline 110.000 & 21.233 & 21.247 & 744.881 & 750.080 & -0.693 & 1.000 \\
\hline 110.000 & 19.505 & 21.225 & 735.242 & 733.530 & 0.233 & 1.000 \\
\hline 110.000 & 16.226 & 21.183 & 717.420 & 716.280 & 0.159 & 1.000 \\
\hline 110.000 & 12.728 & 21.137 & 699.037 & 701.350 & -0.330 & 1.000 \\
\hline 110.000 & 9.357 & 21.092 & 681.899 & 679.320 & 0.380 & 1.000 \\
\hline 110.000 & 6.522 & 21.053 & 667.903 & 675.320 & -1.098 & 1.000 \\
\hline 110.000 & 3.900 & 21.017 & 655.279 & 659.390 & -0.623 & 1.000 \\
\hline 110.000 & 1.479 & 20.983 & 643.884 & 643.780 & 0.016 & 1.000 \\
\hline 100.000 & 31.549 & 21.701 & 1144.445 & 1130.240 & 1.257 & 1.000 \\
\hline 100.000 & 27.871 & 21.660 & 1107.439 & 1106.280 & 0.105 & 1.000 \\
\hline 100.000 & 24.358 & 21.620 & 1073.829 & 1075.970 & -0.199 & 1.000 \\
\hline 100.000 & 22.203 & 21.595 & 1053.995 & 1047.810 & 0.590 & 1.000 \\
\hline 100.000 & 22.180 & 21.595 & 1053.782 & 1046.400 & 0.705 & 1.000 \\
\hline 100.000 & 18.958 & 21.557 & 1025.160 & 1025.810 & -0.063 & 1.000 \\
\hline 100.000 & 15.432 & 21.516 & 995.174 & 999.900 & -0.473 & 1.000 \\
\hline 100.000 & 11.018 & 21.463 & 959.450 & 971.220 & -1.212 & 1.000 \\
\hline 100.000 & 7.204 & 21.416 & 930.068 & 931.960 & -0.203 & 1.000 \\
\hline 100.000 & 3.274 & 21.367 & 901.133 & 906.500 & -0.592 & 1.000 \\
\hline 95.000 & $0.362 E-05$ & 21.503 & 1057.128 & 1073.360 & -1.512 & 1.000 \\
\hline 100.000 & $0.111 \mathrm{E}-04$ & 21.325 & 877.990 & 868.420 & 1.102 & 1.000 \\
\hline 105.000 & $0.303 E-04$ & 21.144 & 742.077 & 743.510 & -0.193 & 1.000 \\
\hline 110.000 & $0.747 E-04$ & 20.962 & 637.039 & 632.120 & 0.778 & 1.000 \\
\hline 115.000 & $0.169 \mathrm{E}-03$ & 20.779 & 554.363 & 552.050 & 0.419 & 1.000 \\
\hline 120.000 & $0.355 E-03$ & 20.595 & 488.153 & 483.220 & 1.021 & 1.000 \\
\hline
\end{tabular}


Table 14. VISCOSITY AT ELEVATED PRESSURES (continued)

Data from Diller et al. [91] (continued)

\begin{tabular}{|c|c|c|c|c|c|c|}
\hline $\begin{array}{l}\mathrm{T} \\
\mathrm{K}\end{array}$ & $\begin{array}{c}\mathrm{P} \\
\mathrm{MPa}\end{array}$ & $\stackrel{\rho}{\mathrm{mol} \cdot \mathrm{dm}^{-3}}$ & $\begin{array}{c}\eta, \text { calc } \\
\mu \mathrm{Pa} \cdot \mathrm{s}\end{array}$ & $\begin{array}{c}\eta, \text { expt } \\
\mu \mathrm{Pa} \cdot \mathrm{s}\end{array}$ & $\begin{array}{c}\text { Dev } \\
8\end{array}$ & wt \\
\hline 125.000 & $0.697 \mathrm{E}-03$ & 20.411 & 434.269 & 428.110 & 1.439 & 1.000 \\
\hline 130.000 & $0.129 \mathrm{E}-02$ & 20.225 & 389.763 & 390.190 & -0.109 & 1.000 \\
\hline 135.000 & $0.228 \mathrm{E}-02$ & 20.038 & 352.501 & 357.380 & -1.365 & 1.000 \\
\hline 140.000 & $0.383 \mathrm{E}-02$ & 19.850 & 320.913 & 318.910 & 0.628 & 1.000 \\
\hline 145.000 & $0.620 \mathrm{E}-02$ & 19.661 & 293.830 & 297.350 & -1.184 & 1.000 \\
\hline 150.000 & $0.967 \mathrm{E}-02$ & 19.470 & 270.366 & 271.600 & -0.454 & 1.000 \\
\hline 155.000 & $0.146 \mathrm{E}-01$ & 19.277 & 249.843 & 251.690 & -0.734 & 1.000 \\
\hline 160.000 & $0.215 \mathrm{E}-01$ & 19.083 & 231.736 & 233.440 & -0.730 & 1.000 \\
\hline 165.000 & $0.307 \mathrm{E}-01$ & 18.886 & 215.632 & 216.930 & -0.598 & 1.000 \\
\hline 170.000 & $0.429 E-01$ & 18.686 & 201.206 & 202.390 & -0.585 & 1.000 \\
\hline 175.000 & $0.587 \mathrm{E}-01$ & 18.484 & 188.197 & 185.700 & 1.345 & 1.000 \\
\hline 180.000 & $0.787 \mathrm{E}-01$ & 18.279 & 176.393 & 173.890 & 1.440 & 1.000 \\
\hline 185.000 & 0.104 & 18.071 & 165.625 & 162.630 & 1.841 & 1.000 \\
\hline 190.000 & 0.135 & 17.860 & 155.750 & 154.330 & 0.920 & 1.000 \\
\hline 195.000 & 0.172 & 17.644 & 146.652 & 143.910 & 1.905 & 1.000 \\
\hline 200.000 & 0.217 & 17.425 & 138.234 & 137.350 & 0.644 & 1.000 \\
\hline 205.000 & 0.271 & 17.201 & 130.414 & 128.970 & 1.120 & 1.000 \\
\hline 210.000 & 0.341 & 16.972 & 123.132 & 121.750 & 1.135 & 1.000 \\
\hline 215.000 & 0.414 & 16.738 & 116.310 & 114.900 & 1.227 & 1.000 \\
\hline 220.000 & 0.499 & 16.498 & 109.906 & 108.150 & 1.624 & 1.000 \\
\hline 225.000 & 0.596 & 16.252 & 103.876 & 102.120 & 1.720 & 1.000 \\
\hline 230.000 & 0.707 & 15.999 & 98.181 & 97.270 & 0.936 & 1.000 \\
\hline 235.000 & 0.832 & 15.737 & 92.785 & 91.840 & 1.029 & 1.000 \\
\hline 240.000 & 0.973 & 15.467 & 87.658 & 87.080 & 0.664 & 1.000 \\
\hline 245.000 & 1.130 & 15.186 & 82.770 & 82.380 & 0.474 & 1.000 \\
\hline 250.000 & 1.304 & 14.894 & 78.092 & 77.810 & 0.363 & 1.000 \\
\hline 255.000 & 1.499 & 14.589 & 73.603 & 73.350 & 0.345 & 1.000 \\
\hline 260.000 & 1.716 & 14.268 & 69.280 & 68.900 & 0.551 & 1.000 \\
\hline 265.000 & 1.954 & 13.928 & 65.087 & 64.970 & 0.180 & 1.000 \\
\hline 270.000 & 2.214 & 13.565 & 60.996 & 60.790 & 0.339 & 1.000 \\
\hline 275.000 & 2.498 & 13.173 & 56.971 & 56.930 & 0.072 & 1.000 \\
\hline 280.000 & 2.809 & 12.742 & 52.966 & 52.850 & 0.219 & 1.000 \\
\hline 285.000 & 3.147 & 12.259 & 48.910 & 49.000 & -0.183 & 1.000 \\
\hline 290.000 & 3.517 & 11.697 & 44.703 & 44.570 & 0.299 & 1.000 \\
\hline 295.000 & 3.919 & 11.010 & 40.163 & 40.710 & -1.343 & 1.000 \\
\hline 300.000 & 4.357 & 10.084 & 34.918 & 35.160 & -0.687 & 1.000 \\
\hline
\end{tabular}


Data from Diller et al. [91] (continued)

Comparisons based on experimental pressures:

Number of Points [91] 164

$$
\begin{aligned}
& \mathrm{AAD} ;=0.84 \quad \mathrm{BIAS} \%=-0.17 \quad \mathrm{RMS} \%=1.05 \\
& \mathrm{AAD}=1.84 \mathrm{BIAS}=0.02 \mathrm{RMS}=3.40 \mu \mathrm{Pa} \cdot \mathrm{s}
\end{aligned}
$$

Weighted Data:

Number of Points [91] 144

$$
\begin{aligned}
& \mathrm{AAD} \%=0.69 \quad \mathrm{BIAS} \%=-0.02 \quad \mathrm{RMS} \%=0.84 \\
& \mathrm{AAD}=1.93 \mathrm{BIAS}=-0.10 \mathrm{RMS}=3.18 \mu \mathrm{Pa} \cdot \mathrm{s}
\end{aligned}
$$

Comparisons based on experimental densities:

Number of Points [91] 164

$$
\begin{aligned}
& \mathrm{AAD}=0.87 \quad \mathrm{BIAS} \%=-0.09 \quad \mathrm{RMS} \%=1.09 \\
& \mathrm{AAD}=1.85 \quad \mathrm{BIAS}=0.24 \mathrm{RMS}=3.39 \mu \mathrm{Pa} \cdot \mathrm{s}
\end{aligned}
$$

Weighted Data:

Number of Points [91] 144

$$
\begin{aligned}
& \mathrm{AAD} \%=0.73 \quad \text { BIAS } 8=0.06 \quad \text { RMS } 8=0.88 \\
& \mathrm{AAD}=1.94 \quad \mathrm{BIAS}=0.15 \mathrm{RMS}=3.20 \mu \mathrm{Pa} \cdot \mathrm{s}
\end{aligned}
$$

Data from Diller [92]

\begin{tabular}{crrrrrr}
\hline $\mathrm{T}$ & $\begin{array}{c}\mathrm{P} \\
\mathrm{MPa}\end{array}$ & $\begin{array}{c}\rho \\
\mathrm{mol} \cdot \mathrm{dm}^{-3}\end{array}$ & $\begin{array}{c}\eta \text {, calc } \\
\mu \mathrm{Pa} \cdot \mathrm{s}\end{array}$ & $\begin{array}{c}\eta \text { expt } \\
\mu \mathrm{Pa} \cdot \mathrm{s}\end{array}$ & $\begin{array}{c}\text { Dev } \\
8\end{array}$ & wt \\
\hline 294.800 & 51.914 & 16.052 & 101.086 & 100.200 & 0.884 & 1.000 \\
294.800 & 48.358 & 15.907 & 97.875 & 96.900 & 1.006 & 1.000 \\
294.600 & 41.470 & 15.607 & 91.666 & 90.700 & 1.066 & 1.000 \\
294.600 & 34.663 & 15.260 & 85.206 & 84.200 & 1.195 & 1.000 \\
294.600 & 27.789 & 14.846 & 78.351 & 78.300 & 0.065 & 1.000 \\
& & & & & & \\
294.600 & 20.908 & 14.334 & 70.924 & 71.500 & -0.806 & 1.000 \\
294.600 & 14.103 & 13.654 & 62.519 & 63.100 & -0.921 & 1.000 \\
294.600 & 7.639 & 12.603 & 52.037 & 52.600 & -1.070 & 1.000 \\
319.180 & 47.808 & 15.131 & 84.196 & 84.800 & -0.712 & 1.000 \\
319.160 & 40.922 & 14.765 & 78.183 & 75.300 & 3.829 & 0.000
\end{tabular}


Table 14. VISCOSITY AT ELEVATED PRESSURES (continued)

Data from Diller [92] (continued)

\begin{tabular}{|c|c|c|c|c|c|c|}
\hline $\begin{array}{l}\mathrm{T} \\
\mathrm{K}\end{array}$ & $\begin{array}{c}\mathrm{P} \\
\mathrm{MPa}\end{array}$ & $\stackrel{\rho}{\mathrm{mol}} \cdot \mathrm{dm}^{-3}$ & $\begin{array}{c}\eta, \text { calc } \\
\mu \mathrm{Pa} \cdot \mathrm{s}\end{array}$ & $\begin{array}{c}\eta, \text { expt } \\
\mu \mathrm{Pa} \cdot \mathrm{s}\end{array}$ & $\begin{array}{c}\text { Dev } \\
\%\end{array}$ & wt \\
\hline 319.140 & 34.752 & 14.383 & 72.550 & 73.700 & -1.560 & 1.000 \\
\hline 319.140 & 31.182 & 14.128 & 69.131 & 70.300 & -1.663 & 1.000 \\
\hline 319.110 & 20.984 & 13.186 & 58.279 & 59.100 & -1.389 & 1.000 \\
\hline 319.410 & 13.918 & 12.093 & 48.477 & 49.500 & -2.067 & 1.000 \\
\hline 319.110 & 10.506 & 11.214 & 42.126 & 42.500 & -0.880 & 1.000 \\
\hline 319.060 & 8.709 & 10.423 & 37.300 & 37.300 & -0.001 & 1.000 \\
\hline 319.640 & 8.658 & 10.316 & 36.720 & 36.800 & -0.218 & 1.000 \\
\hline 319.640 & 8.327 & 10.100 & 35.548 & 35.600 & -0.145 & 1.000 \\
\hline 319.100 & 7.352 & 9.308 & 31.599 & 31.400 & 0.635 & 1.000 \\
\hline 319.630 & 7.056 & 8.756 & 29.170 & 29.000 & 0.588 & 1.000 \\
\hline 319.040 & 6.886 & 8.609 & 28.541 & 28.200 & 1.209 & 1.000 \\
\hline 319.630 & 6.853 & 8.361 & 27.554 & 27.700 & -0.528 & 1.000 \\
\hline 319.630 & 6.574 & 7.606 & 24.734 & 25.000 & -1.065 & 1.000 \\
\hline 319.040 & 6.427 & 7.334 & 23.780 & 23.300 & 2.061 & 1.000 \\
\hline 319.150 & 6.188 & 6.281 & 20.505 & 20.400 & 0.515 & 1.000 \\
\hline 319.170 & 5.905 & 5.159 & 17.554 & 17.300 & 1.471 & 1.000 \\
\hline 319.630 & 5.201 & 3.494 & 14.086 & 14.000 & 0.615 & 1.000 \\
\hline 319.640 & 5.160 & 3.430 & 13.973 & 13.800 & 1.252 & 1.000 \\
\hline 319.170 & 4.547 & 2.676 & 12.724 & 12.700 & 0.192 & 1.000 \\
\hline 319.580 & 3.631 & 1.857 & 11.620 & 11.500 & 1.042 & 1.000 \\
\hline 319.170 & 3.565 & 1.814 & 11.555 & 11.500 & 0.480 & 1.000 \\
\hline 319.670 & 3.498 & 1.758 & 11.504 & 11.300 & 1.808 & 1.000 \\
\hline 319.690 & 1.706 & 0.722 & 10.461 & 10.400 & 0.582 & 1.000 \\
\hline 399.910 & 54.893 & 13.209 & 61.838 & 61.900 & -0.100 & 1.000 \\
\hline 399.790 & 54.201 & 13.168 & 61.384 & 61.500 & -0.188 & 1.000 \\
\hline 399.880 & 48.925 & 12.796 & 57.609 & 57.600 & 0.016 & 1.000 \\
\hline 399.790 & 48.144 & 12.739 & 57.063 & 57.500 & -0.761 & 1.000 \\
\hline 399.880 & 41.419 & 12.171 & 51.988 & 52.200 & -0.407 & 1.000 \\
\hline 399.790 & 41.318 & 12.164 & 51.929 & 52.400 & -0.899 & 1.000 \\
\hline 399.840 & 34.725 & 11.466 & 46.558 & 46.600 & -0.090 & 1.000 \\
\hline 399.790 & 34.504 & 11.441 & 46.380 & 46.800 & -0.898 & 1.000 \\
\hline 399.950 & 27.949 & 10.503 & 40.357 & 40.500 & -0.353 & 1.000 \\
\hline 399.700 & 27.858 & 10.498 & 40.318 & 40.700 & -0.939 & 1.000 \\
\hline 399.790 & 27.574 & 10.446 & 40.019 & 40.400 & -0.943 & 1.000 \\
\hline 399.660 & 24.387 & 9.844 & 36.739 & 36.700 & 0.106 & 1.000 \\
\hline
\end{tabular}


Table 14. VISCOSITY AT ELEVATED PRESSURES (continued)

Data from Diller [92] (continued)

\begin{tabular}{|c|c|c|c|c|c|c|}
\hline $\begin{array}{l}\mathrm{T} \\
\mathrm{K}\end{array}$ & $\begin{array}{c}\mathrm{P} \\
\mathrm{MPa}\end{array}$ & $\stackrel{\rho}{\mathrm{mol} \cdot \mathrm{dm}^{-3}}$ & $\begin{array}{c}\eta, \text { calc } \\
\mu \mathrm{Pa} \cdot \mathrm{s}\end{array}$ & $\begin{array}{c}\eta, \operatorname{expt} \\
\mu \mathrm{Pa} \cdot \mathrm{s}\end{array}$ & $\begin{array}{c}\mathrm{Dev} \\
\frac{8}{\delta}\end{array}$ & wt \\
\hline 399.740 & 20.593 & 8.911 & 32.333 & 32.500 & -0.514 & 1.000 \\
\hline 399.660 & 17.589 & 7.940 & 28.434 & 28.600 & -0.582 & 1.000 \\
\hline 399.760 & 17.205 & 7.790 & 27.889 & 27.900 & -0.041 & 1.000 \\
\hline 399.750 & 13.758 & 6.249 & 22.939 & 23.100 & -0.697 & 1.000 \\
\hline 399.600 & 10.628 & 4.556 & 18.693 & 18.800 & -0.570 & 1.000 \\
\hline 399.540 & 10.314 & 4.384 & 18.319 & 18.300 & 0.106 & 1.000 \\
\hline 399.100 & 6.815 & 2.579 & 15.014 & 15.300 & -1.872 & 1.000 \\
\hline 399.750 & 6.765 & 2.547 & 14.983 & 15.300 & -2.069 & 1.000 \\
\hline 399.730 & 6.695 & 2.514 & 14.934 & 15.500 & -3.653 & 0.000 \\
\hline 399.930 & 6.497 & 2.420 & 14.799 & 15.200 & -2.636 & 1.000 \\
\hline 399.730 & 3.658 & 1.235 & 13.274 & 13.400 & -0.942 & 1.000 \\
\hline 400.110 & 3.475 & 1.164 & 13.208 & 13.100 & 0.827 & 1.000 \\
\hline 499.600 & 50.427 & 10.402 & 43.111 & 42.900 & 0.491 & 1.000 \\
\hline 499.530 & 47.482 & 10.111 & 41.432 & 41.300 & 0.320 & 1.000 \\
\hline 499.530 & 42.747 & 9.586 & 38.646 & 38.400 & 0.642 & 1.000 \\
\hline 499.530 & 38.697 & 9.073 & 36.180 & 35.900 & 0.780 & 1.000 \\
\hline 499.490 & 34.488 & 8.463 & 33.527 & 33.100 & 1.291 & 1.000 \\
\hline 499.080 & 30.982 & 7.891 & 31.266 & 30.800 & 1.513 & 1.000 \\
\hline 499.600 & 27.538 & 7.229 & 28.930 & 28.600 & 1.154 & 1.000 \\
\hline 499.630 & 24.072 & 6.486 & 26.570 & 26.300 & 1.027 & 1.000 \\
\hline 499.630 & 20.488 & 5.621 & 24.137 & 23.900 & 0.992 & 1.000 \\
\hline 500.100 & 18.308 & 5.040 & 22.687 & 22.600 & 0.384 & 1.000 \\
\hline 499.650 & 17.141 & 4.729 & 21.944 & 21.800 & 0.660 & 1.000 \\
\hline 499.660 & 13.681 & 3.748 & 19.857 & 19.700 & 0.797 & 1.000 \\
\hline 500.010 & 13.532 & 3.700 & 19.774 & 19.700 & 0.373 & 1.000 \\
\hline 499.660 & 9.906 & 2.653 & 17.904 & 17.700 & 1.151 & 1.000 \\
\hline 500.020 & 9.614 & 2.566 & 17.774 & 17.800 & -0.145 & 1.000 \\
\hline 499.710 & 6.814 & 1.775 & 16.612 & 16.400 & 1.293 & 1.000 \\
\hline 500.020 & 6.649 & 1.727 & 16.557 & 16.500 & 0.347 & 1.000 \\
\hline 499.730 & 3.458 & 0.868 & 15.535 & 15.100 & 2.879 & 0.000 \\
\hline 500.120 & 3.425 & 0.859 & 15.535 & 15.100 & 2.879 & 0.000 \\
\hline
\end{tabular}


Table 14. VISCOSITY AT ELEVATED PRESSURES (continued)

Data from Diller [92] (continued)

Comparisons based on experimental pressures:

Number of Points [92] 76

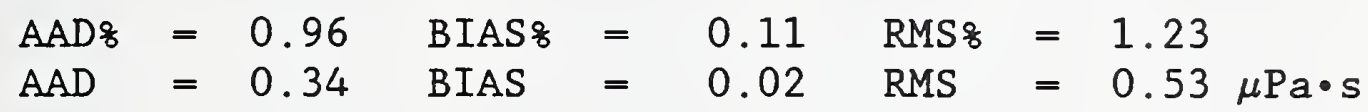

Weighted Data:

Number of Points [92] 72

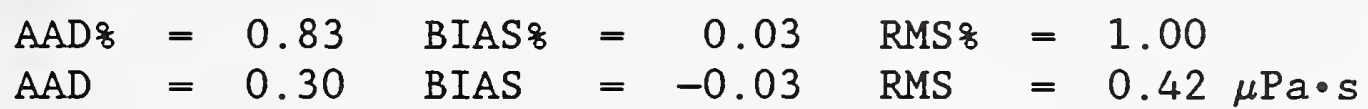

Comparisons based on experimental densities:

Number of Points [92] 76

$\mathrm{AAD} \&=1.02 \quad$ BIAS\& $=0.07$ RMS $8=1.30$

$\mathrm{AAD}=0.37$ BIAS $=0.00 \quad \mathrm{RMS}=0.58 \mu \mathrm{Pa} \cdot \mathrm{s}$

Weighted Data:

Number of Points [92] 72

$\mathrm{AAD} \&=0.89 \quad \mathrm{BIAS} \&=-0.01 \quad \mathrm{RMS} \%=1.07$

$\mathrm{AAD}=0.33 \mathrm{BIAS}=-0.04 \mathrm{RMS}=0.46 \mu \mathrm{Pa} \cdot \mathrm{s}$

Data from Eakin et al. [71]

\begin{tabular}{ccccccc}
\hline $\mathrm{T}$ & $\begin{array}{c}\mathrm{P} \\
\mathrm{MPa}\end{array}$ & $\begin{array}{c}\rho \\
\mathrm{mol} \cdot \mathrm{dm}^{-3}\end{array}$ & $\begin{array}{c}\eta, \mathrm{calc} \\
\mu \mathrm{Pa} \cdot \mathrm{s}\end{array}$ & $\begin{array}{c}\eta \text { expt } \\
\mu \mathrm{Pa} \cdot \mathrm{s}\end{array}$ & $\begin{array}{c}\text { Dev } \\
8\end{array}$ & wt \\
\hline 294.261 & 0.690 & 0.299 & 9.389 & 9.300 & 0.962 & 0.000 \\
294.261 & 1.379 & 0.639 & 9.625 & 9.500 & 1.320 & 0.000 \\
294.261 & 2.068 & 1.040 & 9.958 & 9.800 & 1.612 & 0.000 \\
294.261 & 2.758 & 1.541 & 10.456 & 10.100 & 3.524 & 0.000 \\
294.261 & 3.447 & 2.255 & 11.321 & 10.400 & 8.853 & 0.000 \\
& & & & & & \\
294.261 & 4.137 & 11.345 & 42.338 & 44.000 & -3.777 & 0.000 \\
294.261 & 5.516 & 12.024 & 47.249 & 47.500 & -0.528 & 0.000 \\
294.261 & 6.895 & 12.449 & 50.697 & 50.500 & 0.391 & 0.000 \\
294.261 & 10.342 & 13.150 & 57.164 & 56.700 & 0.818 & 0.000 \\
294.261 & 13.790 & 13.635 & 62.295 & 62.100 & 0.314 & 0.000
\end{tabular}


Table 14. VISCOSITY AT ELEVATED PRESSURES (continued)

Data from Eakin et al. [71] (continued)

\begin{tabular}{|c|c|c|c|c|c|c|}
\hline $\begin{array}{l}\mathrm{T} \\
\mathrm{K}\end{array}$ & $\begin{array}{c}\mathrm{P} \\
\mathrm{MPa}\end{array}$ & $\stackrel{\rho}{\mathrm{mol} \cdot \mathrm{dm}^{-3}}$ & $\begin{array}{c}\eta, \text { calc } \\
\mu \mathrm{Pa} \cdot \mathrm{s}\end{array}$ & $\begin{array}{c}\eta, \text { expt } \\
\mu \mathrm{Pa} \cdot \mathrm{s}\end{array}$ & $\begin{array}{c}\text { Dev } \\
\frac{8}{8}\end{array}$ & wt \\
\hline 294.261 & 20.684 & 14.331 & 70.865 & 70.500 & 0.518 & 0.000 \\
\hline 294.261 & 27.579 & 14.845 & 78.330 & 78.000 & 0.423 & 0.000 \\
\hline 294.261 & 34.474 & 15.261 & 85.221 & 84.500 & 0.853 & 0.000 \\
\hline 294.261 & 41.368 & 15.613 & 91.774 & 90.700 & 1.184 & 0.000 \\
\hline 294.261 & 48.263 & 15.920 & 98.117 & 96.000 & 2.206 & 0.000 \\
\hline 294.261 & 55.158 & 16.193 & 104.331 & 101.300 & 2.992 & 0.000 \\
\hline 294.261 & 62.053 & 16.439 & 110.467 & 106.200 & 4.018 & 0.000 \\
\hline 294.261 & 68.948 & 16.664 & 116.566 & 111.300 & 4.732 & 0.000 \\
\hline 310.928 & 0.690 & 0.280 & 9.873 & 9.800 & 0.749 & 0.000 \\
\hline 310.928 & 1.379 & 0.591 & 10.094 & 10.000 & 0.938 & 0.000 \\
\hline 310.928 & 2.068 & 0.943 & 10.385 & 10.300 & 0.830 & 0.000 \\
\hline 310.928 & 2.758 & 1.353 & 10.781 & 10.600 & 1.707 & 0.000 \\
\hline 310.928 & 3.447 & 1.852 & 11.342 & 10.900 & 4.057 & 0.000 \\
\hline 310.928 & 4.137 & 2.509 & 12.213 & 11.900 & 2.634 & 0.000 \\
\hline 310.928 & 5.516 & 7.517 & 24.157 & 24.900 & -2.984 & 0.000 \\
\hline 310.928 & 6.895 & 10.421 & 37.059 & 37.100 & -0.111 & 0.000 \\
\hline 310.928 & 10.342 & 11.894 & 46.692 & 46.800 & -0.231 & 0.000 \\
\hline 310.928 & 13.790 & 12.630 & 52.728 & 52.800 & -0.135 & 0.000 \\
\hline 310.928 & 20.684 & 13.550 & 61.870 & 62.100 & -0.371 & 0.000 \\
\hline 310.928 & 27.579 & 14.176 & 69.418 & 69.600 & -0.261 & 0.000 \\
\hline 310.928 & 34.474 & 14.662 & 76.220 & 76.200 & 0.027 & 0.000 \\
\hline 310.928 & 41.368 & 15.063 & 82.598 & 82.300 & 0.362 & 0.000 \\
\hline 310.928 & 48.263 & 15.407 & 88.714 & 87.700 & 1.156 & 0.000 \\
\hline 310.928 & 55.158 & 15.709 & 94.665 & 93.200 & 1.572 & 0.000 \\
\hline 310.928 & 62.053 & 15.980 & 100.511 & 98.700 & 1.835 & 0.000 \\
\hline 310.928 & 68.948 & 16.225 & 106.297 & 103.800 & 2.405 & 0.000 \\
\hline 327.594 & 0.690 & 0.264 & 10.350 & 10.300 & 0.489 & 0.000 \\
\hline 327.594 & 1.379 & 0.551 & 10.558 & 10.500 & 0.557 & 0.000 \\
\hline 327.594 & 2.068 & 0.868 & 10.822 & 10.700 & 1.140 & 0.000 \\
\hline 327.594 & 2.758 & 1.223 & 11.159 & 11.000 & 1.444 & 0.000 \\
\hline 327.594 & 3.447 & 1.628 & 11.597 & 11.300 & 2.629 & 0.000 \\
\hline 327.594 & 4.137 & 2.104 & 12.184 & 12.000 & 1.530 & 0.000 \\
\hline 327.594 & 5.516 & 3.437 & 14.241 & 14.300 & -0.415 & 0.000 \\
\hline 327.594 & 6.895 & 6.045 & 20.109 & 19.700 & 2.076 & 0.000 \\
\hline 327.594 & 10.342 & 10.278 & 36.744 & 36.500 & 0.669 & 0.000 \\
\hline 327.594 & 13.790 & 11.483 & 44.202 & 44.300 & -0.220 & 0.000 \\
\hline
\end{tabular}


Table 14. VISCOSITY AT ELEVATED PRESSURES (continued)

Data from Eakin et al. [71] (continued)

\begin{tabular}{|c|c|c|c|c|c|c|}
\hline $\begin{array}{l}\mathrm{T} \\
\mathrm{K}\end{array}$ & $\begin{array}{c}\mathrm{P} \\
\mathrm{MPa}\end{array}$ & $\begin{array}{c}\rho \\
\mathrm{mol} \cdot \mathrm{dm}^{-3}\end{array}$ & $\begin{array}{c}\eta, \text { calc } \\
\mu \mathrm{Pa} \cdot \mathrm{s}\end{array}$ & $\begin{array}{c}\eta, \text { expt } \\
\mu \mathrm{Pa} \cdot \mathrm{s}\end{array}$ & $\begin{array}{c}\text { Dev } \\
8\end{array}$ & wt \\
\hline 327.594 & 20.684 & 12.725 & 54.122 & 54.300 & -0.327 & 0.000 \\
\hline 327.594 & 27.579 & 13.489 & 61.806 & 61.700 & 0.172 & 0.000 \\
\hline 327.594 & 34.474 & 14.056 & 68.548 & 68.500 & 0.070 & 0.000 \\
\hline 327.594 & 41.368 & 14.512 & 74.777 & 74.300 & 0.642 & 0.000 \\
\hline 327.594 & 48.263 & 14.896 & 80.696 & 80.000 & 0.870 & 0.000 \\
\hline 327.594 & 55.158 & 15.230 & 86.416 & 85.500 & 1.071 & 0.000 \\
\hline 327.594 & 62.053 & 15.526 & 92.007 & 91.300 & 0.775 & 0.000 \\
\hline 327.594 & 68.948 & 15.792 & 97.519 & 96.300 & 1.265 & 0.000 \\
\hline 344.261 & 0.690 & 0.249 & 10.820 & 10.800 & 0.189 & 0.000 \\
\hline 344.261 & 1.379 & 0.517 & 11.018 & 11.000 & 0.167 & 0.000 \\
\hline 344.261 & 2.068 & 0.807 & 11.261 & 11.200 & 0.543 & 0.000 \\
\hline 344.261 & 2.758 & 1.123 & 11.559 & 11.500 & 0.510 & 0.000 \\
\hline 344.261 & 3.447 & 1.472 & 11.927 & 11.800 & 1.076 & 0.000 \\
\hline 344.261 & 4.137 & 1.862 & 12.387 & 12.200 & 1.532 & 0.000 \\
\hline 344.261 & 5.516 & 2.810 & 13.722 & 13.700 & 0.160 & 0.000 \\
\hline 344.261 & 6.895 & 4.113 & 16.053 & 15.700 & 2.250 & 0.000 \\
\hline 344.261 & 10.342 & 8.263 & 27.923 & 27.400 & 1.909 & 0.000 \\
\hline 344.261 & 13.790 & 10.181 & 36.725 & 36.700 & 0.069 & 0.000 \\
\hline 344.261 & 20.684 & 11.859 & 47.470 & 47.300 & 0.358 & 0.000 \\
\hline 344.261 & 27.579 & 12.788 & 55.300 & 54.800 & 0.913 & 0.000 \\
\hline 344.261 & 34.474 & 13.445 & 61.992 & 61.700 & 0.473 & 0.000 \\
\hline 344.261 & 41.368 & 13.961 & 68.087 & 67.500 & 0.870 & 0.000 \\
\hline 344.261 & 48.263 & 14.389 & 73.827 & 73.000 & 1.132 & 0.000 \\
\hline 344.261 & 55.158 & 14.756 & 79.339 & 78.300 & 1.326 & 0.000 \\
\hline 344.261 & 62.053 & 15.078 & 84.702 & 84.500 & 0.239 & 0.000 \\
\hline 344.261 & 68.948 & 15.366 & 89.968 & 89.000 & 1.088 & 0.000 \\
\hline 360.928 & 0.690 & 0.237 & 11.284 & 11.300 & -0.146 & 0.000 \\
\hline 360.928 & 1.379 & 0.488 & 11.473 & 11.500 & -0.237 & 0.000 \\
\hline 360.928 & 2.068 & 0.756 & 11.699 & 11.700 & -0.012 & 0.000 \\
\hline 360.928 & 2.758 & 1.043 & 11.968 & 12.000 & -0.264 & 0.000 \\
\hline 360.928 & 3.447 & 1.353 & 12.291 & 12.300 & -0.077 & 0.000 \\
\hline 360.928 & 4.137 & 1.690 & 12.677 & 12.600 & 0.608 & 0.000 \\
\hline 360.928 & 5.516 & 2.460 & 13.703 & 13.600 & 0.755 & 0.000 \\
\hline 360.928 & 6.895 & 3.397 & 15.219 & 15.000 & 1.461 & 0.000 \\
\hline 360.928 & 10.342 & 6.458 & 22.339 & 22.200 & 0.625 & 0.000 \\
\hline 360.928 & 13.790 & 8.805 & 30.647 & 30.800 & -0.498 & 0.000 \\
\hline
\end{tabular}


Table 14. VISCOSITY AT ELEVATED PRESSURES (continued)

Data from Eakin et al. [71] (continued)

\begin{tabular}{|c|c|c|c|c|c|c|}
\hline $\begin{array}{l}\mathrm{T} \\
\mathrm{K}\end{array}$ & $\begin{array}{c}\mathrm{P} \\
\mathrm{MPa}\end{array}$ & $\stackrel{\rho}{\mathrm{mol} \cdot \mathrm{dm}^{-3}}$ & $\begin{array}{c}\eta, \text { calc } \\
\mu \mathrm{Pa} \cdot \mathrm{s}\end{array}$ & $\begin{array}{c}\eta, \operatorname{expt} \\
\mu \mathrm{Pa} \cdot \mathrm{s}\end{array}$ & $\begin{array}{c}\text { Dev } \\
8\end{array}$ & wt \\
\hline 360.928 & 20.684 & 10.963 & 41.832 & 41.500 & 0.800 & 0.000 \\
\hline 360.928 & 27.579 & 12.077 & 49.759 & 49.500 & 0.523 & 0.000 \\
\hline 360.928 & 34.474 & 12.835 & 56.390 & 56.000 & 0.696 & 0.000 \\
\hline 360.928 & 41.368 & 13.414 & 62.354 & 62.000 & 0.570 & 0.000 \\
\hline 360.928 & 48.263 & 13.887 & 67.924 & 67.000 & 1.379 & 0.000 \\
\hline 360.928 & 55.158 & 14.288 & 73.244 & 72.200 & 1.445 & 0.000 \\
\hline 360.928 & 62.053 & 14.637 & 78.397 & 77.500 & 1.158 & 0.000 \\
\hline 360.928 & 68.948 & 14.947 & 83.441 & 82.300 & 1.386 & 0.000 \\
\hline 377.594 & 0.690 & 0.225 & 11.740 & 11.700 & 0.339 & 0.000 \\
\hline 377.594 & 1.379 & 0.462 & 11.921 & 11.900 & 0.179 & 0.000 \\
\hline 377.594 & 2.068 & 0.712 & 12.134 & 12.200 & -0.543 & 0.000 \\
\hline 377.594 & 2.758 & 0.977 & 12.382 & 12.500 & -0.946 & 0.000 \\
\hline 377.594 & 3.447 & 1.258 & 12.671 & 12.800 & -1.008 & 0.000 \\
\hline 377.594 & 4.137 & 1.557 & 13.008 & 13.000 & 0.060 & 0.000 \\
\hline 377.594 & 5.516 & 2.219 & 13.857 & 13.800 & 0.415 & 0.000 \\
\hline 377.594 & 6.895 & 2.977 & 15.009 & 14.900 & 0.735 & 0.000 \\
\hline 377.594 & 10.342 & 5.302 & 19.753 & 19.700 & 0.268 & 0.000 \\
\hline 377.594 & 13.790 & 7.548 & 26.341 & 26.200 & 0.537 & 0.000 \\
\hline 377.594 & 20.684 & 10.066 & 37.176 & 36.800 & 1.023 & 0.000 \\
\hline 377.594 & 27.579 & 11.368 & 45.077 & 45.000 & 0.170 & 0.000 \\
\hline 377.594 & 34.474 & 12.228 & 51.613 & 51.300 & 0.610 & 0.000 \\
\hline 377.594 & 41.368 & 12.873 & 57.438 & 57.000 & 0.768 & 0.000 \\
\hline 377.594 & 48.263 & 13.392 & 62.843 & 62.000 & 1.359 & 0.000 \\
\hline 377.594 & 55.158 & 13.828 & 67.980 & 66.700 & 1.919 & 0.000 \\
\hline 377.594 & 62.053 & 14.204 & 72.938 & 71.700 & 1.727 & 0.000 \\
\hline 377.594 & 68.948 & 14.536 & 77.777 & 76.100 & 2.203 & 0.000 \\
\hline 394.261 & 0.690 & 0.215 & 12.189 & 12.200 & -0.089 & 0.000 \\
\hline 394.261 & 1.379 & 0.439 & 12.364 & 12.400 & -0.292 & 0.000 \\
\hline 394.261 & 2.068 & 0.674 & 12.565 & 12.600 & -0.278 & 0.000 \\
\hline 394.261 & 2.758 & 0.920 & 12.796 & 12.900 & -0.808 & 0.000 \\
\hline 394.261 & 3.447 & 1.179 & 13.060 & 13.200 & -1.062 & 0.000 \\
\hline 394.261 & 4.137 & 1.450 & 13.361 & 13.400 & -0.289 & 0.000 \\
\hline 394.261 & 5.516 & 2.037 & 14.095 & 14.200 & -0.742 & 0.000 \\
\hline 394.261 & 6.895 & 2.686 & 15.038 & 15.000 & 0.253 & 0.000 \\
\hline 394.261 & 10.342 & 4.576 & 18.575 & 18.500 & 0.407 & 0.000 \\
\hline 394.261 & 13.790 & 6.540 & 23.619 & 23.300 & 1.368 & 0.000 \\
\hline
\end{tabular}


Table 14. VISCOSITY AT ELEVATED PRESSURES (continued)

Data from Eakin et al. [71] (continued)

\begin{tabular}{|c|c|c|c|c|c|c|}
\hline $\begin{array}{l}\mathrm{T} \\
\mathrm{K}\end{array}$ & $\begin{array}{c}\mathrm{P} \\
\mathrm{MPa}\end{array}$ & $\stackrel{\rho}{\mathrm{mol}} \cdot \mathrm{dm}^{-3}$ & $\begin{array}{c}\eta, \text { calc } \\
\mu \mathrm{Pa} \cdot \mathrm{s}\end{array}$ & $\begin{array}{c}\eta, \operatorname{expt} \\
\mu \mathrm{Pa} \cdot \mathrm{s}\end{array}$ & $\begin{array}{c}\text { Dev } \\
\frac{o}{z}\end{array}$ & wt \\
\hline 394.261 & 20.684 & 9.205 & 33.469 & 33.300 & 0.509 & 0.000 \\
\hline 394.261 & 27.579 & 10.672 & 41.165 & 41.000 & 0.403 & 0.000 \\
\hline 394.261 & 34.474 & 11.632 & 47.556 & 47.300 & 0.542 & -0.000 \\
\hline 394.261 & 41.368 & 12.342 & 53.226 & 52.700 & 0.999 & 0.000 \\
\hline 394.261 & 48.263 & 12.907 & 58.464 & 57.800 & 1.149 & 0.000 \\
\hline 394.261 & 55.158 & 13.377 & 63.424 & 62.000 & 2.297 & 0.000 \\
\hline 394.261 & 62.053 & 13.780 & 68.198 & 66.600 & 2.399 & 0.000 \\
\hline 394.261 & 68.948 & 14.134 & 72.845 & 70.800 & 2.888 & 0.000 \\
\hline 410.928 & 0.690 & 0.206 & 12.632 & 12.600 & 0.252 & 0.000 \\
\hline 410.928 & 1.379 & 0.419 & 12.800 & 12.800 & 0.003 & 0.000 \\
\hline 410.928 & 2.068 & 0.641 & 12.992 & 13.100 & -0.825 & 0.000 \\
\hline 410.928 & 2.758 & 0.871 & 13.208 & 13.400 & -1.429 & 0.000 \\
\hline 410.928 & 3.447 & 1.111 & 13.453 & 13.600 & -1.084 & 0.000 \\
\hline 410.928 & 4.137 & 1.361 & 13.727 & 13.800 & -0.529 & 0.000 \\
\hline 410.928 & 5.516 & 1.892 & 14.378 & 14.500 & -0.845 & 0.000 \\
\hline 410.928 & 6.895 & 2.466 & 15.184 & 15.200 & -0.102 & 0.000 \\
\hline 410.928 & 10.342 & 4.078 & 18.021 & 17.800 & 1.243 & 0.000 \\
\hline 410.928 & 13.790 & 5.778 & 21.985 & 21.700 & 1.315 & 0.000 \\
\hline 410.928 & 20.684 & 8.417 & 30.625 & 30.500 & 0.410 & 0.000 \\
\hline 410.928 & 27.579 & 10.003 & 37.944 & 37.500 & 1.185 & 0.000 \\
\hline 410.928 & 34.474 & 11.053 & 44.130 & 43.700 & 0.985 & 0.000 \\
\hline 410.928 & 41.368 & 11.824 & 49.625 & 49.000 & 1.275 & 0.000 \\
\hline 410.928 & 48.263 & 12.433 & 54.691 & 54.000 & 1.279 & 0.000 \\
\hline 410.928 & 55.158 & 12.937 & 59.476 & 58.100 & 2.369 & 0.000 \\
\hline 410.928 & 62.053 & 13.367 & 64.073 & 62.500 & 2.517 & 0.000 \\
\hline 410.928 & 68.948 & 13.742 & 68.538 & 66.600 & 2.910 & 0.000 \\
\hline 444.261 & 0.690 & 0.189 & 13.498 & 13.500 & -0.017 & 0.000 \\
\hline 444.261 & 1.379 & 0.384 & 13.656 & 13.800 & -1.047 & 0.000 \\
\hline 444.261 & 2.068 & 0.584 & 13.831 & 14.000 & -1.207 & 0.000 \\
\hline 444.261 & 2.758 & 0.790 & 14.025 & 14.300 & -1.920 & 0.000 \\
\hline 444.261 & 3.447 & 1.001 & 14.240 & 14.500 & -1.795 & 0.000 \\
\hline 444.261 & 4.137 & 1.218 & 14.475 & 14.700 & -1.530 & 0.000 \\
\hline 444.261 & 5.516 & 1.671 & 15.014 & 15.200 & -1.223 & 0.000 \\
\hline 444.261 & 6.895 & 2.146 & 15.652 & 15.800 & -0.939 & 0.000 \\
\hline 444.261 & 10.342 & 3.421 & 17.718 & 17.800 & -0.459 & 0.000 \\
\hline 444.261 & 13.790 & 4.753 & 20.445 & 20.400 & 0.223 & 0.000 \\
\hline
\end{tabular}


Table 14. VISCOSITY AT ELEVATED PRESSURES (continued)

Data from Eakin et al. [71] (continued)

\begin{tabular}{|c|c|c|c|c|c|c|}
\hline $\begin{array}{l}\mathrm{T} \\
\mathrm{K}\end{array}$ & $\begin{array}{c}\mathrm{P} \\
\mathrm{MPa}\end{array}$ & $\stackrel{\rho}{\mathrm{mol} \cdot \mathrm{dm}^{-3}}$ & $\begin{array}{c}\eta, \text { calc } \\
\mu \mathrm{Pa} \cdot \mathrm{s}\end{array}$ & $\begin{array}{c}\eta, \text { expt } \\
\mu \mathrm{Pa} \cdot \mathrm{s}\end{array}$ & $\begin{array}{c}\mathrm{Dev} \\
8\end{array}$ & wt \\
\hline 444.261 & 20.684 & 7.118 & 26.940 & 26.500 & 1.661 & 0.000 \\
\hline 444.261 & 27.579 & 8.795 & 33.237 & 32.800 & 1.334 & 0.000 \\
\hline 444.261 & 34.474 & 9.969 & 38.859 & 38.100 & 1.993 & 0.000 \\
\hline 444.261 & 41.368 & 10.842 & 43.939 & 43.100 & 1.947 & 0.000 \\
\hline 444.261 & 48.263 & 11.530 & 48.641 & 47.700 & 1.972 & 0.000 \\
\hline 444.261 & 55.158 & 12.095 & 53.081 & 51.800 & 2.474 & 0.000 \\
\hline 444.261 & 62.053 & 12.574 & 57.339 & 55.500 & 3.314 & 0.000 \\
\hline 444.261 & 68.948 & 12.990 & 61.468 & 59.600 & 3.134 & 0.000 \\
\hline 477.594 & 0.690 & 0.175 & 14.339 & 14.400 & -0.426 & 0.000 \\
\hline 477.594 & 1.379 & 0.355 & 14.487 & 14.700 & -1.447 & 0.000 \\
\hline 477.594 & 2.068 & 0.537 & 14.650 & 14.900 & -1.678 & 0.000 \\
\hline 477.594 & 2.758 & 0.724 & 14.827 & 15.200 & -2.451 & 0.000 \\
\hline 477.594 & 3.447 & 0.914 & 15.020 & 15.400 & -2.467 & 0.000 \\
\hline 477.594 & 4.137 & 1.108 & 15.228 & 15.600 & -2.382 & 0.000 \\
\hline 477.594 & 5.516 & 1.506 & 15.695 & 16.000 & -1.909 & 0.000 \\
\hline 477.594 & 6.895 & 1.918 & 16.230 & 16.500 & -1.638 & 0.000 \\
\hline 477.594 & 10.342 & 2.994 & 17.882 & 17.800 & 0.459 & 0.000 \\
\hline 477.594 & 13.790 & 4.099 & 19.965 & 19.500 & 2.386 & 0.000 \\
\hline 477.594 & 20.684 & 6.161 & 24.994 & 24.400 & 2.436 & 0.000 \\
\hline 477.594 & 27.579 & 7.791 & 30.264 & 29.700 & 1.901 & 0.000 \\
\hline 477.594 & 34.474 & 9.013 & 35.235 & 34.700 & 1.542 & 0.000 \\
\hline 477.594 & 41.368 & 9.950 & 39.847 & 39.200 & 1.649 & 0.000 \\
\hline 477.594 & 48.263 & 10.696 & 44.165 & 43.100 & 2.471 & 0.000 \\
\hline 477.594 & 55.158 & 11.311 & 48.264 & 47.200 & 2.254 & 0.000 \\
\hline 477.594 & 62.053 & 11.832 & 52.200 & 50.700 & 2.959 & 0.000 \\
\hline 477.594 & 68.948 & 12.284 & 56.017 & 54.300 & 3.163 & 0.000 \\
\hline 510.928 & 0.690 & 0.164 & 15.156 & 15.200 & -0.289 & 0.000 \\
\hline 510.928 & 1.379 & 0.330 & 15.297 & 15.500 & -1.312 & 0.000 \\
\hline 510.928 & 2.068 & 0.498 & 15.449 & 15.800 & -2.223 & 0.000 \\
\hline 510.928 & 2.758 & 0.669 & 15.613 & 16.100 & -3.027 & 0.000 \\
\hline 510.928 & 3.447 & 0.843 & 15.788 & 16.300 & -3.138 & 0.000 \\
\hline 510.928 & 4.137 & 1.019 & 15.977 & 16.500 & -3.172 & 0.000 \\
\hline 510.928 & 5.516 & 1.377 & 16.391 & 16.800 & -2.435 & 0.000 \\
\hline 510.928 & 6.895 & 1.743 & 16.856 & 17.200 & -1.997 & 0.000 \\
\hline 510.928 & 10.342 & 2.685 & 18.248 & 18.100 & 0.816 & 0.000 \\
\hline 510.928 & 13.790 & 3.640 & 19.945 & 19.500 & 2.284 & 0.000 \\
\hline
\end{tabular}


Table 14. VISCOSITY AT ELEVATED PRESSURES (continued)

Data from Eakin et a1. [71] (continued)

\begin{tabular}{ccccccc}
\hline $\mathrm{T}$ & $\begin{array}{c}\mathrm{P} \\
\mathrm{MPa}\end{array}$ & $\begin{array}{c}\rho \\
\mathrm{mol} \cdot \mathrm{dm}^{-3}\end{array}$ & $\begin{array}{c}\eta \text {, calc } \\
\mu \mathrm{Pa} \cdot \mathrm{s}\end{array}$ & $\begin{array}{c}\eta \text { expt } \\
\mu \mathrm{Pa} \cdot \mathrm{s}\end{array}$ & $\begin{array}{c}\text { Dev } \\
8\end{array}$ & wt \\
\hline 510.928 & 20.684 & 5.453 & 24.012 & 23.300 & 3.056 & 0.000 \\
510.928 & 27.579 & 6.982 & 28.435 & 27.700 & 2.655 & 0.000 \\
510.928 & 34.474 & 8.196 & 32.785 & 32.200 & 1.818 & 0.000 \\
510.928 & 41.368 & 9.160 & 36.929 & 36.300 & 1.732 & 0.000 \\
510.928 & 48.263 & 9.942 & 40.866 & 39.700 & 2.938 & 0.000 \\
510.928 & 55.158 & 10.592 & 44.633 & 43.200 & 3.317 & 0.000 \\
510.928 & 62.053 & 11.146 & 48.265 & 46.700 & 3.351 & 0.000 \\
510.928 & 68.948 & 11.626 & 51.794 & 50.200 & 3.176 & 0.000
\end{tabular}

Comparisons based on experimental pressures:

Number of Points [71] 198

$$
\begin{aligned}
& \mathrm{AAD} \&=1.33 \quad \text { BIAS\& }=0.69 \text { RMS } 8=1.61 \\
& \mathrm{AAD}=0.53 \mathrm{BIAS}=0.41 \mathrm{RMS}=0.77 \mu \mathrm{Pa} \cdot \mathrm{s}
\end{aligned}
$$

Comparisons based on experimental densities:

Number of Points [71] 198

$$
\begin{aligned}
& \mathrm{AAD} \%=1.07 \text { BIAS } 8=0.41 \text { RMS } 8=1.46 \\
& \mathrm{AAD}=0.36 \mathrm{BIAS}=0.22 \mathrm{RMS}=0.61 \mu \mathrm{Pa} \cdot \mathrm{s}
\end{aligned}
$$


Table 14. VISCOSITY AT ELEVATED PRESSURES (continued)

Data from Gerf et al. [93]

\begin{tabular}{|c|c|c|c|c|c|c|}
\hline $\begin{array}{l}\mathrm{T} \\
\mathrm{K}\end{array}$ & $\begin{array}{c}\mathrm{P} \\
\mathrm{MPa}\end{array}$ & $\stackrel{\rho}{\mathrm{mol} \cdot \mathrm{dm}^{-3}}$ & $\begin{array}{c}\eta, \text { calc } \\
\mu \mathrm{Pa} \cdot \mathrm{s}\end{array}$ & $\begin{array}{c}\eta, \operatorname{expt} \\
\mu \mathrm{Pa} \cdot \mathrm{s}\end{array}$ & $\begin{array}{c}\text { Dev } \\
\frac{8}{8}\end{array}$ & wt \\
\hline 101.200 & $0.143 E-04$ & 21.280 & 841.053 & 878.000 & -4.208 & 0.000 \\
\hline 103.300 & $0.218 E-04$ & 21.204 & 783.604 & 787.000 & -0.431 & 0.000 \\
\hline 105.700 & $0.345 E-04$ & 21.118 & 725.311 & 729.000 & -0.506 & 0.000 \\
\hline 108.000 & $0.526 E-04$ & 21.035 & 675.757 & 675.000 & 0.112 & 0.000 \\
\hline 111.100 & $0.900 E-04$ & 20.922 & 617.205 & 624.000 & -1.089 & 0.000 \\
\hline 111.400 & $0.946 \mathrm{E}-04$ & 20.911 & 611.982 & 615.000 & -0.491 & 0.000 \\
\hline 112.000 & $0.105 E-03$ & 20.890 & 601.753 & 570.000 & 5.571 & 0.000 \\
\hline 113.400 & $0.131 E-03$ & 20.839 & 578.951 & 556.000 & 4.128 & 0.000 \\
\hline 115.400 & $0.180 E-03$ & 20.766 & 548.771 & 526.000 & 4.329 & 0.000 \\
\hline 121.000 & $0.408 E-03$ & 20.560 & 476.852 & 458.000 & 4.116 & 0.000 \\
\hline 126.800 & $0.875 E-03$ & 20.346 & 417.631 & 412.000 & 1.367 & 0.000 \\
\hline 127.200 & $0.920 E-03$ & 20.331 & 414.008 & 403.000 & 2.731 & 0.000 \\
\hline 141.200 & $0.431 E-02$ & 19.806 & 314.155 & 310.000 & 1.340 & 0.000 \\
\hline 149.500 & $0.926 \mathrm{E}-02$ & 19.489 & 272.561 & 277.000 & -1.603 & 0.000 \\
\hline 150.300 & $0.992 E-02$ & 19.458 & 269.038 & 271.000 & -0.724 & 0.000 \\
\hline 150.800 & $0.104 \mathrm{E}-01$ & 19.439 & 266.874 & 270.000 & -1.158 & 0.000 \\
\hline 159.800 & $0.211 E-01$ & 19.089 & 232.310 & 236.000 & -1.564 & 0.000 \\
\hline 160.100 & $0.216 E-01$ & 19.077 & 231.284 & 225.000 & 2.793 & 0.000 \\
\hline 166.800 & $0.347 E-01$ & 18.812 & 210.119 & 207.000 & 1.507 & 0.000 \\
\hline 167.300 & $0.359 \mathrm{E}-01$ & 18.792 & 208.662 & 203.000 & 2.789 & 0.000 \\
\hline
\end{tabular}

Comparisons based on experimental pressures:

Number of Points [93] 20

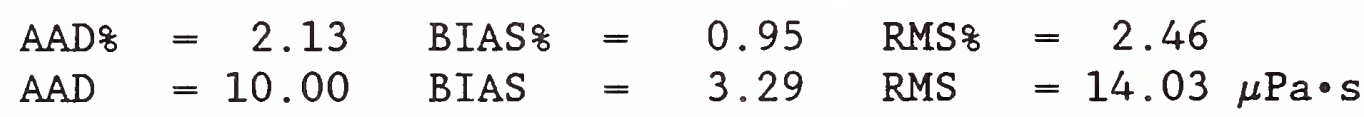

Comparisons based on experimental densities:

Number of Points [93] 20

$$
\begin{array}{llllll}
\mathrm{AAD} \% & =21.17 & \mathrm{BIAS} \% & =-18.84 & \mathrm{RMS} \% & =39.44 \\
\mathrm{AAD}=51.25 & \mathrm{BIAS}=-39.63 & \mathrm{RMS} & =87.70 \mu \mathrm{Pa} \cdot \mathrm{S}
\end{array}
$$


Table 14. VISCOSITY AT ELEVATED PRESSURES (continued)

Data from Gerf et al. [94]

\begin{tabular}{|c|c|c|c|c|c|c|}
\hline $\begin{array}{l}\mathrm{T} \\
\mathrm{K}\end{array}$ & $\begin{array}{c}\mathrm{P} \\
\mathrm{MPa}\end{array}$ & $\stackrel{\rho}{\mathrm{mol}} \stackrel{\mathrm{dm}^{-3}}{ }$ & $\begin{array}{c}\eta, \text { calc } \\
\mu \mathrm{Pa} \cdot \mathrm{s}\end{array}$ & $\begin{array}{c}\eta, \text { expt } \\
\mu \mathrm{Pa} \cdot \mathrm{s}\end{array}$ & $\begin{array}{c}\text { Dev } \\
q\end{array}$ & wt \\
\hline 172.800 & $0.512 \mathrm{E}-01$ & 18.571 & 193.610 & 175.000 & 10.634 & 0.000 \\
\hline 186.000 & 0.109 & 18.026 & 163.457 & 137.000 & 19.312 & 0.000 \\
\hline 201.000 & 0.227 & 17.379 & 136.564 & 114.000 & 19.793 & 0.000 \\
\hline 215.400 & 0.414 & 16.719 & 115.769 & 97.000 & 19.349 & 0.000 \\
\hline 230.000 & 0.700 & 15.999 & 98.189 & 90.000 & 9.099 & 0.000 \\
\hline 231.800 & 0.744 & 15.906 & 96.213 & 92.000 & 4.580 & 0.000 \\
\hline 243.400 & 1.073 & 15.277 & 84.297 & 82.000 & 2.801 & 0.000 \\
\hline 247.600 & 1.214 & 15.034 & 80.287 & 76.000 & 5.640 & 0.000 \\
\hline 270.000 & 2.210 & 13.551 & 60.847 & 67.000 & -9.183 & 0.000 \\
\hline 273.100 & 2.383 & 13.310 & 58.333 & 63.000 & -7.409 & 0.000 \\
\hline 288.000 & 3.363 & 11.916 & 46.281 & 55.000 & -15.853 & 0.000 \\
\hline
\end{tabular}

Comparisons based on experimental pressures:

Number of Points [94] 11

$\begin{array}{ll}\mathrm{AAD} \% & =11.24 \quad \mathrm{BIAS} 8=5.34 \\ \mathrm{AAD}=11.36 & \mathrm{BMS} \%=11.57 \\ \mathrm{BIAS} & =7.80 \quad \mathrm{RMS}=11.61 \mu \mathrm{Pa} \cdot \mathrm{S}\end{array}$

Comparisons based on experimental densities:

Number of Points [94] 11

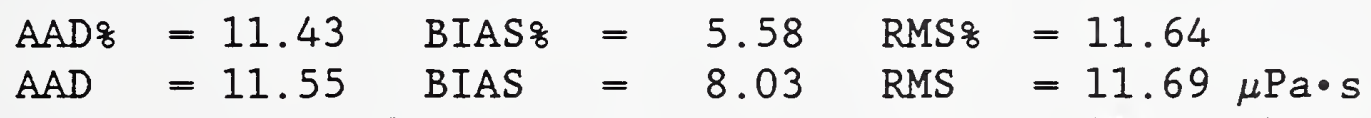

Data from Iwasaki et al. [73]

\begin{tabular}{ccccccc}
\hline $\mathrm{T}$ & $\begin{array}{c}\mathrm{P} \\
\mathrm{MPa}\end{array}$ & $\begin{array}{c}\rho \\
\mathrm{mol} \cdot \mathrm{dm}^{-3}\end{array}$ & $\begin{array}{c}\eta, \text { calc } \\
\mu \mathrm{Pa} \cdot \mathrm{s}\end{array}$ & $\begin{array}{c}\eta \text { expt } \\
\mu \mathrm{Pa} \cdot \mathrm{s}\end{array}$ & $\begin{array}{c}\text { Dev } \\
\text { \% }\end{array}$ & wt \\
\hline 298.150 & 0.201 & 0.082 & 9.377 & 9.270 & 1.152 & 0.000 \\
298.150 & 0.343 & 0.142 & 9.411 & 9.290 & 1.299 & 0.000 \\
298.150 & 0.491 & 0.206 & 9.448 & 9.300 & 1.596 & 0.000 \\
298.150 & 0.644 & 0.273 & 9.490 & 9.310 & 1.934 & 0.000 \\
298.150 & 0.834 & 0.360 & 9.546 & 9.340 & 2.203 & 0.000 \\
& & & & & & \\
298.150 & 1.036 & 0.455 & 9.611 & 9.390 & 2.348 & 0.000 \\
298.150 & 1.264 & 0.568 & 9.691 & 9.450 & 2.552 & 0.000 \\
298.150 & 1.523 & 0.702 & 9.793 & 9.510 & 2.979 & 0.000
\end{tabular}


Table 14. VISCOSITY AT ELEVATED PRESSURES (continued)

Data from Iwasaki et al. [73] (continued)

\begin{tabular}{|c|c|c|c|c|c|c|}
\hline $\begin{array}{l}\mathrm{T} \\
\mathrm{K}\end{array}$ & $\begin{array}{c}\mathrm{P} \\
\mathrm{MPa}\end{array}$ & $\stackrel{\rho}{\mathrm{mol} \cdot \mathrm{dm}^{-3}}$ & $\begin{array}{c}\eta, \text { calc } \\
\mu \mathrm{Pa} \cdot \mathrm{s}\end{array}$ & $\begin{array}{c}\eta, \text { expt } \\
\mu \mathrm{Pa} \cdot \mathrm{s}\end{array}$ & $\begin{array}{c}\text { Dev } \\
8\end{array}$ & wt \\
\hline 298.150 & 1.788 & 0.848 & 9.912 & 9.600 & 3.249 & 0.000 \\
\hline 298.150 & 2.070 & 1.015 & 10.057 & 9.720 & 3.469 & 0.000 \\
\hline 298.150 & 2.351 & 1.196 & 10.225 & 9.840 & 3.915 & 0.000 \\
\hline 298.150 & 2.624 & 1.388 & 10.417 & 10.000 & 4.169 & 0.000 \\
\hline 298.150 & 2.920 & 1.620 & 10.666 & 10.210 & 4.469 & 0.000 \\
\hline 298.150 & 3.243 & 1.913 & 11.008 & 10.510 & 4.743 & 0.000 \\
\hline 298.150 & 3.477 & 2.163 & 11.325 & 10.780 & 5.052 & 0.000 \\
\hline 298.150 & 3.795 & 2.588 & 11.914 & 11.310 & 5.343 & 0.000 \\
\hline 298.150 & 4.029 & 3.026 & 12.587 & 11.800 & 6.674 & 0.000 \\
\hline 298.150 & 4.134 & 3.303 & 13.050 & 12.330 & 5.838 & 0.000 \\
\hline 305.650 & 0.298 & 0.120 & 9.619 & 9.480 & 1.466 & 0.000 \\
\hline 305.650 & 0.599 & 0.246 & 9.696 & 9.530 & 1.740 & 0.000 \\
\hline 305.650 & 0.906 & 0.382 & 9.785 & 9.580 & 2.138 & 0.000 \\
\hline 305.650 & 1.210 & 0.523 & 9.884 & 9.650 & 2.429 & 0.000 \\
\hline 305.650 & 1.566 & 0.699 & 10.019 & 9.750 & 2.758 & 0.000 \\
\hline 305.650 & 1.939 & 0.897 & 10.184 & 9.860 & 3.288 & 0.000 \\
\hline 305.650 & 2.335 & 1.128 & 10.394 & 10.010 & 3.838 & 0.000 \\
\hline 305.650 & 2.688 & 1.356 & 10.620 & 10.220 & 3.912 & 0.000 \\
\hline 305.650 & 3.059 & 1.624 & 10.909 & 10.470 & 4.195 & 0.000 \\
\hline 305.650 & 3.358 & 1.870 & 11.196 & 10.710 & 4.541 & 0.000 \\
\hline 305.650 & 3.696 & 2.191 & 11.603 & 11.060 & 4.914 & 0.000 \\
\hline 305.650 & 4.045 & 2.598 & 12.172 & 11.570 & 5.205 & 0.000 \\
\hline 305.650 & 4.256 & 2.905 & 12.641 & 12.010 & 5.251 & 0.000 \\
\hline 305.650 & 4.360 & 3.084 & 12.928 & 12.270 & 5.361 & 0.000 \\
\hline 305.650 & 4.453 & 3.266 & 13.233 & 12.580 & 5.188 & 0.000 \\
\hline 305.650 & 4.477 & 3.317 & 13.320 & 12.660 & 5.216 & 0.000 \\
\hline 305.650 & 4.536 & 3.452 & 13.556 & 12.850 & 5.498 & 0.000 \\
\hline 305.650 & 4.606 & 3.636 & 13.889 & 13.230 & 4.982 & 0.000 \\
\hline 305.650 & 4.666 & 3.820 & 14.233 & 13.570 & 4.889 & 0.000 \\
\hline 305.650 & 4.718 & 4.010 & 14.602 & 13.950 & 4.672 & 0.000 \\
\hline 305.650 & 4.759 & 4.192 & 14.965 & 14.340 & 4.360 & 0.000 \\
\hline 305.650 & 4.793 & 4.375 & 15.345 & 14.750 & 4.035 & 0.000 \\
\hline 305.650 & 4.819 & 4.544 & 15.707 & 15.210 & 3.264 & 0.000 \\
\hline 305.650 & 4.839 & 4.704 & 16.060 & 15.660 & 2.555 & 0.000 \\
\hline 305.650 & 4.853 & 4.841 & 16.369 & 16.110 & 1.610 & 0.000 \\
\hline 305.650 & 4.862 & 4.954 & 16.630 & 16.550 & 0.485 & 0.000 \\
\hline
\end{tabular}


Table 14. VISCOSITY AT ELEVATED PRESSURES (continued)

Data from Iwasaki et al. [73] (continued)

\begin{tabular}{ccccccc}
\hline $\mathrm{T}$ & $\begin{array}{c}\mathrm{P} \\
\mathrm{K}\end{array}$ & $\begin{array}{c}\rho \\
\mathrm{MPa}\end{array}$ & $\begin{array}{c}\eta, \mathrm{d} \text { calc } \\
\mu \mathrm{Pa} \cdot \mathrm{s}\end{array}$ & $\begin{array}{c}\eta, \text { expt } \\
\mu \mathrm{Pa} \cdot \mathrm{s}\end{array}$ & $\begin{array}{c}\text { Dev } \\
8\end{array}$ & wt \\
\hline 305.650 & 4.868 & 5.041 & 16.832 & 16.950 & -0.693 & 0.000 \\
& & & & & & \\
305.650 & 4.874 & 5.124 & 17.030 & 17.440 & -2.353 & 0.000 \\
305.650 & 4.877 & 5.189 & 17.187 & 17.940 & -4.199 & 0.000 \\
305.650 & 4.879 & 5.216 & 17.251 & 18.190 & -5.159 & 0.000 \\
305.650 & 4.880 & 5.243 & 17.320 & 18.530 & -6.532 & 0.000 \\
305.650 & 4.881 & 5.258 & 17.355 & 18.850 & -7.930 & 0.000 \\
& & & & & & \\
305.650 & 4.882 & 5.279 & 17.408 & 19.200 & -9.336 & 0.000 \\
305.650 & 4.882 & 5.290 & 17.435 & 19.580 & -10.957 & 0.000 \\
305.650 & 4.883 & 5.315 & 17.496 & 20.010 & -12.563 & 0.000 \\
305.650 & 4.884 & 5.325 & 17.519 & 20.460 & -14.373 & 0.000 \\
305.650 & 4.884 & 5.337 & 17.549 & 20.920 & -16.114 & 0.000 \\
& & & & & & \\
305.650 & 4.885 & 5.349 & 17.579 & 21.380 & -17.777 & 0.000 \\
305.650 & 4.885 & 5.351 & 17.585 & 21.810 & -19.370 & 0.000 \\
305.650 & 4.885 & 5.361 & 17.610 & 22.210 & -20.710 & 0.000 \\
305.650 & 4.885 & 5.364 & 17.617 & 22.560 & -21.913 & 0.000 \\
305.650 & 4.886 & 5.377 & 17.648 & 22.870 & -22.831 & 0.000 \\
& & & & & & \\
305.650 & 4.886 & 5.382 & 17.661 & 23.080 & -23.477 & 0.000 \\
305.650 & 4.887 & 5.395 & 17.695 & 23.260 & -23.927 & 0.000 \\
305.650 & 4.887 & 5.406 & 17.722 & 23.450 & -24.427 & 0.000 \\
305.650 & 4.888 & 5.417 & 17.750 & 23.670 & -25.012 & 0.000 \\
305.650 & 4.888 & 5.431 & 17.785 & 23.850 & -25.429 & 0.000 \\
305.650 & 4.888 & 5.443 & 17.814 & 24.080 & -26.020 & 0.000 \\
305.650 & 4.889 & 5.464 & 17.867 & 24.280 & -26.411 & 0.000 \\
305.650 & 4.890 & 5.495 & 17.947 & 24.550 & -26.895 & 0.000 \\
305.650 & 4.891 & 5.543 & 18.069 & 24.840 & -27.260 & 0.000 \\
305.650 & 4.893 & 5.615 & 18.256 & 25.100 & -27.268 & 0.000 \\
305.650 & 4.896 & 5.725 & 18.544 & 25.490 & -27.252 & 0.000 \\
305.650 & 4.900 & 5.977 & 19.220 & 25.860 & -25.679 & 0.000 \\
305.650 & 4.904 & 6.638 & 21.133 & 26.240 & -19.464 & 0.000 \\
305.650 & 4.910 & 7.679 & 24.567 & 26.670 & -7.884 & 0.000 \\
305.650 & 4.917 & 8.081 & 26.050 & 27.110 & -3.911 & 0.000 \\
305.650 & 4.928 & 8.335 & 27.034 & 27.600 & -2.049 & 0.000 \\
305.650 & 4.940 & 8.532 & 27.827 & 28.050 & -0.795 & 0.000 \\
305.650 & 4.956 & 8.700 & 28.526 & 28.580 & -0.188 & 0.000 \\
305.650 & 4.979 & 8.875 & 29.269 & 29.080 & 0.649 & 0.000 \\
305.650 & 5.011 & 9.059 & 30.079 & 29.660 & 1.413 & 0.000
\end{tabular}


Table 14. VISCOSITY AT ELEVATED PRESSURES (continued)

Data from Iwasaki et al. [73] (continued)

\begin{tabular}{|c|c|c|c|c|c|c|}
\hline $\begin{array}{l}\mathrm{T} \\
\mathrm{K}\end{array}$ & $\begin{array}{c}\mathrm{P} \\
\mathrm{MPa}\end{array}$ & $\stackrel{\rho}{\mathrm{mol} \cdot \mathrm{dm}^{-3}}$ & $\begin{array}{c}\eta, \text { calc } \\
\mu \mathrm{Pa} \cdot \mathrm{s}\end{array}$ & $\begin{array}{c}\eta, \text { expt } \\
\mu \mathrm{Pa} \cdot \mathrm{s}\end{array}$ & $\begin{array}{c}\text { Dev } \\
\frac{8}{8}\end{array}$ & wt \\
\hline 305.650 & 5.032 & 9.155 & 30.512 & 30.220 & 0.965 & 0.000 \\
\hline 305.650 & 5.068 & 9.297 & 31.159 & 30.850 & 1.002 & 0.000 \\
\hline 305.650 & 5.113 & 9.438 & 31.824 & 31.450 & 1.188 & 0.000 \\
\hline 305.650 & 5.169 & 9.585 & 32.528 & 32.150 & 1.174 & 0.000 \\
\hline 305.650 & 5.232 & 9.723 & 33.210 & 32.790 & 1.280 & 0.000 \\
\hline 305.650 & 5.307 & 9.863 & 33.919 & 33.540 & 1.131 & 0.000 \\
\hline 305.650 & 5.398 & 10.009 & 34.675 & 34.230 & 1.299 & 0.000 \\
\hline 305.850 & 0.351 & 0.142 & 9.638 & 9.500 & 1.449 & 0.000 \\
\hline 305.850 & 0.598 & 0.246 & 9.701 & 9.540 & 1.692 & 0.000 \\
\hline 305.850 & 0.878 & 0.369 & 9.782 & 9.580 & 2.106 & 0.000 \\
\hline 305.850 & 1.174 & 0.505 & 9.878 & 9.640 & 2.465 & 0.000 \\
\hline 305.850 & 1.468 & 0.649 & 9.986 & 9.730 & 2.627 & 0.000 \\
\hline 305.850 & 1.817 & 0.830 & 10.133 & 9.840 & 2.974 & 0.000 \\
\hline 305.850 & 2.170 & 1.028 & 10.307 & 9.980 & 3.278 & 0.000 \\
\hline 305.850 & 2.482 & 1.219 & 10.488 & 10.130 & 3.533 & 0.000 \\
\hline 305.850 & 2.888 & 1.494 & 10.772 & 10.350 & 4.073 & 0.000 \\
\hline 305.850 & 3.359 & 1.867 & 11.199 & 10.700 & 4.663 & 0.000 \\
\hline 305.850 & 3.680 & 2.169 & 11.581 & 11.050 & 4.807 & 0.000 \\
\hline 305.850 & 4.044 & 2.587 & 12.164 & 11.580 & 5.039 & 0.000 \\
\hline 305.850 & 4.416 & 3.174 & 13.084 & 12.430 & 5.259 & 0.000 \\
\hline 305.850 & 4.531 & 3.420 & 13.506 & 12.840 & 5.185 & 0.000 \\
\hline 305.850 & 4.669 & 3.797 & 14.196 & 13.520 & 4.999 & 0.000 \\
\hline 305.850 & 4.768 & 4.177 & 14.943 & 14.300 & 4.497 & 0.000 \\
\hline 305.850 & 4.822 & 4.473 & 15.560 & 14.980 & 3.875 & 0.000 \\
\hline 305.850 & 4.867 & 4.847 & 16.388 & 16.050 & 2.105 & 0.000 \\
\hline 305.850 & 4.877 & 4.954 & 16.636 & 16.300 & 2.064 & 0.000 \\
\hline 305.850 & 4.882 & 5.018 & 16.787 & 16.660 & 0.760 & 0.000 \\
\hline 305.850 & 4.890 & 5.136 & 17.066 & 17.200 & -0.780 & 0.000 \\
\hline 305.850 & 4.894 & 5.209 & 17.243 & 17.540 & -1.695 & 0.000 \\
\hline 305.850 & 4.898 & 5.278 & 17.411 & 17.990 & -3.221 & 0.000 \\
\hline 305.850 & 4.901 & 5.333 & 17.546 & 18.370 & -4.484 & 0.000 \\
\hline 305.850 & 4.902 & 5.374 & 17.649 & 18.790 & -6.074 & 0.000 \\
\hline 305.850 & 4.903 & 5.403 & 17.722 & 19.100 & -7.216 & 0.000 \\
\hline 305.850 & 4.904 & 5.426 & 17.779 & 19.410 & -8.402 & 0.000 \\
\hline 305.850 & 4.905 & 5.453 & 17.846 & 19.760 & -9.685 & 0.000 \\
\hline 305.850 & 4.906 & 5.469 & 17.888 & 20.110 & -11.049 & 0.000 \\
\hline
\end{tabular}


Táble 14. VISCOSITY AT ELEVATED PRESSURES (continued)

Data from Iwasaki et al. [73] (continued)

\begin{tabular}{|c|c|c|c|c|c|c|}
\hline $\begin{array}{l}\mathrm{T} \\
\mathrm{K}\end{array}$ & $\begin{array}{c}\mathrm{P} \\
\mathrm{MPa}\end{array}$ & $\stackrel{\rho}{\mathrm{mol} \cdot \mathrm{dm}^{-3}}$ & $\begin{array}{c}\eta, \text { calc } \\
\mu \mathrm{Pa} \cdot \mathrm{s}\end{array}$ & $\begin{array}{c}\eta, \operatorname{expt} \\
\mu \mathrm{Pa} \cdot \mathrm{s}\end{array}$ & $\begin{array}{c}\text { Dev } \\
\frac{8}{8}\end{array}$ & wt \\
\hline 305.850 & 4.907 & 5.507 & 17.983 & 20.480 & -12.190 & 0.000 \\
\hline 305.850 & 4.908 & 5.513 & 17.999 & 20.830 & -13.592 & 0.000 \\
\hline 305.850 & 4.908 & 5.528 & 18.038 & 21.180 & -14.836 & 0.000 \\
\hline 305.850 & 4.908 & 5.541 & 18.070 & 21.510 & -15.993 & 0.000 \\
\hline 305.850 & 4.909 & 5.560 & 18.120 & 21.820 & -16.959 & 0.000 \\
\hline 305.850 & 4.909 & 5.577 & 18.162 & 22.190 & -18.150 & 0.000 \\
\hline 305.850 & 4.910 & 5.605 & 18.234 & 22.440 & -18.743 & 0.000 \\
\hline 305.850 & 4.911 & 5.619 & 18.271 & 22.770 & -19.757 & 0.000 \\
\hline 305.850 & 4.911 & 5.645 & 18.339 & 23.040 & -20.402 & 0.000 \\
\hline 305.850 & 4.912 & 5.669 & 18.400 & 23.270 & -20.926 & 0.000 \\
\hline 305.850 & 4.913 & 5.697 & 18.475 & 23.570 & -21.614 & 0.000 \\
\hline 305.850 & 4.914 & 5.745 & 18.603 & 23.830 & -21.936 & 0.000 \\
\hline 305.850 & 4.915 & 5.793 & 18.730 & 24.100 & -22.283 & 0.000 \\
\hline 305.850 & 4.917 & 5.885 & 18.977 & 24.440 & -22.352 & 0.000 \\
\hline 305.850 & 4.918 & 6.002 & 19.297 & 24.710 & -21.907 & 0.000 \\
\hline 305.850 & 4.921 & 6.204 & 19.861 & 25.080 & -20.811 & 0.000 \\
\hline 305.850 & 4.924 & 6.597 & 21.013 & 25.400 & -17.270 & 0.000 \\
\hline 305.850 & 4.929 & 7.253 & 23.100 & 25.900 & -10.811 & 0.000 \\
\hline 305.850 & 4.934 & 7.724 & 24.735 & 26.290 & -5.916 & 0.000 \\
\hline 305.850 & 4.941 & 8.031 & 25.866 & 26.690 & -3.086 & 0.000 \\
\hline 305.850 & 4.949 & 8.258 & 26.737 & 27.170 & -1.595 & 0.000 \\
\hline 305.850 & 4.961 & 8.447 & 27.487 & 27.630 & -0.517 & 0.000 \\
\hline 305.850 & 4.974 & 8.608 & 28.146 & 28.140 & 0.021 & 0.000 \\
\hline 305.850 & 4.993 & 8.773 & 28.838 & 28.660 & 0.621 & 0.000 \\
\hline 305.850 & 5.018 & 8.938 & 29.552 & 29.280 & 0.929 & 0.000 \\
\hline 305.850 & 5.048 & 9.090 & 30.221 & 29.870 & 1.174 & 0.000 \\
\hline 305.850 & 5.082 & 9.230 & 30.855 & 30.490 & 1.198 & 0.000 \\
\hline 305.850 & 5.123 & 9.368 & 31.499 & 31.060 & 1.415 & 0.000 \\
\hline 305.850 & 5.173 & 9.509 & 32.165 & 31.730 & 1.371 & 0.000 \\
\hline 305.850 & 5.232 & 9.649 & 32.849 & 32.400 & 1.386 & 0.000 \\
\hline 305.850 & 5.303 & 9.791 & 33.558 & 33.110 & 1.352 & 0.000 \\
\hline 306.150 & 0.359 & 0.145 & 9.648 & 9.510 & 1.454 & 0.000 \\
\hline 306.150 & 0.595 & 0.244 & 9.709 & 9.550 & 1.668 & 0.000 \\
\hline 306.150 & 0.847 & 0.354 & 9.781 & 9.590 & 1.992 & 0.000 \\
\hline 306.150 & 1.119 & 0.479 & 9.867 & 9.650 & 2.253 & 0.000 \\
\hline 306.150 & 1.486 & 0.657 & 10.001 & 9.740 & 2.677 & 0.000 \\
\hline
\end{tabular}


Table 14. VISCOSITY AT ELEVATED PRESSURES (continued)

Data from Iwasaki et al. [73] (continued)

\begin{tabular}{|c|c|c|c|c|c|c|}
\hline $\begin{array}{l}\mathrm{T} \\
\mathrm{K}\end{array}$ & $\begin{array}{c}\mathrm{P} \\
\mathrm{MPa}\end{array}$ & $\begin{array}{c}\rho \\
\mathrm{mol} \cdot \mathrm{dm}^{-3}\end{array}$ & $\begin{array}{c}\eta, \text { calc } \\
\mu \mathrm{Pa} \cdot \mathrm{s}\end{array}$ & $\begin{array}{c}\eta, \text { expt } \\
\mu \mathrm{Pa} \cdot \mathrm{s}\end{array}$ & $\begin{array}{c}\text { Dev } \\
8\end{array}$ & wt \\
\hline 306.150 & 1.872 & 0.858 & 10.166 & 9.860 & 3.102 & 0.000 \\
\hline 306.150 & 2.248 & 1.073 & 10.357 & 10.000 & 3.571 & 0.000 \\
\hline 306.150 & 2.589 & 1.286 & 10.564 & 10.180 & 3.768 & 0.000 \\
\hline 306.150 & 3.058 & 1.617 & 10.917 & 10.470 & 4.265 & 0.000 \\
\hline 306.150 & 3.459 & 1.950 & 11.310 & 10.910 & 4.628 & 0.000 \\
\hline 306.150 & 3.853 & 2.346 & 11.829 & 11.270 & 4.964 & 0.000 \\
\hline 306.150 & 4.090 & 2.636 & 12.245 & 11.650 & 5.109 & 0.000 \\
\hline 306.150 & 4.326 & 2.989 & 12.791 & 12.160 & 5.187 & 0.000 \\
\hline 306.150 & 4.530 & 3.386 & 13.456 & 12.780 & 5.293 & 0.000 \\
\hline 306.150 & 4.651 & 3.693 & 14.011 & 13.330 & 5.107 & 0.000 \\
\hline 306.150 & 4.754 & 4.040 & 14.677 & 14.000 & 4.834 & 0.000 \\
\hline 306.150 & 4.816 & 4.325 & 15.258 & 14.620 & 4.365 & 0.000 \\
\hline 306.150 & 4.851 & 4.538 & 15.712 & 15.140 & 3.775 & 0.000 \\
\hline 306.150 & 4.869 & 4.670 & 16.000 & 15.470 & 3.423 & 0.000 \\
\hline 306.150 & 4.887 & 4.823 & 16.343 & 15.920 & 2.657 & 0.000 \\
\hline 306.150 & 4.902 & 4.989 & 16.728 & 16.430 & 1.811 & 0.000 \\
\hline 306.150 & 4.911 & 5.102 & 16.995 & 16.830 & 0.983 & 0.000 \\
\hline 306.150 & 4.917 & 5.191 & 17.209 & 17.160 & 0.285 & 0.000 \\
\hline 306.150 & 4.921 & 5.259 & 17.373 & 17.490 & -0.667 & 0.000 \\
\hline 306.150 & 4.925 & 5.338 & 17.568 & 17.880 & -1.743 & 0.000 \\
\hline 306.150 & 4.928 & 5.401 & 17.725 & 18.250 & -2.875 & 0.000 \\
\hline 306.150 & 4.931 & 5.456 & 17.864 & 18.620 & -4.059 & 0.000 \\
\hline 306.150 & 4.933 & 5.519 & 18.024 & 19.080 & -5.533 & 0.000 \\
\hline 306.150 & 4.934 & 5.555 & 18.115 & 19.380 & -6.528 & 0.000 \\
\hline 306.150 & 4.936 & 5.604 & 18.242 & 19.710 & -7.446 & 0.000 \\
\hline 306.150 & 4.937 & 5.635 & 18.323 & 20.030 & -8.524 & 0.000 \\
\hline 306.150 & 4.938 & 5.671 & 18.416 & 20.360 & -9.547 & 0.000 \\
\hline 306.150 & 4.939 & 5.702 & 18.497 & 20.700 & -10.642 & 0.000 \\
\hline 306.150 & 4.940 & 5.734 & 18.583 & 21.000 & -11.511 & 0.000 \\
\hline 306.150 & 4.941 & 5.764 & 18.663 & 21.340 & -12.545 & 0.000 \\
\hline 306.150 & 4.941 & 5.784 & 18.715 & 21.640 & -13.517 & 0.000 \\
\hline 306.150 & 4.943 & 5.851 & 18.894 & 21.960 & -13.960 & 0.000 \\
\hline 306.150 & 4.944 & 5.883 & 18.979 & 22.280 & -14.814 & 0.000 \\
\hline 306.150 & 4.944 & 5.887 & 18.992 & 22.580 & -15.890 & 0.000 \\
\hline 306.150 & 4.946 & 5.981 & 19.248 & 22.870 & -15.836 & 0.000 \\
\hline 306.150 & 4.947 & 6.042 & 19.415 & 23.160 & -16.169 & 0.000 \\
\hline
\end{tabular}


Table 14. VISCOSITY AT ELEVATED PRESSURES (continued)

Data from Iwasaki et al. [73] (continued)

\begin{tabular}{|c|c|c|c|c|c|c|}
\hline $\begin{array}{l}\mathrm{T} \\
\mathrm{K}\end{array}$ & $\begin{array}{c}\mathrm{P} \\
\mathrm{MPa}\end{array}$ & $\begin{array}{c}\rho \\
\mathrm{mol} \cdot \mathrm{dm}^{-3}\end{array}$ & $\begin{array}{c}\eta, \text { calc } \\
\mu \mathrm{Pa} \cdot \mathrm{s}\end{array}$ & $\begin{array}{c}\eta, \text { expt } \\
\mu \mathrm{Pa} \cdot \mathrm{s}\end{array}$ & $\begin{array}{c}\text { Dev } \\
8\end{array}$ & $w t$ \\
\hline 306.150 & 4.948 & 6.113 & 19.615 & 23.490 & -16.497 & 0.000 \\
\hline 306.150 & 4.950 & 6.233 & 19.953 & 23.770 & -16.058 & 0.000 \\
\hline 306.150 & 4.954 & 6.542 & 20.858 & 24.410 & -14.553 & 0.000 \\
\hline 306.150 & 4.960 & 7.116 & 22.656 & 25.190 & -10.059 & 0.000 \\
\hline 306.150 & 4.970 & 7.750 & 24.838 & 25.940 & -4.250 & 0.000 \\
\hline 306.150 & 4.976 & 7.993 & 25.731 & 26.350 & -2.348 & 0.000 \\
\hline 306.150 & 4.985 & 8.193 & 26.495 & 26.800 & -1.140 & 0.000 \\
\hline 306.150 & 4.996 & 8.378 & 27.219 & 27.260 & -0.149 & 0.000 \\
\hline 306.150 & 5.009 & 8.537 & 27.865 & 27.720 & 0.524 & 0.000 \\
\hline 306.150 & 5.024 & 8.684 & 28.473 & 28.250 & 0.791 & 0.000 \\
\hline 306.150 & 5.044 & 8.829 & 29.087 & 28.790 & 1.030 & 0.000 \\
\hline 306.150 & 5.069 & 8.969 & 29.694 & 29.300 & 1.345 & 0.000 \\
\hline 306.150 & 5.098 & 9.107 & 30.306 & 29.860 & 1.494 & 0.000 \\
\hline 306.150 & 5.135 & 9.248 & 30.950 & 30.390 & 1.841 & 0.000 \\
\hline 306.150 & 5.178 & 9.385 & 31.585 & 31.120 & 1.495 & 0.000 \\
\hline 306.150 & 5.229 & 9.522 & 32.239 & 31.760 & 1.508 & 0.000 \\
\hline 306.150 & 5.291 & 9.661 & 32.914 & 32.430 & 1.492 & 0.000 \\
\hline 306.150 & 5.364 & 9.802 & 33.618 & 33.120 & 1.504 & 0.000 \\
\hline 306.150 & 5.449 & 9.941 & 34.332 & 33.850 & 1.423 & 0.000 \\
\hline 306.450 & 0.297 & 0.119 & 9.642 & 9.520 & 1.281 & 0.000 \\
\hline 306.450 & 0.592 & 0.243 & 9.717 & 9.560 & 1.644 & 0.000 \\
\hline 306.450 & 0.895 & 0.375 & 9.804 & 9.610 & 2.021 & 0.000 \\
\hline 306.450 & 1.224 & 0.528 & 9.912 & 9.690 & 2.291 & 0.000 \\
\hline 306.450 & 1.588 & 0.707 & 10.050 & 9.780 & 2.760 & 0.000 \\
\hline 306.450 & 2.095 & 0.982 & 10.283 & 9.950 & 3.348 & 0.000 \\
\hline 306.450 & 2.525 & 1.242 & 10.529 & 10.150 & 3.732 & 0.000 \\
\hline 306.450 & 2.823 & 1.441 & 10.734 & 10.320 & 4.013 & 0.000 \\
\hline 306.450 & 3.255 & 1.769 & 11.101 & 10.640 & 4.330 & 0.000 \\
\hline 306.450 & 3.644 & 2.117 & 11.533 & 11.020 & 4.655 & 0.000 \\
\hline 306.450 & 3.978 & 2.481 & 12.030 & 11.470 & 4.880 & 0.000 \\
\hline 306.450 & 4.209 & 2.788 & 12.485 & 11.880 & 5.092 & 0.000 \\
\hline 306.450 & 4.438 & 3.169 & 13.095 & 12.470 & 5.011 & 0.000 \\
\hline 306.450 & 4.586 & 3.483 & 13.637 & 13.000 & 4.903 & 0.000 \\
\hline 306.450 & 4.679 & 3.729 & 14.087 & 13.450 & 4.733 & 0.000 \\
\hline 306.450 & 4.756 & 3.984 & 14.577 & 13.920 & 4.719 & 0.000 \\
\hline 306.450 & 4.818 & 4.244 & 15.100 & 14.560 & 3.706 & 0.000 \\
\hline
\end{tabular}


Table 14. VISCOSITY AT ELEVATED PRESSURES (continued)

Data from Iwasaki et al. [73] (continued)

\begin{tabular}{|c|c|c|c|c|c|c|}
\hline $\begin{array}{l}\mathrm{T} \\
\mathrm{K}\end{array}$ & $\begin{array}{c}\mathrm{P} \\
\mathrm{MPa}\end{array}$ & $\begin{array}{c}\rho \\
\mathrm{mol} \cdot \mathrm{dm}^{-3}\end{array}$ & $\begin{array}{c}\eta, \text { calc } \\
\mu \mathrm{Pa} \cdot \mathrm{s}\end{array}$ & $\begin{array}{c}\eta, \text { expt } \\
\mu \mathrm{Pa} \cdot \mathrm{s}\end{array}$ & $\begin{array}{c}\text { Dev } \\
\frac{8}{8}\end{array}$ & wt \\
\hline 306.450 & 4.861 & 4.471 & 15.576 & 15.140 & 2.880 & 0.000 \\
\hline 306.450 & 4.886 & 4.641 & 15.946 & 15.600 & 2.217 & 0.000 \\
\hline 306.450 & 4.907 & 4.812 & 16.328 & 16.130 & 1.230 & 0.000 \\
\hline 306.450 & 4.921 & 4.953 & 16.653 & 16.620 & 0.196 & 0.000 \\
\hline 306.450 & 4.931 & 5.067 & 16.920 & 17.130 & -1.227 & 0.000 \\
\hline 306.450 & 4.938 & 5.156 & 17.132 & 17.830 & -3.912 & 0.000 \\
\hline 306.450 & 4.943 & 5.233 & 17.320 & 18.370 & -5.717 & 0.000 \\
\hline 306.450 & 4.946 & 5.283 & 17.443 & 18.970 & -8.050 & 0.000 \\
\hline 306.450 & 4.946 & 5.285 & 17.447 & 19.180 & -9.035 & 0.000 \\
\hline 306.450 & 4.948 & 5.312 & 17.515 & 19.600 & -10.639 & 0.000 \\
\hline 306.450 & 4.949 & 5.335 & 17.572 & 19.870 & -11.565 & 0.000 \\
\hline 306.450 & 4.950 & 5.350 & 17.608 & 20.190 & -12.787 & 0.000 \\
\hline 306.450 & 4.951 & 5.375 & 17.669 & 20.490 & -13.768 & 0.000 \\
\hline 306.450 & 4.953 & 5.404 & 17.742 & 20.920 & -15.191 & 0.000 \\
\hline 306.450 & 4.954 & 5.420 & 17.782 & 21.220 & -16.200 & 0.000 \\
\hline 306.450 & 4.953 & 5.406 & 17.747 & 21.540 & -17.609 & 0.000 \\
\hline 306.450 & 4.956 & 5.464 & 17.894 & 21.870 & -18.182 & 0.000 \\
\hline 306.450 & 4.957 & 5.490 & 17.961 & 22.150 & -18.914 & 0.000 \\
\hline 306.450 & 4.958 & 5.513 & 18.019 & 22.470 & -19.810 & 0.000 \\
\hline 306.450 & 4.959 & 5.544 & 18.097 & 22.780 & -20.556 & 0.000 \\
\hline 306.450 & 4.961 & 5.584 & 18.200 & 23.090 & -21.179 & 0.000 \\
\hline 306.450 & 4.964 & 5.662 & 18.403 & 23.700 & -22.351 & 0.000 \\
\hline 306.450 & 4.969 & 5.831 & 18.849 & 24.490 & -23.033 & 0.000 \\
\hline 306.450 & 4.977 & 6.150 & 19.727 & 25.290 & -21.996 & 0.000 \\
\hline 306.450 & 4.989 & 6.884 & 21.918 & 26.100 & -16.021 & 0.000 \\
\hline 306.450 & 5.007 & 7.810 & 25.064 & 27.040 & -7.306 & 0.000 \\
\hline 306.450 & 5.032 & 8.341 & 27.084 & 27.940 & -3.062 & 0.000 \\
\hline 306.450 & 5.050 & 8.543 & 27.895 & 28.480 & -2.055 & 0.000 \\
\hline 306.450 & 5.072 & 8.726 & 28.656 & 29.030 & -1.288 & 0.000 \\
\hline 306.450 & 5.099 & 8.894 & 29.375 & 29.560 & -0.626 & 0.000 \\
\hline 306.450 & 5.131 & 9.049 & 30.056 & 30.090 & -0.114 & 0.000 \\
\hline 306.450 & 5.170 & 9.203 & 30.750 & 30.720 & 0.097 & 0.000 \\
\hline 306.450 & 5.216 & 9.352 & 31.437 & 31.360 & 0.246 & 0.000 \\
\hline 306.450 & 5.272 & 9.500 & 32.141 & 32.010 & 0.410 & 0.000 \\
\hline 306.450 & 5.338 & 9.647 & 32.857 & 32.670 & 0.571 & 0.000 \\
\hline 306.450 & 5.415 & 9.794 & 33.589 & 33.400 & 0.565 & 0.000 \\
\hline
\end{tabular}


Table 14. VISCOSITY AT ELEVATED PRESSURES (continued)

Data from Iwasaki et al. [73] (continued)

\begin{tabular}{|c|c|c|c|c|c|c|}
\hline $\begin{array}{l}\mathrm{T} \\
\mathrm{K}\end{array}$ & $\begin{array}{c}\mathrm{P} \\
\mathrm{MPa}\end{array}$ & $\begin{array}{c}\rho \\
\mathrm{mol} \cdot \mathrm{dm}^{-3}\end{array}$ & $\begin{array}{c}\eta, \text { calc } \\
\mu \mathrm{Pa} \cdot \mathrm{s}\end{array}$ & $\begin{array}{c}\eta, \text { expt } \\
\mu \mathrm{Pa} \cdot \mathrm{s}\end{array}$ & $\begin{array}{c}\text { Dev } \\
8\end{array}$ & wt \\
\hline 308.150 & 0.349 & 0.140 & 9.704 & 9.570 & 1.401 & 0.000 \\
\hline 308.150 & 0.590 & 0.240 & 9.766 & 9.600 & 1.728 & 0.000 \\
\hline 308.150 & 0.833 & 0.345 & 9.835 & 9.640 & 2.018 & 0.000 \\
\hline 308.150 & 1.062 & 0.448 & 9.906 & 9.690 & 2.226 & 0.000 \\
\hline 308.150 & 1.345 & 0.582 & 10.004 & 9.760 & 2.495 & 0.000 \\
\hline 308.150 & 1.551 & 0.683 & 10.082 & 9.820 & 2.668 & 0.000 \\
\hline 308.150 & 1.868 & 0.847 & 10.217 & 9.910 & 3.100 & 0.000 \\
\hline 308.150 & 2.167 & 1.013 & 10.364 & 10.030 & 3.329 & 0.000 \\
\hline 308.150 & 2.534 & 1.234 & 10.574 & 10.220 & 3.463 & 0.000 \\
\hline 308.150 & 2.911 & 1.485 & 10.834 & 10.410 & 4.069 & 0.000 \\
\hline 308.150 & 3.346 & 1.816 & 11.210 & 10.750 & 4.283 & 0.000 \\
\hline 308.150 & 3.745 & 2.175 & 11.664 & 11.180 & 4.325 & 0.000 \\
\hline 308.150 & 4.234 & 2.741 & 12.467 & 11.870 & 5.033 & 0.000 \\
\hline 308.150 & 4.584 & 3.311 & 13.391 & 12.750 & 5.028 & 0.000 \\
\hline 308.150 & 4.855 & 3.996 & 14.655 & 13.980 & 4.830 & 0.000 \\
\hline 308.150 & 4.992 & 4.580 & 15.868 & 15.270 & 3.913 & 0.000 \\
\hline 308.150 & 5.070 & 5.154 & 17.185 & 16.860 & 1.928 & 0.000 \\
\hline 308.150 & 5.079 & 5.248 & 17.411 & 17.130 & 1.641 & 0.000 \\
\hline 308.150 & 5.093 & 5.413 & 17.821 & 17.680 & 0.797 & 0.000 \\
\hline 308.150 & 5.103 & 5.544 & 18.153 & 18.130 & 0.128 & 0.000 \\
\hline 308.150 & 5.111 & 5.672 & 18.483 & 18.580 & -0.524 & 0.000 \\
\hline 308.150 & 5.120 & 5.828 & 18.897 & 19.150 & -1.319 & 0.000 \\
\hline 308.150 & 5.125 & 5.920 & 19.145 & 19.490 & -1.772 & 0.000 \\
\hline 308.150 & 5.130 & 6.015 & 19.406 & 19.900 & -2.482 & 0.000 \\
\hline 308.150 & 5.133 & 6.089 & 19.609 & 20.180 & -2.829 & 0.000 \\
\hline 308.150 & 5.137 & 6.169 & 19.836 & 20.500 & -3.241 & 0.000 \\
\hline 308.150 & 5.140 & 6.244 & 20.047 & 20.790 & -3.572 & 0.000 \\
\hline 308.150 & 5.143 & 6.309 & 20.234 & 21.090 & -4.059 & 0.000 \\
\hline 308.150 & 5.147 & 6.411 & 20.533 & 21.440 & -4.231 & 0.000 \\
\hline 308.150 & 5.151 & 6.497 & 20.786 & 21.730 & -4.346 & 0.000 \\
\hline 308.150 & 5.155 & 6.589 & 21.061 & 22.080 & -4.614 & 0.000 \\
\hline 308.150 & 5.159 & 6.694 & 21.382 & 22.420 & -4.628 & 0.000 \\
\hline 308.150 & 5.163 & 6.803 & 21.718 & 22.750 & -4.537 & 0.000 \\
\hline 308.150 & 5.172 & 7.020 & 22.406 & 23.400 & -4.247 & 0.000 \\
\hline 308.150 & 5.183 & 7.283 & 23.270 & 24.100 & -3.445 & 0.000 \\
\hline 308.150 & 5.196 & 7.551 & 24.191 & 24.770 & -2.337 & 0.000 \\
\hline
\end{tabular}


Table 14. VISCOSITY AT ELEVATED PRESSURES (continued)

Data from Iwasaki et al. [73] (continued)

\begin{tabular}{|c|c|c|c|c|c|c|}
\hline $\begin{array}{l}\mathrm{T} \\
\mathrm{K}\end{array}$ & $\begin{array}{c}\mathrm{P} \\
\mathrm{MPa}\end{array}$ & $\stackrel{\rho}{\mathrm{mol} \cdot \mathrm{dm}^{-3}}$ & $\begin{array}{c}\eta, \text { calc } \\
\mu \mathrm{Pa} \cdot \mathrm{s}\end{array}$ & $\begin{array}{c}\eta, \text { expt } \\
\mu \mathrm{Pa} \cdot \mathrm{s}\end{array}$ & $\begin{array}{c}\text { Dev } \\
\frac{8}{z}\end{array}$ & wt \\
\hline 308.150 & 5.212 & 7.837 & 25.212 & 25.520 & -1.205 & 0.000 \\
\hline 308.150 & 5.234 & 8.120 & 26.270 & 26.380 & -0.416 & 0.000 \\
\hline 308.150 & 5.249 & 8.270 & 26.853 & 26.780 & 0.274 & 0.000 \\
\hline 308.150 & 5.267 & 8.420 & 27.448 & 27.300 & 0.542 & 0.000 \\
\hline 308.150 & 5.289 & 8.571 & 28.062 & 27.790 & 0.980 & 0.000 \\
\hline 308.150 & 5.313 & 8.711 & 28.642 & 28.340 & 1.064 & 0.000 \\
\hline 308.150 & 5.342 & 8.848 & 29.226 & 28.840 & 1.339 & 0.000 \\
\hline 308.150 & 5.375 & 8.984 & 29.819 & 29.380 & 1.493 & 0.000 \\
\hline 308.150 & 5.415 & 9.121 & 30.427 & 29.990 & 1.456 & 0.000 \\
\hline 308.150 & 5.462 & 9.257 & 31.048 & 30.610 & 1.432 & 0.000 \\
\hline 308.150 & 5.516 & 9.394 & 31.683 & 31.220 & 1.482 & 0.000 \\
\hline 308.150 & 5.585 & 9.542 & 32.388 & 31.940 & 1.403 & 0.000 \\
\hline 308.150 & 5.661 & 9.681 & 33.069 & 32.590 & 1.471 & 0.000 \\
\hline 308.150 & 5.749 & 9.821 & 33.773 & 33.300 & 1.420 & 0.000 \\
\hline 308.150 & 5.850 & 9.962 & 34.498 & 34.000 & 1.466 & 0.000 \\
\hline 308.150 & 5.968 & 10.106 & 35.255 & 34.810 & 1.279 & 0.000 \\
\hline 308.150 & 6.100 & 10.247 & 36.018 & 35.620 & 1.117 & 0.000 \\
\hline 323.150 & 0.202 & 0.076 & 10.103 & 10.020 & 0.824 & 0.000 \\
\hline 323.150 & 0.349 & 0.133 & 10.137 & 10.020 & 1.168 & 0.000 \\
\hline 323.150 & 0.497 & 0.191 & 10.173 & 10.040 & 1.330 & 0.000 \\
\hline 323.150 & 0.746 & 0.291 & 10.239 & 10.090 & 1.480 & 0.000 \\
\hline 323.150 & 1.026 & 0.407 & 10.321 & 10.140 & 1.781 & 0.000 \\
\hline 323.150 & 1.358 & 0.551 & 10.428 & 10.220 & 2.032 & 0.000 \\
\hline 323.150 & 1.650 & 0.684 & 10.533 & 10.300 & 2.265 & 0.000 \\
\hline 323.150 & 1.960 & 0.832 & 10.658 & 10.390 & 2.578 & 0.000 \\
\hline 323.150 & 2.268 & 0.988 & 10.797 & 10.500 & 2.832 & 0.000 \\
\hline 323.150 & 2.544 & 1.134 & 10.936 & 10.620 & 2.979 & 0.000 \\
\hline 323.150 & 2.892 & 1.331 & 11.135 & 10.770 & 3.390 & 0.000 \\
\hline 323.150 & 3.274 & 1.565 & 11.389 & 10.990 & 3.627 & 0.000 \\
\hline 323.150 & 3.848 & 1.962 & 11.863 & 11.410 & 3.968 & 0.000 \\
\hline 323.150 & 4.266 & 2.297 & 12.304 & 11.830 & 4.007 & 0.000 \\
\hline 323.150 & 4.639 & 2.640 & 12.797 & 12.290 & 4.123 & 0.000 \\
\hline 323.150 & 4.972 & 2.995 & 13.350 & 12.790 & 4.375 & 0.000 \\
\hline 323.150 & 5.258 & 3.351 & 13.948 & 13.360 & 4.404 & 0.000 \\
\hline 323.150 & 5.528 & 3.748 & 14.666 & 14.070 & 4.238 & 0.000 \\
\hline 323.150 & 5.774 & 4.178 & 15.509 & 14.880 & 4.225 & 0.000 \\
\hline
\end{tabular}


Table 14. VISCOSITY AT ELEVATED PRESSURES (continued)

Data from Iwasaki et al. [73] (continued)

\begin{tabular}{|c|c|c|c|c|c|c|}
\hline $\begin{array}{l}\mathrm{T} \\
\mathrm{K}\end{array}$ & $\begin{array}{c}\mathrm{P} \\
\mathrm{MPa}\end{array}$ & $\stackrel{\rho}{\mathrm{mol} \cdot \mathrm{dm}^{-3}}$ & $\begin{array}{c}\eta, \text { calc } \\
\mu \mathrm{Pa} \cdot \mathrm{s}\end{array}$ & $\begin{array}{c}\eta, \text { expt } \\
\mu \mathrm{Pa} \cdot \mathrm{s}\end{array}$ & $\begin{array}{c}\text { Dev } \\
\frac{8}{8}\end{array}$ & wt \\
\hline $\begin{array}{l}323.150 \\
323.150 \\
323.150\end{array}$ & $\begin{array}{l}5.895 \\
6.066 \\
6.237\end{array}$ & $\begin{array}{l}4.421 \\
4.810 \\
5.259\end{array}$ & $\begin{array}{l}16.014 \\
16.867 \\
17.924\end{array}$ & $\begin{array}{l}15.410 \\
16.250 \\
17.340\end{array}$ & $\begin{array}{l}3.922 \\
3.796 \\
3.370\end{array}$ & $\begin{array}{l}0.000 \\
0.000 \\
0.000\end{array}$ \\
\hline $\begin{array}{l}323.150 \\
323.150 \\
323.150 \\
323.150 \\
323.150\end{array}$ & $\begin{array}{l}6.327 \\
6.401 \\
6.446 \\
6.525 \\
6.562\end{array}$ & $\begin{array}{l}5.522 \\
5.748 \\
5.890 \\
6.145 \\
6.265\end{array}$ & $\begin{array}{l}18.581 \\
19.166 \\
19.546 \\
20.245 \\
20.586\end{array}$ & $\begin{array}{l}17.990 \\
18.630 \\
19.000 \\
19.700 \\
20.050\end{array}$ & $\begin{array}{l}3.283 \\
2.877 \\
2.871 \\
2.768 \\
2.672\end{array}$ & $\begin{array}{l}0.000 \\
0.000 \\
0.000 \\
0.000 \\
0.000\end{array}$ \\
\hline $\begin{array}{l}323.150 \\
323.150 \\
323.150 \\
323.150 \\
323.150\end{array}$ & $\begin{array}{l}6.596 \\
6.624 \\
6.653 \\
6.682 \\
6.712\end{array}$ & $\begin{array}{l}6.375 \\
6.468 \\
6.561 \\
6.657 \\
6.754\end{array}$ & $\begin{array}{l}20.904 \\
21.175 \\
21.452 \\
21.740 \\
22.036\end{array}$ & $\begin{array}{l}20.330 \\
20.630 \\
20.960 \\
21.230 \\
21.530\end{array}$ & $\begin{array}{l}2.823 \\
2.641 \\
2.345 \\
2.404 \\
2.352\end{array}$ & $\begin{array}{l}0.000 \\
0.000 \\
0.000 \\
0.000 \\
0.000\end{array}$ \\
\hline $\begin{array}{l}323.150 \\
323.150 \\
323.150 \\
323.150 \\
323.150\end{array}$ & $\begin{array}{l}6.744 \\
6.775 \\
6.809 \\
6.846 \\
6.846\end{array}$ & $\begin{array}{l}6.853 \\
6.951 \\
7.054 \\
7.165 \\
7.166\end{array}$ & $\begin{array}{l}22.344 \\
22.655 \\
22.982 \\
23.345 \\
23.347\end{array}$ & $\begin{array}{l}21.850 \\
22.140 \\
22.450 \\
22.810 \\
22.800\end{array}$ & $\begin{array}{l}2.260 \\
2.327 \\
2.369 \\
2.345 \\
2.399\end{array}$ & $\begin{array}{l}0.000 \\
0.000 \\
0.000 \\
0.000 \\
0.000\end{array}$ \\
\hline $\begin{array}{l}323.150 \\
323.150 \\
323.150 \\
323.150 \\
323.150\end{array}$ & $\begin{array}{l}6.925 \\
6.965 \\
7.069 \\
7.275 \\
7.547\end{array}$ & $\begin{array}{l}7.390 \\
7.497 \\
7.760 \\
8.207 \\
8.670\end{array}$ & $\begin{array}{l}24.097 \\
24.464 \\
25.390 \\
27.058 \\
28.914\end{array}$ & $\begin{array}{l}23.550 \\
23.900 \\
24.830 \\
26.460 \\
28.350\end{array}$ & $\begin{array}{l}2.324 \\
2.359 \\
2.257 \\
2.262 \\
1.991\end{array}$ & $\begin{array}{l}0.000 \\
0.000 \\
0.000 \\
0.000 \\
0.000\end{array}$ \\
\hline $\begin{array}{l}323.150 \\
323.150 \\
323.150 \\
348.150 \\
348.150\end{array}$ & $\begin{array}{l}7.821 \\
8.110 \\
8.355 \\
0.202 \\
0.345\end{array}$ & $\begin{array}{l}9.038 \\
9.354 \\
9.581 \\
0.070 \\
0.121\end{array}$ & $\begin{array}{l}30.494 \\
31.931 \\
33.011 \\
10.812 \\
10.845\end{array}$ & $\begin{array}{l}29.940 \\
31.440 \\
32.550 \\
10.720 \\
10.750\end{array}$ & $\begin{array}{l}1.849 \\
1.563 \\
1.417 \\
0.861 \\
0.883\end{array}$ & $\begin{array}{l}0.000 \\
0.000 \\
0.000 \\
0.000 \\
0.000\end{array}$ \\
\hline $\begin{array}{l}348.150 \\
348.150 \\
348.150 \\
348.150 \\
348.150\end{array}$ & $\begin{array}{l}0.591 \\
0.880 \\
1.175 \\
1.507 \\
1.749\end{array}$ & $\begin{array}{l}0.210 \\
0.317 \\
0.430 \\
0.561 \\
0.660\end{array}$ & $\begin{array}{l}10.904 \\
10.979 \\
11.063 \\
11.166 \\
11.247\end{array}$ & $\begin{array}{l}10.780 \\
10.830 \\
10.900 \\
10.970 \\
11.030\end{array}$ & $\begin{array}{l}1.152 \\
1.379 \\
1.494 \\
1.784 \\
1.967\end{array}$ & $\begin{array}{l}0.000 \\
0.000 \\
0.000 \\
0.000 \\
0.000\end{array}$ \\
\hline $\begin{array}{l}348.150 \\
348.150 \\
348.150\end{array}$ & $\begin{array}{l}2.115 \\
2.456 \\
2.729\end{array}$ & $\begin{array}{l}0.814 \\
0.965 \\
1.090\end{array}$ & $\begin{array}{l}11.381 \\
11.519 \\
11.640\end{array}$ & $\begin{array}{l}11.150 \\
11.260 \\
11.380\end{array}$ & $\begin{array}{l}2.073 \\
2.302 \\
2.287\end{array}$ & $\begin{array}{l}0.000 \\
0.000 \\
0.000\end{array}$ \\
\hline
\end{tabular}


Table 14. VISCOSITY AT ELEVATED PRESSURES (continued)

Data from Iwasaki et al. [73] (continued)

\begin{tabular}{|c|c|c|c|c|c|c|}
\hline $\begin{array}{l}\mathrm{T} \\
\mathrm{K}\end{array}$ & $\underset{\mathrm{MPa}}{\mathrm{P}}$ & $\stackrel{\rho}{\mathrm{mol} \cdot \mathrm{dm}^{-3}}$ & $\begin{array}{c}\eta, \text { calc } \\
\mu \mathrm{Pa} \cdot \mathrm{s}\end{array}$ & $\begin{array}{c}\eta, \underset{\mu \mathrm{expt}}{\mu \mathrm{Pa} \cdot \mathrm{s}} \\
\end{array}$ & $\begin{array}{c}\text { Dev } \\
\frac{8}{8}\end{array}$ & wt \\
\hline 348.150 & 3.067 & 1.251 & 11.804 & 11.520 & 2.467 & 0.000 \\
\hline 348.150 & 3.333 & 1.383 & 11.945 & 11.630 & 2.709 & 0.000 \\
\hline 348.150 & 3.598 & 1.520 & 12.098 & 11.790 & 2.611 & 0.000 \\
\hline 348.150 & 3.819 & 1.639 & 12.235 & 11.900 & 2.812 & 0.000 \\
\hline 348.150 & 4.243 & 1.878 & 12.525 & 12.160 & 3.005 & 0.000 \\
\hline 348.150 & 4.595 & 2.090 & 12.799 & 12.440 & 2.887 & 0.000 \\
\hline 348.150 & 5.073 & 2.400 & 13.227 & 12.840 & 3.014 & 0.000 \\
\hline 348.150 & 5.735 & 2.880 & 13.952 & 13.520 & 3.199 & 0.000 \\
\hline 348.150 & 6.366 & 3.403 & 14.831 & 14.320 & 3.572 & 0.000 \\
\hline 348.150 & 6.922 & 3.926 & 15.806 & 15.240 & 3.715 & 0.000 \\
\hline 348.150 & 7.362 & 4.388 & 16.744 & 16.150 & 3.679 & 0.000 \\
\hline 348.150 & 7.640 & 4.701 & 17.423 & 16.790 & 3.773 & 0.000 \\
\hline 348.150 & 7.951 & 5.067 & 18.264 & 17.610 & 3.715 & 0.000 \\
\hline 348.150 & 8.171 & 5.336 & 18.912 & 18.220 & 3.798 & 0.000 \\
\hline 348.150 & 8.496 & 5.738 & 19.938 & 19.240 & 3.627 & 0.000 \\
\hline 348.150 & 8.681 & 5.968 & 20.553 & 19.860 & 3.488 & 0.000 \\
\hline 348.150 & 8.924 & 6.268 & 21.387 & 20.670 & 3.470 & 0.000 \\
\hline 348.150 & 9.105 & 6.487 & 22.022 & 21.290 & 3.440 & 0.000 \\
\hline 348.150 & 9.188 & 6.585 & 22.314 & 21.540 & 3.592 & 0.000 \\
\hline 348.150 & 9.391 & 6.820 & 23.030 & 22.350 & 3.041 & 0.000 \\
\hline 348.150 & 9.481 & 6.921 & 23.346 & 22.590 & 3.345 & 0.000 \\
\hline 348.150 & 9.653 & 7.110 & 23.950 & 23.220 & 3.144 & 0.000 \\
\hline 348.150 & 9.760 & 7.223 & 24.320 & 23.600 & 3.052 & 0.000 \\
\hline 348.150 & 9.865 & 7.333 & 24.684 & 23.970 & 2.979 & 0.000 \\
\hline 348.150 & 10.075 & 7.541 & 25.394 & 24.740 & 2.645 & 0.000 \\
\hline 348.150 & 10.339 & 7.787 & 26.264 & 25.570 & 2.716 & 0.000 \\
\hline 348.150 & 10.528 & 7.953 & 26.871 & 26.190 & 2.602 & 0.000 \\
\hline 348.150 & 10.799 & 8.176 & 27.710 & 27.030 & 2.515 & 0.000 \\
\hline 348.150 & 11.089 & 8.398 & 28.577 & 27.910 & 2.388 & 0.000 \\
\hline 348.150 & 11.497 & 8.682 & 29.731 & 29.050 & 2.343 & 0.000 \\
\hline 348.150 & 11.869 & 8.916 & 30.726 & 30.090 & 2.114 & 0.000 \\
\hline 348.150 & 12.267 & 9.145 & 31.736 & 31.120 & 1.979 & 0.000 \\
\hline 348.150 & 12.624 & 9.334 & 32.599 & 32.090 & 1.587 & 0.000 \\
\hline 348.150 & 12.941 & 9.489 & 33.331 & 32.840 & 1.495 & 0.000 \\
\hline
\end{tabular}


Table 14. VISCOSITY AT ELEVATED PRESSURES (continued)

Data from Iwasaki et al. [73] (continued)

Comparisons based on experimental pressures:

Number of Points [73] 402

$$
\begin{array}{ll}
\mathrm{AAD} \gamma=5.64 & \mathrm{BIAS} \%=-2.03 \quad \mathrm{RMS} \%=8.30 \\
\mathrm{AAD}=1.15 \quad \mathrm{BIAS}=-0.60 \mathrm{RMS}=1.84 \mu \mathrm{Pa} \cdot \mathrm{S}
\end{array}
$$

Comparisons based on experimental densities:

Number of Points [73] 402

$$
\begin{array}{ll}
\mathrm{AAD} \gamma & =2.42 \quad \mathrm{BIAS} \%=2.12 \quad \mathrm{RMS} \%=1.89 \\
\mathrm{AAD}=0.40 \quad \mathrm{BIAS}=0.33 \mathrm{RMS}=0.30 \mu \mathrm{Pa} \cdot \mathrm{s}
\end{array}
$$

\begin{tabular}{|c|c|c|c|c|c|c|}
\hline $\begin{array}{l}\mathrm{T} \\
\mathrm{K}\end{array}$ & $\begin{array}{c}\mathrm{P} \\
\mathrm{MPa}\end{array}$ & $\begin{array}{c}\rho \\
\mathrm{mol} \cdot \mathrm{dm}^{-3}\end{array}$ & $\begin{array}{c}\eta, \text { calc } \\
\mu \mathrm{Pa} \cdot \mathrm{s}\end{array}$ & $\begin{array}{c}\eta, \text { expt } \\
\mu \mathrm{Pa} \cdot \mathrm{s}\end{array}$ & $\begin{array}{c}\text { Dev } \\
\frac{8}{8}\end{array}$ & wt \\
\hline 290.064 & 3.994 & 11.935 & 46.466 & 41.400 & 12.237 & 0.000 \\
\hline 290.064 & 9.986 & 13.375 & 59.359 & 55.300 & 7.340 & 0.000 \\
\hline 290.064 & 19.973 & 14.463 & 72.539 & 70.000 & 3.627 & 0.000 \\
\hline 305.330 & 4.491 & 3.382 & 13.422 & 13.700 & -2.026 & 0.000 \\
\hline 305.330 & 9.986 & 12.266 & 49.463 & 45.200 & 9.432 & 0.000 \\
\hline 305.330 & 19.973 & 13.746 & 63.906 & 59.800 & 6.866 & 0.000 \\
\hline 320.597 & 4.988 & 3.134 & 13.496 & 13.200 & 2.243 & 0.000 \\
\hline 320.597 & 9.986 & 10.868 & 39.970 & 34.600 & 15.522 & 0.000 \\
\hline 320.597 & 19.973 & 12.988 & 56.369 & 50.500 & 11.622 & 0.000 \\
\hline 335.863 & 7.487 & 5.773 & 19.640 & 17.600 & 11.589 & 0.000 \\
\hline 335.863 & 9.986 & 9.052 & 30.942 & 26.300 & 17.649 & 0.000 \\
\hline 335.863 & 19.973 & 12.190 & 49.791 & 43.300 & 14.990 & 0.000 \\
\hline 381.663 & 4.988 & 1.915 & 13.569 & 11.800 & 14.987 & 0.000 \\
\hline 381.663 & 9.986 & 4.852 & 18.810 & 15.700 & 19.808 & 0.000 \\
\hline 381.663 & 19.973 & 9.664 & 35.248 & 29.100 & 21.126 & 0.000 \\
\hline
\end{tabular}

Data from Smith et al. [95] 
Table 14. VISCOSITY AT ELEVATED PRESSURES (continued)

Data from Smith et al. [95] (continued)

Comparisons based on experimental pressures:

Number of Points [95] 15

$$
\begin{aligned}
& \mathrm{AAD} \%=11.40 \quad \mathrm{BIAS} \%=11.13 \quad \mathrm{RMS} \%=6.39 \\
& \mathrm{AAD}=3.74 \mathrm{BIAS}=3.70 \mathrm{RMS}=2.00 \mu \mathrm{Pa} \cdot \mathrm{s}
\end{aligned}
$$

Comparisons based on experimental densities:

Number of Points [95] 15

\begin{tabular}{|c|c|c|c|c|c|c|}
\hline $\begin{array}{l}\mathrm{T} \\
\mathrm{K}\end{array}$ & $\begin{array}{c}\mathrm{P} \\
\mathrm{MPa}\end{array}$ & $\stackrel{\rho}{\mathrm{mol}} \cdot \mathrm{dm}^{-3}$ & $\begin{array}{c}\eta, \text { calc } \\
\mu \mathrm{Pa} \cdot \mathrm{s}\end{array}$ & $\begin{array}{c}\eta, \exp t \\
\mu \mathrm{Pa} \cdot \mathrm{s}\end{array}$ & $\begin{array}{c}\text { Dev } \\
8\end{array}$ & wt \\
\hline 316.482 & 6.002 & 6.652 & 21.516 & 18.900 & 13.844 & 0.000 \\
\hline 310.918 & 5.438 & 6.654 & 21.346 & 18.880 & 13.063 & 0.000 \\
\hline 308.141 & 5.156 & 6.655 & 21.261 & 18.880 & 12.611 & 0.000 \\
\hline 307.140 & 5.055 & 6.655 & 21.231 & 19.010 & 11.681 & 0.000 \\
\hline 306.139 & 4.954 & 6.655 & 21.200 & 19.200 & 10.418 & 0.000 \\
\hline 305.741 & 4.913 & 6.655 & 21.188 & 19.460 & 8.878 & 0.000 \\
\hline 305.640 & 4.903 & 6.655 & 21.185 & 19.630 & 7.919 & 0.000 \\
\hline 305.538 & 4.893 & 6.655 & 21.181 & 19.940 & 6.225 & 0.000 \\
\hline 305.442 & 4.883 & 6.655 & 21.178 & 20.300 & 4.327 & 0.000 \\
\hline 305.395 & 4.878 & 6.656 & 21.178 & 20.840 & 1.621 & 0.000 \\
\hline 322.032 & 6.566 & 6.651 & 21.686 & 18.930 & 14.561 & 0.000 \\
\hline 319.139 & 6.272 & 6.652 & 21.598 & 18.900 & 14.276 & 0.000 \\
\hline 316.472 & 6.001 & 6.652 & 21.516 & 18.890 & 13.902 & 0.000 \\
\hline 313.643 & 5.714 & 6.653 & 21.429 & 18.890 & 13.441 & 0.000 \\
\hline 305.445 & 4.883 & 6.655 & 21.178 & 20.250 & 4.585 & 0.000 \\
\hline 305.411 & 4.880 & 6.656 & 21.178 & 20.580 & 2.908 & 0.000 \\
\hline 305.392 & 4.878 & 6.656 & 21.178 & 20.810 & 1.767 & 0.000 \\
\hline 305.382 & 4.877 & 6.656 & 21.177 & 20.980 & 0.941 & 0.000 \\
\hline 316.472 & 6.058 & 6.964 & 22.487 & 19.930 & 12.830 & 0.000 \\
\hline 313.638 & 5.754 & 6.965 & 22.402 & 19.930 & 12.402 & 0.000 \\
\hline 311.139 & 5.487 & 6.966 & 22.326 & 19.920 & 12.078 & 0.000 \\
\hline 308.142 & 5.169 & 6.967 & 22.235 & 19.990 & 11.229 & 0.000 \\
\hline
\end{tabular}

$$
\begin{aligned}
& \mathrm{AAD} \%=11.46 \quad \text { BIAS } 8=11.23 \text { RMS } 8=6.43 \\
& \mathrm{AAD}=3.75 \mathrm{BIAS}=3.72 \mathrm{RMS}=2.01 \mu \mathrm{Pa} \cdot \mathrm{s}
\end{aligned}
$$

Data from Strumpf et al. [96] 
Table 14. VISCOSITY AT ELEVATED PRESSURES (continued)

Data from Strumpf et al. [96] (continued)

\begin{tabular}{|c|c|c|c|c|c|c|}
\hline $\begin{array}{l}\mathrm{T} \\
\mathrm{K}\end{array}$ & $\begin{array}{c}\mathrm{P} \\
\mathrm{MPa}\end{array}$ & $\stackrel{\rho}{\mathrm{mol}} \cdot \mathrm{dm}^{-3}$ & $\begin{array}{c}\eta, \text { calc } \\
\mu \mathrm{Pa} \cdot \mathrm{s}\end{array}$ & $\begin{array}{c}\eta, \text { expt } \\
\mu \mathrm{Pa} \cdot \mathrm{s}\end{array}$ & $\begin{array}{c}\text { Dev } \\
\%\end{array}$ & wt \\
\hline 307.138 & 5.063 & 6.967 & 22.205 & 20.050 & 10.746 & 0.000 \\
\hline 306.141 & 4.957 & 6.967 & 22.175 & 20.310 & 9.182 & 0.000 \\
\hline 305.737 & 4.915 & 6.968 & 22.163 & 20.620 & 7.484 & 0.000 \\
\hline 305.643 & 4.905 & 6.968 & 22.160 & 20.900 & 6.030 & 0.000 \\
\hline 305.539 & 4.894 & 6.968 & 22.157 & 21.140 & 4.811 & 0.000 \\
\hline 305.424 & 4.882 & 6.968 & 22.154 & 22.010 & 0.652 & 0.000 \\
\hline 305.411 & 4.880 & 6.968 & 22.153 & 22.200 & -0.211 & 0.000 \\
\hline 305.393 & 4.878 & 6.968 & 22.153 & 22.510 & -1.588 & 0.000 \\
\hline 305.385 & 4.878 & 6.968 & 22.152 & 22.640 & -2.154 & 0.000 \\
\hline 322.139 & 6.669 & 6.963 & 22.659 & 20.020 & 13.183 & 0.000 \\
\hline 319.144 & 6.346 & 6.963 & 22.567 & 19.900 & 13.405 & 0.000 \\
\hline 316.471 & 6.058 & 6.964 & 22.487 & 19.900 & 13.000 & 0.000 \\
\hline 305.457 & 4.885 & 6.968 & 22.155 & 21.560 & 2.758 & 0.000 \\
\hline 305.440 & 4.883 & 6.968 & 22.154 & 21.740 & 1.905 & 0.000 \\
\hline 305.421 & 4.881 & 6.968 & 22.153 & 21.960 & 0.881 & 0.000 \\
\hline 305.398 & 4.879 & 6.968 & 22.153 & 22.380 & -1.015 & 0.000 \\
\hline 305.390 & 4.878 & 6.968 & 22.153 & 22.520 & -1.632 & 0.000 \\
\hline 310.919 & 6.139 & 9.640 & 32.947 & 31.090 & 5.971 & 0.000 \\
\hline 308.142 & 5.637 & 9.641 & 32.875 & 30.860 & 6.530 & 0.000 \\
\hline 306.144 & 5.281 & 9.642 & 32.823 & 30.910 & 6.189 & 0.000 \\
\hline 305.371 & 5.144 & 9.640 & 32.792 & 30.970 & 5.883 & 0.000 \\
\hline 304.141 & 4.930 & 9.643 & 32.773 & 30.860 & 6.198 & 0.000 \\
\hline 303.632 & 4.841 & 9.643 & 32.761 & 30.840 & 6.227 & 0.000 \\
\hline 303.123 & 4.754 & 9.644 & 32.748 & 30.890 & 6.016 & 0.000 \\
\hline 302.742 & 4.688 & 9.644 & 32.738 & 30.860 & 6.085 & 0.000 \\
\hline 302.642 & 4.671 & 9.644 & 32.735 & 30.880 & 6.008 & 0.000 \\
\hline 302.544 & 4.654 & 9.644 & 32.733 & 30.980 & 5.657 & 0.000 \\
\hline 302.444 & 4.637 & 9.644 & 32.731 & 31.020 & 5.517 & 0.000 \\
\hline 302.248 & 4.603 & 9.644 & 32.726 & 31.300 & 4.556 & 0.000 \\
\hline 301.852 & 4.536 & 9.644 & 32.715 & 30.860 & 6.012 & 0.000 \\
\hline 322.146 & 8.229 & 9.635 & 33.246 & 30.960 & 7.383 & 0.000 \\
\hline 319.142 & 7.662 & 9.637 & 33.165 & 30.740 & 7.887 & 0.000 \\
\hline 316.472 & 7.163 & 9.638 & 33.092 & 30.830 & 7.337 & 0.000 \\
\hline 313.645 & 6.638 & 9.639 & 33.017 & 30.790 & 7.234 & 0.000 \\
\hline 310.925 & 6.140 & 9.640 & 32.947 & 31.000 & 6.280 & 0.000 \\
\hline 303.151 & 4.758 & 9.644 & 32.749 & 30.820 & 6.259 & 0.000 \\
\hline
\end{tabular}


Table 14. VISCOSITY AT ELEVATED PRESSURES (continued)

Data from Strumpf et al. [96] (continued)

\begin{tabular}{|c|c|c|c|c|c|c|}
\hline $\begin{array}{l}\mathrm{T} \\
\mathrm{K}\end{array}$ & $\begin{array}{c}\mathrm{P} \\
\mathrm{MPa}\end{array}$ & $\stackrel{\rho}{\mathrm{mol} \cdot \mathrm{dm}^{-3}}$ & $\begin{array}{c}\eta, \text { calc } \\
\mu \mathrm{Pa} \cdot \mathrm{s}\end{array}$ & $\begin{array}{c}\eta ; \operatorname{expt} \\
\mu \mathrm{Pa} \cdot \mathrm{s}\end{array}$ & $\begin{array}{c}\text { Dev } \\
\frac{\gamma}{z}\end{array}$ & wt \\
\hline 303.045 & 4.740 & 9.644 & 32.746 & 30.920 & 5.906 & 0.000 \\
\hline 302.900 & 4.715 & 9.644 & 32.742 & 30.980 & 5.688 & 0.000 \\
\hline 301.946 & 4.552 & 9.644 & 32.718 & 30.860 & 6.021 & 0.000 \\
\hline 301.847 & 4.535 & $9: 644$ & 32.715 & 30.810 & 6.184 & 0.000 \\
\hline 316.471 & 6.102 & 7.193 & 23.227 & 20.630 & 12.590 & 0.000 \\
\hline 313.642 & 5.786 & 7.194 & 23.143 & 21.020 & 10.099 & 0.000 \\
\hline 311.146 & 5.509 & 7.194 & 23.068 & 20.660 & 11.654 & 0.000 \\
\hline 308.144 & 5.178 & 7.195 & 22.978 & 20.700 & 11.006 & 0.000 \\
\hline 307.152 & 5.070 & 7.196 & 22.949 & 20.810 & 10.278 & 0.000 \\
\hline 306.141 & 4.960 & 7.196 & 22.919 & 21.010 & 9.085 & 0.000 \\
\hline 305.747 & 4.917 & 7.196 & 22.907 & 21.250 & 7.796 & 0.000 \\
\hline 305.639 & 4.905 & 7.196 & 22.903 & 21.450 & 6.776 & 0.000 \\
\hline 305.551 & 4.896 & 7.196 & 22.901 & 21.730 & 5.387 & 0.000 \\
\hline 305.443 & 4.884 & 7.196 & 22.897 & 22.090 & 3.655 & 0.000 \\
\hline 305.421 & 4.882 & 7.196 & 22.897 & 22.210 & 3.092 & 0.000 \\
\hline 305.405 & 4.880 & 7.196 & 22.896 & 22.410 & 2.170 & 0.000 \\
\hline 305.393 & 4.879 & 7.196 & 22.896 & 22.570 & 1.444 & 0.000 \\
\hline 305.387 & 4.878 & 7.196 & 22.896 & 22.620 & 1.219 & 0.000 \\
\hline 305.379 & 4.877 & 7.196 & 22.895 & 22.770 & 0.551 & 0.000 \\
\hline 305.369 & 4.876 & 7.196 & 22.895 & 22.970 & -0.326 & 0.000 \\
\hline 322.143 & 6.741 & 7.191 & 23.398 & 20.910 & 11.900 & 0.000 \\
\hline 319.138 & 6.401 & 7.192 & 23.307 & 20.640 & 12.921 & 0.000 \\
\hline 316.479 & 6.103 & 7.193 & 23.227 & 20.580 & 12.864 & 0.000 \\
\hline 315.152 & 5.954 & 7.193 & 23.187 & 20.630 & 12.396 & 0.000 \\
\hline 314.157 & 5.843 & 7.193 & 23.158 & 20.680 & 11.981 & 0.000 \\
\hline 313.642 & 5.786 & 7.194 & 23.143 & 21.010 & 10.151 & 0.000 \\
\hline 313.149 & 5.731 & 7.194 & 23.127 & 20.710 & 11.673 & 0.000 \\
\hline 305.643 & 4.906 & 7.196 & 22.904 & 21.410 & 6.976 & 0.000 \\
\hline 316.474 & 6.070 & 7.027 & 22.686 & 20.100 & 12.867 & 0.000 \\
\hline 313.640 & 5.763 & 7.028 & 22.601 & 20.190 & 11.942 & 0.000 \\
\hline 311.141 & 5.493 & 7.028 & 22.526 & 20.120 & 11.956 & 0.000 \\
\hline 308.146 & 5.172 & 7.029 & 22.436 & 20.170 & 11.233 & 0.000 \\
\hline 307.143 & 5.065 & 7.030 & 22.406 & 20.230 & 10.755 & 0.000 \\
\hline 306.144 & 4.958 & 7.030 & 22.375 & 20.480 & 9.252 & 0.000 \\
\hline 305.746 & 4.916 & 7.030 & 22.364 & 20.810 & 7.465 & 0.000 \\
\hline 305.645 & 4.905 & 7.030 & 22.360 & 21.080 & 6.074 & 0.000 \\
\hline
\end{tabular}


Table 14. VISCOSITY AT ELEVATED PRESSURES (continued)

Data from Strumpf et al. [96] (continued)

\begin{tabular}{|c|c|c|c|c|c|c|}
\hline $\begin{array}{l}\mathrm{T} \\
\mathrm{K}\end{array}$ & $\begin{array}{c}\mathrm{P} \\
\mathrm{MPa}\end{array}$ & $\begin{array}{c}\rho \\
\mathrm{mol}\end{array} \mathrm{dm}^{-3}$ & $\begin{array}{c}\eta, \mathrm{calc} \\
\mu \mathrm{Pa} \cdot \mathrm{s}\end{array}$ & $\begin{array}{c}\eta, \text { expt } \\
\mu \mathrm{Pa} \cdot \mathrm{s}\end{array}$ & $\begin{array}{c}\text { Dev } \\
8\end{array}$ & wt \\
\hline 305.542 & 4.894 & 7.030 & 22.357 & 21.340 & 4.767 & 0.000 \\
\hline 305.443 & 4.884 & 7.030 & 22.354 & 21.870 & 2.214 & 0.000 \\
\hline 305.422 & 4.882 & 7.030 & 22.354 & 22.120 & 1.056 & 0.000 \\
\hline 305.410 & 4.880 & 7.030 & 22.353 & 22.300 & 0.238 & 0.000 \\
\hline 305.401 & 4.879 & 7.030 & 22.353 & 22.450 & -0.433 & 0.000 \\
\hline 305.390 & 4.878 & 7.030 & 22.353 & 22.630 & -1.226 & 0.000 \\
\hline 305.382 & 4.877 & 7.030 & 22.352 & 22.790 & -1.921 & 0.000 \\
\hline 322.149 & 6.689 & 7.025 & 22.858 & 20.240 & 12.937 & 0.000 \\
\hline 319.151 & 6.362 & 7.026 & 22.767 & 20.110 & 13.211 & 0.000 \\
\hline 316.478 & 6.070 & 7.027 & 22.686 & 20.110 & 12.812 & 0.000 \\
\hline 313.648 & 5.764 & 7.028 & 22.601 & 20.170 & 12.055 & 0.000 \\
\hline \multicolumn{7}{|c|}{$\begin{array}{l}\text { Pressures were not tabulated in [96]. } \\
\text { The pressures were computed from our equation of state. } \\
\text { are included in the overall pressure comparisons below. }\end{array}$} \\
\hline \multicolumn{7}{|c|}{ Comparisons based on experimental densities: } \\
\hline Number of $P$ & ints [96] & 105 & & & & \\
\hline$A A D \&=$ & BIAS \% & 7.15 & RMS \& & 4.65 & & \\
\hline$A A D$ & 1.63 & 1.59 & RMS & $0.94 \mu \mathrm{Pa} \cdot \mathrm{s}$ & & \\
\hline
\end{tabular}


Table 14. VISCOSITY AT ELEVATED PRESSURES (continued)

Data from Swift et al. [97]

\begin{tabular}{ccccrrr}
$\mathrm{T}$ & $\mathrm{P}$ & $\begin{array}{c}\rho \\
\mathrm{mol} \cdot \mathrm{dm}^{-3}\end{array}$ & $\begin{array}{c}\eta, \mathrm{calc} \\
\mu \mathrm{Pa} \cdot \mathrm{s}\end{array}$ & $\begin{array}{c}\eta \text { expt } \\
\mu \mathrm{Pa} \cdot \mathrm{s}\end{array}$ & $\begin{array}{c}\text { Dev } \\
\text { \& }\end{array}$ & wt \\
\hline 193.150 & 0.172 & 17.725 & 149.955 & 147.000 & 2.010 & 0.000 \\
193.150 & 4.137 & 17.867 & 155.446 & 149.000 & 4.326 & 0.000 \\
213.150 & 0.448 & 16.829 & 118.868 & 119.000 & -0.111 & 0.000 \\
213.150 & 4.082 & 17.001 & 123.732 & 121.000 & 2.258 & 0.000 \\
233.150 & 0.793 & 15.836 & 94.762 & 95.900 & -1.187 & 0.000 \\
& & & & & & \\
253.150 & 1.586 & 14.721 & 75.506 & 75.500 & 0.008 & 0.000 \\
253.150 & 4.137 & 14.980 & 79.436 & 78.600 & 1.063 & 0.000 \\
273.150 & 2.551 & 13.356 & 58.810 & 58.000 & 1.396 & 0.000 \\
273.150 & 4.102 & 13.645 & 61.935 & 60.400 & 2.542 & 0.000 \\
293.150 & 3.895 & 11.382 & 42.559 & 41.800 & 1.816 & 0.000 \\
& & & & & & \\
293.150 & 4.137 & 11.540 & 43.651 & 42.800 & 1.989 & 0.000 \\
298.150 & 4.309 & 10.637 & 37.966 & 37.000 & 2.610 & 0.000 \\
303.150 & 4.792 & 9.738 & 33.216 & 32.700 & 1.577 & 0.000 \\
305.450 & 4.970 & 9.003 & 29.826 & 30.600 & -2.530 & 0.000
\end{tabular}

Comparisons based on experimental pressures:

Number of Points [97] 14

$$
\begin{aligned}
& \mathrm{AAD} \%=1.82 \quad \mathrm{BIAS} \%=1.27 \text { RMS }=1.67 \\
& \mathrm{AAD}=1.46 \mathrm{BIAS}=1.17 \mathrm{RMS}=1.83 \mu \mathrm{Pa} \cdot \mathrm{s}
\end{aligned}
$$

Comparisons based on experimental densities:

Number of Points [97] 14

$$
\begin{aligned}
& \mathrm{AAD} \%=6.62 \quad \text { BIAS } 8=-3.31 \text { RMS } 8=18.21 \\
& \mathrm{AAD}=3.04 \mathrm{BIAS}=-0.06 \mathrm{RMS}=6.11 \mu \mathrm{Pa} \cdot \mathrm{s}
\end{aligned}
$$


Table 14. VISCOSITY AT ELEVATED PRESSURES (continued)

Data from Van Itterbeek et a1. [98]

\begin{tabular}{|c|c|c|c|c|c|c|}
\hline $\begin{array}{l}\mathrm{T} \\
\mathrm{K}\end{array}$ & $\begin{array}{c}\mathrm{P} \\
\mathrm{MPa}\end{array}$ & $\stackrel{\rho}{\mathrm{mol} \cdot \mathrm{dm}^{-3}}$ & $\begin{array}{c}\eta, \text { calc } \\
\mu \mathrm{Pa} \cdot \mathrm{s}\end{array}$ & $\begin{array}{c}\eta, \text { expt } \\
\mu \mathrm{Pa} \cdot \mathrm{s}\end{array}$ & $\begin{array}{c}\text { Dev } \\
8\end{array}$ & wt \\
\hline 126.000 & $0.792 \mathrm{E}-03$ & 20.376 & 425.041 & 402.000 & 5.732 & 0.000 \\
\hline 128.100 & $0.103 E-02$ & 20.298 & 406.046 & 369.000 & 10.040 & 0.000 \\
\hline 134.100 & $0.206 \mathrm{E}-02$ & 20.074 & 358.986 & 333.000 & 7.804 & 0.000 \\
\hline 141.200 & $0.431 E-02$ & 19.806 & 314.155 & 281.000 & 11.799 & 0.000 \\
\hline 155.000 & $0.146 \mathrm{E}-01$ & 19.277 & 249.772 & 215.000 & 16.173 & 0.000 \\
\hline 160.000 & $0.215 \mathrm{E}-01$ & 19.081 & 231.625 & 207.000 & 11.896 & 0.000 \\
\hline 168.300 & $0.384 \mathrm{E}-01$ & 18.752 & 205.795 & 181.000 & 13.699 & 0.000 \\
\hline 168.700 & $0.394 \mathrm{E}-01$ & 18.736 & 204.665 & 178.000 & 14.980 & 0.000 \\
\hline
\end{tabular}

Comparisons based on experimental pressures:

Number of Points [98] 8

$$
\begin{array}{ll}
\mathrm{AAD} \& & =11.52 \quad \mathrm{BIAS} \&=11.52 \quad \mathrm{RMS} \%=3.32 \\
\mathrm{AAD}=28.76 \quad \mathrm{BIAS}=28.76 \mathrm{RMS}=5.02 \mu \mathrm{Pa} \cdot \mathrm{S}
\end{array}
$$

Comparisons based on experimental densities:

Number of Points [98] 8

$$
\begin{aligned}
& \mathrm{AAD} \%=10.25 \quad \text { BIAS\% }=10.25 \quad \mathrm{RMS} \%=4.57 \\
& \mathrm{AAD}=26.05 \quad \mathrm{BIAS}=26.05 \mathrm{RMS}=9.47 \mu \mathrm{Pa} \cdot \mathrm{s}
\end{aligned}
$$


Table 14. VISCOSITY AT ELEVATED PRESSURES (continued)

Comparisons based on experimental pressures:

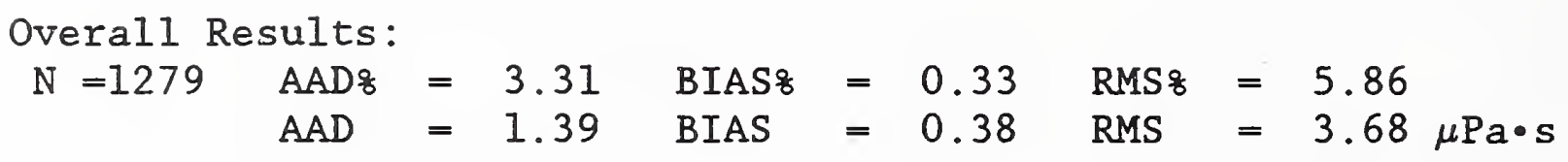

Overall Results (excluding Gerf, Iwasaki, Smith, Strumpf, and Van Itterbeek): $\mathrm{N}=718 \quad \mathrm{AAD} \%=1.05$ BIAS $=0.20$ RMS $8=1.42$ $\mathrm{AAD}=0.75$ BIAS $=0.16 \mathrm{RMS}=1.76 \mu \mathrm{Pa} \cdot \mathrm{S}$

Weighted Data:

$\mathrm{N}=438 \quad \mathrm{AAD} 8=0.75 \quad$ BIAS $8=-0.01 \quad$ RMS $8=0.96$

$\mathrm{AAD}=0.78 \quad \mathrm{BIAS}=-0.04 \quad \mathrm{RMS}=1.85 \mu \mathrm{Pa} \cdot \mathrm{s}$

Comparisons based on experimental densities:

Overall Results:

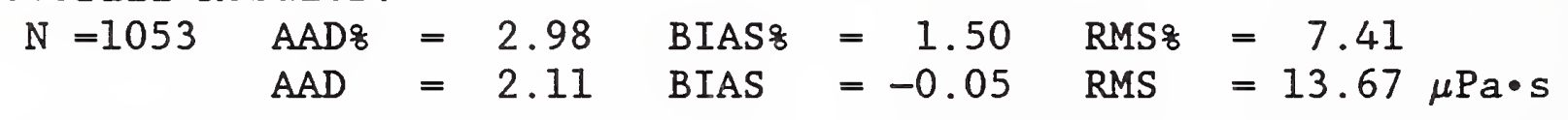

Weighted Data:

$\begin{array}{ll}\mathrm{N}=216 \quad \mathrm{AAD} 8=0.78 & \mathrm{BIAS} 8=0.04 \\ \mathrm{AAD} & =1.40 \quad \mathrm{BIAS}=0.09 \mathrm{RMS}=0.95 \\ & \mathrm{RMS}=2.63 \mu \mathrm{Pa} \cdot \mathrm{s}\end{array}$


Table 15. THERMAL CONDUCTIVITY AT ELEVATED PRESSURES

Data from Carmichael et al. [99]

\begin{tabular}{|c|c|c|c|c|c|c|}
\hline $\begin{array}{l}\mathrm{T} \\
\mathrm{K}\end{array}$ & $\begin{array}{c}\mathrm{P} \\
\mathrm{MPa}\end{array}$ & $\begin{array}{c}\rho \\
\mathrm{mol} \cdot \mathrm{dm}^{-3}\end{array}$ & $\begin{array}{c}\lambda, \operatorname{expt} \\
\mathrm{mW} \cdot \mathrm{m}^{-1} \cdot \mathrm{K}^{-1}\end{array}$ & $\begin{array}{c}\lambda, \operatorname{calc} \\
\mathrm{mW} \cdot \mathrm{m}^{-1} \cdot \mathrm{K}^{-1}\end{array}$ & $\begin{array}{c}\text { Dev } \\
8\end{array}$ & wt \\
\hline 277.594 & 0.12 & 0.054 & 18.988 & 18.492 & -2.609 & 0.000 \\
\hline 277.594 & 1.51 & 0.780 & 20.507 & 20.613 & 0.515 & 0.000 \\
\hline 277.594 & 2.27 & 1.346 & 22.667 & 22.980 & 1.379 & 0.000 \\
\hline 277.594 & 3.30 & 13.114 & 86.151 & 89.003 & 3.311 & 0.000 \\
\hline 277.594 & 6.94 & 13.762 & 92.028 & 96.226 & 4.561 & 0.000 \\
\hline 277.594 & 20.42 & 15.053 & 112.430 & 114.307 & 1.670 & 0.000 \\
\hline 277.594 & 33.13 & 15.788 & 122.283 & 127.116 & 3.953 & 0.000 \\
\hline 310.928 & 0.12 & 0.046 & 22.859 & 22.652 & -0.906 & 0.000 \\
\hline 310.928 & 2.83 & 1.398 & 26.513 & 26.580 & 0.253 & 0.000 \\
\hline 310.928 & 5.00 & 3.964 & 43.681 & 43.338 & -0.785 & 0.000 \\
\hline 310.928 & 6.93 & 10.447 & 66.345 & 70.569 & 6.367 & 0.000 \\
\hline 310.928 & 13.44 & 12.569 & 83.456 & 86.292 & 3.398 & 0.000 \\
\hline 310.928 & 20.67 & 13.548 & 92.627 & 96.919 & 4.634 & 0.000 \\
\hline 310.928 & 34.76 & 14.680 & 106.646 & 112.408 & 5.403 & 0.000 \\
\hline 344.261 & 0.12 & 0.041 & 27.407 & 27.301 & -0.389 & 0.000 \\
\hline 344.261 & 2.88 & 1.180 & 29.896 & 30.085 & 0.630 & 0.000 \\
\hline 344.261 & 6.73 & 3.932 & 40.074 & 41.751 & 4.184 & 0.000 \\
\hline 344.261 & 14.07 & 10.284 & 68.487 & 71.590 & 4.531 & 0.000 \\
\hline 344.261 & 20.77 & 11.872 & 79.690 & 83.397 & 4.651 & 0.000 \\
\hline 344.261 & 36.61 & 13.617 & 95.606 & 101.889 & 6.572 & 0.000 \\
\hline 410.928 & 0.12 & 0.036 & 36.810 & 37.841 & 2.800 & 0.000 \\
\hline 410.928 & 2.93 & 0.930 & 38.529 & 39.588 & 2.750 & 0.000 \\
\hline 410.928 & 7.15 & 2.577 & 42.679 & 44.261 & 3.707 & 0.000 \\
\hline 410.928 & 20.68 & 8.417 & 65.002 & 68.704 & 5.696 & 0.000 \\
\hline 410.928 & 35.14 & 11.137 & 80.547 & 86.125 & 6.926 & 0.000 \\
\hline 444.261 & 0.12 & 0.034 & 41.230 & 43.611 & 5.775 & 0.000 \\
\hline 444.261 & 2.91 & 0.836 & 42.868 & 45.049 & 5.089 & 0.000 \\
\hline 444.261 & 6.60 & 2.042 & 45.656 & 48.012 & 5.160 & 0.000 \\
\hline 444.261 & 19.82 & 6.860 & 62.018 & 66.010 & 6.438 & 0.000 \\
\hline 444.261 & 20.68 & 7.118 & 63.008 & 67.172 & 6.609 & 0.000 \\
\hline 444.261 & 32.58 & 9.683 & 75.896 & 80.818 & 6.484 & 0.000 \\
\hline
\end{tabular}


Table 15. THERMAL CONDUCTIVITY AT ELEVATED PRESSURES (continued)

Data from Carmichael et al. [99] (continued)

Comparisons based on experimental pressures:

Number of Points [99] 31

$\mathrm{AAD} \%=3.81 \quad \mathrm{BIAS} \%=3.51$ RMS $8=2.59$

$\mathrm{AAD}=2.59 \mathrm{BIAS}=2.52 \mathrm{RMS}=1.99 \mathrm{~mW} \cdot \mathrm{m}^{-1} \cdot \mathrm{K}^{-1}$

Densities were not tabulated in [99].

Data from Desmarest et al. [100]

\begin{tabular}{|c|c|c|c|c|c|c|}
\hline $\begin{array}{l}\mathrm{T} \\
\mathrm{K}\end{array}$ & $\begin{array}{c}\mathrm{P} \\
\mathrm{MPa}\end{array}$ & $\begin{array}{c}\rho \\
\mathrm{mol} \cdot \mathrm{dm}^{-3}\end{array}$ & $\begin{array}{c}\lambda, \underset{\mathrm{mW} \cdot \mathrm{m}^{-1} \cdot \mathrm{K}^{-1}}{\operatorname{expt}}\end{array}$ & $\begin{array}{c}\lambda, \text { calc } \\
\mathrm{mW} \cdot \mathrm{m}^{-1} \cdot \mathrm{K}^{-1}\end{array}$ & $\begin{array}{c}\text { Dev } \\
\%\end{array}$ & wt \\
\hline 308.390 & 9.58 & 11.902 & 78.210 & 80.103 & 2.421 & 0.380 \\
\hline 308.750 & 5.23 & 6.901 & 83.770 & 79.491 & -5.108 & 0.400 \\
\hline 308.750 & 5.21 & 6.553 & 80.740 & 78.549 & -2.713 & 0.410 \\
\hline 308.820 & 5.19 & 5.964 & 70.690 & 73.092 & 3.398 & 0.470 \\
\hline 308.870 & 5.17 & 5.596 & 64.630 & 67.687 & 4.729 & 0.490 \\
\hline 308.990 & 5.13 & 5.092 & 57.350 & 59.345 & 3.479 & 0.480 \\
\hline 309.010 & 5.05 & 4.564 & 49.360 & 51.426 & 4.185 & 0.490 \\
\hline 309.050 & 4.90 & 3.991 & 42.850 & 44.226 & 3.210 & 0.490 \\
\hline 309.170 & 4.62 & 3.294 & 36.320 & 37.418 & 3.024 & 0.500 \\
\hline 309.190 & 4.04 & 2.460 & 30.600 & 31.496 & 2.930 & 0.530 \\
\hline 308.750 & 2.49 & 1.203 & 24.970 & 25.609 & 2.559 & 0.580 \\
\hline 308.800 & 1.00 & 0.418 & 22.960 & 23.246 & 1.247 & 0.640 \\
\hline 311.330 & 9.28 & 11.538 & 76.000 & 77.469 & 1.934 & 0.380 \\
\hline 311.310 & 7.39 & 10.696 & 71.150 & 71.906 & 1.063 & 0.390 \\
\hline 311.350 & 6.53 & 10.024 & 68.910 & 68.691 & -0.318 & 0.390 \\
\hline 311.360 & 6.06 & 9.384 & 68.040 & 67.040 & -1.470 & 0.400 \\
\hline 311.350 & 5.80 & 8.764 & 68.630 & 67.236 & -2.031 & 0.400 \\
\hline 311.330 & 5.65 & 8.174 & 69.370 & 68.898 & -0.680 & 0.410 \\
\hline 311.300 & 5.56 & 7.542 & 71.600 & 70.772 & -1.157 & 0.420 \\
\hline 311.320 & 5.51 & 7.025 & 72.220 & 70.939 & -1.774 & 0.420 \\
\hline 311.340 & 5.47 & 6.550 & 70.500 & 69.578 & -1.308 & 0.420 \\
\hline 311.340 & 5.41 & 5.910 & 64.920 & 65.184 & 0.407 & 0.440 \\
\hline 311.380 & 5.33 & 5.230 & 56.470 & 57.673 & 2.130 & 0.470 \\
\hline 311.420 & 5.21 & 4.577 & 48.590 & 49.804 & 2.499 & 0.480 \\
\hline 311.450 & 5.03 & 3.957 & 41.780 & 43.136 & 3.246 & 0.490 \\
\hline 311.510 & 4.75 & 3.349 & 36.250 & 37.711 & 4.030 & 0.520 \\
\hline
\end{tabular}


Table 15. THERMAL CONDUCTIVITY AT ELEVATED PRESSURES (continued)

Data from Desmarest et al. [100] (continued)

\begin{tabular}{|c|c|c|c|c|c|c|}
\hline $\begin{array}{l}\mathrm{T} \\
\mathrm{K}\end{array}$ & $\begin{array}{c}\mathrm{P} \\
\mathrm{MPa}\end{array}$ & $\stackrel{\rho}{\mathrm{mol} \cdot \mathrm{dm}^{-3}}$ & $\begin{array}{c}\lambda, \underset{\mathrm{mW} \cdot \mathrm{m}^{-1} \cdot \mathrm{K}^{-1}}{\operatorname{expt}}\end{array}$ & $\begin{array}{c}\lambda, \quad \operatorname{calc} \\
\mathrm{mW} \cdot \mathrm{m}^{-1} \cdot \mathrm{K}^{-1}\end{array}$ & $\begin{array}{c}\text { Dev } \\
q\end{array}$ & wt \\
\hline 311.570 & 4.30 & 2.672 & 31.870 & 32.913 & 3.272 & 0.530 \\
\hline 311.580 & 4.05 & 2.396 & 30.260 & 31.281 & 3.374 & 0.540 \\
\hline 315.190 & 10.90 & 11.687 & 77.150 & 78.918 & 2.291 & 0.380 \\
\hline 315.150 & 9.86 & 11.366 & 74.920 & 76.468 & 2.067 & 0.380 \\
\hline 315.150 & 8.29 & 10.693 & 71.160 & 72.018 & 1.205 & 0.390 \\
\hline 315.150 & 7.32 & 10.035 & 68.440 & 68.597 & 0.230 & 0.400 \\
\hline 315.170 & 6.72 & 9.378 & 67.110 & 66.295 & -1.215 & 0.400 \\
\hline 315.150 & 6.36 & 8.748 & 66.360 & 65.370 & -1.492 & 0.400 \\
\hline 315.160 & 6.15 & 8.120 & 66.600 & 65.457 & -1.716 & 0.410 \\
\hline 315.190 & 5.99 & 7.361 & 65.860 & 65.409 & -0.685 & 0.420 \\
\hline 315.150 & 5.92 & 6.970 & 64.990 & 64.800 & -0.293 & 0.430 \\
\hline 315.170 & 5.84 & 6.443 & 62.830 & 62.897 & 0.106 & 0.440 \\
\hline 315.220 & 5.73 & 5.792 & 58.280 & 58.801 & 0.894 & 0.450 \\
\hline 315.240 & 5.60 & 5.126 & 51.780 & 53.084 & 2.519 & 0.470 \\
\hline 315.280 & 5.47 & 4.612 & 46.950 & 48.239 & 2.746 & 0.480 \\
\hline 315.350 & 5.26 & 4.006 & 41.360 & 42.760 & 3.384 & 0.500 \\
\hline 315.380 & 4.90 & 3.321 & 36.110 & 37.346 & 3.423 & 0.510 \\
\hline 315.440 & 4.42 & 2.666 & 32.090 & 33.078 & 3.078 & 0.530 \\
\hline 315.480 & 4.05 & 2.289 & 30.010 & 31.010 & 3.332 & 0.540 \\
\hline 322.510 & 13.17 & 11.705 & 77.380 & 79.697 & 2.994 & 0.380 \\
\hline 322.510 & 11.81 & 11.336 & 75.420 & 76.828 & 1.866 & 0.380 \\
\hline 322.520 & 10.06 & 10.697 & 71.230 & 72.449 & 1.711 & 0.390 \\
\hline 322.530 & 8.97 & 10.123 & 67.590 & 69.132 & 2.281 & 0.400 \\
\hline 322.540 & 7.98 & 9.337 & 65.570 & 65.574 & 0.007 & 0.410 \\
\hline 322.540 & 7.44 & 8.645 & 64.250 & 63.423 & -1.287 & 0.410 \\
\hline 322.580 & 7.10 & 8.009 & 62.640 & 62.034 & -0.967 & 0.420 \\
\hline 322.550 & 6.84 & 7.346 & 60.730 & 60.636 & -0.155 & 0.430 \\
\hline 322.580 & 6.71 & 6.930 & 59.560 & 59.451 & -0.183 & 0.430 \\
\hline 322.580 & 6.61 & 6.597 & 58.020 & 58.252 & 0.400 & 0.440 \\
\hline 322.610 & 6.40 & 5.906 & 54.520 & 54.913 & 0.721 & 0.450 \\
\hline 322.630 & 6.20 & 5.270 & 50.060 & 50.925 & 1.727 & 0.470 \\
\hline 322.560 & 5.96 & 4.643 & 45.300 & 46.513 & 2.677 & 0.480 \\
\hline 322.710 & 5.65 & 3.998 & 40.720 & 41.875 & 2.836 & 0.500 \\
\hline 322.730 & 5.19 & 3.280 & 35.950 & 37.120 & 3.255 & 0.520 \\
\hline 322.790 & 4.65 & 2.662 & 32.670 & 33.559 & 2.722 & 0.530 \\
\hline 322.820 & 4.05 & 2.127 & 30.200 & 30.926 & 2.405 & 0.540 \\
\hline
\end{tabular}


Data from Desmarest et al. [100] (continued)

\begin{tabular}{|c|c|c|c|c|c|c|}
\hline $\begin{array}{l}\mathrm{T} \\
\mathrm{K}\end{array}$ & $\begin{array}{c}\mathrm{P} \\
\mathrm{MPa}\end{array}$ & $\begin{array}{c}\rho \\
\mathrm{mol} \cdot \mathrm{dm}^{-3}\end{array}$ & 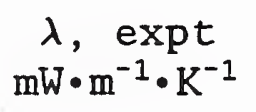 & $\begin{array}{c}\lambda, \quad \operatorname{calc} \\
\mathrm{mW} \cdot \mathrm{m}^{-1} \cdot \mathrm{K}^{-1}\end{array}$ & $\begin{array}{l}\text { Dev } \\
\%\end{array}$ & wt \\
\hline 335.230 & 15.53 & 11.374 & 76.490 & 78.301 & 2.367 & 0.390 \\
\hline 335.230 & 13.01 & 10.650 & 71.840 & 73.128 & 1.793 & 0.400 \\
\hline 335.250 & 11.39 & 9.981 & 68.030 & 69.054 & 1.505 & 0.400 \\
\hline 335.280 & 10.24 & 9.313 & 65.080 & 65.603 & 0.803 & 0.410 \\
\hline 335.280 & 9.40 & 8.635 & 62.600 & 62.649 & 0.078 & 0.420 \\
\hline 335.290 & 8.78 & 7.960 & 60.310 & 60.119 & -0.316 & 0.420 \\
\hline 335.280 & 8.31 & 7.295 & 57.940 & 57.791 & -0.257 & 0.430 \\
\hline 335.290 & 8.10 & 6.949 & 56.270 & 56.534 & 0.470 & 0.440 \\
\hline 335.310 & 7.92 & 6.649 & 54.970 & 55.377 & 0.740 & 0.440 \\
\hline 335.330 & 7.55 & 5.967 & 51.810 & 52.413 & 1.165 & 0.460 \\
\hline 335.340 & 7.17 & 5.293 & 48.250 & 49.012 & 1.580 & 0.470 \\
\hline 335.370 & 6.79 & 4.655 & 44.600 & 45.469 & 1.949 & 0.480 \\
\hline 335.380 & 6.52 & 4.253 & 42.080 & 43.171 & 2.593 & 0.500 \\
\hline 335.410 & 5.75 & 3.328 & 37.150 & 38.019 & 2.340 & 0.510 \\
\hline 335.430 & 5.06 & 2.667 & 34.120 & 34.700 & 1.701 & 0.520 \\
\hline 335.470 & 4.64 & 2.334 & 32.600 & 33.192 & 1.815 & 0.540 \\
\hline 335.510 & 4.05 & 1.921 & 30.990 & 31.488 & 1.606 & 0.550 \\
\hline 335.220 & 2.52 & 1.056 & 28.180 & 28.485 & 1.083 & 0.580 \\
\hline 335.250 & 0.99 & 0.376 & 26.570 & 26.712 & 0.533 & 0.620 \\
\hline 364.440 & 28.19 & 12.007 & 84.840 & 87.103 & 2.668 & 0.380 \\
\hline 364.490 & 23.83 & 11.367 & 79.590 & 81.579 & 2.499 & 0.390 \\
\hline 364.490 & 20.14 & 10.650 & 74.620 & 76.181 & 2.092 & 0.400 \\
\hline 364.490 & 17.57 & 9.985 & 70.500 & 71.803 & 1.848 & 0.410 \\
\hline 364.490 & 15.56 & 9.306 & 67.050 & 67.849 & 1.192 & 0.420 \\
\hline 364.450 & 14.12 & 8.684 & 63.850 & 64.599 & 1.172 & 0.430 \\
\hline 364.490 & 12.77 & 7.951 & 60.570 & 61.131 & 0.926 & 0.440 \\
\hline 364.490 & 11.75 & 7.281 & 57.520 & 58.186 & 1.159 & 0.450 \\
\hline 364.490 & 11.33 & 6.965 & 56.050 & 56.838 & 1.406 & 0.450 \\
\hline 364.510 & 10.91 & 6.635 & 54.830 & 55.438 & 1.109 & 0.460 \\
\hline 364.540 & 10.16 & 6.001 & 51.960 & 52.721 & 1.465 & 0.470 \\
\hline 364.530 & 9.42 & 5.350 & 49.160 & 49.855 & 1.414 & 0.480 \\
\hline 364.570 & 8.66 & 4.681 & 46.280 & 46.837 & 1.204 & 0.490 \\
\hline 364.580 & 7.89 & 4.041 & 43.520 & 43.923 & 0.926 & 0.500 \\
\hline 364.590 & 6.98 & 3.350 & 40.420 & 40.856 & 1.078 & 0.510 \\
\hline 364.620 & 5.97 & 2.673 & 37.700 & 38.047 & 0.920 & 0.530 \\
\hline 364.650 & 5.41 & 2.337 & 36.430 & 36.762 & 0.910 & 0.540 \\
\hline
\end{tabular}


Table 15. THERMAL CONDUCTIVITY AT ELEVATED PRESSURES (continued)

Data from Desmarest et al. [100] (continued)

\begin{tabular}{ccccccc}
\hline $\mathrm{T}$ & $\begin{array}{c}\mathrm{P} \\
\mathrm{MPa}\end{array}$ & $\begin{array}{c}\rho \\
\mathrm{mol} \cdot \mathrm{dm}^{-3}\end{array}$ & $\begin{array}{c}\lambda, \operatorname{expt} \\
\mathrm{mW} \cdot \mathrm{m}^{-1} \cdot \mathrm{K}^{-1}\end{array}$ & $\begin{array}{c}\lambda, \mathrm{calc} \\
\mathrm{mW} \cdot \mathrm{m}^{-1} \cdot \mathrm{K}^{-1}\end{array}$ & $\begin{array}{c}\text { Dev } \\
8\end{array}$ & wt \\
\hline 364.670 & 4.57 & 1.876 & 34.880 & 35.127 & 0.709 & 0.550 \\
364.720 & 2.53 & 0.933 & 31.660 & 32.332 & 2.124 & 0.700 \\
& & & & & & \\
364.770 & 1.00 & 0.345 & 30.270 & 30.975 & 2.328 & 1.970 \\
308.450 & 7.97 & 11.354 & 76.760 & 75.943 & -1.065 & 0.370 \\
308.530 & 6.73 & 10.693 & 72.010 & 71.836 & -0.242 & 0.390 \\
308.590 & 6.03 & 10.076 & 70.170 & 69.094 & -1.533 & 0.390 \\
308.630 & 5.57 & 9.338 & 69.300 & 67.958 & -1.936 & 0.400 \\
308.630 & 5.42 & 8.892 & 71.030 & 68.978 & -2.889 & 0.400 \\
308.750 & 5.33 & 8.286 & 73.600 & 72.383 & -1.653 & 0.410 \\
308.690 & 5.29 & 7.983 & 77.310 & 74.916 & -3.097 & 0.400 \\
308.730 & 5.27 & 7.661 & 79.430 & 77.191 & -2.818 & 0.400 \\
308.730 & 5.26 & 7.505 & 81.300 & 78.136 & -3.892 & 0.400 \\
308.750 & 5.25 & 7.291 & 82.330 & 78.982 & -4.066 & 0.400
\end{tabular}

Comparisons based on experimental pressures:

Number of Points [100] 111

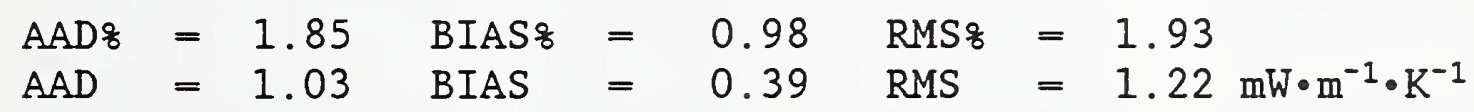

Weighted Data:

Number of Points [100] 111

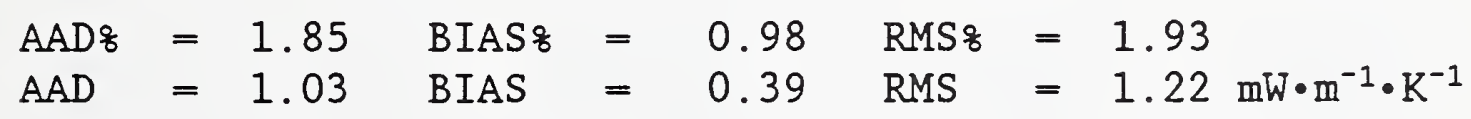


Data from Desmarest et al. [100] (continued)

Comparisons based on experimental densities:

Number of Points [100] 111

$$
\begin{aligned}
& \mathrm{AAD} \&=1.88 \quad \text { BIAS\% }=1.08 \text { RMS } 8=2.04 \\
& \mathrm{AAD}=1.05 \text { BIAS }=0.46 \text { RMS }=1.28 \mathrm{~mW} \cdot \mathrm{m}^{-1} \cdot \mathrm{K}^{-1}
\end{aligned}
$$

Weighted Data:

Number of Points [100] 111

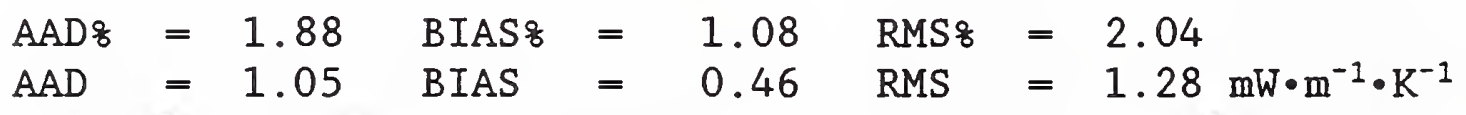

Data from Fleeter et al. [72]

\begin{tabular}{ccccccc}
\hline $\begin{array}{c}\mathrm{T} \\
\mathrm{K}\end{array}$ & $\begin{array}{c}\mathrm{P} \\
\mathrm{MPa}\end{array}$ & $\begin{array}{c}\rho \\
\mathrm{mol} \cdot \mathrm{dm}^{-3}\end{array}$ & $\begin{array}{c}\lambda, \text { expt } \\
\mathrm{mW} \cdot \mathrm{m}^{-1} \cdot \mathrm{K}^{-1}\end{array}$ & $\begin{array}{c}\lambda, \mathrm{calc} \\
\mathrm{mW} \cdot \mathrm{m}^{-1} \cdot \mathrm{K}^{-1}\end{array}$ & $\begin{array}{c}\text { Dev } \\
8\end{array}$ & $w t$ \\
\hline 301.470 & 0.60 & 0.251 & 22.560 & 21.893 & -2.954 & 0.000 \\
301.370 & 0.80 & 0.340 & 22.750 & 22.102 & -2.847 & 0.000 \\
301.270 & 1.00 & 0.432 & 22.890 & 22.327 & -2.457 & 0.000 \\
301.190 & 1.19 & 0.524 & 23.130 & 22.563 & -2.452 & 0.000 \\
301.080 & 1.39 & 0.623 & 23.400 & 22.830 & -2.437 & 0.000 \\
300.980 & 1.60 & 0.733 & 23.600 & 23.140 & -1.949 & 0.000 \\
300.940 & 1.85 & 0.870 & 24.000 & 23.562 & -1.825 & 0.000 \\
300.940 & 2.10 & 1.016 & 24.280 & 24.045 & -0.967 & 0.000 \\
300.850 & 2.33 & 1.161 & 24.730 & 24.544 & -0.752 & 0.000 \\
300.730 & 2.60 & 1.344 & 25.300 & 25.228 & -0.284 & 0.000 \\
300.760 & 2.83 & 1.512 & 25.990 & 25.929 & -0.235 & 0.000 \\
300.370 & 3.41 & 2.030 & 28.400 & 28.428 & 0.099 & 0.000
\end{tabular}

Comparisons based on experimental pressures:

Number of Points [72] 12

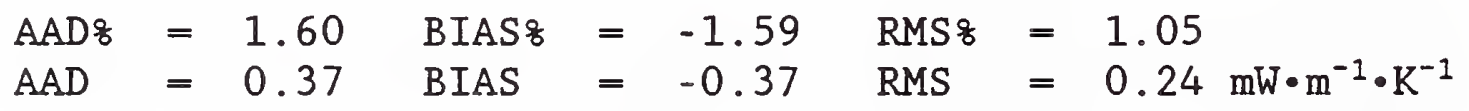


Table 15. THERMAL CONDUCTIVITY AT ELEVATED PRESSURES (continued)

Data from Fleeter et al. [72] (continued)

Comparisons based on experimental densities:

Number of Points [72] 12

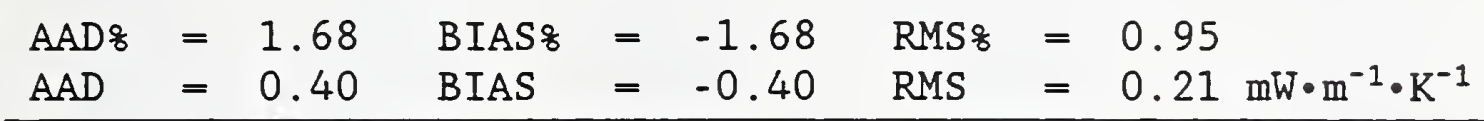

Data from Gilmore et al. [80]

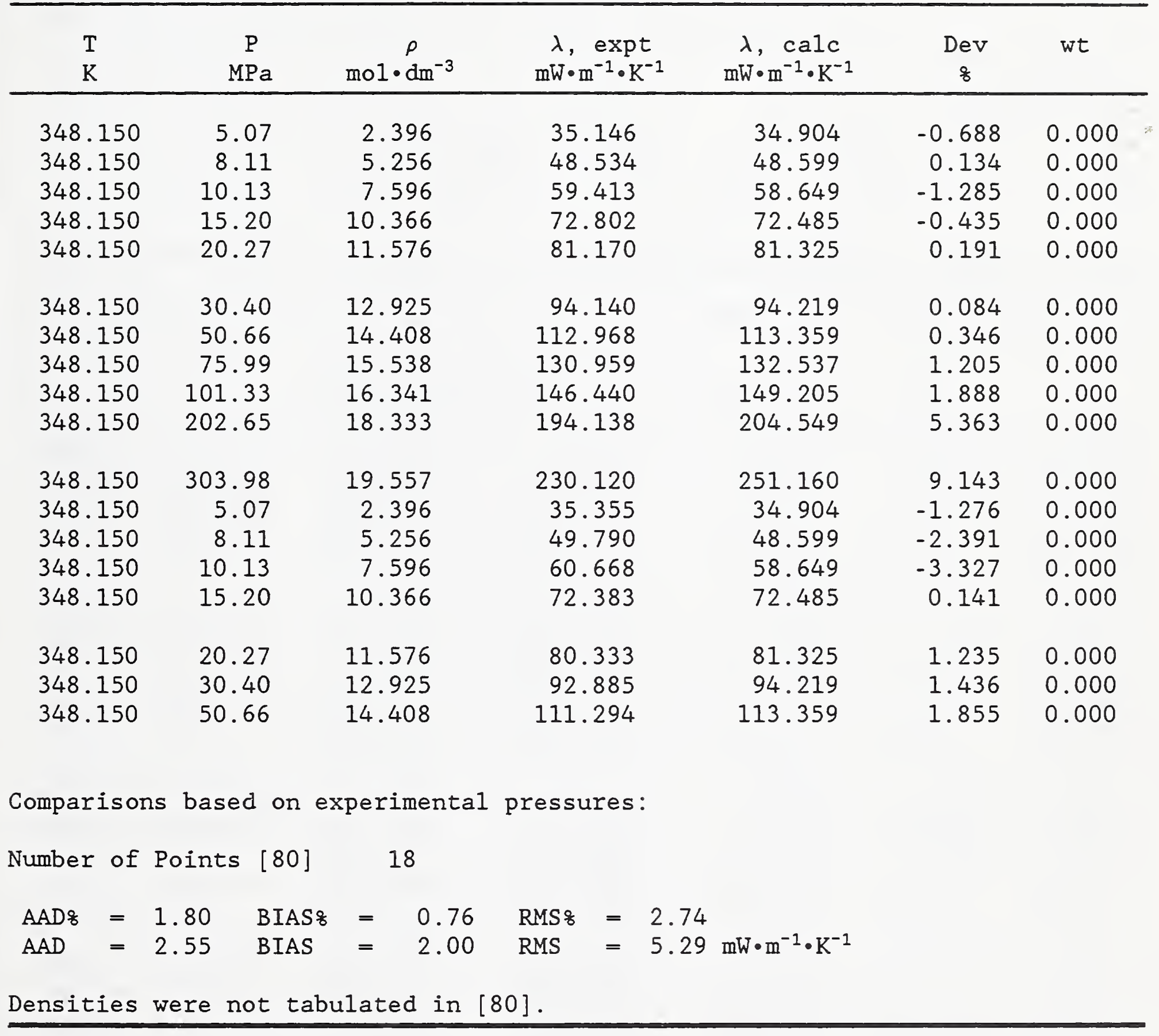


Table 15. THERMAL CONDUCTIVITY AT ELEVATED PRESSURES (continued)

Data from Keyes [81]

\begin{tabular}{ccccccc}
\hline $\mathrm{T}$ & $\mathrm{P}$ & $\begin{array}{c}\rho \\
\mathrm{MPa}\end{array}$ & $\begin{array}{c}\lambda, \operatorname{expt} \\
\mathrm{mol} \cdot \mathrm{dm}^{-3}\end{array}$ & $\begin{array}{c}\lambda, \mathrm{calc} \\
\mathrm{mW} \cdot \mathrm{m}^{-1} \cdot \mathrm{K}^{-1}\end{array}$ & $\begin{array}{c}\text { Dev } \\
\mathrm{mW} \cdot \mathrm{m}^{-1} \cdot \mathrm{K}^{-1}\end{array}$ & wt \\
\hline 325.050 & 0.99 & 0.390 & 25.983 & 25.332 & -2.504 & 0.000 \\
325.050 & 1.72 & 0.712 & 26.861 & 26.149 & -2.650 & 0.000 \\
325.050 & 2.50 & 1.101 & 27.865 & 27.283 & -2.092 & 0.000 \\
325.050 & 3.24 & 1.526 & 30.041 & 28.725 & -4.380 & 0.000 \\
325.050 & 3.99 & 2.039 & 32.049 & 30.772 & -3.986 & 0.000
\end{tabular}

Comparisons based on experimental pressures:

Number of Points [81] 5

$$
\begin{aligned}
& \mathrm{AAD}=3.12 \quad \text { BIAS }=-3.12 \quad \mathrm{RMS} 8=0.89 \\
& \mathrm{AAD}=0.91 \quad \text { BIAS }=-0.91 \quad \mathrm{RMS}=0.32 \mathrm{~mW} \cdot \mathrm{m}^{-1} \cdot \mathrm{K}^{-1}
\end{aligned}
$$

Densities were not tabulated in [81].

Data from Le Neindre et al. [82]

\begin{tabular}{crcrrrr}
\hline $\mathrm{T}$ & $\begin{array}{c}\mathrm{P} \\
\mathrm{MPa}\end{array}$ & $\begin{array}{c}\rho \\
\mathrm{mol} \cdot \mathrm{dm}^{-3}\end{array}$ & $\begin{array}{c}\lambda, \begin{array}{c}\text { expt } \\
\mathrm{mW} \cdot \mathrm{m}^{-1} \cdot \mathrm{K}^{-1}\end{array} \\
\mathrm{~mW} \cdot \mathrm{m}^{-1} \cdot \mathrm{K}^{-1}\end{array}$ & $\begin{array}{c}\text { Dev } \\
\text { o }\end{array}$ & wt \\
\hline 306.550 & 1.93 & 0.888 & 24.300 & 24.295 & -0.020 & 0.000 \\
307.350 & 3.31 & 1.799 & 28.100 & 27.872 & -0.813 & 0.000 \\
307.250 & 7.86 & 11.442 & 76.600 & 76.500 & -0.130 & 0.000 \\
309.250 & 10.79 & 12.144 & 82.700 & 82.225 & -0.575 & 0.000 \\
309.250 & 14.70 & 12.883 & 89.500 & 89.276 & -0.250 & 0.000 \\
& & & & & & \\
309.050 & 19.20 & 13.480 & 96.300 & 95.898 & -0.417 & 0.000 \\
308.850 & 24.90 & 14.043 & 103.400 & 102.991 & -0.395 & 0.000 \\
308.650 & 30.10 & 14.454 & 109.400 & 108.730 & -0.613 & 0.000 \\
308.350 & 35.40 & 14.812 & 114.700 & 114.135 & -0.492 & 0.000 \\
308.150 & 41.20 & 15.146 & 120.300 & 119.586 & -0.594 & 0.000 \\
307.450 & 47.80 & 15.493 & 126.600 & 125.596 & -0.793 & 0.000 \\
307.350 & 56.00 & 15.847 & 132.600 & 132.293 & -0.232 & 0.000 \\
307.050 & 68.50 & 16.312 & 141.800 & 141.858 & 0.041 & 0.000 \\
307.050 & 78.50 & 16.628 & 148.800 & 148.943 & 0.096 & 0.000 \\
347.250 & 2.40 & 0.943 & 29.900 & 29.825 & -0.251 & 0.000 \\
343.450 & 7.80 & 5.315 & 49.600 & 48.848 & -1.517 & 0.000 \\
344.050 & 9.20 & 7.127 & 58.200 & 56.757 & -2.480 & 0.000 \\
342.050 & 11.10 & 9.086 & 66.000 & 64.851 & -1.740 & 0.000 \\
341.150 & 14.70 & 10.729 & 74.900 & 74.201 & -0.933 & 0.000
\end{tabular}


Table 15. THERMAL CONDUCTIVITY AT ELEVATED PRESSURES (continued)

Data from Le Neindre et al. [82] (continued)

\begin{tabular}{|c|c|c|c|c|c|c|}
\hline $\begin{array}{l}\mathrm{T} \\
\mathrm{K} \\
\end{array}$ & $\begin{array}{c}\mathrm{P} \\
\mathrm{MPa} \\
\end{array}$ & $\stackrel{\rho}{\mathrm{mol} \cdot \mathrm{dm}^{-3}}$ & $\begin{array}{c}\lambda, \quad \operatorname{expt} \\
\mathrm{mW} \cdot \mathrm{m}^{-1} \cdot \mathrm{K}^{-1}\end{array}$ & $\begin{array}{c}\lambda, \quad \operatorname{calc} \\
\mathrm{mW} \cdot \mathrm{m}^{-1} \cdot \mathrm{K}^{-1}\end{array}$ & $\begin{array}{c}\text { Dev } \\
\text { \& }\end{array}$ & wt \\
\hline 337.850 & 17.20 & 11.576 & 80.700 & 80.178 & -0.647 & 0.000 \\
\hline 336.050 & 18.00 & 11.842 & 83.000 & 82.210 & -0.951 & 0.000 \\
\hline 339.450 & 21.80 & 12.281 & 86.700 & 86.566 & -0.155 & 0.000 \\
\hline 410.050 & 0.10 & 0.029 & 37.400 & 37.681 & 0.752 & 0.000 \\
\hline 408.950 & 3.70 & 1.210 & 40.200 & 39.932 & -0.668 & 0.000 \\
\hline 408.250 & 6.10 & 2.155 & 43.000 & 42.482 & -1.204 & 0.000 \\
\hline 407.050 & 11.30 & 4.674 & 52.100 & 51.597 & -0.966 & 0.000 \\
\hline 407.450 & 16.40 & 7.092 & 62.800 & 61.901 & -1.432 & 0.000 \\
\hline 407.150 & 20.30 & 8.475 & 69.300 & 68.498 & -1.157 & 0.000 \\
\hline 406.850 & 24.70 & 9.601 & 75.400 & 74.775 & -0.829 & 0.000 \\
\hline 406.550 & 32.40 & 10.930 & 84.500 & 83.849 & -0.770 & 0.000 \\
\hline 403.950 & 38.10 & 11.710 & 91.000 & 89.948 & -1.157 & 0.000 \\
\hline 406.250 & 45.60 & 12.349 & 97.100 & 96.409 & -0.712 & 0.000 \\
\hline 403.450 & 46.40 & 12.497 & 98.400 & 97.505 & -0.910 & 0.000 \\
\hline 402.950 & 51.40 & 12.891 & 102.600 & 101.732 & -0.846 & 0.000 \\
\hline 405.550 & 66.50 & 13.742 & 113.500 & 112.747 & -0.663 & 0.000 \\
\hline 405.250 & 76.80 & 14.245 & 120.200 & 119.906 & -0.245 & 0.000 \\
\hline 404.950 & 87.90 & 14.708 & 127.200 & 127.184 & -0.013 & 0.000 \\
\hline 404.650 & 98.30 & 15.087 & 133.700 & 133.685 & -0.011 & 0.000 \\
\hline 404.350 & 108.40 & 15.416 & 139.200 & 139.756 & 0.400 & 0.000 \\
\hline 404.050 & 119.40 & 15.740 & 144.800 & 146.135 & 0.922 & 0.000 \\
\hline 482.250 & 0.30 & 0.075 & 50.400 & 50.568 & 0.333 & 0.000 \\
\hline 482.050 & 3.70 & 0.973 & 51.700 & 52.088 & 0.750 & 0.000 \\
\hline 481.950 & 6.40 & 1.746 & 53.700 & 53.811 & 0.207 & 0.000 \\
\hline 481.350 & 9.90 & 2.816 & 57.000 & 56.660 & -0.597 & 0.000 \\
\hline 480.950 & 13.70 & 4.018 & 61.100 & 60.523 & -0.944 & 0.000 \\
\hline 480.550 & 17.50 & 5.190 & 65.600 & 64.780 & -1.250 & 0.000 \\
\hline 484.550 & 21.10 & 6.105 & 69.500 & 69.115 & -0.555 & 0.000 \\
\hline 484.250 & 27.90 & 7.680 & 76.400 & 76.134 & -0.348 & 0.000 \\
\hline 484.050 & 34.80 & 8.895 & 82.600 & 82.501 & -0.119 & 0.000 \\
\hline 483.850 & 41.80 & 9.846 & 88.200 & 88.334 & 0.152 & 0.000 \\
\hline 483.550 & 48.00 & 10.530 & 92.900 & 93.106 & 0.221 & 0.000 \\
\hline 483.550 & 54.70 & 11.140 & 97.600 & 97.944 & 0.352 & 0.000 \\
\hline 483.550 & 55.40 & 11.198 & 97.900 & 98.433 & 0.544 & 0.000 \\
\hline 483.350 & 59.00 & 11.486 & 100.600 & 100.911 & 0.309 & 0.000 \\
\hline 483.150 & 67.60 & 12.086 & 106.200 & 106.578 & 0.356 & 0.000 \\
\hline
\end{tabular}


Data from Le Neindre et al. [82]

\begin{tabular}{|c|c|c|c|c|c|c|}
\hline $\begin{array}{l}\mathrm{T} \\
\mathrm{K}\end{array}$ & $\begin{array}{c}\mathrm{P} \\
\mathrm{MPa}\end{array}$ & $\begin{array}{c}\rho \\
\mathrm{mol} \cdot \mathrm{dm}^{-3}\end{array}$ & 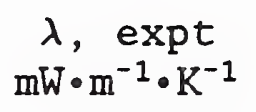 & $\begin{array}{c}\lambda, \quad \operatorname{calc} \\
\mathrm{mW} \cdot \mathrm{m}^{-1} \cdot \mathrm{K}^{-1}\end{array}$ & $\begin{array}{c}\text { Dev } \\
\&\end{array}$ & wt \\
\hline 482.950 & 81.30 & 12.864 & 114.200 & 115.032 & 0.728 & 0.000 \\
\hline 482.750 & 93.70 & 13.442 & 121.000 & 122.225 & 1.012 & 0.000 \\
\hline 482.550 & 106.70 & 13.958 & 128.000 & 129.405 & 1.098 & 0.000 \\
\hline 482.450 & 115.50 & 14.267 & 132.400 & 134.091 & 1.277 & 0.000 \\
\hline 482.450 & 116.70 & 14.306 & 132.800 & 134.720 & 1.446 & 0.000 \\
\hline 571.550 & 0.30 & 0.063 & 65.900 & 67.736 & 2.786 & 0.000 \\
\hline 571.350 & 3.70 & 0.797 & 67.400 & 68.771 & 2.034 & 0.000 \\
\hline 570.950 & 8.80 & 1.940 & 70.400 & 70.957 & 0.791 & 0.000 \\
\hline 570.850 & 15.50 & 3.453 & 73.900 & 74.918 & 1.378 & 0.000 \\
\hline 570.650 & 23.10 & 5.059 & 80.000 & 80.169 & 0.212 & 0.000 \\
\hline 570.450 & 32.20 & 6.689 & 86.100 & 86.600 & 0.580 & 0.000 \\
\hline 570.550 & 39.60 & 7.762 & 91.000 & 91.614 & 0.675 & 0.000 \\
\hline 571.850 & 47.20 & 8.653 & 96.000 & 96.582 & 0.607 & 0.000 \\
\hline 571.350 & 56.40 & 9.569 & 101.400 & 102.078 & 0.668 & 0.000 \\
\hline 572.650 & 66.50 & 10.363 & 106.700 & 107.899 & 1.123 & 0.000 \\
\hline 572.450 & 78.20 & 11.143 & 112.300 & 114.175 & 1.670 & 0.000 \\
\hline 570.750 & 82.20 & 11.403 & 114.500 & 116.173 & 1.461 & 0.000 \\
\hline 572.150 & 93.20 & 11.959 & 119.600 & 121.804 & 1.843 & 0.000 \\
\hline 571.950 & 107.90 & 12.618 & 126.300 & 128.929 & 2.082 & 0.000 \\
\hline 571.950 & 108.90 & 12.659 & 126.800 & 129.404 & 2.053 & 0.000 \\
\hline 571.950 & 115.70 & 12.924 & 129.600 & 132.595 & 2.311 & 0.000 \\
\hline 571.950 & 117.70 & 12.999 & 130.500 & 133.523 & 2.316 & 0.000 \\
\hline 571.550 & 119.00 & 13.052 & 131.000 & 134.115 & 2.378 & 0.000 \\
\hline 647.650 & 0.40 & 0.074 & 80.400 & 83.136 & 3.403 & 0.000 \\
\hline 647.050 & 3.70 & 0.694 & 82.100 & 83.824 & 2.100 & 0.000 \\
\hline 649.450 & 3.90 & 0.729 & 82.000 & 84.367 & 2.887 & 0.000 \\
\hline 649.350 & 6.50 & 1.219 & 83.200 & 85.131 & 2.321 & 0.000 \\
\hline 649.250 & 12.50 & 2.347 & 85.400 & 87.344 & 2.276 & 0.000 \\
\hline 649.250 & 19.30 & 3.579 & 88.600 & 90.435 & 2.071 & 0.000 \\
\hline 649.050 & 39.90 & 6.662 & 99.500 & 101.169 & 1.678 & 0.000 \\
\hline 648.950 & 50.10 & 7.801 & 104.500 & 106.461 & 1.876 & 0.000 \\
\hline 648.850 & 59.70 & 8.691 & 109.100 & 111.265 & 1.985 & 0.000 \\
\hline 648.750 & 71.60 & 9.607 & 114.100 & 116.990 & 2.533 & 0.000 \\
\hline 648.750 & 81.80 & 10.267 & 118.300 & 121.723 & 2.893 & 0.000 \\
\hline 648.850 & 93.90 & 10.937 & 123.300 & 127.158 & 3.129 & 0.000 \\
\hline
\end{tabular}


Table 15. THERMAL CONDUCTIVITY AT ELEVATED PRESSURES (continued)

Data from Le Neindre et al. [82]

\begin{tabular}{|c|c|c|c|c|c|c|}
\hline $\begin{array}{l}\mathrm{T} \\
\mathrm{K}\end{array}$ & $\begin{array}{c}\mathrm{P} \\
\mathrm{MPa}\end{array}$ & $\begin{array}{c}\rho \\
\mathrm{mol} \cdot \mathrm{dm}^{-3}\end{array}$ & $\begin{array}{c}\lambda, \underset{ }{\operatorname{expt} t} \\
\mathrm{~mW} \cdot \mathrm{m}^{-1} \cdot \mathrm{K}^{-1}\end{array}$ & $\begin{array}{c}\lambda, \quad \operatorname{calc} \\
\mathrm{mW} \cdot \mathrm{m}^{-1} \cdot \mathrm{K}^{-1}\end{array}$ & $\begin{array}{c}\text { Dev } \\
8\end{array}$ & wt \\
\hline 648.950 & 114.50 & 11.877 & 131.300 & 136.014 & 3.590 & 0.000 \\
\hline 649.250 & 116.40 & 11.950 & 132.200 & 136.832 & 3.504 & 0.000 \\
\hline 649.450 & 118.50 & 12.030 & 132.500 & 137.722 & 3.941 & 0.000 \\
\hline 725.450 & 4.00 & 0.663 & 96.100 & 100.056 & 4.116 & 0.000 \\
\hline 725.250 & 8.40 & 1.390 & 97.500 & 101.139 & 3.732 & 0.000 \\
\hline 724.950 & 19.40 & 3.134 & 101.500 & 104.789 & 3.240 & 0.000 \\
\hline 724.550 & 29.40 & 4.563 & 105.200 & 108.774 & 3.398 & 0.000 \\
\hline 724.250 & 40.60 & 5.942 & 110.100 & 113.552 & 3.136 & 0.000 \\
\hline 724.150 & 50.30 & 6.954 & 113.700 & 117.737 & 3.551 & 0.000 \\
\hline 724.850 & 51.00 & 7.013 & 114.500 & 118.151 & 3.188 & 0.000 \\
\hline 724.750 & 58.50 & 7.688 & 117.300 & 121.329 & 3.434 & 0.000 \\
\hline 724.550 & 72.20 & 8.740 & 122.400 & 126.992 & 3.752 & 0.000 \\
\hline 724.550 & 80.70 & 9.297 & 125.500 & 130.431 & 3.929 & 0.000 \\
\hline 724.450 & 91.10 & 9.901 & 129.300 & 134.537 & 4.050 & 0.000 \\
\hline 724.450 & 101.80 & 10.449 & 133.200 & 138.682 & 4.115 & 0.000 \\
\hline 724.550 & 111.80 & 10.906 & 136.700 & 142.489 & 4.235 & 0.000 \\
\hline 800.650 & 71.90 & 7.966 & 131.700 & 138.632 & 5.264 & 0.000 \\
\hline 800.250 & 86.50 & 8.885 & 136.100 & 143.728 & 5.605 & 0.000 \\
\hline 799.950 & 99.00 & 9.559 & 140.200 & 148.008 & 5.569 & 0.000 \\
\hline 799.750 & 113.60 & 10.243 & 144.800 & 152.931 & 5.616 & 0.000 \\
\hline
\end{tabular}

Comparisons based on experimental pressures:

Number of Points [82] 113

$\begin{array}{ll}\mathrm{AAD} 8 & =1.63 \quad \mathrm{BIAS} \%=1.07 \\ \mathrm{AAD}=1.74 & \mathrm{RMS} \%=1.88 \\ \mathrm{BIAS} & =1.34 \mathrm{RMS}=2.17 \mathrm{~mW} \cdot \mathrm{m}^{-1} \cdot \mathrm{K}^{-1}\end{array}$

Comparisons based on experimental densities:

Number of Points [82] 113

\begin{tabular}{|c|c|c|c|c|}
\hline$A D$ & & & & 0.09 \\
\hline$A D$ & $=1.89$ & BIAS & $=$ & 0.22 \\
\hline
\end{tabular}


Table 15. THERMAI CONDUCTIVITY AT ELEVATED PRESSURES (continued)

Data from Leng et a1. [83]

\begin{tabular}{ccccccc}
\hline $\begin{array}{c}\mathrm{T} \\
\mathrm{K}\end{array}$ & $\begin{array}{c}\mathrm{P} \\
\mathrm{MPa}\end{array}$ & $\begin{array}{c}\rho \\
\mathrm{mol} \cdot \mathrm{dm}^{-3}\end{array}$ & $\begin{array}{c}\lambda, \begin{array}{c}\text { expt } \\
\mathrm{mW} \cdot \mathrm{m}^{-1} \cdot \mathrm{K}^{-1}\end{array} \\
\mathrm{~mW} \cdot \mathrm{m}^{-1} \cdot \mathrm{K}^{-1}\end{array}$ & $\begin{array}{c}\text { Dev } \\
8\end{array}$ & wt \\
\hline 340.928 & 0.96 & 0.357 & 28.037 & 27.474 & -2.008 & 0.000 \\
340.928 & 2.07 & 0.818 & 28.730 & 28.599 & -0.454 & 0.000 \\
340.928 & 3.24 & 1.389 & 30.806 & 30.282 & -1.701 & 0.000 \\
340.928 & 4.37 & 2.050 & 32.191 & 32.646 & 1.414 & 0.000 \\
340.928 & 6.23 & 3.576 & 38.941 & 39.694 & 1.934 & 0.000 \\
340.928 & 7.95 & 5.833 & 50.536 & 51.402 & 1.712 & 0.000 \\
340.928 & 9.66 & 8.068 & 60.575 & 60.424 & -0.249 & 0.000 \\
340.928 & 11.04 & 9.176 & 66.978 & 65.222 & -2.622 & 0.000 \\
340.928 & 14.21 & 10.591 & 74.593 & 73.257 & -1.792 & 0.000 \\
340.928 & 18.27 & 11.606 & 81.170 & 80.761 & -0.503 & 0.000 \\
340.928 & 21.89 & 12.219 & 88.958 & 86.156 & -3.150 & 0.000 \\
340.928 & 26.45 & 12.804 & 94.842 & 92.007 & -2.990 & 0.000
\end{tabular}

Comparisons based on experimental pressures:

Number of Points [83] 12

$\mathrm{AAD} 8=1.71 \quad$ BIAS\% $=-0.87$ RMS $8=1.73$

$\mathrm{AAD}=1.05$ BIAS $=-0.70$ RMS $=1.20 \mathrm{~mW} \cdot \mathrm{m}^{-1} \cdot \mathrm{K}^{-1}$

Densities were not tabulated in [83].

Data from Lenoir et a1. [84]

\begin{tabular}{ccccccc}
\hline $\mathrm{T}$ & $\begin{array}{c}\mathrm{P} \\
\mathrm{MPa}\end{array}$ & $\begin{array}{c}\rho \\
\mathrm{mol} \cdot \mathrm{dm}^{-3}\end{array}$ & $\begin{array}{c}\lambda, \text { expt } \\
\mathrm{mW} \cdot \mathrm{m}^{-1} \cdot \mathrm{K}^{-1}\end{array}$ & $\begin{array}{c}\lambda, \mathrm{calc} \\
\mathrm{mW} \cdot \mathrm{m}^{-1} \cdot \mathrm{K}^{-1}\end{array}$ & $\begin{array}{c}\text { Dev } \\
8\end{array}$ & $w t$ \\
\hline 315.206 & 1.16 & 0.477 & 25.268 & 24.229 & -4.111 & 0.000 \\
315.206 & 2.00 & 0.884 & 26.307 & 25.358 & -3.606 & 0.000 \\
315.206 & 2.84 & 1.365 & 28.210 & 26.956 & -4.448 & 0.000 \\
315.206 & 3.83 & 2.093 & 30.980 & 30.016 & -3.109 & 0.000 \\
315.206 & 4.33 & 2.574 & 32.883 & 32.531 & -1.072 & 0.000 \\
315.206 & 4.71 & 3.045 & 35.306 & 35.436 & 0.367 & 0.000 \\
315.206 & 5.14 & 3.758 & 40.844 & 40.702 & -0.350 & 0.000 \\
315.206 & 5.38 & 4.346 & 44.306 & 45.802 & 3.378 & 0.000 \\
315.206 & 5.66 & 5.424 & 55.036 & 55.800 & 1.388 & 0.000 \\
315.206 & 6.02 & 7.515 & 71.478 & 65.504 & -8.358 & 0.000 \\
315.206 & 6.45 & 8.927 & 68.882 & 65.487 & -4.928 & 0.000 \\
315.206 & 6.66 & 9.281 & 68.536 & 66.061 & -3.612 & 0.000
\end{tabular}


Table 15. THERMAL CONDUCTIVITY AT ELEVATED PRESSURES (continued)

Data from Lenoir et al. [84] (continued)

\begin{tabular}{|c|c|c|c|c|c|c|}
\hline $\begin{array}{l}\mathrm{T} \\
\mathrm{K}\end{array}$ & $\begin{array}{c}\mathrm{P} \\
\mathrm{MPa}\end{array}$ & $\begin{array}{c}\rho \\
\mathrm{mol} \cdot \mathrm{dm}^{-3}\end{array}$ & 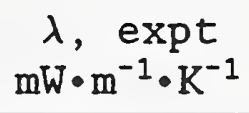 & $\begin{array}{c}\lambda, \quad \text { calc } \\
\mathrm{mW} \cdot \mathrm{m}^{-1} \cdot \mathrm{K}^{-1}\end{array}$ & $\begin{array}{c}\text { Dev } \\
8\end{array}$ & wt \\
\hline $\begin{array}{l}315.206 \\
315.206 \\
315.206\end{array}$ & $\begin{array}{l}7.11 \\
7.88 \\
8.96\end{array}$ & $\begin{array}{r}9.837 \\
10.447 \\
11.011\end{array}$ & $\begin{array}{l}68.882 \\
70.093 \\
72.862\end{array}$ & $\begin{array}{l}67.773 \\
70.626 \\
74.009\end{array}$ & $\begin{array}{r}-1.609 \\
0.759 \\
1.574\end{array}$ & $\begin{array}{l}0.000 \\
0.000 \\
0.000\end{array}$ \\
\hline $\begin{array}{l}315.206 \\
315.206 \\
315.206 \\
315.206 \\
315.206\end{array}$ & $\begin{array}{l}10.21 \\
11.57 \\
12.87 \\
14.10 \\
15.42\end{array}$ & $\begin{array}{l}11.479 \\
11.866 \\
12.165 \\
12.409 \\
12.637\end{array}$ & $\begin{array}{l}77.535 \\
80.131 \\
82.554 \\
84.977 \\
86.535\end{array}$ & $\begin{array}{l}77.312 \\
80.363 \\
82.940 \\
85.176 \\
87.378\end{array}$ & $\begin{array}{r}-0.288 \\
0.289 \\
0.468 \\
0.233 \\
0.974\end{array}$ & $\begin{array}{l}0.000 \\
0.000 \\
0.000 \\
0.000 \\
0.000\end{array}$ \\
\hline $\begin{array}{l}315.206 \\
315.206 \\
315.094 \\
315.094 \\
315.094\end{array}$ & $\begin{array}{r}17.46 \\
19.52 \\
0.86 \\
1.82 \\
2.43\end{array}$ & $\begin{array}{r}12.941 \\
13.206 \\
0.348 \\
0.796 \\
1.122\end{array}$ & $\begin{array}{l}89.996 \\
95.881 \\
23.884 \\
24.922 \\
26.480\end{array}$ & $\begin{array}{l}90.504 \\
93.401 \\
23.895 \\
25.083 \\
26.098\end{array}$ & $\begin{array}{r}0.564 \\
-2.586 \\
0.034 \\
0.634 \\
-1.453\end{array}$ & $\begin{array}{l}0.000 \\
0.000 \\
0.000 \\
0.000 \\
0.000\end{array}$ \\
\hline $\begin{array}{l}315.094 \\
315.094 \\
315.094 \\
315.094 \\
315.094\end{array}$ & $\begin{array}{l}3.07 \\
3.48 \\
3.96 \\
4.36 \\
4.66\end{array}$ & $\begin{array}{l}1.518 \\
1.808 \\
2.213 \\
2.611 \\
2.982\end{array}$ & $\begin{array}{l}26.999 \\
28.557 \\
30.634 \\
32.192 \\
34.269\end{array}$ & $\begin{array}{l}27.517 \\
28.703 \\
30.593 \\
32.737 \\
35.016\end{array}$ & $\begin{array}{r}1.904 \\
0.499 \\
-0.144 \\
1.684 \\
2.171\end{array}$ & $\begin{array}{l}0.000 \\
0.000 \\
0.000 \\
0.000 \\
0.000\end{array}$ \\
\hline $\begin{array}{l}315.094 \\
315.094 \\
315.094 \\
315.094 \\
315.094\end{array}$ & $\begin{array}{l}5.02 \\
5.33 \\
5.58 \\
5.74 \\
5.91\end{array}$ & $\begin{array}{l}3.533 \\
4.224 \\
5.086 \\
5.861 \\
6.947\end{array}$ & $\begin{array}{l}38.249 \\
44.134 \\
53.480 \\
59.883 \\
72.344\end{array}$ & $\begin{array}{l}38.936 \\
44.720 \\
52.808 \\
59.441 \\
64.805\end{array}$ & $\begin{array}{r}1.784 \\
1.319 \\
-1.268 \\
-0.749 \\
-10.434\end{array}$ & $\begin{array}{l}0.000 \\
0.000 \\
0.000 \\
0.000 \\
0.000\end{array}$ \\
\hline $\begin{array}{l}315.094 \\
315.094 \\
315.094 \\
315.094 \\
315.094\end{array}$ & $\begin{array}{l}6.19 \\
6.53 \\
6.99 \\
7.84 \\
8.99\end{array}$ & $\begin{array}{r}8.294 \\
9.095 \\
9.734 \\
10.437 \\
11.036\end{array}$ & $\begin{array}{l}71.479 \\
66.287 \\
66.287 \\
68.883 \\
71.479\end{array}$ & $\begin{array}{l}65.417 \\
65.734 \\
67.390 \\
70.569 \\
74.172\end{array}$ & $\begin{array}{r}-8.491 \\
-0.846 \\
1.653 \\
2.436 \\
3.756\end{array}$ & $\begin{array}{l}0.000 \\
0.000 \\
0.000 \\
0.000 \\
0.000\end{array}$ \\
\hline $\begin{array}{l}315.094 \\
315.094 \\
315.094 \\
315.094 \\
329.817\end{array}$ & $\begin{array}{r}11.23 \\
13.56 \\
16.29 \\
19.86 \\
0.90\end{array}$ & $\begin{array}{r}11.785 \\
12.313 \\
12.780 \\
13.253 \\
0.346\end{array}$ & $\begin{array}{l}79.787 \\
85.844 \\
92.421 \\
93.806 \\
26.307\end{array}$ & $\begin{array}{l}79.694 \\
84.268 \\
88.807 \\
93.918 \\
25.884\end{array}$ & $\begin{array}{r}-0.128 \\
-1.847 \\
-3.922 \\
0.108 \\
-1.617\end{array}$ & $\begin{array}{l}0.000 \\
0.000 \\
0.000 \\
0.000 \\
0.000\end{array}$ \\
\hline 329.817 & 1.72 & 0.698 & 26.999 & 26.752 & -0.927 & 0.000 \\
\hline
\end{tabular}


Table 15. THERMAL CONDUCTIVITY AT ELEVATED PRESSURES (continued)

Data from Lenoir et al. [84] (continued)

\begin{tabular}{|c|c|c|c|c|c|c|}
\hline $\begin{array}{l}\mathrm{T} \\
\mathrm{K}\end{array}$ & $\begin{array}{c}\mathrm{P} \\
\mathrm{MPa}\end{array}$ & $\begin{array}{c}\rho \\
\mathrm{mol} \cdot \mathrm{dm}^{-3}\end{array}$ & $\begin{array}{c}\lambda, \quad \operatorname{expt} \\
\mathrm{mW} \cdot \mathrm{m}^{-1} \cdot \mathrm{K}^{-1}\end{array}$ & $\begin{array}{c}\lambda, \operatorname{calc} \\
\mathrm{mW} \cdot \mathrm{m}^{-1} \cdot \mathrm{K}^{-1}\end{array}$ & $\begin{array}{l}\text { Dev } \\
\&\end{array}$ & wt \\
\hline 329.817 & 2.43 & 1.038 & 27.346 & 27.711 & 1.326 & 0.000 \\
\hline 329.817 & 3.06 & 1.375 & 28.730 & 28.788 & 0.190 & 0.000 \\
\hline 329.817 & 3.31 & 1.523 & 28.903 & 29.301 & 1.365 & 0.000 \\
\hline 329.817 & 3.84 & 1.857 & 29.596 & 30.559 & 3.246 & 0.000 \\
\hline 329.817 & 4.37 & 2.238 & 31.326 & 32.157 & 2.642 & 0.000 \\
\hline 329.817 & 5.00 & 2.776 & 33.057 & 34.716 & 5.005 & 0.000 \\
\hline 329.817 & 5.34 & 3.124 & 35.480 & 36.553 & 3.011 & 0.000 \\
\hline 329.817 & 5.68 & 3.524 & 37.038 & 38.817 & 4.792 & 0.000 \\
\hline 329.817 & 5.95 & 3.876 & 38.076 & 40.910 & 7.432 & 0.000 \\
\hline 329.817 & 6.19 & 4.243 & 40.499 & 43.168 & 6.577 & 0.000 \\
\hline 329.817 & 6.40 & 4.604 & 44.480 & 45.411 & 2.081 & 0.000 \\
\hline 329.817 & 6.93 & 5.673 & 48.460 & 51.683 & 6.637 & 0.000 \\
\hline 329.817 & 7.71 & 7.379 & 56.249 & 58.766 & 4.464 & 0.000 \\
\hline 329.817 & 8.71 & 8.814 & 62.999 & 63.399 & 0.623 & 0.000 \\
\hline 329.817 & 11.28 & 10.477 & 70.441 & 71.585 & 1.613 & 0.000 \\
\hline 329.817 & 13.82 & 11.326 & 79.267 & 77.410 & -2.355 & 0.000 \\
\hline 329.817 & 16.48 & 11.928 & 83.940 & 82.291 & -1.976 & 0.000 \\
\hline 329.817 & 18.07 & 12.215 & 85.152 & 84.859 & -0.355 & 0.000 \\
\hline 340.372 & 1.12 & 0.422 & 28.384 & 27.541 & -2.981 & 0.000 \\
\hline 340.372 & 2.36 & 0.954 & 29.769 & 28.894 & -2.949 & 0.000 \\
\hline 340.372 & 3.24 & 1.393 & 30.980 & 30.222 & -2.458 & 0.000 \\
\hline 340.372 & 4.63 & 2.236 & 33.922 & 33.329 & -1.760 & 0.000 \\
\hline 340.372 & 5.64 & 3.032 & 37.211 & 36.916 & -0.805 & 0.000 \\
\hline 340.372 & 6.65 & 4.068 & 41.884 & 42.271 & 0.913 & 0.000 \\
\hline 340.372 & 8.87 & 7.240 & 54.518 & 57.280 & 5.054 & 0.000 \\
\hline 340.372 & 11.23 & 9.350 & 63.172 & 66.045 & 4.536 & 0.000 \\
\hline 340.372 & 13.78 & 10.494 & 69.229 & 72.565 & 4.806 & 0.000 \\
\hline 340.372 & 16.33 & 11.221 & 74.421 & 77.663 & 4.343 & 0.000 \\
\hline 340.372 & 19.44 & 11.857 & 78.402 & 82.816 & 5.618 & 0.000 \\
\hline
\end{tabular}


Table 15. THERMAL CONDUCTIVITY AT ELEVATED PRESSURES (continued)

Data from Lenoir et al. [84] (continued)

Comparisons based on experimental pressures:

Number of Points [84] 75

$$
\begin{array}{ll}
\mathrm{AAD} \&=2.53 \quad \mathrm{BIAS} \%=0.26 \quad \mathrm{RMS} \%=3.36 \\
\mathrm{AAD}=1.36 \quad \mathrm{BIAS}=0.05 \quad \mathrm{RMS}=2.01 \mathrm{~mW} \cdot \mathrm{m}^{-1} \cdot \mathrm{K}^{-1}
\end{array}
$$

Densities were not tabulated in [84].

Data from Millat et al. [85]

These data were not available during the development of the correlations [1].

\begin{tabular}{|c|c|c|c|c|c|c|}
\hline $\begin{array}{l}\mathrm{T} \\
\mathrm{K}\end{array}$ & $\begin{array}{c}\mathrm{P} \\
\mathrm{MPa}\end{array}$ & $\stackrel{\rho}{\mathrm{mol} \cdot \mathrm{dm}^{-3}}$ & $\begin{array}{c}\lambda, \underset{\mathrm{mW} \cdot \mathrm{m}^{-1} \cdot \mathrm{K}^{-1}}{\operatorname{expt}}\end{array}$ & $\begin{array}{c}\lambda, \quad \text { calc } \\
\mathrm{mW} \cdot \mathrm{m}^{-1} \cdot \mathrm{K}^{-1}\end{array}$ & $\begin{array}{l}\mathrm{Dev} \\
\frac{\gamma}{8}\end{array}$ & wt \\
\hline 425.780 & 0.65 & 0.186 & 42.320 & 40.623 & -4.011 & 0.000 \\
\hline 425.630 & 0.98 & 0.283 & 42.480 & 40.763 & -4.041 & 0.000 \\
\hline 425.630 & 1.34 & 0.391 & 42.430 & 40.956 & -3.474 & 0.000 \\
\hline 425.580 & 1.67 & 0.491 & 42.530 & 41.134 & -3.282 & 0.000 \\
\hline 425.590 & 2.05 & 0.608 & 42.730 & 41.364 & -3.197 & 0.000 \\
\hline 425.560 & 2.35 & 0.703 & 42.900 & 41.549 & -3.149 & 0.000 \\
\hline 425.590 & 2.76 & 0.834 & 43.300 & 41.829 & -3.398 & 0.000 \\
\hline 425.620 & 3.49 & 1.073 & 43.990 & 42.368 & -3.687 & 0.000 \\
\hline 425.670 & 3.78 & 1.171 & 44.120 & 42.605 & -3.434 & 0.000 \\
\hline 425.580 & 4.24 & 1.329 & 44.740 & 42.974 & -3.947 & 0.000 \\
\hline 425.660 & 4.60 & 1.455 & 44.790 & 43.305 & -3.316 & 0.000 \\
\hline 425.690 & 4.82 & 1.532 & 45.260 & 43.511 & -3.863 & 0.000 \\
\hline 425.960 & 5.62 & 1.821 & 45.930 & 44.338 & -3.465 & 0.000 \\
\hline 425.920 & 6.16 & 2.024 & 46.550 & 44.911 & -3.522 & 0.000 \\
\hline 425.830 & 6.25 & 2.059 & 46.520 & 44.998 & -3.271 & 0.000 \\
\hline 425.880 & 6.64 & 2.208 & 47.180 & 45.452 & -3.663 & 0.000 \\
\hline 425.880 & 6.64 & 2.208 & 47.300 & 45.452 & -3.908 & 0.000 \\
\hline 331.500 & 0.66 & 0.248 & 26.590 & 25.899 & -2.598 & 0.000 \\
\hline 331.560 & 0.87 & 0.332 & 26.610 & 26.093 & -1.943 & 0.000 \\
\hline 331.610 & 1.12 & 0.433 & 27.050 & 26.336 & -2.641 & 0.000 \\
\hline 331.690 & 1.48 & 0.586 & 27.210 & 26.718 & -1.807 & 0.000 \\
\hline 331.800 & 2.16 & 0.895 & 28.110 & 27.559 & -1.960 & 0.000 \\
\hline 331.780 & 2.47 & 1.047 & 28.520 & 27.999 & -1.827 & 0.000 \\
\hline 331.790 & 2.84 & 1.239 & 28.940 & 28.595 & -1.193 & 0.000 \\
\hline 331.480 & 3.25 & 1.470 & 29.880 & 29.325 & -1.856 & 0.000 \\
\hline
\end{tabular}


Table 15. THERMAL CONDUCTIVITY AT ELEVATED PRESSURES (continued)

Data from Millat et al. [85] (continued)

\begin{tabular}{|c|c|c|c|c|c|c|}
\hline $\begin{array}{l}\mathrm{T} \\
\mathrm{K}\end{array}$ & $\begin{array}{c}\mathrm{P} \\
\mathrm{MPa}\end{array}$ & $\begin{array}{c}\rho \\
\mathrm{mol} \cdot \mathrm{dm}^{-3}\end{array}$ & 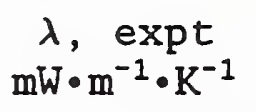 & $\begin{array}{c}\lambda, \text { calc } \\
\mathrm{mW} \cdot \mathrm{m}^{-1} \cdot \mathrm{K}^{-1}\end{array}$ & $\begin{array}{c}\text { Dev } \\
\frac{8}{8}\end{array}$ & wt \\
\hline 331.530 & 3.55 & 1.649 & 30.480 & 29.972 & -1.667 & 0.000 \\
\hline 331.800 & 4.01 & 1.945 & 31.750 & 31.141 & -1.918 & 0.000 \\
\hline 331.770 & 4.30 & 2.151 & 32.400 & 31.991 & -1.262 & 0.000 \\
\hline 331.550 & 4.82 & 2.568 & 34.580 & 33.852 & -2.104 & 0.000 \\
\hline 331.930 & 5.69 & 3.419 & 39.200 & 38.308 & -2.274 & 0.000 \\
\hline 331.920 & 6.31 & 4.241 & 44.010 & 43.114 & -2.036 & 0.000 \\
\hline 380.110 & 0.54 & 0.174 & 34.220 & 33.028 & -3.482 & 0.000 \\
\hline 380.010 & 0.72 & 0.234 & 34.450 & 33.125 & -3.848 & 0.000 \\
\hline 379.490 & 0.94 & 0.308 & 34.460 & 33.186 & -3.697 & 0.000 \\
\hline 379.970 & 1.16 & 0.383 & 34.600 & 33.411 & -3.437 & 0.000 \\
\hline 379.990 & 1.36 & 0.452 & 34.660 & 33.556 & -3.185 & 0.000 \\
\hline 380.090 & 1.62 & 0.544 & 34.850 & 33.765 & -3.113 & 0.000 \\
\hline 379.710 & 1.88 & 0.638 & 35.110 & 33.912 & -3.412 & 0.000 \\
\hline 380.020 & 1.91 & 0.648 & 35.160 & 33.984 & -3.346 & 0.000 \\
\hline 380.020 & 2.47 & 0.857 & 35.520 & 34.468 & -2.963 & 0.000 \\
\hline 380.020 & 2.78 & 0.977 & 35.920 & 34.761 & -3.227 & 0.000 \\
\hline 379.850 & 3.50 & 1.268 & 36.890 & 35.497 & -3.776 & 0.000 \\
\hline 379.980 & 3.80 & 1.394 & 37.090 & 35.867 & -3.298 & 0.000 \\
\hline 380.090 & 4.48 & 1.693 & 38.030 & 36.764 & -3.330 & 0.000 \\
\hline 379.930 & 4.78 & 1.833 & 38.420 & 37.175 & -3.241 & 0.000 \\
\hline 307.710 & 0.62 & 0.253 & 23.270 & 22.700 & -2.451 & 0.000 \\
\hline 307.690 & 0.71 & 0.292 & 23.460 & 22.790 & -2.855 & 0.000 \\
\hline 307.800 & 0.82 & 0.340 & 23.400 & 22.922 & -2.043 & 0.000 \\
\hline 307.730 & 0.95 & 0.398 & 23.550 & 23.058 & -2.088 & 0.000 \\
\hline 308.160 & 1.08 & 0.457 & 23.750 & 23.263 & -2.049 & 0.000 \\
\hline 307.590 & 1.22 & 0.523 & 23.850 & 23.366 & -2.030 & 0.000 \\
\hline 307.650 & 1.36 & 0.590 & 23.950 & 23.555 & -1.649 & 0.000 \\
\hline 307.630 & 1.53 & 0.674 & 24.160 & 23.788 & -1.541 & 0.000 \\
\hline 307.500 & 1.70 & 0.761 & 24.280 & 24.025 & -1.050 & 0.000 \\
\hline 307.630 & 1.87 & 0.851 & 24.550 & 24.311 & -0.975 & 0.000 \\
\hline 307.620 & 2.00 & 0.922 & 24.870 & 24.531 & -1.362 & 0.000 \\
\hline 307.640 & 2.14 & 1.000 & 24.980 & 24.787 & -0.771 & 0.000 \\
\hline 307.620 & 2.30 & 1.094 & 25.320 & 25.098 & -0.878 & 0.000 \\
\hline 307.640 & 3.23 & 1.730 & 27.250 & 27.586 & 1.233 & 0.000 \\
\hline 307.750 & 3.33 & 1.809 & 27.560 & 27.956 & 1.439 & 0.000 \\
\hline
\end{tabular}


Table 15. THERMAL CONDUCTIVITY AT ELEVATED PRESSURES (continued)

Data from Millat et al. [85] (continued)

\begin{tabular}{|c|c|c|c|c|c|c|}
\hline $\begin{array}{l}\mathrm{T} \\
\mathrm{K}\end{array}$ & $\begin{array}{c}\mathrm{P} \\
\mathrm{MPa}\end{array}$ & $\begin{array}{c}\rho \\
\mathrm{mol} \cdot \mathrm{dm}^{-3}\end{array}$ & $\begin{array}{c}\lambda, \quad \operatorname{expt} \\
\mathrm{mW} \cdot \mathrm{m}^{-1} \cdot \mathrm{K}^{-1}\end{array}$ & $\begin{array}{c}\lambda, \quad \text { calc } \\
\mathrm{mW} \cdot \mathrm{m}^{-1} \cdot \mathrm{K}^{-1}\end{array}$ & $\begin{array}{c}\text { Dev } \\
\frac{8}{8}\end{array}$ & $w t$ \\
\hline 307.670 & 3.61 & 2.057 & 28.670 & 29.153 & 1.685 & 0.000 \\
\hline 307.600 & 3.67 & 2.115 & 28.890 & 29.452 & 1.946 & 0.000 \\
\hline 307.520 & 3.77 & 2.217 & 29.340 & 29.992 & 2.222 & 0.000 \\
\hline 307.540 & 3.90 & 2.354 & 30.000 & 30.773 & 2.576 & 0.000 \\
\hline
\end{tabular}

Comparisons based on experimental pressures:

Number of Points [85] 64

\begin{tabular}{|c|c|c|c|c|}
\hline AI & 2.62 & BIAS 8 & -2.28 & RMS क्ष \\
\hline$A D$ & 0.95 & BIAS & -0.85 & RMS \\
\hline
\end{tabular}

Comparisons based on experimental densities:

Number of Points [85] 64

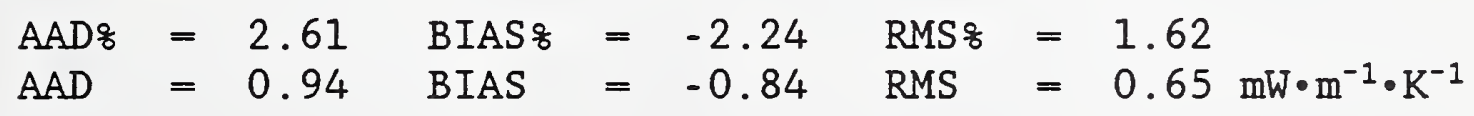

Data from Mostert et a1. [101]

These data were not available during the development of the correlations [1].

\begin{tabular}{ccccrrr}
\hline $\mathrm{T}$ & $\begin{array}{c}\mathrm{P} \\
\mathrm{MPa}\end{array}$ & $\begin{array}{c}\rho \\
\mathrm{mol} \cdot \mathrm{dm}^{-3}\end{array}$ & $\begin{array}{c}\lambda, \begin{array}{c}\text { expt } \\
\mathrm{mW} \cdot \mathrm{m}^{-1} \cdot \mathrm{K}^{-1}\end{array} \\
\mathrm{~mW} \cdot \mathrm{m}^{-1} \cdot \mathrm{K}^{-1}\end{array}$ & $\begin{array}{c}\text { Dev } \\
\text { o }\end{array}$ & wt \\
\hline 333.140 & 1.95 & 0.789 & 27.500 & 27.449 & -0.185 & 0.000 \\
319.983 & - & 0.789 & 25.700 & 25.694 & -0.024 & 0.000 \\
312.028 & - & 0.789 & 24.600 & 24.674 & 0.300 & 0.000 \\
308.037 & - & 0.789 & 24.200 & 24.174 & -0.107 & 0.000 \\
306.995 & - & 0.789 & 24.000 & 24.045 & 0.187 & 0.000 \\
305.962 & - & 0.789 & 23.900 & 23.917 & 0.073 & 0.000 \\
305.620 & - & 0.789 & 23.900 & 23.875 & -0.103 & 0.000 \\
333.132 & 4.81 & 2.519 & 33.100 & 33.779 & 2.050 & 0.000 \\
333.120 & 4.81 & 2.520 & 34.100 & 33.779 & -0.942 & 0.000 \\
320.005 & - & 2.520 & 32.500 & 32.588 & 0.270 & 0.000 \\
319.993 & - & 2.520 & 32.300 & 32.587 & 0.888 & 0.000 \\
312.007 & - & 2.520 & 32.100 & 32.019 & -0.254 & 0.000 \\
308.041 & - & 2.520 & 31.200 & 31.799 & 1.919 & 0.000 \\
308.041 & - & 2.520 & 31.500 & 31.799 & 0.949 & 0.000 \\
306.997 & - & 2.520 & 31.700 & 31.750 & 0.156 & 0.000
\end{tabular}


Data from Mostert et al. [101] (continued)

\begin{tabular}{|c|c|c|c|c|c|c|}
\hline $\begin{array}{l}\mathrm{T} \\
\mathrm{K}\end{array}$ & $\begin{array}{c}\mathrm{P} \\
\mathrm{MPa}\end{array}$ & $\begin{array}{c}\rho \\
\mathrm{mol} \cdot \mathrm{dm}^{-3}\end{array}$ & $\begin{array}{c}\lambda, \underset{\operatorname{expt}}{\exp } \cdot \mathrm{m}^{-1} \cdot \mathrm{K}^{-1}\end{array}$ & $\begin{array}{c}\lambda, \quad \text { calc } \\
\mathrm{mW} \cdot \mathrm{m}^{-1} \cdot \mathrm{K}^{-1}\end{array}$ & $\begin{array}{l}\text { Dev } \\
\frac{8}{8}\end{array}$ & wt \\
\hline 305.966 & - & 2.520 & 31.900 & 31.705 & -0.612 & 0.000 \\
\hline 305.613 & - & 2.520 & 31.900 & 31.691 & -0.657 & 0.000 \\
\hline 305.494 & - & 2.520 & 31.800 & 31.686 & -0.359 & 0.000 \\
\hline 305.488 & - & 2.520 & 32.100 & 31.686 & -1.291 & 0.000 \\
\hline 333.114 & 6.22 & 4.022 & 41.600 & 41.814 & 0.514 & 0.000 \\
\hline 319.962 & - & 4.020 & 42.300 & 42.253 & -0.111 & 0.000 \\
\hline 308.021 & - & 4.020 & 44.400 & 44.971 & 1.286 & 0.000 \\
\hline 306.974 & - & 4.020 & 44.600 & 45.461 & 1.931 & 0.000 \\
\hline 305.942 & - & 4.020 & 45.500 & 46.019 & 1.140 & 0.000 \\
\hline 305.592 & - & 4.020 & 45.300 & 46.227 & 2.046 & 0.000 \\
\hline 305.471 & - & 4.020 & 45.300 & 46.301 & 2.210 & 0.000 \\
\hline 305.459 & - & 4.020 & 44.400 & 46.309 & 4.299 & 0.000 \\
\hline 333.109 & 7.02 & 5.315 & 50.000 & 49.309 & -1.381 & 0.000 \\
\hline 319.946 & - & 5.320 & 52.200 & 52.226 & 0.049 & 0.000 \\
\hline 311.985 & - & 5.320 & 57.600 & 57.963 & 0.629 & 0.000 \\
\hline 308.012 & - & 5.320 & 65.500 & 65.560 & 0.092 & 0.000 \\
\hline 306.961 & - & 5.320 & 70.800 & 69.355 & -2.041 & 0.000 \\
\hline 305.978 & - & 5.320 & 74.000 & 74.642 & 0.868 & 0.000 \\
\hline 305.925 & - & 5.320 & 77.700 & 75.004 & -3.470 & 0.000 \\
\hline 305.924 & - & 5.320 & 74.600 & 75.011 & 0.550 & 0.000 \\
\hline 305.576 & - & 5.320 & 78.700 & 77.666 & -1.314 & 0.000 \\
\hline 305.576 & - & 5.320 & 83.600 & 77.666 & -7.098 & 0.000 \\
\hline 333.103 & 7.42 & 6.104 & 54.700 & 53.317 & -2.528 & 0.000 \\
\hline 319.948 & - & 6.110 & 56.900 & 57.319 & 0.737 & 0.000 \\
\hline 311.992 & - & 6.110 & 66.100 & 65.565 & -0.809 & 0.000 \\
\hline 308.022 & - & 6.110 & 70.900 & 79.116 & 11.588 & 0.000 \\
\hline 306.961 & - & 6.110 & 92.600 & 88.494 & -4.434 & 0.000 \\
\hline 305.922 & - & 6.110 & 123.000 & 110.583 & -10.095 & 0.000 \\
\hline 305.575 & - & 6.110 & 134.000 & 129.721 & -3.193 & 0.000 \\
\hline 305.574 & - & 6.110 & 142.000 & 129.802 & -8.590 & 0.000 \\
\hline 305.455 & - & 6.110 & 162.000 & 141.356 & -12.743 & 0.000 \\
\hline 333.171 & 7.82 & 6.869 & 58.300 & 56.487 & -3.110 & 0.000 \\
\hline 319.947 & - & 6.870 & 62.400 & 60.602 & -2.881 & 0.000 \\
\hline 314.860 & - & 6.870 & 65.200 & 64.869 & -0.508 & 0.000 \\
\hline 312.008 & - & 6.870 & 70.900 & 69.208 & -2.387 & 0.000 \\
\hline 308.011 & - & 6.870 & 85.800 & 83.998 & -2.101 & 0.000 \\
\hline
\end{tabular}


Table 15. THERMAL CONDUCTIVITY AT ELEVATED PRESSURES (continued)

Data from Mostert et al. [101] (continued)

\begin{tabular}{|c|c|c|c|c|c|c|}
\hline $\begin{array}{l}\mathrm{T} \\
\mathrm{K}\end{array}$ & $\begin{array}{c}\mathrm{P} \\
\mathrm{MPa}\end{array}$ & $\begin{array}{c}\rho \\
\mathrm{mol} \cdot \mathrm{dm}^{-3}\end{array}$ & $\begin{array}{c}\lambda, \underset{\exp t}{\mathrm{~mW} \cdot \mathrm{m}^{-1} \cdot \mathrm{K}^{-1}}\end{array}$ & $\begin{array}{c}\lambda, \quad \text { calc } \\
\mathrm{mW} \cdot \mathrm{m}^{-1} \cdot \mathrm{K}^{-1}\end{array}$ & $\begin{array}{c}\text { Dev } \\
\frac{8}{8}\end{array}$ & wt \\
\hline 306.972 & - & 6.870 & 103.000 & 94.772 & -7.989 & 0.000 \\
\hline 306.964 & - & 6.870 & 98.900 & 94.891 & -4.053 & 0.000 \\
\hline 305.910 & - & 6.870 & 131.000 & 127.629 & -2.573 & 0.000 \\
\hline 305.743 & - & 6.870 & 174.000 & 142.387 & -18.169 & 0.000 \\
\hline 305.724 & - & 6.870 & 160.000 & 144.631 & -9.606 & 0.000 \\
\hline 305.624 & - & 6.870 & 169.000 & 159.790 & -5.449 & 0.000 \\
\hline 305.588 & - & 6.870 & 195.000 & 167.281 & -14.215 & 0.000 \\
\hline 305.567 & - & 6.870 & 204.000 & 172.411 & -15.485 & 0.000 \\
\hline 305.567 & - & 6.870 & 205.000 & 172.411 & -15.897 & 0.000 \\
\hline 305.522 & - & 6.900 & 206.000 & 185.565 & -9.920 & 0.000 \\
\hline 305.482 & - & 6.900 & 231.000 & 202.370 & -12.394 & 0.000 \\
\hline 305.447 & - & 6.900 & 245.000 & 223.609 & -8.731 & 0.000 \\
\hline 305.442 & - & 6.900 & 255.000 & 227.425 & -10.814 & 0.000 \\
\hline 333.114 & 8.03 & 7.257 & 59.700 & 57.882 & -3.045 & 0.000 \\
\hline 319.938 & - & 7.260 & 63.500 & 61.667 & -2.887 & 0.000 \\
\hline 311.980 & - & 7.260 & 71.700 & 69.732 & -2.745 & 0.000 \\
\hline 308.021 & - & 7.260 & 88.700 & 83.116 & -6.296 & 0.000 \\
\hline 306.953 & - & 7.260 & 102.000 & 93.060 & -8.764 & 0.000 \\
\hline 305.566 & - & 7.260 & 172.000 & 155.241 & -9.744 & 0.000 \\
\hline 333.116 & 8.53 & 8.015 & 66.900 & 60.431 & -9.670 & 0.000 \\
\hline 319.944 & - & 8.030 & 63.700 & 62.939 & -1.195 & 0.000 \\
\hline 311.993 & - & 8.030 & 74.700 & 68.509 & -8.288 & 0.000 \\
\hline 308.007 & - & 8.030 & 76.900 & 76.654 & -0.320 & 0.000 \\
\hline 306.952 & - & 8.030 & 80.100 & 81.363 & 1.577 & 0.000 \\
\hline 305.914 & - & 8.030 & 86.500 & 89.963 & 4.004 & 0.000 \\
\hline 305.565 & - & 8.030 & 93.200 & 95.121 & 2.061 & 0.000 \\
\hline 305.448 & - & 8.030 & 89.400 & 97.392 & 8.940 & 0.000 \\
\hline 305.447 & - & 8.030 & 92.500 & 97.413 & 5.312 & 0.000 \\
\hline 333.105 & 9.45 & 8.995 & 67.200 & 64.111 & -4.596 & 0.000 \\
\hline 319.945 & - & 9.020 & 65.400 & 64.741 & -1.007 & 0.000 \\
\hline 311.980 & - & 9.020 & 66.500 & 66.651 & 0.228 & 0.000 \\
\hline 308.005 & - & 9.020 & 72.200 & 69.033 & -4.387 & 0.000 \\
\hline 306.958 & - & 9.020 & 71.700 & 70.033 & -2.325 & 0.000 \\
\hline 305.924 & - & 9.020 & 73.100 & 71.292 & -2.474 & 0.000 \\
\hline 305.571 & - & 9.020 & 73.500 & 71.805 & -2.307 & 0.000 \\
\hline 305.461 & - & 9.020 & 75.500 & 71.975 & -4.669 & 0.000 \\
\hline
\end{tabular}


Table 15. THERMAL CONDUCTIVITY AT ELEVATED PRESSURES (continued)

Data from Mostert et a1. [101] (continued)

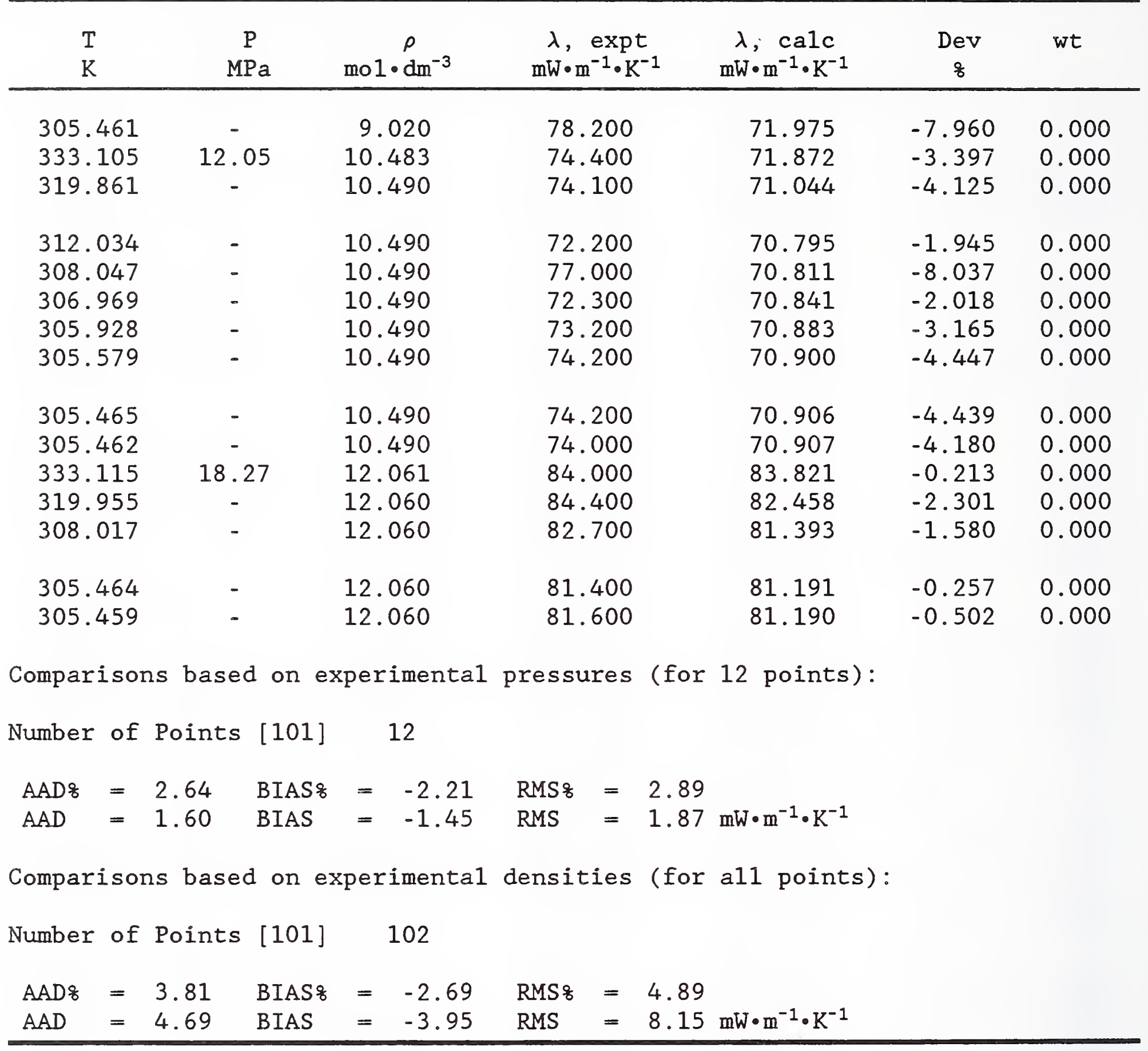


Table 15. THERMAL CONDUCTIVITY AT ELEVATED PRESSURES (continued)

Data from Mostert et al. [102]

These data were not available during the development of the correlations [1].

\begin{tabular}{ccccrrr}
\hline $\begin{array}{c}\mathrm{T} \\
\mathrm{K}\end{array}$ & $\begin{array}{c}\mathrm{P} \\
\mathrm{MPa}\end{array}$ & $\begin{array}{c}\rho \\
\mathrm{mol} \cdot \mathrm{dm}^{-3}\end{array}$ & $\begin{array}{c}\lambda, \text { expt } \\
\mathrm{mW} \cdot \mathrm{m}^{-1} \cdot \mathrm{K}^{-1}\end{array}$ & $\begin{array}{c}\lambda, \text { calc } \\
\mathrm{mW} \cdot \mathrm{m}^{-1} \cdot \mathrm{K}^{-1}\end{array}$ & $\begin{array}{c}\text { Dev } \\
8\end{array}$ & wt \\
\hline 305.455 & 4.88 & 6.170 & 161.980 & 149.377 & -7.781 & 0.000 \\
305.574 & 4.89 & 6.134 & 141.590 & 131.965 & -6.798 & 0.000 \\
305.575 & 4.89 & 6.121 & 134.300 & 130.716 & -2.669 & 0.000 \\
305.922 & 4.93 & 6.157 & 123.100 & 112.774 & -8.389 & 0.000 \\
306.961 & 5.02 & 6.127 & 92.632 & 88.821 & -4.114 & 0.000 \\
308.022 & 5.12 & 6.124 & 70.913 & 79.291 & 11.814 & 0.000 \\
311.992 & 5.49 & 6.089 & 66.106 & 65.405 & -1.060 & 0.000 \\
319.948 & 6.22 & 6.088 & 56.916 & 57.199 & 0.497 & 0.000 \\
333.103 & 7.41 & 6.090 & 54.732 & 53.252 & -2.703 & 0.000
\end{tabular}

Comparisons based on experimental pressures:

Number of Points [102] 9

$\begin{array}{ll}\mathrm{AAD} 8 & =5.09 \quad \mathrm{BIAS} 8=-2.36 \quad \mathrm{RMS} 8=5.77 \\ \mathrm{AAD}=5.64 & \mathrm{BIAS}=-3.72 \mathrm{RMS}=6.09 \mathrm{~mW} \cdot \mathrm{m}^{-1} \cdot \mathrm{K}^{-1}\end{array}$

Comparisons based on experimental densities:

Number of Points [102] 9

\begin{tabular}{|c|c|c|c|c|c|}
\hline & 55 & S & 92 & RMS \& & 7.00 \\
\hline$A I$ & 7.79 & BIAS & -5.94 & RMS & $\mathrm{m}^{-1} \cdot \mathrm{K}^{-1}$ \\
\hline
\end{tabular}

Data from Prasad et al. [86]

\begin{tabular}{ccccrrr}
\hline $\begin{array}{c}\mathrm{T} \\
\mathrm{K}\end{array}$ & $\begin{array}{c}\mathrm{P} \\
\mathrm{MPa}\end{array}$ & $\begin{array}{c}\rho \\
\mathrm{mol} \cdot \mathrm{dm}^{-3}\end{array}$ & $\begin{array}{c}\lambda, \text { expt } \\
\mathrm{mW} \cdot \mathrm{m}^{-1} \cdot \mathrm{K}^{-1}\end{array}$ & $\begin{array}{r}\lambda, \mathrm{calc} \\
\mathrm{mW} \cdot \mathrm{m}^{-1} \cdot \mathrm{K}^{-1}\end{array}$ & $\begin{array}{c}\text { Dev } \\
8\end{array}$ & wt \\
\hline 293.850 & 1.44 & 0.675 & 21.910 & 22.111 & 0.917 & 0.060 \\
293.720 & 1.44 & 0.675 & 22.160 & 22.095 & -0.292 & 0.050 \\
293.590 & 1.44 & 0.676 & 22.060 & 22.081 & 0.097 & 0.050 \\
293.200 & 3.12 & 1.896 & 25.630 & 27.158 & 5.961 & 0.000 \\
297.160 & 18.98 & 14.041 & 103.840 & 101.651 & -2.108 & 0.030 \\
& & & & & & \\
295.490 & 17.64 & 13.993 & 101.470 & 100.838 & -0.623 & 0.030 \\
295.390 & 20.05 & 14.223 & 105.170 & 103.912 & -1.196 & 0.030 \\
295.360 & 20.09 & 14.229 & 105.430 & 103.979 & -1.376 & 0.030 \\
295.360 & 25.13 & 14.631 & 111.790 & 109.763 & -1.813 & 0.030 \\
295.370 & 29.98 & 14.957 & 116.750 & 114.832 & -1.643 & 0.030
\end{tabular}


Table 15. THERMAL CONDUCTIVITY AT ELEVATED PRESSURES (continued)

Data from Prasad et al. [86] (continued)

\begin{tabular}{|c|c|c|c|c|c|c|}
\hline $\begin{array}{l}\mathrm{T} \\
\mathrm{K}\end{array}$ & $\begin{array}{c}\mathrm{P} \\
\mathrm{MPa}\end{array}$ & $\begin{array}{c}\rho \\
\mathrm{mol}\end{array} \mathrm{dm}^{-3}$ & 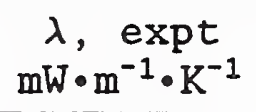 & $\begin{array}{c}\lambda, \quad \text { calc } \\
\mathrm{mW} \cdot \mathrm{m}^{-1} \cdot \mathrm{K}^{-1}\end{array}$ & $\begin{array}{c}\text { Dev } \\
\frac{8}{8}\end{array}$ & wt \\
\hline 294.170 & 3.78 & 2.822 & 31.740 & 34.527 & 8.781 & 0.000 \\
\hline 294.140 & 3.81 & 2.879 & 33.120 & 35.183 & 6.229 & 0.000 \\
\hline 294.250 & 4.48 & 11.563 & 80.990 & 76.831 & -5.136 & 0.040 \\
\hline 294.300 & 5.01 & 11.818 & 82.080 & 78.592 & -4.250 & 0.040 \\
\hline 294.340 & 5.02 & 11.815 & 81.730 & 78.571 & -3.865 & 0.040 \\
\hline 294.420 & 6.03 & 12.181 & 83.840 & 81.443 & -2.859 & 0.040 \\
\hline 294.510 & 7.03 & 12.462 & 85.640 & 83.889 & -2.045 & 0.040 \\
\hline 294.610 & 8.02 & 12.687 & 87.920 & 86.004 & -2.179 & 0.040 \\
\hline 294.700 & 9.00 & 12.883 & 89.630 & 87.937 & -1.888 & 0.030 \\
\hline 294.810 & 10.11 & 13.074 & 91.310 & 89.930 & -1.511 & 0.030 \\
\hline 294.910 & 15.01 & 13.743 & 98.300 & 97.600 & -0.712 & 0.030 \\
\hline 294.510 & 18.88 & 14.160 & 103.690 & 102.946 & -0.718 & 0.030 \\
\hline 294.400 & 30.00 & 14.995 & 117.210 & 115.331 & -1.603 & 0.030 \\
\hline 294.350 & 35.00 & 15.287 & 122.450 & 120.202 & -1.836 & 0.030 \\
\hline 294.310 & 39.99 & 15.545 & 126.650 & 124.779 & -1.477 & 0.030 \\
\hline 294.250 & 45.01 & 15.780 & 130.520 & 129.161 & -1.041 & 0.030 \\
\hline 294.250 & 50.03 & 15.993 & 136.250 & 133.328 & -2.145 & 0.030 \\
\hline 294.240 & 55.04 & 16.189 & 139.860 & 137.328 & -1.810 & 0.030 \\
\hline 294.240 & 60.04 & 16.370 & 143.730 & 141.185 & -1.771 & 0.030 \\
\hline 294.250 & 65.01 & 16.538 & 146.960 & 144.903 & -1.400 & 0.030 \\
\hline 294.270 & 69.92 & 16.694 & 150.640 & 148.459 & -1.448 & 0.030 \\
\hline 293.150 & 0.69 & 0.300 & 20.660 & 20.978 & 1.539 & 0.070 \\
\hline 293.050 & 1.37 & 0.638 & 21.680 & 21.904 & 1.034 & 0.060 \\
\hline 293.140 & 2.07 & 1.048 & 23.100 & 23.270 & 0.734 & 0.050 \\
\hline 294.720 & 2.08 & 1.046 & 22.670 & 23.437 & 3.383 & 0.060 \\
\hline 294.720 & 2.08 & 1.047 & 22.790 & 23.439 & 2.849 & 0.060 \\
\hline 294.740 & 2.76 & 1.536 & 24.440 & 25.436 & 4.075 & 0.060 \\
\hline 294.820 & 3.46 & 2.247 & 29.380 & 29.471 & 0.311 & 0.050 \\
\hline 295.740 & 0.69 & 0.297 & 21.410 & 21.289 & -0.565 & 0.050 \\
\hline 295.840 & 1.38 & 0.634 & 21.930 & 22.223 & 1.337 & 0.060 \\
\hline 295.980 & 0.18 & 0.073 & 20.540 & 20.778 & 1.159 & 0.080 \\
\hline 318.030 & 59.93 & 15.702 & 134.450 & 131.045 & -2.532 & 0.300 \\
\hline 318.360 & 60.11 & 15.700 & 133.720 & 131.056 & -1.992 & 0.300 \\
\hline 318.440 & 65.14 & 15.892 & 136.640 & 134.829 & -1.326 & 0.300 \\
\hline 318.500 & 70.10 & 16.068 & 139.770 & 138.445 & -0.948 & 0.300 \\
\hline 318.680 & 55.27 & 15.490 & 130.160 & 127.150 & -2.312 & 0.310 \\
\hline
\end{tabular}


Table 15. THERMAL CONDUCTIVITY AT ELEVATED PRESSURES (continued)

Data from Prasad et al. [86] (continued)

\begin{tabular}{|c|c|c|c|c|c|c|}
\hline $\begin{array}{l}\mathrm{T} \\
\mathrm{K}\end{array}$ & $\begin{array}{c}\mathrm{P} \\
\mathrm{MPa}\end{array}$ & $\stackrel{\rho}{\mathrm{mol} \cdot \mathrm{dm}^{-3}}$ & $\begin{array}{c}\lambda, \underset{\mathrm{mW} \cdot \mathrm{m}^{-1} \cdot \mathrm{K}^{-1}}{\exp t} \\
\end{array}$ & $\begin{array}{c}\lambda, \quad \text { calc } \\
\mathrm{mW} \cdot \mathrm{m}^{-1} \cdot \mathrm{K}^{-1}\end{array}$ & $\begin{array}{c}\text { Dev } \\
8\end{array}$ & wt \\
\hline 318.690 & 55.00 & 15.479 & 129.800 & 126.938 & -2.205 & 0.310 \\
\hline 318.710 & 55.00 & 15.478 & 128.900 & 126.930 & -1.528 & 0.310 \\
\hline 318.740 & 50.03 & 15.252 & 124.930 & 122.877 & -1.643 & 0.310 \\
\hline 318.760 & 45.07 & 15.005 & 121.100 & 118.656 & -2.018 & 0.320 \\
\hline 318.790 & 40.04 & 14.726 & 116.380 & 114.154 & -1.913 & 0.320 \\
\hline 318.980 & 34.97 & 14.403 & 111.840 & 109.276 & -2.292 & 0.330 \\
\hline 318.990 & 30.04 & 14.047 & 106.620 & 104.261 & -2.213 & 0.330 \\
\hline 319.010 & 25.02 & 13.614 & 102.010 & 98.650 & -3.293 & 0.340 \\
\hline 319.100 & 20.14 & 13.085 & 95.900 & 92.477 & -3.569 & 0.340 \\
\hline 319.260 & 15.05 & 12.323 & 86.850 & 84.765 & -2.400 & 0.360 \\
\hline 319.520 & 15.02 & 12.300 & 87.150 & 84.578 & -2.951 & 0.360 \\
\hline 315.570 & 3.94 & 2.179 & 30.350 & 30.466 & 0.381 & 0.500 \\
\hline 314.990 & 3.82 & 2.089 & 29.820 & 29.975 & 0.520 & 0.510 \\
\hline 314.990 & 5.00 & 3.505 & 37.890 & 38.724 & 2.201 & 0.500 \\
\hline 315.500 & 7.01 & 9.672 & 72.090 & 67.146 & -6.858 & 0.380 \\
\hline 315.580 & 8.20 & 10.588 & 74.540 & 71.427 & -4.177 & 0.380 \\
\hline 315.690 & 8.07 & 10.494 & 73.470 & 70.898 & -3.500 & 0.380 \\
\hline 315.750 & 9.04 & 10.986 & 75.480 & 73.881 & -2.118 & 0.380 \\
\hline 315.860 & 10.05 & 11.365 & 78.170 & 76.514 & -2.118 & 0.370 \\
\hline 316.410 & 13.90 & 12.291 & 86.700 & 84.187 & -2.898 & 0.360 \\
\hline 316.550 & 20.10 & 13.208 & 96.150 & 93.575 & -2.678 & 0.340 \\
\hline 315.530 & 0.18 & 0.069 & 23.070 & 23.316 & 1.068 & 0.660 \\
\hline 316.130 & 0.35 & 0.134 & 23.340 & 23.539 & 0.854 & 1.020 \\
\hline 316.280 & 0.69 & 0.274 & 23.740 & 23.875 & 0.569 & 0.620 \\
\hline 316.400 & 1.38 & 0.576 & 24.410 & 24.643 & 0.955 & 0.600 \\
\hline 316.790 & 2.07 & 0.915 & 25.770 & 25.652 & -0.457 & 0.510 \\
\hline 316.880 & 2.76 & 1.301 & 26.830 & 26.928 & 0.363 & 0.530 \\
\hline 316.990 & 3.45 & 1.759 & 28.620 & 28.700 & 0.279 & 0.510 \\
\hline 317.140 & 4.07 & 2.260 & 30.690 & 31.010 & 1.042 & 0.510 \\
\hline 317.430 & 4.09 & 2.271 & 30.640 & 31.092 & 1.476 & 0.520 \\
\hline 314.630 & 3.75 & 2.038 & 29.330 & 29.697 & 1.252 & 0.520 \\
\hline 315.120 & 4.83 & 3.218 & 37.520 & 36.615 & -2.411 & 0.460 \\
\hline 316.470 & 5.17 & 3.703 & 39.300 & 40.153 & 2.170 & 0.490 \\
\hline 316.550 & 5.18 & 3.715 & 39.270 & 40.236 & 2.460 & 0.490 \\
\hline 316.620 & 5.20 & 3.735 & 39.270 & 40.387 & 2.845 & 0.500 \\
\hline 316.700 & 5.18 & 3.688 & 39.760 & 40.014 & 0.638 & 0.480 \\
\hline
\end{tabular}


Table 15. THERMAL CONDUCTIVITY AT ELEVATED PRESSURES (continued)

Data from Prasad et al. [86] (continued)

\begin{tabular}{|c|c|c|c|c|c|c|}
\hline $\begin{array}{l}\mathrm{T} \\
\mathrm{K}\end{array}$ & $\begin{array}{c}\mathrm{P} \\
\mathrm{MPa}\end{array}$ & $\stackrel{\rho}{\mathrm{mol} \cdot \mathrm{dm}^{-3}}$ & $\begin{array}{c}\lambda, \underset{\mathrm{mW}}{\exp \mathrm{m}^{-1} \cdot \mathrm{K}^{-1}}\end{array}$ & $\begin{array}{c}\lambda, \quad \operatorname{calc} \\
\mathrm{mW} \cdot \mathrm{m}^{-1} \cdot \mathrm{K}^{-1}\end{array}$ & $\begin{array}{c}\text { Dev } \\
\%\end{array}$ & wt \\
\hline 318.680 & 5.72 & 4.692 & 48.430 & 47.775 & -1.353 & 0.450 \\
\hline 318.660 & 5.89 & 5.245 & 52.650 & 52.221 & -0.815 & 0.450 \\
\hline 318.640 & 6.22 & 6.660 & 64.720 & 60.715 & -6.188 & 0.400 \\
\hline 318.630 & 5.52 & 4.193 & 43.080 & 43.756 & 1.569 & 0.480 \\
\hline 318.650 & 5.18 & 3.530 & 38.350 & 38.760 & 1.069 & 0.490 \\
\hline 318.700 & 4.85 & 3.050 & 35.130 & 35.556 & 1.214 & 0.500 \\
\hline 318.740 & 4.49 & 2.630 & 32.300 & 33.087 & 2.437 & 0.520 \\
\hline 318.790 & 4.34 & 2.477 & 31.870 & 32.263 & 1.233 & 0.510 \\
\hline 318.820 & 7.12 & 9.066 & 70.920 & 65.007 & -8.337 & 0.000 \\
\hline 350.490 & 4.39 & 1.936 & 33.690 & 33.424 & -0.789 & 0.510 \\
\hline 350.330 & 4.82 & 2.200 & 34.710 & 34.395 & -0.909 & 0.500 \\
\hline 348.420 & 47.28 & 14.205 & 111.570 & 110.410 & -1.040 & 0.330 \\
\hline 349.530 & 35.02 & 13.299 & 101.820 & 98.684 & -3.080 & 0.340 \\
\hline 349.920 & 45.15 & 14.027 & 111.220 & 108.111 & -2.796 & 0.330 \\
\hline 350.780 & 45.61 & 14.030 & 111.230 & 108.273 & -2.659 & 0.330 \\
\hline 351.160 & 50.19 & 14.293 & 113.720 & 112.085 & -1.438 & 0.330 \\
\hline 351.280 & 54.99 & 14.549 & 117.760 & 115.979 & -1.512 & 0.320 \\
\hline 351.330 & 60.04 & 14.797 & 122.010 & 119.930 & -1.705 & 0.320 \\
\hline 350.600 & 10.06 & 7.241 & 60.600 & 57.277 & -5.484 & 0.410 \\
\hline 350.780 & 10.16 & 7.317 & 60.280 & 57.592 & -4.459 & 0.410 \\
\hline 350.900 & 15.06 & 10.115 & 73.620 & 71.176 & -3.319 & 0.390 \\
\hline 350.910 & 15.05 & 10.111 & 74.100 & 71.158 & -3.970 & 0.390 \\
\hline 350.980 & 20.02 & 11.374 & 82.700 & 80.015 & -3.246 & 0.370 \\
\hline 351.180 & 24.93 & 12.159 & 89.640 & 86.821 & -3.145 & 0.360 \\
\hline 349.170 & 22.96 & 11.970 & 87.560 & 84.843 & -3.103 & 0.360 \\
\hline 349.030 & 25.07 & 12.276 & 90.160 & 87.665 & -2.768 & 0.360 \\
\hline 349.000 & 30.07 & 12.857 & 96.060 & 93.596 & -2.565 & 0.350 \\
\hline 348.990 & 35.32 & 13.344 & 101.890 & 99.151 & -2.688 & 0.340 \\
\hline 349.050 & 40.01 & 13.707 & 106.410 & 103.698 & -2.548 & 0.340 \\
\hline 349.070 & 7.02 & 3.977 & 42.650 & 42.294 & -0.834 & 0.480 \\
\hline 349.070 & 7.19 & 4.151 & 43.350 & 43.163 & -0.431 & 0.480 \\
\hline 349.160 & 6.15 & 3.183 & 38.290 & 38.468 & 0.464 & 0.500 \\
\hline 349.210 & 8.03 & 5.076 & 48.620 & 47.757 & -1.776 & 0.450 \\
\hline 349.310 & 9.03 & 6.266 & 54.480 & 53.223 & -2.307 & 0.440 \\
\hline 350.380 & 7.31 & 4.202 & 43.460 & 43.499 & 0.089 & 0.480 \\
\hline 351.150 & 68.78 & 15.185 & 129.330 & 126.516 & -2.176 & 0.310 \\
\hline
\end{tabular}


Table 15. THERMAL CONDUCTIVITY AT ELEVATED PRESSURES (continued)

Data from Prasad et al. [86] (continued)

\begin{tabular}{|c|c|c|c|c|c|c|}
\hline $\begin{array}{l}\mathrm{T} \\
\mathrm{K}\end{array}$ & $\begin{array}{c}\mathrm{P} \\
\mathrm{MPa}\end{array}$ & $\begin{array}{c}\rho \\
\mathrm{mol} \cdot \mathrm{dm}^{-3}\end{array}$ & 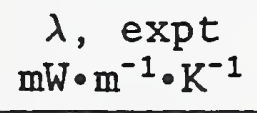 & $\begin{array}{c}\lambda, \quad \operatorname{calc} \\
\mathrm{mW} \cdot \mathrm{m}^{-1} \cdot \mathrm{K}^{-1}\end{array}$ & $\begin{array}{c}\text { Dev } \\
\frac{8}{8}\end{array}$ & wt \\
\hline $\begin{array}{l}351.210 \\
349.870\end{array}$ & $\begin{array}{r}70.08 \\
0.34\end{array}$ & $\begin{array}{r}15.236 \\
0.120\end{array}$ & $\begin{array}{r}130.580 \\
28.000\end{array}$ & $\begin{array}{r}127.441 \\
28.281\end{array}$ & $\begin{array}{r}-2.404 \\
1.004\end{array}$ & $\begin{array}{l}0.310 \\
1.210\end{array}$ \\
\hline 349.980 & 1.38 & 0.506 & 28.970 & 29.120 & 0.516 & 0.620 \\
\hline 349.990 & 2.07 & 0.789 & 30.000 & 29.809 & -0.637 & 0.530 \\
\hline 350.000 & 2.76 & 1.094 & 30.880 & 30.632 & -0.803 & 0.520 \\
\hline 350.010 & 3.45 & 1.429 & 31.610 & 31.638 & 0.090 & 0.540 \\
\hline 350.020 & 3.79 & 1.608 & 32.140 & 32.220 & 0.250 & 0.540 \\
\hline 350.050 & 4.14 & 1.797 & 32.820 & 32.869 & 0.151 & 0.530 \\
\hline 350.220 & 4.13 & 1.793 & 32.870 & 32.880 & 0.029 & 0.530 \\
\hline 350.140 & 4.48 & 1.995 & 33.660 & 33.593 & -0.199 & 0.520 \\
\hline 350.100 & 4.83 & 2.205 & 34.390 & 34.384 & -0.016 & 0.520 \\
\hline 350.090 & 5.17 & 2.433 & 35.370 & 35.289 & -0.228 & 0.510 \\
\hline 350.090 & 5.43 & 2.606 & 36.300 & 36.005 & -0.813 & 0.500 \\
\hline 350.080 & 5.42 & 2.603 & 35.800 & 35.993 & 0.538 & 0.520 \\
\hline 394.170 & 4.92 & 1.777 & 39.640 & 39.146 & -1.247 & 0.520 \\
\hline 395.900 & 4.98 & 1.789 & 40.580 & 39.445 & -2.796 & 0.490 \\
\hline 396.200 & 4.94 & 1.769 & 40.810 & 39.434 & -3.372 & 0.470 \\
\hline 396.420 & 5.03 & 1.804 & 40.790 & 39.573 & -2.984 & 0.480 \\
\hline 396.730 & 10.04 & 4.318 & 48.560 & 48.794 & 0.482 & 0.500 \\
\hline 397.750 & 14.98 & 6.949 & 59.830 & 60.056 & 0.378 & 0.460 \\
\hline 397.540 & 19.99 & 8.844 & 69.110 & 69.189 & 0.114 & 0.430 \\
\hline 398.330 & 29.99 & 10.890 & 80.670 & 82.359 & 2.093 & 0.400 \\
\hline 397.980 & 33.90 & 11.431 & 84.470 & 86.649 & 2.580 & 0.390 \\
\hline 397.750 & 33.84 & 11.432 & 84.820 & 86.619 & 2.121 & 0.390 \\
\hline 397.660 & 35.04 & 11.579 & 86.250 & 87.867 & 1.874 & 0.390 \\
\hline 397.670 & 45.06 & 12.557 & 95.350 & 97.256 & 1.999 & 0.370 \\
\hline 397.700 & 45.14 & 12.562 & 95.680 & 97.317 & 1.711 & 0.370 \\
\hline 397.750 & 50.04 & 12.937 & 99.810 & 101.457 & 1.650 & 0.360 \\
\hline 397.850 & 59.95 & 13.572 & 107.470 & 109.233 & 1.640 & 0.350 \\
\hline 397.870 & 70.01 & 14.100 & 114.810 & 116.531 & 1.499 & 0.340 \\
\hline 397.960 & 69.81 & 14.088 & 113.770 & 116.370 & 2.285 & 0.340 \\
\hline 397.960 & 40.21 & 12.116 & 91.500 & 92.842 & 1.467 & 0.380 \\
\hline 397.970 & 25.08 & 10.060 & 76.380 & 76.455 & 0.098 & 0.410 \\
\hline 397.980 & 20.08 & 8.849 & 68.860 & 69.273 & 0.600 & 0.430 \\
\hline 397.800 & 15.02 & 6.963 & 60.250 & 60.125 & -0.207 & 0.450 \\
\hline 397.710 & 10.01 & 4.273 & 48.710 & 48.739 & 0.059 & 0.500 \\
\hline
\end{tabular}


Table 15. THERMAL CONDUCTIVITY AT ELEVATED PRESSURES (continued)

Data from Prasad et al. [86] (continued)

\begin{tabular}{|c|c|c|c|c|c|c|}
\hline $\begin{array}{l}\mathrm{T} \\
\mathrm{K}\end{array}$ & $\begin{array}{c}\mathrm{P} \\
\mathrm{MPa}\end{array}$ & $\begin{array}{c}\rho \\
\mathrm{mol}\end{array} \mathrm{dm}^{-3}$ & 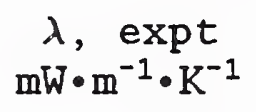 & $\begin{array}{c}\lambda, \quad \text { calc } \\
\mathrm{mW} \cdot \mathrm{m}^{-1} \cdot \mathrm{K}^{-1}\end{array}$ & $\begin{array}{l}\text { Dev } \\
8\end{array}$ & wt \\
\hline 398.210 & 4.73 & 1.668 & 40.940 & 39.449 & -3.643 & 0.470 \\
\hline 398.160 & 4.83 & 1.707 & 40.030 & 39.555 & -1.188 & 0.520 \\
\hline 398.350 & 4.49 & 1.569 & 39.730 & 39.189 & -1.362 & 0.520 \\
\hline 398.350 & 4.18 & 1.443 & 39.300 & 38.840 & -1.172 & 0.530 \\
\hline 398.360 & 4.14 & 1.429 & 39.180 & 38.804 & -0.959 & 0.530 \\
\hline 398.830 & 0.38 & 0.115 & 35.780 & 35.954 & 0.486 & 1.960 \\
\hline 398.880 & 0.69 & 0.212 & 36.190 & 36.132 & -0.159 & 0.570 \\
\hline 398.930 & 1.38 & 0.433 & 36.740 & 36.558 & -0.496 & 0.540 \\
\hline 398.990 & 2.07 & 0.663 & 37.460 & 37.042 & -1.115 & 0.510 \\
\hline 399.030 & 2.76 & 0.905 & 37.910 & 37.589 & -0.848 & 0.540 \\
\hline 399.070 & 3.45 & 1.158 & 38.910 & 38.207 & -1.808 & 0.500 \\
\hline 399.090 & 3.44 & 1.157 & 39.120 & 38.208 & -2.332 & 0.490 \\
\hline 398.080 & 5.15 & 1.842 & 40.320 & 39.941 & -0.940 & 0.530 \\
\hline 397.920 & 5.52 & 2.002 & 41.010 & 40.408 & -1.469 & 0.510 \\
\hline 397.890 & 6.21 & 2.311 & 41.950 & 41.393 & -1.327 & 0.510 \\
\hline 397.870 & 6.90 & 2.634 & 43.050 & 42.488 & -1.306 & 0.510 \\
\hline 500.660 & 4.10 & 1.034 & 55.370 & 55.642 & 0.492 & 0.690 \\
\hline 500.920 & 30.03 & 7.676 & 77.820 & 78.904 & 1.393 & 0.470 \\
\hline 500.300 & 35.15 & 8.546 & 81.790 & 83.291 & 1.836 & 0.450 \\
\hline 499.820 & 40.11 & 9.253 & 87.130 & 87.286 & 0.179 & 0.430 \\
\hline 498.590 & 60.02 & 11.241 & 100.030 & 101.447 & 1.416 & 0.400 \\
\hline 497.380 & 15.70 & 4.362 & 65.500 & 64.553 & -1.446 & 0.500 \\
\hline 497.080 & 10.03 & 2.711 & 59.740 & 59.135 & -1.013 & 0.540 \\
\hline 494.960 & 4.97 & 1.285 & 56.300 & 55.109 & -2.116 & 0.500 \\
\hline 495.720 & 45.01 & 9.932 & 90.730 & 90.941 & 0.233 & 0.420 \\
\hline 495.740 & 50.00 & 10.446 & 94.670 & 94.591 & -0.084 & 0.410 \\
\hline 495.790 & 49.96 & 10.441 & 94.710 & 94.561 & -0.157 & 0.410 \\
\hline 501.150 & 3.74 & 0.939 & 55.170 & 55.545 & 0.680 & 0.740 \\
\hline 500.400 & 65.13 & 11.574 & 103.840 & 104.731 & 0.858 & 0.390 \\
\hline 500.260 & 65.07 & 11.573 & 104.070 & 104.697 & 0.602 & 0.390 \\
\hline 499.930 & 60.27 & 11.233 & 101.130 & 101.610 & 0.474 & 0.390 \\
\hline 499.790 & 55.09 & 10.820 & 97.410 & 98.168 & 0.778 & 0.400 \\
\hline 497.370 & 49.54 & 10.365 & 94.460 & 94.278 & -0.193 & 0.410 \\
\hline 497.100 & 45.10 & 9.911 & 90.930 & 91.033 & 0.113 & 0.420 \\
\hline 497.080 & 40.12 & 9.318 & 87.420 & 87.214 & -0.235 & 0.430 \\
\hline 497.120 & 35.20 & 8.629 & 83.420 & 83.209 & -0.253 & 0.440 \\
\hline
\end{tabular}


Table 15. THERMAL CONDUCTIVITY AT ELEVATED PRESSURES (continued)

Data from Prasad et al. [86] (continued)

\begin{tabular}{|c|c|c|c|c|c|c|}
\hline $\begin{array}{l}\mathrm{T} \\
\mathrm{K}\end{array}$ & $\begin{array}{c}\mathrm{P} \\
\mathrm{MPa}\end{array}$ & $\begin{array}{c}\rho \\
\mathrm{mol} \cdot \mathrm{dm}^{-3}\end{array}$ & $\begin{array}{c}\lambda, \operatorname{expt} \\
\mathrm{mW} \cdot \mathrm{m}^{-1} \cdot \mathrm{K}^{-1}\end{array}$ & $\begin{array}{c}\lambda, \quad \operatorname{calc} \\
\mathrm{mW} \cdot \mathrm{m}^{-1} \cdot \mathrm{K}^{-1}\end{array}$ & $\begin{array}{c}\text { Dev } \\
\frac{8}{8}\end{array}$ & $w t$ \\
\hline 497.170 & 30.15 & 7.787 & 79.080 & 78.816 & -0.334 & 0.450 \\
\hline 497.190 & 25.07 & 6.764 & 74.280 & 74.046 & -0.315 & 0.470 \\
\hline 497.420 & 20.00 & 5.539 & 69.330 & 68.974 & -0.514 & 0.490 \\
\hline 97.400 & 15.01 & 4.164 & 65.160 & 63.858 & -1.998 & 0.490 \\
\hline 497.480 & 10.22 & 2.764 & 60.210 & 59.361 & -1.411 & 0.530 \\
\hline 497.540 & 8.98 & 2.403 & 59.250 & 58.344 & -1.530 & 0.530 \\
\hline 497.590 & 7.06 & 1.854 & 57.890 & 56.915 & -1.685 & 0.520 \\
\hline 498.110 & 6.24 & 1.623 & 57.510 & 56.452 & -1.840 & 0.520 \\
\hline 498.130 & 5.04 & 1.294 & 56.320 & 55.713 & -1.077 & 0.550 \\
\hline 498.200 & 5.11 & 1.311 & 56.720 & 55.764 & -1.686 & 0.520 \\
\hline 498.220 & 5.53 & 1.425 & 57.130 & 56.019 & -1.945 & 0.510 \\
\hline 498.220 & 5.55 & 1.431 & 57.310 & 56.031 & -2.231 & 0.500 \\
\hline 495.660 & 3.88 & 0.987 & 55.710 & 54.621 & -1.956 & 0.490 \\
\hline 495.610 & 4.09 & 1.044 & 55.170 & 54.724 & -0.808 & 0.560 \\
\hline 495.580 & 4.05 & 1.036 & 55.420 & 54.702 & -1.296 & 0.530 \\
\hline 495.540 & 4.88 & 1.260 & 56.510 & 55.162 & -2.386 & 0.480 \\
\hline 495.520 & 0.34 & 0.084 & 54.090 & 53.055 & -1.913 & 0.220 \\
\hline 495.530 & 0.69 & 0.169 & 54.110 & 53.180 & -1.719 & 0.300 \\
\hline 495.540 & 1.38 & 0.340 & 54.350 & 53.443 & -1.668 & 0.400 \\
\hline 495.550 & 2.07 & 0.515 & 54.360 & 53.731 & -1.157 & 0.490 \\
\hline 495.580 & 2.76 & 0.693 & 55.080 & 54.047 & -1.875 & 0.460 \\
\hline 495.600 & 3.45 & 0.873 & 55.220 & 54.387 & -1.509 & 0.500 \\
\hline 495.640 & 3.45 & 0.873 & 55.200 & 54.39 & -1.460 & 0.510 \\
\hline 600.270 & 34.55 & 6.593 & 91.560 & 91.583 & 0.025 & 0.490 \\
\hline 600.350 & 40.24 & 7.366 & 95.140 & 95.075 & -0.068 & 0.470 \\
\hline 600.230 & 40.08 & 7.348 & 94.400 & 94.967 & 0.600 & 0.480 \\
\hline 600.140 & 45.03 & 7.948 & 97.640 & 97.910 & 0.277 & 0.460 \\
\hline 599.760 & 49.76 & 8.468 & 99.930 & 100.624 & 0.694 & 0.460 \\
\hline 599.750 & 50.02 & 8.495 & 99.700 & 100.769 & 1.072 & 0.460 \\
\hline 599.750 & 54.98 & 8.978 & 102.110 & 103.567 & 1.427 & 0.450 \\
\hline 599.790 & 59.95 & 9.416 & 105.180 & 106.307 & 1.072 & 0.440 \\
\hline 599.760 & 62.99 & 9.665 & 106.650 & 107.944 & 1.213 & 0.430 \\
\hline 599.710 & 64.99 & 9.822 & 107.600 & 109.004 & 1.305 & 0.430 \\
\hline 599.630 & 50.04 & 8.499 & 100.050 & 100.770 & 0.720 & 0.450 \\
\hline 599.580 & 35.06 & 6.676 & 92.250 & 91.814 & -0.472 & 0.480 \\
\hline 599.560 & 25.03 & 5.061 & 86.400 & 85.487 & -1.057 & 0.500 \\
\hline
\end{tabular}


Table 15. THERMAL CONDUCTIVITY AT ELEVATED PRESSURES (continued)

Data from Prasad et al. [86] (continued)

\begin{tabular}{crccccc}
\hline $\mathrm{T}$ & $\begin{array}{c}\mathrm{P} \\
\mathrm{K}\end{array}$ & $\begin{array}{c}\rho \\
\mathrm{MPa}\end{array}$ & $\mathrm{mol} \cdot \mathrm{dm}^{-3}$ & $\begin{array}{c}\lambda, \begin{array}{c}\text { expt } \\
\mathrm{mW} \cdot \mathrm{m}^{-1} \cdot \mathrm{K}^{-1}\end{array} \\
\begin{array}{c}\lambda, \text { calc } \\
\mathrm{mW} \cdot \mathrm{m}^{-1} \cdot \mathrm{K}^{-1}\end{array}\end{array}$ & $\begin{array}{c}\text { Dev } \\
\text { o }\end{array}$ & wt \\
\hline 599.520 & 15.04 & 3.128 & 80.630 & 79.434 & -1.483 & 0.530 \\
599.490 & 10.00 & 2.076 & 77.910 & 76.821 & -1.398 & 0.540 \\
599.270 & 10.02 & 2.080 & 78.060 & 76.787 & -1.631 & 0.530 \\
599.320 & 14.99 & 3.120 & 81.050 & 79.375 & -2.066 & 0.510 \\
& & & & & & \\
599.360 & 10.03 & 2.083 & 77.770 & 76.812 & -1.232 & 0.550 \\
599.450 & 3.97 & 0.811 & 75.290 & 74.371 & -1.220 & 0.510 \\
599.240 & 19.93 & 4.111 & 83.530 & 82.259 & -1.522 & 0.510 \\
597.770 & 6.81 & 1.409 & 76.670 & 75.095 & -2.055 & 0.490 \\
597.770 & 6.22 & 1.283 & 76.450 & 74.856 & -2.084 & 0.480 \\
597.770 & 5.54 & 1.140 & 76.560 & 74.595 & -2.566 & 0.450 \\
597.770 & 4.84 & 0.995 & 76.220 & 74.342 & -2.464 & 0.440 \\
597.840 & 4.13 & 0.847 & 75.800 & 74.108 & -2.233 & 0.440 \\
597.900 & 4.14 & 0.849 & 76.080 & 74.122 & -2.573 & 0.420
\end{tabular}

Comparisons based on experimental pressures:

Number of Points [86] 239

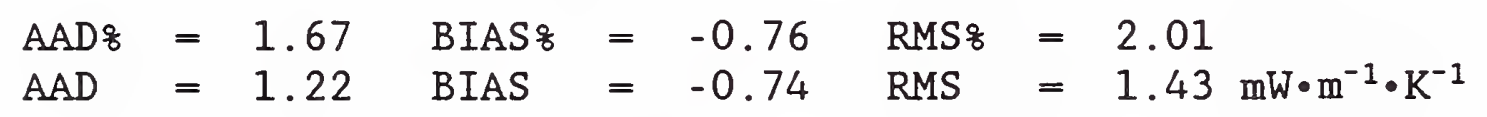

Weighted Data:

Number of Points [86] 235

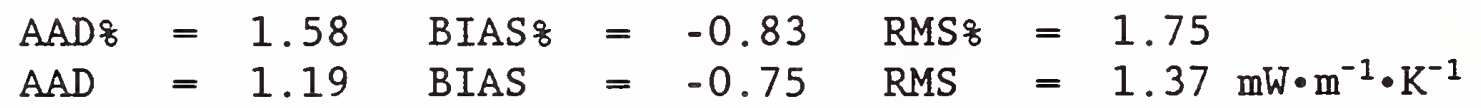


Table 15. THERMAL CONDUCTIVITY AT ELEVATED PRESSURES (continued)

Data from Prasad et al. [86] (continued)

Comparisons based on experimental densities:

Number of Points [86] 239

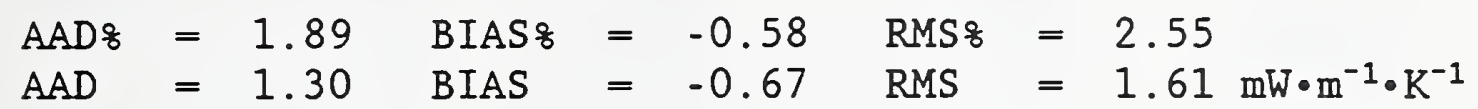

Weighted Data:

Number of Points [82] 235

$\mathrm{AAD} \%=1.78 \quad \mathrm{BIAS} \%=-0.65$ RMS $8=2.32$

$\mathrm{AAD}=1.26$ BIAS $=-0.68$ RMS $=1.54 \mathrm{~mW} \cdot \mathrm{m}^{-1} \cdot \mathrm{K}^{-1}$

Data from Roder [103]

\begin{tabular}{|c|c|c|c|c|c|c|}
\hline $\begin{array}{l}\mathrm{T} \\
\mathrm{K}\end{array}$ & $\begin{array}{c}\mathrm{P} \\
\mathrm{MPa}\end{array}$ & $\begin{array}{c}\rho \\
\mathrm{mol} \cdot \mathrm{dm}^{-3}\end{array}$ & $\begin{array}{c}\lambda, \operatorname{expt} \\
\mathrm{mW} \cdot \mathrm{m}^{-1} \cdot \mathrm{K}^{-1}\end{array}$ & $\begin{array}{c}\lambda, \operatorname{calc} \\
\mathrm{mW} \cdot \mathrm{m}^{-1} \cdot \mathrm{K}^{-1}\end{array}$ & $\begin{array}{c}\text { Dev } \\
\frac{8}{8}\end{array}$ & wt \\
\hline 112.472 & 0.72 & 20.882 & 237.280 & 237.668 & 0.164 & 0.240 \\
\hline 112.265 & 0.72 & 20.890 & 237.650 & 237.855 & 0.086 & 0.240 \\
\hline 112.125 & 0.71 & 20.895 & 238.420 & 237.981 & -0.184 & 0.240 \\
\hline 111.929 & 0.71 & 20.902 & 238.520 & 238.158 & -0.152 & 0.240 \\
\hline 112.488 & 14.44 & 21.073 & 244.360 & 244.655 & 0.121 & 0.240 \\
\hline 112.263 & 14.43 & 21.081 & 244.540 & 244.844 & 0.124 & 0.240 \\
\hline 111.945 & 14.43 & 21.092 & 244.550 & 245.111 & 0.230 & 0.240 \\
\hline 111.866 & 14.42 & 21.095 & 246.050 & 245.176 & -0.355 & 0.240 \\
\hline 112.380 & 27.53 & 21.247 & 250.660 & 251.187 & 0.210 & 0.240 \\
\hline 112.271 & 27.52 & 21.250 & 250.830 & 251.269 & 0.175 & 0.240 \\
\hline 112.016 & 27.51 & 21.258 & 251.070 & 251.468 & 0.159 & 0.240 \\
\hline 111.860 & 27.51 & 21.264 & 251.580 & 251.589 & 0.003 & 0.240 \\
\hline 112.368 & 41.11 & 21.411 & 256.640 & 257.662 & 0.398 & 0.230 \\
\hline 112.085 & 41.09 & 21.420 & 256.440 & 257.865 & 0.556 & 0.230 \\
\hline 111.867 & 41.11 & 21.427 & 256.710 & 258.033 & 0.515 & 0.230 \\
\hline 111.688 & 41.09 & 21.433 & 256.980 & 258.161 & 0.460 & 0.230 \\
\hline 112.228 & 54.61 & 21.569 & 261.860 & 263.998 & 0.817 & 0.230 \\
\hline 112.043 & 54.60 & 21.574 & 262.270 & 264.126 & 0.708 & 0.230 \\
\hline 111.958 & 54.59 & 21.577 & 262.800 & 264.180 & 0.525 & 0.230 \\
\hline 111.824 & 54.58 & 21.581 & 262.770 & 264.270 & 0.571 & 0.230 \\
\hline 111.681 & 69.19 & 21.740 & 268.690 & 270.900 & 0.823 & 0.230 \\
\hline 112.147 & 69.19 & 21.727 & 268.530 & 270.598 & 0.770 & 0.230 \\
\hline 112.291 & 69.18 & 21.722 & 268.080 & 270.501 & 0.903 & 0.230 \\
\hline
\end{tabular}


Table 15. THERMAL CONDUCTIVITY AT ELEVATED PRESSURES (continued)

Data from Roder [103] (continued)

\begin{tabular}{|c|c|c|c|c|c|c|}
\hline $\begin{array}{l}\mathrm{T} \\
\mathrm{K}\end{array}$ & $\begin{array}{c}\mathrm{P} \\
\mathrm{MPa}\end{array}$ & $\underset{\mathrm{mol}}{\stackrel{\rho}{\mathrm{dm}^{-3}}}$ & $\begin{array}{c}\lambda, \underset{\mathrm{mWpt}}{\exp } \mathrm{m}^{-1} \cdot \mathrm{K}^{-1}\end{array}$ & $\begin{array}{c}\lambda ; \text { calc } \\
\mathrm{mW} \cdot \mathrm{m}^{-1} \cdot \mathrm{K}^{-1}\end{array}$ & $\begin{array}{c}\text { Dev } \\
8\end{array}$ & wt \\
\hline 111.984 & 69.18 & 21.731 & 268.730 & 270.702 & 0.734 & 0.230 \\
\hline 134.971 & 0.44 & 20.048 & 217.240 & 216.123 & -0.514 & 0.250 \\
\hline 134.640 & 0.44 & 20.060 & 217.700 & 216.446 & -0.576 & 0.250 \\
\hline 134.361 & 0.44 & 20.070 & 217.620 & 216.717 & -0.415 & 0.250 \\
\hline 134.071 & 0.44 & 20.081 & 218.170 & 217.000 & -0.536 & 0.250 \\
\hline 134.854 & 13.90 & 20.287 & 225.580 & 224.378 & -0.533 & 0.240 \\
\hline 134.565 & 13.90 & 20.297 & 225.910 & 224.643 & -0.561 & 0.240 \\
\hline 134.184 & 13.89 & 20.310 & 225.790 & 224.992 & -0.353 & 0.240 \\
\hline 134.078 & 13.89 & 20.314 & 226.670 & 225.086 & -0.699 & 0.240 \\
\hline 134.744 & 27.79 & 20.510 & 233.210 & 232.479 & -0.314 & 0.240 \\
\hline 134.456 & 27.78 & 20.520 & 233.570 & 232.726 & -0.361 & 0.240 \\
\hline 134.246 & 27.78 & 20.527 & 233.950 & 232.906 & -0.446 & 0.240 \\
\hline 134.088 & 27.77 & 20.532 & 234.340 & 233.041 & -0.554 & 0.240 \\
\hline 134.918 & 40.17 & 20.685 & 239.970 & 239.179 & -0.329 & 0.240 \\
\hline 134.690 & 40.16 & 20.692 & 240.010 & 239.363 & -0.270 & 0.240 \\
\hline 134.471 & 40.15 & 20.699 & 240.290 & 239.542 & -0.311 & 0.240 \\
\hline 134.226 & 40.15 & 20.707 & 240.660 & 239.743 & -0.381 & 0.240 \\
\hline 134.401 & 53.22 & 20.878 & 247.020 & 246.569 & -0.182 & 0.240 \\
\hline 134.180 & 53.22 & 20.885 & 247.090 & 246.743 & -0.140 & 0.240 \\
\hline 134.994 & 53.21 & 20.860 & 246.470 & 246.091 & -0.154 & 0.240 \\
\hline 134.748 & 53.20 & 20.868 & 246.770 & 246.283 & -0.197 & 0.240 \\
\hline 134.363 & 66.73 & 21.050 & 253.550 & 253.573 & 0.009 & 0.230 \\
\hline 135.047 & 66.73 & 21.030 & 253.890 & 253.053 & -0.330 & 0.230 \\
\hline 134.838 & 66.73 & 21.036 & 253.060 & 253.211 & 0.060 & 0.230 \\
\hline 134.711 & 66.73 & 21.040 & 253.380 & 253.307 & -0.029 & 0.230 \\
\hline 156.011 & 0.95 & 19.260 & 197.210 & 195.701 & -0.765 & 0.260 \\
\hline 155.629 & 0.95 & 19.275 & 197.660 & 196.078 & -0.800 & 0.250 \\
\hline 155.403 & 0.95 & 19.283 & 197.830 & 196.301 & -0.773 & 0.250 \\
\hline 155.076 & 0.95 & 19.296 & 198.150 & 196.623 & -0.771 & 0.250 \\
\hline 156.240 & 13.36 & 19.521 & 205.470 & 204.084 & -0.675 & 0.250 \\
\hline 155.886 & 13.37 & 19.534 & 205.890 & 204.419 & -0.715 & 0.250 \\
\hline 155.578 & 13.37 & 19.544 & 206.010 & 204.705 & -0.633 & 0.250 \\
\hline 155.271 & 13.37 & 19.556 & 206.550 & 204.997 & -0.752 & 0.250 \\
\hline 156.006 & 27.36 & 19.799 & 214.550 & 213.422 & -0.526 & 0.250 \\
\hline 155.801 & 27.34 & 19.805 & 214.830 & 213.588 & -0.578 & 0.250 \\
\hline
\end{tabular}


Table 15. THERMAL CONDUCTIVITY AT ELEVATED PRESSURES (continued)

Data from Roder [103] (continued)

\begin{tabular}{|c|c|c|c|c|c|c|}
\hline $\begin{array}{l}\mathrm{T} \\
\mathrm{K}\end{array}$ & $\begin{array}{c}\mathrm{P} \\
\mathrm{MPa}\end{array}$ & $\stackrel{\rho}{\mathrm{mol} \cdot \mathrm{dm}^{-3}}$ & 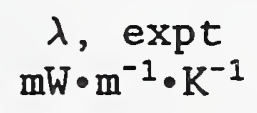 & $\begin{array}{c}\lambda, \quad \text { calc } \\
\mathrm{mW} \cdot \mathrm{m}^{-1} \cdot \mathrm{K}^{-1}\end{array}$ & $\begin{array}{c}\text { Dev } \\
8\end{array}$ & wt \\
\hline 155.382 & 27.36 & 19.820 & 215.340 & 213.974 & -0.634 & 0.250 \\
\hline 155.255 & 27.34 & 19.824 & 215.460 & 214.079 & -0.641 & 0.250 \\
\hline 155.870 & 40.84 & 20.037 & 222.500 & 221.863 & -0.286 & 0.240 \\
\hline 155.644 & 40.84 & 20.044 & 223.090 & 222.058 & -0.463 & 0.240 \\
\hline 155.268 & 40.85 & 20.056 & 223.150 & 222.382 & -0.344 & 0.240 \\
\hline 155.129 & 40.87 & 20.061 & 223.450 & 222.508 & -0.422 & 0.240 \\
\hline 156.283 & 53.81 & 20.231 & 229.870 & 229.193 & -0.294 & 0.240 \\
\hline 155.908 & 53.81 & 20.242 & 230.180 & 229.498 & -0.296 & 0.240 \\
\hline 155.688 & 53.81 & 20.248 & 230.560 & 229.677 & -0.383 & 0.240 \\
\hline 155.405 & 53.81 & 20.257 & 230.660 & 229.905 & -0.327 & 0.240 \\
\hline 156.172 & 67.36 & 20.433 & 237.580 & 236.993 & -0.247 & 0.240 \\
\hline 155.796 & 67.36 & 20.443 & 237.700 & 237.288 & -0.173 & 0.240 \\
\hline 155.602 & 67.35 & 20.449 & 238.140 & 237.438 & -0.295 & 0.240 \\
\hline 155.390 & 67.35 & 20.455 & 238.020 & 237.602 & -0.176 & 0.240 \\
\hline 175.465 & 1.02 & 18.493 & 177.140 & 176.685 & -0.257 & 0.270 \\
\hline 175.148 & 1.02 & 18.506 & 177.540 & 176.991 & -0.309 & 0.260 \\
\hline 174.727 & 1.01 & 18.523 & 178.160 & 177.396 & -0.429 & 0.260 \\
\hline 174.391 & 1.01 & 18.537 & 178.520 & 177.722 & -0.447 & 0.260 \\
\hline 175.765 & 13.93 & 18.827 & 187.210 & 186.387 & -0.440 & 0.260 \\
\hline 175.298 & 13.93 & 18.844 & 187.330 & 186.812 & -0.277 & 0.260 \\
\hline 174.912 & 13.92 & 18.858 & 187.700 & 187.162 & -0.287 & 0.260 \\
\hline 174.645 & 13.92 & 18.868 & 188.130 & 187.402 & -0.387 & 0.260 \\
\hline 175.447 & 27.80 & 19.158 & 197.030 & 196.610 & -0.213 & 0.260 \\
\hline 175.218 & 27.79 & 19.165 & 197.260 & 196.808 & -0.229 & 0.260 \\
\hline 174.668 & 27.80 & 19.184 & 197.830 & 197.288 & -0.274 & 0.260 \\
\hline 174.450 & 27.80 & 19.191 & 198.220 & 197.477 & -0.375 & 0.250 \\
\hline 175.339 & 41.32 & 19.435 & 205.910 & 205.777 & -0.064 & 0.250 \\
\hline 174.999 & 41.32 & 19.446 & 206.350 & 206.060 & -0.141 & 0.250 \\
\hline 174.650 & 41.32 & 19.457 & 206.810 & 206.350 & -0.222 & 0.250 \\
\hline 174.355 & 41.31 & 19.466 & 207.170 & 206.591 & -0.279 & 0.250 \\
\hline 175.581 & 53.96 & 19.659 & 213.720 & 213.644 & -0.036 & 0.250 \\
\hline 175.267 & 53.96 & 19.668 & 213.950 & 213.892 & -0.027 & 0.250 \\
\hline 175.004 & 53.95 & 19.676 & 214.260 & 214.102 & -0.074 & 0.250 \\
\hline 174.706 & 53.95 & 19.685 & 214.300 & 214.338 & 0.018 & 0.250 \\
\hline 175.540 & 66.41 & 19.869 & 221.040 & 221.285 & 0.111 & 0.250 \\
\hline 175.276 & 66.40 & 19.877 & 221.360 & 221.486 & 0.057 & 0.250 \\
\hline
\end{tabular}


Table 15. THERMAL CONDUCTIVITY AT ELEVATED PRESSURES (continued)

Data from Roder [103] (continued)

\begin{tabular}{|c|c|c|c|c|c|c|}
\hline $\begin{array}{l}\mathrm{T} \\
\mathrm{K}\end{array}$ & $\begin{array}{c}\mathrm{P} \\
\mathrm{MPa}\end{array}$ & $\begin{array}{c}\rho \\
\mathrm{mol} \cdot \mathrm{dm}^{-3}\end{array}$ & 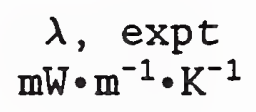 & $\begin{array}{c}\lambda, \operatorname{calc} \\
\mathrm{mW} \cdot \mathrm{m}^{-1} \cdot \mathrm{K}^{-1}\end{array}$ & $\begin{array}{c}\text { Dev } \\
8\end{array}$ & wt \\
\hline 174.808 & 66.38 & 19.889 & 221.670 & 221.838 & 0.076 & 0.240 \\
\hline 174.635 & 66.38 & 19.894 & 222.020 & 221.971 & -0.022 & 0.240 \\
\hline 195.294 & 0.95 & 17.661 & 157.720 & 157.669 & -0.032 & 0.280 \\
\hline 194.868 & 0.95 & 17.679 & 158.030 & 158.069 & 0.025 & 0.280 \\
\hline 194.550 & 0.95 & 17.693 & 158.490 & 158.368 & -0.077 & 0.280 \\
\hline 194.219 & 0.95 & 17.707 & 158.920 & 158.678 & -0.152 & 0.280 \\
\hline 195.460 & 14.52 & 18.110 & 169.380 & 169.280 & -0.059 & 0.270 \\
\hline 195.134 & 14.52 & 18.122 & 170.050 & 169.564 & -0.286 & 0.270 \\
\hline 194.717 & 14.52 & 18.138 & 170.510 & 169.929 & -0.341 & 0.270 \\
\hline 194.349 & 14.52 & 18.152 & 170.750 & 170.249 & -0.293 & 0.270 \\
\hline 195.334 & 27.38 & 18.472 & 179.820 & 179.442 & -0.210 & 0.260 \\
\hline 194.895 & 27.37 & 18.487 & 180.350 & 179.800 & -0.305 & 0.260 \\
\hline 194.640 & 27.37 & 18.496 & 180.520 & 180.008 & -0.283 & 0.260 \\
\hline 194.243 & 27.36 & 18.509 & 180.980 & 180.334 & -0.357 & 0.260 \\
\hline 195.664 & 39.33 & 18.751 & 187.980 & 187.850 & -0.069 & 0.260 \\
\hline 195.269 & 39.33 & 18.763 & 188.600 & 188.161 & -0.233 & 0.260 \\
\hline 194.871 & 39.33 & 18.776 & 188.880 & 188.479 & -0.212 & 0.260 \\
\hline 194.474 & 39.32 & 18.788 & 189.340 & 188.791 & -0.290 & 0.260 \\
\hline 195.893 & 53.39 & 19.045 & 197.320 & 197.312 & -0.004 & 0.260 \\
\hline 195.491 & 53.39 & 19.057 & 197.620 & 197.621 & 0.000 & 0.260 \\
\hline 195.045 & 53.39 & 19.071 & 198.090 & 197.962 & -0.064 & 0.260 \\
\hline 194.639 & 53.38 & 19.083 & 198.420 & 198.270 & -0.076 & 0.260 \\
\hline 195.695 & 67.21 & 19.316 & 205.940 & 206.433 & 0.239 & 0.250 \\
\hline 195.277 & 67.21 & 19.328 & 206.100 & 206.742 & 0.312 & 0.250 \\
\hline 194.985 & 67.21 & 19.336 & 206.460 & 206.961 & 0.243 & 0.250 \\
\hline 194.585 & 67.22 & 19.347 & 206.920 & 207.262 & 0.165 & 0.250 \\
\hline 195.273 & 0.93 & 17.661 & 157.020 & 157.667 & 0.412 & 0.280 \\
\hline 194.811 & 0.93 & 17.681 & 157.550 & 158.102 & 0.350 & 0.280 \\
\hline 194.391 & 0.93 & 17.699 & 157.940 & 158.497 & 0.353 & 0.280 \\
\hline 194.165 & 0.93 & 17.709 & 158.320 & 158.710 & 0.246 & 0.280 \\
\hline 195.518 & 11.51 & 18.015 & 166.330 & 166.742 & 0.248 & 0.270 \\
\hline 195.096 & 11.51 & 18.031 & 166.770 & 167.114 & 0.207 & 0.270 \\
\hline 194.602 & 11.50 & 18.050 & 167.400 & 167.543 & 0.086 & 0.270 \\
\hline 194.370 & 11.50 & 18.059 & 167.410 & 167.745 & 0.200 & 0.270 \\
\hline 195.405 & 20.84 & 18.295 & 174.220 & 174.376 & 0.089 & 0.270 \\
\hline 195.011 & 20.84 & 18.309 & 174.630 & 174.708 & 0.045 & 0.270 \\
\hline
\end{tabular}


Table 15. THERMAL CONDUCTIVITY AT ELEVATED PRESSURES (continued)

Data from Roder [103] (continued)

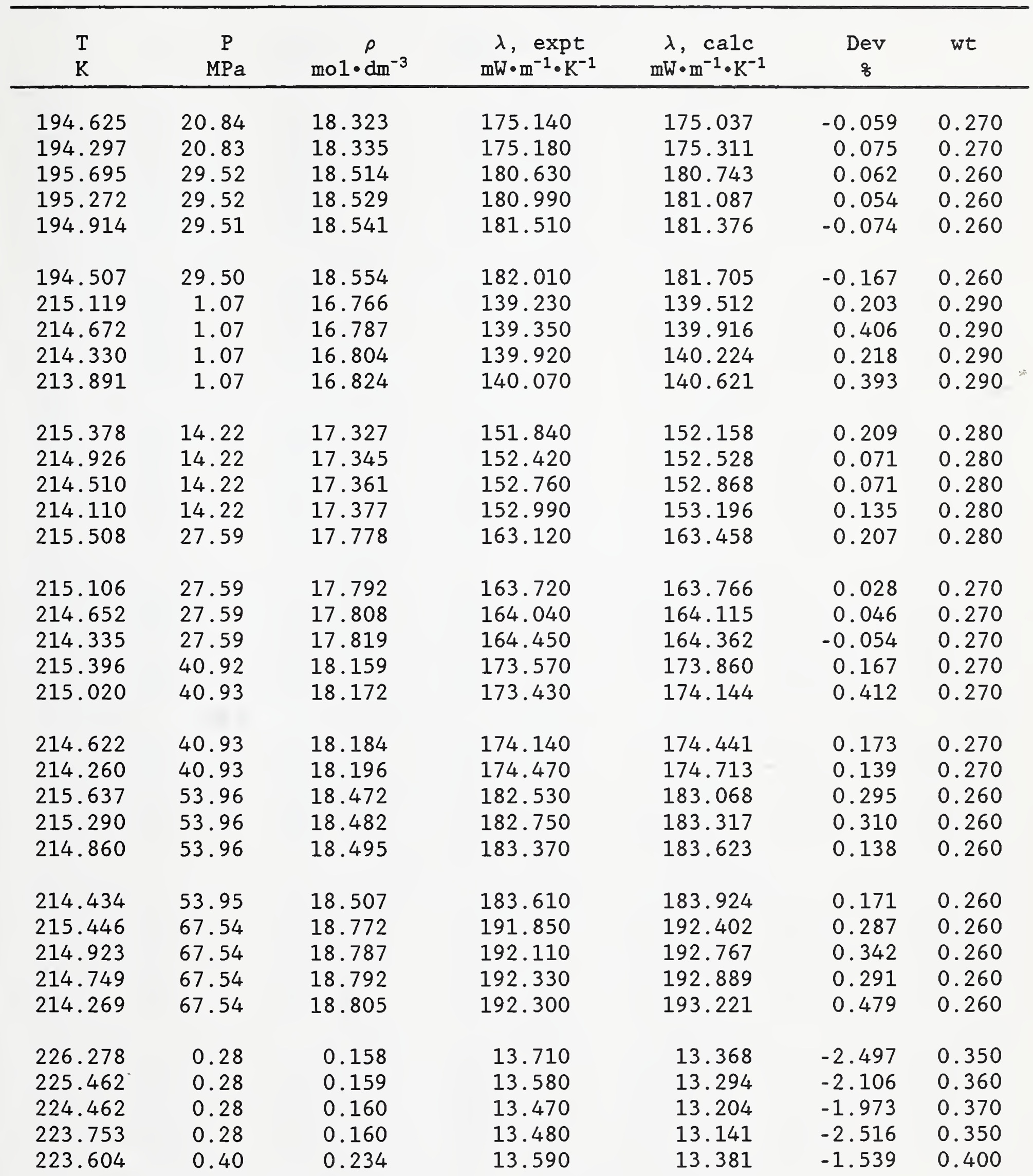


Table 15. THERMAL CONDUCTIVITY AT ELEVATED PRESSURES (continued)

Data from Roder [103] (continued)

\begin{tabular}{|c|c|c|c|c|c|c|}
\hline $\begin{array}{l}\mathrm{T} \\
\mathrm{K}\end{array}$ & $\begin{array}{c}\mathrm{P} \\
\mathrm{MPa}\end{array}$ & $\stackrel{\rho}{\mathrm{mol} \cdot \mathrm{dm}^{-3}}$ & $\begin{array}{c}\lambda, \operatorname{expt} \\
\mathrm{mW} \cdot \mathrm{m}^{-1} \cdot \mathrm{K}^{-1}\end{array}$ & $\begin{array}{c}\lambda, \text { calc } \\
\mathrm{mW} \cdot \mathrm{m}^{-1} \cdot \mathrm{K}^{-1}\end{array}$ & $\begin{array}{c}\text { Dev } \\
\%\end{array}$ & wt \\
\hline 226.046 & 0.41 & 0.234 & 13.880 & 13.602 & -2.000 & 0.390 \\
\hline 225.279 & 0.41 & 0.237 & 13.800 & 13.543 & -1.865 & 0.390 \\
\hline 224.256 & 0.41 & 0.239 & 13.730 & 13.459 & -1.973 & 0.390 \\
\hline 232.979 & 0.27 & 0.146 & 14.110 & 13.964 & -1.038 & 0.400 \\
\hline 235.295 & 0.27 & 0.144 & 14.460 & 14.183 & -1.916 & 0.360 \\
\hline 234.388 & 0.27 & 0.145 & 14.330 & 14.098 & -1.616 & 0.380 \\
\hline 233.661 & 0.27 & 0.146 & 14.250 & 14.030 & -1.546 & 0.380 \\
\hline 235.876 & 0.41 & 0.223 & 14.740 & 14.490 & -1.693 & 0.400 \\
\hline 235.098 & 0.41 & 0.226 & 14.630 & 14.424 & -1.405 & 0.410 \\
\hline 234.239 & 0.42 & 0.229 & 14.510 & 14.352 & -1.092 & 0.420 \\
\hline 233.532 & 0.42 & 0.231 & 14.460 & 14.291 & -1.169 & 0.420 \\
\hline 235.696 & 0.60 & 0.342 & 15.060 & 14.874 & -1.238 & 0.430 \\
\hline 234.802 & 0.61 & 0.347 & 14.960 & 14.806 & -1.032 & 0.430 \\
\hline 234.098 & 0.61 & 0.350 & 14.890 & 14.752 & -0.924 & 0.440 \\
\hline 233.468 & 0.61 & 0.354 & 14.810 & 14.708 & -0.686 & 0.440 \\
\hline 235.623 & 1.74 & 15.769 & 121.040 & 122.177 & 0.940 & 0.310 \\
\hline 235.120 & 1.74 & 15.796 & 121.560 & 122.612 & 0.866 & 0.310 \\
\hline 234.688 & 1.74 & 15.819 & 121.800 & 122.986 & 0.974 & 0.310 \\
\hline 235.837 & 14.81 & 16.517 & 135.840 & 136.557 & 0.528 & 0.300 \\
\hline 235.390 & 14.81 & 16.535 & 136.100 & 136.890 & 0.581 & 0.300 \\
\hline 234.896 & 14.81 & 16.556 & 136.450 & 137.265 & 0.597 & 0.290 \\
\hline 234.395 & 14.79 & 16.576 & 136.310 & 137.634 & 0.972 & 0.300 \\
\hline 236.015 & 29.75 & 17.127 & 149.320 & 150.123 & 0.538 & 0.290 \\
\hline 235.493 & 29.75 & 17.145 & 149.830 & 150.489 & 0.440 & 0.280 \\
\hline 235.039 & 29.74 & 17.161 & 149.970 & 150.806 & 0.558 & 0.280 \\
\hline 234.575 & 29.74 & 17.177 & 150.370 & 151.131 & 0.506 & 0.280 \\
\hline 235.715 & 46.69 & 17.678 & 163.100 & 163.897 & 0.488 & 0.280 \\
\hline 235.230 & 46.68 & 17.692 & 163.140 & 164.218 & 0.660 & 0.280 \\
\hline 234.817 & 46.68 & 17.705 & 163.440 & 164.493 & 0.644 & 0.280 \\
\hline 234.553 & 46.67 & 17.713 & 163.340 & 164.666 & 0.812 & 0.280 \\
\hline 235.860 & 67.10 & 18.197 & 177.020 & 178.478 & 0.824 & 0.270 \\
\hline 235.408 & 67.09 & 18.209 & 177.300 & 178.765 & 0.826 & 0.270 \\
\hline 235.000 & 67.10 & 18.221 & 177.760 & 179.029 & 0.714 & 0.270 \\
\hline 234.578 & 67.10 & 18.233 & 178.150 & 179.302 & 0.646 & 0.270 \\
\hline 246.118 & 0.29 & 0.150 & 15.480 & 15.281 & -1.289 & 0.400 \\
\hline 245.283 & 0.29 & 0.150 & 15.370 & 15.197 & -1.126 & 0.400 \\
\hline
\end{tabular}


Table 15. THERMAL CONDUCTIVITY AT ELEVATED PRESSURES (continued)

Data from Roder [103] (continued)

\begin{tabular}{|c|c|c|c|c|c|c|}
\hline $\begin{array}{l}\mathrm{T} \\
\mathrm{K}\end{array}$ & $\begin{array}{c}\mathrm{P} \\
\mathrm{MPa}\end{array}$ & $\stackrel{\rho}{\mathrm{mol} \cdot \mathrm{dm}^{-3}}$ & $\begin{array}{c}\lambda, \operatorname{expt} \\
\mathrm{mW} \cdot \mathrm{m}^{-1} \cdot \mathrm{K}^{-1}\end{array}$ & $\begin{array}{c}\lambda, \quad \operatorname{calc} \\
\mathrm{mW} \cdot \mathrm{m}^{-1} \cdot \mathrm{K}^{-1}\end{array}$ & $\begin{array}{l}\text { Dev } \\
\frac{8}{8}\end{array}$ & wt \\
\hline 244.378 & 0.29 & 0.151 & 15.250 & 15.107 & -0.939 & 0.410 \\
\hline 243.664 & 0.30 & 0.152 & 15.250 & 15.038 & -1.393 & 0.390 \\
\hline 245.875 & 0.48 & 0.253 & 15.780 & 15.568 & -1.344 & 0.420 \\
\hline 245.065 & 0.48 & 0.254 & 15.530 & 15.490 & -0.259 & 0.470 \\
\hline 244.183 & 0.48 & 0.255 & 15.580 & 15.405 & -1.121 & 0.430 \\
\hline 243.466 & 0.48 & 0.256 & 15.410 & 15.337 & -0.474 & 0.450 \\
\hline 245.735 & 0.63 & 0.342 & 15.900 & 15.839 & -0.385 & 0.460 \\
\hline 244.967 & 0.64 & 0.345 & 15.820 & 15.774 & -0.292 & 0.470 \\
\hline 244.163 & 0.64 & 0.350 & 15.760 & 15.710 & -0.316 & 0.460 \\
\hline 243.571 & 0.65 & 0.353 & 15.690 & 15.665 & -0.162 & 0.470 \\
\hline 253.199 & 0.31 & 0.153 & 16.100 & 16.025 & -0.466 & 0.450 \\
\hline 255.368 & 0.31 & 0.151 & 16.300 & 16.251 & -0.301 & 0.460 \\
\hline 254.535 & 0.31 & 0.152 & 16.290 & 16.164 & -0.774 & 0.430 \\
\hline 253.732 & 0.31 & 0.152 & 16.140 & 16.080 & -0.370 & 0.460 \\
\hline 255.761 & 0.71 & 0.367 & 17.010 & 16.934 & -0.449 & 0.470 \\
\hline 254.898 & 0.71 & 0.368 & 16.930 & 16.850 & -0.474 & 0.470 \\
\hline 254.158 & 0.71 & 0.370 & 16.840 & 16.778 & -0.366 & 0.470 \\
\hline 253.565 & 0.71 & 0.371 & 16.720 & 16.721 & 0.008 & 0.480 \\
\hline 253.366 & 1.08 & 0.607 & 17.500 & 17.517 & 0.096 & 0.480 \\
\hline 255.373 & 1.08 & 0.601 & 17.690 & 17.688 & -0.013 & 0.480 \\
\hline 254.680 & 1.09 & 0.605 & 17.650 & 17.637 & -0.071 & 0.480 \\
\hline 253.937 & 1.09 & 0.610 & 17.570 & 17.584 & 0.079 & 0.480 \\
\hline 254.449 & 2.68 & 14.756 & 106.450 & 107.504 & 0.991 & 0.320 \\
\hline 253.940 & 2.67 & 14.787 & 106.960 & 107.918 & 0.896 & 0.320 \\
\hline 253.325 & 2.66 & 14.825 & 107.290 & 108.421 & 1.054 & 0.320 \\
\hline 252.902 & 2.65 & 14.850 & 107.310 & 108.760 & 1.351 & 0.320 \\
\hline 254.110 & 12.53 & 15.581 & 119.720 & 120.672 & 0.795 & 0.310 \\
\hline 253.594 & 12.53 & 15.605 & 120.220 & 121.037 & 0.679 & 0.310 \\
\hline 253.101 & 12.52 & 15.628 & 120.810 & 121.384 & 0.475 & 0.310 \\
\hline 252.573 & 12.52 & 15.653 & 120.850 & 121.766 & 0.758 & 0.310 \\
\hline 254.403 & 20.23 & 16.019 & 127.990 & 128.731 & 0.579 & 0.300 \\
\hline 253.858 & 20.22 & 16.041 & 128.230 & 129.093 & 0.673 & 0.300 \\
\hline 253.388 & 20.23 & 16.061 & 128.610 & 129.412 & 0.623 & 0.300 \\
\hline 252.994 & 20.22 & 16.076 & 129.210 & 129.674 & 0.359 & 0.300 \\
\hline 254.159 & 33.11 & 16.616 & 140.450 & 140.933 & 0.344 & 0.290 \\
\hline 253.660 & 33.11 & 16.634 & 140.540 & 141.249 & 0.504 & 0.290 \\
\hline
\end{tabular}


Table 15. THERMAL CONDUCTIVITY AT ELEVATED PRESSURES (continued)

Data from Roder [103] (continued)

\begin{tabular}{|c|c|c|c|c|c|c|}
\hline $\begin{array}{l}\mathrm{T} \\
\mathrm{K}\end{array}$ & $\begin{array}{c}\mathrm{P} \\
\mathrm{MPa}\end{array}$ & $\stackrel{\rho}{\mathrm{mol}} \cdot \mathrm{dm}^{-3}$ & $\begin{array}{c}\lambda, \underset{\mathrm{mW}}{\operatorname{expt}} \mathrm{m}^{-1} \cdot \mathrm{K}^{-1}\end{array}$ & $\begin{array}{c}\lambda ; \text { calc } \\
\mathrm{mW} \cdot \mathrm{m}^{-1} \cdot \mathrm{K}^{-1}\end{array}$ & $\begin{array}{l}\text { Dev } \\
8\end{array}$ & wt \\
\hline 253.187 & 33.11 & 16.650 & 140.810 & 141.549 & 0.525 & 0.290 \\
\hline 253.031 & 33.12 & 16.656 & 141.070 & 141.652 & 0.413 & 0.290 \\
\hline 254.116 & 48.19 & 17.154 & 152.680 & 153.354 & 0.442 & 0.280 \\
\hline 253.881 & 48.19 & 17.162 & 153.050 & 153.498 & 0.293 & 0.280 \\
\hline 253.385 & 48.19 & 17.177 & 153.260 & 153.799 & 0.352 & 0.280 \\
\hline 253.005 & 48.19 & 17.189 & 153.350 & 154.025 & 0.440 & 0.280 \\
\hline 254.209 & 67.25 & 17.696 & 166.120 & 167.367 & 0.750 & 0.280 \\
\hline 253.703 & 67.25 & 17.710 & 166.460 & 167.662 & 0.722 & 0.270 \\
\hline 253.193 & 67.25 & 17.724 & 166.700 & 167.961 & 0.756 & 0.270 \\
\hline 252.879 & 67.25 & 17.733 & 166.920 & 168.145 & 0.734 & 0.270 \\
\hline 265.102 & 0.25 & 0.116 & 17.310 & 17.216 & -0.542 & 0.440 \\
\hline 264.337 & 0.25 & 0.117 & 17.220 & 17.132 & -0.511 & 0.440 \\
\hline 263.542 & 0.25 & 0.117 & 17.100 & 17.045 & -0.323 & 0.460 \\
\hline 262.977 & 0.25 & 0.117 & 17.060 & 16.983 & -0.451 & 0.450 \\
\hline 265.752 & 0.48 & 0.231 & 17.570 & 17.599 & 0.167 & 0.510 \\
\hline 264.908 & 0.48 & 0.231 & 17.590 & 17.509 & -0.462 & 0.470 \\
\hline 264.097 & 0.48 & 0.232 & 17.460 & 17.422 & -0.217 & 0.480 \\
\hline 263.393 & 0.48 & 0.233 & 17.440 & 17.347 & -0.532 & 0.460 \\
\hline 265.436 & 0.85 & 0.429 & 18.190 & 18.149 & -0.226 & 0.490 \\
\hline 264.620 & 0.85 & 0.431 & 18.060 & 18.067 & 0.039 & 0.500 \\
\hline 263.885 & 0.85 & 0.433 & 17.960 & 17.994 & 0.188 & 0.500 \\
\hline 263.258 & 0.85 & 0.434 & 17.880 & 17.932 & 0.289 & 0.500 \\
\hline 265.203 & 1.15 & 0.608 & 18.640 & 18.707 & 0.359 & 0.500 \\
\hline 264.354 & 1.16 & 0.612 & 18.580 & 18.634 & 0.292 & 0.500 \\
\hline 263.693 & 1.16 & 0.617 & 18.520 & 18.581 & 0.331 & 0.500 \\
\hline 263.140 & 1.16 & 0.621 & 18.460 & 18.539 & 0.427 & 0.500 \\
\hline 264.825 & 1.52 & 0.861 & 19.610 & 19.612 & 0.011 & 0.490 \\
\hline 264.132 & 1.52 & 0.867 & 19.590 & 19.570 & -0.100 & 0.480 \\
\hline 263.478 & 1.53 & 0.873 & 19.490 & 19.536 & 0.236 & 0.490 \\
\hline 262.955 & 1.53 & 0.880 & 19.430 & 19.514 & 0.434 & 0.490 \\
\hline 275.153 & 0.33 & 0.149 & 18.420 & 18.444 & 0.133 & 0.530 \\
\hline 274.297 & 0.33 & 0.150 & 18.380 & 18.347 & -0.180 & 0.490 \\
\hline 273.611 & 0.33 & 0.150 & 18.280 & 18.269 & -0.061 & 0.500 \\
\hline 273.020 & 0.33 & 0.150 & 18.010 & 18.202 & 1.066 & 0.730 \\
\hline 275.662 & 0.70 & 0.328 & 19.010 & 18.982 & -0.145 & 0.500 \\
\hline 274.744 & 0.70 & 0.329 & 18.870 & 18.882 & 0.063 & 0.510 \\
\hline
\end{tabular}


Table 15. THERMAL CONDUCTIVITY AT ELEVATED PRESSURES (continued)

Data from Roder [103] (continued)

\begin{tabular}{|c|c|c|c|c|c|c|}
\hline $\begin{array}{l}\mathrm{T} \\
\mathrm{K}\end{array}$ & $\begin{array}{c}\mathrm{P} \\
\mathrm{MPa}\end{array}$ & $\stackrel{\rho}{\mathrm{mol} \cdot \mathrm{dm}^{-3}}$ & $\begin{array}{c}\lambda, \underset{\operatorname{expt}}{\operatorname{mW} \mathrm{m}^{-1} \cdot \mathrm{K}^{-1}}\end{array}$ & $\begin{array}{c}\lambda, \quad \text { calc } \\
\mathrm{mW} \cdot \mathrm{m}^{-1} \cdot \mathrm{K}^{-1}\end{array}$ & $\begin{array}{c}\text { Dev } \\
8\end{array}$ & $w t$ \\
\hline 274.038 & 0.70 & 0.330 & 18.790 & 18.805 & 0.079 & 0.510 \\
\hline 273.395 & 0.70 & 0.331 & 18.700 & 18.735 & 0.187 & 0.520 \\
\hline 275.327 & 1.04 & 0.508 & 19.520 & 19.473 & -0.239 & 0.490 \\
\hline 274.544 & 1.04 & 0.510 & 19.400 & 19.393 & -0.037 & 0.500 \\
\hline 273.897 & 1.04 & 0.512 & 19.230 & 19.327 & 0.503 & 0.520 \\
\hline 273.274 & 1.04 & 0.514 & 19.160 & 19.263 & 0.538 & 0.520 \\
\hline 275.168 & 1.30 & 0.660 & 19.890 & 19.942 & 0.260 & 0.510 \\
\hline 274.374 & 1.30 & 0.665 & 19.840 & 19.872 & 0.162 & 0.500 \\
\hline 273.772 & 1.31 & 0.674 & 19.760 & 19.836 & 0.384 & 0.510 \\
\hline 273.259 & 1.32 & 0.678 & 19.710 & 19.796 & 0.437 & 0.510 \\
\hline 275.682 & 1.61 & 0.855 & 20.650 & 20.679 & 0.140 & 0.500 \\
\hline 274.976 & 1.61 & 0.861 & 20.590 & 20.631 & 0.198 & 0.500 \\
\hline 274.238 & 1.62 & 0.869 & 20.490 & 20.584 & 0.458 & 0.500 \\
\hline 273.649 & 1.62 & 0.874 & 20.440 & 20.539 & 0.487 & 0.500 \\
\hline 275.238 & 2.06 & 1.196 & 22.120 & 22.045 & -0.337 & 0.480 \\
\hline 274.610 & 2.06 & 1.204 & 22.170 & 22.029 & -0.637 & 0.480 \\
\hline 273.904 & 2.07 & 1.214 & 22.110 & 22.012 & -0.444 & 0.480 \\
\hline 273.369 & 2.07 & 1.223 & 22.060 & 22.007 & -0.239 & 0.480 \\
\hline 276.523 & 4.96 & 13.531 & 92.360 & 93.433 & 1.161 & 0.350 \\
\hline 275.924 & 4.96 & 13.577 & 92.740 & 93.906 & 1.258 & 0.340 \\
\hline 275.201 & 4.96 & 13.631 & 93.560 & 94.480 & 0.983 & 0.340 \\
\hline 274.611 & 4.96 & 13.675 & 93.960 & 94.948 & 1.052 & 0.340 \\
\hline 274.197 & 4.96 & 13.706 & 94.370 & 95.277 & 0.961 & 0.340 \\
\hline 276.148 & 11.79 & 14.419 & 103.740 & 104.632 & 0.860 & 0.330 \\
\hline 275.546 & 11.79 & 14.451 & 104.070 & 105.029 & 0.921 & 0.330 \\
\hline 274.955 & 11.79 & 14.483 & 104.550 & 105.421 & 0.833 & 0.330 \\
\hline 274.440 & 11.79 & 14.510 & 105.030 & 105.760 & 0.695 & 0.330 \\
\hline 275.840 & 19.38 & 15.057 & 113.520 & 114.185 & 0.586 & 0.320 \\
\hline 275.289 & 19.38 & 15.082 & 113.810 & 114.520 & 0.623 & 0.320 \\
\hline 274.798 & 19.38 & 15.103 & 114.230 & 114.815 & 0.512 & 0.320 \\
\hline 274.335 & 19.38 & 15.124 & 114.550 & 115.096 & 0.476 & 0.320 \\
\hline 274.466 & 32.56 & 15.872 & 128.020 & 128.325 & 0.238 & 0.300 \\
\hline 276.141 & 32.56 & 15.812 & 126.810 & 127.392 & 0.459 & 0.310 \\
\hline 275.511 & 32.56 & 15.835 & 127.240 & 127.742 & 0.394 & 0.310 \\
\hline
\end{tabular}


Table 15. THERMAL CONDUCTIVITY AT ELEVATED PRESSURES (continued)

Data from Roder [103] (continued)

\begin{tabular}{|c|c|c|c|c|c|c|}
\hline $\begin{array}{l}\mathrm{T} \\
\mathrm{K}\end{array}$ & $\underset{\mathrm{MPa}}{\mathrm{P}}$ & $\begin{array}{c}\rho \\
\mathrm{mol} \cdot \mathrm{dm}^{-3}\end{array}$ & $\begin{array}{c}\lambda, \underset{\mathrm{mW} \cdot \mathrm{m}^{-1} \cdot \mathrm{K}^{-1}}{\operatorname{expt}}\end{array}$ & $\begin{array}{c}\lambda, \operatorname{calc} \\
\mathrm{mW} \cdot \mathrm{m}^{-1} \cdot \mathrm{K}^{-1}\end{array}$ & $\begin{array}{c}\text { Dev } \\
8\end{array}$ & wt \\
\hline 274.956 & 32.56 & 15.854 & 127.620 & 128.049 & 0.336 & 0.310 \\
\hline 276.942 & 48.70 & 16.470 & 140.240 & 140.849 & 0.434 & 0.290 \\
\hline 276.326 & 48.70 & 16.489 & 140.600 & 141.174 & 0.408 & 0.290 \\
\hline 275.745 & 48.70 & 16.507 & 140.850 & 141.480 & 0.448 & 0.290 \\
\hline 275.265 & 48.70 & 16.521 & 141.120 & 141.734 & 0.435 & 0.290 \\
\hline 277.211 & 65.59 & 17.021 & 152.810 & 153.585 & 0.507 & 0.290 \\
\hline 276.677 & 65.59 & 17.035 & 153.200 & 153.859 & 0.430 & 0.290 \\
\hline 276.110 & 65.62 & 17.052 & 153.450 & 154.169 & 0.469 & 0.280 \\
\hline 275.551 & 65.62 & 17.067 & 153.750 & 154.457 & 0.460 & 0.280 \\
\hline 275.160 & 65.62 & 17.078 & 154.230 & 154.662 & 0.280 & 0.280 \\
\hline 286.164 & 0.25 & 0.106 & 19.600 & 19.642 & 0.217 & 0.580 \\
\hline 285.222 & 0.25 & 0.106 & 19.530 & 19.529 & -0.003 & 0.520 \\
\hline 284.404 & 0.25 & 0.107 & 19.370 & 19.431 & 0.317 & 0.600 \\
\hline 283.648 & 0.25 & 0.108 & 19.330 & 19.342 & 0.064 & 0.540 \\
\hline 283.344 & 0.82 & 0.377 & 19.910 & 20.008 & 0.493 & 0.540 \\
\hline 285.524 & 0.82 & 0.374 & 20.190 & 20.254 & 0.319 & 0.540 \\
\hline 284.734 & 0.82 & 0.375 & 20.030 & 20.165 & 0.673 & 0.560 \\
\hline 284.032 & 0.82 & 0.376 & 20.100 & 20.086 & -0.072 & 0.510 \\
\hline 283.715 & 1.45 & 0.716 & 20.810 & 21.068 & 1.240 & 0.550 \\
\hline 285.881 & 1.45 & 0.707 & 21.160 & 21.285 & 0.590 & 0.530 \\
\hline 285.110 & 1.45 & 0.711 & 21.120 & 21.209 & 0.422 & 0.520 \\
\hline 284.391 & 1.45 & 0.714 & 21.030 & 21.137 & 0.509 & 0.520 \\
\hline 286.150 & 2.19 & 1.187 & 23.010 & 23.062 & 0.226 & 0.500 \\
\hline 285.324 & 2.19 & 1.196 & 22.980 & 23.014 & 0.146 & 0.500 \\
\hline 284.054 & 2.19 & 1.208 & 22.920 & 22.938 & 0.081 & 0.500 \\
\hline 283.033 & 2.19 & 1.219 & 22.740 & 22.885 & 0.638 & 0.510 \\
\hline 284.680 & 2.19 & 1.204 & 22.880 & 22.981 & 0.443 & 0.500 \\
\hline 285.413 & 2.78 & 1.712 & 25.650 & 25.559 & -0.355 & 0.480 \\
\hline 284.073 & 2.78 & 1.741 & 25.850 & 25.644 & -0.797 & 0.470 \\
\hline 286.061 & 2.78 & 1.699 & 25.620 & 25.528 & -0.360 & 0.480 \\
\hline 284.671 & 2.78 & 1.728 & 25.730 & 25.603 & -0.495 & 0.480 \\
\hline 296.023 & 0.29 & 0.120 & 20.800 & 20.893 & 0.447 & 0.660 \\
\hline 295.044 & 0.29 & 0.121 & 20.680 & 20.771 & 0.441 & 0.650 \\
\hline 294.331 & 0.29 & 0.121 & 20.450 & 20.683 & 1.139 & 1.370 \\
\hline 293.565 & 0.29 & 0.121 & 20.460 & 20.588 & 0.627 & 0.730 \\
\hline 296.326 & 0.86 & 0.373 & 21.450 & 21.556 & 0.496 & 0.560 \\
\hline
\end{tabular}


Table 15. THERMAL CONDUCTIVITY AT ELEVATED PRESSURES (continued)

Data from Roder [103] (continued)

\begin{tabular}{|c|c|c|c|c|c|c|}
\hline $\begin{array}{l}\mathrm{T} \\
\mathrm{K}\end{array}$ & $\begin{array}{c}\mathrm{P} \\
\mathrm{MPa}\end{array}$ & $\begin{array}{c}\rho \\
\mathrm{mol}\end{array} \mathrm{dm}^{-3}$ & 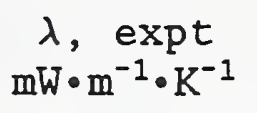 & $\begin{array}{c}\lambda, \quad c a l c \\
\mathrm{~mW} \cdot \mathrm{m}^{-1} \cdot \mathrm{K}^{-1}\end{array}$ & $\begin{array}{c}\text { Dev } \\
\frac{8}{8}\end{array}$ & wt \\
\hline 295.392 & 0.86 & 0.374 & 21.310 & 21.445 & 0.635 & 0.570 \\
\hline 294.709 & 0.86 & 0.375 & 21.320 & 21.364 & 0.208 & 0.540 \\
\hline 293.982 & 0.86 & 0.376 & 21.200 & 21.278 & 0.370 & 0.550 \\
\hline 295.892 & 1.38 & 0.633 & 22.080 & 22.228 & 0.668 & 0.550 \\
\hline 295.161 & 1.38 & 0.639 & 21.990 & 22.156 & 0.755 & 0.550 \\
\hline 294.429 & 1.38 & 0.641 & 21.940 & 22.076 & 0.618 & 0.540 \\
\hline 293.742 & 1.39 & 0.645 & 21.680 & 22.007 & 1.508 & 0.580 \\
\hline 293.441 & 2.12 & 1.078 & 23.220 & 23.410 & 0.818 & 0.520 \\
\hline 295.410 & 2.12 & 1.066 & 23.410 & 23.585 & 0.749 & 0.530 \\
\hline 294.658 & 2.12 & 1.072 & 23.350 & 23.525 & 0.750 & 0.520 \\
\hline 294.060 & 2.13 & 1.078 & 23.280 & 23.479 & 0.854 & 0.530 \\
\hline 295.407 & 2.97 & 1.705 & 26.210 & 26.309 & 0.376 & 0.500 \\
\hline 294.702 & 2.97 & 1.721 & 26.320 & 26.329 & 0.035 & 0.490 \\
\hline 294.072 & 2.97 & 1.735 & 26.210 & 26.344 & 0.513 & 0.500 \\
\hline 293.616 & 2.98 & 1.745 & 26.400 & 26.361 & -0.148 & 0.490 \\
\hline 293.665 & 3.51 & 2.364 & 30.670 & 30.308 & -1.181 & 0.470 \\
\hline 294.579 & 3.51 & 2.326 & 30.270 & 30.027 & -0.803 & 0.470 \\
\hline 294.115 & 3.51 & 2.345 & 30.400 & 30.163 & -0.779 & 0.470 \\
\hline 295.195 & 3.51 & 2.301 & 30.020 & 29.863 & -0.522 & 0.480 \\
\hline 296.227 & 0.31 & 0.129 & 20.830 & 20.940 & 0.530 & 0.680 \\
\hline 295.398 & 0.31 & 0.130 & 20.630 & 20.837 & 1.005 & 0.980 \\
\hline 294.659 & 0.31 & 0.130 & 20.600 & 20.746 & 0.708 & 0.750 \\
\hline 293.906 & 0.31 & 0.131 & 20.510 & 20.653 & 0.696 & 0.740 \\
\hline 293.702 & 0.84 & 0.370 & 21.160 & 21.226 & 0.314 & 0.550 \\
\hline 295.810 & 0.84 & 0.366 & 21.390 & 21.476 & 0.404 & 0.560 \\
\hline 295.069 & 0.84 & 0.367 & 21.270 & 21.388 & 0.556 & 0.570 \\
\hline 294.373 & 0.84 & 0.369 & 21.150 & 21.306 & 0.736 & 0.580 \\
\hline 293.470 & 1.36 & 0.633 & 21.740 & 21.939 & 0.915 & 0.550 \\
\hline 295.500 & 1.36 & 0.627 & 22.050 & 22.162 & 0.510 & 0.540 \\
\hline 294.790 & 1.36 & 0.629 & 21.850 & 22.084 & 1.070 & 0.560 \\
\hline 294.083 & 1.36 & 0.631 & 21.890 & 22.006 & 0.530 & 0.540 \\
\hline 293.755 & 2.14 & 1.092 & 23.260 & 23.499 & 1.029 & 0.530 \\
\hline 295.763 & 2.15 & 1.079 & 23.470 & 23.672 & 0.860 & 0.530 \\
\hline 294.979 & 2.15 & 1.084 & 23.430 & 23.605 & 0.746 & 0.520 \\
\hline 294.412 & 2.15 & 1.088 & 23.460 & 23.557 & 0.412 & 0.520 \\
\hline 293.954 & 2.92 & 1.690 & 25.940 & 26.108 & 0.648 & 0.500 \\
\hline
\end{tabular}


Table 15. THERMAL CONDUCTIVITY AT ELEVATED PRESSURES (continued)

Data from Roder [103] (continued)

\begin{tabular}{|c|c|c|c|c|c|c|}
\hline $\begin{array}{l}\mathrm{T} \\
\mathrm{K}\end{array}$ & $\begin{array}{c}\mathrm{P} \\
\mathrm{MPa}\end{array}$ & $\begin{array}{c}\rho \\
\mathrm{mol} \cdot \mathrm{dm}^{-3}\end{array}$ & $\begin{array}{c}\lambda, \underset{\mathrm{mW}}{\operatorname{expt}} \mathrm{m}^{-1} \cdot \mathrm{K}^{-1}\end{array}$ & $\begin{array}{c}\lambda, \quad \operatorname{calc} \\
\mathrm{mW} \cdot \mathrm{m}^{-1} \cdot \mathrm{K}^{-1}\end{array}$ & $\begin{array}{c}\text { Dev } \\
\frac{8}{\partial}\end{array}$ & wt \\
\hline 295.783 & 2.92 & 1.660 & 25.930 & 26.117 & 0.722 & 0.510 \\
\hline 295.168 & 2.92 & 1.670 & 25.990 & 26.111 & 0.467 & 0.500 \\
\hline 294.546 & 2.92 & 1.681 & 25.790 & 26.113 & 1.252 & 0.510 \\
\hline 295.866 & 3.39 & 2.128 & 28.720 & 28.726 & 0.020 & 0.490 \\
\hline 295.244 & 3.39 & 2.148 & 28.820 & 28.817 & -0.010 & 0.480 \\
\hline 294.681 & 3.39 & 2.165 & 28.830 & 28.909 & 0.275 & 0.490 \\
\hline 294.168 & 3.39 & 2.184 & 29.010 & 29.011 & 0.002 & 0.480 \\
\hline 295.599 & 5.51 & 11.862 & 78.770 & 78.971 & 0.255 & 0.370 \\
\hline 295.240 & 5.51 & 11.904 & 79.180 & 79.276 & 0.122 & 0.370 \\
\hline 294.776 & 5.50 & 11.959 & 79.390 & 79.672 & 0.355 & 0.370 \\
\hline 294.211 & 5.50 & 12.024 & 79.980 & 80.156 & 0.220 & 0.370 \\
\hline 293.548 & 5.50 & 12.100 & 80.400 & 80.724 & 0.403 & 0.370 \\
\hline 296.074 & 11.96 & 13.283 & 91.600 & 92.312 & 0.777 & 0.350 \\
\hline 295.642 & 11.97 & 13.310 & 91.770 & 92.576 & 0.879 & 0.350 \\
\hline 294.974 & 11.97 & 13.352 & 92.020 & 92.985 & 1.049 & 0.350 \\
\hline 294.359 & 11.97 & 13.390 & 92.210 & 93.358 & 1.244 & 0.350 \\
\hline 293.856 & 11.97 & 13.421 & 92.790 & 93.667 & 0.946 & 0.350 \\
\hline 296.312 & 18.85 & 14.070 & 101.340 & 101.935 & 0.587 & 0.340 \\
\hline 295.386 & 18.85 & 14.114 & 101.670 & 102.431 & 0.748 & 0.340 \\
\hline 294.831 & 18.85 & 14.141 & 102.040 & 102.732 & 0.678 & 0.340 \\
\hline 294.201 & 18.85 & 14.171 & 102.460 & 103.071 & 0.597 & 0.330 \\
\hline 293.719 & 18.85 & 14.194 & 102.450 & 103.332 & 0.861 & 0.330 \\
\hline 295.782 & 25.89 & 14.668 & 109.750 & 110.367 & 0.563 & 0.330 \\
\hline 295.166 & 25.89 & 14.693 & 110.080 & 110.678 & 0.543 & 0.330 \\
\hline 294.560 & 25.88 & 14.718 & 110.210 & 110.981 & 0.700 & 0.330 \\
\hline 293.962 & 25.89 & 14.742 & 110.780 & 111.288 & 0.459 & 0.320 \\
\hline 296.260 & 35.69 & 15.257 & 119.730 & 119.928 & 0.166 & 0.320 \\
\hline 295.539 & 35.69 & 15.282 & 119.700 & 120.275 & 0.481 & 0.320 \\
\hline 294.984 & 35.69 & 15.302 & 120.550 & 120.540 & -0.009 & 0.310 \\
\hline 294.374 & 35.69 & 15.323 & 120.170 & 120.835 & 0.553 & 0.310 \\
\hline 296.694 & 49.71 & 15.905 & 131.480 & 131.932 & 0.344 & 0.300 \\
\hline 295.818 & 49.71 & 15.932 & 131.870 & 132.336 & 0.353 & 0.300 \\
\hline 295.280 & 49.71 & 15.949 & 132.130 & 132.585 & 0.344 & 0.300 \\
\hline 294.618 & 49.70 & 15.968 & 132.240 & 132.889 & 0.491 & 0.300 \\
\hline 296.067 & 66.76 & 16.546 & 144.580 & 145.359 & 0.539 & 0.290 \\
\hline 295.429 & 66.76 & 16.564 & 144.920 & 145.649 & 0.503 & 0.290 \\
\hline
\end{tabular}


Table 15. THERMAL CONDUCTIVITY AT ELEVATED PRESSURES (continued)

Data from Roder [103] (continued)

\begin{tabular}{|c|c|c|c|c|c|c|}
\hline $\begin{array}{l}\mathrm{T} \\
\mathrm{K}\end{array}$ & $\begin{array}{c}\mathrm{P} \\
\mathrm{MPa}\end{array}$ & $\stackrel{\rho}{\mathrm{mol} \cdot \mathrm{dm}^{-3}}$ & $\begin{array}{c}\lambda, \operatorname{expt} \\
\mathrm{mW} \cdot \mathrm{m}^{-1} \cdot \mathrm{K}^{-1}\end{array}$ & $\begin{array}{c}\lambda, \operatorname{calc} \\
\mathrm{mW} \cdot \mathrm{m}^{-1} \cdot \mathrm{K}^{-1}\end{array}$ & $\begin{array}{l}\text { Dev } \\
\frac{8}{8}\end{array}$ & wt \\
\hline 294.891 & 66.77 & 16.578 & 145.120 & 145.898 & 0.536 & 0.290 \\
\hline 294.396 & 66.78 & 16.592 & 145.530 & 146.128 & 0.411 & 0.290 \\
\hline 302.965 & 0.24 & 0.097 & 22.180 & 21.724 & -2.055 & 0.310 \\
\hline 303.561 & 0.24 & 0.097 & 22.060 & 21.801 & -1.176 & 0.360 \\
\hline 304.449 & 0.24 & 0.097 & 21.960 & 21.915 & -0.206 & 0.490 \\
\hline 305.309 & 0.24 & 0.097 & 22.210 & 22.026 & -0.830 & 0.400 \\
\hline 302.718 & 0.64 & 0.266 & 22.320 & 22.090 & -1.032 & 0.460 \\
\hline 303.335 & 0.64 & 0.266 & 22.500 & 22.167 & -1.481 & 0.430 \\
\hline 304.854 & 0.64 & 0.264 & 22.560 & 22.358 & -0.898 & 0.460 \\
\hline 304.006 & 0.64 & 0.265 & 22.520 & 22.251 & -1.195 & 0.450 \\
\hline 305.696 & 0.96 & 0.406 & 23.070 & 22.817 & -1.097 & 0.050 \\
\hline 302.745 & 0.96 & 0.411 & 22.500 & 22.456 & -0.197 & 0.520 \\
\hline 303.313 & 0.96 & 0.410 & 22.660 & 22.525 & -0.596 & 0.500 \\
\hline 304.074 & 0.96 & 0.408 & 22.760 & 22.618 & -0.625 & 0.500 \\
\hline 304.938 & 0.96 & 0.407 & 22.860 & 22.724 & -0.596 & 0.500 \\
\hline 306.007 & 1.52 & 0.675 & 23.950 & 23.587 & -1.518 & 0.050 \\
\hline 303.238 & 1.52 & 0.684 & 23.460 & 23.270 & -0.811 & 0.500 \\
\hline 305.362 & 1.52 & 0.677 & 23.820 & 23.512 & -1.292 & 0.050 \\
\hline 304.514 & 1.52 & 0.680 & 23.750 & 23.415 & -1.411 & 0.480 \\
\hline 306.272 & 1.85 & 0.845 & 24.580 & 24.128 & -1.841 & 0.050 \\
\hline 305.761 & 1.85 & 0.848 & 24.530 & 24.072 & -1.867 & 0.050 \\
\hline 304.422 & 1.85 & 0.854 & 24.260 & 23.928 & -1.370 & 0.480 \\
\hline 304.209 & 1.85 & 0.855 & 24.230 & 23.905 & -1.342 & 0.480 \\
\hline 303.597 & 1.85 & 0.857 & 23.750 & 23.840 & 0.377 & 0.530 \\
\hline 303.292 & 1.86 & 0.864 & 24.180 & 23.823 & -1.477 & 0.480 \\
\hline 305.293 & 1.86 & 0.855 & 24.220 & 24.037 & -0.757 & 0.500 \\
\hline 304.494 & 1.86 & 0.858 & 24.020 & 23.951 & -0.288 & 0.510 \\
\hline 303.861 & 1.86 & 0.861 & 23.970 & 23.883 & -0.362 & 0.510 \\
\hline 306.756 & 2.50 & 1.224 & 25.810 & 25.452 & -1.388 & 0.050 \\
\hline 304.906 & 2.50 & 1.239 & 25.470 & 25.293 & -0.693 & 0.500 \\
\hline 304.332 & 2.50 & 1.244 & 25.410 & 25.246 & -0.647 & 0.500 \\
\hline 306.052 & 2.50 & 1.229 & 25.560 & 25.391 & -0.662 & 0.050 \\
\hline 304.396 & 3.16 & 1.721 & 27.620 & 27.221 & -1.445 & 0.480 \\
\hline 304.645 & 3.16 & 1.717 & 27.640 & 27.228 & -1.492 & 0.480 \\
\hline 305.885 & 3.16 & 1.698 & 27.670 & 27.266 & -1.459 & 0.050 \\
\hline 305.553 & 3.16 & 1.703 & 27.690 & 27.255 & -1.570 & 0.050 \\
\hline
\end{tabular}


Table 15. THERMAL CONDUCTIVITY AT ELEVATED PRESSURES (continued)

Data from Roder [103] (continued)

\begin{tabular}{|c|c|c|c|c|c|c|}
\hline $\begin{array}{l}\mathrm{T} \\
\mathrm{K}\end{array}$ & $\begin{array}{c}\mathrm{P} \\
\mathrm{MPa}\end{array}$ & $\underset{\mathrm{mol}}{\rho} \cdot \mathrm{dm}^{-3}$ & $\begin{array}{c}\lambda, \underset{\mathrm{mW}}{\operatorname{expt}} \\
\mathrm{mW} \cdot \mathrm{m}^{-1} \cdot \mathrm{K}^{-1}\end{array}$ & $\begin{array}{c}\lambda, \quad \text { calc } \\
\mathrm{mW} \cdot \mathrm{m}^{-1} \cdot \mathrm{K}^{-1}\end{array}$ & $\begin{array}{c}\text { Dev } \\
8\end{array}$ & wt \\
\hline 305.536 & 3.79 & 2.297 & 31.260 & 30.307 & -3.047 & 0.050 \\
\hline 307.396 & 3.79 & 2.243 & 31.220 & 30.127 & -3.500 & 0.050 \\
\hline 306.273 & 3.79 & 2.276 & 31.100 & 30.235 & -2.782 & 0.050 \\
\hline 304.935 & 3.79 & 2.316 & 31.100 & 30.387 & -2.294 & 0.460 \\
\hline 304.128 & 3.79 & 2.342 & 31.460 & 30.497 & -3.061 & 0.450 \\
\hline 303.535 & 3.79 & 2.362 & 31.440 & 30.588 & -2.709 & 0.460 \\
\hline 315.524 & 0.12 & 0.045 & 22.830 & 23.265 & 1.906 & 0.030 \\
\hline 314.484 & 0.12 & 0.045 & 22.820 & 23.126 & 1.339 & 0.040 \\
\hline 313.667 & 0.12 & 0.045 & 22.690 & 23.016 & 1.438 & 0.040 \\
\hline 312.814 & 0.12 & 0.047 & 22.180 & 22.906 & 3.272 & 0.000 \\
\hline 314.792 & 0.64 & 0.254 & 23.940 & 23.631 & -1.291 & 0.040 \\
\hline 313.738 & 0.64 & 0.255 & 23.840 & 23.493 & -1.454 & 0.040 \\
\hline 313.154 & 0.64 & 0.256 & 23.690 & 23.418 & -1.147 & 0.040 \\
\hline 312.499 & 0.64 & 0.256 & 23.610 & 23.333 & -1.172 & 0.040 \\
\hline 314.460 & 1.08 & 0.443 & 24.410 & 24.045 & -1.493 & 0.050 \\
\hline 313.728 & 1.08 & 0.445 & 24.240 & 23.954 & -1.180 & 0.050 \\
\hline 313.012 & 1.08 & 0.446 & 24.050 & 23.864 & -0.774 & 0.050 \\
\hline 312.439 & 1.08 & 0.447 & 24.060 & 23.792 & -1.115 & 0.050 \\
\hline 314.805 & 1.92 & 0.846 & 25.530 & 25.193 & -1.321 & 0.050 \\
\hline 313.965 & 1.92 & 0.849 & 25.250 & 25.097 & -0.607 & 0.050 \\
\hline 313.310 & 1.92 & 0.854 & 25.260 & 25.029 & -0.916 & 0.050 \\
\hline 312.571 & 1.92 & 0.858 & 24.870 & 24.947 & 0.309 & 0.050 \\
\hline 314.525 & 2.58 & 1.211 & 26.690 & 26.329 & -1.354 & 0.050 \\
\hline 313.751 & 2.57 & 1.214 & 26.600 & 26.247 & -1.329 & 0.050 \\
\hline 313.090 & 2.57 & 1.219 & 26.540 & 26.184 & -1.341 & 0.050 \\
\hline 312.484 & 2.57 & 1.224 & 26.530 & 26.127 & -1.517 & 0.050 \\
\hline 314.137 & 3.12 & 1.563 & 28.050 & 27.586 & -1.656 & 0.050 \\
\hline 313.535 & 3.12 & 1.570 & 27.880 & 27.546 & -1.199 & 0.050 \\
\hline 312.497 & 3.12 & 1.583 & 27.950 & 27.479 & -1.685 & 0.050 \\
\hline 312.873 & 3.12 & 1.578 & 27.920 & 27.503 & -1.494 & 0.050 \\
\hline 314.577 & 3.87 & 2.144 & 30.830 & 30.202 & -2.038 & 0.050 \\
\hline 313.834 & 3.87 & 2.160 & 30.850 & 30.217 & -2.053 & 0.050 \\
\hline 313.183 & 3.87 & 2.175 & 30.690 & 30.234 & -1.487 & 0.050 \\
\hline 312.670 & 3.87 & 2.185 & 30.620 & 30.241 & -1.239 & 0.050 \\
\hline 314.380 & 4.02 & 2.281 & 31.480 & 30.880 & -1.907 & 0.050 \\
\hline 313.708 & 4.02 & 2.299 & 31.420 & 30.915 & -1.608 & 0.050 \\
\hline
\end{tabular}


Table 15. THERMAL CONDUCTIVITY AT ELEVATED PRESSURES (continued)

Data from Roder [103] (continued)

\begin{tabular}{|c|c|c|c|c|c|c|}
\hline $\begin{array}{l}\mathrm{T} \\
\mathrm{K}\end{array}$ & $\begin{array}{c}\mathrm{P} \\
\mathrm{MPa}\end{array}$ & $\stackrel{\rho}{\mathrm{mol}} \cdot \stackrel{\mathrm{dm}^{-3}}{ }$ & 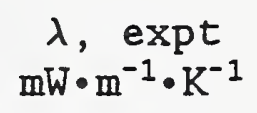 & $\begin{array}{c}\lambda, \quad \operatorname{calc} \\
\mathrm{mW} \cdot \mathrm{m}^{-1} \cdot \mathrm{K}^{-1}\end{array}$ & $\begin{array}{c}\text { Dev } \\
\frac{8}{8}\end{array}$ & wt \\
\hline 313.054 & 4.02 & 2.316 & 31.210 & 30.955 & -0.818 & 0.050 \\
\hline 309.155 & 4.02 & 2.432 & 32.050 & 31.328 & -2.254 & 0.050 \\
\hline 311.722 & 4.56 & 3.014 & 34.840 & 35.185 & 0.990 & 0.050 \\
\hline 312.873 & 4.56 & 2.952 & 35.500 & 34.768 & -2.063 & 0.050 \\
\hline 311.752 & 4.56 & 3.012 & 35.280 & 35.173 & -0.303 & 0.050 \\
\hline 312.101 & 4.56 & 2.993 & 35.390 & 35.039 & -0.992 & 0.050 \\
\hline 312.375 & 4.56 & 2.978 & 35.040 & 34.939 & -0.289 & 0.050 \\
\hline 312.983 & 4.75 & 3.235 & 37.280 & 36.772 & -1.363 & 0.050 \\
\hline 312.000 & 4.75 & 3.306 & 36.990 & 37.342 & 0.951 & 0.050 \\
\hline 311.899 & 4.75 & 3.313 & 38.290 & 37.406 & -2.308 & 0.050 \\
\hline 312.323 & 4.75 & 3.280 & 38.310 & 37.130 & -3.080 & 0.040 \\
\hline 316.546 & 4.75 & 3.028 & 36.930 & 35.351 & -4.275 & 0.040 \\
\hline 312.203 & 4.90 & 3.569 & 39.660 & 39.466 & -0.488 & 0.050 \\
\hline 313.324 & 4.91 & 3.486 & 39.030 & 38.686 & -0.882 & 0.050 \\
\hline 312.858 & 4.91 & 3.528 & 39.450 & 39.056 & -0.999 & 0.050 \\
\hline 312.449 & 4.91 & 3.566 & 39.410 & 39.411 & 0.003 & 0.050 \\
\hline 313.386 & 5.11 & 3.910 & 42.620 & 42.263 & -0.837 & 0.050 \\
\hline 312.085 & 5.11 & 4.099 & 44.150 & 44.365 & 0.488 & 0.050 \\
\hline 312.980 & 5.11 & 3.964 & 43.090 & 42.839 & -0.582 & 0.050 \\
\hline 312.558 & 5.11 & 4.025 & 43.580 & 43.509 & -0.163 & 0.050 \\
\hline 311.923 & 5.11 & 4.127 & 44.090 & 44.689 & 1.359 & 0.050 \\
\hline 312.042 & 5.20 & 4.407 & 47.040 & 47.568 & 1.122 & 0.050 \\
\hline 313.007 & 5.20 & 4.215 & 45.130 & 45.232 & 0.225 & 0.050 \\
\hline 312.519 & 5.20 & 4.306 & 45.830 & 46.314 & 1.055 & 0.050 \\
\hline 312.071 & 5.20 & 4.400 & 46.240 & 47.485 & 2.692 & 0.050 \\
\hline 312.095 & 5.43 & 5.514 & 59.620 & 59.939 & 0.536 & 0.040 \\
\hline 312.909 & 5.43 & 5.120 & 55.470 & 54.791 & -1.224 & 0.040 \\
\hline 312.497 & 5.43 & 5.300 & 56.260 & 57.143 & 1.570 & 0.050 \\
\hline 312.524 & 5.43 & 5.287 & 57.890 & 56.975 & -1.581 & 0.040 \\
\hline 313.140 & 5.42 & 4.983 & 54.820 & 53.141 & -3.063 & 0.040 \\
\hline 312.429 & 5.42 & 5.277 & 55.180 & 56.974 & 3.252 & 0.050 \\
\hline 312.065 & 5.42 & 5.467 & 56.880 & 59.483 & 4.577 & 0.000 \\
\hline 311.837 & 5.42 & 5.607 & 57.700 & 61.298 & 6.236 & 0.000 \\
\hline 312.754 & 5.50 & 5.636 & 60.680 & 60.271 & -0.674 & 0.040 \\
\hline 312.452 & 5.50 & 5.830 & 62.560 & 62.489 & -0.114 & 0.040 \\
\hline 312.430 & 5.50 & 5.845 & 64.330 & 62.658 & -2.599 & 0.000 \\
\hline
\end{tabular}


Table 15. THERMAL CONDUCTIVITY AT ELEVATED PRESSURES (continued)

Data from Roder [103] (continued)

\begin{tabular}{|c|c|c|c|c|c|c|}
\hline $\begin{array}{l}\mathrm{T} \\
\mathrm{K}\end{array}$ & $\begin{array}{c}\mathrm{P} \\
\mathrm{MPa}\end{array}$ & $\begin{array}{c}\rho \\
\mathrm{mol} \cdot \mathrm{dm}^{-3}\end{array}$ & 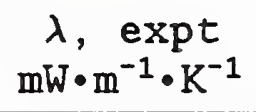 & $\begin{array}{c}\lambda, \text { calc } \\
\mathrm{mW} \cdot \mathrm{m}^{-1} \cdot \mathrm{K}^{-1}\end{array}$ & $\begin{array}{c}\text { Dev } \\
z\end{array}$ & wt \\
\hline 311.620 & 5.50 & 6.551 & 65.160 & 68.950 & 5.816 & 0.000 \\
\hline 312.283 & 5.58 & 6.655 & 67.440 & 68.019 & 0.859 & 0.040 \\
\hline 311.910 & 5.58 & 7.029 & 68.220 & 69.698 & 2.167 & 0.040 \\
\hline 311.810 & 5.58 & 7.134 & 69.300 & 70.005 & 1.018 & 0.040 \\
\hline 311.444 & 5.58 & 7.517 & 70.080 & 70.541 & 0.658 & 0.040 \\
\hline 312.282 & 5.60 & 6.909 & 68.770 & 68.771 & 0.001 & 0.040 \\
\hline 311.992 & 5.60 & 7.198 & 68.970 & 69.693 & 1.048 & 0.040 \\
\hline 311.693 & 5.60 & 7.499 & 70.080 & 70.109 & 0.041 & 0.040 \\
\hline 311.488 & 5.60 & 7.706 & 70.840 & 70.082 & -1.070 & 0.040 \\
\hline 312.441 & 5.67 & 7.348 & 69.330 & 68.932 & -0.575 & 0.040 \\
\hline 312.179 & 5.67 & 7.585 & 69.530 & 69.179 & -0.505 & 0.040 \\
\hline 311.801 & 5.67 & 7.909 & 70.280 & 69.083 & -1.703 & 0.040 \\
\hline 311.720 & 5.67 & 7.980 & 69.920 & 68.997 & -1.319 & 0.040 \\
\hline 312.490 & 5.77 & 7.979 & 69.200 & 68.034 & -1.684 & 0.040 \\
\hline 312.170 & 5.77 & 8.198 & 68.980 & 67.871 & -1.607 & 0.040 \\
\hline 311.442 & 5.77 & 8.628 & 69.100 & 67.449 & -2.389 & 0.040 \\
\hline 311.609 & 5.77 & 8.537 & 69.760 & 67.530 & -3.196 & 0.040 \\
\hline 312.513 & 5.83 & 8.263 & 69.210 & 67.381 & -2.642 & 0.040 \\
\hline 312.199 & 5.83 & 8.445 & 69.380 & 67.258 & -3.059 & 0.040 \\
\hline 311.858 & 5.83 & 8.627 & 68.930 & 67.146 & -2.587 & 0.040 \\
\hline 311.788 & 5.83 & 8.662 & 69.590 & 67.129 & -3.536 & 0.040 \\
\hline 312.939 & 5.92 & 8.408 & 68.830 & 66.748 & -3.025 & 0.040 \\
\hline 312.434 & 5.92 & 8.659 & 68.910 & 66.711 & -3.191 & 0.040 \\
\hline 312.183 & 5.92 & 8.774 & 69.490 & 66.717 & -3.990 & 0.040 \\
\hline 311.910 & 5.92 & 8.891 & 68.770 & 66.748 & -2.940 & 0.040 \\
\hline 313.330 & 6.07 & 8.697 & 68.630 & 66.167 & -3.589 & 0.040 \\
\hline 313.030 & 6.07 & 8.820 & 68.350 & 66.250 & -3.072 & 0.040 \\
\hline 312.630 & 6.07 & 8.974 & 68.940 & 66.391 & -3.698 & 0.040 \\
\hline 312.361 & 6.07 & 9.072 & 68.840 & 66.506 & -3.391 & 0.040 \\
\hline 312.057 & 6.07 & 9.177 & 69.300 & 66.654 & -3.818 & 0.040 \\
\hline 313.149 & 6.21 & 9.098 & 68.620 & 66.256 & -3.445 & 0.040 \\
\hline 312.903 & 6.21 & 9.178 & 68.930 & 66.398 & -3.673 & 0.040 \\
\hline 312.603 & 6.21 & 9.272 & 68.970 & 66.584 & -3.459 & 0.040 \\
\hline 311.863 & 6.21 & 9.489 & 69.020 & 67.090 & -2.797 & 0.040 \\
\hline 314.216 & 6.24 & 8.803 & 68.830 & 65.745 & -4.482 & 0.040 \\
\hline 313.237 & 6.24 & 9.139 & 68.300 & 66.265 & -2.979 & 0.040 \\
\hline
\end{tabular}


Table 15. THERMAL CONDUCTIVITY AT ELEVATED PRESSURES (continued)

Data from Roder [103] (continued)

\begin{tabular}{|c|c|c|c|c|c|c|}
\hline $\begin{array}{l}\mathrm{T} \\
\mathrm{K}\end{array}$ & $\begin{array}{c}\mathrm{P} \\
\mathrm{MPa}\end{array}$ & $\begin{array}{c}\rho \\
\mathrm{mol} \cdot \mathrm{dm}^{-3}\end{array}$ & $\begin{array}{c}\lambda, \underset{\operatorname{expt}}{\mathrm{mW} \cdot \mathrm{m}^{-1} \cdot \mathrm{K}^{-1}}\end{array}$ & $\begin{array}{c}\lambda, \quad \text { calc } \\
\mathrm{mW} \cdot \mathrm{m}^{-1} \cdot \mathrm{K}^{-1}\end{array}$ & $\begin{array}{c}\text { Dev } \\
\frac{8}{8}\end{array}$ & wt \\
\hline 312.994 & 6.24 & 9.216 & 68.250 & 66.414 & -2.689 & 0.040 \\
\hline 312.566 & 6.24 & 9.345 & 68.380 & 66.695 & -2.465 & 0.040 \\
\hline 314.753 & 6.72 & 9.478 & 69.230 & 66.612 & -3.781 & 0.040 \\
\hline 313.885 & 6.72 & 9.679 & 69.470 & 67.299 & -3.125 & 0.040 \\
\hline 313.249 & 6.72 & 9.818 & 69.560 & 67.815 & -2.509 & 0.040 \\
\hline 312.504 & 6.72 & 9.973 & 70.080 & 68.430 & -2.354 & 0.040 \\
\hline 315.859 & 7.16 & 9.761 & 71.240 & 67.448 & -5.323 & 0.040 \\
\hline 314.022 & 6.59 & 9.489 & 70.680 & 66.735 & -5.582 & 0.040 \\
\hline 313.425 & 7.16 & 10.207 & 71.270 & 69.401 & -2.622 & 0.040 \\
\hline 313.000 & 7.16 & 10.280 & 71.440 & 69.745 & -2.372 & 0.040 \\
\hline 315.405 & 8.21 & 10.617 & 73.050 & 71.583 & -2.008 & 0.040 \\
\hline 314.630 & 8.21 & 10.719 & 73.700 & 72.149 & -2.104 & 0.040 \\
\hline 313.835 & 8.21 & 10.820 & 73.910 & 72.723 & -1.606 & 0.040 \\
\hline 313.032 & 8.21 & 10.920 & 74.150 & 73.309 & -1.135 & 0.040 \\
\hline 316.074 & 9.43 & 11.114 & 76.570 & 74.760 & -2.364 & 0.040 \\
\hline 315.284 & 9.43 & 11.197 & 77.150 & 75.275 & -2.430 & 0.040 \\
\hline 314.756 & 9.43 & 11.251 & 77.770 & 75.618 & -2.767 & 0.040 \\
\hline 313.377 & 9.43 & 11.390 & 77.120 & 76.515 & -0.784 & 0.040 \\
\hline 316.248 & 11.27 & 11.702 & 80.270 & 79.119 & -1.434 & 0.040 \\
\hline 315.470 & 11.27 & 11.766 & 80.750 & 79.567 & -1.465 & 0.040 \\
\hline 314.704 & 11.27 & 11.828 & 80.820 & 80.012 & -0.999 & 0.040 \\
\hline 314.003 & 11.27 & 11.885 & 81.230 & 80.421 & -0.996 & 0.040 \\
\hline 316.056 & 13.58 & 12.252 & 84.370 & 83.801 & -0.675 & 0.040 \\
\hline 315.417 & 13.58 & 12.295 & 84.820 & 84.136 & -0.807 & 0.040 \\
\hline 314.603 & 13.58 & 12.350 & 85.090 & 84.564 & -0.618 & 0.040 \\
\hline 314.061 & 13.58 & 12.386 & 85.350 & 84.850 & -0.586 & 0.040 \\
\hline 316.350 & 16.87 & 12.794 & 90.060 & 89.078 & -1.090 & 0.040 \\
\hline 315.570 & 16.87 & 12.838 & 90.570 & 89.453 & -1.233 & 0.040 \\
\hline 314.803 & 16.87 & 12.881 & 90.550 & 89.824 & -0.802 & 0.040 \\
\hline 314.136 & 16.87 & 12.918 & 90.540 & 90.147 & -0.435 & 0.040 \\
\hline 314.309 & 21.23 & 13.445 & 96.840 & 96.065 & -0.800 & 0.030 \\
\hline 316.080 & 21.23 & 13.360 & 95.980 & 95.265 & -0.744 & 0.030 \\
\hline 315.357 & 21.23 & 13.395 & 96.550 & 95.591 & -0.993 & 0.030 \\
\hline 314.639 & 21.23 & 13.430 & 96.490 & 95.919 & -0.592 & 0.030 \\
\hline 315.862 & 26.24 & 13.860 & 102.390 & 101.398 & -0.969 & 0.030 \\
\hline 315.135 & 26.24 & 13.891 & 102.550 & 101.711 & -0.818 & 0.030 \\
\hline
\end{tabular}


Table 15. THERMAL CONDUCTIVITY AT ELEVATED PRESSURES (continued)

Data from Roder [103] (continued)

\begin{tabular}{|c|c|c|c|c|c|c|}
\hline $\begin{array}{l}\mathrm{T} \\
\mathrm{K}\end{array}$ & $\begin{array}{c}\mathrm{P} \\
\mathrm{MPa}\end{array}$ & $\stackrel{\rho}{\mathrm{mol}} \cdot \mathrm{dm}^{-3}$ & $\begin{array}{c}\lambda, \underset{\mathrm{mW} \cdot \mathrm{m}^{-1} \cdot \mathrm{K}^{-1}}{\operatorname{expt}}\end{array}$ & $\begin{array}{c}\lambda, \quad \text { calc } \\
\mathrm{mW} \cdot \mathrm{m}^{-1} \cdot \mathrm{K}^{-1}\end{array}$ & $\begin{array}{c}\text { Dev } \\
8\end{array}$ & wt \\
\hline 314.700 & 26.24 & 13.909 & 102.730 & 101.899 & -0.808 & 0.030 \\
\hline 313.834 & 26.24 & 13.946 & 102.730 & 102.277 & -0.441 & 0.030 \\
\hline 314.272 & 32.90 & 14.435 & 109.700 & 109.153 & -0.499 & 0.030 \\
\hline 315.836 & 32.90 & 14.377 & 109.580 & 108.501 & -0.985 & 0.030 \\
\hline 315.195 & 32.90 & 14.401 & 109.840 & 108.768 & -0.976 & 0.030 \\
\hline 314.436 & 32.90 & 14.429 & 109.830 & 109.083 & -0.680 & 0.030 \\
\hline 316.300 & 41.60 & 14.898 & 117.750 & 116.564 & -1.007 & 0.030 \\
\hline 315.651 & 41.60 & 14.920 & 117.830 & 116.825 & -0.853 & 0.030 \\
\hline 314.843 & 41.60 & 14.946 & 118.040 & 117.150 & -0.754 & 0.030 \\
\hline 314.511 & 41.59 & 14.957 & 118.150 & 117.281 & -0.735 & 0.030 \\
\hline 315.977 & 52.11 & 15.431 & 126.950 & 125.667 & -1.010 & 0.030 \\
\hline 315.292 & 52.10 & 15.450 & 127.020 & 125.928 & -0.860 & 0.030 \\
\hline 314.743 & 52.09 & 15.466 & 127.190 & 126.138 & -0.827 & 0.030 \\
\hline 314.133 & 52.07 & 15.483 & 127.370 & 126.368 & -0.786 & 0.030 \\
\hline 315.536 & 64.28 & 15.938 & 136.180 & 135.317 & -0.634 & 0.030 \\
\hline 315.223 & 64.27 & 15.945 & 136.370 & 135.428 & -0.691 & 0.030 \\
\hline 314.630 & 64.27 & 15.961 & 136.700 & 135.661 & -0.760 & 0.030 \\
\hline 314.152 & 64.27 & 15.974 & 136.950 & 135.851 & -0.802 & 0.030 \\
\hline 315.798 & 68.12 & 16.069 & 139.040 & 138.051 & -0.712 & 0.030 \\
\hline 315.147 & 68.12 & 16.086 & 139.230 & 138.305 & -0.664 & 0.030 \\
\hline 314.575 & 68.12 & 16.101 & 139.850 & 138.529 & -0.945 & 0.030 \\
\hline 313.879 & 68.12 & 16.119 & 140.240 & 138.803 & -1.025 & 0.030 \\
\hline 315.282 & 2.58 & 1.207 & 26.200 & 26.408 & 0.793 & 0.000 \\
\hline 314.669 & 2.58 & 1.212 & 26.050 & 26.349 & 1.147 & 0.000 \\
\hline 314.116 & 2.58 & 1.216 & 26.010 & 26.296 & 1.099 & 0.000 \\
\hline 313.470 & 2.58 & 1.221 & 26.270 & 26.235 & -0.135 & 0.000 \\
\hline 313.851 & 5.39 & 4.645 & 51.310 & 49.189 & -4.134 & 0.000 \\
\hline 313.411 & 5.39 & 4.761 & 52.410 & 50.614 & -3.427 & 0.000 \\
\hline 313.264 & 5.39 & 4.804 & 53.970 & 51.148 & -5.229 & 0.000 \\
\hline 313.035 & 5.39 & 4.874 & 55.280 & 52.049 & -5.846 & 0.000 \\
\hline 312.901 & 5.39 & 4.918 & 56.540 & 52.619 & -6.936 & 0.000 \\
\hline 312.630 & 5.39 & 5.014 & 57.730 & 53.884 & -6.661 & 0.000 \\
\hline 313.283 & 5.49 & 5.319 & 60.080 & 56.525 & -5.918 & 0.000 \\
\hline 313.023 & 5.49 & 5.443 & 61.830 & 58.045 & -6.121 & 0.000 \\
\hline 312.802 & 5.49 & 5.560 & 63.530 & 59.474 & -6.385 & 0.000 \\
\hline 312.702 & 5.49 & 5.618 & 64.540 & 60.162 & -6.783 & 0.000 \\
\hline
\end{tabular}


Table 15. THERMAL CONDUCTIVITY AT ELEVATED PRESSURES (continued)

Data from Roder [103] (continued)

\begin{tabular}{|c|c|c|c|c|c|c|}
\hline $\begin{array}{l}\mathrm{T} \\
\mathrm{K}\end{array}$ & $\begin{array}{c}\mathrm{P} \\
\mathrm{MPa}\end{array}$ & $\underset{\mathrm{mol}}{\rho} \cdot \mathrm{dm}^{-3}$ & $\underset{\mathrm{m} W \cdot \mathrm{m}^{-1} \cdot \mathrm{K}^{-1}}{\operatorname{expt}}$ & $\begin{array}{c}\lambda, \operatorname{calc} \\
\mathrm{mW} \cdot \mathrm{m}^{-1} \cdot \mathrm{K}^{-1}\end{array}$ & $\begin{array}{l}\text { Dev } \\
\frac{\gamma}{\delta}\end{array}$ & wt \\
\hline 312.541 & 5.49 & 5.717 & 65.810 & 61.326 & -6.814 & 0.000 \\
\hline 313.209 & 5.57 & 5.864 & 65.120 & 61.678 & -5.285 & 0.000 \\
\hline 313.050 & 5.57 & 5.970 & 66.270 & 62.744 & -5.321 & 0.000 \\
\hline 312.735 & 5.57 & 6.212 & 67.580 & 64.952 & -3.888 & 0.000 \\
\hline 312.613 & 5.57 & 6.312 & 68.080 & 65.773 & -3.388 & 0.000 \\
\hline 312.552 & 5.57 & 6.363 & 69.420 & 66.176 & -4.674 & 0.000 \\
\hline 313.181 & 5.60 & 6.126 & 66.700 & 63.679 & -4.530 & 0.000 \\
\hline 313.013 & 5.60 & 6.252 & 67.570 & 64.752 & -4.171 & 0.000 \\
\hline 312.859 & 5.60 & 6.375 & 68.710 & 65.714 & -4.361 & 0.000 \\
\hline 312.694 & 5.60 & 6.516 & 69.290 & 66.696 & -3.744 & 0.000 \\
\hline 312.525 & 5.60 & 6.668 & 68.960 & 67.621 & -1.941 & 0.000 \\
\hline 313.310 & 5.63 & 6.275 & 67.570 & 64.431 & -4.646 & 0.000 \\
\hline 313.221 & 5.63 & 6.343 & 68.680 & 64.955 & -5.423 & 0.000 \\
\hline 312.929 & 5.63 & 6.582 & 69.200 & 66.584 & -3.780 & 0.000 \\
\hline 312.704 & 5.63 & 6.781 & 69.740 & 67.681 & -2.953 & 0.000 \\
\hline 312.570 & 5.63 & 6.905 & 69.890 & 68.241 & -2.359 & 0.000 \\
\hline 313.253 & 5.74 & 7.144 & 68.580 & 67.554 & -1.497 & 0.000 \\
\hline 312.657 & 5.74 & 7.642 & 68.440 & 68.404 & -0.053 & 0.000 \\
\hline 313.187 & 5.74 & 7.207 & 69.000 & 67.718 & -1.859 & 0.000 \\
\hline 312.924 & 5.74 & 7.428 & 68.680 & 68.177 & -0.732 & 0.000 \\
\hline 312.745 & 5.74 & 7.577 & 68.300 & 68.349 & 0.072 & 0.000 \\
\hline 322.509 & 0.21 & 0.080 & 24.490 & 24.290 & -0.817 & 0.040 \\
\hline 325.164 & 0.21 & 0.079 & 24.870 & 24.655 & -0.865 & 0.040 \\
\hline 324.231 & 0.21 & 0.080 & 24.880 & 24.526 & -1.422 & 0.030 \\
\hline 323.528 & 0.21 & 0.080 & 24.840 & 24.430 & -1.652 & 0.030 \\
\hline 322.741 & 0.21 & 0.080 & 24.170 & 24.322 & 0.628 & 0.200 \\
\hline 322.892 & 0.78 & 0.304 & 25.020 & 24.836 & -0.736 & 0.050 \\
\hline 324.818 & 0.78 & 0.302 & 25.510 & 25.095 & -1.628 & 0.040 \\
\hline 324.104 & 0.78 & 0.303 & 25.440 & 24.999 & -1.734 & 0.040 \\
\hline 323.142 & 0.78 & 0.304 & 25.290 & 24.870 & -1.660 & 0.040 \\
\hline 324.110 & 1.33 & 0.535 & 26.160 & 25.560 & -2.295 & 0.040 \\
\hline 324.425 & 1.33 & 0.534 & 25.890 & 25.600 & -1.119 & 0.050 \\
\hline 325.134 & 1.33 & 0.533 & 26.210 & 25.693 & -1.971 & 0.050 \\
\hline 325.667 & 1.33 & 0.532 & 26.220 & 25.763 & -1.745 & 0.050 \\
\hline 323.528 & 2.42 & 1.066 & 27.510 & 26.980 & -1.928 & 0.050 \\
\hline 324.158 & 2.42 & 1.063 & 27.710 & 27.050 & -2.383 & 0.050 \\
\hline
\end{tabular}


Table 15. THERMAL CONDUCTIVITY AT ELEVATED PRESSURES (continued)

Data from Roder [103] (continued)

\begin{tabular}{|c|c|c|c|c|c|c|}
\hline $\begin{array}{l}\mathrm{T} \\
\mathrm{K}\end{array}$ & $\begin{array}{c}\mathrm{P} \\
\mathrm{MPa}\end{array}$ & $\begin{array}{c}\rho \\
\mathrm{mol} \cdot \mathrm{dm}^{-3}\end{array}$ & $\begin{array}{c}\lambda, \underset{\mathrm{mW}}{\exp \mathrm{m}^{-1} \cdot \mathrm{K}^{-1}}\end{array}$ & $\begin{array}{c}\lambda, \operatorname{calc} \\
\mathrm{mW} \cdot \mathrm{m}^{-1} \cdot \mathrm{K}^{-1}\end{array}$ & $\begin{array}{c}\text { Dev } \\
8\end{array}$ & wt \\
\hline 324.601 & 2.42 & 1.060 & 27.800 & 27.099 & -2.521 & 0.050 \\
\hline 325.203 & 2.42 & 1.057 & 27.590 & 27.167 & -1.533 & 0.050 \\
\hline 326.009 & 2.42 & 1.053 & 27.720 & 27.258 & -1.667 & 0.050 \\
\hline 323.991 & 3.09 & 1.442 & 28.710 & 28.292 & -1.457 & 0.050 \\
\hline 325.322 & 3.09 & 1.430 & 28.730 & 28.413 & -1.103 & 0.050 \\
\hline 325.307 & 3.09 & 1.430 & 28.980 & 28.412 & -1.961 & 0.050 \\
\hline 325.584 & 3.09 & 1.427 & 28.880 & 28.437 & -1.533 & 0.050 \\
\hline 324.024 & 4.00 & 2.061 & 31.260 & 30.759 & -1.601 & 0.050 \\
\hline 324.589 & 4.00 & 2.053 & 31.270 & 30.783 & -1.558 & 0.050 \\
\hline 325.140 & 4.00 & 2.043 & 31.370 & 30.800 & -1.816 & 0.050 \\
\hline 325.733 & 4.00 & 2.033 & 31.350 & 30.821 & -1.688 & 0.050 \\
\hline 324.464 & 4.44 & 2.413 & 33.000 & 32.435 & -1.711 & 0.050 \\
\hline 324.965 & 4.44 & 2.403 & 33.010 & 32.431 & -1.754 & 0.050 \\
\hline 325.494 & 4.44 & 2.388 & 33.280 & 32.411 & -2.611 & 0.050 \\
\hline 326.066 & 4.44 & 2.376 & 33.000 & 32.410 & -1.789 & 0.050 \\
\hline 323.930 & 4.93 & 2.913 & 35.490 & 35.007 & -1.360 & 0.050 \\
\hline 324.510 & 4.93 & 2.890 & 35.550 & 34.920 & -1.771 & 0.050 \\
\hline 325.048 & 4.93 & 2.871 & 35.520 & 34.851 & -1.882 & 0.050 \\
\hline 325.295 & 4.93 & 2.862 & 35.430 & 34.819 & -1.725 & 0.050 \\
\hline 324.150 & 5.35 & 3.420 & 38.710 & 38.022 & -1.777 & 0.050 \\
\hline 324.621 & 5.35 & 3.388 & 38.570 & 37.838 & -1.897 & 0.050 \\
\hline 325.147 & 5.35 & 3.359 & 38.550 & 37.672 & -2.277 & 0.050 \\
\hline 326.750 & 5.35 & 3.276 & 38.170 & 37.240 & -2.438 & 0.050 \\
\hline 323.776 & 5.66 & 3.912 & 41.750 & 41.229 & -1.248 & 0.050 \\
\hline 324.289 & 5.66 & 3.868 & 41.460 & 40.916 & -1.312 & 0.050 \\
\hline 324.542 & 5.66 & 3.848 & 41.300 & 40.781 & -1.257 & 0.050 \\
\hline 324.907 & 5.66 & 3.819 & 41.230 & 40.580 & -1.578 & 0.050 \\
\hline 323.874 & 5.89 & 4.316 & 44.790 & 44.009 & -1.744 & 0.050 \\
\hline 324.053 & 5.89 & 4.296 & 44.440 & 43.848 & -1.333 & 0.050 \\
\hline 324.312 & 5.89 & 4.268 & 44.100 & 43.636 & -1.053 & 0.050 \\
\hline 324.771 & 5.89 & 4.219 & 43.770 & 43.258 & -1.169 & 0.050 \\
\hline 323.525 & 6.13 & 4.898 & 48.730 & 48.136 & -1.218 & 0.040 \\
\hline 323.874 & 6.13 & 4.839 & 48.410 & 47.662 & -1.545 & 0.040 \\
\hline 324.345 & 6.13 & 4.765 & 47.850 & 47.065 & -1.640 & 0.040 \\
\hline 324.549 & 6.13 & 4.734 & 47.080 & 46.821 & -0.550 & 0.050 \\
\hline 323.904 & 6.26 & 5.171 & 51.040 & 49.917 & -2.200 & 0.040 \\
\hline
\end{tabular}


Table 15. THERMAL CONDUCTIVITY AT ELEVATED PRESSURES (continued)

Data from Roder [103] (continued)

\begin{tabular}{|c|c|c|c|c|c|c|}
\hline $\begin{array}{l}\mathrm{T} \\
\mathrm{K}\end{array}$ & $\begin{array}{c}\mathrm{P} \\
\mathrm{MPa}\end{array}$ & $\begin{array}{c}\rho \\
\mathrm{mol} \cdot \mathrm{dm}^{-3}\end{array}$ & $\begin{array}{c}\lambda, \underset{m W}{\operatorname{expt}} \mathrm{m}^{-1} \cdot \mathrm{K}^{-1} \\
\mathrm{~m}\end{array}$ & $\begin{array}{c}\lambda, \text { calc } \\
\mathrm{mW} \cdot \mathrm{m}^{-1} \cdot \mathrm{K}^{-1}\end{array}$ & $\begin{array}{c}\text { Dev } \\
8\end{array}$ & wt \\
\hline $\begin{array}{l}324.325 \\
324.505\end{array}$ & $\begin{array}{l}6.26 \\
6.26\end{array}$ & $\begin{array}{l}5.091 \\
5.058\end{array}$ & $\begin{array}{l}50.190 \\
50.210\end{array}$ & $\begin{array}{l}49.286 \\
49.029\end{array}$ & $\begin{array}{l}-1.801 \\
-2.353\end{array}$ & $\begin{array}{l}0.040 \\
0.040\end{array}$ \\
\hline $\begin{array}{l}324.897 \\
325.266 \\
325.846 \\
324.760 \\
324.005\end{array}$ & $\begin{array}{l}6.26 \\
6.26 \\
6.27 \\
6.27 \\
6.27\end{array}$ & $\begin{array}{l}4.990 \\
4.929 \\
4.861 \\
5.038 \\
5.178\end{array}$ & $\begin{array}{l}49.510 \\
48.660 \\
49.520 \\
49.680 \\
50.000\end{array}$ & $\begin{array}{l}48.494 \\
48.019 \\
47.473 \\
48.839 \\
49.936\end{array}$ & $\begin{array}{l}-2.053 \\
-1.317 \\
-4.135 \\
-1.692 \\
-0.127\end{array}$ & $\begin{array}{l}0.040 \\
0.040 \\
0.040 \\
0.040 \\
0.050\end{array}$ \\
\hline $\begin{array}{l}323.494 \\
324.172 \\
326.309 \\
325.022 \\
324.185\end{array}$ & $\begin{array}{l}6.27 \\
6.46 \\
6.46 \\
6.46 \\
6.46\end{array}$ & $\begin{array}{l}5.282 \\
5.667 \\
5.221 \\
5.476 \\
5.667\end{array}$ & $\begin{array}{l}50.460 \\
54.020 \\
51.230 \\
52.000 \\
53.330\end{array}$ & $\begin{array}{l}50.757 \\
52.974 \\
49.714 \\
51.584 \\
52.968\end{array}$ & $\begin{array}{r}0.589 \\
-1.937 \\
-2.958 \\
-0.801 \\
-0.678\end{array}$ & $\begin{array}{l}0.050 \\
0.040 \\
0.040 \\
0.040 \\
0.040\end{array}$ \\
\hline $\begin{array}{l}323.175 \\
325.811 \\
324.856 \\
323.931 \\
323.040\end{array}$ & $\begin{array}{l}6.46 \\
6.78 \\
6.78 \\
6.79 \\
6.79\end{array}$ & $\begin{array}{l}5.930 \\
6.187 \\
6.448 \\
6.731 \\
7.021\end{array}$ & $\begin{array}{l}54.950 \\
56.400 \\
57.420 \\
58.270 \\
59.100\end{array}$ & $\begin{array}{l}54.826 \\
55.242 \\
56.740 \\
58.206 \\
59.543\end{array}$ & $\begin{array}{r}-0.225 \\
-2.053 \\
-1.183 \\
-0.110 \\
0.749\end{array}$ & $\begin{array}{l}0.040 \\
0.040 \\
0.040 \\
0.040 \\
0.040\end{array}$ \\
\hline $\begin{array}{l}325.765 \\
324.914 \\
324.042 \\
323.136 \\
325.695\end{array}$ & $\begin{array}{l}6.88 \\
6.88 \\
6.88 \\
6.88 \\
7.18\end{array}$ & $\begin{array}{l}6.459 \\
6.705 \\
6.972 \\
7.265 \\
7.274\end{array}$ & $\begin{array}{l}57.700 \\
58.950 \\
59.870 \\
60.560 \\
61.250\end{array}$ & $\begin{array}{l}56.487 \\
57.753 \\
58.999 \\
60.200 \\
59.376\end{array}$ & $\begin{array}{l}-2.103 \\
-2.031 \\
-1.455 \\
-0.594 \\
-3.060\end{array}$ & $\begin{array}{l}0.040 \\
0.040 \\
0.040 \\
0.040 \\
0.040\end{array}$ \\
\hline $\begin{array}{l}324.714 \\
323.969 \\
323.059 \\
325.677 \\
324.723\end{array}$ & $\begin{array}{l}7.18 \\
7.18 \\
7.18 \\
7.41 \\
7.41\end{array}$ & $\begin{array}{l}7.558 \\
7.774 \\
8.037 \\
7.805 \\
8.055\end{array}$ & $\begin{array}{l}61.850 \\
62.700 \\
63.010 \\
62.980 \\
63.410\end{array}$ & $\begin{array}{l}60.422 \\
61.147 \\
61.961 \\
60.796 \\
61.614\end{array}$ & $\begin{array}{l}-2.309 \\
-2.477 \\
-1.666 \\
-3.467 \\
-2.833\end{array}$ & $\begin{array}{l}0.040 \\
0.040 \\
0.040 \\
0.040 \\
0.040\end{array}$ \\
\hline $\begin{array}{l}323.985 \\
323.084 \\
326.504 \\
325.489 \\
324.602\end{array}$ & $\begin{array}{l}7.41 \\
7.41 \\
7.62 \\
7.62 \\
7.62\end{array}$ & $\begin{array}{l}8.247 \\
8.475 \\
7.984 \\
8.228 \\
8.439\end{array}$ & $\begin{array}{l}63.860 \\
64.240 \\
63.380 \\
64.100 \\
64.550\end{array}$ & $\begin{array}{l}62.217 \\
62.919 \\
61.092 \\
61.904 \\
62.592\end{array}$ & $\begin{array}{l}-2.572 \\
-2.056 \\
-3.609 \\
-3.426 \\
-3.033\end{array}$ & $\begin{array}{l}0.040 \\
0.040 \\
0.040 \\
0.040 \\
0.040\end{array}$ \\
\hline $\begin{array}{l}323.783 \\
326.641 \\
325.669 \\
324.766\end{array}$ & $\begin{array}{l}7.62 \\
8.06 \\
8.06 \\
8.06\end{array}$ & $\begin{array}{l}8.628 \\
8.637 \\
8.830 \\
9.005\end{array}$ & $\begin{array}{l}64.470 \\
65.620 \\
66.230 \\
66.550\end{array}$ & $\begin{array}{l}63.208 \\
62.943 \\
63.637 \\
64.284\end{array}$ & $\begin{array}{l}-1.957 \\
-4.080 \\
-3.915 \\
-3.405\end{array}$ & $\begin{array}{l}0.040 \\
0.040 \\
0.040 \\
0.040\end{array}$ \\
\hline
\end{tabular}


Table 15. THERMAL CONDUCTIVITY AT ELEVATED PRESSURES (continued)

Data from Roder [103] (continued)

\begin{tabular}{|c|c|c|c|c|c|c|}
\hline $\begin{array}{l}\mathrm{T} \\
\mathrm{K}\end{array}$ & $\begin{array}{c}\mathrm{P} \\
\mathrm{MPa}\end{array}$ & $\begin{array}{c}\rho \\
\mathrm{mol} \cdot \mathrm{dm}^{-3}\end{array}$ & 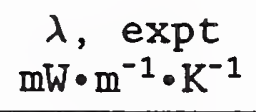 & $\begin{array}{c}\lambda, \quad \text { calc } \\
\mathrm{mW} \cdot \mathrm{m}^{-1} \cdot \mathrm{K}^{-1}\end{array}$ & $\begin{array}{c}\text { Dev } \\
z\end{array}$ & wt \\
\hline 323.950 & 8.06 & 9.160 & 66.690 & 64.870 & -2.730 & 0.040 \\
\hline 327.097 & 8.77 & 9.313 & 68.690 & 65.407 & -4.779 & 0.040 \\
\hline 326.417 & 8.77 & 9.420 & 69.240 & 65.861 & -4.880 & 0.040 \\
\hline 325.389 & 8.77 & 9.577 & 69.690 & 66.544 & -4.514 & 0.040 \\
\hline 324.053 & 8.77 & 9.775 & 69.520 & 67.438 & -2.995 & 0.040 \\
\hline 328.172 & 9.52 & 9.733 & 71.790 & 67.353 & -6.180 & 0.000 \\
\hline 327.339 & 9.52 & 9.842 & 72.450 & 67.866 & -6.326 & 0.000 \\
\hline 327.636 & 9.52 & 9.804 & 73.380 & 67.687 & -7.759 & 0.000 \\
\hline 328.307 & 9.52 & 9.717 & 75.110 & 67.277 & -10.428 & 0.000 \\
\hline 327.433 & 10.69 & 10.461 & 74.640 & 71.318 & -4.450 & 0.040 \\
\hline 327.360 & 10.69 & 10.468 & 75.290 & 71.357 & -5.224 & 0.040 \\
\hline 325.038 & 10.69 & 10.706 & 74.770 & 72.677 & -2.799 & 0.040 \\
\hline 323.933 & 10.69 & 10.817 & 74.460 & 73.314 & -1.539 & 0.040 \\
\hline 327.970 & 11.99 & 10.921 & 78.030 & 74.327 & -4.746 & 0.040 \\
\hline 327.699 & 11.99 & 10.945 & 79.260 & 74.468 & -6.046 & 0.000 \\
\hline 326.348 & 11.99 & 11.063 & 79.110 & 75.177 & -4.972 & 0.040 \\
\hline 325.540 & 11.99 & 11.133 & 78.700 & 75.603 & -3.936 & 0.040 \\
\hline 324.688 & 11.99 & 11.206 & 78.780 & 76.056 & -3.457 & 0.040 \\
\hline 324.292 & 14.47 & 11.876 & 82.320 & 81.291 & -1.250 & 0.040 \\
\hline 326.347 & 14.47 & 11.735 & 81.240 & 80.310 & -1.145 & 0.040 \\
\hline 325.441 & 14.47 & 11.798 & 81.480 & 80.742 & -0.905 & 0.040 \\
\hline 324.670 & 14.47 & 11.850 & 81.590 & 81.111 & -0.587 & 0.040 \\
\hline 324.214 & 17.22 & 12.396 & 86.230 & 85.969 & -0.303 & 0.040 \\
\hline 324.757 & 17.22 & 12.365 & 86.650 & 85.725 & -1.068 & 0.040 \\
\hline 326.145 & 17.22 & 12.284 & 85.750 & 85.111 & -0.746 & 0.040 \\
\hline 325.425 & 17.22 & 12.326 & 86.080 & 85.427 & -0.758 & 0.040 \\
\hline 324.063 & 20.96 & 12.938 & 92.720 & 91.435 & -1.386 & 0.040 \\
\hline 326.081 & 20.96 & 12.837 & 91.900 & 90.591 & -1.424 & 0.040 \\
\hline 325.404 & 20.96 & 12.871 & 92.270 & 90.873 & -1.514 & 0.040 \\
\hline 325.014 & 20.96 & 12.890 & 92.960 & 91.036 & -2.070 & 0.040 \\
\hline 323.871 & 25.56 & 13.454 & 98.710 & 97.283 & -1.446 & 0.030 \\
\hline 325.636 & 25.56 & 13.378 & 97.610 & 96.582 & -1.053 & 0.030 \\
\hline 324.837 & 25.56 & 13.413 & 97.890 & 96.900 & -1.011 & 0.030 \\
\hline 324.029 & 25.56 & 13.448 & 97.830 & 97.225 & -0.618 & 0.030 \\
\hline 326.318 & 31.50 & 13.877 & 104.130 & 102.911 & -1.171 & 0.030 \\
\hline 325.408 & 31.50 & 13.912 & 104.420 & 103.258 & -1.113 & 0.030 \\
\hline
\end{tabular}


Table 15. THERMAL CONDUCTIVITY AT ELEVATED PRESSURES (continued)

Data from Roder [103] (continued)

\begin{tabular}{ccccccc}
\hline $\mathrm{T}$ & $\begin{array}{c}\mathrm{P} \\
\mathrm{MPa}\end{array}$ & $\begin{array}{c}\rho \\
\mathrm{mo} \cdot \mathrm{dm}^{-3}\end{array}$ & $\begin{array}{c}\lambda, \begin{array}{c}\text { expt } \\
\mathrm{mW} \cdot \mathrm{m}^{-1} \cdot \mathrm{K}^{-1}\end{array} \\
\mathrm{MW} \cdot \mathrm{m}^{-1} \cdot \mathrm{K}^{-1}\end{array}$ & $\begin{array}{c}\text { Dev } \\
\text { o }\end{array}$ & wt \\
\hline 324.644 & 31.50 & 13.942 & 104.800 & 103.550 & -1.192 & 0.030 \\
324.054 & 31.50 & 13.964 & 104.790 & 103.780 & -0.964 & 0.030 \\
323.917 & 38.52 & 14.460 & 112.650 & 110.770 & -1.669 & 0.030 \\
326.025 & 38.52 & 14.388 & 111.360 & 109.979 & -1.240 & 0.030 \\
325.271 & 38.52 & 14.414 & 111.610 & 110.260 & -1.209 & 0.030 \\
& & & & & & \\
323.442 & 38.52 & 14.477 & 112.270 & 110.950 & -1.176 & 0.030 \\
323.767 & 47.48 & 14.973 & 120.370 & 118.821 & -1.287 & 0.030 \\
327.091 & 47.48 & 14.871 & 118.640 & 117.600 & -0.877 & 0.030 \\
324.947 & 47.48 & 14.937 & 119.840 & 118.383 & -1.216 & 0.030 \\
324.170 & 47.48 & 14.961 & 120.200 & 118.671 & -1.272 & 0.030 \\
326.213 & 51.75 & 15.111 & 123.220 & 121.495 & -1.400 & 0.030 \\
325.387 & 51.76 & 15.136 & 123.340 & 121.800 & -1.248 & 0.030 \\
324.761 & 51.76 & 15.155 & 123.570 & 122.030 & -1.246 & 0.030 \\
324.049 & 51.76 & 15.175 & 123.480 & 122.290 & -0.963 & 0.030 \\
325.860 & 64.21 & 15.658 & 133.130 & 131.371 & -1.321 & 0.030 \\
325.163 & 64.21 & 15.677 & 133.280 & 131.622 & -1.244 & 0.030 \\
324.496 & 64.20 & 15.694 & 133.300 & 131.864 & -1.078 & 0.030 \\
324.124 & 64.20 & 15.704 & 133.310 & 131.997 & -0.985 & 0.030 \\
325.753 & 67.17 & 15.774 & 134.990 & 133.608 & -1.024 & 0.030 \\
325.167 & 67.18 & 15.790 & 135.150 & 133.824 & -0.981 & 0.030 \\
324.588 & 67.17 & 15.805 & 135.150 & 134.034 & -0.826 & 0.030 \\
323.892 & 67.17 & 15.823 & 135.300 & 134.288 & -0.748 & 0.030
\end{tabular}

Comparisons based on experimental pressures:

Number of Points [103] 797

$$
\begin{aligned}
& \mathrm{AAD}=1.19 \quad \mathrm{BIAS} \%=-0.76 \quad \mathrm{RMS} \%=1.60 \\
& \mathrm{AAD}=0.81 \quad \mathrm{BIAS}=-0.44 \text { RMS }=1.13 \mathrm{~mW} \cdot \mathrm{m}^{-1} \cdot \mathrm{K}^{-1}
\end{aligned}
$$

Weighted Data:

Number of Points [103] 752

$$
\begin{aligned}
& \mathrm{AAD} \%=1.01 \quad \mathrm{BIAS} \%=-0.61 \quad \mathrm{RMS} \%=1.25 \\
& \mathrm{AAD}=0.70 \text { BIAS }=-0.33 \text { RMS }=0.92 \mathrm{~mW} \cdot \mathrm{m}^{-1} \cdot \mathrm{K}^{-1}
\end{aligned}
$$


Table 15. THERMAL CONDUCTIVITY AT ELEVATED PRESSURES (continued)

Data from Roder [103] (continued)

Comparisons based on experimental densities:

Number of Points [103] 797

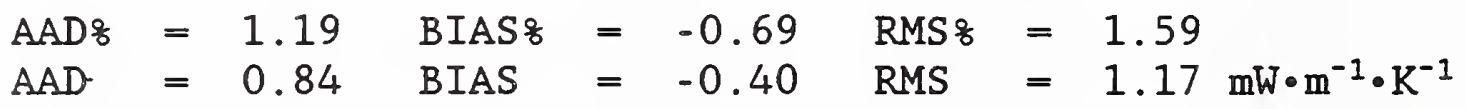

Weighted Data:

Number of Points [103] 752

$\mathrm{AAD} \%=1.03 \quad \mathrm{BIAS} \%=-0.56 \quad \mathrm{RMS} \%=1.28$

$\mathrm{AAD}=0.74$ BIAS $=-0.30 \quad \mathrm{RMS}=0.99 \mathrm{~mW} \cdot \mathrm{m}^{-1} \cdot \mathrm{K}^{-1}$

Data from Tufeu et al. [88]

( $P$ not given)

\begin{tabular}{|c|c|c|c|c|c|c|}
\hline $\begin{array}{l}\mathrm{T} \\
\mathrm{K}\end{array}$ & $\begin{array}{c}\mathrm{P} \\
\mathrm{MPa}\end{array}$ & $\stackrel{\rho}{\mathrm{mol} \cdot \mathrm{dm}^{-3}}$ & 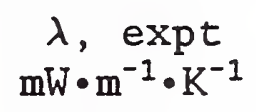 & $\begin{array}{c}\lambda, \quad \operatorname{calc} \\
\mathrm{mW} \cdot \mathrm{m}^{-1} \cdot \mathrm{K}^{-1}\end{array}$ & $\begin{array}{c}\text { Dev } \\
\frac{8}{8}\end{array}$ & wt \\
\hline 500.300 & - & 0.121 & 52.150 & 54.008 & 3.562 & 0.000 \\
\hline 500.300 & - & 0.251 & 52.500 & 54.198 & 3.235 & 0.000 \\
\hline 500.300 & - & 0.648 & 53.000 & 54.849 & 3.489 & 0.000 \\
\hline 500.300 & - & 1.264 & 54.450 & 56.050 & 2.938 & 0.000 \\
\hline 500.300 & - & 1.929 & 56.150 & 57.596 & 2.575 & 0.000 \\
\hline 500.300 & - & 2.627 & 58.000 & 59.472 & 2.537 & 0.000 \\
\hline 500.300 & - & 3.359 & 60.050 & 61.681 & 2.716 & 0.000 \\
\hline 500.300 & - & 4.024 & 62.600 & 63.878 & 2.041 & 0.000 \\
\hline 500.300 & - & 4.756 & 65.000 & 66.479 & 2.275 & 0.000 \\
\hline 500.300 & - & 5.421 & 67.800 & 68.999 & 1.769 & 0.000 \\
\hline 500.300 & - & 6.019 & 70.150 & 71.397 & 1.778 & 0.000 \\
\hline 500.300 & - & 6.718 & 72.800 & 74.365 & 2.150 & 0.000 \\
\hline 500.300 & - & 7.150 & 74.750 & 76.308 & 2.084 & 0.000 \\
\hline 500.300 & - & 7.582 & 76.700 & 78.344 & 2.144 & 0.000 \\
\hline 500.300 & - & 7.715 & 77.150 & 78.992 & 2.387 & 0.000 \\
\hline 307.050 & - & 6.870 & 99.800 & 93.646 & -6.166 & 0.000 \\
\hline 307.800 & - & 6.870 & 91.000 & 85.638 & -5.893 & 0.000 \\
\hline 311.800 & - & 6.870 & 73.000 & 69.629 & -4.618 & 0.000 \\
\hline 313.350 & - & 6.870 & 67.800 & 66.884 & -1.350 & 0.000 \\
\hline 322.750 & - & 6.870 & 58.500 & 59.179 & 1.161 & 0.000 \\
\hline 343.150 & - & 6.870 & 56.600 & 55.762 & -1.481 & 0.000 \\
\hline
\end{tabular}


Table 15. THERMAL CONDUCTIVITY AT ELEVATED PRESSURES (continued)

Data from Tufeu et al. [88] (continued)

\begin{tabular}{|c|c|c|c|c|c|c|}
\hline $\begin{array}{l}\mathrm{T} \\
\mathrm{K}\end{array}$ & $\begin{array}{c}\mathrm{P} \\
\mathrm{MPa}\end{array}$ & $\underset{\mathrm{mol} \cdot \stackrel{\rho}{\mathrm{dm}^{-3}}}{ }$ & $\begin{array}{c}\lambda, \underset{\mathrm{mW} \cdot \mathrm{m}^{-1} \cdot \mathrm{K}^{-1}}{\exp t} \\
\end{array}$ & $\begin{array}{c}\lambda, \quad \text { calc } \\
\mathrm{mW} \cdot \mathrm{m}^{-1} \cdot \mathrm{K}^{-1}\end{array}$ & $\begin{array}{c}\text { Dev } \\
\frac{8}{8}\end{array}$ & wt \\
\hline 406.150 & - & 6.870 & 61.000 & 60.745 & -0.419 & 0.000 \\
\hline 434.150 & - & 6.870 & 64.000 & 64.564 & 0.882 & 0.000 \\
\hline 500.300 & - & 6.870 & 73.300 & 75.040 & 2.373 & 0.000 \\
\hline
\end{tabular}

Comparisons based on experimental densities:

Number of Points [88] 24

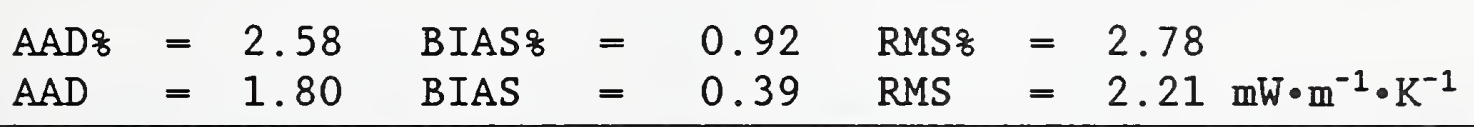

Comparisons based on experimental pressures

Overal1 Results:

$$
\begin{aligned}
& \mathrm{N}=1498 \quad \mathrm{AAD} \%=1.59 \quad \mathrm{BIAS} \%=-0.44 \quad \mathrm{RMS} \%=2.18 \\
& \mathrm{AAD}=1.09 \text { BIAS }=-0.22 \quad \mathrm{RMS}=1.73 \quad \mathrm{~mW} \cdot \mathrm{m}^{-1} \cdot \mathrm{K}^{-1}
\end{aligned}
$$

Weighted Data:

$$
\begin{aligned}
& \mathrm{N}=1098 \quad \mathrm{AAD} 8=1.21 \quad \text { BIAS } 8=-0.50 \quad \text { RMS } 8=1.53 \\
& \mathrm{AAD}=0.84 \text { BIAS }=-0.35 \quad \mathrm{RMS}=1.10 \mathrm{~mW} \cdot \mathrm{m}^{-1} \cdot \mathrm{K}^{-1}
\end{aligned}
$$

Comparisons based on experimental pressures (excluding Keyes, Lenoir, Millat, and Mostert; also experimental densities are used for Tufeu):

Overa11 Results:

$$
\begin{aligned}
& \mathrm{N}=1357 \quad \mathrm{AAD} \%=1.47 \quad \text { BIAS\& }=-0.33 \quad \text { RMS } 8=2.02 \\
& \mathrm{AAD}=1.06 \text { BIAS }=-0.16 \quad \mathrm{RMS}=1.65 \mathrm{~mW} \cdot \mathrm{m}^{-1} \cdot \mathrm{K}^{-1}
\end{aligned}
$$

Comparisons based on experimental densities

Overall Results:

$$
\begin{aligned}
& \mathrm{N}=1471 \quad \mathrm{AAD} \%=1.71 \quad \text { BIAS } 8=-0.69 \quad \text { RMS } 8=2.56 \\
& \mathrm{AAD}=1.34 \text { BIAS }=-0.62 \quad \mathrm{RMS}=2.88 \mathrm{~mW} \cdot \mathrm{m}^{-1} \cdot \mathrm{K}^{-1}
\end{aligned}
$$

Weighted Data:

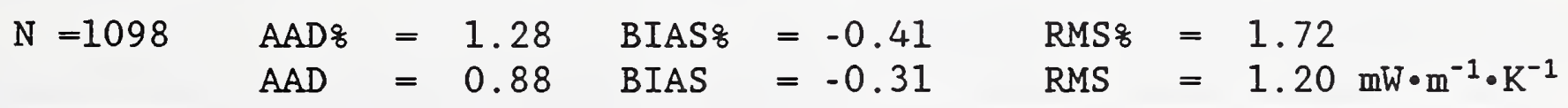


We gratefully acknowledge support from the Office of Standard Reference Data of the National Institute of Standards and Technology. We thank B.S. Coultrip and M. Nguyen for assistance with data compilation.

7. References

[1] D.G. Friend, H. Ingham, J.F. Ely, Thermophysical Properties of Ethane, J. Phys. Chem. Ref. Data 20(2), 275-8 (1991).

[2] D.G. Friend, J.F. Ely, H. Ingham, Tables for the Thermophysical Properties of Ethane, Nat. Inst. Stand. Technol., Tech. Note 1346 (1990).

[3] D.G. Friend, J.F. E1y, H. Ingham, J. Phys. Chem. Ref. Data 18, 583 (1989).

[4] D.G. Friend, J.F. Ely, H. Ingham, Tables for the Thermophysical Properties of Methane, Nat. Inst. of Stand. Techno1., Tech. Note 1325 (1989).

[5] C.H. Barkelew, J.L. Valentine, C.O. Hurd, Trans. Amer. Inst. Chem. Eng. 43,25 (1947).

[6] G.F. Carruth and R. Kobayashi, J. Chem. Eng. Data 18, 115 (1973).

[7] C.H. Chui and F.B. Canfield, Trans. Faraday Soc. 67, 2933 (1971); M.Y. Shana'a and F.B. Canfield, Trans. Faraday Soc. 64, 2281 (1968).

[8] L. Djordjevich and R.A. Budenholzer, J. Chem. Eng. Data 15, 10 (1970).

[9] D.R. Douslin and R.H. Harrison, J. Chem. Thermodynamics 5, 491 (1973).

[10] L.C. Kahre, J. Chem. Eng. Data 18, 267 (1973).

[11] A.G. Loomis and J.E. Walters, J. Amer. Chem. Soc. 48, 2051 (1926).

[12] V.M. Miniovich and G.A. Sorina, Russ. J. Phys. Chem. 45, 306 (1971).

[13] A.K. Pal, G.A. Pope, Y. Arai, N.F. Carnahan, and R. Kobayashi, J. Chem. Eng. Data 21, 394 (1976).

[14] G.A. Pope, Ph.D. Thesis (Dept. Chem. Eng.), Rice Univ. (1971).

[15] F. Porter, J. Amer. Chem. Soc. 48, 2055 (1926).

[16] J. Regnier, J. Chimie Physique 69, 942 (1972).

[17] W.T. Ziegler, B.S. Kirk, J.C. Mullins, and A.R. Berquist, Tech. Report No. 2, Proj. A-764, Eng. Exp. Sta., Georgia Inst. Tech., Atlanta, GA (Dec. 1964). 
[18] A.W. Tickner and F.P. Lossing, J. Phys. Colloid Chem. 55, 733 (1951).

[19] G.C. Straty and R. Tsumura, Nat. Bur. Stand. J. Research (U.S) 80A, 35 (1976).

[20] R.D. Goodwin, H.M. Roder, and G.C. Straty, Nat. Bur. Stand. (U.S.), Technical Note 684 (1976).

[21] R.J. Gugnoni, J.W. Eldridge, V.C. Okay, and T.J. Lee, AIChE J. 20, 358 (1974).

[22] W.M. Haynes and M.J. Hiza, J. Chem. Thermodyn. 9, 179 (1977).

[23] J. Klosek and C. McKinley, Proc. Ist Int1. Conf. on LNG, Paper 22, Chicago (1968).

[24] C.R. McClune, Cryogenics 16, 289 (1976).

[25] J.E. Orrit and J.M. Laupretre, Adv. Cryo. Eng. 23, 573 (1978); J.E. Orrit and J.F. Olives, Density of Liquefied Natural Gas and its Components, 4 th Intl. Conf. LNG, Algeria (1974).

[26] M.W. Pestak, R.E. Goldstein, M.H.W. Chan, J.R. de Bruyn, and D.A. Balzarini, and N.W. Ashcroft, Phys. Rev. B 36, 599 (1987).

[27] J.B. Rodosevich and R.C. Miller, AIChE J. 19, 729 (1973).

[28] P. Sliwinski, Z. Phys. Chem. (Neue Folge) 63, 263 (1969).

[29] J.R. Tomlinson, Natural Gas Processors Assoc. Tech. Publ. TP-1, Tulsa, OK (1971).

[30] F. Porter, J. Am. Chem. Soc. 48, 2055 (1926).

[31] J. Chao, R.C. Wilhoit, and B.J. Zwolinski, J. Phys. Chem. Ref. Data 2, 427 (1973).

[32] K.M Pamidimukkala, D. Rogers, and G.B. Skinner, J. Phys. Chem. Ref. Data 11,83 (1982).

[33] TRC Thermodynamic Tables-Hydrocarbons, Thermodynamics Research Center, Texas A\&M Univ., College Station, TX (Oct. 31, 1985).

[34] K. Bier, J. Kunze, and G. Maurer, J. Chem. Thermodynamics 8, 857 (1976); K. Bier, J. Kunze, and G. Maurer, and H. Sand, J. Chem. Eng. Data 21,5 (1976).

[35] A. Furtado, Ph.D. Thesis (Dept. Chem. Eng.), University of Michigan, Ann Arbor, MI (1973). 
[36] J.H. Dymond and E.B. Smith, The Virial Coefficients of Pure Gases and Mixtures, (Clarendon Press, Oxford, 1980).

[37] A. Eucken and A. Parts, Z. Phys. Chem. B20, 184 (1933).

[38] H.E. Tester in Thermodynamic Functions of Gases, Vo1. 3, F. Din, ed., (Butterworths Scientific Publications, London, 1961).

[39] R.D. Gunn, M.S. Thesis, Univ. Calif. (Berkeley) 1958.

[40] J.A. Huff and T.M. Reed III, J. Chem. Eng. Data, 8, 306 (1963).

[41] S.D. Hamann and W.J. McManamey, Trans. Faraday Soc. 49, 149 (1953).

[42] H.H. Reamer, R.H. Olds, B.H. Sage, and W.N. Lacey, Ind. Eng. Chem. 36, 957 (1944).

[43] A.E. Hoover, I. Nagata, T.W. Leland, and R. Kobayashi, J. Chem. Phys. 48, 2633 (1968).

[44] J.D. Lambert, G.A.H. Roberts, J.S. Rowlinson, and V.J. Wilkinson, Proc. R. Soc. London A196, 113 (1949).

[45] H. Mansoorian, K.R. Hall, and P.T. Eubank, Proc. 7th Symp. on Thermophysical Properties, A. Cezairliyan, ed., (Amer. Soc. Mech. Eng., 1977) p. 456.

[46] M.L. McGlashan and D.J. Potter, Proc. R. Soc. London A267, 478 (1962).

[47] A. Michels, W. Van Straaten, and J. Dawson, Physica 20, 17 (1954).

[48] V.K. Strein, R.N. Lichtenthaler, B. Schramm, and Kl. Schäfer, Ber. Bunsenges. Phys. Chem. 75, 1308 (1971).

[49] J.A. Beattie, C. Hadlock, N. Poffenberger, J. Chem. Phys. 3, 93 (1935).

[50] G.J. Besserer and D.B. Robinson, J. Chem. Eng. Data 18, 137 (1973).

[51] Ye.A. Golovskiy, E.P. Mitsevich, V.A. Tsymarnyy, Measurement of the density of ethane at 90.24-270.21 $\mathrm{K}$ and at pressures to 604.09 bar, VNIIEGazprom Dep. No. 39M USSR (1978); data have been tabulated in [52].

[52] V.V. Sychev, A.A. Vasserman, A.D. Kozlov, V.A. Zagoruchenko, G.A. Spiridonov, and V.A. Tsymarny, Thermodynamic Properties of Ethane, (Hemisphere Publishing, Washington, DC 1987), T.B. Selover Jr., ed., English Language Edition.

[53] N.E. Khazanova and E.E. Sominskaya, Russ. J. Phys. Chem. 45, 87 (1971). 
[54] W.W.R. Law, A continuously weighed pycnometer providing densities for $\mathrm{CO}_{2}$ + Ethane mixtures between 240 and $350 \mathrm{~K}$ at pressures to $35 \mathrm{MPa}$, Ph.D. Thesis, Texas A\&M Univ. (1986).

[55] W.R. Parrish, Fluid Phase Equil. 18, 279 (1984).

[56] J.V. Sengers, personal communication, Univ. Maryland, Inst. Phys. Sci. Tech., College Park, MD, 1988.

[57] C.B. Wallace Jr., I.H. Silberberg, J.J. McKetta, Petrol. Refiner, Hydrocarbon Processing 43 No. 10, 177 (1964).

[58] H.M. Roder, Nat. Bur. Stand. J. Research 80A, 739 (1976).

[59] T. Miyazaki, A.V. Hejmaki, and J.E. Powers, J. Chem. Thermo. 12, 105 (1980).

[60] R. Wiebe, K.H. Hubbard, and M.J. Brevoort, J. Am. Chem. Soc. 52, 611 (1930).

[61] R.K. Witt and J.D. Kemp, J. Am. Chem. Soc. 59, 273 (1937).

[62] V.E. Terres, W. Jahn, and H. Reissmann, Brennstoff-Chemie 38, 129 (1957).

[63] R. Tsumura and G.C. Straty, Cryogenics 17, 195 (1977).

[64] G.R. Poole and R.A. Aziz, Can. J. Phys. 50, 721 (1972).

[65] E. Vangeel, Katholieke Universiteit, Leuven, Belgium, private communication to D. Diller, Natl. Inst. Stand. Technol., Boulder, CO (1974); data were tabulated in Table 17 of [20].

[66] Y. Abe, J. Kestin, and H.E. Khalifa, Physica 93A, 155 (1978).

[67] H. Adzumi, Bul1. Chem. Soc. Japan 12, 199 (1937).

[68] L.T. Carmichael and B.H. Sage, J. Chem. Eng. Data 8, 94 (1963).

[69] P.M. Craven and J.D. Lambert, Proc. R. Soc. London 205A, 439 (1951).

[70] A.G. De Rocco and J.O. Halford, J. Chem. Phys. 28(6), 1152 (1958).

[71] E. Eakin, K.E. Starling, J.P. Dolan, and R.T. Ellington, J. Chem. Eng. Data 7, 33 (1962).

[72] R. Fleeter, J. Kestin, and W.A. Wakeham, Physica 103A, 521 (1980).

[73] H. Iwasaki and M. Takahashi, J. Chem. Phys. 74, 1930 (1981).

[74] J. Kestin, S.T. Ro, and W.A. Wakeham, Trans. Faraday Soc. 67, 2308 (1971). 
[75] J. Kestin, H.E. Khalifa, and W.A. Wakeham, J. Chem. Phys. 66(3), 1132 (1977).

[76] J.D. Lambert, K.J. Cotton, M.W. Pailthorpe, A.M. Robinson, J. Scrivins, W.R.F. Vale, and R.M. Young, Proc. R. Soc. Faraday 231A, 280 (1955).

[77] V.M. Trautz and K.G. Sorg, Ann. Phys. 10, 81 (1931).

[78] H. Voge1, Ann. Phy. 43, 1235 (1914).

[79] A.A. Clifford, E. Dickinson, and P. Gray, J. Chem. Soc. London, Faraday Trans. I72, 1997 (1976).

[80] T.F. Gilmore and E.W. Comings, AIChE J. 12, 1172 (1966).

[81] F.G. Keyes, Trans. ASME, 809 (1954).

[82] B. Le Neindre, R. Tufeu, P. Bury, P. Jahannin, and B. Vodar, in Proc. 8th Int. Conf. Therm. Conduct., C.Y. Ho and R.E. Taylor, eds. (Plenum, NY, 1969), p. 229.

[83] D.E. Leng and E.W. Comings, Ind. Eng. Chem. 49, 2042 (1957).

[84] J.M. Lenoir, W.A. Junk, and E.W. Comings, Chem. Eng. Progress 49(10), 539 (1953).

[85] J. Millat, M. Ross, W.A. Wakeham, and M. Zalaf, Int. J. Thermophys. 9(4), 481 (1988).

[86] R.C. Prasad and J.E. Venart, Int. J. Thermophys. 5, 367 (1984).

[87] H.M. Roder and C.A. Nieto de Castro, High Temp.-High Pressures 17, 453 (1985).

[88] R. Tufeu, Y. Garrabos, and B. Le Neindre, in Proc. 16th Conf. Therm. Conduct., D.C. Larsen, ed. (Plenum, NY, 1983), p. 605.

[89] L.V. Yakush, N.A. Vanicheva, and L.S. Zaitseva, Inz.-Fiz. Zhurna1, 37, 1071 (1979); trans. in J. Eng. Phys. 37, 1071 (1979).

[90] J.D. Baron, J.G. Roof, and F.W. Wells, J. Chem. Eng. Data 4, 283 (1959).

[91] D.E. Diller and J.M. Saber, Physica 108A, 143 (1981); and D.E. Diller, Proc. 8th Symp. on Thermophysical Properties, J.V. Sengers, ed., (Amer. Soc. Mech. Eng., NY 1982), p. 219.

[92] D.E. Diller, unpublished data, Nat. Inst. Stand. Technol., Boulder, Co.

[93] S.F. Gerf and G.I. Galkov, Zh. Tekh. Fiz. 10(9), 725 (1940).

[94] S.F. Gerf and G.I. Galkov, Zh. Tekh. Fiz. 11(9), 801 (1941). 



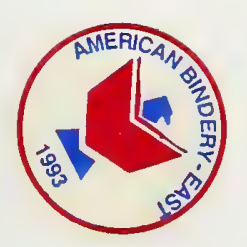


